\title{
Markus Reuther
}

\section{Forschungssteuerung zwischen Staat und Wissenschaft}

\author{
Ein Fallbeispiel \\ aus der bayerischen \\ Waldschadensforschung
}




\title{
Forschungssteuerung zwischen Staat und Wissenschaft

\author{
Ein Fallbeispiel aus der \\ bayerischen Waldschadensforschung
}

\begin{abstract}
Dissertation zur Erlangung des Doktorgrades an der Fakultät für Forstwissenschaften und Waldökologie der Georg-August-Universität Göttingen
\end{abstract}

\author{
vorgelegt von \\ Markus Reuther
}

geboren in

Hof a.d. Saale (Bayern)

Göttingen, 1999 

D 7

1. Berichterstatter: Prof. Dr. Krott

2. Berichterstatter: $\quad$ Prof. Dr. Möhring

Tag der mündlichen Prüfung: 16.4.1999

Die vorliegende Arbeit ist in Buchform unter der ISBN 3-89712-511-0 im

Cuvillier Verlag Göttingen veröffentlicht.

Dienstliche Anschrift des Verfassers:

Markus Reuther

Lehrstuhl für Bodenkunde und Standortslehre der

Ludwig-Maximilians-Universität München

Am Hochanger 13, D-85354 Freising

Tel: 08161/71-4674; Fax: -4738

E-mail: reuther@boku.forst.uni-muenchen.de 


\section{Vorwort und Danksagung}

Die vorliegende Arbeit beschäftigt sich mit einem Projekt des Bayerischen Umweltministeriums zur Koordinierung und Verstärkung der Waldschadensforschung in den Jahren 1984 bis 1993. Ihr Autor gehörte dem Projekt in den ersten Jahren als wissenschaftlicher Mitarbeiter, anschlieBend als Leiter der ,Projektgruppe Bayern zur Erforschung der Wirkung von Umweltschadstoffen (PBWU)“ an der ehemaligen Gesellschaft für Strahlen- und Umweltforschung, heute GSF-Forschungszentrum für Umwelt und Gesundheit GmbH, Neuherberg, an.

Die 9 Jahre hautnah erlebter Projektdurchführung inmitten des Spannungsbogens zwischen politischen und wissenschaftlichen Interessen zu dem damals gesellschaftlich hochaktuellen Thema „Waldsterben“ drängten den Autor förmlich zu einer wissenschaftlichen Aufarbeitung. Dies führte zu einem ersten Gespräch mit dem damaligen Inhaber des Lehrstuhls für Forstpolitik und Forstgeschichte an der Forstwissenschaftlichen Fakultät der LMU München in Freising, Herrn Prof. K.-R. Volz. Die ermutigenden Worte und der konstruktive Verweis auf seinen Kollegen, den künftigen Betreuer der Arbeit, Herrn Prof. M. Krott, gaben der Arbeit erst die entsprechende Ausrichtung. Nicht die naturwissenschaftlichen Ergebnisse der Waldschadensforschung, sondern der zugrundeliegende Forschungsprozeß und dessen Steuerung sind Schwerpunkt der 1994 begonnenen Arbeit.

Als besondere Vorteile werden dabei gesehen, daß der Autor als ehemaliger „Bestandteil“ der Projektgruppe detaillierte Kenntnisse über das Projekt verfügt, und ihm vom GSF-Forschungszentrum sowie dem Bayerischen Umweltministerium alle Dokumente aus der 9 jährigen Laufzeit des PBWU-Projektes zur Verfügung gestellt wurden. Weiterhin bestehen auch über die Projektlaufzeit hinaus gute Kontakte zu beteiligten Wissenschaftlern und Verwaltungsangehörigen, die das umfangreiche Datenmaterial durch weitere Dokumente und persönliche Erlebniswerte ergänzten.

Bedauerlicherweise - aus Sicht ihres Verfassers - mußte die Arbeit in einer fachfremden Umgebung durchgeführt werden. Sie entstand außerdienstlich neben meiner beruflichen Betätigung als Projektkoordinator zu naturwissenschaftlichen Forschungsvorhaben - wenn auch im weiteren Sinne zum Thema „Waldschäden“ - an der Bayerischen Landesanstalt für Wald und Forstwirtschaft und anschließend am Lehrstuhl für Bodenkunde und Standortslehre der Forstwissenschaftlichen Fakultät in Freising. Um so mehr bin ich meinem beruflichen und privaten Umfeld, welches unter den gegebenen Umständen der Durchführung der Arbeit nicht nur zuließ, sondern sogar förderte, und den fachlichen Beratern und Begleitern der Arbeit, zu Dank verpflichtet.

Dies betrifft an erster Stelle den Promotor und Betreuer der Arbeit, Herrn Prof. Max Krott, Leiter des Instituts für Forstpolitik, Forstgeschichte und Naturschutz an der Universität Göttingen. Er nahm sich trotz räumlicher Distanz zwischen Göttingen, ehemals Wien, und Ismaning/Freising der Betreuung meiner Arbeit umfassend, zeit- und arbeitsintensiv, bis ins wissenschaftlich-theoretische wie redaktionelle Detail und immer motivierend an. Mein Dank gebührt auch den Präsidenten der Bayerischen Landesanstalt für Wald und Forstwirtschaft, vormals 
Herrn Dr. R. Holzapfl und anschließend Herrn Dr. G. Braun, sowie dem Inhaber des Lehrstuhls für Bodenkunde und Standortslehre, Herrn Prof. K.E. Rehfuess. Sie brachten im Rahmen meiner dortigen Beschäftigungsverhältnisse nicht nur Verständnis für meine außerdienstliche Betätigung auf, sondern unterstützten diese auch mit Zuspruch und fachlich-thematischer Diskussion. Die Nähe zum Lehrstuhl für Forstpolitik und Forstgeschichte an der Forstwissenschaftlichen Fakultät, zu dessen Leiter, Prof. M. Suda, zu Prof. E. Gundermann und Forstrat R. Beck, gestattete mir dankenswerterweise, fachspezifische Anregungen oder Literatur über kurze Wege zu akquirieren und eine am Lehrstuhl durchgeführten Diplomarbeit zum Thema zu betreuen.

Mein besonderer Dank gilt auch dem damaligen Vorstand und Mitarbeitern des Instituts für Wissenschaftstheorie und Wissenschaftsforschung der Universität Wien, Frau Prof. H. Nowotny, Frau Dr. U. Felt und Herrn K. Taschwer. Ihre freundliche Unterstützung erleichterte mir den theoretischen Zugang zur Arbeit.

Die mit dem PBWU-Projekt vertrauten Herren Professoren K.E. Rehfuess, E.-D. Schulze und Emeritus H. Ziegler waren gerne bereit, mir über mündliche Information oder schriftliche Dokumente Hinter- oder Vordergründiges aus ihrer Sicht zu erhellen. Gleichermaßen danken möchte ich Herrn Regierungsdirektor K. Weißgerber als stets wohlwollendem Ansprechpartner am Bayerischen Umweltministerium. Er und Herr Dr. F. Perzl, der im Jahr 1996 leider viel zu früh verstorbene Prokurist und Leiter der Wissenschaftlich-Technischen Abteilung des GSFForschungszentrums, gleichzeitig eine Art „Vaterfigur“ für die damalige PBWU-Projektgruppe, unterstützten die Arbeit in Form von Diskussionen, Rekonstruktionen und durch die Überlassung von Unterlagen.

Wesentliche Unterstützung und Zuarbeit fand ich durch die quantitative Analyse der PBWUForschungsförderung im Rahmen der Diplomarbeit von Diplom-Forstwirt D. Steuer. Für die Bearbeitung und Auswertung des umfangreichen Materials an Forschungsanträgen, Gutachten und Forschungsberichten sei ihm herzlich gedankt. Hinsichtlich der Recherchen zur "Entdeckung des Waldsterbens", welche in der vorliegenden Arbeit allerdings nurmehr am Rande berücksichtigt wird, möchte ich Herrn emerit. Prof. O. Kandler, LMU München, und Herrn Prof. W. Elling, FH Weihenstephan, für die informativ-kritischen Gespräche und die Unterstützung bei Literaturrecherchen danken. Eine weitere Bereicherung waren die Diskussionen mit der Diplom-Soziologin K. Dressel, die ihrerseits im Rahmen einer Diplomarbeit den Waldschadensdiskurs wissenssoziologisch analysierte und mich als einen ihrer Interviewpartner auswählte.

Abschließend möchte ich die „Anteilnahme“ meiner Familie an der vorliegenden Arbeit würdigen. Da diese außerhalb meiner beruflichen Tätigkeit und damit in der Freizeit durchgeführt wurde, waren zwangsläufig erhebliche Belastungen des Familienlebens die Folge. Daß meine Frau Maria trotz familiärer Beeinträchtigung und eigenen beruflichen Engagements mich fortwährend unterstützte, motivierte und mir über Längen und Durststrecken hinweghalf, unsere beiden Kinder Kristof und Lukas in dieser Zeit geboren wurden und ihre ersten Lebensjahre absolvieren mußten, legt nahe, den Dreien die Arbeit mit herzlichem Dank zu widmen. 
Ismaning bei München, Januar 1999 


\section{Inhaltsverzeichnis}

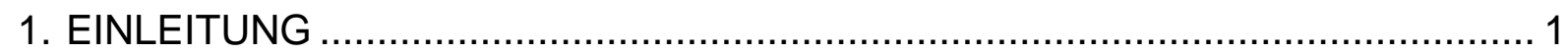

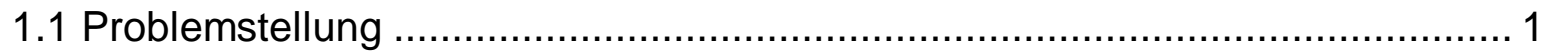

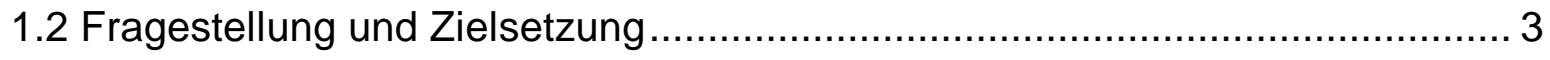

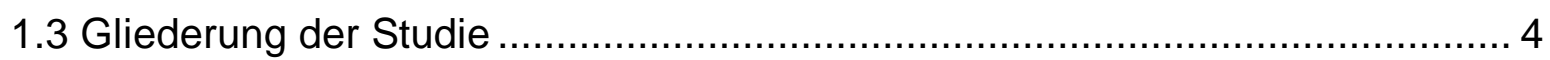

2. THEORIE

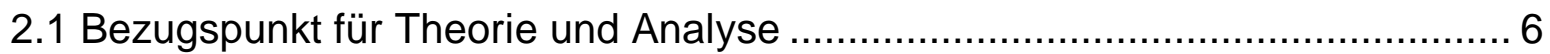

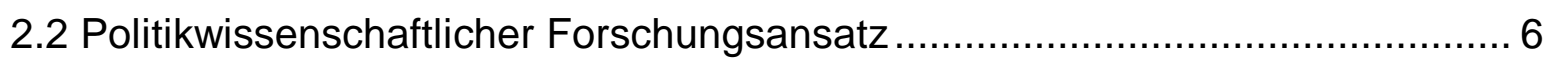

2.3 Theoretische Grundlagen zur Politikfeld-Analyse ...................................... 7

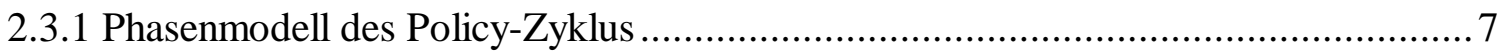

2.3.1.1 Problemdefinition und Agendagestaltung............................................................. 9

2.3.1.2 Politik-(Programm-)formulierung......................................................................10

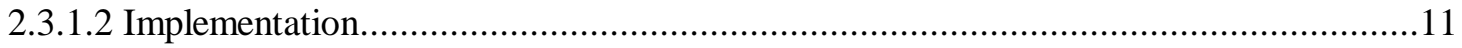

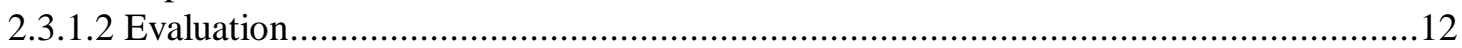

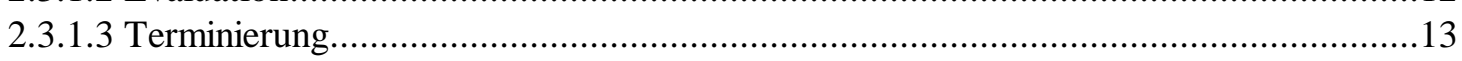

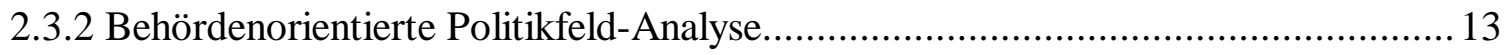

2.3.3 Kritische Bewertung der Politikfeld-Analyse......................................................... 14

2.3.4 Verschachtelung verschiedener Policies (Programmschachteln) ............................ 16

2.4 Organisations- und wissenschaftssoziologische Theorie ........................... 19

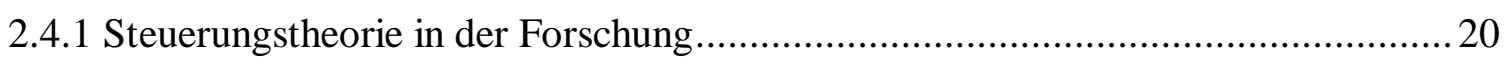

2.4.2 Thesen zur Steuerung mittels intermediärer Systeme …………………............... 21

2.4.3 Angewandte Forschung versus Grundlagenforschung .........................................2 22

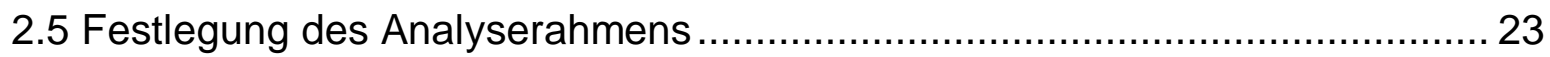

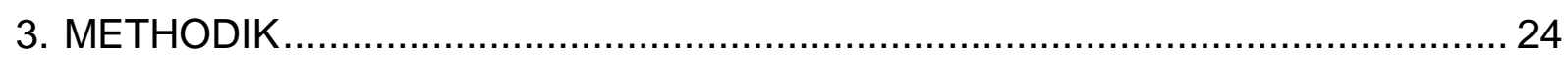

3.1 Retrospektive teilnehmende Beobachtung................................................. 24

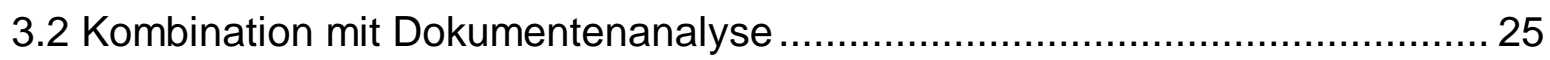

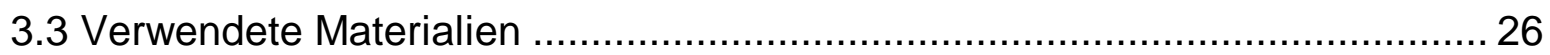

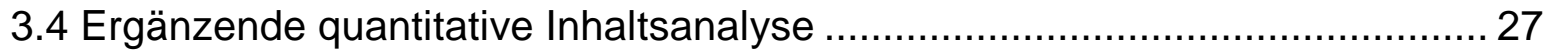

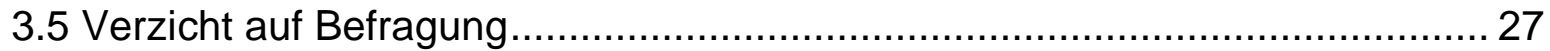

3.6 Methodische Kontrollinstrumente und Anonymisierung personen-

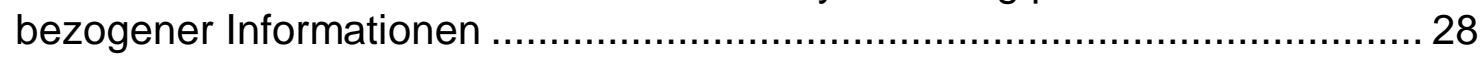

3.7 Forschungsansatz Fallstudie .......................................................... 28 


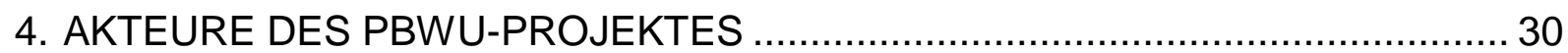

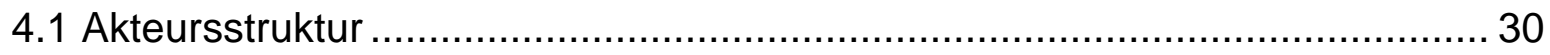

4.2 Charakterisierung der einzelnen Akteure ................................................ 31

4.2.1 Projektgruppe Bayern zur Erforschung der Wirkung von

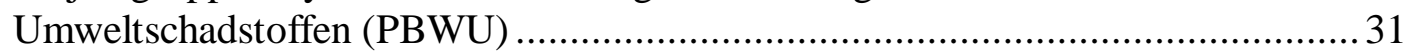

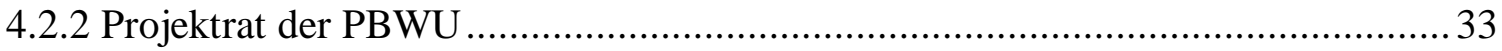

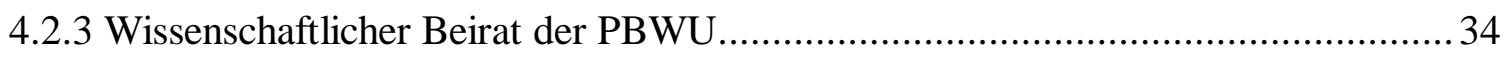

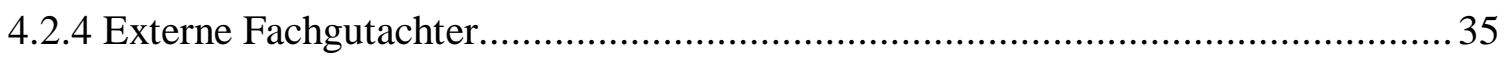

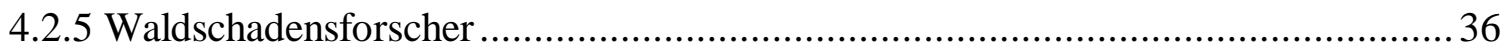

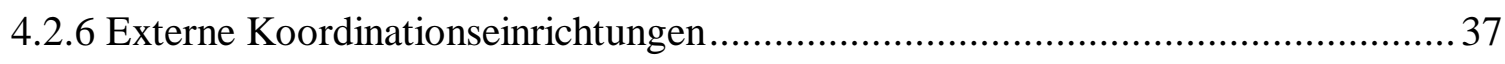

4.3 Theorieorientiertes Zwischenergebnis .................................................. 38

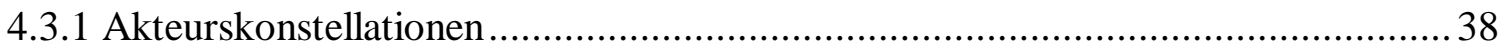

4.3.2 Akteursspezifische Steuerungskonflikte............................................................ 40

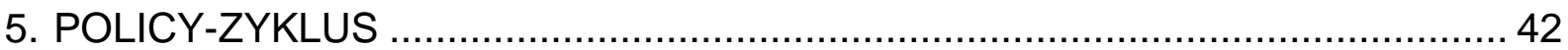

5.1 Gründung des PBWU-Projektes............................................................ 42

5.1.1 Thematisierung der Waldschadensforschung (Problemdefinition) ........................ 42

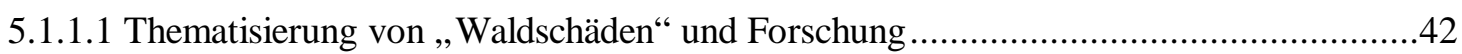

5.1.1.2 Anfängliche Struktur der bayerischen Waldschadensforschung ..................................44

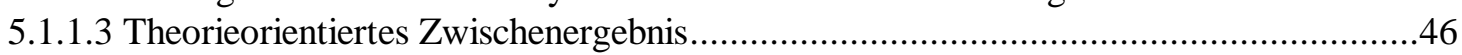

5.1.2 Politische Behandlung der Forschungskoordination und Beschluß zur

PBWU-Gründung (Agendagestaltung) ......................................................... 48

5.1.2.1 Waldschadensforschung auf der politischen Tagesordnung .....................................48

5.1.2.2 Bund/Länder-Bemühungen zur Koordinierung der Waldschadensforschung .................50

5.1.2.3 Bayerischer Vorschlag zur Bildung einer bundesweiten Koordinierungsstelle ...............52

5.1.2.4 Beschluß zur Schaffung einer Koordinierungsstelle für die bayerische

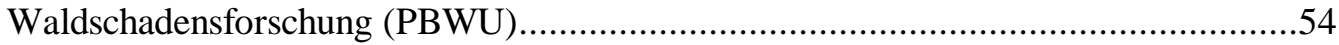

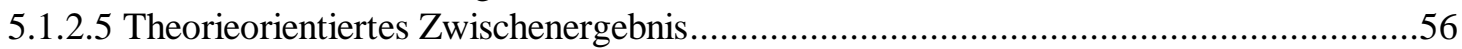

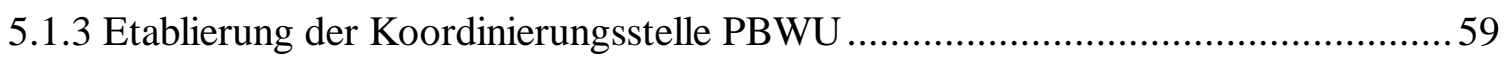

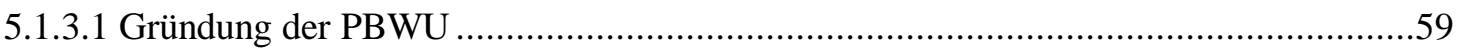

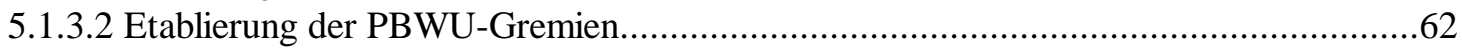

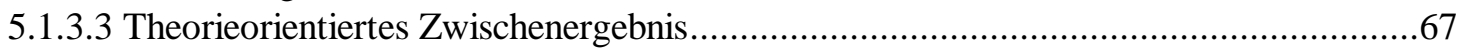

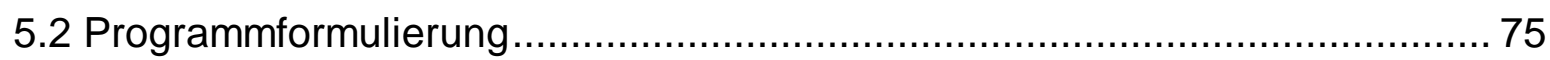

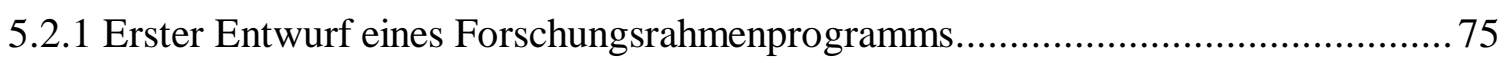

5.2.2 Kritische Diskussion des Rahmenprogramm-Entwurfs in den PBWU-Gremien ..... 76

5.2.3 Beschluß und Ausschreibung des PBWU-Rahmenprogramms ............................. 78

5.2.4 Theorieorientiertes Zwischenergebnis ........................................................... 79

5.2.4.1 Einbindung bestehender Forschungsprogramme als Strategie....................................79

5.2.4.2 Ressortübergreifender Koordinationsanspruch der Projektgruppe ..............................80

5.2.4.3 Forschungssteuerung: Top down- versus bottom up-Ansatz.....................................82 


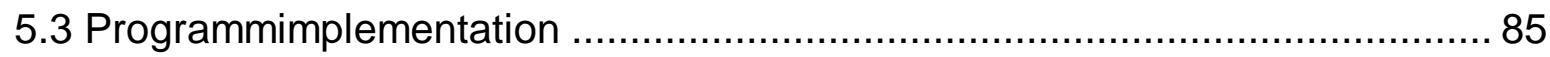

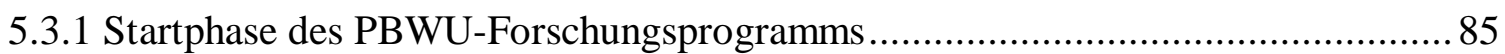

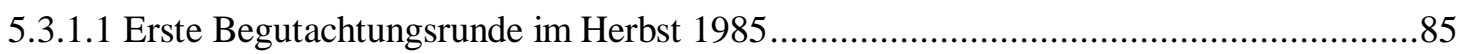

5.3.1.2 Zweite Begutachtungsrunde im Frühjahr 1986 ........................................................91

5.3.1.3 Vertrauenskrise im Wissenschaftlichen Beirat ..........................................................94

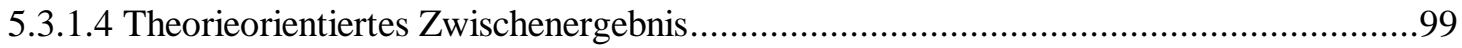

5.3.2 Weitere Programmimplementation und Projekt-Neuordnung ............................ 102

5.3.2.1 Förderverfahren zu Forschungsanträgen des Jahres 1987.......................................103

5.3.2.2 Förderverfahren zu Forschungsanträgen des Jahres 1988_.......................................106

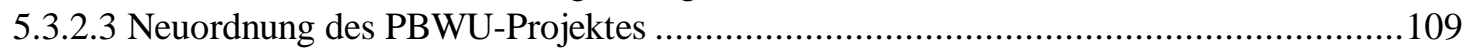

5.3.2.4 Förderverfahren im PBWU-Projekt nach dessen Neuordnung .................................113

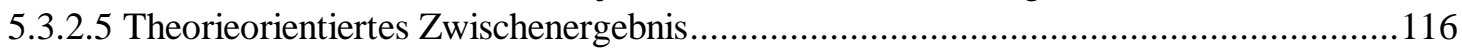

5.3.3 Programmimplementation am alpinen Forschungsschwerpunkt Wank ................. 123

5.3.3.1 Etablierung des Forschungsschwerpunktes „Wank“ ................................................123

5.3.3.2 Wissenschaftliches Steuerungskomitee für den Wank .............................................128

5.3.3.3 Kritik externer Wissenschaftler an der Wank-Forschung .........................................132

5.3.3.4 Weitere Behandlung des alpinen Forschungskonzepts .............................................134

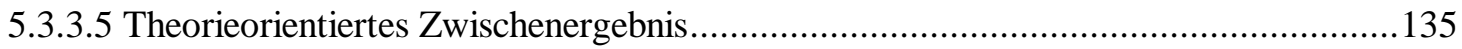

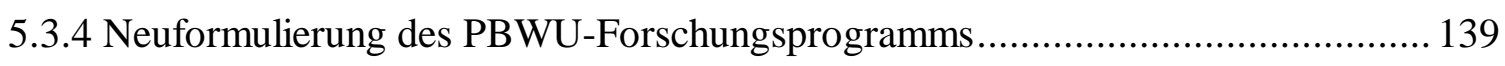

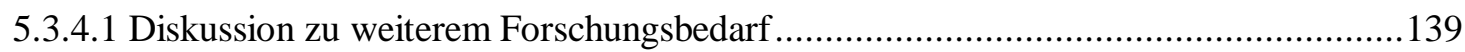

5.3.4.2 Entwicklung eines Forschungskonzeptes zur künftigen Waldschadens-

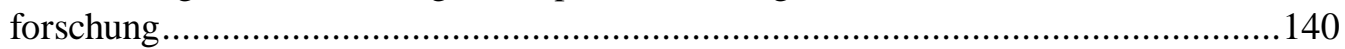

5.3.4.3 Vorstellung des Forschungskonzepts gegenüber dem Bayerischen

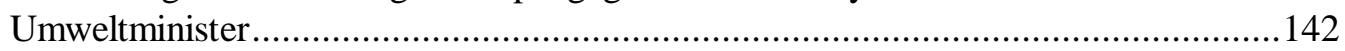

5.3.4.4 Vorstudien zur Umsetzung des Forschungskonzeptes .............................................143

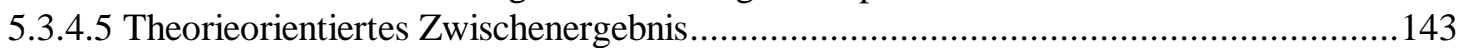

5.3.5 Förder- und Begutachtungsverfahren im PBWU-Projekt ................................. 146

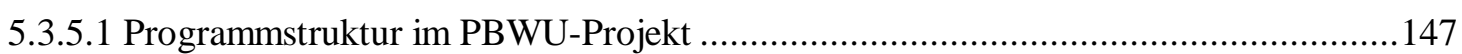

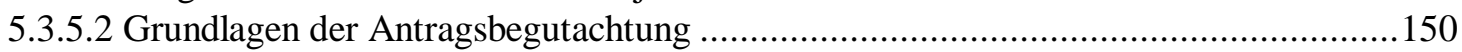

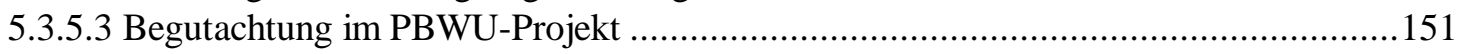

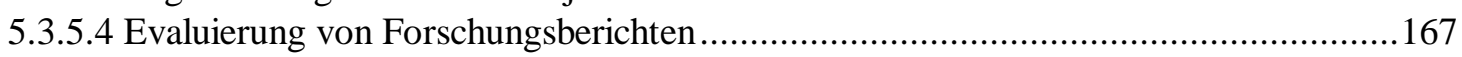

5.4 Terminierung und Evaluierung des PBWU-Projekts ................................ 170

5.4.1 Einschätzung der Waldschadensforschung durch das Bayerische

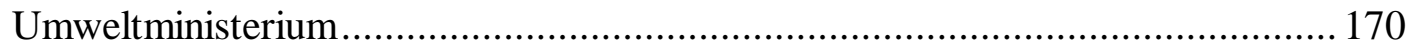

5.4.2 Landtagsanhörung zur Waldschadensforschung .......................................... 171

5.4.3 Ressortabstimmungsversuche zwischen Umwelt- und Landwirtschafts-

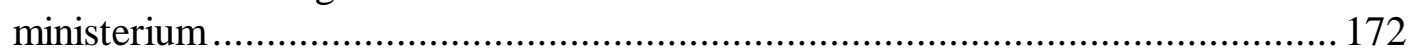

5.4.4 Einspar-Maßnahmen des Umweltministeriums in der Forschung ....................... 173

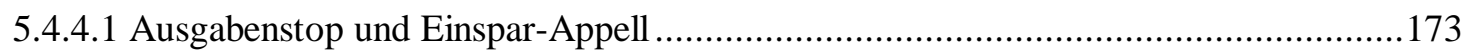

5.4.4.2 Stellungnahme des Wissenschaftlichen Beirats zu den Sparmaßnahmen....................173

5.4.4.3 Externe politische Unterstützung gegen die Sparmaßnahmen ...................................174

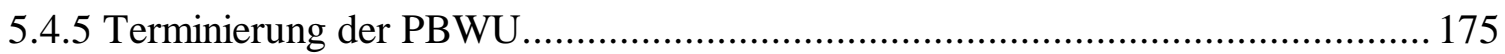

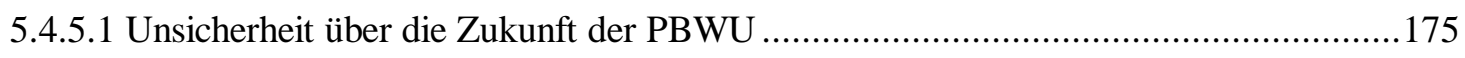

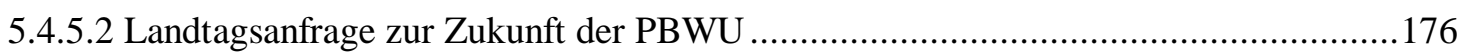

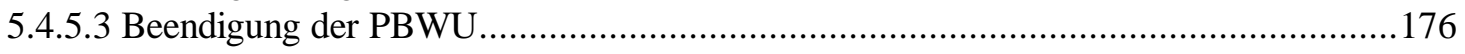




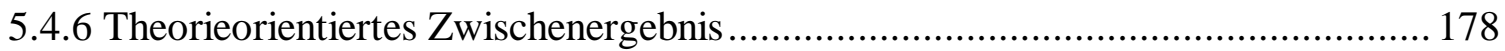

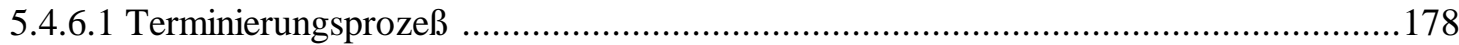

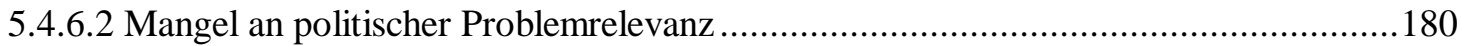

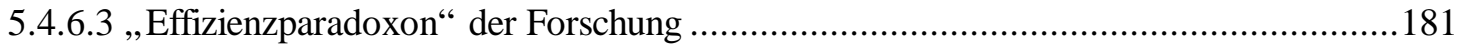

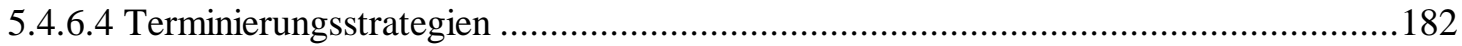

5.4.6.5 Nutzenentgang durch kurzfristige Programmterminierung ......................................184

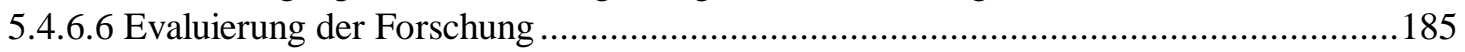

6. POLITISCHE FAKTOREN DER ENTWICKLUNG UND STEUERUNG DES

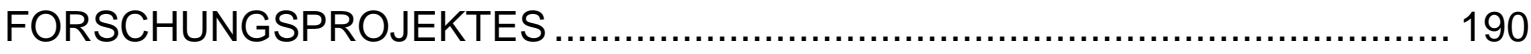

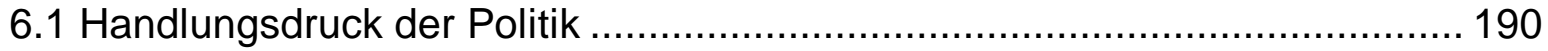

6.2 Vermengung politischer und fachlicher Beiträge der Akteure ..................... 191

6.3 Territorial-Verhalten als Hindernis für Koordination................................... 193

6.4 Politische Dominanz in der Etablierung und Terminierung der

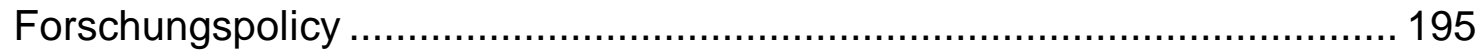

6.5 Wissenschaftliche Dominanz im Verlauf der Programmimplementation ....... 198

6.6 Externe Fachbegutachtung zur Sicherung der Forschungsqualität .............. 200

6.7 Koordinierungsstelle zwischen Programmsteuerung und Kooperation......... 203

6.8 Vorhabenbetreuung statt Forschungskoordination ...................................... 204

6.9 MangeInde Erfolgsbeurteilung der Forschungspolicy ................................ 206

7. EXKURS: EMPFEHLUNGEN ZU DURCHFÜHRUNG UND KOORDINATION

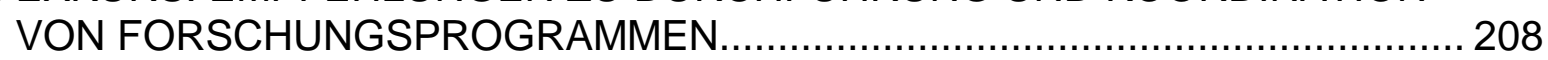

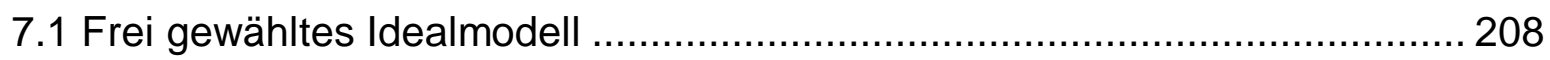

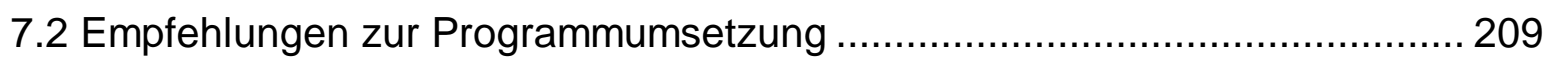

7.2.1 Politische Gestaltung zeitgerechter Forschung ………...................................... 209

7.2.2 Integration eigenständiger Forschungsfinanziers .............................................. 210

7.2.3 Trennung politischer und wissenschaftlicher Interessen bei der

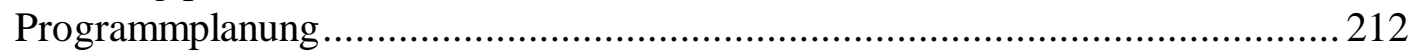

7.2.4 Verfahren der Programmerstellung und Forschungsakquisition ............................213

7.2.5 Kompetenz der Koordinierungsstelle zur Programmkoordination........................ 214

7.2.6 Ausgleich zwischen Programmvorgaben und Forschungsflexibilität......................215

7.2.7 Verfahren zur Begutachtung von Forschungsanträgen ...................................... 216

7.2.8 Kontrolle und Erfolgsbeurteilung der Forschung................................................. 218

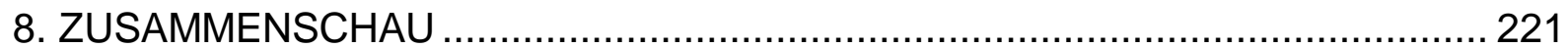

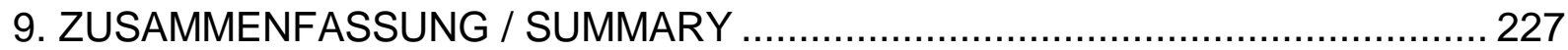


10. LITERATUR. 


\section{Abbildungsverzeichnis}

Abb. 1: $\quad$ Phasenmodell des Policy-Zyklus.................................................................... 8

Abb. 2: $\quad$ Elemente und Struktur der behördenorientierten Politikfeld-Analyse................ 14

Abb. 3: Programmverschachtelung der Waldschadensforschung ................................19

Abb. 4: $\quad$ Organisations- und Verfahrensstruktur im PBWU-Projekt................................31

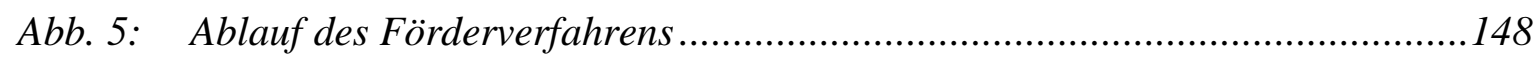

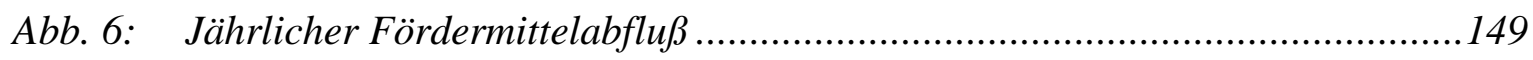

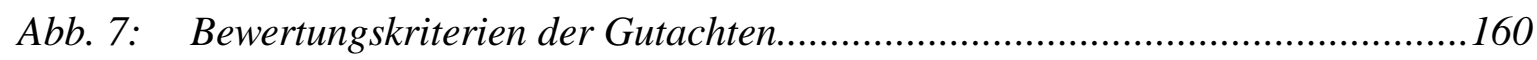

Abb. 8: Zusammenhang zwischen Forschungsqualität und Fördermitteln..................209

\section{Tabellenverzeichnis}

Tab. 1: "Programmschachteln" in der Phasenabfolge des Policy-Zyklus .................... 18

Tab. 2: Forschungsanträge und Antragsteller (Sommer 1985) ...................................86

Tab. 3: Forschungsanträge und Fachdisziplinen (Sommer 1985)................................86

Tab. 4: Forschungsanträge und Forschungsstandorte (Sommer 1985)........................87

Tab. 5: $\quad$ Forschungsanträge und Forschungseinrichtungen (Frühjahr 1986) .................91

Tab. 6: Forschungsanträge und Fachdisziplinen (Frühjahr 1986) .............................92

Tab. 7: $\quad$ Forschungsanträge und Standorte (Frühjahr 1986).......................................92

Tab. 8: Forschungsanträge und Forschungseinrichtungen (1987) .......................... 103

Tab. 9: Forschungsanträge und Fachdisziplinen (1987) ....................................... 104

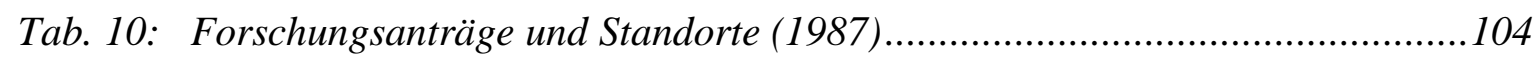

Tab. 11: Forschungsvorhaben und Forschungseinrichtungen .....................................150

Tab. 12: Forschungsvorhaben und Fachdisziplinen ..................................................150

Tab. 13: Forschungsvorhaben und Standorte............................................................. 150 


\title{
$1 \quad$ Einleitung
}

\author{
„Was bringt den Waldschadensforscher \\ um sein Brot? \\ Des Waldes Gesundheit oder sein Tod. \\ Drum hält der Forscher, auf daß er lebe, \\ ihn zwischen beiden in der Schwebe. " \\ (frei nach Eugen Roth)
}

\subsection{Problemstellung}

Das Thema „Waldsterben“ prägte so anhaltend wie kaum ein anderes Thema in den 80er Jahren die Umweltdiskussion in Deutschland und dem benachbarten Ausland. Es fällt in eine Zeit und Region mit bereits hoher Umweltsensibilität in Öffentlichkeit, Politik und Wissenschaft und betrifft mit dem Objekt Wald zudem ein Symbol hoher Wertschätzung. Daß am Wald, einem „existentiellen Kulturgut“ der Menschheit, Schäden beobachtet werden, muß daher besorgniserregend stimmen.

Je weniger zu Beginn das Ausmaß des Umweltproblems exakt beurteilt werden kann, desto mehr verbleiben Freiräume für - meist negative - Spekulationen. So kommt nicht nur in der von Medien stark beeinflußten Öffentlichkeit, sondern auch bei vielen Fachwissenschaftlern die Befürchtung auf, ,es könne in Form eines flächendeckenden Waldsterbens eine Umweltkatastrophe unvorstellbaren Ausmaßes bevorstehen“ [UMWELTBUNDESAMT 1996, S. 1]. Allein vor dem Hintergrund der gesellschaftlichen Umweltsensibilisierung einerseits, der bestehenden umweltpolitischen, wirtschaftlichen - nicht nur forstwirtschaftlicher - und wissenschaftlichen Interessen andererseits, verwundert es nicht, daß das „Waldsterben“ rasch zum Politikum wird.

Inzwischen ist es wieder „still geworden“ um den Wald, die Sorge um ihn wurde aus den öffentlichen Schlagzeilen verdrängt [MöHRING 1992, S. 6]. Auch heute noch, Ende der 90er Jahre, werden jährlich wiederkehrend und europaweit Waldschadenserhebungen durchgeführt und die Ergebnisse von der Politik der Öffentlichkeit präsentiert. Sie werden zwar überwiegend als Waldzustands- und nicht mehr als Waldschadensbericht bezeichnet, und die früheren pessimistischen Prognosen wurden aufgegeben. Nach wie vor wird jedoch nicht nur die Bundesrepublik Deutschland, sondern auch viele andere europäische Staaten als von neuartigen Waldschäden betroffen bezeichnet und von einer Gefährdung der Wälder gesprochen [z.B. BuNDESMINISTERIUM FÜR ERNÄHRUNG, LANDWIRTSCHAFT UND FORSTEN 1996, S. 3]. Die kritische Diskussion in der Wissenschaft um die Gefährdung der Wälder, die Existenz über das Normalmaß hinausgehender Schäden, die Eignung der verwendeten Schadenskriterien sowie die Rolle von Luftschadstoffen als Schadensursache hat sich inzwischen allerdings verstärkt [s. z.B. Ellenberg 1994, 1996 S. 89-102, SCHLAEPFER 1993, S. 87ff; SKELly \& INNES, 1994; KANDLER 1994, LANDMANN 1995; SPIEKER et al. 1996, BAYERISCHES STAATSMINISTERIUM FÜR ERNÄHRUNG, LANDWIRTSCHAFT UND FORSTEN 1995].

Dazwischen liegen nahezu 20 Jahre intensiver Waldschadensforschung. Die Politik jedenfalls bescheinigt der Wissenschaft - und sich selbst - den Erfolg: Der deutschen Forschung sei es in 
relativ kurzem Zeitraum gelungen, wesentliche Fragen der Ursachen-Wirkungsbeziehungen aufzuklären und damit Grundlagen für politische Entscheidungen erarbeitet $\mathrm{zu}$ haben [BUNDESMINISTERIUM FÜR ERNÄHRUNG, LANDWIRTSCHAFT UND FORSTEN 1996, S. 50].

Der Wissensstand zu Beginn der Diskussion reichte bei weitem nicht aus, den Erklärungsbedarf zu befriedigen, zudem das Problem „Waldsterben“ mitsamt dem zugrundeliegenden Objekt Wald alsbald als äußerst komplex und vielschichtig erkannt wurde. Damit ist der Beitrag verschiedener Fachdisziplinen in einer interdisziplinären Zusammenarbeit gefordert. Dies stellt nicht nur die Waldschadensforscher vor eine schwierige Aufgabe. Auch von der Politik wird umfangreiche, nicht nur kurzfristige Mittelbereitstellung und sinnvolle Mittelverteilung gefordert.

Eine weitere Schwierigkeit besteht darin, daß die Waldschadensforschung in einem speziellen politischen und psychologischen Umfeld zu arbeiten hatte, das von z.T. übertriebenen oder falschen Erwartungen von Politik und Öffentlichkeit auf der einen, Versprechungen der Wissenschaft auf der anderen Seite geprägt war. Aus diesem Anspruch dürfte eine oftmals beobachtete Unzufriedenheit über den Erfolg der Waldschadensforschung resultieren, insbesondere hinsichtlich der Erwartung, Luftschadstoffe als die schadensverursachende Schlüsselrolle nachweislich identifizieren zu können [s.a. SCHLAEPFER 1995, S. 56]. Diese Schwierigkeit bei der Ursachenforschung nimmt noch zu, je mehr sich die ursprünglich angenommene Problemschwere des Waldschadens als tatsächlich nicht zutreffend erweist, wie viele Kritiker proklamieren, oder ,die Gefahren in weiten Bereichen unseres Landes und anderer europäischer Länder gar nicht bestanden“ [ELLENBERG 1996, S. 102].

Aber die Forschung kam zum Zuge. Allein in der Bundesrepublik Deutschland finanzieren Bund, Länder und andere Förderer in der Zeit von 1982 bis 1995 die Waldschadensforschung mit etwa 850 Forschungsvorhaben und über 460 Mio. DM, die eigenen Haushaltsmittel von Bundes- und Landesforschungseinrichtungen und weiteren Institutionen noch nicht miteinberechnet [BAYERISCHES STAATSMINISTERIUM UND BUNDESMINISTERIUM FÜR ERNÄHRUNG, LANDWIRTSCHAFT UND FORSTEN 1995, S. 4 bzw. 1996, S. 6]. Eine nahezu unüberschaubare Vielzahl überwiegend staatlicher, universitärer wie außeruniversitärer Forschungsinstitutionen verschiedener Fachrichtungen beteiligen sich an der Forschung (s. jährliche Dokumentationen der Interministeriellen Arbeitsgruppe des Bundes und der Länder IMA).

Auf Bundes- und Länderebene werden in den frühen 80er Jahren spezielle Förder- bzw. Koordinationsinstitutionen zur Durchführung bzw. Abstimmung von Programmen zur Waldschadensforschung etabliert. $\mathrm{Zu}$ nennen sind auf Bundesebene insbesondere das Aktionsprogramm des Bundes „Rettet den Wald“, und die „Interministerielle Arbeitsgruppe des Bundes und der Länder Waldschäden/Luftverunreinigungen“ (IMA) [UMWELTBUNDESAMT 1996, S. 4]. Auf Länderebene werden das „Projekt Europäisches Forschungszentrum für Maßnahmen zur Luftreinhaltung (PEF) in Baden-Württemberg, der Forschungsschwerpunkt „Luftverunreinigungen und Waldschäden“ des Landes Nordrhein-Westfalen oder das „Forschungszentrum Waldökosysteme / Waldsterben“ der Universität Göttingen / Niedersachsen gegründet. 
Viele mitteleuropäische Länder folgen der Bundesrepublik mit vergleichbaren Programmen, z.B. Österreich („Forschungsinitiative gegen das Waldsterben“), die Schweiz (SanasilvaProgramm, Nationales Forschungsprogramm NFP 14/14+) und Frankreich (Depérissement des Forêts et Pollution Atmosphérique - Deforpa -).

Auch im Freistaat Bayern, welcher vergleichsweise frühzeitig Waldschäden konstatiert, führt das Thema zu einer drastischen Verstärkung der Forschung und deren finanzieller Förderung. Dies betrifft bereits Ende der 70er Jahre in erster Linie die vom Landwirtschaftsressort finanzierte forstliche Forschung, aber auch Förderaktivitäten des Kultus- und Umweltressorts in den frühen 80er Jahren.

Auf Ministerratsbeschluß wird an der damaligen Gesellschaft für Strahlen- und Umweltforschung (GSF) bei München eigens eine Koordinationsstelle für die bayerische Waldschadensforschung etabliert, die „Projektgruppe Bayern zur Erforschung der Wirkung von Umweltschadstoffen (PBWU). Das von ihr in den Jahren 1984 bis 1993 betreute Forschungsprogramm, das sich allerdings im wesentlichen auf den umweltministeriellen Anteil der gesamtbayerischen Waldschadensforschung beschränkt, umfaßt bei rund 80 Forschungsvorhaben und ergänzenden Serviceleistungen ein Fördervolumen in Höhe von etwa 35 Mio. DM [REUTHER et al. 1993, S. 2]. Damit nimmt das PBWU-Programm neben der vom Landwirtschaftsressort geförderten Forschung und gegenüber den weiteren beteiligten bayerischen wie außerbayerischen Förderbeiträgen eine herausragende Stellung in der bayerischen Waldschadensforschung ein [s.a. INTERMINISTERIELLE ARBEITSGRUPPE WALDSCHÄDEN / LUFTVERUNREINIGUNGEN IMA 1993, S. 9/10; REUTHER \& KIRCHNER 1990, S. 8].

\subsection{Fragestellung und Zielsetzung}

Die Waldschadensforschung bietet aus verschiedenen Gründen ein erfolgversprechendes Studienobjekt politikwissenschaftlichen Arbeit in Hinblick auf das Management moderner Umweltforschung. Sie beschäftigt sich in erheblichem finanziellen, zeitlichen, fachlich-inhaltlichen wie institutionellen Umfang mit einem Umweltthema von hoher gesellschaftlicher Relevanz. Sowohl die Politik, als auch die Wissenschaft haben ein vitales, wenn auch unterschiedliches Interesse an der Forschung über das Thema.

Die Politik verfolgt in der Öffentlichkeit das Ziel, naturwissenschaftliche Forschung finanziell zu fördern, welche ihr wissenschaftlich fundierte Maßnahmenvorschläge zur Lösung des bestehenden Waldschadensproblems liefern soll. Hierzu ist es naheliegend, die Identifizierung der Schadensursachen dahingehend $\mathrm{zu}$ fordern, daß eine politische, effektive Bekämpfung bzw. Beseitigung der Ursachen erfolgen kann. Dies setzt eine problemorientiert-angewandte, multidisziplinäre Ursachenforschung voraus, die als politische Zielsetzung der staatlich finanzierten Forschung formuliert und verfolgt werden wird.

Auch der Wissenschaft ist an der Erforschung der Waldschäden sowie deren Ursachen aufgrund ihres intrinsischen Interesses der Erkenntnisproduktion und -erweiterung gelegen. Dabei steht jedoch das naturwissenschaftliche Interesse im Vordergrund. Dieses verlangt überwiegend nach Forschungsansätzen, die zum grundlegenden Verständnis von Systemen, Vorgän- 
gen, Prozessen und Wirkungsmechanismen führen, damit wiederum aber nicht deckungsgleich mit den Förderabsichten der Forschungspolitik sein müssen.

Trotz unterschiedlicher Interessen sind beide Akteursgruppen zur Verfolgung ihrer Zielsetzungen aufeinander angewiesen. Die Wissenschaft bedarf zur Finanzierung und Durchführung der von ihr beabsichtigten Forschung umfangreicher staatlicher Förderung. Die Politik stellt diese zur Verfügung, um von der Forschung zur Verfolgung ihrer politischen Ziele umsetzbare Ergebnisse zu erhalten.

Aus dem einleitend nur kurz angedeuteten Spannungsfeld zwischen Interessenlagen von Politik und Forschung ergibt sich die der Studie zugrundeliegende, forschungspolitisch relevante Fragestellung:

- Wie läßt sich Forschung organisieren und politisch steuern, daß sie zum einen den selbstgesetzten Standards der Wissenschaft in hohem Maße entspricht, und zum anderen problemorientiert-angewandt und interdisziplinär durchgeführt wird, um zu Ergebnissen zu führen, die den politisch gesetzten Zielen dienen?

Diese Frage soll anhand des Fallbeispiels des PBWU-Projektes als einem staatlichen Förderprogramm bearbeitet und beantwortet werden. Hierzu zielt die vorliegende Studie auf die sozialwissenschaftlich fundierte Analyse der Forschungsförderung ab. Diese muß die spezifischen forschungsseitigen, organisatorischen und verwaltungsmäßigen Strukturen ebenso berücksichtigen wie die Interessenlagen und Handlungspläne der Beteiligten aus Politik, Verwaltung und Forschung. Die Frage nach der Forschungskoordination gewinnt bei der gegebenen Komplexität der Forschung und der im Projekt eigens eingerichteten Koordinierungsstelle besonderes Interesse.

Dabei wird unterstellt, daß forschungspolitische Prozesse stattfinden, die zu Abweichungen vom Idealtyp wissenschaftlichen Forschung ebenso wie vom Idealtyp politischer Forschungsförderung führen. Anhand des Beispiels wird theorieorientiert erklärt, warum derartige Abweichungen eintreten. Das Wissen über die Prozeß-steuernden Faktoren kann abschließend dazu dienen, alternative Steuerungsstrategien für Forschungspolitik und Förderprogramme zu entwerfen. Darin wird der praktische Nutzen der Studie gesehen.

\subsection{Gliederung der Studie}

Der Aufbau der vorliegenden Arbeit folgt der genannten Fragestellung. Die theoretische Grundlegung (Kapitel 2) sucht nach einem geeigneten Analyseinstrument. Hierzu wird der „Policy-Ansatz“ gewählt, der geeignet ist, die Abfolge der verschiedenen Teilschritte (Phasen) der politischen Maßnahme PBWU-Projekt und des daraus resultierenden Forschungsprozesses $\mathrm{zu}$ beschreiben und theorieorientiert zu analysieren. In Kapitel 3 wird das methodische Instrumentarium zur Durchführung der Studie dargestellt.

Vor der Beschreibung des Prozesses und der theorieorientieren Analyse werden in Kapitel 4 die politischen und wissenschaftlichen Akteure im einzelnen vorgestellt, die im PBWU-Projekt 
beteiligt sind. Die jeweiligen Akteursstrukturen und -konstellationen, ihre Interessenlagen und Handlungspläne werden skizziert.

Das Hauptinteresse der Studie richtet sich auf die Durchführung des PBWU-Projektes als politische Maßnahme und hier insbesondere auf den daraus resultierenden Forschungsprozeß. Dies ist in dem zentralen Kapitel 5 ausführlich dargestellt. Einleitend wird kurz auf die Entdeckung und Diskussion des „Waldsterbens“ im politischen und gesellschaftlichen Kontext sowie die ersten Ansätze einer Waldschadensforschung und deren Koordinierung eingegangen. Die Beschreibung der Phasen der Problemerkennung und Thematisierung als Forschungsaufgabe sind nicht nur eine historische Darstellung, sondern bereits Bestandteile der „Policy-Analyse“ zum PBWU-Projekt. Nach den Phasen Gründung und Etablierung des PBWU-Projektes rückt die Beschreibung des PBWU-Projektes und damit der Forschungsprozeß und das Forschungsförderverfahren in den Mittelpunkt. Dabei folgt die Gliederung wiederum empirisch wie theoriegeleitet den Phasen der Forschungspolicy. Theorieorientierte Zwischenergebnisse werden in Unterkapiteln behandelt.

Der Forschungsprozeß beginnt mit der Erarbeitung und Formulierung eines Forschungsrahmenprogramms. Dessen Umsetzung reicht von der Einreichung und Behandlung von Forschungsanträgen über die Durchführung des Förderverfahrens und der Forschung selbst bis hin zur Evaluierung und Terminierung des Gesamtprojektes. Detailuntersuchungen greifen das Förderverfahren unter besonderer Berücksichtigung der Qualitätssicherung der Forschung und des Steuerungseinflusses der beteiligten politischen wie wissenschaftlichen Gremien heraus.

Kapitel 6 bildet den Abschluß der Analyse. Hier werden die zentralen politischen Faktoren der Entwicklung und Steuerung des Forschungsprojektes zusammenfassend dargestellt. Der Forschungsprozeß im PBWU-Projekt wird im Kräftespiel politischer wie wissenschaftlicher Interessen und Steuerungseinflüsse erklärt.

Die Studie endet exkursorisch mit Empfehlungen für das Forschungsmanagement (Kapitel 7). Aufbauend auf den Ergebnissen der Analyse und orientiert an einem Idealmodell werden praxistaugliche Maßnahmen zur Verbesserung der Durchführung anwendungsorientierter, interdisziplinärer Forschungsprogramme entwickelt und vorgeschlagen. 


\section{Theorie}

\subsection{Bezugspunkt für Theorie und Analyse}

Die Waldschadensforschung ist, wie für staatlich geförderte Forschung allgemein gültig, inmitten des Spannungsfeldes zwischen Wissenschaft und Staat gelegen. Dieses ist „,von grundlegenden Interessenkonflikten geprägt“ "FELT et al. 1995, S. 209]. Spezifischen Interessen der Wissenschaftler stehen die des Staates gegenüber. Beiderseitiger, wenn auch unterschiedlicher Nutzen, muß jedoch für Forschung wie Politik gegeben sein, damit die staatliche Forschungsförderung umgesetzt werden kann.

Die Verfolgung und Durchsetzung der Akteursinteressen erfolgt im „Kampf“ um und in den Institutionen und Gremien des Projektes sowie den dort verwendeten Verfahren zur Programmplanung und Forschungsförderung. Die Existenz der „Projektgruppe Bayern zur Erforschung der Wirkung von Umweltschadstoffen“ (PBWU) zur Koordinierung der Forschung mit den zugehörigen Gremien der Politik und Wissenschaft schafft dabei als eigene Institution eine besondere Ausgangslage. Die Studie wählt zwei zentrale Bezugspunkte, die bei der Findung des theoretischen Analyserahmens berücksichtigt werden müssen.

Erster Bezugspunkt ist, daß im Projekt eine Gestaltung der Forschung stattfindet. Der Bezugspunkt kann in dieser allgemeinen Formulierung vorab angenommen werden, da er über Art, Intensität und Zielrichtung der Forschungsgestaltung noch keine Aussagen macht. Inwieweit und durch wen hierbei eine aktive „Steuerung“ der Forschung, eine normative „Regelung“ des Forschungsverlaufes oder eine passive „Anpassung“ der Rahmenbedingungen an das faktische Forschungsgeschehen erfolgt, bleibt offen.

Als zweiter Bezugspunkt der Analyse wird unterstellt, daß die Forschung als Erkenntnisproduktion für die im Projekt beteiligten Akteure von unterschiedlichem Interesse ist. Dies drückt sich in den Strategien aus und nimmt wiederum Einfluß auf die Gestaltung bzw. Steuerung der Forschung. Das PBWU-Projekt kann daher in ihrer intermediären Struktur zwischen Staat und Wissenschaft zur Koordination der Forschung sowie Überbrückung interessenbedingter Gegensätze analysiert werden. Die Steuerungswirkungen werden mit Blick auf die wissenschaftlich-fachlichen und politischen Faktoren untersucht.

\subsection{Politikwissenschaftlicher Forschungsansatz}

Die Analyse von Politikinhalten, wie sie auch die Steuerung durch die PBWU kennzeichnen, erlaubt der Policy-Ansatz [s.a. KROTT 1993, S. 101]. Er wurde in den USA in der Nachkriegszeit auf dem Easton`schen Theorie-Fundament [EASTON 1965] entwickelt und wird heute auch zur Analyse politischer Prozesse in Deutschland eingesetzt [WINDHOFF-HÉRITIER 1987; SCHUBERT 1991]. Nach KROTT [1990, S. 29] vereinfacht und präzisiert der Policy-Ansatz den politischen Prozeß zu einem analytischen Rahmen. Er stellt ein analytisch-erklärendes Instrumentarium zur Untersuchung von Politikinhalten dar. 
Die Politikfeld-Analyse fragt nach der Entstehung, Durchführung und Beendigung einer Policy. Dazu wird der Politikinhalt in verschiedene, logisch aufeinander folgende Phasen zerlegt, die insgesamt das Modell des „Policy-Zyklus“ ergeben. Dieser reicht von der Wahrnehmung eines Problems über die Formulierung von Handlungsalternativen oder Lösungsprogrammen über deren Vollzug bis hin zur Wirkungskontrolle, Terminierung oder Neuformulierung [WindHOFF-HÉRITIER 1987, S. 64ff; vON PRITTwitz 1990, S. 93 ff; KROTT 1990, S. 30].

Durch die zusätzliche Einführung des analytischen Begriffs „Politiknetz“ (,Policy-Netz“) können alle Akteure und deren Beziehungen in einem Politikfeld berücksichtigt werden. Der Begriff „Politikarena“ hebt indes auf den politischen Prozeß, Konflikt und Konsens während Entstehung und Durchführung einer Policy ab [WINDHOFF-HÉRITIER 1987, S. 43].

Die Festlegung dieses ,akteurstheoretischen Bezugsrahmens“ erlaubt es, Akteurskonstellationen und -strategien vor dem Hintergrund bestehender Interessenlagen, Einfluß- und Machtpotentiale zu analysieren. Die Frage der Forschungssteuerung unter Berücksichtigung der Interessen von Politik und Wissenschaft kann so - wie noch ausgeführt werden wird - erfolgversprechend bearbeitet werden [s.a. HOHN \& SCHIMANK 1990, S. 25ff].

Das zielorientierte Handeln der politisch-administrativen wie der Forscher-Akteure führt unweigerlich zu Interdependenzen, die bspw. Kooperations-, Konsens-, Interessenkonflikte oder Konkurrenzen untereinander bedingen können. Dabei ist zu berücksichtigen, daß das Verhältnis zwischen verschiedenen Akteuren durch eine Policy nicht völlig neu gestaltet wird. Es beruht i.a. auf einer gewissen Tradition. So bestimmen Sympathien und Antipathien, die sich aus dem Zusammenwirken in anderen Kontexten entwickelt haben, auch das Verhalten der Akteure bei der gemeinsamen Durchführung späterer Programme [NETSCH, 1989, S. 55]. In der Fallstudie wird hierbei die Klientelbildung zwischen bestimmten Forschergruppen und Forschungsförderressorts, aber auch die wissenschaftsinterne ,Zunftmentalität“ [CLARK 1983, zit. in: BRAUN 1997, S. 58/59] zu berücksichtigen sein. Zudem verursacht die gegenseitige Beeinflussung von Akteursstrategien antizipierendes Verhalten in Hinblick auf das beabsichtigte Handlungsergebnis: Das Wissen oder die Vermutungen der Akteure über Akteurskonstellationen und daraus erwartete Handlungsstrategien anderer Akteure gehen wiederum in die eigene Handlungsstrategie ein [s.a. HoHn \& SCHIMANK 1990, S. 33ff]. So ist die Frage zu stellen, inwieweit opportunistisches Verhalten der Forscher von forschungspolitischer, finanzierender Seite antizipiert werden könnte [NOWOTNY 1994, persönliche Mitteilung].

\subsection{Theoretische Grundlagen zur Politikfeld-Analyse}

Im folgenden soll der Prozeß einer Policy nach dem Ansatz des Policy-Zyklus bzw. der daran anknüpfenden Politikfeld-Analyse (Policy-Analyse) in seinen theoretischen Grundlagen näher dargestellt, auf das als Fallbeispiel gewählte PBWU-Forschungsprojekt übertragen und kritisch diskutiert werden. 


\subsubsection{Phasenmodell des Policy-Zyklus}

Das Phasenmodell des Policy-Zyklus wird von verschiedenen Politikwissenschaftlern geringfügig unterschiedlich terminologisch definiert. So werden in der deutschen Verwaltungswissenschaft der 80er Jahre die Prozeß-Elemente „Problemwahrnehmung - Politikformulierung - Programmentwicklung - Politik-/Programmvollzug (Implementation) - Wirkungskontrolle (Evaluation)““ definiert [MAYNTZ 1980; HeSSE 1985, zit. in: VON PRITTWITZ 1990, S. 94].

WINDHOFF-HÉRITIER [1987, S. 65] versteht ihr Phasenmodell als Zusammenfassung der terminologischen Vielfalt. Sie definiert die Phasen des Politikzyklus als „Problemdefinition (-formulierung) - Agenda-Gestaltung - Politikformulierung - Politikimplementation - PolitikNovellierung oder Terminierung“, die von einer „Politik-Evaluation“ begleitet bzw. verursacht werden können (s. Abbildung 1). Die Ergebnisse und Wirkungen der Policy rufen eine "politische Reaktion und eine politische Verarbeitung“ hervor, die wiederum zur „Terminierung oder Fortsetzung" führen können.

Von PrITTwitz [1990, S. 93ff] übernimmt den Ansatz von BREWER \& DE LEON [1983] und beschreibt den Policy-Zyklus als Phasen der ,Problemwahrnehmung (Initiation) - Vorabschätzung von Handlungsalternativen (Estimation) - Programmbildung/Entscheidung (Selection) Programmvollzug (Implementation) -Wirkungskontrolle (Evaluation) - Handlungskorrektur/Lernen (Termination)“ (s. Abb. 1). Die von WINDHOFF-HÉRITIER als Agenda-Gestaltung definierte Phase werde hier weiter differenziert nach der ,noch informellen Politikdefinition und formal verbindlichen Entscheidungsfindung“. 
Phaseneinteilung des Policy-Zyklus nach WINDHOFF-HÉRITIER 1987
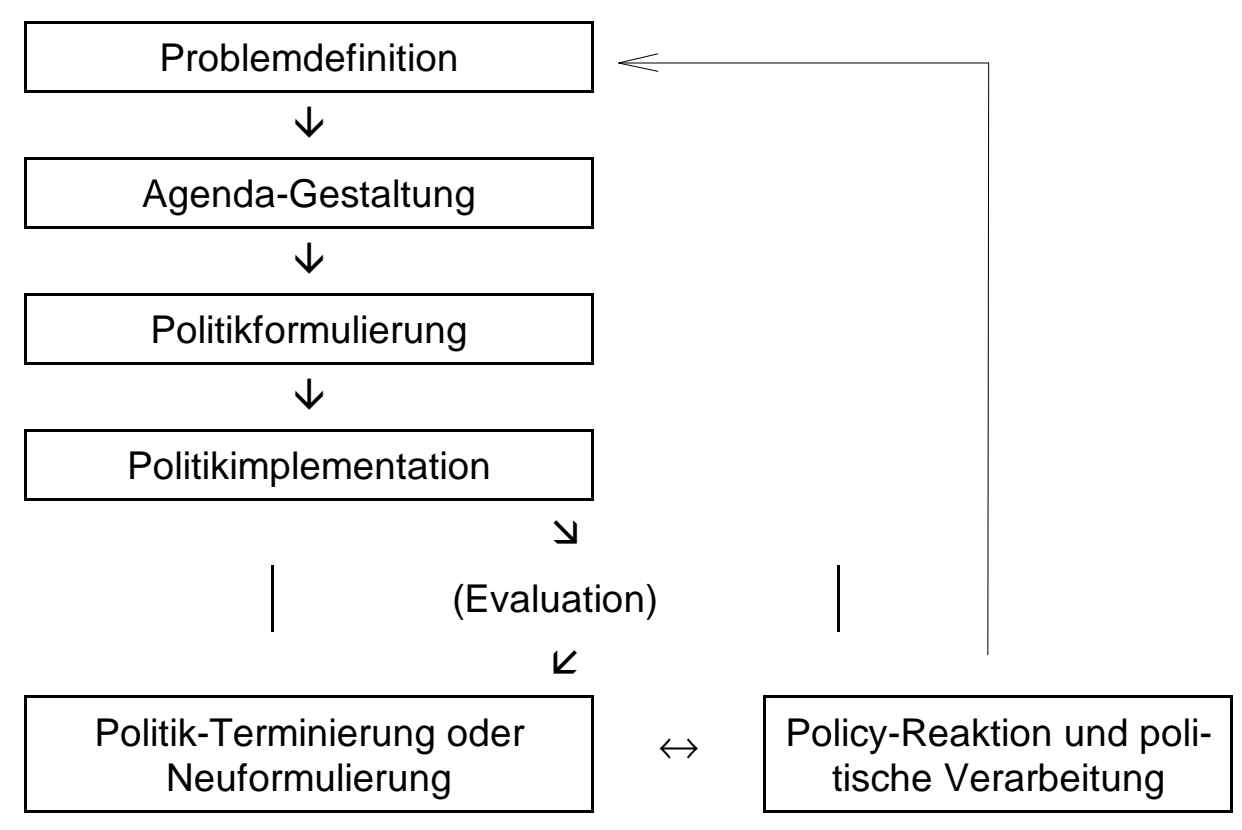

Abb. 1: Phasenmodell des Policy-Zyklus nach WINDHOFF-HÉRITIER [1987, S. 65] (geringfügig verändert)

KrotT [1989, S. 8-13; 1990, S. 61-75, 1993, S. 102-110] legt Wert auf die generelle Differenzierung des politischen Prozesses in die übergreifenden Phasen der 
- Politikformulierung und der

- Politikimplementation (Programmvollzug).

In Hinblick auf die Zielsetzung der vorliegenden Arbeit soll auf eine grundlegende Diskussion oder Neuformulierung der Begriffsdefinitionen verzichtet werden. In ausreichendem Maß kann auf Gemeinsamkeiten der genannten Autoren zurückgegriffen werden, so daß eine weitgehend allgemeingültige Beschreibung der einzelnen Phasen möglich ist. Die einzeln ausgewiesenen Phasen bezeichnen dabei nicht nur die empirisch zu beschreibende Prozeß-Abfolge eines Policy-Zyklus, sondern können im wesentlichen auch als Analyseschritte des methodisches Instrumentariums Policy-Analyse der praktischen Politikberatung dienen [WINDHOFF-HÉRITIER 1987, S. 115/116]

\subsubsection{Problemdefinition und Agenda-Gestaltung}

Die „Problemdefinition“ als erste Phase der Politikformulierung legt fest, welches der bestehenden gesellschaftlichen Problem als behandlungsrelevant erklärt wird [KROTT 1993, S. 103]. Daran schließt die von WINDHOFF-HÉRITIER [1987, S. 67ff] eigens ausgewiesene Phase der „Agenda-Gestaltung“ an, in der ein Thema im politischen Feld behandelt, d.h. auf die politische Tagesordnung gesetzt wird. Es handelt sich hierbei um einen ,z.T. diffusen, schwer faßbaren Auswahlprozeß“. Wie am Fallbeispiel der Waldschadensforschung dargestellt werden wird, ist dieser eng mit der generellen Diskussion zum Thema „Waldschäden/Waldsterben“ und somit mit der übergeordneten Ebene einer Waldschadens-Policy verknüpft. Bei den gegebenen Unsicherheiten in Kenntnis und Bewertung des Problems Waldschäden wird der Ruf nach Waldschadensforschung, der letztlich und unter anderem zu dem PBWU-Projekt führt, nahezu zeitgleich und häufig in direkter Koppelung an das Problem selbst artikuliert. Der Prozeß reicht somit zeitlich noch weit vor die Gründung des PBWU-Forschungsprojektes zurück.

Die Auswahl von Problemen ist abhängig von den beteiligten Akteuren und deren Handeln, den Eigenschaften des Problems und dem Thematisierungsprozeß [PETERS 1982, zit. in: KROTT 1990, S. 63 u.a.a.O.]. Bezogen auf die Waldschadensforschung betrifft dies in erster Linie das Verhalten der Wissenschaft und Politik, des weiteren die politische Frage, ob Forschung einen Beitrag zur Problembewältigung beitragen kann und nicht zuletzt die Thematisierung des der Forschung zugrundeliegenden Phänomens „,Waldsterben“ in Öffentlichkeit und Medien.

Die Thematisierung setzt Probleme auf die politische Agenda, die beteiligten Akteure konkurrieren darum, ihre Sichtweise des Problems zu proklamieren und sich politisch durchzusetzen. Es bedarf also politischer Macht und politischen Einflusses, um ein Thema auf die Agenda zu setzen [KROTT 1993, S. 104; WindHOFF-HÉRITIER, 1987, S. 68]. Die politische Behandlung des Themas Waldschadensforschung hat hierbei insbesondere die Rolle von Politik und Verwaltung sowie die Einbeziehung der Wissenschaft zu analysieren.

Weitere Voraussetzungen wie eine breite und andauernde, öffentliche Problem-Aufmerksamkeit sowie die Zuständigkeit und Handlungsbereitschaft betroffener Verwaltungen [COBB \& ElDER 1976, zit. in: WindHOFF-HÉRITIER 1987, S. 71] sind gegeben. Sie resultieren weitenteils aus der Gesamtproblematik Waldschäden und führen einerseits zu hohem politi- 
schen Handlungsdruck, andererseits zu Konkurrenzsituationen verschiedener Ressorts bzgl. der Zuständigkeit für die Waldschadensforschung.

Die Medien spielen während des Thematisierungsprozesses für das spezifische Problembewußtsein eines politischen Themas in der Öffentlichkeit eine erhebliche Rolle. Die Forschung selbst ist zwar überwiegend nur indirekt und weit weniger stark als das zugrunde liegende Thema „Waldsterben“ von medialer Aufmerksamkeit betroffen, kann aber von der intensiven und anhaltenden Thematisierung profitieren. Gerade die umfangreiche und dramatisierende Behandlung des Themas Waldsterben in den Medien hat „Lehrstück“-Charakter und ihren Niederschlag in eigenen, z.T. kritischen Abhandlungen hierzu gefunden [z.B. HolzBERGER 1996; KROTT 1987; ZIERHOFER 1998; ZIMMERMANN 1991]. Anzumerken ist, daß die „Medienkarriere“ eines Themas nicht mit der tatsächlichen Problemschwere oder der Intensität der politischen Behandlung direkt synchronisiert sein muß [KROTT 1990, S. 65; WINDHOFF-HÉRITIER 1987, S. 69 und 73; vON PRITTwitz 1990, S. 13-30; Russ-MoHL 1993].

\subsubsection{Politik-(Programm-)formulierung}

Auf die Phase der politischen Problemdefinition und -behandlung folgt entsprechend der Phasenheuristik des Policy-Ansatzes von WindHOFF-HÉritier [1987, S. 74 ff, s.a. KrotT 1990, S. 66 ff] die Formulierung und Entscheidung von Programmen. Diese Phase besteht aus der Verarbeitung von Informationen, Erarbeitung von Programmvorschlägen und politischen Entscheidung darüber. Zudem beinhalten die Programme Zielvorstellungen und Instrumente zur anschließenden Umsetzung.

Übertragen auf die Forschungspolicy im Rahmen des PBWU-Projektes kann die Phase der Politik- bzw. Programmformulierung auf zwei Ebenen gesehen werden. Zum einen umfaßt sie als Politikformulierung die politische Vorbereitung und Entscheidung zum PBWU-Projekt an sich. Zum anderen ist die Programmformulierung in der Erarbeitung des PBWU-Forschungsrahmenprogramms unterhalb der Ebene der vorstehend genannten politischen Entscheidung zu sehen, welche dann die Basis für die anschließende Phase der Programmimplementation, der Umsetzung des Forschungsprogrammes, darstellt.

Die Focussierung auf den Forschungsprozeß an sich erlaubt es, in der vorliegenden Studie letztgenannte Rahmenprogramm-Formulierung in den Mittelpunkt der hier behandelten Phase zu stellen. Aus diesem Blickwinkel kann die politische Programmformulierung zur Gründung der PBWU einschließlich deren Umsetzung und Etablierung der Projektgruppe der vorangehenden Phase der Agenda-Gestaltung zugeordnet werden.

Bereits bei der politischen Entscheidung zum PBWU-Projekt finden maßgebliche Weichenstellungen hinsichtlich der Projekt- bzw. Programmorganisation und -struktur statt, die aus Steuerungsbemühungen zwischen politischen und wissenschaftlichen Akteuren, aber auch zwischen den verschiedenen politischen Ressorts resultieren. Ebenso wie bei den im vorangegangenen beschriebenen Phasen der Problemdefinition und Agendagestaltung muß daher in Beschreibung und Analyse auf die Zeit noch vor Gründung des PBWU-Projektes zurückgegriffen werden. 
Gerade die Programmbildung erfordert detaillierte Fachkenntnis. Neben der Problembehandlung durch die Fachverwaltungen werden daher häufig externe Experten hinzugezogen. Diese bringen zwar formal ihr Fachwissen, aber auch informal ihre Interessenpositionen mit ein, bzw. können beide argumentativ miteinander verknüpfen [KROTT 1990, S. 69; 1993, S. 106]. Die Interessenpositionen der Wissenschaft werden im gewählten Fallbeispiel daher - neben denen der verschiedenen politischen Ressorts - sowohl bei der Gründung des PBWU-Projektes an sich, als auch gerade bei der Erstellung und Aushandlung dessen Rahmenprogramms zu berücksichtigen sein.

Als Folge der unterschiedlichen Interressenpositionen wird die Programmformulierung häufig nur vage und wenig präzisiert als eine Art „Rahmenentscheidung“ vorgegeben. Die Ausgestaltung und Konkretisierung der politischen Entscheidung bzw. des Programmes - und damit auch die eventuell zugrundeliegende Konfliktbewältigung - werden in die anschließende Phase der Implementation verschoben [WINDHOFF-HÉRITIER 1987, S. 83; KROTT 1990, S. 66].

\subsubsection{Implementation}

Die Implementation ist die Umsetzung des in der Formulierungsphase entschiedenen Handlungsauftrages. Die vorliegende Studie stellt wiederum entsprechend ihrer Zielsetzung und in Konsequenz zu der bisherigen Phaseneinteilung den Forschungsprozeß und damit die Umsetzung des PBWU-Forschungsrahmenprogramms in den Mittelpunkt.

Die Wirkung eines Programmes ist abhängig von dem Verhalten der beteiligten Akteure, den Programmeigenschaften und der Implementationsstruktur [WINDHOFF-HÉRITIER 1987, S. 86 ff; KROTT 1993, S. 106, s.a. MAYNTZ 1980, S. 236]. Im gewählten Beispiel steht die Umsetzung des PBWU-Forschungsprogramms im Mittelpunkt. Die Existenz einer eigenen, intermediären Koordinierungsstelle stellt hierbei eine strukturelle Besonderheit dar.

Die Akteure sind bestrebt, Ressourcen zu mehren und/oder Bestandssicherung zu betreiben, und sie haben Eigeninteressen zur Steuerung des Programmes. Die Wahrnehmung des eigenen Handlungsspielraumes erschwert dabei die Durchsetzung einheitlicher Programmziele [KROTT 1990, S. 70; 1993, S. 106/107]. Die Implementationsakteure versuchen zudem bereits während der Phase der Politik- bzw. Programmformulierung, Einfluß auf die Ausgestaltung des ,politischen Auftrages“ zu nehmen [WINDHOFF-HÉRITIER 1987, S. 89]. Dies kann die Konfliktverlagerung aus der Programmformulierung in die Implementationsphase noch verstärken. Im gewählten Fallbeispiel sind politische wie wissenschaftliche Akteure in beiden Phasen und sowohl bei der Gründung des PBWU-Projektes als auch bei der Erstellung eines Forschungsrahmenprogramms beteiligt. Aufgrund der genannten Phasenüberlappungen muß daher nicht zwangsläufig die jeweils zu erwartende Akteursdominanz der betreffenden Phase steuerungsentscheidend sein.

In diesem Zusammenhang ist eine Differenzierung der Programmimplementierung in den zentral von oben gesteuerten top down-Ansatz gegenüber dem bottom up-Ansatz, der eine Implementation unter Beteiligung der Betroffenen beschreibt, von Bedeutung [VON PRITTWITZ 1990, S. 96; WindHOFF-HÉRITIER 1987, S. 96/97; HILl \& RIESER 1983, S. 335ff]. Anhand des 
PBWU-Forschungsrahmenprogramms wird zu prüfen sein, inwieweit die Voraussetzungen für einen top down-Ansatz der Implementation, ein präzise formuliertes und faktisch umsetzbares Handlungsprogramm, überhaupt gegeben sind, und die gegebenen Kräfteverhältnisse zwischen Forschungspolitik und Wissenschaft den Ansatz determinieren.

Das Zusammenwirken mehrerer Akteure erfordert Mechanismen zur Konfliktregelung und Koordination. Hierzu wurde im PBWU-Projekt in erster Linie die intermediäre Koordinierungsstelle eingerichtet. Als Instrumente verfügen die Akteure über verschiedene Machtmittel, insbesondere Informationsmittel. Der Einsatz und die Wirkung der verschiedenen Machtmittel stellt ein wichtiges Analysekriterium in der vorliegenden Studie dar. So steht der Sachautorität der Wissenschaft die Finanzierungshoheit der Politik gegenüber. Für die intermediäre Projektgruppe wird der Machtfaktor Information als zentrales Instrument zur Koordination eine wichtige Rolle spielen [s.a. KROTT 1990, S. 71/72; BRAUN 1997, S. 362, 365].

\subsubsection{Evaluation}

Aus der Phase der Programmimplementierung folgen Wirkungen und Ergebnisse der Policy, die letztlich politische Reaktionen wie die Entscheidung über Weiterführung, Veränderung oder Terminierung der Maßnahme hervorrufen. Damit schließt sich der Policy-Zyklus. Eine wissenschaftliche Evaluierung ist im Rahmen einer Policy allerdings die Ausnahme. Lediglich für Politikberatung ist die Durchführung einer wissenschaftlichen Evaluation obligatorisch [s.a. WINDHOFF-HÉRITIER 1987, S. 65, 107, 115ff].

Die Evaluation erfolgt in der Regel durch die beteiligten Akteure. Sie versuchen während des gesamten Projektes, dessen Erfolg zu bewerten. Dies ist für eine „objektive“ Erfolgsbewertung im Rahmen der Policy-Analyse zwar nur von begrenztem Nutzen. Die vorliegende Studie beschreibt und analysiert daher, ob bzw. in welcher Art eine Programmevaluation durchgeführt wurde, und welche Kriterien und Argumente die Grundlage für die Beendigung des Projektes im Jahr 1993 darstellten. Zudem geht sie auf die im Forschungssystem auftretenden Sonderfälle der Evaluation ein. Dabei handelt es sich im wesentlichen um die Begutachtung von Forschungsanträgen (ex ante-) sowie von Forschungsberichten (ex post-Evaluation). Einzelheiten hierzu werden im Rahmen der empirischen Darstellung und Analyse des Förderverfahrens im Rahmen der Implementation behandelt.

Wissenschaftliche Instrumente zur Erfolgsbeurteilung werden in der politischen Praxis nur selten benutzt. Dabei handelt es sich bei der Evaluation generell um ein politikwissenschaftliches Analyseinstrument, mit dem die Programmwirkung sowie sämtliche Prozesse und Phasen mittels geeigneter Indikatoren überprüft werden [WINDHOFF-HÉRITIER 1987, S. 126]. Methodisch differenziert die Evaluation zwischen einer Zielerreichungs- und Ursachenanalyse [HELLSTERN \& Wollmann 1984, S. 24]. Dabei stellt die hier einschlägige Zielerreichungsevaluation einen Ist/Soll-Vergleich zwischen Programmzielsetzung - oder einzelner Forschungsteilziele - und deren tatsächlicher Erreichung an. Offenheit der Programme mit entsprechend vagen Zielsetzungen erschweren dabei jedoch die Festlegung präziser Erfolgsmaßstäbe und -indikatoren. Bezogen auf die Evaluationsphase des PBWU-Projektes wird die Analyse berücksichtigen müssen, inwiefern der Erfolg der Programmimplementation nach subjektiven Kriterien und 
Erfolgsmaßstäben, je nach Klientelgebundenheit zu Forschung oder Politik, bewertet wurde [s.a. KROTT 1993, S. 109].

Hinsichtlich des Einsatzes von Kosten/Nutzen-Analysen zur Bestimmung der Programmeffizienz [RossI et al. 1988, S. 164ff] sind von vornherein nur geringe Erfolgsaussichten zu erwarten. Läßt sich zwar der Kostenaufwand für die Durchführung der Forschung ohne weiteres ermitteln, ist der Nutzen oder die Wirkung von Forschungsergebnissen für den Bereich der Forschung nahezu unberechenbar.

\subsubsection{Terminierung}

Die Terminierung bezeichnet die Beendigung eines Policy-Programmes [WiNDHOFF-HÉRITIER 1987, S. 195]. Sie stellt nur eine von verschiedenen Möglichkeiten politischer Entscheidungen über eine Policy dar. Neben der Programmbeendigung kann eine Policy auch in modifizierter Form weitergeführt werden [WINDHOFF-HÉRITIER 1987, S. 65; VON PRITTWITZ 1990, S. 98]. Der Policy-Zyklus endet dann nicht, sondern geht in eine erneute Politikformulierungsphase über.

WiNDHOFF-HÉRITIER [1987, S. 106-110] führt verschiedene Gründe an, die zu einer PolicyTerminierung führen können und analysiert die jeweils zugrunde liegenden Gegebenheiten und Strategien der beteiligten Akteure. Im vorliegenden Fall müssen hinsichtlich der Beendigung des PBWU-Projektes und damit des PBWU-Forschungsprogramms im engeren Sinne wissenschaftliche wie forschungspolitische Argumente, Finanzengpässe, personelle Veränderungen an der ressortpolitischen Spitze, ressortspezifische Fachargumente und der Grad der Thematisierung berücksichtigt werden. Der Widerstand der Wissenschaft gegen eine politisch argumentierte Terminierung verdeutlicht hierbei die bestehenden Interessenverhältnisse. Die Terminierungswirkung wird wiederum in Zusammenhang mit der Evaluation des Forschungs- bzw. Projekterfolges zu diskutieren sein.

\subsubsection{Behördenorientierte Politikfeld-Analyse}

KRotT [1989, S. 4-7; 1990, S. 45ff] modifiziert den allgemeinen Policy-Ansatz unter Berücksichtigung sozialwissenschaftlicher Theorien über den politischen Prozeß und über Organisationen zu einem behördenorientierten Policy-Ansatz. Dabei wird zur Begründung unter anderem herausgestellt, daß ein Großteil der Policies ohnehin vom politisch-administrativen System ausgehe und somit das politische Handeln von Behörden adäquat analysiert werden könne.

Die behördenorientierte Politikfeld-Analyse übernimmt die in der allgemeinen Form strukturierte politische Konfliktregelung bzw. Problembearbeitung des Policy-Zyklus. Hinsichtlich der Akteure differenziert der Ansatz nach einer frei wählbaren „Hauptbehörde“ und den ,sonstigen Akteuren“, die über „Handlungspläne“ miteinander verbunden sind. Das Modell der behördenorientierten Politikfeld-Analyse ist in Abbildung 2 schematisch dargestellt.

Die nahezu willkürliche Festlegung eines Hauptakteurs (Hauptbehörde) entbindet unter anderem von der empirisch schwer lösbaren Aufgabe, a priori den wichtigsten Akteur zu ermitteln 
[KROTT 1990, S. 45] und erlaubt so die Zentrierung der Akteurskonstellationen je nach Fragestellung der Untersuchung. Den Ansatz der behördenorientierten Politikfeld-Analyse auch für die vorliegende Studie zur staatlich finanzierten Forschung zu übernehmen, erscheint daher ebenso pragmatisch wie sinnvoll. Bezogen auf die Zielsetzung der vorliegenden Studie, welche die Organisation und Steuerung der Forschung analysiert und Empfehlungen zu deren Optimierung abgeben will, ist es sinnvoll, die explizit für Koordinierungsfunktionen eingerichtete PBWU-Projektgruppe als Hauptakteur auszuwählen. Zudem sprechen methodische Vorteile hierfür.

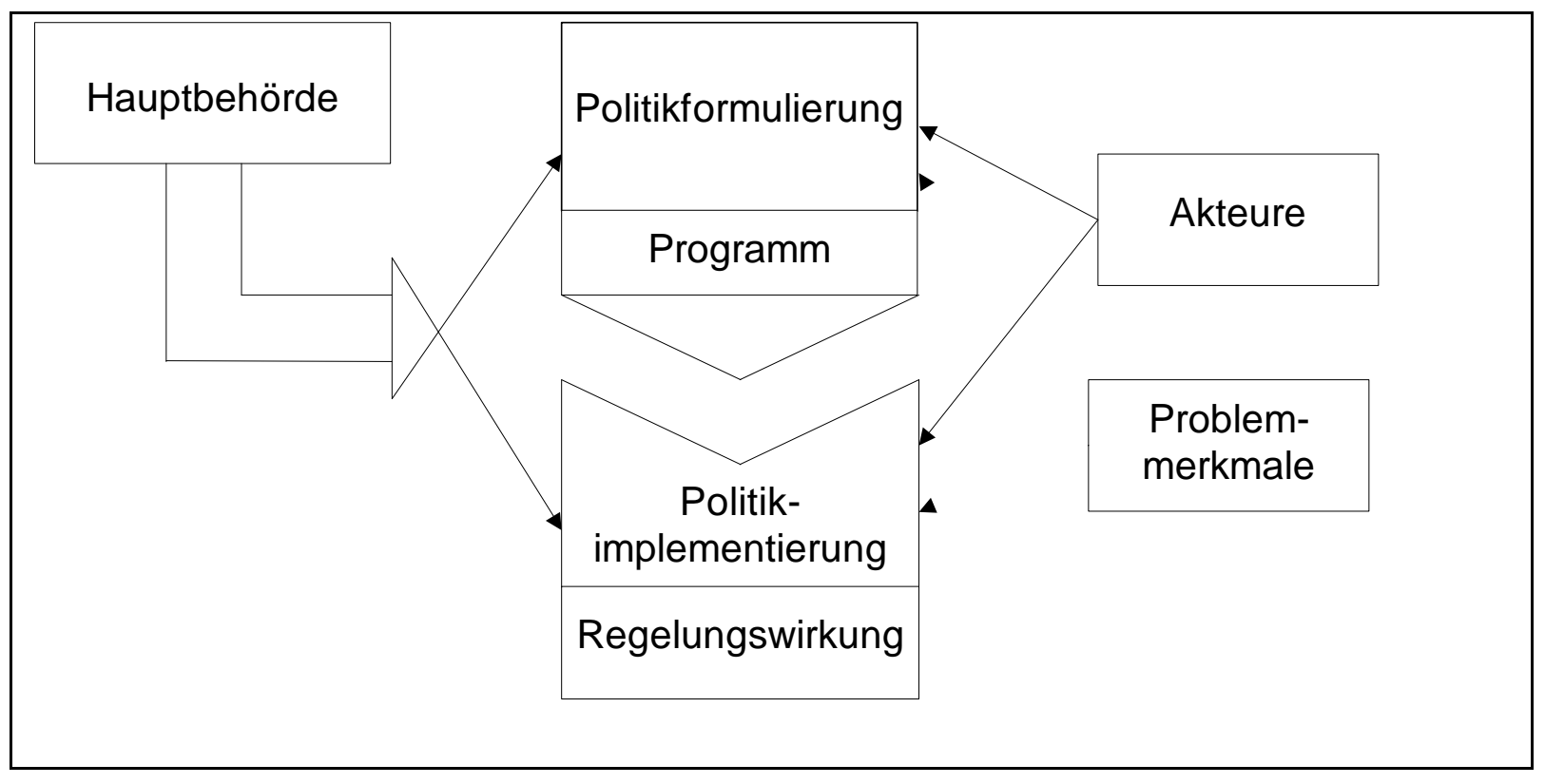

Abb. 2: Elemente und Struktur der behördenorientierten Politikfeld-Analyse [nach KROTT 1989, S.5, geringfügig verändert). Die Verbindungslinien zwischen den Elementen charakterisieren die Handlungspläne der Akteure, geprägt durch Ziele und Instrumente wie Information und Macht

\subsubsection{Kritische Bewertung der Politikfeld-Analyse}

Die Politikfeld-Analyse wird in der deutschen Verwaltungsforschung mehr in ihrer beschreibend-erklärenden als in ihrer beratenden Funktion eingesetzt [WINDHOFF-HÉRITIER 1987, S. 8/9]. Die Forschungsansätze betreffen überwiegend Bereiche der Wirtschafts- und Sozial-, aber auch die Umweltpolitik, z.T. reduziert auf den Teilaspekt der Implementationsforschung. Als Fallstudien, empirische Forschungsberichte und Ansätze zur Theoriebildung und -weiterentwicklung bei der Implementation politischer Programme können grundlegend für Deutschland die von MAYNTZ [1980 und 1983] genannt werden. Dort werden jedoch auch Grenzen und Kritikpunkte an der Policy-Analyse deutlich gemacht. Unter anderem führen dort Zweifel an der „Erklärungskraft ihrer steuerungstheoretischen Annahmen“ und dem „Nutzen der verwendeten Begrifflichkeit“ zu einem Überdenken und einer Neuorientierung der bisherigen Ansätze [HÉRITIER 1993a]. Einige der Kritikpunkte sollen beispielhaft und mit Relevanz für die vorliegende Fallstudie aufgegriffen werden. 
Während die Policy-Analyse in den ersten Ansätzen das politische System in den Mittelpunkt stellte, wurde in der Folge verstärkt der gesamtgesellschaftliche Kontext berücksichtigt. Die Schwierigkeiten derart umfangreicher empirischer Erfaßbarkeit machten jedoch wiederum Partialanalysen unumgänglich, die den Anspruch auf gesamtgesellschaftliche Relevanz wiederum schmälerten [KROTT 1990, S. 31].

Aus der anfänglich starken Betonung des politische Systems resultiert zudem der Vorwurf, die Policy-Analyse legte ein zu stark steuerungstheoretisch-instrumentell orientiertes Politikverständnis an, welches verschiedene ,menschliche“ Aspekte der Demokratie wie Fähigkeiten, Ideen etc. zu wenig berücksichtigte. Nicht zuletzt die allein auf den Programmerfolg ausgerichteten Evaluationsansätze der Policy-Analyse würden es wiederum unmöglich machen, die gesamtgesellschaftlichen Auswirkungen einer Policy zu erfassen [HÉRITIER 1993b, S. 14/15]. Auch die in dem Policy-Ansatz implizierte top down-Sicht der Programmimplementation wird als häufig nicht realitätsnah und daher analytisch weniger fruchtbar angesehen als der bottom up-Ansatz, der von politischen Entscheidungsinstanzen nicht völlig dominiert wird [VON PRITTWITZ 1990, S. 100].

Derartigen Kritikpunkten wird in der vorliegenden Fallstudie wiederum durch die bewußte Wahl einer Partialanalyse der PBWU-Forschungspolicy als Ausschnitt einer gesamtgesellschaftlichen Waldschadens-Policy und den Verzicht auf einen gesamtgesellschaftlichen Analyseanspruch begegnet. Steuerungsansätze nach dem top down- bzw. bottom up-Modell können zudem durch die im folgenden dargestellten moderneren Steuerungstheorien auf der intermediären Ebene zwischen Politik und Wissenschaft [BRAUN 1997, S. 45] ergänzt oder sogar ersetzt werden. Die Einbeziehung organisations- und wissenschaftssoziologischer Theorien sowie die genannten Vorteile der Zentrierung der Studie auf die Koordinierungsstelle PBWU in Anlehnung an den Ansatz der behördenorientierten Policy-Analyse sollen dem genannten Vorwurf eines zu stark steuerungstheoretisch orientierten Politikverständnisses entgegenwirken.

Einen zentralen Kritikpunkt stellt das stark schematisierende und damit vereinfachende Modell der Phasenabfolge der Politikgestaltung dar [WINDHOFF-HÉRITIER, 1987, S. 89; VON PRITTWITZ 1990, S. 99]. Die einzelnen Phasen würden sich in der Realität ,nicht funktional getrennt und logisch aneinanderreihen“, sondern sich , überschneiden, wiederholen und simultan verlaufen" oder überhaupt nicht auftreten. Vergleichbares gilt für die Akteure, welche mit ihrem Verhalten zu einer Überlagerung der unterschiedlichen Phasen beitrügen, in ihren Konstellationen und Handlungsplänen vielfältig seien und ständigem Wandel unterlägen [HÉRITIER 1993b, S. 11; WiNDHOFF-HÉRITIER 1987, S. 89; VON PRITTWITZ 1990, S. 100].

Die Neuorientierung versucht nun, die zentralen Kritikpunkte zu berücksichtigen und den analytischen Ansatz der Policy-Analyse entsprechend zu modifizieren [HÉRITIER 1993b, S. 16-19 und verschiedene Beiträge in HÉRITIER [1993a]. Dabei käme unter anderem das Phasensequenzschema ... nur noch als Hintergrundfolie zur Anwendung“. Die „Verflechtung von Akteuren würde ... als ein sehr viel komplexerer Prozeß betrachtet“", dem durch die Anwendung der sog. „Netzwerkanalyse“ und die Ent-Schematisierung des Zusammenwirkens staatlicher und privater Akteure Rechnung getragen werden könne. Dabei wird die Vorstellung revidiert, 
daß die staatlichen Akteure in einem Politikfeld den gesellschaftlichen Akteuren gegenüber immer eine dominante Rolle einnähmen. Ähnliches gilt auch für die Verflechtung einzelner Policies, die nur durch eine Analyse gegenseitiger Interdependenzen adäquat berücksichtigt werden könnten. Auch das „,zielgerichtete rationale Verhalten der Akteure“ wird ,als nur eine handlungsleitende Orientierung unter anderen“ betrachtet. Ideen, Werte und Überzeugungen sollten verstärkt einbezogen werden, da sie eine Schlüsselrolle bei der Politikgestaltung spielten.

Die vorliegende Studie greift die Kritik bzw. Neuorientierung der Policy-Theorie auf. Das starre Phasensequenzschema wird auf die Funktion eines groben, deskriptiv-historisch wie auswertungsleitenden Analyserahmens reduziert, das, wie bereits im Vorangegangenen dargestellt, Phasenüberschneidungen durchaus berücksichtigen kann. Auch die kritisierte Focussierung auf die politischen Akteure wird durch adäquate Berücksichtigung der Wissenschaft und deren formale, aber auch informale Interessenlage überwunden.

\subsubsection{Verschachtelung verschiedener Policies (Programmschachteln)}

Neben der Überschneidung von Phasen des Policy-Zyklus soll gesondert auf die Verflechtung verschiedener Policies eingegangen werden. Sie betrifft in vorliegender Fallstudie insbesondere die Einordnung der Forschungspolicy des PBWU-Projektes in den Rahmen einer übergeordneten Waldschadenspolicy. Wie sich bereits bei der theoriegestützten Charakterisierung des Policy-Zyklus, bezogen auf die Fallstudie, andeutete, lassen sich Bezüge und Zuordnungen einzelner Phasen zu verschiedenen Policies herstellen.

So nimmt die Thematisierung und Agenda-Gestaltung zur Forschungspolicy Bezug auf die allgemeine Thematisierung und Behandlung der übergeordneten Policy „Waldsterben“ allgemein, da Problem und Forschung hierzu eng miteinander verwoben sind. Eine Vermengung beider Policies in der historischen Darstellung und Analyse ist daher unvermeidlich. Zudem bestehen quasi unter dem Dach der Waldschadenspolicy - parallel mehrere Forschungspolicies in Form von Förderprogrammen verschiedener Ressorts, zwischen denen Interdependenzen bestehen.

In ähnlicher Weise treten Zuordnungsunschärfen zwischen verschiedenen Policies bspw. bei der Festlegung des PBWU-Forschungsrahmenprogramms auf. Die Erstellung des Rahmenprogramms kann bei Übertragung des Policy-Zyklus auf den im Zentrum der Studie stehenden PBWU-Forschungsprozeß problemlos als zentraler Bestandteil der Programmformulierung definiert werden, auf den als Implementationsphase die Umsetzung des Forschungsprogrammes folgt. Wird allerdings die Forschungspolicy auf das gesamte PBWU-Projekt bezogen, muß bereits der politische Beschluß zur Gründung des PBWU-Projektes als Politikformulierung verstanden werden; demgegenüber würde das Rahmenprogramm bereits ein Umsetzungsinstrument im Rahmen der Politikimplementation darstellen.

Vergleichbares gilt für die in der Endphase der Programmimplementation durchgeführte Neukonzeption des PBWU-Forschungsprogramms. Theorieorientiert stellt diese zwar eine „Neuformulierung“ dar, die als Variante zur „Policy-Terminierung“ im Rahmen dieser abschließenden Policy-Phase [WINDHOFF-HÉRITIER 1987, S. 65, 105ff] bearbeitet werden könn- 
te. Zumindest bei der Beschränkung der vorliegenden Studie auf den reinen Forschungsprozeß des PBWU-Programms wäre diese Zuordnung auch richtig. Die Studie greift jedoch weiter und macht das gesamte PBWU-Projekt, dessen zentraler Bestandteil allerdings das Forschungsprogramm ist, zum Gegenstand der empirischen Beschreibung und Policy-Analyse. Damit wird die Terminierungsphase die Beendigung des PBWU-Projektes als politische Maßnahme in den Mittelpunkt stellen, während die Neuformulierung des Forschungsprogramms noch als Bestandteil der Programmimplementation, der sie auch zeitlich zuzuordnen ist, gesehen und dort behandelt werden wird. Beide Beispiele beruhen - neben Effekten der Phasenüberlappung - wiederum auf unterschiedlicher Definition, Abgrenzung und Verflechtung zwischen verschiedenen Policies bzw. Policy-Ebenen.

Die skizzierten Befunde lassen sich mit dem Begriff der „Programm- oder PolicyVerschachtelung“ bezeichnen. Dies lehnt sich an die Begriffsdefinition von SCHARPF 1988 und TSEBELIS 1990 [zit. in: HÉRITIER 1993b, S. 19] an, welche politische Entscheidungsprozesse aufgrund der Verknüpfung verschiedener Policies als „verschachtelt“ (,nested“) bezeichnen. Die hier zu definierende Programmverschachtelung hebt weniger auf die Entscheidungsprozesse ab. Sie charakterisiert vielmehr die hierarchische (vertikale) Über- oder Unterordnung unterschiedlicher, aber inhaltlich benachbarter Policies bzw. Programme mit jeweiligen Verflechtungszusammenhängen. Über den Aspekt des parallelen (horizontalen) Nebeneinanders von Policies mitsamt den bestehenden, gegenseitiger Interdependenzen geht der Begriff der Verschachtelung erheblich hinaus.

Exemplarisch und mit Bezug auf die vorliegende Studie lassen sich derartige Programm- bzw. Politikverschachtelungen als Waldschadenspolicy $\rightarrow$ Waldschadens-Forschungspolicy $\rightarrow$ PBWU-Projekt $\rightarrow$ PBWU-Forschungsprogramm skizzieren. Dabei stellt die Waldschadenspolicy die äußerste Programmschale dar, das Forschungsprogramm die innerste. Auf die mögliche Erweiterung im Hinblick auf die Einbettung der Waldschadenspolicy in eine umfassende Umweltpolicy auf der einen Seite, die Ausweisung einer weiteren Sub-Policy auf der Ebene einzelner Forschungsvorhaben im Rahmen des PBWU-Forschungsprogrammes auf der anderen, soll zur Verdeutlichung des Konzeptes lediglich hingewiesen werden.

Eine schematische Darstellung der vertikalen Verschachtelung sowie der horizontalen ParallelPolicies wurde in Abbildung 3 vorgenommen. Dabei können Interdependenzen vertikal wie horizontal zwischen den einzelnen Policies bzw. Programm-Schachteln bestehen. Diese werden zudem überprägt durch die bereits erwähnten Überlappungen zwischen den verschiedenen Phasen der jeweiligen Policy-Zyklen. In Tabelle 1 wurde versucht, den verschiedenen Programmschachteln die Inhalte der jeweiligen Policy-Zyklen zuzuordnen.

Für die Schwerpunktsetzung der vorliegenden Studie auf den Prozeß der Programm- und Forschungssteuerung zwischen Politik und Wissenschaft wird die kombinierte Darstellung und Analyse der beiden die PBWU betreffenden Policies in den Mittelpunkt gestellt. Diese bezieht sich auf das PBWU-Projekt als Maßnahmenpolicy an sich, sowie das im Rahmen des Projektes durchgeführte Forschungsprogramm. Die weiteren, übergeordneten Policies (Programmschachteln) werden bedarfsweise dann, wenn es für die historische Entwicklung, das Verständnis und die Analyse des PBWU-Projektes erforderlich ist, miteinbezogen. 
Gewisse Kompromisse, die gegenüber dem idealtypischen Phasenmodell des Policy-Zyklus einerseits, der Abgrenzung verschiedener Policies voneinander andererseits eingegangen müssen, sind nötig. Sie erleichtern es, den empirischen Politikverlauf mit wichtigen Hypothesen zu verbinden und erhöhen den Erkenntniswert der Studie. 
Tab. 1: „Programmschachteln“ in der Phasenabfolge des Policy-Zyklus

\begin{tabular}{|c|c|c|c|c|}
\hline \multirow[b]{2}{*}{$\begin{array}{l}\text { Policy- } \\
\text { Zyklus }\end{array}$} & \multicolumn{4}{|c|}{ Policy-Programmschachteln } \\
\hline & $\begin{array}{l}\text { Waldschadens- } \\
\text { policy }\end{array}$ & $\begin{array}{l}\text { Waldschadens- } \\
\text { Forschungspolicy }\end{array}$ & $\begin{array}{c}\text { PBWU- } \\
\text { Forschungspolicy }\end{array}$ & $\begin{array}{l}\text { PBWU- } \\
\text { Forschungspro- } \\
\text { gramm }\end{array}$ \\
\hline $\begin{array}{l}\text { Problem- } \\
\text { definition } \\
\qquad \preceq\end{array}$ & $\begin{array}{l}\text { Erkennung von } \\
\text { Waldschäden als } \\
\text { Problem }\end{array}$ & $\begin{array}{l}\text { Waldschäden bestehen } \\
\text { als Problem; For- } \\
\text { schung als Problemlö- } \\
\text { sungsbeitrag }\end{array}$ & $\begin{array}{l}\text { Waldschäden bestehen } \\
\text { als Problem; ange- } \\
\text { wandte Ursachenfor- } \\
\text { schung als Problemlö- } \\
\text { sungsbeitrag }\end{array}$ & $\begin{array}{l}\text { Waldschäden und For- } \\
\text { schungsbedarf zur Ur- } \\
\text { sachenklärung beste- } \\
\text { hen }\end{array}$ \\
\hline $\begin{array}{l}\text { Agenda- } \\
\text { Gestaltung } \\
\qquad \downarrow\end{array}$ & $\begin{array}{l}\text { politische Behand- } \\
\text { lung des Themas } \\
\text { Waldschäden (Um- } \\
\text { fang, Ursachen, } \\
\text { Maßnahmen etc.) }\end{array}$ & $\begin{array}{l}\text { politische Behandlung } \\
\text { des Forschungsbedarfs }\end{array}$ & $\begin{array}{l}\text { politische Behandlung } \\
\text { des Forschungsbedarfs } \\
\text { bei Focussierung auf } \\
\text { angewandte, interdis- } \\
\text { ziplinäre und koordi- } \\
\text { nierte Forschung }\end{array}$ & $\begin{array}{c}\text { wissenschaftliche und } \\
\text { politische Diskussion } \\
\text { des bestehenden For- } \\
\text { schungsbedarfs bzw. } \\
\text { politischer For- } \\
\text { schungsrahmen- } \\
\text { bedingungen }\end{array}$ \\
\hline $\begin{array}{l}\text { Programm- } \\
\text { formulie- } \\
\text { rung } \\
\qquad\end{array}$ & $\begin{array}{l}\text { Maßnahmen- } \\
\text { formulierung: Zu- } \\
\text { standserhebung, } \\
\text { Luftreinhaltung, Sa- } \\
\text { nierung, Entschädi- } \\
\text { gung, Forschung etc. }\end{array}$ & $\begin{array}{l}\text { Maßnahmen- } \\
\text { formulierung im Be- } \\
\text { reich Forschung: Pro- } \\
\text { gramme versch. Res- } \\
\text { sorts etc. }\end{array}$ & $\begin{array}{l}\text { Formulierung und Be- } \\
\text { schluß zur Durchfüh- } \\
\text { rung des PBWU- } \\
\text { Projektes }\end{array}$ & $\begin{array}{l}\text { wissenschaftliche (und } \\
\text { politische) Formulie- } \\
\text { rung des Forschungs- } \\
\text { rahmenprogramms der } \\
\text { PBWU }\end{array}$ \\
\hline $\begin{array}{l}\text { Implemen- } \\
\text { tation } \\
\Omega\end{array}$ & $\begin{array}{l}\text { Umsetzung der Maß- } \\
\text { nahmenprogramme } \\
\text { in den o.g. Bereichen }\end{array}$ & $\begin{array}{l}\text { Umsetzung der Pro- } \\
\text { gramme (z.B. Kurato- } \\
\text { rium der LWF, } \\
\text { Forsch.- gruppe } \\
\text { Forsttoxikologie, } \\
\text { MAGL, PBWU etc.) }\end{array}$ & $\begin{array}{l}\text { Gründung, Etablie- } \\
\text { rung und Umsetzung } \\
\text { des PBWU-Projektes } \\
\text { auf Grundlage eines } \\
\text { Forschungsrahmen- } \\
\text { programms }\end{array}$ & $\begin{array}{l}\text { Umsetzung der } \\
\text { PBWU-Waldscha- } \\
\text { densforschung ent- } \\
\text { sprechend dem Pro- } \\
\text { grammrahmen }\end{array}$ \\
\hline $\begin{array}{l}\text { Evaluie- } \\
\text { rung } \\
\qquad \preceq\end{array}$ & $\begin{array}{l}\text { Bewertung der Maß- } \\
\text { nahmenwirkungen in } \\
\text { Hinblick auf das Pro- } \\
\text { blem Waldschäden }\end{array}$ & $\begin{array}{c}\text { Bewertung der jewei- } \\
\text { ligen Programmwir- } \\
\text { kungen }\end{array}$ & $\begin{array}{l}\text { Bewertung des } \\
\text { PBWU-Projektes }\end{array}$ & $\begin{array}{c}\text { Bewertung der } \\
\text { PBWU-Forschung }\end{array}$ \\
\hline $\begin{array}{l}\text { Terminie- } \\
\text { rung }\end{array}$ & $\begin{array}{c}\text { Beendigung der } \\
\text { Waldschadenspolicy } \\
\text { oder Neuformulie- } \\
\text { rung weiterer Poli- } \\
\text { cies }\end{array}$ & $\begin{array}{l}\text { Beendigung oder } \\
\text { Neuformulierung von } \\
\text { Programmen }\end{array}$ & $\begin{array}{l}\text { Beendigung des } \\
\text { PBWU-Projektes }\end{array}$ & $\begin{array}{l}\text { Beendigung des } \\
\text { PBWU-Forschungs- } \\
\text { programms oder } \\
\text { Neuformulierung der } \\
\text { Forschungskonzeption }\end{array}$ \\
\hline
\end{tabular}




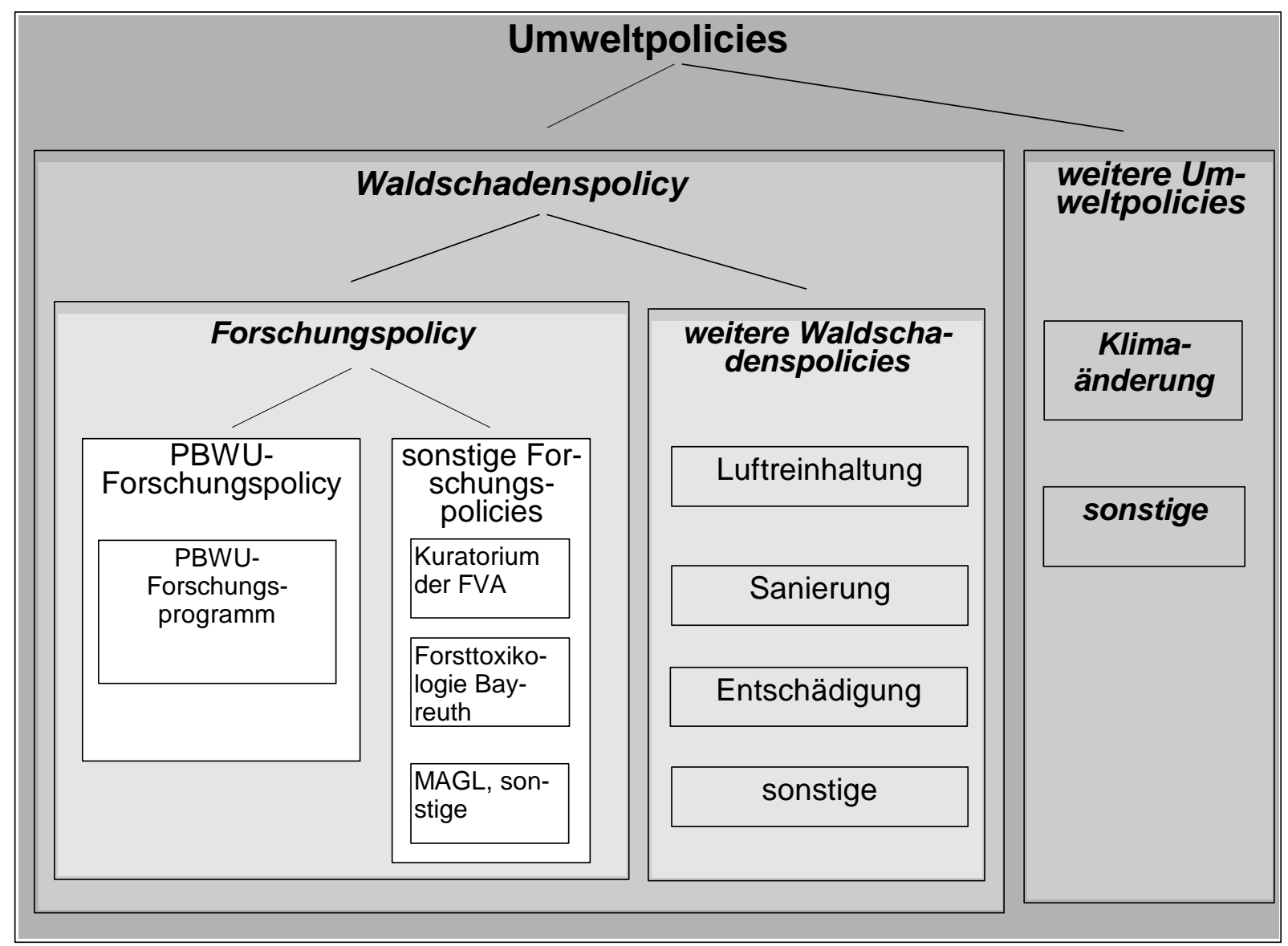

Abb. 3: Programmverschachtelung der Waldschadensforschung

\subsection{Organisations- und wissenschaftssoziologische Theorie}

Der Policy-Ansatz erlaubt es, Theorien der Organisationssoziologie, Wissenschaftsforschung und Forschungssteuerung in die Analyse miteinzubeziehen. Für die vorliegende Fallstudie ist dies zum Verständnis von staatlich-administrativem und Forscher-Handeln sogar zwingend notwendig.

Organisationssoziologische Bürokratie-Theorien sind von MAYNTZ [1985, S. 82ff] und wiederum von KROTT [1990, S. 34-42] ausführlich referiert und von letzterem hinsichtlich ihrer Eignung als Analysegrundlage für das politische Handeln von Behörden vergleichend bewertet. Beispielhaft sollen hier der Bürokratieansatz von MAX WEBER [1980] sowie der von DownS [1967] genannt werden. Während erstgenannter sich auf die „legale Herrschaft“ und damit unpolitisches Verwaltungshandeln stützt, sieht Downs die eigeninteressengesteuerten Verhaltensweisen der Beamten mit entsprechenden Machtressourcen als treibende Kraft. Auf derartigen theoretischen Grundlagen basierend kann im PBWU-Fallbeispiel ressortspezifisches Behördenhandeln analysiert werden, woraus letztlich Steuerungseinflüsse der Politik resultieren.

Theorien der soziologischen Wissenschaftsforschung über soziale Aspekte der Forschung betreffen in erster Linie die Interessengeleitetheit und soziale Bedingtheit wissenschaftlicher Erkenntnisproduktion, das Abhängigkeitsverhältnis zwischen Wissenschaft und Staat, sowie die 
Wissenschaft als Konkurrenzkampf [FELT et al. 1995, S. 75 ff; zum Waldschadensdiskurs s.a. DRESSEL 1995]. Diese Aspekte sollen hier nur erwähnt werden. Auf die Frage nach der Objektivität wissenschaftlicher Erkenntnisproduktion wird im Rahmen dieser Policy-Studie bei der gewählten Focussierung nicht näher eingegangen. Die weiteren genannten Aspekte werden in der Folge sowie bei der Analyse des Forschungsprozesses noch vertieft.

\subsubsection{Steuerungstheorie in der Forschung}

Die Studie zielt auf die Analyse des Forschungs- und Förderprozesses als Kräftespiel zwischen den Interessen von Forschungspolitik und Wissenschaft ab. Damit ist generell das Problem der Steuerbarkeit der Forschung berührt. Dabei handelt es sich um eine der zentralen Fragen der Wissenschaftsforschung bzw. der Wissenschafts- und Forschungspolitik, der weitenteils nur als „diffus“ zu beschreibende Prozesse zugrunde liegen [FELT et al. 1995, S. 213]. Nach BRAUN [1997, S. 19], der im europäischen Ländervergleich die staatlich institutionalisierten, intermediären Fördersysteme als Vermittlungsmechanismus der Forschungsförderung zwischen Politik und Wissenschaft untersucht, zeigen verschiedentlich durchgeführte Analysen nur vage, ,,wer wen steuert".

Dem Steuerungseinfluß des Finanzgebers, der über die Verteilung von Fördermitteln im Rahmen einer distributiven Policy erfolgt, steht der grundsätzliche Autonomieanspruch der Forscher zur wissenschaftlichen Erkenntnis gegenüber. Daraus ergibt sich das bereits erwähnte wechselseitige Abhängigkeitsverhältnis, in dem die Wissenschaft vom Staat die Finanzierung der Forschung erwartet, an der sie selbst interessiert ist, der Staat hingegen die Fördermittel unter der Bedingung vergibt, daß daraus ein kollektiver Nutzen entsteht [FELT et al. 1995, S. 209].

Die Forscher können sich auf ihre „monopolisierte kognitive Domäne“ berufen, allein für die Beurteilung von Wahrheitsfragen kompetent zu sein. Sie sind diesbezüglich steuerndem Einfluß in der Regel durch verfassungsrechtlichen Schutz entzogen. Während eine Einflußnahme in diesem Bereich lediglich innerhalb des Forschungsprozesses, wie der wissenschaftlichen Evaluierung der Forschung in der Antrags- oder Ergebnisphase, erfolgt, können forschungspolitische Steuerungsmaßnahmen forschungsextern darauf einwirken, ,, wo überhaupt, für wen und in welchem Umfang geforscht wird [HOHN \& SCHIMANK, 1990, S. 13 ff].

BRAUN [1997, S. 18 ff] arbeitet theoriegestützt heraus, daß das „Wissensmonopol“ der Wissenschaft mittels „freiwilliger Mitarbeit des Monopolisten“ aufgebrochen werden muß, wenn die Politik die Ressource Wissen nutzen will. Unter Berücksichtigung system- wie akteurstheoretischer Ansätze wird Forschungssteuerung - in Anlehnung an LUHMANN - kybernetisch als „Verringerung einer Differenz“ in den betreffenden Teilsystemen bzw. als „absichtsvolle Beeinflussung" der beteiligten Akteure definiert. Die politische Steuerung wird dabei als kollektiv organisierter und institutionalisierter Prozeß der strukturellen Kopplung zwischen politischen und teilsystemischen Akteuren verstanden [BRAUN 1997, S. 43-45]. Die Durchsetzungsfähigkeit der Politik ist dabei abhängig von verfügbaren Machtressourcen, möglichen Koalitionen, institutionellen Bedingungen und den verwendeten Strategien. 


\subsubsection{Thesen zur Steuerung mittels intermediärer Systeme}

Im gewählten Fallbeispiel erhebt der Staat mit Planung und Implementation des Waldschadensforschungsprogrammes in einer Art problemlösungsorientierter Auftragsforschung den Anspruch an Forschungssteuerung prioritär für sich. Die Installierung einer intermediären Projektgruppe soll es im Grundsatz ermöglichen, die Wissenschaft flexibler in staatliche Steuerungsziele einzubeziehen [BRAUN 1997, S. 81]. Andererseits gewährt sie der Forschung eine stärkere Einflußnahme auf den Förderprozeß und die Beibehaltung ihrer Autonomie.

Die wenn auch nur temporär installierte PBWU-Projektgruppe weist durchaus vergleichbare Strukturen, Interessenlagen und Handlungsmuster auf wie die von BRAUN untersuchten, langfristig etablierten Intermediär-Institutionen der nationalen Forschungsförderung. Seine zentralen Thesen zur Funktion und Aufgabe intermediärer Systeme in der Forschungssteuerung im Rahmen des Kräftespiels zwischen staatlicher Forschungspolitik und Wissenschaft [BRAUN 1997, S. 23, 47-65, 316] können daher auf das PBWU-Projekt übertragen werden.

Danach sind intermediäre Systeme als ,rational begründbare Steuerungserweiterung des politischen Handelns“ zu sehen. Dem liegt ein steuerungsoptimistischer Ansatz zugrunde, über zentral koordinierte Planungs- und Durchführungsverfahren im Verbund Politik und Wissenschaft forschungspolitisch selektieren zu können. Die politischen Akteure wollen die Wissenschaft sowie die intermediären Organisationen vereinnahmen, um eigene forschungspolitische Ziele zu definieren und umzusetzen (These 1).

Demgegenüber versucht die Wissenschaft, sich staatliche Kompetenzen und Ressourcen anzueignen und damit Definitionsmacht über die staatliche Mittelverteilung der Forschungsförderung zu erlangen oder zu erweitern. Die Vereinnahmungsabsicht gegenüber intermediären Systemen bestehen also gleichermaßen von Seiten der Wissenschaft wie der Politik (These 2).

Dieser moderne Ansatz, auf der Ebene struktureller Kopplungen und Verhandlungssysteme zwischen Politik und gesellschaftlichen Teilsystemen politisches Handeln sowie wechselseitige Einflußnahmen zu untersuchen [s.a. BRAUn 1997, S. 19, 44/45; s.a. HOHN \& SCHIMANK 1990, S. 15], kann auch in das Zentrum der vorliegenden Studie gestellt werden. Wechselnde Steuerungsdominanzen zwischen Politik und Wissenschaft, die das PBWU-Projekt in den verschiedenen Phasen des Forschungsprozesses prägen, können damit erklärt und bewertet werden.

Die These 3 formuliert, daß intermediäre Systeme eine Eigendynamik und Verselbständigungstendenzen gegenüber politischer Fremdbestimmung entwickeln und damit eine „Grauzone zwischen Politik und Wissenschaft" bilden [BRAun 1997, S. 60]. Sie trifft für das Fallbeispiel der intermediären PBWU-Projektgruppe zwar im Grundsatz, in Hinblick auf deren vergleichsweise geringen Institutionalisierungs- und Etablierungsgrad sowie temporären Charakter jedoch nur bedingt zu. Dennoch werden verschiedene Handlungsstrategien der Projektgruppe gegenüber Politik und Wissenschaft gerade unter dem Aspekt der Schaffung oder Sicherung ihrer Kompetenzen, Zuständigkeiten, Eigenständigkeit oder Existenz zu analysieren sein.

Der Ansatz wird ergänzt durch die Verwendung von bereits mehrfach erwähnten top downbzw. bottom up-Steuerungsmodellen der Implementationsforschung [s.a. WINDHOFF-HÈRITIER 
1987, S. 96/97]. Ohnehin korrespondieren politische Steuerungserweiterung eng mit dem top down-Ansatz politischer Rationalität, wissenschaftliche Einflußnahme mit dem bottom upAnsatz wissenschaftlicher Rationalität, wie in der Analyse des Forschungs- bzw. Förderprozesses noch vertieft werden wird.

\subsubsection{Angewandte Forschung versus Grundlagenforschung}

Hinsichtlich der Steuerungsproblematik muß grundsätzlich zwischen dem Typ der angewandten Forschung und dem der Grundlagenforschung unterschieden werden. Staatlich geförderte Industrieforschung soll in diesem Zusammenhang nicht berücksichtigt werden. Während angewandte Forschung spezifische, praktisch nutzbare Zielsetzungen oder Problemlösungen verfolgt, und in Form staatlicher Auftragsforschung durch die zielgerichtete Mittelvergabe charakterisiert ist, will die überwiegend an Hochschulen durchgeführte Grundlagenforschung neue Erkenntnisse über Phänomene und Tatsachen gewinnen, ohne daß damit auf konkrete An- oder Verwendung abgezielt wird [BUNDESMINISTERIUM FÜR FORSCHUNG UND TECHNOLOGIE 1982, zit. in: BRAUN 1997, S. 418 ff]. Letztgenannte ist daher hinsichtlich forschungspolitischer Steuerungseinflüsse als vergleichsweise autonom zu bezeichnen. Demgegenüber ist angewandte Forschung in stärkerem Maße vom Auftraggeber abhängig, was sich insbesondere bei der Auswahl der Forschungsthemen und der Ressourcenakquisition äußert [s.a. FELT et al. 1995, S. 226ff].

Der inzwischen stark gestiegene Ressourcenbedarf heutiger Forschung macht es jedoch für jede Art der Forschung erforderlich, ihren Finanzbedarf über die Forschungsleistung zu legitimieren. Naheliegend ist daher, auch deren Steuerung in erster Linie über die Finanzierung zu bewerkstelligen. Für angewandte oder Auftragsforschung ist dies ohnehin charakteristisch. Jedoch kann auch die Grundlagenforschung bspw. im Rahmen sog. Drittmittelförderung (Projektfinanzierung) durch Forschungsprogramme und Schwerpunktsetzung gesteuert werden“ [s.a. FELT et al. 1995, S. 226ff]. BRAUN [1997, S. 422] verwendet in diesem Zusammenhang den Begriff ,,problemorientierter Grundlagenforschung“, welche zwar aus wissenschaftlichem und Reputationsinteresse, aber gleichzeitig anwendungsorientiert betrieben würde.

Derartige, inhaltlich, zeitlich und finanziell begrenzte Projektfinanzierung bietet für den Auftraggeber neben dem zentralen Charakteristikum aktiver, zielgerichteter Forschungsthematisierung zur beabsichtigten Problemlösung weitere Vorteile: Die gegebene Mittelkonkurrenz verstärkt Ansporn und Erfolgsdruck auf die meist aktiven, flexiblen und jungen Forschergruppen; hohe Kreativität und Produktivität ist zu erwarten. Einer Leistungsbezogenheit der Forschung wird hier am ehesten Rechnung getragen. Allerdings setzen die Forscher vornehmlich auf relativ erfolgssichere Projekte, um die fachliche Beurteilung zu begünstigen [HILL \& RIESER 1983 S. 150].

Den „Antagonismus“ zwischen wissenschaftlichen Interessen der Selbstbestimmung und Grundlagenorientierung und politischen Interessen der Anwendungsorientierung und Forschungssteuerung [BRAUN 1997, S. 338] wird auch die Analyse des Fallbeispiels der PBWUWaldschadensforschung berücksichtigen müssen. Die Interessenkopplung grundlagen- mit anwendungsorientierter Forschung ist Strategie der Forscher und gleichzeitig Voraussetzung, 
sich am Programm zu beteiligen. Hier ist es die Entscheidung der Förderpolitik, ob und wieviel an Grundlagenforschung sie zum Verständnis und zur Aufklärung des Waldschadensphänomens zuläßt, um die Forscher für eine Beteiligung am Programm zu gewinnen.

\subsection{Festlegung des Analyserahmens}

Die obigen Ausführungen lassen erwarten, daß die empirische Studie zum PBWU-Projekt mit Hilfe des theoretischen Ansatzes der Politikfeldanalyse unter Berücksichtigung organisationsund wissenschaftssoziologischer einschließlich steuerungstheoretischer Grundlagen erfolgreich durchgeführt werden kann. Die Voraussetzungen hierfür sind gegeben. So handelt es sich bei der Waldschadensforschung um eine Maßnahme, die weitestgehend der Prozeßabfolge politischer Konfliktregelung entspricht. Es können, die Kritik an der starren Phasensequenz des Policy-Ansatzes durchaus im Hintergrund, im wesentlichen alle Phasen identifiziert und bearbeitet werden. Weiterhin können die Akteure bestimmt werden, die die Policy maßgeblich beeinflussen. Ihre Zielsetzungen, Instrumente und Handlungspläne zur Durchsetzung ihrer Interessen können analysiert werden. Die spezifischen Problemmerkmale des Themas Waldschäden generell, wie speziell der Waldschadensforschung, können hinlänglich beschrieben und bei der Analyse berücksichtigt werden.

In Hinblick auf die Verschachtelung verschiedener Policies kann die im Mittelpunkt stehende Forschungspolicy als Partialanalyse aus der gesamten Waldschadenspolicy herausgelöst werden, ohne den Erfolg der Analyse oder die Eignung der Policy-Analyse als Theoriehintergrund zu gefährden. Der Aspekt des Forschungsmanagements kann in sich geschlossen behandelt, das empirisch zu erfassende Analysematerial noch bewältigt werden.

Daher soll in der empirischen Bearbeitung des PBWU-Projektes entsprechend dem behördenorientierten Policy-Ansatz vorgegangen werden. Zum einen erscheinen die dort ausgearbeiteten theoretischen Grundlagen für die Analyse der an der Forschungspolicy beteiligten politisch-administrativen wie wissenschaftlichen Teilsysteme zutreffend und geeignet. Zum anderen soll von dem zentralen Charakteristikum der behördenorientierten Politikfeld-Analyse Gebrauch gemacht werden, eine Hauptbehörde - nämlich die PBWU-Projektgruppe - frei wählen und den weiteren beteiligten Akteuren gegenüberstellen zu können. Diese Zentrierung nimmt noch keine Aussage über deren Rolle im Prozeß der Forschungspolicy vorweg, erlaubt es aber, die Analyse mit besonderer Blickrichtung auf die zentrale Zielsetzung der Arbeit, nämlich die Analyse des Forschungsmanagements, hin zu orientieren. Daraus können anschließend die relevanten Faktoren, die den Forschungsprozeß bestimmen, abgeleitet werden.

Letztlich können die prozeßbestimmenden Faktoren zu entsprechenden Empfehlungen für die Organisation und Durchführung problemorientiert-angewandter, interdisziplinärer Forschung umgesetzt werden. Mit den als Exkurs bezeichneten Empfehlungen verläßt die Studie den sozialwissenschaftlichen, normativen Ansatz der Analyse und will für die Praxis des Forschungsmanagements werteorientierte, in Politik, Verwaltung und Wissenschaft taugliche Aussagen machen [s.a. KROTT 1996]. 


\section{Methodik}

Für die Policy-Analyse ${ }^{1}$ kann die für die Implementationsforschung getroffene Aussage übernommen werden, wonach diese an keine Festlegung bei den anzuwendenden Methoden gebunden ist [HuCKe \& WollmanN 1980, S. 216/217]. Prinzipiell steht das gesamte Methodenspektrum empirischer Sozialforschung zur Verfügung. Dabei werden jedoch in der Praxis politikwissenschaftlicher Forschung vorrangig wenig standardisierte, offene Verfahren angewendet, wobei generell die Beobachtung, die Inhalts- bzw. Dokumentenanalyse sowie die Befragung im Vordergrund stehen. Aufgrund der jeweiligen methodischen Vor- und Nachteile werden verschiedene empirische Erhebungsmethoden häufig kombinbiert.

Die vorliegende Arbeit entspricht der Empfehlung, verschiedene Verfahren zu einem multimethodischen Ansatz zu kombinieren [HuCKE \& Wollmann 1980, S. 221]. Sie wählt für die Policy-Analyse der Waldschadensforschung im PBWU-Projekt die qualitativ ausgerichteten Ansätze der „teilnehmenden Beobachtung“ in Kombination mit der „Dokumentenanalyse“. Ein besonders herauszuarbeitender Aspekt wird durch eine „,quantitative Inhaltsanalyse“ ergänzt, welche im Rahmen einer Diplomarbeit durchgeführt wurde [STEUER 1997].

\subsection{Retrospektive teilnehmende Beobachtung}

Die Anwendung der teilnehmenden Beobachtung ist die Besonderheit der vorliegenden Analyse. Der Verfasser der Arbeit war über die gesamte Laufzeit des PBWU-Projektes Mitarbeiter bzw. Leiter der Projektgruppe und kann somit als teilnehmender Beobachter verstanden werden. Als besonderes Charakteristikum muß jedoch herausgestellt werden, daß der Verfasser als beteiligter PBWU-Akteur mit dementsprechend ausgerichtetem Handlungsplan und Verhalten systemimmanenter Bestandteil des Untersuchungsgegenstands, der Forschungspolicy, war. Nach der Definition von von ALEMANn [1995, S. 110/111] kann dies als eine teilnehmende Beobachtung im Sinn einer Identifizierung mit der Akteursrolle in der Forschungspolicy verstanden werden.

Die Durchführung einer politik- und sozialwissenschaftlichen Analyse der Forschungspolicy war zum Zeitpunkt der teilnehmenden Beobachtung allerdings keineswegs geplant oder absehbar. Die Perspektive einer Beobachtung als sozialwissenschaftliche Methode mit entsprechender Zielsetzung der Analyse der Forschungspolicy war damals also nicht gegeben. Die teilnehmende Beobachtung muß in dem konkreten Anwendungsfall daher mit dem Zusatz ,retrospektiv“ versehen werden. Erst und ausschließlich nach Ablauf des Projektes und zur Durchführung vorliegender Studie wendet der Verfasser seine Funktion als ehemaliger PBWU-Akteur zu der des - retrospektiv - beobachtenden sozialwissenschaftlichen Forschers.

\footnotetext{
${ }^{1}$ Auch bei der Policy-Analyse selbst handelt es sich streng genommen um ein methodisches Instrumentarium, das auf dem theoretischen Modell des Policy-Zyklus beruht. Aufgrund der Nähe zur Theorie wurde die PolicyAnalyse jedoch im dortigen Kapitel behandelt und hier ausschließlich auf die klassischen, sozialempirischen Methoden abgehoben, die in der Studie zum Einsatz kamen.
} 
Aus dieser personellen Konstellation ergeben sich besondere Vorteile, die kaum auf einen externen, teilnehmenden Beobachter zutreffen dürften und sich für eine Nutzung in Form einer sozialwissenschaftlichen Auswertung geradezu anbieten. Sie bestehen in dem nahezu uneingeschränkten Zugang des Verfassers zum Forschungsfeld, hohen Verständnis- und Detailkenntnisgrad und weitreichenden Einblick in die formale, aber eben auch informale Wirklichkeit im Projekt, welche sich mit keiner anderen sozialempirischen Erhebungsmethodik gleichwertig erschließen ließe. Der Umstand, daß der Verfasser als ehemaliger PBWU-Akteur ohne jedwede sozial- oder politikwissenschaftliche Analyse-Absicht Bestandteil der Policy war, kann als ein weiterer methodischen Vorteil gewertet werden. Die teilnehmende Beobachtung ist somit sozialwissenschaftlich unbelastet, in keiner Weise durch die Einflußnahme des Verfassers als Sozial-Forscher auf das Untersuchungsobjekt verzerrt oder gestört; sie erhält damit einen weitgehend nicht-reaktiven Charakter.

Den genannten Vorteilen stehen jedoch methodische Nachteile gegenüber. So besteht in dem Fall der retrospektiven teilnehmenden Beobachtung keine Möglichkeit mehr, die Vorgehensweise der Datenerhebung zu strukturieren und dem Ziel der Arbeit anzupassen. Die Beobachtung muß wie erfolgt als Analysegrundlage übernommen werden. Weiterhin bedingt der retrospektive Charakter der teilnehmenden Beobachtung, daß z.T. inzwischen weit zurückliegende formale wie informale Tatbestände in Erinnerung gebracht werden müssen. Das methodisch bedingte Problem subjektiven Wahrnehmungs- bzw. Erinnerungsvermögens des Forschers wird allein durch die erhebliche Zeitspanne zwischen Beobachtung und Auswertung noch verstärkt. Auch kann unterstellt werden, daß die Beteiligten-Perspektive zur Verschleierung oder Verheimlichung bestimmter Fakten oder Vorgänge führte. Der PBWU-Akteur wurde im Zuge der Forschungspolicy von der einen oder anderen Beratung oder Schriftstückkenntnis bewußt ausgeschlossen. Dadurch werden Fakten geschaffen, die sich nun der Analyse, zumindest aus der teilnehmenden Beobachtung, entziehen.

Weiterhin muß davon ausgegangen werden, daß der Blickwinkel des ehemaligens Erlebens als PBWU-Akteur den der nun erfolgten, sozialwissenschaftlichen Analyse durch ein und dieselbe Person subjektiv-selektiv überprägt, da er eine Art reflexive wie retrospektive Selbstbeurteilung darstellt. Der Sozialforscher ,fungiert als Methode, die sich selbst anwendet“ [ATTESLANDER 1993, S. 105]; der Forscher beurteilt sich selbst, wenn auch in unterschiedlicher Funktion. Dies kann sowohl für eine bestimmte Wahrnehmungs- oder Beurteilungsperspektive einzelner, während der teilnehmenden Beobachtung akquirierter Tatbestände zutreffen, als auch für die subjektive Gesamteinschätzung ganzer Akteursgruppen, bestanden doch Akteurskonstellationen, die sich als Gegner- oder Verbündeten-Positionen im Projekt beschreiben lassen. Erfordert allein die reflexive Beschreibung der Erfahrungen eines methodisch üblicherweise externen Sozialforschers als teilnehmender Beobachter die Einführung von Kontroll- und Distanzierungsstrategien, so trifft dies für die hier gegebene Situation, Beobachter und Beobachteter in einer Person, in besonderem Maße zu.

\subsection{Kombination mit Dokumentenanalyse}

Die Dokumentenanalyse wurde gleichwertig miteinbezogen, um einerseits umfangreiche zusätzliche Informationen miteinzubeziehen, zum anderen das „Insider-Wissen“ und die Kennt- 
nisse über den Verlauf der Policy aus der teilnehmenden Beobachtung schriftlich belegen zu können. Letztere Vorgehensweise lehnt sich an die sog. „Triangulation“, eine Arbeitstechnik aus dem Journalismus an, Informationen erst dann als wahr zu akzeptieren, wenn sie durch eine andere Quelle bestätigt worden sind [FLICK et al. 1995, S. 414].

Mit dieser zeitstabilen, nicht-reaktiven Erhebungsmethode zumindest für die objektive Erfassung der formalen Wirklichkeit kann der potentielle Einfluß subjektiv-selektiven Wahrnehmungs- und Erinnerungsvermögens des Durchführenden als gleichzeitig Bewertender und Bewerteter korrigiert werden. Zusätzlich können Tatbestände oder Vorgänge, die dem teilnehmenden Beobachter als betroffenem PBWU-Akteur aus welchen strategischen oder anderen Gründen auch immer verborgen blieben, im Rahmen ihrer faktischen Zugänglichkeit berücksichtigt werden. Die Objektivierung des methodischen Gesamtansatzes findet allerdings dort ihre Grenzen, wo es um die Erhebung und Interpretation nicht schriftlich fixierter Fakten oder Handlungen geht, die sich einer Dokumentenanalyse entziehen.

\subsection{Verwendete Materialien}

Dem Verfasser standen, resultierend aus seiner ehemaligen PBWU-Funktion und durch das Entgegenkommen des GSF-Forschungszentrums, nach Abschluß des Projektes das gesamte vorhandene Akten- und sonstiges Dokumentenmaterial der PBWU zur Verfügung. Dies stellt wiederum eine günstige Rahmenbedingung dar, die die Durchführung der Dokumentenanalyse erleichtert und auf eine breite Datenbasis stellt. Weiterhin bestehen eigene Aufzeichnungen des Verfassers aus seiner Zeit als Angehöriger der Projektgruppe PBWU. Ergänzend wurde Dokumentenmaterial von einzelnen, insbesondere in den Gremien der PBWU beteiligter Wissenschaftler auf Anfrage zur Verfügung gestellt.

Bei dem Dokumentenmaterial handelt es sich insbesondere um folgende Dokumenttypen:

- Schriftsätze (Aktendokumente) von Institutionen/Behörden, insbesondere Ministerien und Forschungsinstitutionen, der Projektgruppe PBWU sowie von sonstigenEinzelpersonen,

- Statistiken der PBWU oder beteiligter Ministerien zur Forschungsförderung,

- Aktenvermerke, Telephonnotizen, Randnotizen, etc.,

- Sitzungsprotokolle und Drucksachen des Bayerischen Landtags und Senats,

- Sitzungsprotokolle der PBWU-Gremien, Arbeitsgruppen etc.,

- Forschungsanträge, einschließlich begleitender Schriftwechsel zu Antragstellung, Begutachtung und Förderentscheidung,

- externe Fachgutachten,

- Verträge, Rahmenvereinbarungen etc.

- Forschungsberichte (Zwischen- und Schlußberichte),

- Stellungnahmen zu Forschungsberichten,

- Forschungsprogramme, -konzeptionen etc.,

- einschlägige Veröffentlichungen (wissenschaftl. bzw. populärwissenschaftliche Publikationen, Proceedings zu Status- und Querschnittsseminaren, sog. „graue Literatur“ etc.),

- Medienberichterstattungen. 
Die Heterogenität der Datentypen ließ von einer quantitativen oder qualitativen, stark schematisch-statistisch geprägten Inhaltsanalyse schriftlicher Quellen abraten. Eine derartige Verarbeitung des gesamten Dokumentenbestandes zum Thema, wie sie z.B. von JAKOBS [1994] im Rahmen eines forstpolitischen Beitrags zur Implementationsforschung (,Praxisversuch Energieproduktion") vorgenommen wurde, hätte erhebliche Probleme bei der Erstellung eines operationellen, einheitlichen und kategorisierbaren Erhebungs- bzw. Auswertungsmodus bereitet. Sie hätte weiterhin den Umfang der Arbeit stark erweitert und den Blick nicht unbedingt auf die wesentlichen Textbestandteile und deren kontextualen Zusammenhänge gelenkt. Zudem hätten durch eine schematische Inhaltsanalyse gerade die prozeßorientierte Dynamik der Policy und die akteurstheoretische Betrachtung mit dem Auftreten spezifischer Akteurskonstellationen und der Rolle einzelner Beteiligter, den jeweiligen Interessenlagen und Machtmitteln, nicht ausreichend beschrieben, rekonstruiert und interpretiert werden können. Hier bot sich vielmehr die qualitative Auswertung der Textdokumente vor dem Kenntnishintergrund aus der teilnehmenden Beobachtung an.

\subsection{Ergänzende quantitative Inhaltsanalyse}

Zusätzlich wurde für einen zentralen, abgrenzbaren Ausschnitt der Untersuchung, nämlich den des Verfahrens der PBWU-Forschungsförderung, eine quantitative Inhaltsanalyse im Rahmen der Diplomarbeit „Management von Forschungsprojekten am Beispiel der PBWU-Waldschadensforschung von 1984 bis 1993“ durchgeführt [STEUER 1997]. Im Mittelpunkt dieser Teilstudie steht die Analyse der einzelnen Forschungsvorhaben anhand eines Kategoriensystems. Die statistische Auswertung erlaubt die Charakterisierung des Förderverlaufes und der Förderstruktur insgesamt und speziell der Entscheidungsfindung zur Forschungsförderung unter Berücksichtigung der beteiligten Akteure.

\subsection{Verzicht auf Befragung}

Auf den Einsatz weiterer Erhebungsmethoden empirischer Sozialforschung wurde bewußt verzichtet. Einerseits standen ausreichend andere, geeignete Verfahren zur Verfügung, deren fallspezifische Vorteile insbesondere aufgrund der personellen Konstruktion (Beteiligung des Verfassers an der PBWU) voll genutzt werden konnten. Andererseits wären auch bei der Anwendung einer Befragungstechnik (z.B. Interview) methodische Probleme aufgetreten. So könnte - ähnlich der teilnehmenden Beobachtung - die wiederum retrospektive Einschätzung der Befragten von subjektiv-selektiver Wahrnehmung oder Erinnerung überprägt sein und die Wirklichkeit nur verzerrt wiedergegeben werden. Vergleichbare Verfälschungen der aus einer Befragung ableitbarer Faktenlage dürften sich aufgrund der fallspezifischen Konstellation des Interviewers und des Befragten ergeben. Aus der jeweiligen Zugehörigkeit der Interviewpartner zu einer Akteursgruppe der PBWU-Forschungspolicy resultierte retrospektiv eine wie auch immer geartete persönliche Einstellung zum Fragesteller, die sich subjektiv auf das Interview ausgewirkt hätte. Letztlich ist anzunehmen, daß es beim Interviewpartner ein gewisses Mißtrauen gegenüber dem Interviewer als ehemaligem PBWU-Akteur provoziert hätte, demjenigen Informationen zu liefern, der ohnehin beteiligt war und selbst in hohem Maße über InsiderWissen verfügte. 
Trotz dieser methodischen Vorbehalte wurden im Rahmen vorliegender Arbeit einzelne informativ-ergänzende, aber weitgehend unsystematische Gespräche mit Beteiligten durchgeführt, ohne daß dies jedoch als adäquater Methodikeinsatz der mündlichen Befragung zu bewerten wäre.

\subsection{Methodische Kontrollinstrumente und Anonymisierung personenbezogener Informationen}

Ein Kontrollinstrument zur methodischen Objektivierung der vorliegenden Arbeit, wie es für die teilnehmende Beobachtung gefordert wird [ATTESLANDER 1993, S. 106/110; FRIEDRICHS 1980, S. 289/304], stellt der enge Theoriebezug dar. Die einschlägigen Theoriebausteine, und hier insbesondere die zur Policy-Analyse, zum Handeln von Behörden und der Wissenschaft, sind im Theorie-Kapitel ausführlich dargestellt. Die anschließende, empirisch mit den Methodikinstrumenten teilnehmende Beobachtung und Dokumentenanalyse nachgezeichnete Forschungspolicy nimmt in ihrer Analyse wiederum engen Bezug zur Theorie. Damit kann die bloße historische Aufarbeitung des Themas aus der Sicht eines Betroffenen erweitert und eine theoriegestützte, struktur- und prozeßorientierte Analyse der Forschung auf der Grundlage eines akteurstheoretischen Bezugsrahmens vorgenommen werden.

Letztlich kann die stetige Diskussion und Abstimmung des Verfassers mit dem Betreuer der Arbeit unter anderem als Supervision der Feldforschung verstanden werden. Dies diente dazu, bei Durchführung der Studie in selbstreflektierenden Gespräche die erforderliche „Distanzperspektive“ zu gewährleisten, und die einseitige Identifikation mit der Teilnehmerrolle im Feld (,going native“) zu vermeiden [ATTESLANDER 1993, S. 110, FLICK et al. 1995, S. 192, FRIEDRICHS 1980, S. 304], die beim Verfasser während der teilnehmenden Beobachtung ja tatsächlich bestanden hatte. Grundsätzlich kann unterstellt werden, daß der Verfasser trotz ehemaliger persönlicher Partizipation an dem Untersuchungsobjekt weitestgehend wertneutral, vorurteils- und außerwissenschaftlich-absichtsfrei an die Studie heranging.

Die Nutzung personenbezogener Informationen sowie weiterer, nicht öffentlicher Dokumente oder mündlicher Mitteilungen, die im Rahmen der teilnehmenden Beobachtung bzw. bei der Erstellung vorliegender Studie gewonnen worden waren, macht zum Schutze der Informanten und aus Gründen des Datenschutzes eine Anonymisierung erforderlich. Die Zitierung derartiger Dokumente bzw. mündlicher Informationen erfolgt in anonymisierter Form. Im Text findet sich lediglich der Quellenbezug (,i.Q.“ = interne Quelle) mit Angabe der Informationsquelle und des Entstehungsdatums. Dabei sind Dokumente von Einzelpersonen - soweit möglich unter den Institutionen aufgeführt, denen sie sich zuordnen lassen, z.B. einzelne Mitglieder des Wissenschaftlichen Beirats diesem Gremium. Bei Protokollen ist als Entstehungsdatum der Sitzungstermin angegeben, um die Zuordnung zu erleichtern. Die Zitierung läßt offen, ob und um welche Art von Schriftstücken, schriftlichen Notizen und ähnlichem es sich handelt, oder um mündliche Informationen. 


\subsection{Forschungsansatz Fallstudie}

Die bayerische Waldschadensforschung im Rahmen des PBWU-Projektes stellt insbesondere mit den eindeutig definierten Markierungen der Gründung und Beendigung des Projektes ein in sich geschlossenes Policy-Objekt aus dem Bereich der Umweltforschung dar. Damit eignet sich das Projekt grundsätzlich als Untersuchungsgegenstand nach dem Forschungsansatz einer Fallstudie, welche sich im Gegensatz zu vergleichenden Untersuchungen definitionsgemäß auf die themenorientiere Analyse eines einzelnen Objektes bezieht [VON ALEMANN 1995, S. 364].

Die Fallstudie leistet insbesondere die ,genaue Beschreibung des beobachteten Objektes“ und betrachtet bei der erforderlichen, zudem aber ressourcensparenden Ausschnittsbildung eine „erhebliche Fülle empirischer Vorgänge“ [KROTT 1990, S. 87/88]. Sie „soll einen Einblick in das Zusammenwirken vieler Faktoren liefern und dabei typische Vorgänge der Wirklichkeit erhellen oder die Identifikation von Handlungsmustern ermöglichen“. Meist werden hierfür mehrere Methoden eingesetzt, um methodische Fehler zu minimieren und das Objekt aus verschiedenen Perspektiven zu beleuchten [LAMNEK 1989, zit. in: WEBER 1993, S. 9].

Dabei wird der Typ der ,interpretativen Fallstudie“ gewählt. Diese beinhaltet - im Gegensatz zur deskriptiven Fallstudie - eine Theorie als Bezugspunkt und untersucht das Objekt über die reine Beschreibung hinaus entsprechend theorieorientiert [LIJPHART 1977; ECKSTEIN 1975, beide zit. in: KROTT 1990, S. 88 bzw. 1994a, S. 16; von AlEMANn 1995, S. 364]. Im vorliegenden Fall sind es insbesondere politik- und sozialwissenschaftliche Grundlagen der PolicyTheorie, die unter Berücksichtigung des Akteur-Modells auf den empirisch ermittelten Prozeß der staatlichen Forschungsförderung angewendet werden.

Nach KROTT [1990, S. 90] ist es erforderlich, das Untersuchungsobjekt auf seine Eignung für die Durchführung einer interpretativen Fallstudie hin zu überprüfen. Diese „Auswahl des geeignetsten Falls" bezieht sich auf die Kriterien des theoretischen Ertrags, des praktischen Nutzens und der Durchführbarkeit, welche in hohem Maße erfüllt sein sollen. Bezogen auf das vorliegende Objekt der PBWU-Waldschadensforschung kann konstatiert werden:

- Die theoretischen Grundlagen des Policy-Ansatzes können mittels Policy-Analyse und ergänzender politik- und sozialwissenschaftlicher Theorien in der Fallstudie zur Forschungspolicy zutreffend, sinnvoll und theoretisch gewinnbringend eingesetzt werden.

- Die Forschungspolicy stellt ein relevantes Thema dar mit allgemein gültigen wie spezifischen Eigenschaften und Merkmalen, die eine Behandlung an sich sowie die Übertragung auf vergleichbare Umweltforschung sinnvoll und machbar erscheinen lassen.

- Die Durchführung einer Fallstudie ist realistisch. Umfangreiche, empirisch gewonnene Informationen und Daten aus teilnehmender Beobachtung und aus Dokumentenanalyse liegen vor. Das PBWU-Forschungsprojekt wurde 1993 beendet und kann damit als in sich geschlossener und abgeschlossener Gesamtkomplex gesehen und bearbeitet werden.

- Die Beteiligung des Verfassers an dem PBWU-Projekt als Mitarbeiter bzw. Leiter der Projektgruppe begünstigt methodisch die Durchführung einer Fallstudie.

Damit scheinen die Voraussetzungen zur erfolgversprechenden Durchführung einer interpretativen Fallstudie gegeben zu sein, ein entsprechendes Vorgehen erscheint gerechtfertigt. 


\section{$4 \quad$ Akteure des PBWU-Projektes}

\subsection{Akteursstruktur}

Die gewählte Focussierung der Arbeit auf den Forschungs- und Förderprozeß im PBWUProjekt bestimmt auch die Zusammensetzung der zu berücksichtigenden Akteure. Der politische Beschluß (Beschluß des Bayerischen Ministerrats vom 5.6.1984) zur Gründung des PBWU-Projektes sowie erste Verfahrensvereinbarungen während der Etablierungsphase legen die Organisations- und damit die Akteursstruktur zum Projekt weitgehend fest. Danach können folgende, der Politik bzw. Wissenschaft zuzuordnenden Akteure ausgewiesen und im folgenden kurz charakterisiert werden.

Die zwischen Politik und Wissenschaft an der Großforschungseinrichtung angesiedelte, mit Koordinierungsaufgaben betraute Projektgruppe Bayern zur Erforschung der Wirkung von Umweltschadstoffen (PBWU) wird entsprechend dem behördenorientierten Policy-Ansatz [KROTT 1990, S. 45ff] als Hauptakteur festgelegt. Auch in Hinblick auf die Analyse des Kräftespiels forschungspolitischer Steuerung der Wissenschaft nach steuerungstheoretischen Ansätzen [BRAUN 1997] kommt der intermediären Organisationseinheit eine zentrale Rolle und Bedeutung zu. Nicht zuletzt sprechen methodische Gründe bei der Durchführung vorliegender Arbeit für eine derartige Zentrierung.

Das förderentscheidende Gremium des Projektrates stellt die politisch orientierte Akteursgruppe dar. Neben weiteren mit Forschung befaßten Ressorts, die in dem Gremium vertreten sind, nehmen das federführende und projektfinanzierende Bayerische Umweltministerium sowie die Großforschungseinrichtung GSF, die ehemalige Gesellschaft für Strahlen- und Umweltforschung mbH., Neuherberg (heute: GSF-Forschungszentrum für Umwelt und Gesundheit $\mathrm{GmbH}$ ), als der Projektgruppe dienstvorgesetzte und sie beherbergende Institution eine Sonderstellung ein.

Den politischen stehen die wissenschaftlich orientierten Akteure im PBWU-Projekt gegenüber. Hierzu zählt insbesondere das fachliche Beratungsgremium Wissenschaftlicher Beirat, bestehend aus mehreren renommierten und in der Waldschadensforschung meist selbst involvierten Forscherpersönlichkeiten. Weiterhin zählen zu den wissenschaftlichen Akteuren die in das Förderverfahren regelmäßig einbezogenen externen Fachgutachter. Als Adressaten des Förderprogramms stellen die Waldschadensforscher als Antragsteller und eigentliche Forschungsdurchführende eine weitere relevante, wissenschaftlich orientierte Akteursgruppe des PBWUProjektes dar.

Letzlich sind externe Forschungs- oder Koordinierungseinrichtungen des Bundes oder der Länder zu nennen, mit denen, ihren Aufgaben entsprechend, insbesondere die Projektgruppe in gewisser, wenn auch für das Projekt an sich peripherer Beziehung steht.

Die Abbildung 4 gibt eine Übersicht über die beteiligten Akteure und deren strukturelle Verknüpfungen. 


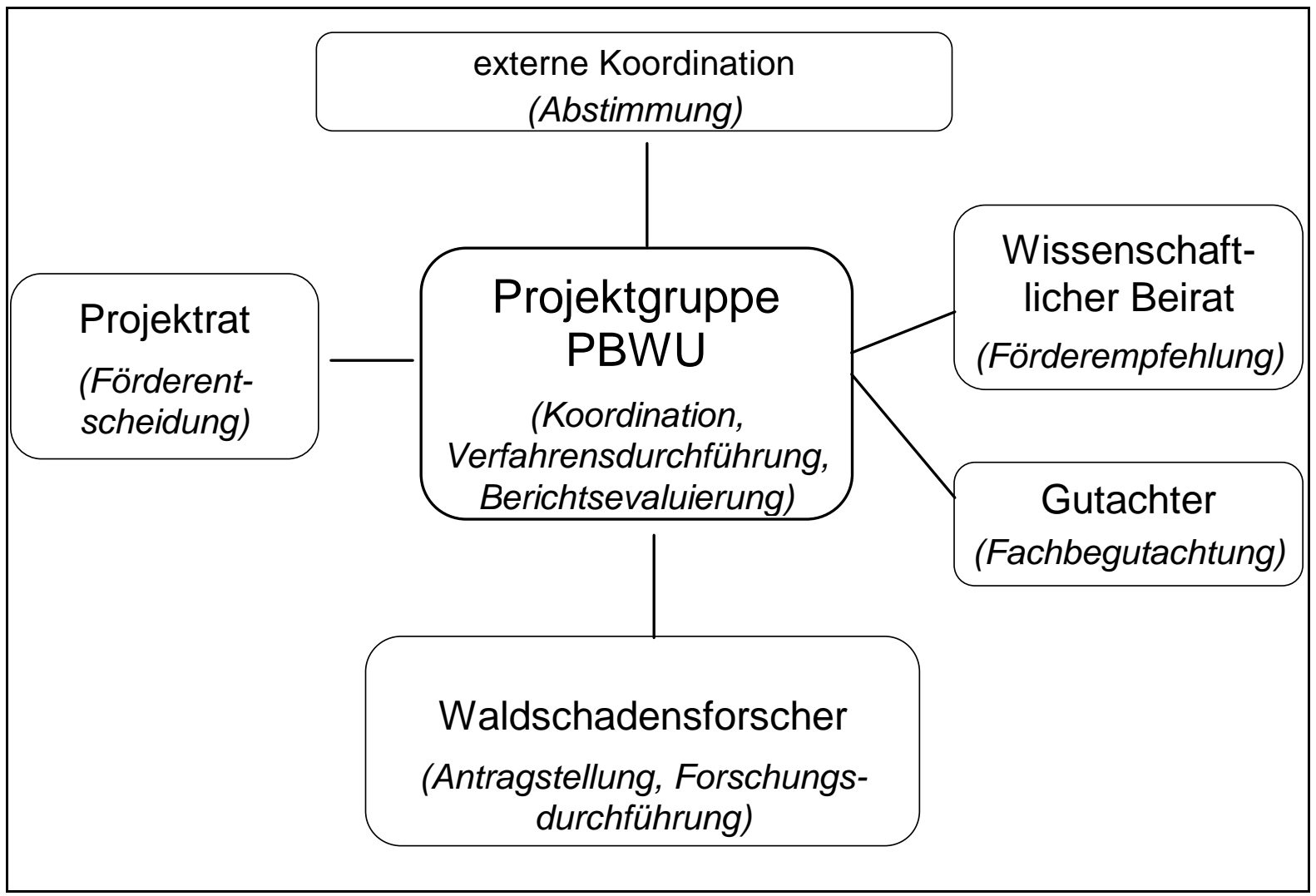

Abb. 4: Übersicht über die Organisations- und Verfahrensstruktur im PBWU-Projekt

Auf eine explizite Darstellung weiterer, bei der gewählten Ausschnittsbildung der Forschungspolicy als extern zu bezeichnende Akteure soll hier verzichtet werden. Damit wird der Einfluß derartiger Akteure wie der Medien und Öffentlichkeit, politischer wie fachlicher Interessenvertreter, der Forstpraxis etc. auf das PBWU-Projekt keinesfalls übersehen. Im Hinblick auf die zentrale Fragestellung nach der Forschungssteuerung im PBWU-Projekt soll sich die Analyse jedoch direkt auf die steuerungsrelevanten Akteure beziehen und die indirekten Einflüsse anderer Akteure nur soweit erfassen, als sie im Prozeß empirisch sichtbar werden.

Der Analyse des Forschungs- bzw. Förderprozesses im PBWU-Projekt wird die Charakterisierung der beteiligten wissenschaftlichen wie politischen Akteure vorangestellt. Diese werden hinsichtlich ihrer spezifischen Aufgaben, Interessenpositionen, formalen wie informalen Handlungspläne und Ressourcen vorgestellt. Auf interessenbedingte Konflikte zwischen den Akteuren wird im Grundsatz eingegangen. Detaillierte Handlungsstrategien bleiben jedoch der anschließenden Analyse vorbehalten. 


\subsection{Charakterisierung der einzelnen Akteure}

\subsubsection{Projektgruppe Bayern zur Erforschung der Wirkung von Umweltschadstoffen (PBWU)}

Die PBWU-Projektgruppe wurde 1984 auf Beschluß des Bayerischen Ministerrats auf Grundlage eines Vertrags des Bayerischen Umweltministeriums mit dem GSF-Forschungszentrum gegründet. Sie setzt sich zusammen aus 3 Naturwissenschaftlern unterschiedlicher, für die Waldschadensforschung relevanter Fachrichtungen und der entsprechenden Infrastruktur (Sekretariat etc.). Die Projektgruppe kann als Projektträger des bayerischen Umweltressorts bezeichnet werden. Wie für derartige Organisations- bzw. Administrationseinheiten zur Forschungsförderung üblich [BRAUN 1997, S. 231ff], ist sie dem Ministerium gegenüber weisungsgebunden, insbesondere in Förderentscheidungen, personalrechtlich und administrativ jedoch in die Großforschungseinrichtung GSF eingegliedert. Entsprechend ihrem intermediären Charakter steht die Projektgruppe zwischen den politischen und wissenschaftlichen Fronten. Sie ist dem Druck, die Interessen aus Politik und Wissenschaft zu vereinbaren, direkt ausgesetzt [BRAUN 1997, S. 351]. Ihre größere Nähe zur Politik ergibt sich aus der vertraglichen Anbindung an ihren Auftraggeber, als dessen ,,verlängerter Arm“ sie handelt.

Aufgabe und damit formaler Handlungsplan der Projektgruppe ist es, für ihren politischen Auftraggeber als Koordinationsstelle für die Waldschadensforschung in Bayern zu fungieren. Dies bedeutet aus steuerungstheoretischer Sicht die Vereinbarung politischer und wissenschaftlicher Interessen, wobei wissenschaftliche Qualitätskriterien der Forschung ebenso zu berücksichtigen sind wie politische Relevanzkriterien [BRAUN 1997, S. 351]. Hierzu wird ein eigenes Förderprogramm aufgelegt, das dem politischen Auftrag zur Verstärkung der Waldschadensforschung Rechnung tragen soll. Wesentliche Aufgaben der Projektgruppe bestehen daher in der entsprechenden Programmerstellung und -implementation sowie der organisatorischen Verfahrensdurchführung zur Forschungsförderung.

Neben den formalen Aufgaben muß der Projektgruppe informal an ihrer Existenzsicherung gelegen sein. Dem dient die möglichst umfassende und verantwortliche Ausfüllung der Koordinierungsaufgaben. Dem Streben nach Existenzsicherung käme ein umfangreiches, möglichst langfristig angelegtes Forschungsprogramm entgegen. Aus einer derartigen Interessenlage könnte ein entsprechender informeller Handlungsplan resultieren. Die enge Anbindung der PBWU an das politische Umweltressort macht zudem Strategien erforderlich, auch bei der Wissenschaft die Akzeptanz und Anerkennung im Sinne beidseitig erfolgreicher Kooperation herzustellen.

Zur Erfüllung ihrer Aufgaben wird die Projektgruppe vom Auftraggeber mit entsprechenden formalen Machtressourcen wie Organisations-, Administrations-, aber auch Lenkungsfunktionen ausgestattet. Letztere finden ihre Grenzen zwar in der Verpflichtung zur Beteiligung ihrer wissenschaftlichen und politischen Gremien, lassen aber bei der nur geringen Verfahrensnormierung im Projekt ausreichend Handlungsfreiräume oder zumindest Interpretationsspielräume offen, die für die Verfolgung informaler Interessen genutzt werden können. 
Aufgrund ihrer Weisungsgebundenheit gegenüber dem Ministerium kann die Projektgruppe indirekt von den Machtressourcen des Auftraggebers profitieren. Die Projektgruppe kann sich in ihrem Handeln formal auf den Auftraggeber Umweltministerium berufen. Ebenso kann die personelle wie administrative Zugehörigkeit zur GSF und die daraus resultierende Nähe zu deren Forschungsinstituten als informale Ressource genutzt werden. Die intermediäre Stellung der Projektgruppe zwischen Politik und Wissenschaft läßt zudem einen hohen Informationsstand erwarten, der als Machtressource gezielt gegenüber anderen Akteuren eingesetzt werden kann.

\subsubsection{Projektrat der PBWU}

Die Vertreter der Politik sind im eigentlichen Entscheidungsgremium des Projektes, dem Projektrat, repräsentiert. Ihm gehören Fachbeamte aus den für Forschung zuständigen Abteilungen der einschlägigen bayerischen Ressorts, sowie der GSF-Geschäftsführer an. Die formale Handlungsrationalität des Gremiums besteht darin, den problemlösungsorientierten Auftrag des Projektes über die Einflußnahme in grundsätzlichen Fragen sowie über die Entscheidungskompetenz in konkreten Fördermaßnahmen zu sichern und für die Berücksichtigung ressortpolitischer Interessen zu sorgen.

Das Bayerische Staatsministerium für Landesentwicklung und Umweltfragen (Umweltministerium) nimmt eine prominente Stellung im Projektrat ein. Noch vor Gründung des Projektes übernimmt es im politischen Raum die Initiative zur Bildung einer Koordinierungsstelle für die Waldschadensforschung und erhält entsprechend dem Bayerischen Ministerratsbeschluß zur PBWU-Gründung die Federführung. Es ist Hauptfinanzgeber des Projekts und Vertragspartner für die forschungsdurchführenden Institutionen sowie speziell für die GSF hinsichtlich der Finanzierung und Durchführung der Projektgruppe PBWU. Daraus ergibt sich formal wie informal eine enge Verbindung zwischen diesen beiden Akteuren. Das politische Ressort bedient sich der externen Projektgruppe in einer Art ,administrativer Brückenkopf-Funktion“, um den direkten Zugang zur Wissenschaft zu verbessern [BRAUn 1997, S. 231]. Maßgebliche Entscheidungen im PBWU-Projekt, insbesondere Förderentscheidungen, bedürfen der Beschlüsse des gesamten Gremiums Projektrat. Sie sind jedoch von der aus der Finanzierungshoheit des Umweltressorts resultierenden, formalen Durchsetzungsmacht bestimmt.

Wie für Behörden und Institutionen allgemein gültig, so prägen neben den allgemeinen, legitim zugeteilten Verwaltungsbefugnissen und der speziellen Finanzierungshoheit zur Forschungsförderung im PBWU-Projekt auch informale Machtressourcen deren Handeln bzw. das ihrer Angehörigen. Zu nennen sind hier behördeninterne soziale Beziehungen, der Umgang mit dem Machtfaktor Information zwischen Politik und Fachverwaltung sowie Entscheidungsfreiräume, die gerade im Bereich der Forschungsadministration aufgrund der Unbestimmtheit und Unvorhersehbarkeit verschiedener Vorgänge bestehen. Diese können von den einzelnen Verwaltungsangehörigen je nach Typus und Interessenlage individuell genutzt werden [DowNS 1967, S. 92-111; KROTT 1990, S. 78-81].

Mit dem Bayerischen Staatsministerium für Ernährung, Landwirtschaft und Forsten ist das für das Objekt Wald zuständige Forstressort im Projektrat der PBWU vertreten. Über das Kultusressort wird die allgemeine Zuständigkeit für Wissenschaft und Hochschulfragen berücksich- 
tigt. Bei beiden Ressorts kommt jedoch der Aspekt ressortspezifischer Forschungsförderung hinzu. So finanziert das Forstressort über das Kuratorium der ehemaligen Bayerischen Forstlichen Versuchs- und Forschungsanstalt (heute: Bayerische Landesanstalt für Wald und Forstwirtschaft) überwiegend forstliche Forschung der Forstwissenschaftlichen Fakultät und der Forschungsanstalt selbst, darunter auch die Tannen- und Fichtenforschung zu Beginn der Waldschadensforschung. Das Kultusressort beteiligt sich ab 1984 für mehrere Jahre maßgeblich an der Finanzierung der „Bayerischen Forschungsgruppe Forsttoxikologie“ mit ihrem nordbayerischen Forschungsschwerpunkt Fichtelgebirge [SCHULZE et al. 1989, S. 6].

Dies führt zu der besonderen und hinsichtlich Forschungskoordination konfliktträchtigen Konstellation, daß politische Vertreter bereits etablierter Forschungsförderungsprogramme in das neu gegründete PBWU-Projekt an entscheidungsbefugter Stelle integriert werden. Die PBWU mit übergreifendem Koordinierungsanspruch trifft auf bestehende und kooperierende Förderbzw. Forscherkoalitionen, deren Vertreter im Projektrat der PBWU - und vergleichbares gilt auch für den Wissenschaftlichen Beirat - ihre Interessen informal zugunsten der von ihnen betreuten Klientela verfolgen können. Neben der formalen Ressource der Entscheidungsmacht im Rahmen des Gremiums Projektrat können die ressortspezifischen Förderer/Forscherkoalitionen als informale, forschungspolitische wie fachliche Ressource eingebracht werden.

Die GSF nimmt hierbei eine Sonderstellung ein. Ihr wissenschaftlich-technischer Geschäftsführer ist im Projektrat der PBWU vertreten. Als Vertragspartner des Umweltministeriums bzgl. der Projektgruppe PBWU und als deren dienstvorgesetzte Instanz ist die Beteiligung der GSF im Projektrat formal unter überwiegend administrativen Gesichtspunkten zu betrachten. Die Verschränkung mit forschungspolitisch-wissenschaftlichen Interessenlagen der Forschungsinstitution GSF stellt jedoch einen erheblichen informalen Macht- bzw. Konkurrenzvorteil zur Einflußnahme auf die Waldschadensforschung dar, der an förderentscheidender Stelle im Projektrat genutzt werden kann. Durch den direkten dienstvorgesetzten Zugang der GSFGeschäftsführung zur Projektgruppe sowie die spätere Mitgliedschaft eines GSF-Institutsleiters im Wissenschaftlichen Beirat kann das formale wie informale Einflußnahmepotential der GSF auf das PBWU-Projekt noch vergrößert werden.

Das Bayerische Staatsministerium des Inneren, welches in den ersten Jahren des PBWUProjektes dem Projektrat angehört, tritt so gut wie nicht in Erscheinung.

\subsubsection{Wissenschaftlicher Beirat der PBWU}

Ungeachtet der Bedeutung der Waldschadensforscher als eigentlich Durchführende der Forschung stellt in der Programm- und Forschungssteuerung das Gremium des Wissenschaftlichen Beirats den wissenschaftlichen Hauptakteur dar. Der Beirat setzt sich aus mehreren namhaften Fachvertretern universitärer und außeruniversitärer Forschungseinrichtungen Bayerns zusammen, die meist selbst in die Waldschadensforschung involviert sind. Der Beirat hat die Aufgabe, bei der Erstellung fachlicher Programme maßgeblich mitzuwirken und Projektgruppe sowie Projektrat insbesondere in Hinblick auf die Förderentscheidungen zu Forschungsanträgen fachlich zu beraten. 
Die formale Handlungsrationalität des Wissenschaftler-Gremiums ist geprägt durch den Anspruch, durch die Einflußnahme auf Forschungskonzeption und Fördermittelvergabe die Produktivität der Wissenschaft zu verbessern [s.a. BRAUN 1997, S. 351]. Die vertraglich vorgesehene, verfahrensmäßige Beteiligung des Gremiums stellt eine erhebliche formale Machtressource dar, die zur wissenschaftlichen Steuerung des Projektes verwendet werden kann. Die hohe wissenschaftliche Reputation der Beiratsmitglieder, die Abdeckung relevanter Forschungsdisziplinen und somit hochrangige fachliche Kompetenz ergänzen das Machtpotential des Gremiums wesentlich in Hinblick auf die Abgabe fachlicher Empfehlungen zu Forschungsanträgen.

Informale Handlungspläne des Gremiums bzw. seiner Mitglieder können die Stärkung der jeweils vertretenen Forschungsdisziplin und Eigeninteressen im Gesamtkontext des Forschungsprogramms betreffen. Koalitionsbildungen im Gremium, z.B. auf fachlicher Ebene, und der hohe Informationsstand können die Position noch verbessern und versprechen wissenschaftliche Konkurrenzvorteile gegenüber externen Forschern. Die Option, daß sich Beiratsmitglieder selbst mit Forschungsvorhaben am PBWU-Projekt beteiligen können und dies auch in erheblichem Umfang nutzen, verdeutlicht diese informale Ressource.

Weitere, informelle Machtressourcen einzelner Beiratsmitglieder sind bereits bestehende Koalitionen mit den weiteren, im Projektrat vertretenen Förderressorts. Der Schulterschluß zwischen Förderer und Forschern, die gleichzeitig Beiratsmitglied sind, begünstigt die jeweilige Interessenvertretung im PBWU-Projekt in politischer wie wissenschaftlicher Hinsicht.

\subsubsection{Externe Fachgutachter}

Um die fachliche Beurteilung von eingereichten Forschungsanträgen auf eine hohe qualitative Basis zu stellen, werden in das Förderverfahren des PBWU-Projektes - wie üblich - externe Gutachter als Experten einbezogen [s.a. BRAUN 1997, S. 339; Felt et al. 1995, S. 235ff; JASANOFF 1990, S. 61ff]. Diese sind als eine weitere, wissenschaftlich orientierte Akteursgruppe im PBWU-Projekt zu nennen. Die fachliche Bewertung durch Gutachter dient dem Wissenschaftlichen Beirat als Grundlage für eine Förderempfehlung an den förderentscheidenden Projektrat.

Formaler Handlungsplan der Gutachter ist die umfassende, wissenschaftlich-fachliche Beurteilung von Forschungsanträgen. Dies soll die Ausgewogenheit und Qualität der fachlichen Einschätzung durch die Entscheidungsgremien erhöhen und ein möglichst objektives Urteil erlauben. Die dominierende, formale Ressource fachlicher Kompetenz wird durch die Auswahl als Gutachter verliehen. Ein persönliches, wissenschaftliches Interesse an den Forschungsinhalten der zu begutachtenden Anträge kann dabei ebenso unterstellt werden wie das Interesse an eigener Reputationssteigerung [BRAUN 1997, S. 339ff].

Mit der formalen Gutachterfunktion ist gleichzeitig in hohem Maße informales Machtpotential verbunden. Die fachspezifische Information kann als wissenschaftlicher Konkurrenzvorteil gegenüber dem zu begutachtenden Antragsteller für Eigeninteressen des Gutachters genutzt werden. Zumindest theoretisch zeigt sich in der gutachterlichen Antragsbeurteilung ein Zielkonflikt 
zwischen formalen und informalen Interessen, nämlich zwischen objektiver, fachlicher Beurteilungskompetenz und subjektiver, persönlicher oder fachlicher Beziehung. Letztere kann sich im Rahmen des Begutachtungsverfahrens als Zu- oder Abneigungsverhalten (Konkurrenz) gegenüber dem Antragsteller äußern.

Auf die generelle Wahrheits-Problematik der Begutachtung wissenschaftlicher Erkenntnis mittels des Urteils von Experten, die bspw. von MoHR [1995, S. 120-136] als „Expertendilemma erster und zweiter Art" bezeichnet wird, soll im Rahmen dieser Arbeit nicht eingegangen werden.

\subsubsection{Waldschadensforscher}

Die eigentliche Durchführung der naturwissenschaftlichen Forschung und somit der Erkenntnisfortschritt zum Thema wird von den Waldschadensforschern, den eigentlichen Adressaten des Forschungsförderprogrammes, geleistet. Im PBWU-Projekt sind über 40 Forschergruppen verschiedener Fachdisziplinen beteiligt, die als Forschungs-Durchführende auftreten. Weitere Antragsteller, die nicht zu einer Umsetzung der vorgeschlagenen Forschungsbeiträge im Rahmen des PBWU-Förderprogrammes gelangen, kommen hinzu. Insgesamt werden bei der PBWU während der Gesamtprojektlaufzeit rund 120 Forschungsanträge eingereicht. Beteiligt sind Forschergruppen staatlicher, universitärer sowie außeruniversitärer Forschungseinrichtungen Bayerns. Zu nennen sind insbesondere Forschergruppen der Münchener Universitäten, der Universitäten Bayreuth und Würzburg, des Fraunhofer-Instituts für Atmosphärische Umweltforschung in Garmisch-Partenkirchen und der GSF, Neuherberg. Vereinzelt werden Forschungsvorhaben vom Max-Planck-Institut für Biochemie, Martinsried, der Fachhochschule Weihenstephan und - überwiegend Serviceleistungen - von der ehemaligen Bayerischen Forstlichen Versuchs- und Forschungsanstalt, München, durchgeführt [REUTHER et al. 1993, Anhang 1].

Die antragstellenden Waldschadensforscher können von den vorhandenen fachlichen, instrumentellen, infrastrukturellen und personellen Ressourcen an ihren Institutionen profitieren; dies umso mehr, je stärker die künftigen Forschungsarbeiten auf den bisherigen aufbauen. Zusätzliche personelle Ressourcen für die im Rahmen des PBWU-Projektes durchzuführenden Forschungsarbeiten, die überwiegend von kostengünstigen, meist hochmotivierten Doktoranden geleistet werden [s.a. KROTT 1994a, S. 149], sind vergleichsweise einfach zu akquirieren. Auch für Geräteinvestitionen bietet das PBWU-Programm grundsätzlich Möglichkeiten.

Formal gilt das Hauptinteresse der Forscher dem Fortschritt in der wissenschaftlichen Erkenntnis. Sie sind dem wissenschaftlichen Ethos als verbindlichem Wertesystem der Wissenschaft verpflichtet [MoHR 1981]. Daneben bestehen jedoch informale Ziele wie Autonomie, Konkurrenzvorteil, Renommee, Prestige und persönliche Anerkennung, die den formalen Handlungsplan des Forschers überprägen. Neben Information, etwa in Form von fachlicher Kompetenz, kann der Forscher auch auf Macht als Durchsetzungsmittel zurückgreifen. Aufgrund bereits geleisteter Forschungsarbeiten in der entsprechenden Disziplin ergibt sich jeweils ein Vorsprung an fachlichem Wissen oder an wissenschaftlichen Daten gegenüber Konkurrenten, welcher die Anerkennung in der „scientific community“ stärkt. 
Zur Verbesserung der Konkurrenzsituation und damit der Bewilligungschancen für einen Forschungsauftrag wird daher bei der Antragstellung häufig auf die Exklusivität des erarbeiteten wissenschaftlichen ,know hows“ verwiesen und mit der beabsichtigten Forschung ein erheblicher Erkenntniszugewinn in Aussicht gestellt. Im Rahmen der über wissenschaftliche Reputation verlaufende Selbststeuerung der Forschung innerhalb der scientific community [BRAUN 1997, S. 66ff] ist deren Interesse allerings primär im Bereich der Grundlagenforschung gelegen.

Darüber hinaus bestehen Möglichkeiten, mit wissenschaftlichen Gruppen desselben Fachbereichs oder im interdisziplinären, fachbereichsübergreifenden Forschungsverbund zu koalieren, um die wissenschaftliche Attraktivität gegenüber dem Finanzgeber zu erhöhen. Entsprechende Forschungsverbünde müssen denn auch bei der Analyse des PBWU-Projektes berücksichtigt werden.

Die Konstellation, daß Mitglieder des Wissenschaftlichen Beirats der PBWU gleichzeitig als Antragsteller bzw. Forschungsnehmer auftreten, muß als ein gesonderter Konkurrenzvorteil gegenüber den sonstigen Antragstellern gewertet werden. Das ohnehin hohe Renommee der Forscher im Beirat kann mit der Machtressource hohen Informationsstandes über das Projekt sowie Beteiligung im Rahmen des Förderverfahrens kombiniert werden.

$\mathrm{Zu}$ erwähnen ist abschließend, daß die wissenschaftlichen Akteure als Mitglieder des Wissenschaftlichen Beirats, des Gutachtergremiums sowie als Antragsteller die Gemeinsamkeit aufweisen, in die scientific community und deren Selbststeuerung über Professions-, Qualifikations-, Bewertungs-(Evaluations-) und Publikationsverfahren eingebunden zu sein [s.a. BRAUN 1997, S. 66ff].

\subsubsection{Externe Koordinationseinrichtungen}

Die bisher dargestellten Akteure sind interner Bestandteil der Organisationsstruktur des PBWU-Projektes. Die Integration in die bundesweite Forschung zum Thema erfolgt über eine interministerielle Arbeitsgruppe. Diese, bezogen auf das PBWU-Projekt externe Koordinationsstruktur soll abschließend skizziert werden.

Zur Abstimmung der Forschungsaktivitäten zwischen Bund und Ländern war zu Beginn der Waldschadensforschung 1982/83 die „Interministerielle Arbeitsgruppe Waldschäden/Luftverunreinigungen“ (IMA) unter Federführung des Umweltbundesamtes (UBA) gegründet worden. Zu ihrer wissenschaftlichen Beratung wurde ein „Forschungsbeirat Waldschäden/ Luftverunreinigungen“ (FBW) eingesetzt [MÖHRING 1992, S. 15; UMWELTBUNDESAMT 1996, S. 4; Der BUNDESMINISTER FÜR ForsChUNG UND TeChNOLOGIE 1985, S. 113-134]. Die PBWU gehört neben weiteren vergleichbaren Länder- und Bundes-Koordinationsstellen bzw. Forschungsförderressorts der IMA an und ist damit in die bundesweite Forschungskoordination eingebunden.

Neben Querschnittsveranstaltungen der IMA zu bestimmten Problemfeldern sind insbesondere die 3 Berichte des FBW in den 80er Jahren und der Internationale Kongreß Waldschadensforschung im Jahr 1989 wichtige Markierungen des Standes der Waldschadensforschung und 
richtungsweisend für künftige Forschungsschwerpunkte [FORSCHUNGSBEIRAT WALDSCHÄDEN 1984, 1986 und 1989a, b].

Das formale Ziel der ressort- und länderübergreifenden Forschungsabstimmung kann allerdings nur mit begrenzten, im wesentlichen auf Information beruhenden Machtressourcen verfolgt werden. Im Rahmen der „IMA-Frühkoordination“ der Forschung findet ein schriftlicher, formalisierter Informationsaustausch über beabsichtigte Forschungsaktivitäten statt. Laufende Forschungsaktivitäten werden dokumentiert [z.B. IMA 1983] und länderübergreifende, forschungskonzeptionelle Empfehlungen der Förderpolitik erarbeitet (s. verschiedene Jahresberichte der IMA). Derartige Koordinierungsmaßnahmen sind jedoch mangels Durchsetzungsmacht weitgehend unverbindlich. Die Finanzierungshoheit der Länderressorts bzgl. Fördermaßnahmen bleibt uneingeschränkt bestehen, länder- bzw. ressortspezifische Förderinteressen können weiterverfolgt werden. So beschränkt sich auch die Interaktion zwischen PBWUProjekt und IMA auf informative Kooperation. Weder in wissenschaftlicher, noch in forschungspolitischer Hinsicht muß sich das Projekt koordinierend-steuernden Einflüssen einer bundesweiten Koordinierungsstelle unterwerfen.

\subsection{Theorieorientiertes Zwischenergebnis}

\subsubsection{Akteurskonstellationen}

Die vorliegende Fallstudie focussiert auf die Forschungssteuerung als Teilsystem der gesamten Waldschadenspolicy. Damit kann sich die Analyse auf die dem Projekt zuzuordnenden Akteure beschränken, welche die Projektstruktur definieren und am Prozeß der Forschungsförderung beteiligt sind. Mit dem gewählten Ansatz der Akteure als analytischen Einheiten erfaßt die Analyse des Forschungsprozesses nicht nur dessen strukturelle Bestandteile wie Programme und Verfahren, sondern auch Akteurskonstellationen und spezifische Rollen individueller Akteure [HoHN \& SCHIMANK 1990, S. 19]. Den einzelnen Akteuren lassen sich jeweils an den zugeteilten Aufgaben orientierte, formale Handlungspäne und formal handlungslegitimierende Ressourcen zur deren Durchsetzung zuordnen. Weitere, informal handlungsleitende, meist persönliche oder klientelspezifische Interessen der Akteure können in der Analyse des tatsächlichen Handelns zusätzlich berücksichtigt werden [KROTT 1990, S. 56ff].

Die Struktur des PBWU-Projektes führt zum Entstehen sog. „Hybridgemeinschaften“ [FELT et al. 1995, S. 214, 288; HoHN \& SCHIMANK, 1990, S. 19]. Akteursgruppen wie Wissenschaftlicher Beirat, Projektrat sowie die Projektgruppe selbst repräsentieren Wissenschaft, Politik, Koordination und Administration. Sie führen zu komplexen Informations- und Steuerungsprozessen. Es kommt zu einer Vermengung politischer und fachlicher Interessenlagen und zu Verständnisproblemen und entsprechendem Vermittlungs-, Übersetzungs- und Transformationsbedarf zwischen Politik und Wissenschaft [s.a. KROTT 1990, S. 75ff]. Die Bewältigung derartiger Probleme ist unter anderem Aufgabe der intermediären Projektgruppe PBWU. Ihr Vermittlungserfolg setzt allerdings ihre Akzeptanz einerseits, Kompetenz andererseits voraus.

Die PBWU-Projektgruppe steht als intermediäre Koordinierungseinheit an der Schnittstelle zwischen den Politik und Wissenschaft. Der formale Handlungsplan der PBWU wird im we- 
sentlichen von den Koordinierungsaufgaben bestimmt, die ihr vom politischen Auftraggeber Umweltministerium zugewiesen wurden. Aus den hiermit verbundenen Handlungsbefugnissen, die formal mit der Weisungsgebundenheit gegenüber dem Auftraggeber gekoppelt sind, ergeben sich die Machtressourcen der Projektgruppe. Diese werden durch die Beteiligung ihrer Beratungsgremien und Entscheidungsgremien begrenzt. Die zentrale, mit hohem Informationspotential ausgestattete Stellung zwischen politischen und wissenschaftlichen Akteuren sowie die im Bereich des Forschungsförderverfahrens erheblichen Handlungs- und Entscheidungsfreiräume verleihen der Projektgruppe jedoch weitreichende informale Machtressourcen. Diese können nicht nur zur Erfüllung der Koordinierungsaufgaben, sondern auch zur Verfolgung informaler, bspw. an Existenzsicherung, Eigenständigkeit und Aufgabenerweiterung gelegener Eigeninteressen der Gruppe eingesetzt werden.

Das politisch administrative System wird durch den Projektrat der PBWU vertreten. Eine besondere Stellung nimmt hierbei das federführende und projektfinanzierende Umweltressort sowie die, die Projektgruppe beherbergende GSF ein. Dem Umweltministerium kommt dabei aufgrund seiner Entscheidungs- und Finanzierungshoheit das überragende formale Machtpotential in dem Projekt zu. Auf informaler Ebene treten Interessen des Verwaltungsapparates hinzu, die unter Einsatz von Machtinstrumenten wie Fachwissen und Informationsbeziehungen der Fachbeamten verfolgt werden können.

Die neben politisch-administrativen Ressorts ebenfalls im Projektrat vertretene GSFGeschäftsführung ist administrativ Vertragspartnerin des Umweltministeriums und Dienstvorgesetzte bzgl. der PBWU-Projektgruppe. Sie besitzt aber gleichzeitig wissenschaftliche Forschungsinteressen, die zudem über die Beteiligung in den PBWU-Gremien vertreten werden. Diese Konstellation stellt eine formale Verfahrensbeteiligung sowohl in politischer wie wissenschaftlicher Hinsicht sicher, erleichert aber gleichzeitig bspw. über Informations- oder Konkurrenzvorteile die informale Interessenverfolgung der GSF im PBWU-Projekt.

Die ebenfalls im Projektrat vertretenen Landwirtschafts- und Kultusressorts stellen gleichzeitig und parallel zur PBWU-Konstruktion Förderinstitutionen für anderweitig bereits etablierte Forschungsverbünde dar. Neben der formalen politischen Entscheidungsfunktion im Rahmen des Projektrates können die bestehenden Klientelbildungen zwischen Förderern und Forschern als Machtinstrumente zur eigenen, informalen Interessenverfolgung im PBWU-Projekt genutzt werden.

Im Verfahren der Forschungsförderung vertreten der Wissenschaftliche Beirat und die externen Fachgutachter die wissenschaftlichen Interessen. Während der Beirat vertragsgemäß formal die grundlegende Konzeptionierung, Programmformulierung und fachliche Beratung der PBWU und der beteiligten Fachressorts zur Aufgabe hat, ergänzen Gutachter die fachliche Bewertung vorgelegter Einzelanträge durch jeweilige Stellungnahmen.

Neben dem formalen, funktionalen Handlungsplan der wissenschaftlich-fachlichen Beratung und Bewertung verfolgen die Wissenschaftler informale Interessen, die bspw. in der Stärkung ihrer eigenen Forschungsrichtung, Schaffung wissenschaftlicher Konkurrenzvorteile und Vergrößerung der wissenschaftlichen Reputation innerhalb der scientific community gelegen sind. 
Das hohe wissenschaftliche Renommee, die Fachkompetenz und Sachautorität dieser Akteure bei hohem Informationsstand über das Projekt können als informale Machtressourcen für Eigeninteressen genutzt werden.

Die Waldschadensforscher sind von den Aushandlungsprozessen wissenschaftlicher wie politischer Akteure im PBWU-Projekt als Antragsteller und Forschungsdurchführende betroffen. Sie können in ihren Handlungsplänen die Erweiterung des wissenschaftlichen Erkenntnisstandes, aber auch die Durchsetzung informaler Interessen, wie z.B. die Vermehrung wissenschaftlicher und finanzieller Ressourcen und persönlichen Renommees, verfolgen. Dazu dienen verschiedene Machtmittel und Durchsetzungsstrategien in Form von Wissens- und Informationsvorteilen, Koalitionsbildung unter Forschern oder mit Forschungsförderern etc.

\subsubsection{Akteursspezifische Steuerungskonflikte}

Die komplexen Akteursstrukturen im PBWU-Projekt bedingen nahezu zwangsläufig Probleme der Forschungssteuerung. Dem politischen Ziel, aus den Ergebnissen der Waldschadensforschung politische Handlungsmaßnahmen ableiten zu können, steht das wissenschaftliche Interesse der Forscher gegenüber, sich an dieser Forschung und damit am Programm der PBWU zu beteiligen. Interessengegensätze bestehen darin, daß die universitären Forscher überwiegend an Grundlagenforschung interessiert sind, der staatliche Finanzgeber jedoch angewandte Waldschadensforschung erwartet. Ein Kompromiß ergibt sich insofern, als zur Produktion von Ergebnissen angewandter Waldschadensforschung vorab oder anteilig ohnehin erst wissenschaftliche Grundlagen erarbeitet werden müssen. Wie die spätere Analyse der Programmimplementation zeigen wird, akzeptiert dies der Auftraggeber auch in weitem Rahmen.

Hinsichtlich der Festlegung und Umsetzung der Forschungsthemen sind daher unterschiedliche Interessenlagen und damit Interessenkonflikte zwischen den verschiedenen Akteuren aus Wissenschaft und Politik zu erwarten. Der Auftraggeber wird bestrebt sein, geeignete Forschergruppen über die finanzielle Förderung für die Beteiligung am Forschungsprojekt zur Ausfüllung des Programmes zu gewinnen. Die Forscher werden sich bemühen, mit den von ihnen jeweils bearbeitbaren Themen sich am Forschungsprogramm zu beteiligen und deren fachliche Bedeutung herauszustellen. Dabei können Konkurrenzsituationen um den Zugang zum Forschungsprogramm auftreten, falls mehrere, am gleichen Thema interessierte Forschergruppen bestehen. Andererseits kann der Fall eintreten, daß vom Auftraggeber ausgewiesene Forschungsthemen allein deswegen nicht bearbeitet werden können, weil Forschergruppen entsprechenden fachlichen Zuschnitts nicht bestehen oder sich - aus welchen Gründen auch immer - nicht am Forschungsprogramm beteiligen wollen.

Damit wird wiederum deutlich, daß die Gestaltung der Waldschadensforschung sowie deren Steuerung von zahlreichen vielschichtigen Interdependenzen zwischen den beteiligten Akteursgruppen wie Einzelakteuren geprägt ist. Jeder Akteur, der beabsichtigt, zielgerichtet auf den Forschungsprozeß einzuwirken, sieht sich mit anderen Akteuren konfrontiert, die fördernd, erschwerend oder verhindernd für sein eigenes Handlungsziel relevant werden. Konkurrenzzwänge, Interessenkonflikte bestehen hier gleichermaßen wie Kooperations- oder Konsenser- 
fordernisse und korporatives Verhalten, d.h. gemeinsame Interessenverfolgung durch Bündnispartner [HOHN \& SCHIMANK 1990, S. 21, 27].

Zur forschungspolitischen Steuerung und Abstimmung der Forschung bedient sich der Auftraggeber im PBWU-Projekt des Projektrats. Über die im Projektrat vertretenen Ministerien können die entsprechenden ressortspezifischen, politischen Zielsetzungen im Forschungsprogramm der PBWU berücksichtigt werden. Daß das Bayerische Umweltministerium, welches die Federführung hinsichtlich der Initiierung und insbesondere auch der Finanzierung des PBWU-Projektes übernimmt, das höchste Machtpotential besitzen und somit den dominierenden Steuerungseinfluß ausüben wird, ist naheliegend. Allerdings bestehen noch weitere ressortspezifische Interessenlagen im Projektrat, die sich - wie an anderer Stelle dargestellt (Kap. 5.1.3.3.5 und Kap. 6.3) - in territorialer Abgrenzung gegen die Bündelung zu einem Programm äußern.

Die eigentliche fachliche Bewertung der Forschung, insbesondere in der Phase der Antragsstellung, findet über die Beteiligung externer Fachgutachter statt. Die Gutachter besitzen erheblichen Steuerungseinfluß auf Inhalt, Qualität und Verlauf der Forschung. Der Auswahl der Gutachter, den Beurteilungskriterien zur Ermittlung der Forschungsqualität sowie der Behandlung der Gutachtervoten durch die PBWU-Gremien wird daher bei der Analyse des PBWU-Projektes besondere Aufmerksamkeit geschenkt werden müssen. Dabei ist im Grundatz davon auszugehen, daß sich mit steigender Einheitlichkeit des externen Begutachtungsergebnisses der Steuerungseinfluß durch den Wissenschaftliche Beirat verringert und umgekehrt mehrdeutige Begutachtungsergebnisse die Einflußnahme durch den Beirat verstärken. 


\section{Policy-Zyklus}

\subsection{Gründung des PBWU-Projektes}

Voraussetzung für die Formulierung und Implementation eines politischen Programms ist nach dem Modell des Policy-Ansatzes das Vorhandensein eines gesellschaftlichen Problems. Dieses muß ins öffentliche Bewußtsein getreten sein, durch die Forderungen bestimmter Akteure und gesellschaftlicher Wertvorstellungen als behandlungsrelevant definiert (Problemdefinition) und - im Wettbewerb zu anderen Themen - auf die politische Handlungs- bzw. Entscheidungsebene gehoben werden (Agenda-Gestaltung) [WINDHOFF-HÉRITIER 1987, S 65-67].

Übertragen auf die Analyse des Forschungsprozesses zum Thema „Waldschäden“ am Fallbeispiel des PBWU-Projektes setzt dies die Definition des Phänomens Waldschäden als Forschungsproblem voraus. Die Waldschadensforschung muß von den beteiligten Akteuren thematisiert und politisch behandelt werden.

Die Analyse dieser Initialphasen der Forschungspolicy muß daher zeitlich noch weit vor die Gründung des PBWU-Projektes und damit in die Zeit der allgemeinen Entdeckung des Phänomens „Waldschäden“ zurückzugehen, da beide Aspekte - Thematisierung Waldschäden und Waldschadensforschung - weitgehend und bereits frühzeitig gekoppelt auftreten. Die analytisch reizvolle Frage, warum und auf welche Weise das Thema „Waldschäden“ allgemein zum gesellschaftlichen Problem erhoben wurde, soll und muß im Rahmen dieser Studie zur Forschungssteuerung ausgeklammert werden. Sie klingt hier allenfalls bei der großenteils kombinierten Thematisierung von Waldschäden und Forschung an. Daß das Thema „Waldschäden“ an sich zu einem gesellschaftlichen Problem erhoben wurde, kann jedoch als zweifelsfrei und für der Studie als extern vorgegebenes Faktum unterstellt werden [HOLZBERGER 1995; ZIERHOFER 1998].

Im folgenden wird die Phase der Problemdefinition und die politische Behandlung des Themas Waldschadensforschung bis hin zum Beschluß des PBWU-Projektes empirisch dargestellt und theorieorientiert analysiert.

\subsubsection{Thematisierung der Waldschadensforschung (Problemdefinition)}

\subsubsection{Thematisierung von , Waldschäden“ und Forschung}

Seit etwa Mitte der 70er Jahre werden in Bayern verstärkt Schäden an Tanne beobachtet [BAYERISCHE STAATSFORSTVERWALTUNG 1983a; S. 1 u.a.a.O.]. Im Rahmen eines ersten Erfahrungsaustausches wird bereits von einem „Tannensterben“ gesprochen [WACHTER 1977; WAGNER 1978]. Ein Münchner Forstbotaniker fordert, ,unverzüglich und konsequent mit Untersuchungen zu beginnen“ [SCHÜTT 1977]. Als Ergebnis einer Ortsbesichtigung geschädigter Tannenbestände mit Vertretern der Bayerischen Staatsforstverwaltung, Forstwissenschaft und Forstpraxis im Oktober 1977 wird ein Sofortprogramm zur Zustandsbeobachtung sowie ein 
Grundlagenforschungsprogramm beschlossen. Ähnliche Maßnahmen erfolgen auch in anderen Bundesländern, insbesondere Baden-Württemberg und Nordrhein-Westfalen [KNABE 1981].

Mehrere Lehrstühle der Forstwissenschaftlichen Fakultät der Universität München sowie die Bayerische Forstliche Versuchs- und Forschungsanstalt beteiligen sich an der Forschung, der 1977 - 1981 über 1 Mio DM zur Tannenforschung zur Verfügung gestellt werden [BAYERISCHE StaAtsforstVerwaltung 1981, S. 3 und 1982a, S. 2/3]. Die forstliche Forschung kann dabei bereits auf gewisse Erfahrungen, wie z.B. zu Immisionsschäden an Wäldern, zurückgreifen [VON SCHÖNBORN \& WEBER 1981].

Nach den Dokumentationen der Bayerischen Staatsforstverwaltung und Berichten von Forstwissenschaftlern werden - nach ersten Anzeichen in 1979 - etwa seit Sommer 1980 in Süddeutschland Kronenverlichtungen und vorzeitiges Absterben in Fichtenbeständen beobachtet [KNABE 1981; SEITSCHEK 1982; BAYERISCHE STAATSFORSTVERWALTUNG 1982b, S. 3, SCHÜTT 1982] ${ }^{1}$. Besorgniserregende Einschätzungen in der Wissenschaft führen zu Beginn des Jahres $1981 \mathrm{zu}$ der Frage, ob dem „Tannensterben“ ein „Fichtensterben“ folgte [SCHÜTT 1981a]. Bei dem gegebenen, weitgehend nur spekulativen Kenntnisstand über Ausmaß und Ursache verstärkte sich der Verdacht auf eine erhöhte Gefährdung der Wälder durch permanente Umweltbelastung. Eine Aufklärung durch verstärkte Forschungsaktivitäten werde gefordert.

Bei der Besichtigung „neuartiger Waldschäden“ an Fichte im einem Forstamt nahe München im Mai 1981 durch namhafte Forstforscher werden Schadensmeldungen hinsichtlich Ausmaß, Bedeutung und Ursachen zwar unterschiedlich eingeschätzt. Man ist sich jedoch darin einig, daß Handlungsbedarf in Form der Installierung eines Beobachtungs- und Forschungsprogramms bestünde [KNABE 1981]. Der Begriff des „Fichtensterbens“ wird geprägt, wenn auch unter heftigem Widerspruch [BRAUN 1981; SCHMIDT-VOGT 1981; REHFUESS 1981]. Schadensmeldungen der Forstämter werden von der BAYERISCHEN STAATSFORSTVERWALTUNG [1981, S. 1/2] zurückhaltend eingeschätzt. Ähnlich dem Vorgehen bei der Tanne werden jedoch Beobachtungsflächen in ausgewählten Fichten-Altbeständen eingerichtet und ein Forschungsprogramm aufgelegt.

Die von Unsicherheiten über die Ursachen geprägte Diskussion zum Thema erhöht den Druck auf die Forschung und wird von den Wissenschaftlern selbst verstärkt. Straff organisierte, koordinierte und interdisziplinäre und integrierte Ursachenforschung müsse einsetzen [SCHÜTT 1982; STAHL 1981], die Belastung durch Schadstoffe quantifiziert und die Auswirkungen überprüft werden [REHFUESS 1981]. Auch wird bereits auf die Langfristigkeit der zu verstärkenden Forschung hingewiesen, um die Wirkungsketten in vernetzten Ökosystemen überhaupt erfassen zu können [Prof. ULRICH in: LEHRINGER 1982].

\footnotetext{
${ }^{1}$ Kurz vorher waren die Forschungsergebnisse eines Göttinger Bodenkundlers zur zunehmenden Bodenversauerung im Solling unter dem Einfluß von Luftschadstoffen publiziert worden [ULRICH et al. 1979]. Mit den Prognosen des Absterbens der dortigen Bestände bei anhaltendem Schadstoffeintrag und die Übertragung dieses Gefährdungspotentials auf vergleichbare Standorte in ganz Mitteleuropa wird die Publikation Ulrichs von vielen als ausschlaggebend für die breite Wahrnehmung des Themas „Waldschäden“ sowie als Grundlage für deren ursächliche Erklärung angesehen [ROQUEPLO 1986; DER RAT VON SACHVERSTÄNDIGEN FÜR UMWELTFRAGEN 1983, S. 13; VerEIN DEUTSCHER INGENIEURE 1983, S. 9; ELL \& LUHMANN 1996; KANDLER 1994].
} 
Das Phänomen wird 1982 von offiziellen Stellen bereits als „Baumsterben“ bezeichnet, da inzwischen auch unerklärbare Schäden an anderen Baumarten gemeldet werden [BAYERISCHE StAATSFORSTVERWALtung 1982a, S. 1 und 1982b, S. 2]. Zum Durchbruch in die Öffentlichkeit gelangt das Thema und der Begriff „Waldsterben“ durch die Medien. Die dreiteilige Serie in der Zeitschrift „,Der Spiegel“ „Säureregen: Da liegt was in der Luft“ vom November 1981 [DER SPIEGEL 1981] gilt als „Alarmauslöser“, als maßgeblicher Initiator der Themenkarriere „Waldsterben“ in den Medien und als Carrier des Problems aus Forschung und Forstpraxis in die Öffentlichkeit [BAuMgartner 1991; HolzBERGER 1995, S. 70]. Unter Berufung auf Forstexperten und Wissenschaftler wird drastisch vermittelt, daß schwefelhaltige Niederschläge Wälder, aber auch Atemluft und Nahrung vergifteten. Nach bereits früherer, sporadischer Verwendung durch den Bund Naturschutz setzt sich nun in Folge des Spiegel-Artikels bei Medien und Öffentlichkeit der Begriff „Waldsterben“ durch [s. KRÄMER \& SUDA 1987, zit. in: HolZBERGER 1995, S. 57; SCHÜTZE in: Süddeutsche Zeitung vom 14./15.9.1996].

In der Diskussion zu den Ursachen des Waldsterbens steht der Faktor Luftschadstoffe im Zentrum. Die genannte Serie in der Zeitschrift „,Der Spiegel“ leistet einen wesentlichen Beitrag zur Behauptung eines kausalen Zusammenhangs zwischen säurehaltigem Regen und Waldsterben [s.a. Verein DeUTSCHER IngENIEURE - VDI 1983, S. 9; FARTHMANn 1983]. Ohne die Beteiligung der Luftschadstoffe sei das Ausmaß der beobachteten Schäden generell nicht erklärbar. In der breiten Öffentlichkeit scheint dieser Zusammenhang sicher zu sein [DER RAT VON SACHVERSTÄNDIGEN FÜR UMWELTFRAGEN, 1983, S. 1; VDI 1983, S. III; LEHRINGER 1982; EISENMANN 1983].

Auf die Frage, inwieweit Luftverunreinigungen ursächlich für die neuen Waldschäden seien, könne, wie der Sachverständigenrat ausführt, jedoch keine allgemein überzeugende Antwort gegeben werden. Diese Einschätzung sei jedoch Anlaß, die Forschung zu intensivieren. Sie biete ein breites Spektrum zu bearbeitender Forschungsfelder, deren vorschnelle Einengung auf einige wenige, vermutete Hauptfaktoren aus wissenschaftlicher Sicht - im Gegensatz zu umweltpolitischer Sicht - abzulehnen sei [DER RAT DER SACHVERSTÄNDIGEN FÜR UMWELTFRAGEN 1983, S. 212, 252].

\subsubsection{Anfängliche Struktur der bayerischen Waldschadensforschung}

Inzwischen sind in der Bundesrepublik Deutschland bereits eine Vielzahl an Forschungsvorhaben angelaufen. Vom Bund wird im April 1983 die Gemeinsame Interministerielle Arbeitsgruppe (IMA) „Waldschäden/Luftverunreinigungen“ des Bundes und der Länder gegründet, um sich mit der „,Koordinierung der Waldschadensforschung zu befassen und Forschungs- und Handlungskonzepte abzustimmen“ [UMWELTBUNDESAMT 1996, S. 4].

Nach einer Zusammenstellung der IMA [1983] befinden sich Mitte 1983 bereits knapp 300 Forschungsvorhaben zum Thema in Bearbeitung oder Planung, die im wesentlichen von verschiedenen Bundes- oder Länderministerien sowie der Deutschen Forschungsgemeinschaft (DFG) finanziert werden. Allein für bayerische Forschungsinstitute werden rund 40 Forschungsvorhaben genannt, von denen etwa $80 \%$ an der Forstwissenschaftlichen Fakultät der Universität München angesiedelt sind. 
Das Umweltressort trägt mit bereits laufenden bzw. erweiterten Bioindikator- und lufthygienische Messungen, Regenwasser- und Bodenuntersuchungen zur Forschung bei. In einer gemeinsamen Dokumentation des BAYERISCHEN UMWELT- UND LANDWIRTSCHAFTSMINISTERIUMS [1982, S. 10] zum „Baumsterben“ werden unter anderem die erfolgten bzw. noch erforderlichen Maßnahmen dargestellt. Bis zu diesem Zeitpunkt seien für Schadenserfassung, forstliche Forschung und Immissionsuntersuchungen beider Ressorts insgesamt ca. 5 Mio. DM aufgewendet worden. Es wird ausgeführt, daß die Koordination der forstlichen Forschungsvorhaben über das Kuratorium der Bayerischen Forstlichen Versuchs- und Forschungsanstalt erfolgte und eine Abstimmung mit benachbarten Landesforstverwaltungen stattfände.

\subsection{Forstliche Forschung an Forstfakultät und Forschungsanstalt}

Die Zusammenarbeit der forstlichen Lehrstühle der Universität München mit der behördlichen Forschungsanstalt läßt sich aufgrund nachbarschaftlicher, institutionell-organisatorischer und personeller Strukturen vergleichsweise leicht bewerkstelligen. Die dem Landwirtschaftsressort zugeordnete Forschungseinrichtung Forstliche Forschungsanstalt (FFA) ist bis 1978 eng mit der Forstwissenschaftlichen Fakultät verbunden [REHFUESS 1978, GoETTLING 1981, ZANG 1981, Holl 1993]. Dies sichert eine enge Kooperation und eine starke Position der Fakultät hinsichtlich der Einflußnahme auf die an der Forschungsanstalt bearbeiteten Themen. Beide Institutionen partizipieren - die Forschungsanstalt nahezu ausschließlich - von den Fördermitteln des Landwirtschaftsministeriums und sind dessen Modalitäten der Forschungsförderung unterworfen.

Um den universitären Forschungs- und Lehrbereich von den praxisorientierten Versuchs- und Forschungsaufgaben der Staatsforstverwaltung abzukoppeln, trennen sich 1979 beide Institutionen (Bayerisches Gesetz- und Verordnungsblatt Nr. 6/65 vom 13.3.1979). Die neu benannte Bayerische Forstliche Versuchs- und Forschungsanstalt (FVA) ist in die Hierarchie der Staatsforstverwaltung voll eingebunden [GoETTLING 1981]. Als Bindeglied zur Forstwissenschaftlichen Fakultät dient neben gemeinsamen Arbeits- und Projektgruppen das Kuratorium der FVA. Diesem gehören unter anderem paritätisch Vertreter der Staatsforstverwaltung und der Fakultät an. Das Kuratorium gibt dem Landwirtschaftsministerium Förderempfehlungen zur Finanzierung eingereichter Forschungsanträge. Es versteht sich als Koordinationsstelle für den Bereich der vom Bayerischen Landwirtschaftsministerium geförderten Forschung [s.a. BAYERISCHE STAATSFORSTVERWALTUNG 1982b, S. 5].

Fakultät und Forschungsanstalt gewinnen durch die gemeinsamen Strukturen in Hinblick auf die Tannen-, Fichten- bzw. in der Folge Waldschadensforschung. Die Koalition zwischer universitärer und angewandt forstlicher, praxisnahen Forschung kann, wie auch die folgende Analyse des PBWU-Forschungsprojektes zeigen wird, kaum hoch genug eingeschätzt werden. Die forstwissenschaftliche Forschung besitzt fachlich-professionell bedingt leichten Zugang zum Objekt Wald und hat über die Forschungsanstalt enge Anbindung an die Forstpraxis. Die frühzeitige Etablierung des forstwissenschaftlichen Verbundprojektes „Höglwald“ ist hierfür ein Beispiel. Bereits zu Ende des Jahres 1981 kann unter Leitung eines Münchner Bodenkunde-Professors der Forstwissenschaftlichen Fakultät mit Mitteln des Kuratoriums die Pilotphase für ein umfangreiches Forschungsexperiment zur Säurewirkung und Düngung (Kalkung) in ei- 
nem Fichtenbestand begonnen werden [BAYERISCHE STAATSFORSTVERWALTUNG 1983a, S. 4]. Das Projekt hat - bei veränderten Fragestellungen - bis heute Bestand.

\subsection{Etablierung weiterer Forschungsverbünde}

Neben der forstlichen, vom Landwirtschaftsressort finanzierten Waldschadensforschung wird im Jahr 1983 auf Initiative des Beirats für Wissenschafts- und Hochschulfragen beim Bayerischen Kultusministerium die „Bayerische Forschungsgruppe Forsttoxikologie“ gegründet [SCHULZE et al. 1989, S. 4ff]. Die Koordination und Umsetzung der einzelnen Forschungsbereiche obliegt drei namhaften Wissenschaftlern nordbayerischer Universitäten. Die Finanzierung in Höhe von mehreren Mio. DM erfolgt maßgeblich und in enger Abstimmung mit dem Kultusressort unter Einbeziehung externer Gutachter. Damit wird mit dem nordbayerischen Fichtelgebirge als regionalem Forschungsschwerpunkt ein multidisziplinärer Forschungsverbund zur Klärung der Wirkung von Stoffeinträgen auf Waldbestände mit etwa 20 Einzelprojekten etabliert. Das Projekt stellt einen wichtigen Eckpfeiler der bayerischen Waldschadensforschung dar.

Eine weitere Forschungsinitiative zur Waldschadensforschung geht von der damaligen Gesellschaft für Strahlen- und Umweltforschung (GSF) in Neuherberg - heute GSF-Forschungszentrum für Umwelt und Gesundheit - aus. Sie legt ebenfalls im Jahr 1983 ein überwiegend auf Laborarbeiten beruhendes Forschungsprogramm über die Wirkung von Luftschadstoffen auf Pflanzen vor (Aktionsprogramm der GSF, Stand 23.3.1983). Zu dessen Umsetzung soll eine koordinierende Arbeitsgemeinschaft, die „Münchner Arbeitsgemeinschaft Luftschadstoffe“ (MAGL) mit einem Lenkungsausschuß etabliert werden, dem führende Wissenschaftler der GSF sowie der beiden Münchner Universitäten angehören. Auch Lehrstuhlinhaber der Forstwissenschaftlichen Fakultät sagen ihre Mitarbeit zu (Arbeitsprogramm der MAGL, Stand 10.6.1993).

Zur Durchführung der Forschungsarbeiten ist der Bau und Betrieb einer technisch wie finanziell anspruchsvollen Experimentieranlage für Luftschadstoff-Belastungsversuche (Expositionskammern) geplant, die von der GSF betreut und den Forschern zur Verfügung gestellt wird. Die Arbeitsgemeinschaft wird am 22.6.1983 gegründet, die Kammern, an deren Finanzierung sich auch das Bayerische Umweltressort beteiligt, werden Ende des Jahres 1984 eingeweiht (s. Aufzeichnungen des GSF-Forschungszentrums zur MAGL). In Verbindung mit der Angliederung der Anlage an ein neu gegründetes Forschungsinstitut an der GSF wird ein weiterer Schwerpunkt der Waldschadensforschung in Bayern geschaffen. In den Folgejahren beteiligen sich zahlreiche Forschergruppen aus dem In- und Ausland und von anderweitigen bayerischen Forschungsschwerpunkten an den GSF-Kammerexperimenten der MAGL [LANGEBARTELS et al. 1997].

\subsubsection{Theorieorientiertes Zwischenergebnis}

\subsection{Hoher Handlungsdruck}

Die anfängliche Waldschadensdiskussion ist geprägt durch die vorherrschende Unkenntnis bzw. Vermutungen über die Problemschwere, die bestehende Verwirrung aufgrund vielfältiger 
Ursachen-Hypothesen und durch widersprüchliche Forschungsergebnisse. Nicht zuletzt durch Beiträge von Forschern und Umweltgruppen entsteht der starke Verdacht, daß die Luftschadstoffe ursächlich zum Waldsterben führen. Die Thematisierung des mit katastrophalen Folgen drohende Ereignis „Waldsterben“ erzeugt Angst und damit hohen Handlungsdruck.

Die Überzeugung, daß überhaupt Lösungen im politisch-administrativen Bereich möglich sind, besteht. Maßnahmen der Luftreinhaltepolitik sowie ergänzende, ursachenklärende Forschung lassen auf eine erfolgreiche Problembewältigung hoffen. Wesentliche Voraussetzungen dafür, daß das Thema Waldschadensforschung zu einem behandlungswürdigen Policy-Problem erhoben wird („Issue-Initiierung“) sind damit gegeben [WINDHOFF-HÉRITIER 1987, S. 67/68].

\subsection{Forschung als Beitrag zur Problem- oder Konfliktlösung}

In dieser Situation ist die Legitimation für Forschung [MAYNTZ 1985, S. 19] ausgesprochen hoch. Das politisch-administrative System ist stark an Forschung interessiert. Generell - und idealisiert dargestellt - ist Forschung dann politisch legitimiert, wenn es - wie im Falle des Waldsterbens - gelingt, ein neues, noch unbekanntes Problem zu definieren. Dessen Beurteilung kann durch entsprechende Forschungsbeiträge versachlicht, Problemlösungen können durch gezielte und wissenschaftlich legitimierte Maßnahmen herbeigeführt werden.

Dies setzt allerdings voraus, daß das politische System das Problem auch rational bearbeiten möchte, die bestehenden Interessenkalküle die rationale Bearbeitung überhaupt zulassen und „gezielte Maßnahmen oder „Versachlichung“ des Problems nicht lediglich politische Schutzbehauptungen darstellen. Weitere Faktoren spielen in der politischen Rationalität eine Rolle. So werden mit der Forschungsverstärkung Aktivitäten unternommen, die politisch weniger konfliktreich sind als konkrete Gegenmaßnahmen, wie z.B. im Bereich der Luftreinhaltung. Die Forschung verschafft der Politik zudem einen Zeitgewinn zur Durchführung von Gegenmaßnahmen; sie bietet eine kurzfristige Entlastung für die Lösung politischer Konflikte.

Forschungsbedarf wird auch von den Wissenschaftlern, und hier von den primär tangierten Forstforschern festgestellt. Trotz der zu erwartenden, objektspezifischen Forschungsprobleme bestehen vermeintlich hohe Erfolgsaussichten für die Forschung, da die Ursachen ja im Grundsatz (in Form der Luftschadstoffe) als bekannt vermutet werden und nur noch wissenschaftlich abgesichert werden müssen. Die Forschung kann daher glaubhaft versprechen, den Verdacht maßgeblicher Beteiligung von Luftschadstoffen am Zustandekommen von Waldschäden rasch zu beweisen und somit zur Problemlösung beizutragen. Das formale wissenschaftliche Interesse an Erkenntnisgewinn zur wissenschaftlichen Beschreibung und ursächlichen Aufklärung des Phänomens kann dabei an die Erwartung zusätzlicher finanzieller Ressourcen geknüpft werden.

In einer Vorphase der bayerischen Waldschadensforschung zu Tannen- und Fichtenschäden kann die forstwissenschaftliche Forschung der Universität München rasch zusätzliche Forschungsfinanzierung durch das Landwirtschaftsministerium erwirken. Eine maßgebliche Rolle spielt dabei ein Münchner Forstbotaniker, der sich nicht nur an der Entdeckung und Ursachendiskussion zum „Waldsterbens“ beteiligt, sondern auch frühzeitig die Notwendigkeit einer umfassenden und koordinierten Erforschung der Phänomene fordert. Die enge Verbindung zwischen universitärer Forschung der Forstwissenschaftlichen Fakultät, der praxisnahen Forstli- 
chen Versuchs- und Forschungsanstalt und dem ressortzuständigen Finanzgeber wirkt sich positiv auf die Durchführung der ersten forstlichen Forschungsprogramme zu Tannen- und Fichtenschäden aus. Sie stellt einen potentiellen Konkurrenzvorteil dar gegenüber anderen, nichtforstlichen Forschungsinstitutionen, welche sich erst in der Folge in großem Umfang an der Waldschadensforschung beteiligen. Bereits bestehende Forschungsansätze, die entweder aus den genannten Programmen resultieren, oder ohnehin betrieben und nun für das Forschungsthema Waldschäden verwendet bzw. modifiziert werden können, erleichtern es in der Folge den betreffenden Forschungsinstitutionen zudem in fachlicher Hinsicht, sich an der Forschung $\mathrm{zu}$ beteiligen. Die erworbene Kompetenz kann wiederum als Konkurrenzvorteil gegenüber „Neueinsteigern“" genutzt werden.

\subsubsection{Politische Behandlung der Forschungskoordination und Beschluß zur PBWU- Gründung (Agendagestaltung)}

Zur Aufnahme der Waldschadensforschung und deren Koordination auf die politische Tagesordnung kann unter anderem auf die Arbeit von SCHNALKE [1986] zur Behandlung des Themas „Waldsterben“ im Bayerischen Landtag und Senat zurückgegriffen werden. Darin wird der Zeitraum zwischen 1981 bis 1983 beschrieben, als das Problem „Waldsterben“ in der forstlichen Wissenschaft und Praxis bereits weitgehend bekannt, definiert, von den Medien bereits aufgegriffen war und nun in den politischen Bereich Eingang findet.

\subsubsection{Waldschadensforschung auf der politischen Tagesordnung}

Bereits im Rahmen der erstmaligen Behandlung des Themas „Waldschäden“ im Bayerischen Landtag am 27.5.1981, die auf Antrag der politischen Opposition (SPD) stattfindet, berichtet der Leiter der Ministerialforstabteilung des Landwirtschaftsressorts nicht nur über die Schadenssituation und bereits eingeleitete Maßnahmen. Auch Bedarf an Forschung wird artikuliert. Die Vermutung, daß neben dem Witterungsverlauf auch Umweltbelastungen eine Rolle spielten, machten weitere Forschungsmaßnahmen notwendig [SCHNALKE 1986, S. 21, 23; BAYERISCHE StAATSFORSTVERWALtung 1981, S. 2-4]. Die Abgeordneten sind sich zwar über die Gefahr der Waldschäden einig, über deren Ursachen aber im unklaren. Konkrete Gegenmaßnahmen erschienen noch als verfrüht; allerdings sei eine Intensivierung der Ursachenforschung notwendig, um konkrete wissenschaftliche Ergebnisse als Grundlage für erforderliche politische Entscheidungen zu liefern.

Auch ein Antrag der Regierungspartei (CSU) wird am gleichen Tag (27.5.1981) dem Landwirtschaftsausschuß vorgelegt. Darin wird ein Forschungsprogramm zur Aufklärung des Fichtensterbens gefordert, welches das laufende Programm zum Tannensterben ergänzen soll. Der Antrag wird unter Hinweis auf die Notwendigkeit sofortiger und umfassender Forschung behandelt und am 4.3.1982 verabschiedet (Drucksache 9/11212).

Die einschlägigen Aktivitäten der Bayerischen Staatsforstverwaltung steigen daraufhin erheblich an [s. BAyerische StAatsforstVerwaltung 1983a, S. 3-7, 1983b, S. 10ff]. Neben Schadenserhebungen wird die Bayerische Forstliche Versuchs- und Forschungsanstalt mit der Erstellung eines Forschungsprogramms in Abstimmung mit der Forstwissenschaftlichen Fa- 
kultät beauftragt. Im August 1981 erhalten drei Lehrstühle zusätzliche Finanzmittel zur Ursachenforschung. Ende 1981 werden, mit 1,2 Mio. DM ausgestattet, 19 Projekte an verschiedene Lehrstühle der Fakultät sowie die Forschungsanstalt vergeben [BAYERISCHE STAATSFORSTVERWALTUNG 1982a, S. 3 und 1983a, S. 4].

Im Jahr 1982 rückt die Immissionsbelastung und deren Reduzierung immer mehr in den Mittelpunkt der Landtagsdiskussionen [SCHNALKE 1986, S. 25]. Dies ist bezeichnend für den inzwischen vermuteten und bei Öffentlichkeit und Medien nahezu unumstößlichen Zusammenhang zwischen Waldsterben und Luftschadstoffen [s.a. DER RAT VON SACHVERSTÄNDIGEN FÜR UMWELTFRAGEN 1983, S. 1; SUDA 1984, S. 41].

Die Notwendigkeit von Forschungsmaßnahmen zur Ursachenklärung der Waldschäden wird im Landtag unterschiedlich beurteilt [SCHNALKE 1986, S. 35ff]. Von Vertretern der Staatsregierung wird die uneinheitliche Einschätzung durch die Wissenschaft beklagt und auf laufende Forschungsaktivitäten verwiesen, deren Ergebnisse erst abgewartet werden müßten. Demgegenüber reichte der Opposition der bisherige Wissensstand bereits aus, um drastische Emissionsminderungen durchzusetzen.

Letztlich setzt sich jedoch die politische Forderung durch, die Ursachenforschung zu den Waldschäden zu intensivieren. Am 22.7.1982 werden dem Bayerischen Landtag sowohl von der Regierungspartei als auch der Opposition vorgelegte Dringlichkeitsanträge beschlossen (Drucksache 9/12848). Neben Emissionsminderungsmaßnahmen und forstlichen Sanierungsmaßnahmen wird die Intensivierung der ,Säure-Wirkungsforschung“ zur Ermittlung der Ursachen gefordert. Der Ministerrat beschließt auf seiner Sitzung am 29.6.1982, die Verbesserung der Luftreinhaltepolitik und die Ursachenforschung verstärkt fortzuführen (Beschluß des Bayerischen Ministerrats vom 29.6.1982 in: BAYERISCHE STAATSFORSTVERWALTUNG [1982b, S. 12]).

Ein weiterer Dringlichkeitsantrag der CSU-Fraktion zum Waldsterben vom 8.2.1983 hat neben einem Maßnahmenkatalog zur Schadstoffreduzierung die „Effizienzsteigerung“ der Forschung zum Inhalt. Diese solle durch einheitliche Schadenserhebung, interdisziplinäre Zusammenarbeit und verstärkte Luftschadstoff-Wirkungsforschung erreicht werden. Dem Antrag wird am 3.5.1983 vom Landtag zugestimmt (Drucksache 10/832).

Die Umsetzung der politisch beschlossenen Verstärkung der Waldschadensforschung wird in den jeweiligen Vollzugsberichten der Staatsregierung präsentiert. Am 17.5.1982 kann der Landwirtschaftsminister bereits auf die Durchführung 15 zusätzlicher Forschungsprojekte der Forstwissenschaftlichen Fakultät in Zusammenarbeit mit der Bayerischen Forstlichen Versuchsund Forschungsanstalt verweisen (Vollzugsbericht der Staatsregierung Nr. V2-FG-555-187, zit. in: SCHNALKE [1986, S. 24/25]). Das Umweltministerium erweitert unter anderem seine bereits 1974 begonnene Messungen zur Klärung möglicher Immissionseinflüsse auf die Vegetation [BAYERISCHES UMWELT- UND LANDWIRTSCHAFTSMINISTERIUM 1982, S. 9]. Im Vollzugsbericht vom 26.1.1983 (Nr. V2-FV100a-115, zit. in: SCHNALKE [1986, S. 33/34]) werden inzwischen 12 weitere bewilligte, überwiegend wiederum von Münchner Forstwissenschaftlern 
bearbeitete Forschungsprojekte erwähnt. Der gesamte Finanzierungsumfang der Forschung betrüge bisher mindestens 11 Mio. DM.

Der aktuelle Stand der Waldschadensforschung im Jahr 1983 wird im Vollzugsbericht vom 30.1. 1984 beschrieben (Nr. V2-FG 558-484, zit. in: SchNALKE [1986, S. 44/45]). Danach habe sich eine verstärkte, interdisziplinäre Zusammenarbeit bereits ergeben. Ursachenforschung werde von der Münchner Forstwirtschaftlichen Fakultät, einer Reihe weiterer Institute und der finanziell und personell verstärkten Forstlichen Versuchs- und Forschungsanstalt betrieben. Zudem sei an der Gesellschaft für Strahlen- und Umweltforschung mbH. (GSF) die „, Münchner Arbeitsgruppe Luftschadstoffe“ (MAGL) konstituiert worden, welche in neu errichteten Expositionskammern verschiedene Forschungsprojekte zum Thema bearbeiteten solle.

Die Einbeziehung der Wissenschaft in die politischen Agenda-Gestaltung zur Forschungspolicy im Landtag erfolgt erstmals während einer Expertenanhörung im Februar 1983 (Landtagsprotokoll zur Gemeinschaftlichen informatorischen Sitzung mehrerer Landtagsausschüsse vom 2./3.2.1983). Vertreten sind u.a. renommierte Waldschadensforscher der beiden Münchner Universitäten, der Universität Bayreuth sowie die Bayerische Forstliche Versuchs- und Forschungsanstalt. Für den Zustand des Waldes könne auch zu Beginn des Jahres 1983 noch kein verläßliches Bild abgegeben werden. Wie ScHNALKE [1986, S. 39ff] die Diskussion um die Schadensursachen zusammenfaßt, werden nach übereinstimmender Auffassung die Luftverunreinigungen als Hauptursache angesehen. Es wird eine Komplexerkrankung durch abiotische und biotische Faktoren angenommen. Die facettenreich vorgestellten Erscheinungsformen der Waldschäden und deren Ursachenhypothesen sind einerseits geeignet, auf erheblichen Forschungsbedarf hinzuweisen. Andererseits stiften sie erhebliche Verwirrung bei den Politikern (Landtagsprotokoll vom 2./3.2.1983, S. 308 ff, 351ff).

\subsubsection{Bund/Länder-Bemühungen zur Koordinierung der Waldschadensforschung}

Die Forderung, die Waldschadensforschung zu koordinieren, wird nicht nur von der Politik, sondern auch von den Waldschadensforschern frühzeitig und wiederholt artikuliert [SCHÜTT 1978, 1982 und 1983; DiE ZEIT, 25.12.1981]. Auch DER RAT VON SACHVERSTÄNDIGEN FÜR UMWELTFRAGEN [1983, S. 265] weist auf die Notwendigkeit der Koordinierung der Forschungsarbeiten hin. Diese müsse nicht nur fachlich zwischen den verschiedenen Disziplinen gefördert, sondern auch institutionell zwischen den einschlägigen Forschungsanstalten des Bundes und der Länder sowie den Universitäten verstärkt werden. Zum damaligen Zeitpunkt vorhandene Ansätze der Bundesregierung werden als nicht ausreichend eingeschätzt.

Erstmals politisch artikuliert wird ein bundesweiter Koordinierungsbedarf der Waldschadensforschung in den Dringlichkeitsanträgen der Regierungspartei und Opposition vom Mai 1982 (Drucksache 9/12848 zum Beschluß der Anträge am 22.7.1982). Das Ersuchen an die Staatsregierung, ,die Forschung ... zu intensivieren und auf eine Koordinierung durch den Bund oder die EG hinzuwirken“, steht an erster Stelle eines 8-Punkte-Forderungskatalogs.

Auch der Bayerische Ministerrat wirft in seiner Sitzung vom 29.6.1982 der Bundesregierung vor, das Land in der Waldschadensforschung nicht ausreichend zu unterstützen. Der Minister- 
rat ,hält es insbesondere für unerträglich, daß die Bundesregierung ... Forschungsarbeiten über die Ursachen des Baumsterbens nicht koordiniert und nicht genügend Mittel für die Ursachenforschung bereitstellt“. Entsprechende Maßnahmen der Forschungsförderung und -koordinierung für die gesamte Bundesrepublik werden gefordert (Beschluß des Bayerischen Ministerrats vom 29.6.1982, abgedruckt in: BAYERISCHE STAATSFORSTVERWALTUNG [1982b, S. 1-2]).

Anläßlich der bereits erwähnten Expertenanhörung im Landtag vom 2./3.2.1983 (s. Protokoll zur Anhörung) sowie einer Haushaltsdebatte im Senat im Februar 1983 [zit. in: SCHNALKE 1986, S. 42] weist der Bayerische Landwirtschaftsminister darauf hin, daß die Forstliche Versuchs- und Forschungsanstalt zwar, ,von Anfang an die Koordinierung der Forschung über das Waldsterben wahrgenommen“ hätte. Eine Koordinierung auf Bundesebene sei jedoch noch nicht verwirklicht, obwohl derzeit bereits über 120 Forschungsvorhaben bundesweit vergeben seien. Die damit verbundene Gefahr der „,Doppelforschung“ sowie die Erfordernis eines verbesserten Ergebnisaustausches werden dabei bereits erkannt.

In der Folge fordert der Bayerische Ministerpräsident, verbunden mit Kritik am Bundeslandwirtschaftsressort hinsichtlich der Behandlung des Themas „Waldsterben“, bundesweite Forschungskoordiniation (i.Q.: Bayer. Staatskanzlei, Februar und Mai 1983 ohne genaue Datumsangabe). Dabei wird vorgeschlagen, in München eine zentrale Forschungseinrichtung zur Erforschung der Wirkungen von Umweltschadstoffen zu gründen. Dieser Vorschlag kann auch damit in Zusammenhang gebracht werden, daß nahezu zeitgleich das Land Baden-Württemberg die Einrichtung eines vergleichbaren „Projektes Europäisches Forschungszentrum für Maßnahmen zur Luftreinhaltung“ (PEF) vorbereitet.

Auf Bundesebene wird die Koordinierung der Waldschadensforschung im Juni 1983 Kabinettssache. Ein entsprechendes Konzept wird beschlossen, worin der Stand der Forschung, deren Finanzierung und Koordinierung dargestellt wird. Zum damaligen Zeitpunkt würden rund 200 Vorhaben bundesweit bearbeitet, an deren Finanzierung in Höhe von insgesamt rund 26 Millionen DM sich Bund, Länder, Deutsche Forschungsgemeinschaft und sonstige staatliche Auftraggeber beteiligten. Angeregt von den Ländern Baden-Württemberg und Bayern ,hält es die Bundesregierung für zweckmäßig, die von Bund und Ländern geförderten Forschungsvorhaben in regionalen Forschungsschwerpunkten mit fachlich differenzierter Thematik zu bündeln und die wissenschaftlichen Aktivitäten gezielt weiter zu verstärken“. Dabei böten sich die Räume Karlsruhe/Freiburg bzw. München/Bayreuth an, um ausgehend von dort ansässigen Großforschungseinrichtungen, Hochschulen und Landesforschungsanstalten regionale Forschungsschwerpunkte mit der notwendigen fachlichen Verzahnung und Koordination zu etablieren (Kabinettsvorlage vom 8. Juni 1983).

Hervorzuheben ist, daß der Bund eine ,, wirksame Koordinierung nur auf freiwilliger und kollegialer Grundlage“ für möglich hält. Die Koordination durch eine einzige zentrale, fachlich ausgewiesene Institution scheide angesichts der großen Selbständigkeit etablierter Forschergruppen, rechtlicher Bedenken sowie in Ermangelung einer geeigneten, auf allen relevanten Disziplinen zum Thema Waldschäden führenden Institution aus (S. 7 der Kabinettsvorlage). 
Der Beschluß des Bundeskabinetts zur Koordinierung der Waldschadensforschung wird den betroffenen bayerischen Ressorts Kultus, Landwirtschaft und Umwelt sowie der GSF mitgeteilt (i.Q.: Bundesforschungsministerium, 16.6.1983). Gebeten wird, Möglichkeiten der Bündelung und Intensivierung der Forschung zu nennen. Auf die drei in Bayern bereits bestehenden Förderschwerpunkte, das Kuratorium der Forstlichen Versuchs- und Forschungsanstalt, die Münchner Arbeitsgruppe Luftschadstoffe an der GSF sowie die Bayerische Forschungsgruppe Forsttoxikologie, wird hingewiesen.

\subsubsection{Bayerischer Vorschlag zur Bildung einer bundesweiten Koordinierungsstelle}

Die betroffenen bayerischen Ressorts reagieren rasch auf die erbetenen Vorschläge (i.Q.: Bayer. Umwelt- und Landwirtschaftsministerium, Ende Juni 1983 ohne genaues Datum). Dem bayerischen Ministerpräsidenten wird ein „Forschungskonzept für eine überregionale zentrale Forschungseinrichtung zur Bekämpfung des Waldsterbens“"vorgelegt, das den bundesweiten Vorschlag inhaltlich weitgehend berücksichtigt.

Das Konzept listet unter anderem auf Bundes- oder Länderebene inzwischen konstituierte Institutionen im Zusammenhang mit der Waldschadensforschung, -förderung und -koordinierung auf: Es handelt sich um

- die Interministerielle Arbeitsgruppe Waldschäden/Luftschadstoffe des Bundes und der Länder (IMA), deren Aufgabe die bundesweite Koordination sämtlicher Forschungsarbeiten ist, und der ein

- wissenschaftlicher „Forschungsbeirat Waldschäden“ (FBW) zugeordnet wurde,

- die Münchner Arbeitsgemeinschaft Luftschadstoffe (MAGL) und deren Lenkungsausschuß bei der Gesellschaft für Strahlen- und Umweltforschung (GSF) in Neuherberg bei München zur gemeinschaftlichen Nutzung von Expositionskammern,

- die bestehenden Projektträgerschaften des Bundesministeriums für Forschung und Technologie bei der Kernforschungsanlage Jülich (KFA) und der GSF zur Koordination der ressortfinanzierten Forschung,

- das Projekt Europäisches Forschungszentrum für Maßnahmen zur Luftreinhaltung (PEF) des Landes Baden-Württemberg, angesiedelt beim Kernforschungszentrum Karlsruhe (KFK), welches im Bereich der Luftschadstoff-Forschung und technischer Verfahren zur Emissionsminderung tätig werden soll,

- die Bayerische Forschungsgruppe Forsttoxikologie auf Initiative des Beirats für Wissenschafts- und Hochschulfragen beim Bayerischen Kultusministerium mit Forschungsschwerpunkt Fichtelgebirge sowie das

- Kuratorium der Bayerischen Forstlichen Versuchs- und Forschungsanstalt des Landwirtschaftsministeriums zur Koordinierung und Finanzierung der forstlichen Forschung in Bayern.

Trotz dieser „Fülle von Koordinationsbemühungen“ des Bundes und der Länder könne jedoch nicht von einer zielgerichteten Wirkungsforschung gesprochen werden. In dem Schreiben wird daher ein integriertes Forschungskonzept vorgeschlagen, für welches unter Einbeziehung der genannten Koordinationseinrichtungen zentral in München die „Deutsche Gesellschaft zur Er- 
forschung der Wirkungen von Umweltschadstoffen mbH.“ (DGWU) gegründet werden solle. Aufgabe sei die bundesweite Lenkung sämtlicher Forschungsarbeiten zum Thema. Mit zeitlicher Priorität sei der Komplex „Waldschäden“ zu bearbeiten. Die bereits bestehenden Aktivitäten von Forschergruppen in Bayern sollten zunächst den Schwerpunkt bilden. Begründet wird die Gesellschaft unter anderem damit, daß die Wirkungsforschung zu verstärken und hierzu eine klare Konzeption und Koordination zu schaffen sei. Damit könne zudem Doppelforschung vermieden und Forschungsaktivitäten gebündelt werden.

Auf den Ministerratssitzungen vom 5. und 12. Juli 1983 wird das bayerische Konzept zur bundesweiten Verstärkung und Koordinierung der Wirkungsforschung behandelt und beschlossen (i.Q.: Bayerische Staatskanzlei, 15.7.1983). Begründet wird der Standort München zur Gründung der bundesweiten Koordinierungsstelle DGWU mit den in Bayern bereits vorhandenen Forschungseinrichtungen an Hochschulen und Behörden sowie der GSF. Mit der Gründung der DGWU werden das Bayerische Umwelt- und Landwirtschaftsministerium beauftragt. Die ländereigenen Finanzmittel solle das Finanzministerium bereitstellen. Als Ansprechpartner für den Bund wird der Ministerialdirigent im Umweltministerium benannt.

Auch der Bayerische Senat unterstützt die Einrichtung der DGWU. Er favorisiert als deren Sitz die GSF in Neuherberg (Senats-Drucksache 115/83 und 128/83, zit. in: SCHNALKE 1986, S. 53). Das Umweltministerium übernimmt die Verhandlungsführung mit den weiteren beteiligten bayerischen Ressorts, dem Bund und der GSF.

Die GSF erklärt grundsätzlich ihre Bereitschaft, die Geschäftsführung der DGWU zu übernehmen (i.Q.: Bayerisches Umweltministerium, 22.8.1983). Die Aufgaben der Forschungskoordination sollten mit mehreren bei der DGWU angestellten Wissenschaftlern verschiedener Fachrichtungen sowie abgeordneten Fachbeamten des Umwelt- und Landwirtschaftsministeriums bearbeitet werden. Die Alternative, statt der DGWU die GSF selbst mit der Lenkung und Koordinierung der Forschung zu betrauen, wird verworfen. Es werde die fehlende Bereitschaft anderer wissenschaftlicher Institutionen befürchtet, die GSF als Lenkungs- und Koordinationsstelle anzuerkennen.

Das Bayerische Umweltministerium sieht sich kurz darauf jedoch veranlaßt, die GSF lediglich für eine „Übergangslösung“ zu gewinnen (i.Q.: Bayer. Umweltministerium, 22.8.1983). Es deute sich nämlich an, daß auf Bundesebene eine ablehnende Haltung bzgl. der DGWU bestünde. So teilt das Bundesforschungsministerium mit, daß Fragen der Neukonzeptionierung der Umwelt-Wirkungsforschung bereits von bestehenden Koordinierungsgremien behandelt würden. Auch sei zu prüfen, ob nicht erforderlichenfalls die Aktivitäten bereits etablierter Einrichtungen des Landes intensiviert werden sollten, statt eine neue zu schaffen (i.Q.: Bundesforschungsministerium, 17.11.1983).

Im Dezember 1983 muß die Bayerische Staatsregierung dem Senat mitteilen, daß der Vorschlag zur DGWU-Gründung vom Bund noch immer nicht positiv aufgenommen sei (SenatsDrucksache 217/83). Daher werde zunächst die Einrichtung einer Koordinierungsleitstelle für die mit bayerischen Mitteln finanzierten Forschung erwogen (Senats-Drucksache 44/84 vom 9.3.1984). Daß Bayern auch im Alleingang eine ,zentrale Forschungsstätte zum Waldsterben“ 
für Bayern mit Sitz bei der GSF einrichten wolle, wenn die gemeinsame Finanzierung mit dem Bund nicht rasch genug zustande käme, kommt auch in einer entsprechenden Presse-Meldung der SÜDDEUTSCHEN ZEITUNG vom 15.2.1984 zum Ausdruck.

\subsubsection{Beschluß zur Schaffung einer Koordinierungsstelle für die bayerische Waldschadensforschung (PBWU)}

Ende November 1983 legt das Bayerische Umweltministerium der GSF eine Leistungsbeschreibung für die Schaffung einer Projektgruppe zur Koordination der Umweltforschung in Bayern vor. Die GSF wird gebeten zu prüfen, ob bzw. unter welchen Bedingungen sie bereit wäre, einen derartigen Forschungsauftrag für den Fall zu übernehmen, daß die bundesweite DGWU nicht oder noch nicht gegründet würde. Als Schwerpunktbereiche der zu verstärkenden und über die Stelle zu koordinierenden Forschung werden Schadenserfassung, Umweltanalyse und Ursachen-Wirkungsbeziehungen genannt (i.Q.: Bayer. Umweltministerium, 30.11. 1983).

Die GSF-Geschäftsführung gibt intern zu der Leistungsbeschreibung allerdings Unwägbarkeiten und Erfolgsunsicherheiten des Projektes zu bedenken (i.Q.: GSF, ohne Datumsangabe). Auch die vom Ministerium formulierte „Lenkungsfunktion“ wird kritisiert und eine allgemeinere Formulierung vorgeschlagen. Dennoch übermittelt die GSF dem Umweltministerium ein Angebot über die Einrichtung einer Projektgruppe und für die Betreuung eines koordinierten Forschungsprogramms ,"Waldschadensforschung und Wirkungen von Umweltschadstoffen“" an (i.Q.: GSF, 2.2.1984). Ziel sei es, „einerseits als Mittler zwischen Geldgebern und Wissenschaftlern effektive Forschung unter möglichst unbürokratischen Bedingungen zu gewährleisten und andererseits die notwendige Koordination des Projekts sicherzustellen, ohne die Eigenverantwortlichkeit der Forscher zu beschneiden“. Die Aufgaben der Projektgruppe werden beschrieben als die

- Ausarbeitung einer Matrix zur Erfassung sämtlicher Belastungspfade der geschädigten Waldbäume nach Art und Ausmaß der Belastung in unterschiedlichen geographischen Räumen Bayerns,

- Durchführung einer status quo-Analyse bisheriger Waldschadensforschung,

- Identifizierung des Forschungsbedarfs,

- Erstellung und Ausschreibung eines Rahmenprogramms an geeignete Forschungsinstitute,

- Programmdefinition (Begutachtung von Forschungsanträgen und Förderempfehlungen an die zu bewilligenden Stellen) und

- fachliche Betreuung und Berichtswesen.

Dieser Aufgabenkatalog entspricht weitgehend den Vorgaben des Umweltministeriums. Auch die drei vorgeschlagenen Schwerpunkte der Forschung werden übernommen. In dem Angebot wird unter anderem auf die an der GSF bereits in Eigenregie eingerichtete Stabsstelle „Wirkungsforschung Waldschäden“ hingewiesen. Diese habe mit organisatorischen Vorarbeiten begonnen. Beabsichtigt sei, die Stabsstelle in der nunmehr geplanten Projektgruppe aufgehen zu lassen. Das Angebot der GSF wurde maßgeblich von der Stabsstelle verfaßt. Ihr Leiter ist gegenüber dem Umweltministerium auch als direkter Ansprechpartner genannt. 
Der geplante Personalansatz beträgt 5 wissenschaftliche Mitarbeiter verschiedener naturwissenschaftlicher und für die Waldschadensforschung relevanter Disziplinen. Zur Finanzierung der Projektgruppe werden pro Jahr Mittel in Höhe von rund 700.000 DM beantragt. Als Auftrag- und Finanzgeber des Forschungsprojektes werden die beteiligten bayerischen Ressorts Umwelt-, Landwirtschafts- und Kultusministerium genannt. Auch ein aus mehreren ausgewiesenen Wissenschaftlern bestehendes Beratungsgremium wird vorgeschlagen.

Das GSF-Angebot sowie ein entsprechender Beschlußvorschlag für den Bayerischen Ministerrat werden vom Umweltministerium den weiteren zu beteiligenden Ressorts zugeleitet (i.Q.: Bayer. Umweltministerium, 16.5.1984). Dort stößt die geplante Gründung der Projektgruppe z.T. auf erhebliche Kritik (i.Q.: GSF, 18.5.1984). Das Finanzministerium moniere die Kosten, das Landwirtschaftsministerium unterbreite einen Gegenvorschlag. Kritisiert werde die Preisgabe bayerischer Zuständigkeiten an eine vom Bund abgängige Einrichtung und damit die fehlende Bindung an bayerische Ressorts. Die Aufgabe könnte auch von Fachbeamten der einschlägigen Ressorts übernommen werden; die Bildung einer interministeriellen Arbeitsgruppe werde vorgeschlagen. Das Umweltministerium weist demgegenüber besonders auf den direkten Kontakt der beabsichtigten Koordinierungsstelle zur Forschung an der GSF und deren hohe fachliche Kompetenz hin. Die Interessenvertretung der beteiligten Ressorts könne durch die Bildung eines entprechenden Projektrates gewährleistet werden.

Das Umweltministerium kann sich mit seinem Vorschlag trotz der Kritik von seiten der Nachbarressorts durchsetzen. Gestützt durch einen einstimmigen Landtagsbeschluß vom 8.5.1984 wird am 5.6.1984 der Ministerratsbeschluß gefaßt, die „Projektgruppe Bayern zur Erforschung der Wirkung von Umweltschadstoffen“ (PBWU) zu gründen². Der Ministerratsbeschluß hat folgenden Wortlaut:

„1. Die Projektgruppe trägt die Bezeichnung Projektgruppe Bayern zur Erforschung der Wirkung von Umweltschadstoffen - PBWU.

2. Die Projektgruppe wird unmittelbar bei der Geschäftsführung der GSF angesiedelt.

3. Zur Aufsicht über die Projektgruppe wird ein Projektrat gebildet, dem neben dem wissenschaftlich-technischen Geschäftsführer der GSF je 1 Vertreter der Staatsministerien für Landesentwicklung und Umweltfragen, für Ernährung, Landwirtschaft und Forsten, für Unterricht und Kultus sowie des Inneren angehören.

4. Die Projektgruppe besteht entsprechend dem mit Schreiben vom 16. Mai übersandten Finanzierungsplan aus 5 Wissenschaftlern verschiedener einschlägiger Fachrichtungen sowie 2 Hilfskräften.

5. Der Projektgruppe steht, soweit erforderlich, das personelle und sächliche Forschungspotential der Großforschungseinrichtung zur Verfügung. “

In den beiden Vollzugsberichten der Staatsregierung vom 30.1. und 31.7.1984 [zit. in: SCHNALKE 1986, S. 44-47] wird die PBWU-Gründung dokumentiert. Beabsichtigt sei, daß die

\footnotetext{
${ }^{2}$ Die Abkürzung PBWU wird entsprechend ihrer realiter erfolgten Verwendung in der Folge sowohl für die „Projektgruppe Bayern zur Erforschung der Wirkung für Umweltschadstoffe“ selbst, als auch für das über die Projektgruppe durchgeführte „PBWU-Projekt“" verwendet.
} 
PBWU die der DGWU zugedachten Aufgaben für Bayern übernehmen und später gegebenenfalls in diese eingebracht werden sollte. Die PBWU habe ihre Arbeit bereits aufgenommen.

\subsubsection{Theorieorientiertes Zwischenergebnis}

\subsection{Politischer Handlungsdruck}

Der politische Handlungsdruck umfaßt bei der gegebenen Ursachendiskussion zum einen die Verstärkung der Luftreinhaltepolitik, zum anderen die Verstärkung der Ursachenforschung, um Gegenmaßnahmen möglichst gezielt - und damit aber auch erst mit entsprechender Verzögerung - vornehmen zu können. Läßt sich die Thematisierung des Themas nicht aufhalten, verspricht zumindest die - forschungsbedingte - Verzögerung von Gegenmaßnahmen gewisse Entspannung im politischen Handlungsdruck und Entlastung von zusätzlichen Maßnahmen der Luftreinhaltung.

Hierbei müssen Regierung und Opposition unterschiedliche Rollen zugeschrieben werden. Während die Staatsregierung überwiegend auf die Verstärkung der Forschung setzt, um formal aufgrund deren Ergebnisse anschließend gezielt handeln zu können und informal einen Zeitgewinn zu erhalten, wirft die Opposition der Regierung unzureichende Luftreinhaltepolitik vor und fordert dementsprechend drastischere Maßnahmen und sofortiges Handeln.

Beide Maßnahmenpfade, Luftreinhaltepolitik und Ursachenforschung, müssen zudem länderübergreifend angegangen werden. Die anfängliche Vorgehensweise Bayerns, Verantwortung und Handlungsnotwendigkeiten auf den Bund abzuwälzen, kann dazu dienen, politische Kritik zu formulieren und sich diesbezüglich zu exkulpieren. Zudem kann daraus der - wenn letztlich auch erfolglose - Vorstoß legitimiert werden, hinsichtlich der zu verstärkenden Waldschadensforschung selbst anstelle des Bundes initiativ zu werden und eine bundesweite Koordinierungsstelle in Bayern zu schaffen. Diese Maßnahme wäre geeignet, zusätzliche Finanzmittel des Bundes in das Land fließen zu lassen und damit eine herausragende und steuernde Stellung in der bundesweiten Forschung zum Thema zu übernehmen.

\subsection{Umweltressort entlastet und konkurriert mit Landwirtschaftsressort}

Das Landwirtschafts-(Forst-)ressort hatte bereits aufgrund dem Waldsterben vorausgehender Tannenschäden auch unter dem Druck der Forstforscher ein entsprechendes Forschungsprogramm aufgelegt. Es sieht die forstliche Forschung zum Thema durch das Kuratorium der Bayerischen Forstlichen Versuchs- und Forschungsanstalt als ausreichend koordiniert an. Da die Forstverwaltung sich von externen Effekten der Luftverunreinigungen betroffen sieht, können verstärkte Forschungsansätze in erster Linie auf die forstlichen und forstwirtschaftlichen Auswirkungen der Waldschäden konzentriert werden sowie auf forstliche Gegen- bzw. Sanierungsmaßnahmen. Andere in den Mittelpunkt gestellte Forschungsbereiche zur Rolle von Luftschadstoffen und entsprechender Wirkungsforschung können - auch zur Entlastung des eigenen Etats - anderen Ressorts überlassen werden.

Bei deutlicher Focussierung der Waldschadensursachen auf den Faktor Luftverunreinigungen kann und muß immer mehr das Umweltministerium die federführende Rolle in der Finanzierung 
und Koordinierung der Waldschadensforschung übernehmen. Das Ministerium versucht, sich durch Verweis auf bereits vor der Waldschadensdiskussion umfangreich erfolgte Maßnahmen der Luftreinhaltepolitik hinsichtlich einer Verantwortlichkeit für die Waldschäden zu entziehen. Es sieht aber auch die Chance, durch die Federführung in der zu verstärkenden und koordinierenden Ursachen-Wirkungsforschung zur Aufklärung der Waldschäden seine Zuständigkeit, Kompetenz und Handlungsbereitschaft in einem neuen aktuellen Umweltthema unter Beweis zu stellen und damit sein Renommee zu verbessern.

Zudem entsteht verstärkt durch die politische Opposition und die Wissenschaftler eine Lobby, die neben der bisher bereits beteiligten, traditionellen Forstforschung auch außerforstliche Forschung etablieren will, soll das Ursachenproblem der Waldschäden erfolgreich gelöst werden. Ohnehin sind die Bestrebungen, eine bundes-, in Wirklichkeit nurmehr bayernweite Institution zur Forschungskoordinierung zu gründen, nur zeitlich vorrangig auf das aktuelle Thema Waldschäden focussiert. Generell ist es Strategie, wie auch in den Bezeichnungen der geplanten bundesdeutschen und später der realisierten bayerischen Projektgruppe zum Ausdruck kommt, eine Koordinationsstelle für die Wirkungsforschung $\mathrm{zu}$ Umweltschadstoffen allgemein $\mathrm{zu}$ gründen. Gerade vor diesem Hintergrund erscheint die Federführung des Umweltressorts bspw. gegenüber dem Landwirtschaftsressort legitim.

Die bereitwillig erfolgte Abtretung der Initiative für die zu verstärkende Waldschadensforschung an das Bayerische Umweltministerium findet allerdings bald ihre Grenzen. Sie bestehen darin, daß sich damit auch die Zuständigkeit für die Koordinierung der gesamtbayerischen Waldschadensforschung via einer eigens vom Landtag beschlossenen und an der bayerischen Großforschungseinrichtung GSF einzurichtenden Projektgruppe ergibt. Die Nachbarressorts befürchten zu Recht, daß zur bayernweiten Koordinierung der Waldschadensforschung ein steuernder Einfluß des Umweltministeriums auch auf die bereits bestehenden Programme des Landwirtschafts- und Kultusressorts ausgeübt werden könnte. Dieser würde zur Aufgabe eigenständiger, ressortspezifischer Forschungsplanung und -umsetzung zwingen. Entsprechende Machtkämpfe zur Wahrung der ressortspezifischen Interessen innerhalb des PBWU-Projekts bestimmen denn auch in der Folge insbesondere während derer Gründungs- und Programmformulierungsphase die Diskussionen.

\subsection{Koordinationsbedarf der Waldschadensforschung}

Daß Waldschadensforschung sinnvoll und effektiv nur in Form umfangreicher Forschungsprogramme unter Beteiligung verschiedenen Fachdisziplinen durchgeführt werden kann, der zudem einen erheblichen Koordinationsaufwand erfordert, wird bald und insbesondere auch von den Wissenschaftlern erkannt und gefordert. Kritik besteht an einer ersten Welle von bundesweiten Forschungsaktivitäten, die nach Aufflammen des Themas allenthalben gestartet wurden und die Gefahr von fachlichen oder regionalen Abstimmungsmängeln sowie von Doppelforschung bergen. Dies kann von den Wissenschaftlern dazu benutzt werden, in künftigen, strukturell $\mathrm{zu}$ verbessernden Forschungsaktivitäten ihre eigene Beteiligung zu stärken. Die Forschungskoordination muß nicht nur organisatorische, verteilungspolitische, sondern insbesondere auch fachliche Aspekte berücksichtigen. Es ist daher aus wissenschaftlicher Sicht naheliegend, nicht nur die Koordination der Forschung zu fordern, sondern auch zu versuchen, bei der 
Koordinierung selbst mitzuwirken und die entsprechenden Eigeninteressen bestmöglich hierin einzubringen. Dahinter steht die generelle steuerungsrelevante Interessenlage der Wissenschaft, Definitionsmacht über die Verteilung der Forschungsfördermittel zu erreichen [BRAUN 1997, S. 316].

Daß Forschungskoordination nicht nur notwendig ist, sondern diese Funktion auch einen erheblichen Macht- und Steuerungseinfluß bedeutet, ist nicht nur den Wissenschaftlern, sondern auch dem politisch administrativem System klar. Dieser Einfluß bezieht sich auf die Definition forschungspolitischer Ziele [BRAUN 1997, S. 316], die Auswahl zu bearbeitender Themen, aber auch die Finanzmittelverteilung sowie generell die Zutrittsentscheidung für verschiedene Forschergruppen bzw. Forschungsinstitutionen auf das Feld der Waldschadensforschung.

Während der Bund vorzieht, die Forschung thematisch und regional zu bündeln und dezentral $\mathrm{zu}$ koordinieren, fordert Bayern jedoch eine bundesweite Koordinationseinrichtung zur Forschungssteuerung im eigenen Lande. Die beiden Koordinierungsansätze repräsentieren unterschiedliche Steuerungsabsichten. Will der Bund die freiwillige Kooperation und Selbststeuerung in der Wissenschaft als Voraussetzung für eine wirksame Koordination berücksichtigen, favorisiert Bayern die Forschungskoordinierung durch die zentrale Lenkung aller bundesweiten Forschungsarbeiten. Dem bayerischen Vorstoß wird vom Bund jedoch eine Absage erteilt. Auch das Angebot, die vorgeschlagene Gesellschaft bei der GSF als Großforschungseinrichtung des Bundes einzurichten, reicht nicht aus, die erforderliche Akzeptanz beim Bund zu erreichen.

\subsection{Ressortstreitigkeiten auf Bundes- und Landesebene}

Auch innerhalb des Landes Bayern führt die Diskussion um die Ansiedlung einer Koordinationsstelle zu politischen bzw. Ressortstreitigkeiten. Während generell die Forderung der Forschungsverstärkung sowie die damit zu verbindende Schaffung einer Koordinationsstelle für die Waldschadensforschung von allen getragen wird, stößt der Vorschlag des Bayerischen Umweltministeriums, die Koordinationsstelle bei der GSF anzusiedeln, auf erhebliche Kritik. Die Befürchtung des Bundes, im Falle der bayerischen Gründung einer DGWU Kompetenzen an das Land abzugeben, ist vergleichbar mit der bayerischer Ministerien gegenüber der PBWUGründung, nämlich eigenstaatliche Zuständigkeiten an die GSF als eine vom Bund abhängige Forschungseinrichtung abzugeben.

Die GSF profitiert letztlich von dem Vorstoß Bayerns, eine bundesweite Koordinationsstelle in Bayern einzurichten. Als Sitz war die Großforschungseinrichtung hierfür bereits frühzeitig empfohlen worden. Dies ist insbesondere aus Sicht einer bundesweiten und generell über die Waldschadensforschung hinausgehende Aufgabenstellung schlüssig zu begründen und erhöht zudem die Chancen, die Zustimmung des Bundes zu gewinnen. Bei erfolgter Ablehnung des Bundes sind allerdings aufgrund fortgeschrittener Verhandlungen zwischen dem federführenden Umweltressort und der GSF inzwischen informale Verpflichtungen entstanden, die das Ministerium unter Zugzwang setzen. Es wäre trotz Beschränkung auf bayerische Belange nunmehr nahezu unmöglich, statt der GSF eine andere - bayerische - Institution mit der For- 
schungskoordination zu beauftragen, wie es von Vertretern benachbarter Ressorts gefordert wird.

Zudem besteht noch Hoffnung, daß die Entscheidung des Bundes eventuell revidiert und die inzwischen zu gründende Projektgruppe in einer späteren Bundeskoordinationsstelle aufgehen könnte. Daß Bayern notfalls einen Alleingang mit der GSF unternehmen würde, wenn die Einigung mit dem Bund nicht rasch genug zustande käme, wie in einer Pressemeldung des Bayerischen Umweltministeriums vom Februar 1984 verlautet, unterstreicht die Handlungsbereitschaft des Ministeriums einerseits, die faktisch beschlossene Beteiligung der GSF andererseits.

\subsubsection{Etablierung der Koordinierungsstelle PBWU}

\subsubsection{Gründung der PBWU}

\subsection{Entwurf eines PBWU-Vertrags durch das Bayerische Umweltministerium}

Bereits eine Woche nach Ministerratsbeschluß vom 5.6.1984 zum PBWU-Projekt übermittelt das Bayerische Umweltministerium der GSF einen Vertragsentwurf zur Einrichtung und Durchführung des Projektes, der eng mit der bereits bestehenden GSF-Stabsstelle abgestimmt ist. Auch die weiteren zu beteiligenden bayerischen Ressorts erhalten den Vertragsentwurf zur Abstimmung (i.Q.: Bayer. Umweltministerium, 14.6. und 3.7.1984). Der Entwurf impliziert die Vorgaben des Ministerratsbeschlusses und orientiert sich weitgehend, z.B. hinsichtlich der zusätzlichen Etablierung eines Wissenschaftlichen Beirats, an dem früheren Angebot der GSF. Die Projektstruktur umfaßt, wie in der Leistungsbeschreibung zum Vertragsentwurf dargestellt wird,

- die Projektgruppe, die der GSF-Geschäftsführung unterstellt und mit den für die Projektabwicklung erforderlichen Entscheidungskompetenzen ausgestattet ist,

- den Projektrat zur ständigen Fühlungnahme, bestehend aus den beteiligten bayerischen Ressorts und der GSF-Geschäftsführung

- den Wissenschaftlichen Beirat, bestehend aus mehreren Wissenschaftlern zur fachlichen Unterstützung der Projektgruppe, Begutachtung von Anträgen, inhaltlichen Mitgestaltung des Projekts und Bewertung der Ergebnisse sowie

- die Forschungsnehmer, d.h. Forschungsinstitutionen, die sich mit der eigentlichen Durchführung von Forschungsvorhaben zum Thema entsprechend einem Rahmenprogramm beteiligen können.

Kernpunkt des Vertragsentwurfs ist die Aufgabenbeschreibung der Projektgruppe, ,die Erforschung der Wirkung von Luftschadstoffen in Bayern zu lenken, zu koordinieren und auszuwerten“, wobei die Waldschadensforschung mit Priorität zu behandeln sei. Eine enge Abstimmung mit den Gremien Projektrat und Wissenschaftlicher Beirat ist vorgesehen. Die Vorgehensweise der Projektgruppe zur Vorbereitung und Ausschreibung eines ForschungsRahmenprogramms, Durchführung eines Begutachtungsverfahrens sowie die fachliche Betreuung, Berichtslegung etc. sind ebenfalls im Vertragsentwurf im Grundsatz festgelegt. Als Pro- 
jektbeginn ist bereits der 1.7.1984 vorgesehen. Die Vergütung beträgt bei dreijähriger Laufzeit insgesamt rund 2 Mio. DM.

Die GSF-Stabsstelle nutzt den vom Umweltressort vorgelegten Vertragsentwurf das Signal dafuir, bereits vor Vertragsunterzeichnung hinsichtlich weiterer Personalrekrutierung und vorbereitender Projektaufgaben in Vorleistung zu treten. Neben dem designierten Leiter der PBWU, einem promovierten Agrarwissenschaftler, ist bereits ein Diplom-Meteorologe und ein Diplom-Forstwirt in der Stabsstelle der GSF beschäftigt. Ein weiterer Diplom-Forstwirt wird bei absehbarer Unterzeichnung des PBWU-Vertrags zum 1.7.1984 eingestellt.

\subsection{Kritik seitens Forschungspolitik und Wissenschaft}

Der GSF-Geschäftsführung erscheint der Vertragsentwurf des Umweltressorts in der vorgelegten Fassung in einigen zentralen Formulierungen jedoch nicht akzeptabel. Sie bittet, auf die Aufgabe, die Forschung zu ,lenken“, zu verzichten. Angesichts der Tatsache, daß die Projektgruppe keine Durchsetzungsbefugnisse und -möglichkeiten hätte, könne eine Lenkung überhaupt nicht erfolgen. Auch die weiteren genannten Aufgaben der Forschungskoordination und -auswertung erscheinen der GSF als zu absolut gefaßt (i.Q.: GSF, 16.7.1984).

Auch das Landwirtschaftsministerium hat gegen die Aufgabenbeschreibung der Projektgruppe „erhebliche Bedenken“ (i.Q.: Bayer. Landwirtschaftsministerium, 24.7.1984). Der Aufgabenumfang ginge weit über den Auftrag des Landtagsbeschlusses vom 8.5.1984 hinaus, die bayerischen Forschungsvorhaben lediglich aufeinander abzustimmen. Lenkung und Ergebnisauswertung seien Aufgabe der jeweiligen Ressorts, wozu die Projektgruppe allenfalls Entscheidungshilfen liefern könne. Die enge Anbindung der Projektgruppe an die weiteren Ressorts werde daher nachdrücklich gefordert. Vom Kultusministerium liegt keine Stellungnahme zum Vertragsentwurf vor. Allerdings werden führende Wissenschaftler der von diesem Ressort betreuten und maßgeblich finanzierten Forschungsgruppe Forsttoxikologie um Stellungnahme gebeten (s. unten).

Die GSF-Geschäftsführung setzt ihrerseits noch während der Vertragsverhandlungen informell mehrere namhafte bayerische Waldschadensforscher über das geplante PBWU-Projekt in Kenntnis (i.Q.: GSF, 30.7.1984). Im Vorgriff auf das zu gründende Gremium des Wissenschaftlichen Beirats wird um ein gemeinsames Gespräch gebeten. Dieses solle helfen, die Akzeptanz von Seiten der Wissenschaft herzustellen und damit die Erfüllung der Aufgaben der PBWU zu sichern. Bei den Wissenschaftlern handelt es sich - ähnlich den vom Kultusressort angefragten Wissenschaftlern - fast ausschließlich um spätere Mitglieder des Wissenschaftlichen Beirats der PBWU. Ein gemeinsamer Termin kommt allerdings nicht zustande.

Ein Sprecher der Bayerischen Forschungsgruppe Forsttoxikologie antwortet auf Bitte des Kultusministeriums der GSF (i.Q.: Forschungsgruppe Forsttoxikologie, 28.8.1984). Neben der Hoffnung, daß auch die von ihm vertretene Forschungsgruppe eine zusätzliche Förderung aus dem PBWU-Projekt erfahren könne, wird der Anspruch auf Freiheit der Wissenschaft zu bedenken gegeben. Der Wissenschaftler hebt besonders hervor, daß in seinem Forschungsverbund die bearbeiteten Fragestellungen und angewandten Methoden entsprechend dem Bayeri- 
schen Hochschulgesetz frei gewählt würden, die Zusammenarbeit nach eigener Entscheidung erfolge und die Ergebnisse in wissenschaftlichen Publikationen vorgelegt würden.

Eine interne, von führenden Wissenschaftlern der genannten Forschungsgruppe unterzeichnete Stellungnahme (i.Q.: Forschungsgruppe Forsttoxikologie, ohne Datumsangabe) macht die kritische Einstellung gegenüber dem PBWU-Projekt noch deutlicher. Zwar wird die Durchführung eines Forschungsprogramms begrüßt. Gegen den Aufbau der Projektgruppe sprächen aber verschiedene Gesichtspunkte: So sei die Begutachtung von Forschungsanträgen durch den Auftraggeber oder von diesem bestellte Gutachter nicht haltbar. Die Koordinationsaufgaben der Projektgruppe stünden im Widerspruch zur Kreativität und Selbständigkeit der Forscher. Es sei eine straffe Auftragsforschung erwünscht, ,ohne jedoch zu wissen, was erforscht werden solle und ohne für das erforschte Ergebnis verantwortlich zeichnen zu wollen“.

Mit der Aufgabe der Ergebnisdokumentation werde zudem ein Eingriff in das Urheberrecht der Wissenschaftler befürchtet und eine rasche Umsetzung wissenschaftlicher Ergebnisse behindert. Generell wird die Sinnhaftigkeit einer derartigen Projektgruppe in Frage gestellt, die bei hohen Kosten und hohem organisatorischem Aufwand „keine eigentliche wissenschaftliche Initiative und Idee" habe. Eine Mitarbeit der Universitäten sei auf breiter interdisziplinärer Ebene nicht zu erwarten.

Interne Anmerkungen der genannten Wissenschaftler zur Leistungsbeschreibung der PBWU erweitern die Kritik noch (i.Q.: Forschungsgruppe Forsttoxikologie, ohne Datumsangabe). So würden der Projektgruppe Kontrollfunktionen gegenüber den Forschern übertragen, ohne wissenschaftlich und sachlich gleichrangig zu sein, ihre Dokumentationsfunktion entzöge sich der Korrekturmöglichkeit der Wissenschaftler. Mit der fachlichen Betreuung sei die Projektgruppe wissenschaftlich schlichtweg überfordert, die freie Entfaltung einer kreativen Forschung hingegen blockiert.

Dem Wissenschaftlichen Beirat hingegen müsse nicht nur beratende Funktion, sondern vielmehr Weisungsbefugnis gegenüber der Projektgruppe erteilt werden. Allerdings müßte er dann entsprechend umstrukturiert werden, um den Eindruck eines „Selbstbedienungsladens“ zu vermeiden. Dieser Verdacht träfe auch für die GSF selbst zu, die sich bei der starken Stellung der Geschäftsführung und Auswahl der Gutachter durch die Projektgruppe eventuell als größter Forschungsnehmer entwickeln könnte. Für den Fall, daß mit dem PBWU-Projekt „Auftragsforschung" im Sinne von Industrieforschung betreut werden sollte, sei gegen die kontrollierenden Aufgaben der PBWU nichts einzuwenden. Für übliche, universitäre „Drittmittelforschung" sei das Konzept jedoch nicht akzeptabel.

Das offizielle Antwortschreiben an die GSF gibt der Sprecher der Forschungsgruppe weiteren, von der GSF angeschriebenen Kollegen zur Kenntnis (i.Q.: Forschungsgruppe Forsttoxikologie, 28.8.1984). Zur Rückversicherung seiner Einschätzung habe er den Präsidenten seiner Universität um eine Stellungnahme gebeten. Dieser hatte die Angelegenheit einer Behandlung in der Bayerischen Rektorenkonferenz zugeführt.

Der Präsident der Universität teilt die Bedenken der Forscher. Er hält eine zentrale Koordinationsstelle, wie geplant, angesichts der Eigenverantwortlichkeit der Universitäten für nicht erfor- 
derlich und befürchtet, daß damit nur die Bürokratie erhöht und die Universitäten bloße Teile der Ressortforschung würden (i.Q.: Forschungsgruppe Forsttoxikologie, 27.9.1984). Die Mitglieder der Rektorenkonferenz beurteilen das PBWU-Projekt jedoch nicht in diesem Maße kritisch (i.Q.: Forschungsgruppe Forsttoxikologie, 23.11.1984). Vielmehr würde die beabsichtigte Vorgehensweise an der GSF dazu beitragen, Doppelarbeiten zu vermeiden. Voraussetzung sei allerdings, daß die Einbindung anderer Forschungsvorhaben nicht als sachliche Einmischung zu verstehen sei und die Freiheit der Wahl der Untersuchungsgegenstände durch die Wissenschaftler gesichert bliebe. Der Universitätspräsident rät daher von einem „Alleingang“ der Universität gegen die geplante PBWU-Gründung ab.

Insgesamt nimmt die heftige externe Kritik der Wissenschaft jedoch keinen direkten Einfluß auf die politischen Vertragsverhandlungen zur PBWU-Gründung. Sie wird erst mit der späteren Installierung des Gremiums des Wissenschaftlichen Beirats offiziell zum Ausdruck gebracht und behandelt.

\subsection{Vertragsabschluß zum PBWU-Projekt}

In einem modifizierten Vertragsentwurf des Bayerischen Umweltministerium sind die geforderten Änderungen des Landwirtschaftsministeriums sowie der GSF allerdings nur zum Teil berücksichtigt; das kritisierte Aufgabenspektrum der PBWU bleibt weitgehend erhalten. Auch der vom Landwirtschaftsressort geforderten Stärkung der Stellung des Projektrats wurde nicht entsprochen (i.Q.: Bayer. Umweltministerium, 9.8.1984). Die Einbeziehung der Änderungswünsche hätte nach Auffassung des Umweltministeriums eine Neuauflage der Abstimmungsgespräche erfordert, was zu weiteren, aufgrund des Handlungsdrucks nicht hinnehmbaren Verzögerungen geführt hätte (i.Q.: PBWU, 12.7.1984).

Die GSF sendet daraufhin zwar eine gegengezeichnete Vertragsausfertigung an das Umweltministerium zurück, erklärt aber ihre Änderungsvorschläge vom 16.7.1984 zum Bestandteil des PBWU-Vertrags (i.Q.: GSF, 22.8.1984). Das PBWU-Projekt beginnt somit offiziell und rückwirkend zum 1.7.1984.

\subsubsection{Etablierung der PBWU-Gremien}

\subsection{Konstituierende Sitzung des „Projektrats“}

Die konstituierende Sitzung des Projektrats der PBWU findet am 8.10.1984 in der GSF statt (i.Q.: Bayer. Umweltministerium, 15.10. und 3.12.1984). Die Fachvertreter der beteiligten Ressorts - i.d.R. hohe Verwaltungsbeamte wie z.B. Forschungsreferenten - waren dem Umweltministerium bereits vorab namentlich benannt worden. Als Vorsitzender des Projektrats wird der Ministerialdirigent des Bayerischen Umweltministeriums bestimmt.

Eine Satzung erscheint nicht erforderlich. Eingangs zur Sitzung kann der inzwischen bestellte Leiter der Projektgruppe auf bereits erfolgte, projektvorbereitende Aktivitäten verweisen. Hinsichtlich der Etablierung des Wissenschaftlichen Beirats empfehlen die Projektratsmitglieder, zunächst ein Gremium zur laufenden Beratung und Zielfindung zu berufen. 11 namhafte Wissenschaftler, die überwiegend an der Waldschadensforschung und hier insbesondere an dem 
vom Kultusressort finanzierten, nordbayerischen Forschungsverbund beteiligt sind, werden vorgeschlagen.

Um aufgrund des erklärten Handlungsdrucks noch vor Etablierung des Wissenschaftlichen Beirats Forschungsfinanzierungen im PBWU-Projekt durchführen zu können, wird allerdings festgelegt, ,die Abstimmung über mögliche Förderungen in pragmatischer Weise zwischen der Projektgruppe und dem zu bewilligenden Ressort kurzhändig“ herbeizuführen. Damit behält sich das finanzierende Umweltministerium vor, auch ohne eine Abstimmung mit dem Wissenschaftlichen Beirat Forschungsvorhaben auf den Weg bringen zu können.

Auf der Sitzung werden verschiedene Kritikpunkte von Mitgliedern des Projektrates am PBWU-Vertrag diskutiert und sollen ergänzend zum Vertrag festgeschrieben werden. Diese beschränken einerseits die „Lenkungsaufgabe“ der PBWU auf die Abgabe von Förderempfehlungen an die zuständigen Ressorts, konkretisieren und stärken andererseits die Funktionen des Projektrats.

Die im Entwurf des Sitzungsprotokolls ausgeführten Vereinbarungen berücksichtigen die Kritikpunkte jedoch nur unzureichend. Der Vertreter des Landwirtschaftsressorts hält daher weitere PBWU-Vertragsinterpretationen und -korrekturen für unabdingbar (i.Q.: Bayer. Landwirtschaftsministerium, 7.11.1984). Die Rolle der PBWU werde lediglich darin gesehen, Forschungsdefizite zu ermitteln und zu versuchen, potentielle Forschungsnehmer dafür zu gewinnen. Hinsichtlich der Überprüfung eingereichter Forschungsanträge solle ihre Funktion zugunsten einer stärkeren Beteiligung der Gremien Projektrat und Wissenschaftlicher Beirat eingeschränkt werden. Auch der Vertreter des Kultusministeriums möchte hinsichtlich der Beurteilung eingehender Forschungsanträge die Projektgruppe auf bloße Verwaltungsfunktionen reduziert wissen (i.Q.: Bayer. Kultusministerium, 24.10.1984).

Die erbetenen Korrekturen der beiden Ministerien werden übernommen. Sie werden allesamt als „Interpretation des Projektrats zum vertraglichen Vollzug des Projekts PBWU, Stand 1.12.1984“ dem Protokoll in seiner Endfassung gesondert beigefügt (i.Q.: Bayer. Umweltministerium, 3.12.1984). Das Kultusministerium setzt hierüber den Sprecher der Bayerischen Forschungsgruppe Forsttoxikologie in Kenntnis (i.Q.: Bayer. Kultusministerium, 18.12.1984).

Auch das Verhältnis zwischen PBWU und anderen bestehenden Förder- bzw. Forschungseinrichtungen, insbesondere der Bayerischen Forstlichen Versuchs- und Forschungsanstalt und der Forschungsgruppe Forsttoxikologie, wird auf der konstituierenden Sitzung vom 8.10.1984 diskutiert (i.Q.: Bayer. Umweltministerium, 3.12.1984). Einigkeit besteht darin, daß die Inhalte und Ergebnisse dortiger Forschungsvorhaben auch der PBWU zur Verfügung stehen müßten. Die Nutzungsrechte, die der Auftraggeber Umweltministerium aus dem PBWUVertrag erwirbt, so wird klargestellt, „beziehen sich ausschließlich auf die Arbeitsergebnisse der Projektgruppe, nicht jedoch auf die vorgelagerten Ergebnisse ressortgebundener Forschungsvorhaben“. Der Leiter der PBWU solle als Gast bei der nächsten Kuratoriumssitzung der Forschungsanstalt geladen werden.

Bei einem ersten informativen Besuch der PBWU bei der Forschungsanstalt am 13.11.1984 werden diese Nutzungsrecht-Vorbehalte deutlich gemacht. Es werden Bedenken geäußert ge- 
genüber der frühzeitigen Überlassung von Informationen über Forschungsprojekte an die PBWU. Eine entsprechende Regelung für einen Informationsfluß der Forschungsanstalt an die Projektgruppe kann nicht vereinbart werden (i.Q.: PBWU, 19.11.1984).

\subsection{Einrichtung des „Wissenschaftlichen Beirats“}

Entsprechend der Leistungsbeschreibung des PBWU-Vertrags (i.d.F. vom 1.12.1984) ist im PBWU-Projekt ein beratendes Wissenschaftler-Gremium einzurichten. Aufgaben des Wissenschaftlichen Beirats sind die

- fachliche Unterstützung und Beratung der PBWU und des Projektrats,

- fachliche Begutachtung der Forschungsanträge,

- inhaltliche Mitgestaltung des Projekts und

- Bewertung der Ergebnisse.

Die auf der konstituierenden Projektratsitzung vom 8.10.1984 vorgeschlagenen Wissenschaftler repräsentieren fast ausschließlich bayerische Universitäten. Vertreten sind führende Wissenschaftler der Universitäten Bayreuth, Würzburg, Erlangen, Regensburg und der beiden Münchener Universitäten. Es wird betont, daß ,es förderlich sei, wenn die Mitglieder dieses Gremiums selbst aktiv in der Waldschadensforschung beteiligt sind“ (i.Q.: Bayer. Umweltministerium, 3.12.1984). Zudem soll nach dem PBWU-Vertrag die Zusammensetzung des Gremiums aufgabenspezifisch orientiert sein.

Mit den Namensnennungen sind relevante wissenschaftliche Disziplinen abgedeckt. Es handelt sich um Vertreter der Botanik, Pflanzenökologie, Ökologischen Chemie, Bodenkunde, Hydrologie, Zoologie, Atmosphärenphysik und Immissionsforschung. Botaniker sind mit allein 4 von 11 Nennungen stark vertreten, was auf den beabsichtigten Förderschwerpunkt der Wirkungsforschung hinweist. Bei den meisten der vorgeschlagenen Beiratsmitglieder handelt es sich um Wissenschaftler, die von der GSF-Geschäftsführung bereits im Vorfeld zur PBWUGründung kontaktiert worden waren, der GSF-nahen Münchner Arbeitsgruppe Luftschadstoffe (MAGL) oder/und der kultusministeriell finanzierten Bayerischen Forschungsgruppe Forsttoxikologie angehören.

Die vorgeschlagenen Wissenschaftler werden vom Bayerischen Umweltminister um Mitarbeit im Wissenschaftlichen Beirat der PBWU gebeten (i.Q.: Bayer. Umweltministerium, 18.12.1984). Mit 2 Ausnahmen bekunden alle Angeschriebenen ihre Zusage. Führende Vertreter des nordbayerischen Forschungsverbundes Forsttoxikologie nutzen ihre Zusage dazu, ihre grundsätzliche Kritik an der Einrichtung der Projektgruppe zum Ausdruck zu bringen (i.Q.: Wissenschaftlicher Beirat, 7.1. und 2.1.1985). Ein weiteres Beiratsmitglied scheidet nach nur kurzer Zugehörigkeit aus. Es könne es sich mit der mehrheitlich getragenen, Luftschadstoffe betonenden Auffassung über die Ursachen der Waldschäden nicht identifizieren (i.Q.: Wissenschaftlicher Beirat, 18.3.1985).

Die erste Sitzung des Wissenschaftlichen Beirats findet gemeinsam mit dem Projektrat am 11.2.1985 in der GSF statt (i.Q.: Bayer. Umweltministerium, 11.2.1985). Der Leiter der PBWU ist nur zeitweise zur Sitzung zugelassen. Eine geschäftsmäßige Organisation des Gre- 
miums erscheint nur im Hinblick auf seine Aufgabenbeschreibung erforderlich. Für das Förderverfahren wird es als nicht zweckmäßig angesehen, wenn der Beirat die Beurteilung von Forschungsanträgen selbst vornähme. Statt dessen solle ein externes Gutachtergremium berufen werden. Dieses könne aus DFG-Gutachtern bestehen und bspw. mit dem für die Bayerischen Forschungsgruppe Forsttoxikologie identisch sein. Der Beirat würde lediglich eine Vorauswahl aus den eingereichten Anträgen treffen, während die eigentliche Bewertung durch das Gutachtergremium erfolgen sollte.

Einige Mitglieder des Beirats äußern ihre Kritik an dem Projekt. Dies führt zu einer kontroversen und grundsätzlichen Diskussion um die PBWU-Aufgaben, wobei die Argumente der Wissenschaftler jedoch weder umfassend noch im einzelnen dokumentiert werden. Im Nachgang zum Protokoll wird denn auch von Seiten eines Beiratsmitglieds eine grundsätzliche Korrektur erbeten (i.Q.: Wissenschaftlicher Beirat, 7.6.1985). Demnach werde das Ergebnis der Diskussion so verstanden, daß der Beirat in seiner Beratungsfunktion nicht der Projektgruppe nachgeordnet sei, wie aus dem Protokoll hervorgehe, sondern parallel zur Projektgruppe dem Projektrat unterstellt sei.

Ausführlich und rechtfertigend wird im Protokoll hingegen die Position der politischen Vertreter dargelegt. Danach mache die Waldschadensproblematik eine erhebliche Intensivierung der Forschung unumgänglich und erfordere eine zügige und flexible administrative Abwicklung. Die Gründung der PBWU wird dabei über den entsprechenden Ministerratsbeschluß sowie als Alternativlösung zu der nicht realisierbaren, bundesweiten DGWU legitimiert. Die wissenschaftlichen Zielsetzungen würden zudem im wesentlichen vom Wissenschaftlichen Beirat festzulegen sein. Der Mittelaufwand für die Projektgruppe werde in Hinblick auf die voraussichtlich eingesetzten - jedoch im Protokoll nicht explizit genannten - Fördermittel als vergleichbar mit dem anderer Fördereinrichtungen gesehen.

Über die Beteiligung der verschiedenen Ressortvertreter an der Diskusssion geben die internen Quellen keinen Aufschluß. Aufgrund der zum Teil kritischen Einstellung des Landwirtschaftsund Kultusministeriums kann allerdings angenommen werden, daß diese überwiegend die Auffassung des federführenden Umweltministeriums widerspiegelt. Mangels Korrekturwünschen muß jedoch die formale Akzeptanz der weiteren Ressorts unterstellt werden.

\subsection{Schaffung einer Analyseeinrichtung für die Waldschadensforschung}

Auf der konstituierenden Sitzung des Wissenschaftlichen Beirats am 11.2.1985 wird auch ein von Mitgliedern des Beirats erwünschtes Analyselabor für die Waldschadensforschung diskutiert. Dessen Installierung an der GSF und Finanzierung durch das Bayerische Umweltministerium wird in Anbetracht des erwartungsgemäß hohen Analyseaufwandes im PBWU-Projekt in Aussicht gestellt.

Bereits Ende März 1985 legt die GSF dem Umweltministerium hierzu ein Angebot vor. Nach verschiedenen Modifikationen am Vertragsentwurf kommt es im September 1985 zum Abschluß (i.Q.: GSF, 29.3.1985 und 9.9.1985). Damit stellt die GSF eine bestimmte Analysenkapazität für die bayerische Waldschadensforschung zur Verfügung. Mitsamt den erforderlichen Analysengeräten, Personal- und Betriebskosten beläuft sich die vertraglich vereinbarte Gesamt- 
summe auf erheblich über 1 Mio DM. Der Vertrag wird in der Folge mehrfach verlängert und der Analysenumfang erweitert.

Die Einrichtung der zentralen Analyseeinrichtung stellt die erste finanzwirksame Maßnahme im Rahmen des PBWU-Projektes dar. Die großzügige Finanzierung der Analytik, die in der Anfangsphase überdies stark von anderweitig finanzierten Forschungsvorhaben genutzt wird (s. jährliche Nutzerprotokolle der Zentralen Analytik), kann neben dem formalen Zweck der Fördermaßnahme als eine Maßnahme der Vertrauensbildung sowie des Entgegenkommens des die PBWU befürwortenden Umweltressorts gegenüber der Kritik aus dem Wissenschaftlichen Beirat bzw. der GSF gesehen werden. Die Analyseeinrichtung wird auch von nutznießenden Mitgliedern des Wissenschaftlichen Beirats, insbesondere der kultusministeriell finanzierten Bayerischen Forschungsgruppe Forsttoxikologie denn auch ausdrücklich positiv quittiert (i.Q.: Forschungsgruppe Forsttoxikologie, 26.3.1985 und PBWU, 20.12.1985).

Von der Analyseeinrichtung können vorerst Vorhaben der Waldschadensforschung außerhalb des PBWU-Projektes profitieren. Dafür muß jedoch gleichzeitig der Verlust der hierfür benötigten Finanzmittel für das Projekt selbst in Kauf genommen werden. Erst im Rahmen späterer Vertragsabschlüsse zur Zentralen Analytik wird mehr und mehr auf das Anliegen prioritärer Nutzung durch Vorhaben aus dem PBWU-Forschungsprogramm gegenüber externen abgestellt.

\subsection{Pressemeldung zur Waldschadensforschung an bayerischen Universitäten}

Eine Pressemeldung erschüttert in der Folge das ohnehin nur mäßige Vertrauen von Wissenschaftlichem Beirat und Projektrat in das Projekt. In einer dpa-Meldung (Deutsche PresseAgentur vom 11.2.1985) wird der Leiter der PBWU-Projektgruppe mit populistischen Aussagen zur Waldschadensforschung zitiert. Unter der Schlagzeile: ,Waldsterben beschert Bayerns Universitäten einen Geldsegen“ (Münchner Merkur vom 12.2.1985) weist der PBWU-Leiter u.a. auf die enorme Höhe der für die Forschung zur Verfügung gestellten Finanzmittel hin. Gleichzeitig werde aber die Koordinierbarkeit der Forschung bezweifelt, wenn bspw. vergleichbare Arbeiten unter unterschiedlichem Titel an verschiedenen Forschungseinrichtungen durchgeführt würden. Er gesteht freimütig ein, daß die öffentliche Diskussion über den $\mathrm{Zu}$ stand der Wälder half, ,, Mittel an Land zu ziehen, ... auch wenn der Wald nur als Alibi herhalten mußte“.

Der Artikel hat GSF-intern eine weitgehende Einschränkung künftiger Pressearbeit der PBWU zur Folge. Gegenüber den PBWU-Gremien sowie der Bayerischen Staatsregierung bedauert der Geschäftsführer der GSF schriftlich den Vorfall und bittet angesichts der allgemeinen Problematik von Pressezitierungen darum, der Angelegenheit „nicht zu viel Gewicht beizumessen“ (s. Schreiben vom 5.3.1985). Die nachfolgende Kontaktaufnahme der GSF mit der Tageszeitung Münchner Merkur hat das Erscheinen eines weiteren, entschärfenden Artikels am 2./3.3.1985 zur Folge.

Insbesondere der Wissenschaftliche Beirat der PBWU muß sich durch derartige Aussagen von seiten der jungen, mit dem Auftrag der Forschungskoordination ausgestatteten und ohnehin stark kritisierten Projektgruppe brüskiert fühlen. Zwar wird der Vorfall im Rahmen der näch- 
sten Beiratssitzung vom 21.3.1985 formal nicht mehr thematisiert. Vorbehalte der Gremienmitglieder beziehen sich seit diesem Zeitpunkt jedoch nicht mehr nur auf das PBWU-Projekt selbst, sondern auch auf die Person ihres Leiters. Eine wesentliche Voraussetzung für eine erfolgreiche Forschungskoordinierung, die Akzeptanz der Koordinierungsstelle durch die Wissenschaft, ist davon schwer betroffen. Umso mehr kann sich der Beirat in seiner Kritik bestärkt sehen und kompensatorisch auf forschungspolitische Zugeständnisse drängen, die seinen Steuerungseinfluß gegenüber der Projektgruppe stärken sollen.

\subsubsection{Theorieorientiertes Zwischenergebnis}

\subsection{Steuerungsanspruch des staatlichen Auftraggebers}

Die Diskussion zur PBWU-Gründung lenkt bereits frühzeitig den Blick auf die Grundproblematik der Forschungssteuerung, je nach dem welcher Typ von Forschung vom Auftrag- bzw. Finanzgeber beabsichtigt bzw. vom Forschungsnehmer erwartet wird. Allein die im PBWUProjekt im Rahmen der späteren Programmimplementation verwendete Terminologie, welche in den Bewilligungen bzw. Förderverträgen mit außeruniversitären Forschungseinrichtungen die Begriffe „Auftraggeber“ und „Auftragnehmer“ verwendet, weist eindeutig in Richtung der vom finanzierenden Umweltressort beabsichtigten Art der Forschung. Ein Großteil der im gesamten PBWU-Projekt bzw. in dessen Analyse zentral bestehenden Steuerungsproblematik muß daher auf vor dem Hintergrund politischer- und wissenschaftlerseits unterschiedlicher Auffassungen über den zumindest beabsichtigten - wenn auch nicht unbedingt realisierten - Typ der Auftragsforschung betrachtet werden.

Die rasche Vorlage eines Vertragsentwurfs zur PBWU-Gründung und die unzureichende Verhandlung von Vertragsmodifikationen, die von den beteiligten Ressorts und der GSF für erforderlich gehalten werden, machen nicht nur den Handlungsdruck deutlich, unter dem sich das Umweltministerium sieht. Sie dokumentieren auch die strikte Interessenverfolgung des Ressorts, um sich die politische Steuerungsdominanz des Projekts via Projektgruppe zu sichern.

Forderungen zur Abschwächung deren Lenkungsfunktion werden nicht vor Vertragsabschluß berücksichtigt, sondern allenfalls nachverhandelt. Auf der konstituierenden Sitzung des Projektrats der PBWU kann die Vormachtstellung des Bayerischen Umweltministeriums trotz der Einwände der beteiligten Ressorts gegen das Aufgabenspektrum der PBWU ausgebaut werden. Der hochrangige Vertreter des Umweltressorts wird Vorsitzender des Projektrats. Das Ministerium behält sich vor, auch ohne Einschaltung des noch zu bestellenden Wissenschaftlichen Beirats Fördermaßnahmen aussprechen zu können.

$\mathrm{Da}$ die Modifizierungswünsche der weiteren beteiligten Ressorts mit Zeitverzug und Handlungsdruck begründet übergangen, auf der konstituierenden Projektratssitzung nur unzureichend nachverhandelt werden und lediglich als „Interpretationen des Projektrats“ den bereits unterzeichneten Vertrag korrigieren, muß die künftige Zusammenarbeit mit der Projektgruppe und deren Akzeptanz belasten. Dies kann als ein „Geburtsfehler“ der PBWU bezeichnet werden. Damit verschlechtern sich die Startbedingungen der PBWU für eine konstruktive, vertrauensvolle Zusammenarbeit nicht nur mit der Politik in Form der benachbarten Ressorts, sondern 
auch mit der Wissenschaft, welche für eine erfolgreiche Erfüllung der Koordinationsfunktionen Voraussetzung ist [BRAUN, 1997, S. 352 ff].

Die Vorgehensweise das Umweltministeriums zur Durchsetzung der PBWU hat weiterhin zur Folge, daß eine Zufinanzierung der beiden benachbarten Ministerien im Rahmen einer ressortübergreifenden, bayerischen Waldschadensforschung nicht zu erwarten ist. Die bestehenden Förderstrukturen über das Kuratorium der Bayerischen Forstlichen Versuchs- und Forschungsanstalt bzw. die Forschungsgruppe Forsttoxikologie bleiben trotz den bayernweiten Koordinierungsauftrages der PBWU weitgehend unberührt, abgesehen davon, daß Ergebnisse daraus zur Verfügung gestellt werden sollen und der PBWU dort bestenfalls ein rein informativer Gaststatus offeriert wird. Die beteiligten Ressorts hingegen erhalten die Möglichkeit, über das Gremium Projektrat die vom Bayerischen Umweltministerium über die PBWU einzuschlagende Forschungskonzeption grundsätzlich und hinsichtlich einzelner Fördermaßnahmen mitzubestimmen. Die Interessendurchsetzung der Fachressorts schwächt im Endergebnis die Koordination durch die PBWU.

\subsection{Anspruch der Wissenschaft auf Forschungsfreiheit}

Als Pendent zu den politischen Akteuren repräsentiert das Gremium Wissenschaftlicher Beirat den wissenschaftlichen Steuerungseinfluß im Kräftespiel um das PBWU-Forschungsprojekt. Die Projektgruppe wird dabei bald zum Zentrum der Auseinandersetzung. Konflikte, die sich aus den vorgesehenen Forschungssteuerungsfunktion der Projektgruppe zur Durchführung von staatlich finanzierter, problemorientiert-angewandter Forschung gegenüber dem idealtypischen Anspruch der Wissenschaft auf Grundlagenforschung und Forschungsfreiheit ergeben, prägen die Interessenkonstellation im Projekt. Noch vor Konstituierung des Beirats wird von seiten hochrangiger Wissenschaftler deutlich die Befürchtung geäußert, die Freiheit und Selbstverantwortlichkeit der Wissenschaft könnte durch die Einrichtung einer derartigen Koordinationsstelle beschnitten werden.

Die Wissenschaftler wollen sich nicht in das Korsett einer straff kontrollierten, ressortspezifisch finanzierten Auftragsforschung (Ressortforschung) zwängen lassen. Sie erkennen sofort, welche Steuerungsinstrumente der Projektgruppe, dem Auftraggeber bzw. der GSF über die Finanzierungs-, Begutachtungs- oder Kontrollfunktion bei einem derartigen Forschungstyp erwachsen würden. Die Kritik an den Steuerungsfunktionen der Projektgruppe resultiert folglich aus der Erwartung, ja dem Anspruch, den die Wissenschaftler aus dem Projekt ableiten, nämlich einer forscherfreundlichen, universitären Drittmittelforschung. Es wird sogar konstatiert, $\mathrm{da}$ bei industrieforschungsähnlicher Auftragsforschung die Kontrollfunktionen der PBWU durchaus legitim seien, dieser Forschungstyp bei den Wissenschaftlern aber anscheinend außer Diskussion steht.

Formal über den Ministerratsbeschluß zur Gründung der Projektgruppe, informal über die Internalisierung der bestehenden fachlich-wissenschaftlichen wie politisch-administrativen Gründungskonflikte kann die Koordinationsstelle PBWU geschaffen und das Waldschadensforschungsprogramm gestartet werden. Das rasche und zielstrebige Handeln des Umweltministeriums, die Forschung zu verstärken, die Bereitschaft und Präsenz der GSF, ad hoc die Koordi- 
nationsstelle bei sich zu installieren, sowie die Beteiligung weiterer Ressortvertreter und führender Wissenschaftler trotz erheblicher Kritik, waren formal Voraussetzungen für diesen Erfolg.

Die Strategie der PBWU-Befürworter GSF und Umweltministerium, die Kritiker als Mitglieder des Beirats zu gewinnen und damit die bestehenden Konflikte zu internalisieren, hat weitgehend Erfolg. Jedoch kann auch von Seiten der Wissenschaft erwartet werden, daß sich ein Steuerungseinfluß auf das Forschungsgeschehen im Beirat leichter und wirkungsvoller realisieren läßt als von außerhalb des PBWU-Projektes.

Kritik zu internalisieren kann zudem für das federführende Umweltressorts als strategisch vorteilhaft gesehen werden. So zeigt das Ministerium, wie aus der ersten gemeinsamen Sitzung hervorgeht, Aufgeschlossenheit gegenüber den Anliegen des Beirats, die Eigeninitiative, den Ideenreichtum und die Nutzungsrechte der Forschung im Rahmen des PBWU-Projekts zu sichern. Mit der festen Zusicherung, den Beirat mit der Festlegung der generellen ForschungsZielsetzung zu betrauen, will die Politik trotz aller Kritik die Basis schaffen für eine Akzeptanz des Projekts in dem Wissenschaftlergremium. Das Angebot des Ministeriums, ein zentrales Analyselabor einzurichten, welches überwiegend bereits bestehenden Forschungsverbünden zugute kommt, kann sowohl als vertrauensbildende Maßnahme des finanzierenden Ministeriums, als auch als erster, eigeninteressengeleiteter Erfolg der Wissenschaft verbucht werden. Die GSF kommt dem Wunsch, das Labor im eigenen Hause anzusiedeln, gerne nach.

Der Wissenschaft wird über die GSF informal und noch weit vor der offiziellen Beteiligung im Beiratsgremium die Möglichkeit der Einflußnahme auf das PBWU-Projekt in seiner Gründungsphase gegeben. Dies unterstreicht die Position der GSF, welche zum einen die politische Absicht zur PBWU-Gründung unterstützt, zum anderen die Akzeptanz der Wissenschaft sucht. Daß ein Großteil der von der GSF kontaktierten Wissenschaftler dem künftigen Beirat zugehören werden, zeugt von dem erheblichen Steuerungseinfluß der GSF bzgl. der personellen Besetzung des Gremiums.

\subsection{Politische und wissenschaftliche Interessenvertetung im PBWU-Projekt}

Mit der Einsetzung des Wissenschaftlichen Beirats sind der für die Waldschadensforschung erforderliche wissenschaftlich-multidisziplinäre Sachverstand sowie die Interessen der Forscher einbezogen. Ausgeprägte Koalitionen sind festzustellen, die bspw. in fachlicher Hinsicht den Bereich der Botanik, in institutioneller Hinsicht Vertreter der Forschergruppe Forsttoxikologie betreffen. Zudem bestehen, wie die Schriftwechsel anläßlich der Kritik an der PBWU-Gründung dokumentieren, gute persönliche Beziehungen zwischen mehreren, im Gremium vertretenen Wissenschaftlern. Eine derartige Koalitionsbildung kann die gemeinsame Interessenverfolgung erheblich erleichtern und auf die fachliche, institutionelle oder regionale Ausrichtung der Forschung Einfluß nehmen.

Bei der personellen Besetzung des Gremiums Wissenschaftlicher Beirat spielen neben fachlichen auch forschungspolitische Aspekte eine wichtige Rolle. Die Ressortvertreter im Projektrat können die von ihnen betreuten Klientela erfolgreich im Beirat integrieren und somit nicht nur auf ressortpolitischer, sondern auch auf wissenschaftlicher Ebene eine entsprechende Inter- 
essenvertretung im PBWU-Projekt erreichen. Die verschiedenen forschungspolitischen Interessenlagen der einzelnen Ressorts bzw. der GSF sind somit nicht nur im Projektrat integriert, sondern werden auch fachlich im Wissenschaftlichen Beirat der PBWU repräsentiert.

Die Besetzung des Beirats mit ausgewiesenen Wissenschaftler-Persönlichkeiten hoher Reputation dient generell der fachlichen wie politischen Akzeptanz und Stellung des Projektes in der bundesweiten Waldschadensforschung. Die beteiligten Ressorts verschaffen sich hiermit einen Zugang zur Wissenschaft, der es ihnen erlaubt, deren Sachverstand zu nutzen, die Wissenschaft für die Definition und Umsetzung ihrer forschungspolitischen Ziele zu gewinnen und die Forschungsmaßnahmen wissenschaftlich zu legitimieren [s.a. BRAUN 1997, S. 316].

Trotz aller Kritik gegen das PBWU-Projekt nützt jedoch die Möglichkeit, im Rahmen der wissenschaftlichen Beratungsfunktion die Geschicke der bayerischen Waldschadensforschung maßgeblich mit zu beeinflussen, die eigenen fachlichen Interessen einbringen und nicht zuletzt selbst von der Forschungsförderung profitieren zu können, den beteiligten Wissenschaftlern im Beirat. Zudem besteht in der Folge Gelegenheit, innerhalb des Gremiums die Kritik an der PBWU zu thematisieren und Veränderungen herbeizuführen. Die hochrangige Einladung und Ernennung durch den Umweltminister ist nicht nur dazu dienlich, den politischen Stellenwert und die Bedeutung des Projekts zu heben, sondern damit auch die renommierten Wissenschaftler für eine ehrenamtliche Mitarbeit zu gewinnen.

Hinsichtlich der fachlichen Beurteilung von Forschungsanträgen erlegt sich der Beirat selbst Beschränkungen auf und sieht seine Beteiligung nur in einer Vorauswahl. Nur so kann der Eindruck eines „Selbstbedienungsladens“ für die im Gremium beteiligten Wissenschaftler vermieden werden. Andererseits verbleibt damit die Möglichkeit, eigene Anträge einzubringen und sich selbst am Projekt in inhaltlicher wie finanzieller Hinsicht zu beteiligen.

Der Vorschlag, das Gutachtergremium überwiegend mit hochrangigen DFG-Gutachtern personell zu besetzen, kann generell dazu dienen, die Qualität der Forschungsarbeiten auf hohem Niveau zu gewährleisten. Auf das Gutachtergremium der Forschungsgruppe Forsttoxikologie zurückzugreifen, verbessert zwar die Abstimmung zwischen beiden Programmen. Er präjudiziert allerdings die Dominanz dieses bestehenden Forschungsverbundes gegenüber dem neu aufzulegenden PBWU-Forschungsprojekt. Zudem erschließt sich damit für die bislang vom Kultusministerium geförderte Forschungsgruppe die Möglichkeit, auf externe Finanzierungsbeiträge aus dem PBWU-Projekt zugreifen zu können.

\subsection{Umweltressort gegen Nachbarressort-Interessen}

Legitimiert durch die Partnerressorts sowie den Bund kann das Bayerische Umweltministerium die Federführung über die Koordination der bayerischen Forschungskoordination nun für sich beanspruchen. Die letztendliche Entscheidungshoheit für die Vergabe für Fördermitteln, der Vorsitz im Projektrat, die enge Koalition mit der GSF sowie die vertragliche Weisungsbefugnis gegenüber der Projektgruppe lassen das Umweltministerium im Forschungssystem der PBWU eine maßgebliche politische Stellung und Steuerungsfunktion einnehmen. 
Dennoch können auch die weiteren beteiligten Ressorts ihre Interessen wahren, ja sogar ihre Ressourcen vermehren. Die im einzelnen noch auszuhandelnde Stellung und Befugnisse des Projektrates sowie Wissenschaftlichen Beirats in der PBWU ermöglichen es grundsätzlich, ressort- sowie fachspezifische Interessen in dem PBWU-Projekt zu berücksichtigen, ohne jedoch auf ihre eigenen Forschungsaktivitäten verzichten oder diese in die PBWU integrieren zu müssen.

Der vertragliche Anspruch, die Waldschadensforschung bayernweit zu koordinieren, wird in seiner Umsetzung nicht dahingehend verstanden, sämtliche Mittelvergabe- oder Förderentscheidungshoheiten bayerischer Förderressorts auf die PBWU zu übertragen. Für das Landwirtschaftsministerium ergibt sich somit die Möglichkeit, in ihrem Bereich bereits bestehende Forschungsprogramme über das Kuratorium der Forstlichen Versuchs- und Forschungsanstalt weiterführen zu können, zusätzlich aber über die PBWU sich Mitsprache- und Mitentscheidungsrecht bei dortigen, vom Umweltressort finanzierten Förderentscheidungen zu sichern. Vergleichbares gilt für das Kultusministerium, welches nach wie vor eigenverantwortlich das Forschungsprogramm der Bayerischen Forschergruppe Forsttoxikologie finanziert, zusätzlich aber über Betreiben im Projektrat nun die Möglichkeit hat, Mittel aus dem PBWU-Projekt dorthin zu steuern. Eigene Ressourcen und Forschungsprogramm-Nischen können trotz des formalen Anspruchs bayernweiter Koordinierung durch die PBWU ohne wesentliche Einschränkung bewahrt werden.

\subsection{Territorialität der PBWU-Akteure}

Die Handlungspläne der Akteure im PBWU-Projekt sind Ausdruck einer ausgeprägten Territorialität in ressortbezogener, regionaler und forschungsthematischer Hinsicht. Diese prägt das PBWU-Projekt seit seiner Gründung bis hin zu seiner Terminierung. Bereits erkennbar ist die Territorialität an dem - vergeblichen - Vorstoß Bayerns, die bundesweite Forschungskoordinierung an sich zu ziehen.

Das Erkennen sowie das Verständnis territorialen Verhaltens der Akteure erleichtert die Analyse der politisch wie wissenschaftlichen Gestaltung bzw. Steuerung der Waldschadensforschung im PBWU-Projekt. So treffen bspw. im Wissenschaftlichen Beirat Vertreter universitärer Forschung sowie von Großforschungseinrichtungen zusammen, deren Interessenlage sich bereits in Hinblick auf die Grundlagen- bzw. Anwendungsorientierung ihrer Forschungsrichtung wesentlich unterscheiden können. Auch die bei einigen Mitgliedern gegebene, problematische Zwitterrolle, einerseits dem wissenschaftlichen Steuerungsgremium anzugehören, andererseits selbst Antragsteller bzw. Waldschadensforscher innerhalb oder außerhalb des PBWU-Projektes zu sein, läßt sich zwar formal entschärfen, prägt aber informal den Handlungsplan des betreffenden Einzelakteurs.

Zur Interpretation des territorialen Verhaltens kann das Konzept der „Bureau Territoriality“ von Downs [1967, S. 211ff] herangezogen werden. Bezogen auf die Analyse von politischen Verwaltungseinheiten geht dieses davon aus, daß zwischen den verschiedenen Institutionen und den betreuten Klientela mannigfache Beziehungen und Einflußnahmen bestehen. Deren Intensität kann räumlich über ein Modell territorialer Zonen, zentriert um die Verwaltungsein- 
heiten (,heartland“" = „Kerngebiet“) dargestellt werden, wobei mit dem Abstand vom Zentrum als Maßstab der Einfluß der betreffenden Behörde sinkt. Aus der Überlappung - umso mehr der der peripheren Zonen - wird unter anderem das Ringen um die eigene Position, insbesondere die Vergrößerung oder die Intensivierung des eigenen Einflußbereiches, in Konkurrenz zu dem anderer Institutionen, visuell deutlich. Dabei wird unterstellt, daß jede Institution danach strebt, die bestehenden territorialen Grenzen zur Sicherung des Überlebens und Schaffung von Autonomie zu verteidigen oder zu erweitern [Downs 1967, S. 215].

Die Verstärkung der Waldschadensforschung und deren Finanzierung, die damit verbundene zusätzliche Administration sowie die explizite Aufgabe der Forschungskoordination bietet grundsätzlich auch für die im PBWU-Projekt beteiligten Akteure die Möglichkeit, die bestehenden territorialen Konstellationen zu ihren Gunsten zu verändern. Über das Modell wird deutlich, daß der Versuch Bayerns, die aus seiner Sicht territorial peripher befindliche, bundesweite Forschungskoordination zu übernehmen, nur geringe Erfolgsaussichten haben muß, da dies zu sehr in das Zentrum (territorial: „Mutterland“) des Einflußbereichs des Bundes eingreifen würde.

Gerade auch die territorialen Verhaltensmuster zur Einflußnahme auf die Waldschadensforschung zwischen den maßgeblich beteiligten bayerischen Ressorts Landwirtschaft, Kultus und Umwelt kann das Modell von DowNS ein Stück weit erklären. Bis zur Neugründung der Koordinationsstelle PBWU, welche neue und zusätzliche Einflußnahmeräume erschließen läßt, ist die territoriale Konstellation weitgehend stabil. Das Bayerische Landwirtschaftsministerium finanziert und koordiniert die forstlich orientierte, überwiegend südbayerische Waldschadensforschung, das Kultusressort die Bayerische Forschergruppe Forsttoxikologie im nordbayerischen Fichtelgebirge und das Umweltministerium ausgewählte, ressortspezifische LuftschadstoffForschungsprojekte. Die territorialen Aktionsräume sind weitgehend voneinander separiert, eine Überlappung der Einflußbereiche besteht weder in finanzieller, fachlicher noch regionaler Hinsicht.

Durch die Gründung der PBWU betritt und besetzt jedoch ein weiterer Akteur das Feld, versucht sich selbst Platz und Raum zu schaffen. Bei Schaffung neuer Funktionen kann dieser Versuch in der weniger kritischen Besetzung von bisherigen Leerräumen (,Niemandsland“) gesehen werden. Bei Übernahme im Grundsatz bereits bestehender Funktionen muß allerdings in die bereits besetzten und verteidigten Territorien der anderen Akteure vorgestoßen werden.

Anderereits versuchen die bereits bestehenden Akteure, ihre mit Einflußpotential besetzten Territorien zu bewahren, ja diese in Richtung der erst neu geschaffenen bzw. durch die PBWU besetzten Freiräume zu erweitern. Dadurch, daß die Machtstrukturen und Einflußpotentiale einer neu auf den Plan getretenen Institution zwangsläufig noch vergleichsweise schwach sind, gelingt es den bereits bestehenden umso leichter, ihre Territorien nicht nur zu behalten, sondern diese in ihrer Ausdehnung und in ihrem jeweiligen Machtpotential gegen das Potentialgefälle in Richtung der neu geschaffenen PBWU auszudehnen. In der modellhaften Vorstellung müßte entsprechend der Aufgabenstellung und Zielsetzung der PBWU diese ihre territoriale Ausdehnung weit in die Territorien der Nachbarressorts vortreiben. 
Dabei stehen aber institutionell schwachen Machtpotentialen der PBWU starke und in Richtung Territoriumszentrum sich verstärkende Positionen der benachbarten Ressorts gegenüber. Die - wie die weitere Anlyse zeigen wird - schwache Koordinationsfunktion der PBWU im Einflußbereich der beteiligten Ressorts Landwirtschaft und Kultus bei demgegenüber starker Einflußnahmemöglichkeit dieses Ressorts im Projektrat läßt sich hiermit erklären. Das bayerische Umweltressort betreibt die Einsetzung der PBWU selbst federführend und tritt diesbezügliche Kompetenz gewollt an die Koordinationsstelle ab. Daher entstehen in dieser Konstellation keinerlei territoriale Komplikationen.

Bei der Alternative, statt der Einrichtung einer neuen Organisationseinheit PBWU deren Funktion einem der bestehenden Ressorts direkt zuzuordnen, wäre die Entwicklung der territorialen Verhältnisse nach dem Modell in abgeschwächter Form verlaufen. So wäre dann kein zusätzlicher Raumanspruch für eine neue Institution zulasten der anderen Ressorts entstanden, die Machtpotentiale wären homogener gewesen. Die territoriale Ausdehnung der bereits bestehenden, mit den Aufgaben betrauten Institution hätte jedoch zu ähnlichen territorialen Überlagerungen mit den erwähnten Interessenkonflikten geführt, da der Anspruch ressortübergreifender Koordination an sich in die Zuständigkeit des oder der benachbarten Ressorts eingreifen muß.

Nur geringer Koordinationsbedarf hätte, wie für langsame und weniger dramatische politische Veränderungen allgemein gilt [DownS 1967, S. 220], ohnehin im bisherigen territorialen „Niemandsland“ der bestehenden Behörden abgedeckt werden können. Der beabsichtigte Umfang der Forschungsverstärkung und -koordination mit den zusätzlich hierfür erforderlichen fachlichen, personellen und finanziellen Ressourcen erhöhte jedoch auch bei den betreffenden Ressorts die Neigung, eine neue, externe Institution zu gründen, zumindest dann, wenn die Einflußnahme auf deren Handeln gesichert werden konnte. Allein der Vorteil, die Personalrekrutierung sowie eventuelle Probleme mit der Personalbindung außerhalb eines Ministeriums an eine diesbezüglich flexiblere Großforschungseinrichtung zu verlagern, erhöhte die Realisierbarkeit der Projektgruppe.

Zudem setzt die Funktion der Koordination ein hohes Maß an Interessenneutralität voraus. Die Entscheidung, die Projektgruppe außerhalb eines Ressorts zu plazieren, die Ressortvertreter jedoch über den Projektrat zu implementieren, diente diesem Anspruch. Daß auch einer Großforschungseinrichtung wie der die PBWU beherbergenden GSF Interessengeleitetheit unterstellt werden muß, welche wiederum das Handeln der PBWU beeinflußt, widerspricht jedoch dem Anspruch auf Neutralität. Daß die GSF über die Projektgruppe PBWU mit der Koordinierung der Waldschadensforschung beauftragt wurde, gereicht dementsprechend auch verschiedentlich zur Kritik. Sie läßt sich ebenfalls in das Konzept der Territorialität einpassen. Von seiten verschiedener Ressorts wurde die institutionelle Abgabe eigenstaatlicher Kompetenz vom Land Bayern auf den Bund - bzw. dessen Großforschungseinrichtung - befürchtet, ein Ausdruck territorialen Übergriffs.

Das Territorialverhalten wird auch bei den im wissenschaftlichen Beiratsgremium der PBWU vertretenen und den jeweiligen Ressortklientela zuzuordnenden Waldschadensforschern deutlich. Die heftige Kritik der im Forschungsverbund Forsttoxikologie zusammengeschlossenen Wissenschaftler gegenüber der PBWU kann als Strategie zur Beibehaltung bestehender fachli- 
cher und regionaler Forscherterritorialität gesehen werden und sollte vor Einflußnahmen von außen schützen.

Es wird deutlich, daß das Territorialitätsmodell gerade bei Bezug auf das Fallbeispiel ressort-, finanzmittel-, fach- und regionalübergreifender Forschungskoordination sehr schnell die bestehenden Grenzen zwischen Anspruch und Realität aufzeigen. Dem formalen Anspruch der zumindest bayernweiten - Forschungskoordination durch die eigens eingerichtete Projektgruppe PBWU stehen informale Territorialitätsbarrieren in den genannten Bereichen von seiten der politischen Ressortvertreter sowie der Forscher gegenüber.

Dadurch, daß das Feld der Waldschadensforschung von territorialen Ansprüchen mehrerer forschungsfinanzierender Ressorts geprägt ist, die ihre Ressortautonomie aufrecht erhalten wollen, sowie mehreren, den Ressorts zuzuordnenden Forschergruppen mit ebenfalls ausgeprägtem Autonomiebestreben, muß eine ressortübergreifende Koordination scheitern. Ein gewisser Erfolg kann sich nur dadurch einstellen, daß Ressorts wie Forscher trotz ihres territorialen Verhaltens an den zusätzlichen Förderressourcen bzw. der Beteiligung an der Erkenntnisproduktion zum Thema partizipieren und finanziell davon profitieren wollen. Nur deshalb werden Kompromisse der Akzeptanz und Beteiligung am PBWU-Projekt eingegangen. Diese bedeuten lediglich in geringem Umfang einen Verlust der Förder- oder Forschungsautonomie und stellen zudem eine eigene Ressourcenmehrung in Aussicht. 


\subsection{Programmformulierung}

\subsubsection{Erster Entwurf eines Forschungsrahmenprogramms}

Die Erstellung eines PBWU-Rahmenprogramms für die Waldschadensforschung stellt - neben weiteren Grundsatzfragen wie den Aufgaben der Projektgruppe und dem Verfahren der Antragsbegutachtung - bereits Gegenstand der ersten gemeinsamen Sitzung der PBWU-Gremien vom 11.2.1985 dar (i.Q.: Bayer. Umweltministerium, 22.2.1985). Die Ankündigung des einladenden Bayerischen Umweltministers, die Projektgruppe werde zur Sitzung zusätzlich zu einem Zwischenbericht zum Stand der Forschung einen Konzeptentwurf zu künftigen Forschungsschwerpunkten in Bayern vorlegen, kann jedoch in letztgenannter Hinsicht nicht erfüllt werden.

Die Erörterung des Themas während der Sitzung beschränkt sich auf die grundlegend konzeptionelle Frage, ob die Forschung auf wenige regionale Schwerpunktstandorte mit umfangreichen Arbeitsprogramm konzentriert, oder aber möglichst viele Standorte weniger umfangreich bearbeitet werden sollten. Ohne daß hierzu jedoch konkrete Festlegungen getroffen werden, wird der Projektgruppe der Auftrag erteilt, die „Konzeption eines Rahmenprogramms“ auszuarbeiten und zur nächsten Sitzung vorzulegen. Dabei solle der Forschungsplan ,,so flexibel gestaltet sein sollte, daß Eigeninitiativen und Ideen potentieller Antragsteller nicht beschränkt würden“.

Kurzfristig wird von der PBWU der erste, etwa 30-seitige Entwurf eines Rahmenprogramms als Diskussionsgrundlage vorgelegt (Rahmenprogramm für Freilandversuche „Projekt Bayern zur Erforschung der Wirkung von Umweltschadstoffen“, Stand 1.3.1985). Darin wird davon ausgegangen, daß nach dem Stand des Wissens „Luftschadstoffe die Hauptursache der Waldschäden sind und in ihrer Kombinationswirkung miteinander und mit anderen Schadfaktoren die neuartigen Waldschäden verursachen“. Da die Kombination der jeweils wirksamen Schadfaktoren als standortsabhängig angesehen wird, wird an ausgewählten Freilandstandorten ein interdisziplinärer Forschungsansatz vorgeschlagen. Dieser soll durch standortunabhängige Untersuchungen zur Aufklärung wirkungsmechanistischer Grundlagen im Labor ergänzt werden, wie in einem gesonderten Rahmenprogrammteil (,Rahmenprogramm für Laborexperimente“, Stand 6.3.1985) mitsamt möglichen Themenschwerpunkten formuliert wird. Belastungsexperimente mit Luftschadstoffen wie in den Klimakammern der GSF komme dabei besondere Bedeutung zu.

Als Hauptkriterien für die Auswahl der Freilandstandorte werden definiert

- bereits vorhandene Untersuchungen bzw. Datensätze,

- die Überprüfbarkeit des Wirkungspfades von Luftschadstoffen auf die Pflanze sowie

- die Vergleichbarkeit der Standorte.

Anschließend werden 4 ,Standorträume“ vorgeschlagen, welche als Forschungsschwerpunkte auf- bzw. ausgebaut werden könnten. Es handelt sich um das Fichtelgebirge, den Bayerischen Wald, den „Höglwald“ und die bayerischen Kalkalpen. 
Die Standorträume werden im einzelnen charakterisiert, die geplanten sowie gegebenenfalls bereits bearbeiteten Untersuchungsthemen ausgeführt und übersichtsartig aufgelistet. Dabei wird berücksichtigt, daß das nordostbayerische Fichtelgebirge bereits von der Bayerischen Forschungsgruppe Forsttoxikologie, dem vom bayerischen Kultusressort sowie vom Bundesforschungsministerium geförderten Forschungsverbund überwiegend nordbayerischer Universitäten, interdisziplinär bearbeitet wird. Auch bezüglich des Standorts Bayerischer Wald wird auf bereits etablierte, von Länder- und Bundesressorts finanzierte Waldschadensforschung zum Thema Stoffeinträge verwiesen [s.a. BUNDESMINISTERIUM FÜR FORSCHUNG UND TECHNOLOGIE 1984, S. 19]. Ähnliches gilt für den Höglwald, einen Versuchs-Waldbestand zwischen München und Augsburg, auf dem umfangreiche Forschungsarbeiten zur Bodenversauerung und Düngung überwiegend vom bayerischen Landwirtschaftsministerium, in der Folge auch von Bund und der EU, gefördert werden. Bei den genannten, bereits etablierten Forschungsstandorten werden daher überwiegend die Verstärkung bzw. Intensivierung vorhandener Forschungsansätze empfohlen und entsprechende Untersuchungsthemen (,Themenlisten“) vorgestellt.

Als einziger, grundlegend neuer Forschungsschwerpunkt wird der bayerische Alpenraum vorgeschlagen. Begründungen hierfür liefern die dort beobachteten und durch die Ergebnisse der offiziellen Waldschadenserhebungen dokumentierten starken Waldschäden, die gefährdete Schutzfunktion der alpinen Wälder, die Besonderheiten der alpinen Immissionssituation sowie die generell unbefriedigende Forschungssituation in dieser Region. Vorliegende lufthygienische Meßreihen des ortsansässigen Forschungsinstitutes der Fraunhofer-Gesellschaft sowie weitere forschungsbegünstigende, infrastrukturelle Gegebenheiten ließen den Raum Garmisch-Partenkirchen als alpinen Forschungsstandort sinnvoll erscheinen. Während die Waldschadensforschung an anderen Schwerpunkten überwiegend die Auswirkungen versauernd wirkender Stoffeinträge auf Waldbestände, Baumindividuen bzw. Böden in den Mittelpunkt stellte, solle dort die Hypothese überprüft werden, wonach Photooxidantien mit der Leitsubstanz Ozon für das Auftreten von Waldschäden verantwortlich sind.

\subsubsection{Kritische Diskussion des Rahmenprogramm-Entwurfs in den PBWU-Gremien}

Die Diskussion des Rahmenprogramm-Vorschlags der PBWU steht im Mittelpunkt der zweiten Sitzung der PBWU-Gremien am 21.3.1985 (i.Q.: PBWU, 13.4.1985). Der Vorschlag wird im Grundsatz akzeptiert. Höchste Priorität erhält der Aufbau des neuen Forschungsschwerpunktes im bayerischen Alpenraum. Forschungsseitige Ergänzungen an den bereits bestehenden Schwerpunktstandorten Fichtelgebirge, Bayerischer Wald und Höglwald sowie standortsunabhängige Versuche sollten folgen.

Die PBWU erhält den Auftrag, das Rahmenprogramm entsprechend der Prioritätensetzung zu modifizieren, unverzüglich einen Ausschreibungstext zu entwerfen und dem Beirat zur Stellungnahme vorzulegen. Die Planung sieht vor, kurzfristig einschlägige Forschungsinstitute direkt anzuschreiben und in geeigneten Bekanntmachungen auf die Ausschreibung hinzuweisen. Die Abgabefrist für Forschungsanträge bei der PBWU wird bereits auf Ende Juni 1985 terminiert. Die Bearbeitung der Anträge, Diskussion im Wissenschaftlichen Beirat und fachliche Begutachtung sollten unverzüglich folgen. 
Terminlich verspätet versendet die PBWU ein nur geringfügig modifiziertes Rahmenprogramm und den Textentwurf einer Ausschreibung (Stand 1.4.1985) mit der Bitte um Stellungnahme an ihre Gremien. In der Ausschreibung sind unter Zuständigkeit der PBWU für organisatorische und fachliche Betreuung die Programmschwerpunkte interdisziplinärer, standortbezogener Forschung zusammenfassend dargestellt. Sie schließt mit Angaben zu Antragsformalitäten einschließlich -terminen (i.Q.: PBWU, 3.4.1985).

Seitens des Wissenschaftlichen Beirats werden daraufhin mehrere schriftliche Stellungnahmen vorgelegt, welche erhebliche Kritik an dem PBWU-Programm zum Ausdruck bringen. So bemängelt ein Mitglied, daß „Laborexperimente wesentlich zu kurz“ kämen (i.Q.: $P B W U$, 2.5.1985). Verschiedene Arbeitsgruppen, die - wie das Beiratsmitglied selbst - hierzu konkrete und inhaltlich benannte Beiträge liefern könnten, würden nicht angesprochen.

Ausführlich äußern sich zwei führende Wissenschaftler der Forschungsgruppe Forsttoxikologie zu Rahmenprogramm und Ausschreibung (i.Q.: PBWU, 3.5. 1985 und andere ohne Datumsangabe). Formal wird die knappe und nicht realisierbare Terminsetzung der Antragseinreichung und fehlende Angaben über die Gesamtorganisation des Projekts sowie über die Form der Antragstellung kritisiert. In aller Deutlichkeit werde darauf hingewiesen, daß entgegen der Auffassung der Projektgruppe der Wissenschaftliche Beirats nicht als ein der PBWU untergeordnetes Gremium zu verstehen sei. Die Funktion der PBWU müsse sich auf die organisatorische Betreuung von Forschungsvorhaben beschränken, während die fachliche Betreuung der Wissenschaft, nämlich dem Wissenschaftlichen Beirat, obläge. Die in Anspruch genommene fachliche Betreuung durch die PBWU könne in Anbetracht des umfangreichen Projekts nicht geleistet werden. Auch das Bayerische Umweltministerium wird mit Schreiben vom selben Tag auf diese „Fehlinterpretation“ der PBWU aufmerksam gemacht und um entsprechende Abhilfe gebeten.

Weitere Anmerkungen der beiden Beiratsmitglieder betreffen die Konzeption und fachliche Inhalte von Ausschreibung und Rahmenprogramm. Kritisiert wird unter anderem, daß das auf der vorangegangenen Gremiensitzung erarbeitete Konzept verschiedener „Profilstudien“, die Verknüpfung verschiedener regionaler Forschungsstandorte, fehle bzw. zu wenig deutlich herausgearbeitet worden sei. Diesem müßten die geplanten oder bereits bearbeiteten Forschungsstandorte integrativ zugeordnet werden, wobei gerade der von den sich äußernden Beiratsmitgliedern betreute Schwerpunktstandort Fichtelgebirge von zentraler regionaler Bedeutung erschiene. Die fehlende Vergleichbarkeit von Standorten, welche als Begründung für die Bearbeitung mehrerer Forschungsstandorte herangezogen worden sei, werde als „Freibrief für jede Mißplanung“ bzw. „legitimierte Doppelforschung“ verstanden. Zudem wird die „völlige Beschränkung auf die Luftschadstoff-Hypothese" kritisiert. Demgegenüber werden weitere, aus Sicht der Beiratsmitglieder für relevant erachtete Forschungsthemen vorgeschlagen.

Letztlich wird die vorgegebene, umfangreiche Themenliste der zu bearbeitenden Forschungsaspekte als „,wissenschaftshemmend“ angesehen. Zur „quantitativen Streichung“ wird angeregt. Die Vorschläge von Forschungsthemen unterdrückten die wissenschaftliche Kreativität und die wissenschaftliche Verantwortung der Forscher sowie den Zwang zur Integration, der sich aus dem fachlich-kollegialen Austausch hinsichtlich der Sinnhaftigkeit der Messung verschiedener 
Parameter ergeben könnte. Die PBWU müsse ansonsten die Verantwortung dafür tragen, daß nur die Parameter gemessen würden, die sie sich ausgedacht hätte.

Auch das Bayerische Landwirtschaftsministerium gibt eine Stellungnahme zu dem Rahmenprogramm der PBWU ab (i.Q.: Bayer. Landwirtschaftsministerium, 18.4.1985). Im wesentlichen handelt es sich dabei um zahlreiche fachlich-inhaltliche Konkretisierungen und Ergänzungen, welche die Stringenz des Forschungsansatzes erhöhen sollten. Auch hier wird die „organisatorische Betreuungsaufgabe“ der PBWU gegenüber der „fachlichen“ als ausreichend erachtet.

Die vorgetragenen Kritikpunkte und Anregungen werden von Projektgruppe in unterschiedlichem Umfang in das Rahmenprogramm und die Ausschreibung eingearbeitet. Formal wird, wie von allen Kritikern gefordert, auf die Aufgabe der ,fachlichen“ Koordination durch die PBWU verzichtet. Den konkret ausformulierten Korrektur-Vorschlägen des Landwirtschaftsministeriums wird weitestgehend gefolgt. Neben überwiegend formalen Modifikationen wird versucht, auch die fachlichen Kritikpunkte, insbesondere an der Darstellung der Gesamtkonzeption, in Form von Ergänzungen mitzuberücksichtigen, ohne dadurch jedoch Ausschreibung oder Rahmenprogramm wesentlich umzustrukturieren. Die kritisierte Auflistung von Forschungsthemen zu den einzelnen Freilandschwerpunkten beispielsweise bleibt im wesentlichen bestehen.

\subsubsection{Beschluß und Ausschreibung des PBWU-Rahmenprogramms}

In der Endfassung liegt ein 14-seitiges Rahmenprogramm zum Projekt „Waldschadensforschung und Wirkungen von Umweltschadstoffen“ (i.Q.: PBWU, 24.5.1985) vor, das grundsätzlich drei Forschungsansätze vorsieht. Dabei handelt es sich um

- standortsbezogene, interdisziplinäre Freilanduntersuchungen,

- Laborexperimente an Waldbäumen und Modellpflanzen sowie ergänzend

- Stichprobenuntersuchungen auf Beobachtungsflächen.

Ausgehend von der bereits im ersten Entwurf vorgenommenen Beschreibung der bestehenden Freilandstandorte und der dort bearbeiteten Forschungsprogramme werden neu in das Programm aufzunehmende Forschungsthemen skizziert. Dies trifft für den Bayerischen Wald und den Höglwald zu. Für das Fichtelgebirge werden demgegenüber keine weiteren Forschungsansätze für erforderlich gehalten werden.

Für den geplanten „Standort Kalkalpen“ wird ein eigenes Forschungsprogramm vorgestellt. Unter Berücksichtigung infrastruktureller Vorteile wird der Raum Garmisch-Partenkirchen vorgeschlagen, um dort schwerpunktmäßig den Einfluß von Photooxidantien auf die Waldvitalität zu untersuchen. Als Versuchsdesign wird ein Höhenprofil, bestehend aus mehreren Untersuchungsflächen in verschiedenen Höhenlagen, avisiert.

Laborexperimente werden als Ergänzungen zu den Freilanduntersuchungen empfohlen, um zu einer „stärker abgesicherten Aussage ... als Beobachtung und Experiment allein“ zu gelangen. Dabei finden insbesondere Experimente zur Schadstoffwirkung und deren -mechanismen sowie Indikatoren zur Früherkennung von Schäden Erwähnung. Beispielhaft werden Themenschwerpunkte aufgelistet. Eine Übersicht über die gesamte Palette der vorgesehenen oder bereits be- 
arbeiteten Untersuchungsthemen, die den vier bayerischen Freilandstandorten zugeordnet werden, schließt das Rahmenprogramm ab.

In der vorangestellten Ausschreibung wird die zur Koordination der PBWU-Waldschadensforschung eingerichtete Projektgruppe vorgestellt. Anschließend wird das im Rahmenprogramm niedergelegte Forschungskonzept umrissen und Prioritäten gesetzt, wie z.B. die Einrichtung des alpinen Forschungsschwerpunktes. Geplanten Untersuchungen zu Schäden in Laubwäldern wird noch Pilotcharakter zugewiesen.

Als Reaktion auf die Kritik aus den Reihen des Wissenschaftlichen Beirats wird besonders betont, daß dem Einfallsreichtum der Antragsteller nicht vorgegriffen, und fachlich sowie standörtlich anders gelagerte Untersuchungen nicht ausgeschlossen werden sollten. Unter Einbeziehung eines unabhängigen Gremiums aus DFG-Gutachtern werde der erwartete Forschungsbeitrag zur Ursachenaufklärung das Hauptentscheidungskriterium für die finanzielle Förderung im PBWU-Projekt darstellen. Abschließend werden Gestaltungsvorgaben zur Antragstellung (z.B. nach den Grundsätzen der Deutschen Forschungsgemeinschaft), die Darstellung geplanter Zusammenarbeiten mit benachbarten Vorhaben, die auf 3 Jahre begrenzte Laufzeit von Forschungsvorhaben und das vorgesehene Begutachtungsverfahren aufgeführt.

In dieser Form werden Ausschreibung und Rahmenprogramm den Mitgliedern des Projektrats vorgelegt (i.Q.: PBWU, 3.6.1985). Mit der Bekräftigung, daß die „wertvollen Anregungen“ aus dem Kreis der PBWU-Gremien in der nun vorliegenden Fassung berücksichtigt werden konnten, wird um Einverständnis zu dem Programm gebeten. Da bis zum vorgegebenen Abstimmungstermin 14.6.1985 keine gegenteiligen Äußerungen eintreffen, wird das Rahmenprogramm in der bestehenden Fassung vom 24.5.1985 von der PBWU in Zusammenarbeit mit dem Bayerischen Umweltministerium an bayerische Forschungseinrichtungen ausgeschrieben (i.Q.: PBWU, 25.6.1985).

\subsubsection{Theorieorientiertes Zwischenergebnis}

\subsubsection{Einbindung bestehender Forschungsprogramme als Strategie}

Dem fortgeschrittenen Stand der Forschung entsprechend muß das Programm, welches in der Folge implementiert und durch die Projektgruppe koordiniert werden soll, einerseits neue Forschungsansätze entwickeln, andererseits die bereits etablierten Forschungsaktivitäten miteinbeziehen. Ein Konzept, bestehend lediglich aus einem neu zu schaffenden Freilandstandort, hätte die Notwendigkeit einer eigens eingerichteten, finanziell aufwendigen Projektgruppe in Frage gestellt. Die PBWU entwirft daher ein Rahmenprogramm, welches nicht nur den ressortspezifischen Anliegen ihres Auftraggebers Rechnung tragen soll, nämlich einen neuen Forschungsstandort zur Klärung der bis dahin noch wenig untersuchten Frage der Photooxidantienwirkung im Alpenraum zu etablieren. Auch die Einbeziehung vorhandener Forschungsschwerpunkte und der dort bearbeiteten Themen können zur Anreicherung und Abrundung des PBWU-Gesamtkonzeptes genutzt werden.

Von den im Beirat beteiligten Forschern zu bereits etablierten Programmen, und hier maßgeblich Vertretern der Forschungsgruppe Forsttoxikologie, wird die Einbeziehung der eigenen 
Forschungsstandorte in das PBWU-Konzept unterstützt. Die Aussicht auf zusätzliche Finanzierungsmöglichkeiten wird einerseits von den beteiligten Wissenschaftlern sowie deren politischen Ressortvertretern sofort erkannt. Sie über ein Anreizprogramm zur Zufinanzierung eigener Themenstellungen für das Gesamtkonzept zu gewinnen, kann aus politischer Sicht auch für die Durchsetzung des beabsichtigten alpinen Forschungsschwerpunktes nur erwünscht sein. Der Standort könnte damit an wissenschaftlicher Qualifikation, Protektion und Akzeptanz gewinnen.

Der Handlungsdruck, einen auf das Umweltressort zugeschnittenen Forschungsschwerpunkt einzurichten, ergibt sich sowohl aus wissenschaftlichen, als auch aus politischen Gründen. Ein Forschungsschwerpunkt zu Photooxidantien wäre unter wissenschaftlichen Aspekten ein sinnvolles Pendent zu der bisher überwiegend säurewirkungsorientierten Waldschadensforschung. Ein eigener Forschungsstandort des Umweltressorts zeigt eigenständiges Handeln des Ressorts. Zudem könnte der Standort gegenüber den anderer, an der Waldschadensforschung bereits beteiligter Ressorts wie dem Landwirtschafts- und Kultusministerium, in Wettbewerb treten und die Ressourcen und die Kompetenz des Umweltministeriums unterstreichen.

\subsubsection{Ressortïbergreifender Koordinationsanspruch der Projektgruppe}

Die Einbindung bestehender Forschungsaktivitäten in das PBWU-Programm ist ein Versuch, den Steuerungseinfluß der PBWU auch auf die anderweitig finanzierten und koordinierten Forschungsschwerpunkte auszudehnen und damit den Anspruch nach zentraler, bayernweiter Koordination zu erfüllen. Die Möglichkeit, aus dem PBWU-Projekt zusätzliche Finanzmittel den bereits etablierten Schwerpunkten zufließen zu lassen, entspricht zwar den Interessenlagen der dort finanzierenden Ressorts und der beteiligten Wissenschaftler. Demgegenüber muß aber ein ressortübergreifendes Steuerungspotential der PBWU als unerwünschte Einmischung in die dortigen Kompetenzen verstanden werden. Zur Vermeidung dieses Konflikts ist die PBWU bemüht, die bestehenden Forschungsschwerpunkte zwar in ihr Gesamtkonzept miteinzubeziehen, ohne dort jedoch den Eindruck einer konzeptionellen Dominanz, sondern vielmehr den einer Ergänzung des bestehenden Forschungsprogrammes zu erwecken.

Das nachträgliche „Überstülpen“ einer mit Koordinations- und Regelungsbefugnissen ausgestatteten Projektgruppe auf bestehende Forschungs- und Finanzierungsstrukturen muß jedoch zu Konflikten führen und kann bestenfalls über eine hohe fachliche und institutionelle Kompetenz der Koordinierungsstelle oder über eine starke Beteiligung derer, die die vorhandenen Forschungsstrukturen fachlich und finanziell vertreten, akzeptiert werden. Dabei muß der PBWU spätestens nach der z.T. harschen Kritik der Wissenschaftler an einer befürchteten Einmischung klar sein, daß eine steuernde Funktion angesichts der dort gegebenen institutionellen, ressort- und fachspezifischen Kompetenz nicht ausgeübt werden kann. Ihre Einflußnahme kann sich hier lediglich auf die inhaltliche und finanzielle Verstärkung der bestehenden Forschungsansätze beziehen.

Somit wird die Rolle der PBWU zugunsten der fachlichen Beratungs- und Steuerungsfunktion der Wissenschaftler auf eine organisatorische Betreuungs- und Koordinationsfunktion in der bayerischen Waldschadensforschung reduziert. Mit Hinweis auf die Freiheit und die Kreativität 
der Forscher können die ohnehin weitläufigen Themenlisten des von der PBWU vorgelegten Forschungsprogramms offen gehalten werden.

In diesem Zusammenhang sieht sich das Landwirtschaftsministerium dazu veranlaßt, die eigene Förderstrategie der Waldschadensforschung klarzustellen (i.Q.: Bayer. Landwirtschaftsministerium, 29.4.1985). So wird zwar die forstfachliche Hilfestellung bei der Umsetzung des PBWU-Konzeptes durch die Forstverwaltung angeboten. Die Auswahl, Begutachtung, Finanzierung und Abwicklung von Forschungsvorhaben des Forstressorts erfolge jedoch nach wie vor in Eigenregie unter Einschaltung des Kuratoriums der Bayerischen Forstlichen Versuchsund Forschungsanstalt. Der PBWU wird lediglich eine beschränkte Möglichkeit zur Stellungnahme eingeräumt. Damit wird offensichtlich, daß die PBWU, als zentrale bayerische Koordinierungsstelle geplant, zur ressortspezifischen Koordinierungsstelle des Bayerischen Umweltministeriums herabgestuft wird. Mit den weiteren beteiligten Ressorts kann lediglich das Niveau informativer Zusammenarbeit erreicht werden.

Der bei Gründung der PBWU beabsichtigte Ansatz, die Forschungsverstärkung und -koordinierung ressortübergreifend $\mathrm{zu}$ bewerkstelligen, unterliegt bereits bei Erstellung des Forschungsprogramms erheblichen Einschränkungen. Die in der Waldschadensforschung bereits maßgeblich tätigen Ressorts wie das Landwirtschafts- und das Kultusministerium können in ihren Vergabepraxen eigenständig fortfahren, ohne wesentlich durch die PBWU hierin koordinierend beeinflußt zu werden. Eher noch besteht Aussicht darauf, daß zusätzliche Fördermittel ergänzend zu den bestehenden Forschungsaktivitäten aus dem neuen und finanziell noch nicht konkretisierten Finanzmitteltopf des Umweltministeriums via PBWU „mitgenommen“ werden können.

Ergebnis der Phase der Programmerstellung ist ein aus mehreren regionalen Forschungsschwerpunkten bestehendes und Laboruntersuchungen berücksichtigendes Forschungsprogramm. Abgesehen von den regionalen Vorgaben läßt das Programm fachlich/inhaltlich großen Spielraum für zu bearbeitende Forschungsthemen. Der Kreativität der angesprochenen Wissenschaftler soll dabei nicht vorgegriffen werden. Die Ausschreibung des Programms erfolgt an alle einschlägigen Forschungseinrichtungen. Es ist $\mathrm{zu}$ erwarten, daß eine Fülle von Forschungsanträgen unterschiedlicher fachlicher Thematik, institutioneller Herkunft und wissenschaftlicher Qualität im Rahmen des PBWU-Projekts vorgelegt werden wird und einer entsprechenden Auswahl zugeführt werden muß.

Die bereits während der Gründungsphase nur als ungenügend zu bezeichnende Akzeptanz der Projektgruppe bei ihren Gremien kann im Zuge der Verhandlungen zur Programmformulierung nicht verbessert werden. Zwar wird der Projektgruppe zugebilligt, ein Rahmenprogramm zu entwerfen und zur Beratung vorzulegen, die fachlich/inhaltliche Kritik daran ist jedoch erheblich. Die entsprechende Umarbeitung des Programms durch die PBWU ist jedoch nur ungenügend und erfolgt zudem mit zeitlichen Verzögerungen. Nach wie vor bestehen Mißverständnisse in der formalen Stellung der PBWU gegenüber ihren Gremien. Entsprechend der von den Gremien zugedachten Rolle kann die Akzeptanz der Gruppe allenfalls in organisatorischer, nicht jedoch in fachlicher Hinsicht hergestellt werden. Dies führt jedoch zu einer Kollision des 
Selbstverständnisses und des Anspruchs der Gremienmitglieder auf Beteiligung bei Grundsatzfragen und Förderentscheidungen mit denen der Projektgruppe auf Forschungskoordination.

\subsubsection{Forschungssteuerung: Top down-versus bottom up-Ansatz}

Dem Bayerischen Umweltministerium ist an einer zügigen, flexiblen Umsetzung des Forschungsprojektes gelegen. Die PBWU selbst wird mit der Erstellung des Rahmenprogramms betraut. Bereits im Vorfeld, nämlich zur PBWU-Gründung, sowie während der Diskussion des Programmentwurfs, sind die in den PBWU-Gremien vertretenen Wissenschaftler und die weiteren politischen Ressortvertreter darauf bedacht, ihre Einflußnahme auf das Rahmenprogramm geltend zu machen. Hieraus läßt sich ein Steuerungskonflikt in der Forschung zwischen politischen und wissenschaftlichen Akteuren ableiten, der über die Modelle des „top down“- bzw. des ,,bottom up“-Ansatzes theoriegestützt beschrieben und analysiert werden kann.

Vom politischen Auftraggeber initiiert, weist das Programm Züge forschungspolitischer Steuerungsabsichten auf. Entsprechend der politischen Entscheidung, durch Verstärkung der Forschung einen Beitrag zur Lösung des Problems Waldschäden zu liefern, beabsichtigt das Bayerische Umweltministerium die Durchführung eines problemorientierten Forschungsprogrammes. Dieses orientiert sich mit der Schwerpunktsetzung Photooxidantien im Alpenraum im wesentlichen an den Zielsetzungen des Förderressorts und dokumentiert damit dessen Anspruch auf Steuerungsdominanz.

Idealtypisch kann dieser Typ der Forschungssteuerung mit dem Ansatz des „top down“Modells der Programmimplementation [s. WINDHOFF-HÉRITIER 1987, S. 96/97] beschrieben werden. Der Ansatz setzt eine klare Problemdefinition und Zielsetzung als Grundlage für ein entsprechendes Forschungsprogramm voraus, welches von einer Zentralinstanz - im vorliegenden Fallbeispiel der Projektgruppe PBWU - inhaltlich und organisatorisch strukturiert den Forschern in Form einer anwendungsorientierten Auftragsforschung zur Bearbeitung übergeben wird. Ziele und Programmschwerpunkte müssen als konzeptionelle Grundlagen von der Politik vorgegeben werden, die forschungsseitige Umsetzung hat sich inhaltlich streng daran zu orientieren. Dies erfordert ein straffes Programmanagement [HILL \& RIESER 1983, S. 171, 335ff].

Demgegenüber bezeichnet der sog. „bottom up“-Ansatz die inhaltlich-politisch weitgehend ungesteuerte, wissenschaftliche Bearbeitung eines Problems. Als Vorgaben bestehen lediglich die Problemdefinition und der zur Verfügung stehende Finanzmittelrahmen [HILL \& RIESER 1983, S. 342ff]. Die Programmausgestaltung obliegt weitestgehend den an der Forschung beteiligten Akteuren.

Beide Ansätze beinhalten in der Aushandlung eines Rahmenprogramms sowohl politische als auch wissenschaftliche Beiträge. Allerdings dominiert im Falle des „top down“-Modells der forschungspolitische Aspekt. Hier soll eine politisch erwünschte Problembearbeitung zielgerichtet durch Vorgabe bestimmter Forschungsinhalte und durch eine straffe Programmorganisation und -durchführung erreicht werden. Im Falle des „,bottom up“-Modells dominiert die Freiheit wissenschaftlicher Ideenfindung im Rahmen der Bearbeitung eines nur allgemein vorgegebenen Forschungsgebietes. Nicht „,von oben herab“, sondern von der wissenschaftlichen 
Basis ausgehend und formuliert sollen in einer Art problemorientierter Grundlagenforschung politisch nutzbare Problemlösungsbeiträge geliefert werden.

Die Wirkungen beider Typen in Hinblick auf die Interessenlage der politischen wie wissenschaftlichen Akteure liegen auf der Hand. Sie spiegeln sich im Aushandlungsprozeß um das Rahmenprogramm der PBWU wider. Den im Beirat vertretenen Forschern ist an einem weniger wissenschaftlich reglementierten Forschungsförderverfahren nach dem „bottom up“-Modell gelegen. Dementsprechend wird die Vorgabe konkreter Forschungsthemen im Rahmenprogramm der PBWU als wissenschaftshemmend, ideen- und kreativitätshinderlich bezeichnet. Ebenso wird die formal starke Stellung der PBWU hinsichtlich der fachlichen Koordination des Projektes kritisiert. Die Eigenverantwortlichkeit der Forscher in Auswahl und wissenschaftlicher Bearbeitung bestimmter Themen sowie freie Nutzungsrechte in der Ergebnisverwertung werden gefordert.

Demgegenüber beabsichtigt der Auftraggeber Bayerisches Umweltministerium im Grundsatz die Finanzierung konkreter Forschungsbeiträge auf Grundlage eines Rahmenprogramms. Dies bedeutet eine Vorgehensweise nach dem „top down“-Modell. Hierfür spricht der erwartete Beitrag zur Ursachenklärung der Waldschäden als Hauptentscheidungskriterium für eine Vorhaben-Förderung, wie in der Ausschreibung zum Rahmenprogramm formuliert wird. Auch die der PBWU zugedachte, steuerungsdominante Stellung ist für dieses Modell charakteristisch. Letztlich spricht auch die Erarbeitung eines Rahmenprogramms durch die PBWU selbst in enger Abstimmung mit dem Ministerium für die Dominanz politischer gegenüber wissenschaftlichen Steuerungsaspekten und somit für den „top down“-Ansatz.

Bereits bei dem Entwurf des Rahmenprogramms durch die PBWU kommt es zu einer Aufweichung des politisch beabsichtigten ,top down“-Ansatzes. Erfordert das vielschichtige Thema Waldschadensforschung ohnehin eine Struktur, die zahlreiche Forschungsdisziplinen tangiert, so umfaßt das PBWU-Rahmenprogramm eine Vielzahl von Forschungsaspekten allein innerhalb des Segments Luftschadstoff-Forschung und beläßt in ausreichendem Maße wissenschaftliche Freiräume. In dieser Breite ermöglicht die inhaltliche Ausgestaltung des Programms einen steuernden Einfluß der Wissenschaft während der folgenden Phase der Programmimplementation. Demgegenüber ist eine nur schwache Steuerungswirkung des politischen Programmanagements bzw. des Auftraggebers zu erwarten [s.a. HILL \& RIESER 1983, S. 313, 350].

Auch die Abstimmung des PBWU-Rahmenprogamms mit dem Wissenschaftlichen Beirat muß zwangsläufig zu einer Vermischung beider Ansätze beitragen. Die Forscher versuchen, mit ihrer Interessendurchsetzung hinsichtlich einer weitgehenden fachlich-inhaltlichen Selbststeuerung der Wissenschaft dem Korsett eines politisch geprägten Rahmenprogramms zu begegnen.

Zudem sind die Mitglieder des Beirats überwiegend der Grundlagenforschung zuzuordnen, wie sich aus der Beteiligung an verschiedenen Forschungsprogrammen der Deutschen Forschungsgemeinschaft (DFG) ablesen läßt. Sie werden daher tendenziell versuchen, das angewandte Forschung favorisierende Programm des Auftraggebers für Forschungsansätze, die dem grundlegenden Verständnis von Ursachen-Wirkungsbeziehungen zum Thema dienen, offen zu halten. Auch der allgemein akzeptierte Vorschlag, für die Begutachtung einzelner Forschungs- 
anträge maßgeblich DFG-Gutachter heranzuziehen, läßt erwarten, daß gegenüber dem maßgeblich politischen Förderkriterium angewandter Forschung wissenschaftliche, ja sogar grundlagenforschungsorientierte Entscheidungskriterien starke Berücksichtigung finden werden.

Somit ist das von den Gremien letztlich verabschiedete Rahmenprogamm der PBWU ein Kompromiß aus beiden Ansätzen, geprägt von dem Grundkonflikt der Autonomieansprüche der Wissenschaft gegenüber politikbestimmten Zielsetzungen der Forschungsförderung [HILL \& RIESER 1983, S. 357]. Das Bayerische Umweltministerium setzt sich mit gewissen, politisch begründeten Forschungsschwerpunkten (Photooxidantien-Wirkungsforschung im Alpenraum) im Grundsatz zwar für eine anwendungsorientierte Waldschadensforschung durch. Die Wissenschaft behält bei der gegebenen Programmbreite und inhaltlichen Offenheit sowie ihrer Beteiligung bei der Antragsbegutachung aber die Option, ihre eigenen fachlichen Forscherinteressen sowie insbesondere Grundlagenforschungsaspekte einbringen zu können. Insofern besitzt das Rahmenprogramm Alibifunktion: Formal verspricht es politisch aktuelle Problemlösungen, informal können sich Grundlagenforscher ihre Beteiligung sichern [HILL \& RIESER 1983, S. $349 \mathrm{ff}]$.

Durch das Abweichen vom strengen ,top down“-Ansatz einer problemorientierten Auftragsforschung zugunsten der Wissenschaftlerinteressen kann der Auftraggeber Steuerungskonflikte zwar nicht beseitigen. Er kann sie jedoch in die Implementationsphase zurückstellen und damit eine Gefährdung des Gesamtprojektes erst einmal vermeiden, die sich aufgrund der grundsätzlichen Kritik an dem Aufgabenspektrum der PBWU ohnehin angebahnt hatte.

Auch der PBWU selbst kann Interesse daran unterstellt werden, einen vergleichsweise offenen Forschungsrahmen abzustecken. Einerseits ist sie damit weniger der Kritik ausgesetzt, aus wissenschaftlicher Sicht für erforderlich gehaltene Aspekte nicht berücksichtigt zu haben. Andererseits öffnet sie dadurch möglichst vielen potentiellen Forschungsrichtungen den Zugang zum Projekt, begünstigt damit dessen Realisation und macht die Wissenschaftler dem Projekt gegenüber geneigt. Gerade auch die Einbeziehung bereits bestehender Forschungsschwerpunkte in das Programm läßt dort die finanzielle Unterstützung des Bayerischen Umweltministeriums im Rahmen des PBWU-Projektes erwarten. Sie muß von den dortigen Forschern begrüßt werden, solange ein markanter Koordinations- bzw. Forschungssteuerungseinfluß der PBWU, der die dortige Selbstverwaltung der Wissenschaft beeinträchtigen würde, abgewehrt werden kann. 


\subsection{Programmimplementation}

Auf die Phase der Programmformulierung folgt in Anlehnung an den Policy-Ansatz von WinDHOFF-HÉRITIER [1987, S. 64ff] die Programmimplementation. Diese bildet im PBWUProjekt den eigentlichen Forschungsprozeß. Er kommt durch die Umsetzung des Rahmenprogramms in Form von konkreten Forschungsbeiträgen, das Förderverfahren zwischen Antragstellung und Förderentscheidung unter Einbeziehung der Antragsselektion, sowie die Koordination der Forschung durch die Projektgruppe PBWU zustande. In der folgenden, empirischen Darstellung des Forschungsprozesses im PBWU-Projekt wird neben der allgemeinen Forschungsimplementation der spezielle Aspekt der alpinen Waldschadensforschung am Forschungsschwerpunkt Wank gesondert behandelt.

\subsubsection{Startphase des PBWU-Forschungsprogramms}

Der Implementationsprozeß im PBWU-Projekt reicht von der Behandlung erster, aufgrund der Ausschreibung des Rahmenprogramms eingereichter Forschungsanträge im Jahr 1985 bis hin zur Bewilligung letzter Anträge zu Beginn der 90er Jahre. Aufgrund der hohen Programmlaufzeit, der unterschiedlichen Selektionsprozesse im Förderverfahren und der variierenden Steuerungsdominanz zwischen Politik und Wissenschaft wird der Implementationsprozeß der Übersichtlichkeit wegen in mehrere Phasen aufgeteilt.

\subsubsection{Erste Begutachtungsrunde im Herbst 1985}

\subsection{Programmausschreibung und Einreichung erster Anträge bei der PBWU}

Das Rahmenprogramm der PBWU zur Verstärkung der bayerischen Waldschadensforschung wird in der Fassung vom 24.5.1985 ausgeschrieben. Die Ausschreibung ist an einschlägige universitäre wie außeruniversitäre Forschungseinrichtungen gerichtet, und zwar ausschließlich an bayerische Institutionen. Die Vorlage von Forschungsanträgen ist kurzfristig bis zum 31.8.1985 befristet.

Zum Stichtag werden 29 Forschungsanträge bei der PBWU eingereicht. Die insgesamt beantragte Mittelhöhe beträgt ca. 14 Mio. DM. Bei den antragstellenden Universitätsinstituten sind sowohl in Hinblick auf Anzahl als auch Finanzhöhe der Anträge die beiden Münchener Universitäten stark vertreten. Weiterhin stammen Anträge von den Universitäten Würzburg, Erlangen/Nürnberg und Bayreuth sowie von der außeruniversitären Fraunhofer-Gesellschaft - Institut für Atmosphärische Umweltforschung in Garmisch-Partenkirchen (FhG-IFU) und der GSF Neuherberg (s. Tabelle 2).

Hinsichtlich der bearbeiteten Fachdisziplinen überwiegen Anträge, die sich mit biochemischen und pflanzenphysiologischen Fragestellungen einschließlich Cuticula-Forschung, Schadstoffentgiftung sowie den Bereichen Luftchemie und Immissionserfassung beschäftigen (s. Tabelle 3). Weitere Anträge betreffen atmogene Schadstofffrachten, den Einfluß von Klima und Boden auf die Waldvitalität, Zustand und Zeigerwerte der Bodenvegetation, Schadstoffanreicherun- 
gen im Holz, Wirt/Parasit-Verhältnisse unter Schadstoffeinfluß sowie moderne, fernerkundungsgestützter Methoden der Waldzustandserfassung.

\begin{tabular}{|l|c|c|}
\hline Antragsteller & Anzahl Anträge & $\begin{array}{c}\text { Kosten } \\
\text { (Mio. DM) }\end{array}$ \\
\hline Uni. München & 5 & 3,2 \\
\hline TU München & 5 & 2,4 \\
\hline Uni. Würzburg & 5 & 0,6 \\
\hline TUM-Weihenstephan & 3 & 1,0 \\
\hline Uni. Erlangen/Nürnbg. & 3 & 3,3 \\
\hline GSF Neuherberg & 3 & 1,2 \\
\hline Uni. Bayreuth & 2 & 1,7 \\
\hline FhG-IFU Garmisch-P. & 2 & 0,5 \\
\hline FH Weihenstephan & 1 & 0,1 \\
\hline Summe: & $\mathbf{2 9}$ & $\mathbf{1 4 , 0}$ \\
\hline
\end{tabular}

Tab. 2: Forschungsanträge und Antragsteller (Sommer 1985) (eigene Erhebung)

\begin{tabular}{|l|c|c|}
\hline Fachdisziplin & Anzahl Anträge & $\begin{array}{c}\text { Kosten } \\
\text { (Mio. DM) }\end{array}$ \\
\hline Luftchemie/Strahlung & 7 & 4,6 \\
\hline Biochemie & 6 & 1,4 \\
\hline Physiologie & 5 & 2,2 \\
\hline Cuticula & 3 & 1,3 \\
\hline Klima & 2 & 1,7 \\
\hline Bodenkunde & 2 & 2,1 \\
\hline Sonstige & 4 & 0,7 \\
\hline Summe: & $\mathbf{2 9}$ & $\mathbf{1 4 , 0}$ \\
\hline
\end{tabular}

Tab. 3: Forschungsanträge und Fachdisziplinen (Sommer 1985) (eigene Erhebung)

Regional beziehen sich zahlreiche Anträge auf den neu einzurichtenden, alpinen Forschungsschwerpunkt „Kalkalpen“ oder stellen weitgehend standortsunabhängige Laboruntersuchungen bzw. nicht lokal zuordenbare Freilanduntersuchungen dar. Einzelne Anträge beziehen sich auf die bestehenden Forschungsschwerpunkte Fichtelgebirge und Bayerischer Wald sowie die in der Ausschreibung vorgesehene Pilotphase zur Konzeption eines Laubwaldstandorts. Für den ebenfalls bereits bestehenden Forschungsstandort Höglwald werden keine Anträge vorgelegt (s. Tabelle 4).

Nahezu alle einschlägigen bayerischen und bisher in der Waldschadensforschung involvierten Forschungseinrichtungen beteiligen sich. Es kann davon ausgegangen werden, daß die Mitglieder des Wissenschaftlichen Beirats für die von ihnen vertretenen oder benachbarte Institute aufgrund der vorangegangenen Beschäftigung mit dem PBWU-Rahmenproramm als zeitlichen Vorteil nutzen konnten und zeitgerecht Anträge vorlegen konnten. Vergleichbares kann für die GSF gelten. Auch den weiteren Ressorts im Projektrat der BWU kommt eine vergleichbare Vermittlerrolle gegenüber den von ihnen jeweils vertretenen Forscherklientela oder bestehenden Forschungsverbünden zu. 


\begin{tabular}{|l|c|c|}
\hline Standort & Anzahl Anträge & $\begin{array}{c}\text { Kosten } \\
\text { (Mio. DM) }\end{array}$ \\
\hline Kalkalpen & 11 & 4,3 \\
\hline Fichtelgebirge & 3 & 2,3 \\
\hline Bayer. Wald & 2 & 1,3 \\
\hline Höglwald & - & - \\
\hline Laubwald & 1 & 0,3 \\
\hline $\begin{array}{l}\text { Labor od. nicht } \\
\text { zuordenbar }\end{array}$ & 12 & 5,8 \\
\hline Summe: & $\mathbf{2 9}$ & $\mathbf{1 4 , 0}$ \\
\hline
\end{tabular}

Tab. 4: Forschungsanträge und Forschungsstandorte (Sommer 1985) (eigene Erhebung)

\subsection{Mündliches Begutachtungsverfahren zur Forschungsförderung}

Die Projektgruppe führt eine interne Bewertung der einzelnen Anträge durch (s. Tätigkeitsbericht der PBWU für den betreffenden Berichtszeitraum 1.7. - 30.11.1985). Darin werden insbesondere die Einpassung in das PBWU-Rahmenprogramm und der zu erwartende Beitrag zur Ursachenklärung der Waldschäden geprüft. Eine Diskussion und Vorauswahl der Anträge durch den Wissenschaftlichen Beirat, wie auf den Sitzungen der PBWU-Gremien vom 11.2. und 21.3.1985 beschlossen worden war (i.Q.: Bayer. Umweltministerium, 11.2.1985 und $P B W U, 21.3 .1985$ ), findet nicht statt. Allerdings lädt die PBWU entsprechend dem Gremienbeschluß vom 11.2.1985 sowie in der Ausschreibung festgelegten Verfahren zu einer gemeinsamen mündlichen Begutachtungsrunde die Antragsteller und externen Gutachtern in die GSF ein. Den Gutachtern werden alle Forschungsanträge, die kurzgefaßten Stellungnahmen der PBWU sowie das Rahmenprogramm übersandt (i.Q.: PBWU, 2.10.1985).

Der Vertreter des Kultusministeriums im Projektrat bringt seine „Überraschung“ und sein „Unverständnis“ über das gewählte Vorgehen zum Ausdruck (i.Q.: Kultusministerium, 10.10.1985). Eine Begründung wird gefordert, warum die Gremienbeschlüsse mißachtet und das Begutachtungsverfahren ohne die koordinierende Vorauswahl und gesamtkonzeptionelle Einordnung der Anträge durch den Wissenschaftlichen Beirat erfolgte. Die externen Gutachter dürften wohl kaum in der Lage sein, über eine reine Einzelbegutachtung hinausgehend die einzelnen Anträge zu koordinieren und den interdisziplinären Ansatz zu wahren. Statt dessen werde ein „beziehungsloses Nebeneinander einzelner Projekte“ befürchtet.

Die PBWU begründet die Abweichungen von der vereinbarten Vorgehensweise insbesondere mit Terminschwierigkeiten und dem Handlungsdruck, der sich aufgrund des möglichst raschen Beginns einer ersten Förderphase ergäbe (i.Q.: PBWU, 21.10.1985). Das Verfahren sei mit dem Vorsitzenden des Projektrats, dem Vertreter des Umweltministeriums, abgestimmt. Daher müsse die Begutachtung der Anträge bereits vor der Beratung in den Gremien der PBWU erfolgen, was jedoch nicht als negativer Einfluß auf das Ergebnis zu bewerten sei.

Bei den externen Experten, die von der PBWU um Bewertung der Anträge gebeten werden, handelt es sich vorschlagsgemäß um DFG-Gutachter. Nahezu alle der angefragten Wissenschaftler erklären ihr Interesse und ihre Bereitschaft zur Beteiligung am Begutachtungsverfah- 
ren. Damit besteht das Gutachtergremium aus 9 hochrangigen und zum Teil selbst in der Waldschadensforschung tätigen Wissenschaftlern aus dem gesamten Bundesgebiet. Sie waren der PBWU vom Bayerischen Kultusministerium genannt worden und bereits bei der Beurteilung der Anträge der Bayerischen Forschungsgruppe Forsttoxikologie beteiligt.

Einige der Gutachter legen wegen terminlicher Verhinderung oder zusätzlich zu ihrer Teilnahme schriftliche Stellungnahmen vor, die z.T. von grundsätzlichen Bemerkungen zum PBWUProjekt begleitet werden. So empfehlen einige Gutachter generell die „Zusammenführung verschiedener, divergierender Projekte" der Bayerischen Staatsregierung. Gleichzeitig wird um ,angemessene Berücksichtigung“ der Arbeiten der Forschungsgruppe Forsttoxikologie auch im PBWU-Projekt gebeten (i.Q.: Fachgutachter, 3.9., 11.9. und 22.10.1985).

Darüber hinaus äußern sich mehrere Gutachter kritisch zur Qualität der vorgelegten Anträge (i.Q.: Fachgutachter, 17.10., 22.10. und 4.11.1985). Dem DFG-üblichen Anspruch an die Forschungsqualität würden nur wenige Anträge gerecht, zudem fielen erhebliche Diskrepanzen zwischen Antragsbegründung und Antragssumme negativ auf. Ein Gutachter zeigt sich enttäuscht darüber, daß „,wichtige Forschungsgebiete völlig (oder fast völlig) fehlen“ und nennt hierfür Beispiele. Das Rahmenprogramm sei zu eng auf direkte Luftschadstoffwirkung zugeschnitten, was dem gegenwärtigen Stand der Erkenntnis nicht mehr gerecht würde. Einzelne Forscher hätten ihre bisherige Arbeitsrichtung zudem nur soweit modifiziert, daß ihr Antrag gerade noch der Ausschreibung gerecht würde. Folglich sei es mit der Ausschreibung nicht gelungen, neue Ansätze zu provozieren, die wesentlich zu einer Kausalanalyse der Schäden beitragen könnten. Die meisten der Vorhaben würden zwar als Grundlagenforschung einen Beitrag zur wissenschaftlichen Erkenntnis liefern, derartige Vorhaben sollten aber nicht in einem angewandten Forschungsprogramm zur Genese von Waldschäden verankert werden.

Zur Begutachtungssitzung am 24./25.10.1985 sind auch die Projektgruppe, Vertreter des Projektrats sowie Gäste anderer Koordinierungseinrichtungen anwesend. Die Anträge werden von den Antragstellern mündlich vorgestellt und diskutiert. Die anschließende Beratung erfolgt unter Ausschluß der Antragsteller. Der Leiter der PBWU moderiert die Sitzung. Er ruft die zu behandelten Vorhaben der Reihe nach auf, verliest gegebenenfalls vorliegende, schriftliche Voten einzelner Gutachter, weist auf die Stellungnahme der PBWU hin, beteiligt sich an der Diskussion und faßt abschließend deren Ergebnis zusammen (i.Q.: PBWU, 24./25.10.1985).

Überwiegend stehen bei der internen Beurteilung fachlich/inhaltliche wie methodische Aspekte im Vordergrund (STEUER [1997, S. 30, 44]). Die jeweils fachlich tangierten Gutachter bestimmen die Diskussion. Im Kontext der Argumente oder durch Proklamation einzelner Gutachter verständigt man sich auf eine Förderempfehlung. Diese geht nur in einzelnen Antragsfällen über das Gesamturteil einer Bewilligung oder Ablehnung hinaus, indem inhaltlich, finanziell oder zeitlich zu konkretisierende Vorgaben bzw. Modifizierungswünsche der Gutachter im Begutachtungsergebnis fixiert werden.

\subsection{Beratung und Entscheidung in den PBWU-Gremien}


Die Ergebnisse der Antragsbegutachtung stehen auf der Tagesordnung der nächsten gemeinsamen Sitzung der PBWU-Gremien am 18.11.1985. Als Sitzungsunterlage versendet die PBWU unter anderem eine Zusammenstellung der beantragten Fördermittel sowie antragsweise eine Zusammenfassung der Gutachterdiskussion. Noch im Vorlauf zur Sitzung legt ein Mitglied des Wissenschaftlichen Beirats, welches selbst zwei Anträge eingereicht hatte, eine ausführliche Stellungnahme zu den übersandten Zusammenfassungen vor. Darin werden verschiedene formale Richtigstellungen vorgenommen, der - negativen - Einschätzung der Gutachter entgegnet und eine Diskussion im Rahmen der kommenden Sitzung vorgeschlagen (i.Q.: Wissenschaftlicher Beirat, 14.11.1985).

Auf der Sitzung rechtfertigt der Leiter der PBWU die kritisierte Verfahrensdurchführung wiederum damit, daß die Einschaltung des Wissenschaftlichen Beirats zur Vorauswahl der Anträge aus Zeitgründen nicht hätte stattfinden können (i.Q.: PBWU, 18.11.1985). Einzelne Gremienmitglieder kritisieren, daß nicht über sämtliche, sondern nur auffällig hohe oder unzureichend begründete Mittelpositionen in den einzelnen Anträgen beraten und entschieden worden sei. Ein möglichst detailliertes Gutachtervotum würde der PBWU und dem verantwortlichen Ministerium die Verhandlungen mit den Antragstellern erleichtern. Auch die Zusammensetzung des DFG-Gutachtergremiums wird als fachlich unausgewogen kritisiert.

Nach intensiver Diskussion akzeptieren die PBWU-Gremien jedoch das Vorgehen der PBWU und die vorliegenden Gutachtervoten. Der Wissenschaftliche Beirat sieht seine Aufgabe nicht in einer „Nach-Begutachtung“, zumal Mitglieder des Gremiums gleichzeitig Antragsteller sind. Unterstrichen wird, daß an der Trennung zwischen Gutachtern und Mitgliedern des Wissenschaftlichen Beirats festgehalten werden müsse. Der Wissenschaftliche Beirat ist um die Neutralität einer fachlichen Beurteilung bemüht. So lagen die Tonbandaufzeichnungen zur Gutachtersitzung zwar der PBWU vor, seien vom Beirat aber nicht verwendet worden (i.Q.: Wissenschaftlicher Beirat, 17.1.1986). Alle Seiten drängen auf eine möglichst rasche Weiterbehandlung der Anträge und Mittelabfluß. Als Förderbeginn für die ersten Antragsbewilligungen des PBWU-Forschungsprogramms beabsichtigt das finanzierende Umweltministerium bereits Anfang des Jahres 1986 (i.Q.: PBWU, 18.11.1985).

Insbesondere in Hinblick auf den zu etablierenden alpinen Forschungsstandort einigt man sich darauf, auf eine zweite Ausschreibung zu verzichten und vielmehr potentielle Antragsteller gezielt anzusprechen. Auf ein erstes Vorgespräch mit mehreren Forschern kann der Leiter der PBWU bereits verweisen. Allerdings wird von einem Mitglied des Wissenschaftlichen Beirats wiederum der im PBWU-Konzept skizzierte Umfang der dort vorgesehenen Forschungsarbeiten kritisiert. Dieser müsse auf ein „finanziell wie arbeitskapazitätsmäßig zu bewältigendes $\mathrm{Ma}$ reduziert" werden.

\subsection{Weiteres Verfahren der Antragsbehandlung}

Den Antragstellern wird das Ergebnis der Begutachtung und der entsprechenden Förderempfehlung der PBWU-Gremien vom Bayerischen Umweltministerium mitgeteilt (i.Q.: Bayer. Umweltministerium, 26.11. bzw. 2.12.1985). Danach werden - vorbehaltlich haushaltsrechtlicher Zustimmung - insgesamt 
- 15 Anträge als „,förderungswürdig“ eingestuft und zur Finanzierung durch das Bayerische Umweltministerium vorgesehen.

- 8 Anträge können nicht zur Förderung empfohlen werden und erfahren eine Ablehnung. Die Ablehnung erfolgt in der Regel schriftlich und ohne konkrete Angabe einer fachlichen Begründung.

- 4 Anträge können in der vorliegenden Form nicht zur Förderung empfohlen werden, allerdings wird eine Antragsmodifizierung anheimgestellt.

- 2 Anträge, die am Schwerpunktstandort Kalkalpen durchgeführt werden sollen, sind grundsätzlich förderungswürdig, müssen aufgrund des dortigen Planungsstands jedoch zurückgestellt und eventuell dem Gesamtprogramm angepaßt werden.

Nachdem die Finanzierung durch das Bayerische Umweltministerium gesichert ist, nimmt die PBWU mit den Antragstellern Kontakt auf, um die weitere Vorgehensweise im Falle einer Vorhabensförderung, Antragsmodifizierung oder -zurückstellung abzustimmen. Da diese Verfahrensweise über die Gesamtlaufzeit des PBWU-Projektes nahezu unverändert bleibt, soll sie an dieser Stelle ausführlich dargestellt werden.

Die Kontaktaufnahme erfolgt in der Regel telephonisch; bei einigen Bewilligungen muß die Akzeptanz der jeweiligen Antragsteller für gutachterseits geforderte Auflagen erst hergestellt werden. Diese betreffen z.B. fachliche Programmkorrekturen oder die Kürzung von Mitteln bzw. Vorhabenlaufzeiten. Die Vorhabenlaufzeiten betragen in der Regel 2 bis maximal 3 Jahre. Meist sind die Auflagenforderungen jedoch vergleichsweise geringfügig und werden zudem zwischen Projektgruppe und Antragsteller verhandelt. So resultiert aus der vom Antragsteller begründeten Unerfüllbarkeit einer Auflage weniger die Nichtbewilligung des gesamten Vorhabens, als vielmehr der Verzicht auf die entsprechende Auflage oder deren Abschwächung.

Anschließend erstellt die PBWU Bewilligungsentwürfe zu den einzelnen Forschungsvorhaben nach einer Mustervorlage des Umweltministeriums. Dem Vertragspartner bleibt unbenommen, die Ergebnisse des Forschungsvorhabens für Forschung und Lehre zu nutzen und zu veröffentlichen ( $\$ 10$ der Musterbewilligung). Das Ministerium sichert sich jedoch die Nutzungsrechte für die erbrachten Leistungen sowie einen redaktionellen Umgestaltungsvorbehalt der schriftlichen Ergebnisdarstellung.

Der Bewilligung vorangestellt ist jeweils ein interner Vermerk für das Bayerische Umweltministerium, welcher das Vorhaben kurz skizziert und dessen Notwendigkeit begründet (s. Bewilligungsentwürfe der PBWU). Die Begründung bezieht sich auf das von den PBWU-Gremien gebilligte Rahmenprogramm und den Beschluß der Gremien zur Finanzierung des Vorhabens entsprechend dem Gutachtervotum. Die von der PBWU dem Umweltministerium vorgelegten Bewilligungsentwürfe werden hausintern geprüft, unterzeichnet und dem Auftragnehmer (bei Universitäten über deren Verwaltung) zur Gegenzeichnung zugesandt.

Entsprechend den Ergebnissen der ersten, mündlichen Begutachtungsrunde können ab 1.4. bzw. 1.5.1986 insgesamt 16 von 29 Forschungsanträgen durch das Bayerische Umweltministerium finanziert werden. Dabei handelt es sich um als förderungswürdig sowie zur Modifizierung empfohlene Anträge. Diese legen für das erste Förderjahr 1986 rund 2,8 Mio. DM an Fördermitteln fest, auf ihre Gesamtlaufzeit von 2-3 Jahren bezogen insgesamt knapp 6 Mio. 
DM. (i.Q.: PBWU, 11.6.1986). Nahezu die Hälfte der bewilligten Vorhaben läßt sich mitteloder unmittelbar dem noch in Planung befindlichen alpinen Forschungsschwerpunkt zuordnen. Weitere Vorhaben betreffen Laborarbeiten mit z.T. weitgehendem Grundlagenforschungscharakter, z.B. zur Schadstoffaufnahme und Schadstoffwirkung in Blattorganen.

\subsubsection{Zweite Begutachtungsrunde im Frühjahr 1986}

\subsection{Einreichung von Forschungsanträgen}

Für die zweite Begutachtungsrunde im Frühjahr 1986 werden wiederum über 20 Anträge vorgelegt. Mit rund 17 Mio. DM beantragter Mittel übertrifft der Finanzumfang dieser Runde die der ersten um etwa 3 Mio. DM. Einige der Antragsteller waren - wie aus Anschreiben zu den eingereichten Anträgen hervorgeht - explizit von der PBWU angesprochen worden. Anzunehmen ist, daß die Mitglieder der PBWU-Gremien für die Verbreitung des PBWU-Rahmenprogramms in Forscherkreisen sorgten und auch die Fördermaßnahmen der ersten Begutachtungsrunde weitere Antragsteller zur Beteiligung am PBWU-Projekt animierten.

Wiederum sind als Antragsteller Münchner sowie nordbayerische Universitäten stark vertreten (s. Tabelle 5). Auch zwei privatrechtliche Firmen reichen nach Vorgesprächen mit der PBWU Anträge ein (i.Q.: PBWU, 30.10. und 18.12.1985). Die zur Bearbeitung vorgesehenen Fachgebiete sind denen der erste Runde ähnlich, Fragen der pflanzenphysiologischen und biochemischen Wirkungsforschung stehen im Vordergrund (s. Tabelle 6). Einen weiteren Schwerpunkt stellen Anträge aus dem Bereich Bodenkunde und Stoffhaushalt dar. Als Besonderheit sind vergleichsweise finanzaufwendige Anträge zur Bewilligung von Analyse-Geräten sowie zur Einrichtung von Versuchsbeständen zu erwähnen.

Gerade im Rahmen der zweiten Begutachtungsrunde wird der geplante alpine Forschungsstandort mit nur drei Anträgen unzureichend berücksichtigt. Die Mehrzahl der Anträge stellt Laborarbeiten dar, läßt sich keinem Schwerpunktstandort zuordnen oder betrifft - als Sammelantrag eingereicht - den bereits etablierten Standort Fichtelgebirge (s. Tabelle 7).

Die PBWU teilt den Antragstellern mit, daß die Begutachtung diesmal in schriftlicher Form erfolgte; die Anträge würden externen Gutachtern zur schriftlichen Bewertung zugeleitet werden. Formal begründet wird dieses Vorgehen mit der etwas geringeren Zahl von Anträgen im Vergleich zur ersten Begutachtungsrunde (i.Q.: PBWU, 8.1.1986). 


\begin{tabular}{|l|c|c|}
\hline Antragsteller & Anzahl Anträge & $\begin{array}{c}\text { Kosten } \\
\text { (Mio. DM) }\end{array}$ \\
\hline Univ. Bayreuth & 7 & 11,2 \\
\hline Univ. München & 5 & 2,3 \\
\hline TU München & 3 & 0,5 \\
\hline Univ. Würzburg & 3 & 0,5 \\
\hline Firmen & 2 & 1,6 \\
\hline Univ. Regensburg & 1 & 0,1 \\
\hline Univ. Erlangen/Nbg. & 1 & 0,3 \\
\hline GSF & 1 & 0,5 \\
\hline Summe: & $\mathbf{2 3}$ & $\mathbf{1 7 , 0}$ \\
\hline
\end{tabular}

Tab. 5: Forschungsanträge und Antragsteller (Frühjahr 1986) (eigene Erhebung)

\begin{tabular}{|l|c|c|}
\hline Fachdisziplin & Anzahl Anträge & $\begin{array}{c}\text { Kosten } \\
\text { (Mio. DM) }\end{array}$ \\
\hline $\begin{array}{l}\text { Physiologie / Pflanzen- } \\
\text { soziologie }\end{array}$ & 6 & 2,7 \\
\hline Biochemie & 5 & 1,9 \\
\hline $\begin{array}{l}\text { Bodenkunde / Stoff- } \\
\text { haushalt }\end{array}$ & 5 & 3,0 \\
\hline $\begin{array}{l}\text { Analysen-Geräte, Flä- } \\
\text { cheneinrichtung }\end{array}$ & 3 & 7,3 \\
\hline sonstige & 4 & 2,1 \\
\hline Summe: & $\mathbf{2 3}$ & $\mathbf{1 7 , 0}$ \\
\hline
\end{tabular}

Tab. 6: Forschungsanträge und Fachdisziplinen bzw. Investitionsanträge (Frühjahr 1986) (eigene Erhebung)

\begin{tabular}{|l|c|c|}
\hline Standort & Anzahl Anträge & $\begin{array}{c}\text { Kosten } \\
\text { (Mio. DM) }\end{array}$ \\
\hline Fichtelgebirge & 7 & 5,4 \\
\hline Kalkalpen & 4 & 1,1 \\
\hline Bayer. Wald & - & - \\
\hline Höglwald & 2 & 0,9 \\
\hline Laubwald & 2 & 1,0 \\
\hline $\begin{array}{l}\text { Labor od. nicht zuorden- } \\
\text { bar }\end{array}$ & 8 & 8,6 \\
\hline Summe: & $\mathbf{2 3}$ & $\mathbf{1 7 , 0}$ \\
\hline
\end{tabular}

Tab. 7: Forschungsanträge und Forschungsstandorte (Frühjahr 1986) (eigene Erhebung)

\subsection{Schriftliches Begutachtungsverfahren zur Forschungsförderung}

Die PBWU erarbeitet wie bisher zu jedem der eingegangenen Anträge eine Stellungnahme. Darin werden wiederum die Einordnung in das Rahmenprogramm, die wissenschaftliche Zielsetzung und Forschungsinhalte sowie ggf. die Anbindung an weitere, bereits bewilligte oder 
beantragte Vorhaben bewertet. Teilweise ist der beantragte Mittel- oder Personalbedarf Bestandteil der PBWU-Bewertung. Mitsamt diesen Stellungnahmen werden die Anträge den externen Fachgutachtern zur schriftlichen Bewertung vorgelegt.

Das Gutachtergremium ist nur teilweise identisch mit dem der ersten Runde. Da nicht alle Gutachter der ersten Runde verfügbar sind und neue Fachgebiete abgedeckt werden müssen, werden zusätzliche Experten einbezogen. Die PBWU tritt hierzu mit der DFG in Kontakt. Dabei bekommen alle Gutachter wiederum sämtliche Anträge zur Kenntnis, um sich eine Bild über den Gesamtrahmen der Antragsrunde machen zu können. Besonders gekennzeichnet werden die dem jeweiligen Gutachter fachspezifisch zugedachten Anträge. Insgesamt werden mindestens 2 Gutachter pro Antrag eingeholt (i.Q.: PBWU, 15.5.1986). Darüber hinaus wird es jedem Gutachter freigestellt, sich zu weiteren Anträgen zu äußern, wovon auch umfangreich Gebrauch gemacht wird.

Die Gutachten werden wiederum von der PBWU schriftlich zusammengefaßt. Dabei werden die Kriterien aufgeführt, auf Grund derer die jeweiligen Voten gefällt wurden. Mit diesen Unterlagen wird der Projektrat der PBWU zur nächsten Sitzung am 15.5.1986 eingeladen (i.Q.: $P B W U$, 15.5.1986). Den Mitgliedern des an der Sitzung nicht beteiligten Wissenschaftlichen Beirats wird neben den eingereichten Anträgen eine Liste der beteiligten Gutachter übersandt.

\subsection{Beratung und Entscheidung im Projektrat der PBWU}

Die Beratung der Forschungsanträge der zweiten Begutachtungsrunde steht im Mittelpunkt der Projektratssitzung vom 15.5.1986. Das einvernehmlich zwischen PBWU und Umweltministerium durchgeführte Verfahren erfordert wiederum erheblichen Aufklärungs- und Rechtfertigungsaufwand gegenüber den weiteren Projektratsmitgliedern (i.Q.: PBWU, 15.5.1986). So muß die Antragsakquisition und das Begutachtungsverfahren im Detail geschildert und die Beteiligung von DFG-Gutachtern versichert werden. Ergänzend stellt das Bayerische Kultusministerium klar, ,daß die Fachgutachter ausschließlich die wissenschaftliche Begutachtung der jeweiligen Anträge zu übernehmen hätten“. Es sei aber Sache des Projektrats, ,zu entscheiden, ob der jeweilige Antrag in das Projekt passe oder nicht“. Die Vorbegutachtung durch die PBWU und Mitteilung an die Gutachter wird als „unüblich und nicht vertretbar“ angesehen (i.Q.: Bayer. Kultusministerium, 20.6.1986).

Im weiteren Verlauf der Sitzung werden die Anträge trotz bestehender Verfahrenskritik auf Grundlage der von der PBWU zusammengefaßten Gutachtervoten weiterbehandelt und Förderentscheidungen getroffen. Dabei folgt der Projektrat wiederum weitestgehend den Empfehlungen der Gutachter [STEUER 1997, S. 46]. Die Ergebnisse sowie eine kurzgefaßte Begründung werden als Übersicht dem Sitzungsprotokoll beigefügt.

Weiterhin wird auf der Sitzung dem Umweltministerium und der PBWU „ungünstige Informationspolitik“ vorgeworfen. So seien Gerüchte im Umlauf, wonach keine weiteren Fördermittel mehr zur Verfügung stünden. Dies wird von Vertretern des Bayerischen Umweltministeriums zwar verneint, aus Finanzgründen seien aber Einschränkungen und Prioritätensetzungen - wie 
die Einrichtung des alpinen Forschungsschwerpunktes - unumgänglich (i.Q.: $P B W U$, 4.6.1986).

\subsection{Zurückstellung bewilligungswürdiger Forschungsanträge}

Im Anschluß an die Projektratsitzung vom 15.5.1986 verfaßt die PBWU im Auftrag des Umweltministerium einen Vermerk über die Programmabwicklung in finanzieller Hinsicht (i.Q.: $P B W U$, 11.6.1986). Darin scheint die auf der Sitzung bereits angedeutete Finanzmittelknappheit auf: Die vom Umweltministerium eingeplanten Fördermittel bis einschließlich 1988 in Höhe von 7 Mio. DM seien durch finanzielle Förderverpflichtungen aus der ersten Begutachtungsrunde bereits weitgehend gebunden. Der noch verbleibende Betrag von nurmehr 1 Mio. DM würde durch die Bewilligung der förderungswürdigen Vorhaben der zweiten Gutachterrunde aufgezehrt. Zudem müßten aber für die Bearbeitung wichtiger Forschungsthemen am alpinen Forschungsschwerpunkt zusätzlich 2 Mio. DM reserviert werden, ohne daß hierfür jedoch bereits konkrete Anträge vorlägen. Mitsamt den förderungswürdigen, aber zurückgestellten Anträgen der zweiten Runde in Höhe von ca. 3,5 Mio. DM ergäbe sich bis einschließlich 1988 ein zusätzlicher Mittelbedarf von insgesamt über 6 Mio. DM.

Die große Diskrepanz zwischen Mittelbedarf und -verfügbarkeit äußert sich in den Mitteilungen des Umweltministeriums an die Antragsteller der zweiten Begutachtungsrunde zum Ergebnis der Förderentscheidung (i.Q.: Bayer. Umweltministerium, 11.6.1986). Neben 12 Förderablehnungen aufgrund negativer Begutachtungsergebnisse wird 6 Antragstellern die $\mathrm{Zu}$ rückstellung ihrer grundsätzlich positiv beurteilten Anträge mitgeteilt. Mehrere Antragsteller, deren Antragsförderung beschlossen worden war, erhalten lediglich eine ,eingeschränkte Zusage“. Den alpinen Forschungsstandort betreffende Antragsteller werden mit erforderlichen Abstimmungen hinsichtlich Konzeption und Forschungsinhalten und eventuell notwendigen Anpassungen an das interdisziplinäre Programm auf ,gegebene Zeit“ vertröstet. Eine definitive Zusage könne derzeit nicht erfolgen. Dieser Typus von Mitteilung kommt faktisch einer Zurückstellung gleich. Letztlich führt die zweite Begutachtungsrunde über insgesamt 23 Einzelanträge vorerst zu keiner einzigen Bewilligung.

\subsubsection{Vertrauenskrise im Wissenschaftlichen Beirat}

\subsection{Kritik des Wissenschaftlichen Beirats am Förderverfahren}

Der Wissenschaftliche Beirat, der bereits während der ersten Begutachtungsrunde verfahrensmäßig übergangen wurde, und dies auch entsprechend kritisiert hatte, wird auch in der zweiten Runde in keiner Weise aktiv beteiligt. Lediglich die gesammelten Anträge, Namensnennungen der Fachgutachter sowie die Zusammenfassungen deren Voten zu den einzelnen Anträgen werden von der PBWU mitgeteilt. In Verbindung mit dem sich abzeichnenden Finanzierungsengpaß und in der Folge nur eingeschränkter Förderzusagen muß dies die Kritik an dem Förderverfahren der PBWU bzw. des Umweltministeriums verstärken.

Die durch den Finanzierungsengpaß in Verbindung mit der Prioritätensetzung am alpinen Forschungsschwerpunkt bedingten negativen Bescheide des Bayerischen Umweltministeriums vom 
Juni 1986 sind Auslöser und Bezugspunkt für den Wissenschaftlichen Beirat, seine Kritik an dem Vorgehen von Projektgruppe und Umweltministerium deutlich zu machen. Einige Beiratsmitglieder sind als Antragsteller selbst von den negativen Förderentscheidungen betroffen.

In einem von nahezu allen Beiratsmitgliedern unterzeichneten Schreiben an den Bayerischen Umweltminister wird an die mehrfach versicherte Aussage des Ministeriums erinnert, ,in erheblichem Rahmen ... einschlägige Forschung“ zu fördern. Dieser Rahmen könne für den behandelten Zeitraum auf eine Größenordnung von etwa 13 Mio. DM hochgerechnet werden. Auch sei ein Appell an die Mitglieder des Beirats ergangen, in den Instituten um sinnvolle Mitarbeit zu werben. Vor diesem Hintergrund werde das Vorgehen, Antragstellern zwar die grundsätzliche Förderungswürdigkeit ihrer Vorhaben mitzuteilen, aufgrund weiterer, erforderlicher Verhandlungen aber keine positive Förderentscheidung fällen zu können, „für unzumutbar gegenüber den antragstellenden Wissenschaftlern und für desavouierend für die Beiratsmitglieder" gehalten. Angesichts der hohen Aufwands in der Konzipierung und Planung wissenschaftlicher Forschung werde es als Pflicht betrachtet, ,angekündigte Versprechen einzulösen“. Verwaltungstechnisch bedingte Zeitspannen und hinhaltende Bescheide verschlimmerten die Situation und widersprächen der in Aussicht gestellten zügigen Abwicklung. „In betroffener Sorge um den Fortgang der Waldschadensforschung in Bayern" werde der Minister um umgehende Einberufung der PBWU-Gremien ersucht, um den weiteren Fortgang persönlich zu erläutern. Davon würden die unterzeichnenden Mitglieder des Beirats eine weitere Identifikation mit dem PBWU-Programm und damit den Verbleib im Gremium abhängig machen (i.Q.: Wissenschaftlicher Beirat, 11.7.1986).

Ein Beiratsmitglied wendet sich zusätzlich mit erheblicher Verfahrenskritik an den Vorsitzenden des Projektrats. Der Wissenschaftler bezieht sich dabei konkret auf die ihm mitgeteilte Förderabsage zu einem als grundsätzlich förderungswürdig eingestuften Forschungsantrag im Rahmen der Bayerischen Forschungsgruppe Forsttoxikologie (i.Q.: Wissenschaftlicher Beirat, 14.7.1986).

In der Antwort des Umweltministers wird darauf hingewiesen, daß bereits zahlreiche Vorhaben mit erheblichem Finanzaufwand bewilligt worden seien und mitsamt den Forschungsmitteln anderer Ressorts den hohen Stellenwert der Waldschadensforschung in Bayern anzeigten. Allerdings hätte die Ausschreibung vorerst nicht den gewünschten Erfolg hinsichtlich der Etablierung des alpinen Forschungsschwerpunktes erzielt. Einschlägige Forschungsinstitute müßten von der PBWU noch gezielt für die dortige Mitarbeit gewonnen werden. Daher sei es nicht möglich, alle wissenschaftlich wünschenswerten Anträge zu fördern, die zur Erreichung der Zielsetzung im Rahmenprogramm zudem nicht unbedingt erforderlich seien. Der Minister schließt mit der Hoffnung, der Wissenschaftliche Beirat würde auch weiterhin seine Fachkompetenz zur Verfügung stellen (i.Q.: Bayer. Umweltministerium, 13.8. bzw. 27.8.1986).

Eine Mitteilung der PBWU an die GSF-Geschäftsführung erhellt die Hintergründe zur aktuellen Fördermittelsituation am Umweltministerium (i.Q.: PBWU, 25.7.1986). So seien rund 1 Mio. DM, die eingeplant gewesen sei, unvorhergesehenerweise zugunsten einer anderen Maßnahme hausintern entzogen worden. Inzwischen habe sich die Lage jedoch etwas entspannt, da zusätzliche Mittel dem Projekt zugeführt werden könnten, wie aus dem Ministerium bekannt 
wurde. Damit könnten jedoch bei weitem nicht alle zur Förderung empfohlenen Anträge finanziert werden. Das Ministerium werde sich in den anstehenden Haushaltsverhandlungen bemühen, weitere Mittel in Höhe von mindestens 6 Mio. DM für die nächsten beiden Jahre zugewiesen zu bekommen. Zudem sei beabsichtigt, zwei Forschungsvorhaben für den Alpenstandort, die zwar noch nicht als Anträge vorlägen, aber ,,absolute Priorität“ besäßen, vorzuziehen. Die PBWU gibt in der Hausmitteilung zu bedenken, daß ohne zusätzliche Mittel bzw. die Bewilligung weiterer Vorhaben nur Ergebnisse produziert würden, deren inhaltliche Zusammenführung durch große Lücken erschwert, wenn nicht unmöglich wäre.

\subsection{Forschungspolitische Prioritätensetzung am alpinen Forschungsstandort}

Bei den beiden prioritär zu finanzierenden Vorhaben am geplanten alpinen Forschungsschwerpunkt handelt es sich zum einen um eine Grundlagenerhebung zur Vitalität der Waldbestände. Diese sollte eine Vorerkundung für die endgültige Standortsfestlegung und die Auswahl von Probeflächen darstellen. Das Vorgehen war insbesondere vom Wissenschaftlichen Beirat im Rahmen verschiedener Sitzungen zur Standortsauswahl gefordert worden. Da es sich überwiegend um eine Serviceleistung handelt, wird auf eine externe Begutachtung des Antrags verzichtet und im November 1986 eine entsprechende Bewilligung des Umweltministeriums ausgesprochen.

Der zweite, prioritär behandelte Antrag betrifft den Aufbau und Betrieb einer meteorologischluftchemischen Meßstation („Basisstation“) am alpinen Forschungsschwerpunkt Wank. Im Hinblick auf die im Mittelpunkt der Arbeiten stehende Luftschadstoff-Hypothese wird dieser Fördermaßnahme von Umweltministerium und PBWU zentrale Bedeutung und hohe zeitliche Priorität beigemessen. Die Maßnahme soll - nach einzelnen Gesprächen der PBWU mit potentiellen Antragstellern - von dem renommierten, standortsnah gelegenen Forschungsinstitut der Fraunhofer-Gesellschaft durchgeführt werden. Dessen neuer Leiter war nachträglich in das Gremium des Wissenschaftlichen Beirats berufen worden (i.Q.: Bayer. Umweltministerium, 18.2.1986), hatte umgehend seine Beteiligung am alpinen Forschungsstandort angeboten und die raschestmögliche Errichtung der Meßstation in Aussicht gestellt (s.a. i.Q.: PBWU, 15.5.1986).

Der Antrag zu Aufbau und Betrieb der Meßstation wird erst im Nachgang zur zweiten Begutachtungsrunde im Juli 1986 vorgelegt und behandelt. Er ist mit der PBWU und dem Umweltministerium abgestimmt (i.Q.: Antragsteller, 7.7.1986) und wird im Einzelverfahren drei externen Fachgutachtern zur schriftlichen Beurteilung vorgelegt (i.Q.: PBWU, 7.7., 14.7. und 21.7.1986). Auf eine eigene Stellungnahme der PBWU an die Gutachter wird nunmehr verzichtet. Die Gutachter befürworten trotz einiger inhaltlich-kritischer Anmerkungen insgesamt das Vorhaben der Meßstation am Wank.

Angesichts der Verstimmung des Wissenschaftlichen Beirats gegenüber dem Förderverfahren im PBWU-Projekt ist die Projektgruppe bemüht, das Gremium nun aktiver an der Förderentscheidung zu beteiligen. Sie versendet den Antrag mitsamt ihrer Zusammenfassung der Gutachtervoten zur Abstimmung an Wissenschaftlichen Beirat und Projektrat. Um baldige Mitteilung von Bedenken, Einwänden oder Anregungen wird gebeten, da das Bayerische Umweltmi- 
nisterium beabsichtige, ,das Vorhaben in einer dem Gutachtervotum entsprechend modifizierten Form in die Förderung aufzunehmen“ (i.Q.: PBWU, 5.9.1986).

Die Reaktion zahlreicher Mitglieder des Wissenschaftlichen Beirats ist heftig. Das Gremium hatte eben erst ohne Mitwirkungsmöglichkeit finanztechnisch begründete Ablehnungen förderungswürdiger Forschungsanträge hinnehmen müssen. Nun wird es mit der politischen Förderabsicht $\mathrm{zu}$ einem finanzaufwendigen Forschungsprojekt für einen konzeptionell und forschungsinhaltlich noch als unausgereift erachteten Standort Wank konfrontiert. Mitglieder des Wissenschaftlichen Beirats sehen die Eignung des geplanten Foschungsstandortes noch nicht als nachgewiesen an und wollen vor Errichtung der Meßstation erst Ergebnisse von Vorerkundungen abwarten (i.Q.: PBWU, 13.1.1986 und Wissenschaftlicher Beirat, 26.3.1986).

Zwei Mitglieder des Wissenschaftlichen Beirats legen überwiegend fachlich-kritische Anmerkungen vor. Weitere Beiratsmitglieder fordern dringend eine Grundsatzdiskussion zum Gesamtkonzept der alpinen Waldschadensforschung. Vorher könne über den vorgelegten Antrag nicht entschieden werden (i.Q.: Wissenschaftlicher Beirat, 12.9., 15.9. und 22.9.1986; PBWU, 22.9.1986). Der Vertreter des Kultusressorts im Projektrat sieht sich außerstande, ohne Kenntnis der einzelnen Gutachten und deren namentlichen Benennung sich zu dem Antrag zu äußern. Aufgrund der Zusammenfassung der Gutachtervoten durch die PBWU kämen Zweifel an der Förderungswürdigkeit des Vorhabens auf (i.Q.: Bayer. Kultusministerium, 1.10.1986).

Die PBWU benennt daraufhin den Gremien die Gutachter und legt deren Stellungnahmen vor. Die fachlichen Kritikpunkte seien dem Antragsteller zur Klärung mitgeteilt worden. Allerdings drängt auch die PBWU auf raschen Vertragsabschluß zum Aufbau der Meßstation, unter anderem um Zeitverluste zu vermeiden. Sie verweist darauf, daß die Grundkonzeption des Forschungsstandorts bereits im Rahmenprogramm niedergelegt und mehrfach gemeinsam diskutiert worden sei, der Standort selbst akzeptiert und die Lage der Meßstation auch von Beiratsmitgliedern als richtig und notwendig erkannt worden war. Auch wenn die Vorerkundungen noch nicht abgeschlossen seien, habe der Antragsteller bereits mit der Planung zur Errichtung der Station begonnen (i.Q.: PBWU, 5.11.1986). Das Umweltministerium unterstreicht gegenüber dem Kultusministerium die Notwendigkeit, den Auftrag nunmehr kurzfristig zu vergeben, „,da weiteres Zuwarten aus haushaltsrechtlichen Gründen zu einer Gefährdung des Gesamtprojekts führen würde“. Eine Diskussion im Wissenschaftlichen Beirat wäre zwar geplant gewesen, konnte aber aus Termingründen nicht realisiert werden (i.Q.: Bayer. Umweltministerium, November 1986 ohne genaue Datumsangabe).

Insgesamt trägt die aktive politische Durchsetzung des Vorhabens Meßstation am Forschungsstandort Wank dazu bei, die ohnehin angespannte Situation zwischen den Allianzen PBWU und Bayerisches Umweltministerium auf der einen Seite, Wissenschaftlicher Beirat und Kultusministerium auf der anderen Seite weiter zu belasten. In einem Schreiben an zwei seiner Kollegen bringt ein selbst von einer Antragsablehnung betroffenes Beiratsmitglied zum Ausdruck, über die PBWU-Angelegenheit ,weiterhin erbost“ zu sein (i.Q.: Wissenschaftlicher Beirat, 22.10.1986). Die höchste Förderpriorität am alpinen Forschungsschwerpunkt sei so vom Wissenschaftlichen Beirat nicht beschlossen worden. Es wird gemutmaßt, daß das PBWU-Projekt ein „Politikum“ mit einer PBWU-erfundenen „Vorzeige-Station“ sei und 
„nichts mit sinnvoller und gezielter Forschung zu tun“ habe. Zu überlegen sei, dem Umweltminister die Beteiligung im Beiratsgremium aufzukündigen, noch ehe auf der nächsten Gremiensitzung darüber debattiert würde.

Mit erheblicher Verspätung gegenüber der umweltministeriellen Ankündigung soll die nächste gemeinsame Sitzung der PBWU-Gremien am 28.11.1986 Möglichkeit zur Diskussion bieten. Die „,weitere Entwicklung des Projekts“ wird im Einladungsschreiben der PBWU als Tagesordnungspunkt vorgemerkt (i.Q.: PBWU, 17.11.1986). Der Vertrag zum Aufbau und Betrieb der Meßstation am Wank ist mit Förderbeginn 5.11.1986 jedoch bereits unter Dach und Fach.

\subsection{Aussprache in den PBWU-Gremien}

Die kritisierte Vorgehensweise des Bayerischen Umweltministeriums und der PBWU zur Forschungsförderung steht denn auch im Mittelpunkt der Sitzung der PBWU-Gremien vom 28.11.1986 (i.Q.: PBWU, 17.12.1986).

Der Leiter der PBWU bestätigt, daß aus der zweiten Begutachtungsrunde aufgrund von Finanzierungsschwierigkeiten bisher keiner der förderungswürdigen Anträge umgesetzt werden konnte. Zur „Grundausstattung“ des alpinen Forschungsschwerpunktes seien jedoch ein Vorerkundungsvorhaben zur Vitalität der Waldbestände am Wank und das Vorhaben „Basisstation" bewilligt worden.

Mitglieder des Gremiums rekapitulieren ihre an den Umweltminister gerichtete Kritik an der Behandlung der Antragsteller der zweiten Begutachtungsrunde. Das ,undurchsichtige und z.T. schwer verständliche“ Verfahren der Begutachtung und die fehlende Einflußnahmemöglichkeit des Gremiums auf die Strukturierung der bayerischen Waldschadensforschung werden beklagt. Letzeres werde gerade angesichts der Bewilligung des Vorhabens „Basisstation“ ohne Zustimmung des Wissenschaftlichen Beirats deutlich. Die bloße Abfrage schriftlicher Stellungnahmen entspräche nicht dem Diskussionsbedürfnis der Beiratsmitglieder. Eindringlich kritisieren zahlreiche Mitglieder die Bevorzugung von Vorhaben zum alpinen Forschungsschwerpunkt. Dies bedeutete eine Neuorientierung des Programms.

Die Vertreter des Bayerischen Umweltministeriums begründen die eingetretenen Verzögerungen und Zurückstellung förderungswürdiger Anträge mit dem bereits erwähnten Finanzmittelengpaß. Für die Zukunft werde die avisierte Gesamtfördersumme des Projekts in Höhe von ca. 30 Mio. DM jedoch auch weiterhin in Aussicht gestellt, womit die bislang zurückgestellten Forschungsanträge alsbald finanziert werden könnten. Die PBWU werde künftig davon Abstand nehmen, eigene Stellungnahmen zu den Anträgen zu verfassen, auch wenn dies hinsichtlich der Einpassung in das Rahmenprogramm durchaus für legitim gehalten werde. Weiterhin sollten den Mitgliedern des Projektrats künftig nicht die PBWU-Zusammenfassungen der Gutachtervoten, sondern die einzelnen Gutachten in anonymisierter Form übermittelt werden.

Den Vorwurf, den Beirat nicht rechtzeitig beteiligt und somit faktisch übergangen zu haben, versucht das Bayerische Umweltministerium mit nicht tolerierbaren Verzögerungen des Projekts zu entschärfen. Zudem habe im Grundsatz Einigkeit darüber bestanden, eine Basisstation vorrangig einzurichten. Der Vorhabenleiter des letztgenannten Projektes nimmt als neu er- 
nanntes Mitglied des Wissenschaftlichen Beirats an der Sitzung teil. Er führt Begründungen für den gewählten Standort ins Feld, schließt sich dem Zeitaspekt-Argument des Ministeriums an und stellt baldmöglichst erste Messungen in Aussicht.

Einlenkend bitten Mitglieder des Projektrats das Wissenschaftlergremium zum Abschluß der heftigen Diskussion, Verständnis und Nachsicht für gewisse Anlaufschwierigkeiten und Koordinationsprobleme aufzubringen, seine Aufgaben aber auch weiterhin wahrzunehmen. Der Beirat stellt die Entscheidung hierzu nach interner Beratung in Aussicht.

Für das weitere Vorgehen verbleibt auf der Sitzung kaum mehr Zeit. Auf eine erneute Programmausschreibung solle verzichtet werden. Die PBWU beabsichtige vielmehr, zur Erweiterung des alpinen Forschungsprogrammes ,die Leiter laufender Vorhaben zusammenzuführen, um so neue Vorhaben zu identifizieren“. Hierbei werde von einigen Beiratsmitgliedern allerdings eine Entscheidungsgruppe bzw. ein Programmleiter nach dem Vorbild der Bayerischen Forschungsgruppe Forsttoxikologie vermißt.

Ein Mitglied des Wissenschaftlichen Beirats, welches zum Sprecher des Gremiums bestimmt worden war, teilt dem Projektratsvorsitzenden das Ergebnis der anschließenden internen Beratung mit (i.Q.: Wissenschaftlicher Beirat, 22.12.1986). Die weitere Mitarbeit wird zwar zugesichert. Gleichzeitig werden aber Vorschläge ,für eine bessere Verständigung zwischen Forschungsbeirat und Projektrat in der Forschungssteuerung " unterbreitet. Diese sehen vor, daß

- ein Beiratsmitglied bei den Projektratssitzungen anwesend sein und die wissenschaftliche Seite vertreten solle,

- ein Beiratsmitglied Einsicht in die Vorbegutachtung durch die PBWU haben und die Interpretation der Gutachten durch die PBWU überprüfen solle (es sei denn, das Beiratsmitglied sei gleichzeitig Antragsteller) und

- die PBWU eine präzisere Aufgabenbeschreibung der Gremien erarbeiten solle.

Die Vorgehensempfehlungen werden hinsichtlich der Bewilligung von Anträgen gegenüber der PBWU konkretisiert (i.Q.: Wissenschaftlicher Beirat, 23.1.1987):

- Neuanträge seien grundsätzlich zurückzustellen, bis der bestehende Antragsstau abgebaut sei. Dort hätten eindeutig positiv begutachtete Anträge höchste Priorität.

- Positiv begutachtete Folgeanträge zu laufenden Arbeiten sollten Vorrang gegenüber Neuanträgen haben, ,um gute Arbeit nicht zu unterbrechen“.

- Nicht eindeutig positiv beurteilte Anträge sollten dem Wissenschaftlichen Beirat zur Diskussion und zur Prioritätensetzung sowie zur Steuerung der Forschungsrichtung vorgelegt werden.

Mit diesen Empfehlungen des Wissenschaftlichen Beirats zum Förderverfahren im PBWUProjekt kann eine erste Phase der Programmimplementation abgegrenzt werden. Trotz Gründungsschwierigkeiten wird über die Durchführung zweier Begutachtungsrunden zu eingegangenen Forschungsanträgen der Forschungsprozeß im PBWU-Projekt in Gang gesetzt. Der Auftraggeber und die Projektgruppe dominieren den Verfahrensgang, das wissenschaftliche Beratungsgremium wird verfahrensmäßig weitgehend übergangen. Dies berechtigt den Beirat 
zu Kritik, die mit Forderungen verknüpft werden kann, künftig stärker am Förderverfahren beteiligt zu werden.

\subsubsection{Theorieorientiertes Zwischenergebnis}

\subsection{Zugang der Forscher zum Forschungsprogramm}

Das Rahmenprogramm der PBWU, in das sich die einzureichenden Anträge einzupassen haben, wird an alle einschlägigen Forschungsinstitutionen Bayerns ausgeschrieben. Der Hinweis auf das Hauptentscheidungskriterium, nämlich der Beitrag zur Ursachenklärung zum Thema Waldschäden, unterstreicht die vom politischen Auftraggeber beabsichtigte, problemorientierte Forschung. Gleichzeitig soll der wissenschaftlichen Qualität Rechnung getragen werden, indem auf die fachliche Selektion der Anträge unter Einbeziehung externer Gutachter hingewiesen wird. Damit wird die kompromißhafte Absicht deutlich, wissenschaftlich hochwertige Forschung unter dem Aspekt angewandter Problemlösung zu fördern.

Aufgrund der offenen Formulierung und breiten Ausschreibung des Rahmenprogramms ist allerdings zu erwarten, daß eher ein Strauß heterogener Einzelanträge vorgelegt werden wird als ein zielgerichtetes, an dem konkreten Forschungsthema orientiertes und aufeinander abgestimmtes Set von Forschungsanträgen. Dies betrifft auch den einzigen neu einzurichtenden Forschungsschwerpunkt im bayerischen Alpenraum. Das generelle Zutrittskriterium zum PBWU-Projekt, sich in das Rahmenprogramm einzupassen, kann leicht erfüllt werden. Die unterstellte Faktorenvielfalt und deren komplexes Zusammenwirken bei der Verursachung von Schäden, die Akzeptanz grundlagenorientierter neben angewandter Forschung sowie die Offenheit gegenüber im Rahmenprogramm unberücksichtigten Forscherideen erleichtern es Antragstellern ganz verschiedener Fachdisziplinen, Beiträge zur Ursachenklärung in Aussicht zu stellen.

Weiterhin sind Antragsteller begünstigt, die bereits im Rahmen anderweitiger Forschungsprogramme Waldschadensforschung betreiben. Das Rahmenprogramm der PBWU läßt dies explizit $\mathrm{zu}$, die Formulierung ist rascher und damit weniger aufwendig als bei Neuanträgen zu bewerkstelligen, bestehende Forschungslücken in dem bereits bearbeiteten Forschungsgebiet könnten über Vorhabenfinanzierung aus dem PBWU-Projekt zu schließen versucht werden. Bereits bearbeitete Fragestellungen fortzuführen, ist zudem effizienter einzuschätzen als das Aufnehmen neuer Fragestellungen. Letztere bergen das Risiko ungewisser Ergebnisse. Die inzwischen erarbeitete, fachliche Expertise einer bereits etablierten Arbeitsgruppe läßt für den Auftraggeber hingegen eine vergleichsweise hohe Erfolgswahrscheinlichkeit der Forschung [s.a. KROTT 1994a, S. 128 ff] und damit bessere Chancen auf positive fachliche Begutachtung erwarten.

Allerdings ist der Vorteil, auf bereits bearbeitete Forschungsfelder aufzubauen, häufig mit dem Nachteil verbunden, die Expertise auf das „Neuland“ des Objektes Wald übertragen zu müssen. Insbesondere nichtforstliche Forschergruppen, die sich nun an der Waldschadensforschung beteiligen wollen, müssen in ihren Arbeitsmethoden und bisher bearbeiteten Objekten oder Modellpflanzen auf das neue Objekt Wald bzw. Kompartimente von Waldbäumen umstellen. 
Hierfür ist meist erheblicher, Grundlagen schaffender Entwicklungsbedarf erforderlich, der von den Forschern leicht unterschätzt wurde und den Forschungserfolg mit Unsicherheiten belastet. Die insgesamt hohe Legitimation der unter hohem politischen Handlungsdruck stehenden Waldschadensforschung kann diesen Nachteil jedoch überwinden und gestattet einer Vielzahl von Forschungsvorhaben, im Rahmen politisch beabsichtigter, problemlösungsorientierter Waldschadensforschung vorab methodische Grundlagenarbeiten durchzuführen.

Somit ist kaum überraschend, daß der Großteil der vorgelegten Forschungsanträge die Fortführung bereits eingeschlagener Forschungsrichtungen in etablierten Arbeitsgruppen vorsieht, häufig eine Verstärkung bestehender Forschungsschwerpunkte darstellt und zudem weitgehend labororientierter Grundlagenforschung zuzuordnen ist. Dies betrifft insbesondere Anträge zur Schadstoff-Entgiftung und zur Cuticula-Forschung.

Auch die Vorlage mehrerer Anträge als Sammelantrag kann die Chancen der Bewilligung erhöhen. Dies bietet sich insbesondere für bereits miteinander kooperierende Forschergruppen an. Dort wird die erwünschte inhaltliche und zeitliche Abstimmung aufeinander sowie die Kooperation verschiedener, u.U. interdisziplinärer Arbeitsgruppen, bereits durch die Antragsteller selbst vermittelt.

Die Projektgruppe verzichtet auf die Zusammenarbeit mit ihrem wissenschaftlichen Beratungsgremium. Sie nimmt eine fachliche Bewertung der Anträge und die grobe Einordnung in das Rahmenprogramm selbst vor und bringt dies - präjudizierend - in die externe Begutachtung ein. Damit geht zwar eine potentiell hohe Steuerungswirkung von der Projektgruppe aus. Die geringe formale Bindungswirkung der Empfehlungen gegenüber dem Votum der Gutachter schwächt das Steuerungspotential jedoch ab. Insgesamt bestehen damit nur geringfügige fachliche wie politische Barrieren und Selektionsmechanismen bei der Zulassung von Forschungsangeboten zu dem Projekt. Eine um so größere Bedeutung kommt allerdings der Antragsselektion im Rahmen der fachlichen Begutachtung zu.

Den Antragstellern muß dieses Verfahren gelegen kommen. Je geringer die Vorgaben zur Beteiligung am Projekt sind, desto mehr können bisherige Forschungsfragestellungen, bestehende thematische oder regionale Forschungsverbünde weiterbetrieben und nun über das neue Förderprogramm finanziert werden. Gerade die rasche Vorlage voll entwickelter Anträge, die ohne Vorerhebungen, Pilotphasen etc. auskommen, spricht dafür, daß der Themenbezug zum neuen Forschungsprogramm rein optisch hergestellt und das bisherige Arbeitsgebiet weitergeführt werden kann [HILL \& RIESER 1983, S. 352].

Selbst Fachgutachter bemängeln im Rahmen der ersten Begutachungsrunde, daß einzelne Antragsteller ihre Arbeitsrichtung nur geringfügig modifiziert hätten, um der Ausschreibung gerade noch gerecht zu werden. Damit würde zwar ein Beitrag zur wissenschaftlichen Erkenntnisproduktion, weniger jedoch zur angewandten, problemlösungsorientierten Waldschadensforschung geliefert (i.Q.: Fachgutachter, 22.10.1985). Der Etablierung neuer Forschungsthemen ist dieses inkrementalistische Verhalten abträglich.

Somit wird das PBWU-Projekt mit einer Fülle von fachlich breit gefächerten Forschungsanträgen gestartet, welche zwar dem hohen politischen Handlungsdruck des Auftraggebers zur Pro- 
grammumsetzung im allgemeinen Rechnung tragen. Dabei werden jedoch überwiegend bereits eingeschlagene Forschungsrichtungen fortgeführt. Diese Antragsstruktur bietet zwar Aussicht auf vergleichsweise hohen Forschungserfolg in den jeweiligen Beiträgen, läßt sich jedoch in inhaltlicher Hinsicht kaum zu einem stringenten, problemlösungsorientierten Gesamtkonzept der PBWU-Waldschadensforschung zusammenfügen.

\subsection{Kompromißhafte Interessenwahrung bei Politik und Wissenschaft}

Die Durchführung der ersten beiden Antragsrunden im PBWU-Projekt der Jahre 1985 und 1986, die bereits zu einem Förderumfang von knapp 10 Mio. DM führen, erfolgt ohne aktive Beteiligung des Wissenschaftlichen Beirats. Der alpine Forschungsschwerpunkt wird gegen den Willen des Beratungsgremiums, bzw. ohne dessen Empfehlungen und Auflagen zu berücksichtigen, etabliert.

Aus der ersten, hinsichtlich der Programmrichtung allerdings entscheidenden Phase der Programmimplementation im PBWU-Projekt geht dennoch die Politik nicht als alleiniger Gewinner hervor. Die politischen Vertreter - respektive der Finanzgeber Bayerisches Umweltministerium und die im Auftrag handelnde Koordinierungsstelle PBWU - können mit der Bewilligung zahlreicher Forschungsvorhaben und Bereitstellung umfangreicher Fördermittel generell das Forschungsprogramm implementieren. Damit kann dem politischen Auftrag, die Waldschadensforschung zu verstärken, entsprochen werden. Doch auch die Wissenschaftsvertreter im PBWU-Projekt können ihre Interessen weitgehend durchsetzen. Insgesamt war unter - wenn auch nur unzureichender - Beteiligung des Beirats an Grundsatzfragen ein Rahmenprogramm verabschiedet und zur Grundlage der Programmimplementation gemacht worden, welches zwar auf problemorientiert-angewandte Forschungsförderung ausgelegt ist. Bei der gegeben Programmbreite und -offenheit läßt es aber einen erheblichen Anteil an Forschungsbeiträgen $\mathrm{zu}$, deren Bearbeitung mehr im Interesse der Wissenschaftler als des politischen Auftraggebers steht. Gerade im Rahmen der beiden ersten Antrags- bzw. Begutachtungsrunden werden zahlreiche Fördermaßnahmen getätigt, die sich stärker auf anderweitig bereits etablierte Programme, bestehende Forschungsrichtungen und überwiegend grundlagenorientierte Forschung beziehen als die beabsichtigten Schwerpunkte im PBWU-Programm.

Der Wissenschaftliche Beirat erkennt den potentiellen Vorwurf, sich durch starke Einflußnahme auf das Förderverfahren selbst mit Fördermitteln im Projekt bedienen zu können und gibt an, um Zurückhaltung und Neutralität bemüht zu sein. Seine generelle Interessenpositionen konnten im Rahmenprogramm jedoch verankert werden, Arbeitsgruppen von Mitgliedern des Beirats können sich, auch ohne aktiv Einfluß auf das Förderverfahren zu nehmen, mit mehreren Forschungsvorhaben am PBWU-Projekt beteiligen [STEUER 1997, S. 61ff].

Diese kompromißhafte Interessenwahrung auf beiden Seiten - Wissenschaft und Politik - geht jedoch zulasten der Effektivität des Forschungsprogrammes. Die thematische wie finanzielle Aufsplitterung der Fördermaßnahmen in politisch beabsichtigte, angewandte Forschung und wissenschaftlich beabsichtigte Grundlagenforschung führt dazu, daß bei begrenzten finanziellen Kapazitäten weder der eine noch der andere Sektor umfassend und abschließend bearbeitet werden kann. 


\subsubsection{Weitere Programmimplementation und Projekt-Neuordnung}

Mit den ersten beiden Begutachtungsrunden 1985/86 konnten eine Reihe von Forschungsvorhaben vom Bayerischen Umweltministerium in die Förderung genommen und der neue alpine Forschungsschwerpunkt Wank gegründet werden. Dessen politische Bevorzugung gegenüber anderen Forschungsvorhaben bei sich gleichzeitig andeutenden Finanzierungsengpä,ssen sowie das weitgehende Übergehen des wissenschaftlichen Beratungsgremiums, hatten jedoch zu einer Vertrauenskrise im PBWU-Projekt geführt. Mit der Zusicherung des auftraggebenden Umweltressorts, den Beirat stärker am Förderverfahren zu beteiligen und die Finanzierung der Waldschadensforschung im PBWU-Projekt zu sichern, konnte Rücktrittsdrohungen des Gremiums begegnet werden.

Die weitere Umsetzung des PBWU-Forschungsprogramms im Jahr 1987 steht jedoch nach wie vor unter der Gegebenheit knapper Fördermittel. Eine zum 1.3.1987 geänderte Geschäftsverteilung am Ministerium führt nicht nur zu einem personellem Wechsel des Vorsitzenden im Projektrat (i.Q.: Bayer. Umweltministerium, 17.3.1987), sondern auch zu Finanzierungsschwierigkeiten, wie anläßlich der Projektratssitzung vom 29.1.1988 ausgeführt wird (i.Q.: $P B W U$, 29.1.1988). Maßnahmen des technischen Umweltschutzes müßten zulasten der vorgesehenen Mittel für die Waldschadensforschung finanziert werden.

Die Mittelknappheit mindert die Möglichkeiten, das Forschungsprogramm inhaltlich auszuweiten und abzurunden, die vom Beirat geforderten Änderungen am Förderverfahren umzusetzen (s. i.Q.: PBWU, 28.11.1986), eine Basis vertrauensvoller Zusammenarbeit zwischen Politik und Wissenschaft zu schaffen und damit das PBWU-Projekt zu stabilisieren. Bezeichnend hierfür ist, daß im Jahr 1987 keine einzige Sitzung der PBWU-Gremien stattfindet. Dennoch bemühen sich PBWU bzw. Bayerisches Umweltministerium, den Kritikpunkten des Beiratsgremiums zu entsprechen und die in Aussicht gestellten Fördermaßnahmen umzusetzen.

\subsubsection{Förderverfahren zu Forschungsanträgen des Jahres 1987}

\subsection{Einreichung von Forschungsanträgen}

Parallel zur Aufarbeitung der zurückgestellten Anträge aus der zweiten Begutachtungsrunde werden im Verlauf des Jahres 1987 mehr als 20 Forschungsanträge mit einem gesamten Antragsvolumen von knapp 10 Mio. DM bei der PBWU behandelt. Während es sich in den meisten Fällen um Neuanträge handelt, werden gegen Ende des Jahres bereits einzelne Fortsetzungsanträge zu Vorhaben der ersten Bewilligungsphase vorgelegt.

Es beteiligen sich fast ausnahmslos in der Waldschadensforschung bereits etablierte bzw. am PBWU-Programm beteiligte Forschungsinstitutionen (s. Tabelle 8). Allein die GSF und das Fraunhofer-Institut in Garmisch-Partenkirchen legen im Lauf des Jahres 1987 je 4 Anträge vor. Ähnlich stark sind Arbeitsgruppen der beiden Münchner Universitäten sowie der Universität Würzburg vertreten. Als ,neue“ Antragsteller treten lediglich das Max-Planck-Institut für Biochemie bei München sowie die Universität Tübingen auf. In einigen Fällen wurden Arbeitsgruppen direkt von der PBWU oder dem Umweltministerium zur Antragstellung für bestimmte Fragestellungen aufgefordert bzw. beraten (i.Q.: PBWU, 2.3. und 29.4.1987). 


\begin{tabular}{|l|c|c|}
\hline Antragsteller & Anzahl Anträge & $\begin{array}{c}\text { Kosten } \\
\text { (Mio. DM) }\end{array}$ \\
\hline Uni. München & 6 & 2,4 \\
\hline TU München & 4 & 0,7 \\
\hline Uni. Würzburg & 3 & 0,3 \\
\hline Uni. Bayreuth & 1 & 0,2 \\
\hline GSF Neuherberg & 4 & 3,0 \\
\hline FhG-IFU Garmisch-P. & 4 & 2,9 \\
\hline sonstige & 2 & 0,3 \\
\hline Summe: & $\mathbf{2 4}$ & $\mathbf{9 , 8}$ \\
\hline
\end{tabular}

Tab. 8: In 1987 behandelte Forschungsanträge und Antragsteller (eigene Erhebung)

Fachlich stehen wiederum pflanzenphysiologische und biochemische Forschungsarbeiten im Mittelpunkt (s. Tabelle 9). Zahlreiche Anträge sehen wirkungsmechanistische, z.T. grundlagenorientierte Untersuchungen im Zusammenhang mit Schadstoffeinfluß auf die Pflanze vor.

\begin{tabular}{|l|c|c|}
\hline Fachdisziplin & Anzahl Anträge & $\begin{array}{c}\text { Kosten } \\
\text { (Mio. DM) }\end{array}$ \\
\hline Luftchemie / Strahlung & 2 & 1,6 \\
\hline Biochemie & 4 & 1,5 \\
\hline Physiologie & 13 & 4,1 \\
\hline Bodenkunde & 2 & 1,5 \\
\hline $\begin{array}{l}\text { Sonstige (Waldzustand, } \\
\text { Pflanzenanzucht) }\end{array}$ & 3 & 1,1 \\
\hline Summe: & $\mathbf{2 4}$ & $\mathbf{9 , 8}$ \\
\hline
\end{tabular}

Tab. 9: In 1987 behandelte Forschungsanträge und Fachdisziplinen (eigene Erhebung)

Entsprechend der Prioritätensetzung und der z.T. erfolgten Kontaktaufnahme mit Antragstellern durch die PBWU überwiegen nunmehr bei weitem Anträge, die den alpinen Forschungsstandort Wank betreffen (s. Tabelle 10). Neben z.T. grundlagenorientieren Fragestellungen (z.B. Eintrag von organischen Komponenten, physiologische oder biochemische Wirkungsmechanismen bzw. Belastungsparameter) haben weitere, durch die PBWU angeregte Anträge überwiegend Dienstleistungscharakter (z.B. Kronenzustandserfassung, Nährstoffversorgung, bodenkundliche Untersuchungen). Sie sollen zur Vervollständigung des dortigen Forschungsprogramms beitragen. 


\begin{tabular}{|l|c|c|}
\hline Standort & Anzahl Anträge & $\begin{array}{c}\text { Kosten } \\
\text { (Mio. DM) }\end{array}$ \\
\hline Kalkalpen (Wank) & 13 & 7,4 \\
\hline Fichtelgebirge & 4 & 0,5 \\
\hline Bayer. Wald & 2 & 0,5 \\
\hline $\begin{array}{l}\text { Labor od. nicht zuorden- } \\
\text { bar }\end{array}$ & 5 & 1,4 \\
\hline Summe: & $\mathbf{2 4}$ & $\mathbf{9 , 8}$ \\
\hline
\end{tabular}

Tab. 10: In 1987 behandelte Forschungsanträge und Forschungsstandorte (eigene Erhebung)

Ein Stichtag zur Einreichung der Anträge des Jahres 1987 besteht nicht, von einer weiteren Antragsrunde kann daher nicht explizit gesprochen werden. Über die verfügbaren Fördermittel liegen keine konkreten Zahlen vor, abgesehen von der Aussage Ende 1986, wonach die vorgesehene Gesamtfördersumme in Höhe von 30 Mio. DM für das PBWU-Projekt auch weiterhin gültig sei (i.Q.: $P B W U, 28.11 .1986)$. Aufgrund der bisherigen Finanzierungsengpässe muß auch in 1987 mit finanziellen Restriktionen gerechnet werden, zumal zurückgestellte Anträge das Budget belasten. Noch vor etwaigen Neubewilligungen beträgt der verpflichtete Mittelbedarf für das Jahr 1987 bereits knapp 6 Mio. DM (i.Q.: PBWU, 7.4.1987).

\subsection{Fallweise schriftliche Begutachtung von Forschungsanträgen}

Die eingegangenen Anträge werden fallweise nach Sichtung durch die PBWU einer schriftlichen Begutachtung durch externe Fachwissenschaftler unterworfen. Bei reinen Serviceleistungen ohne Forschungscharakter (z.B. Waldzustandserfassung, Infrastruktur am Forschungsstandort Wank) hält die PBWU eine Begutachtung für nicht erforderlich.

Zur Auswahl der Gutachter greift die PBWU auf den inzwischen aufgebauten Gutachterstamm zurück. Dieser muß aus fachlichen, terminlichen Gründen oder selbsterklärter persönlicher Beziehung zwischen Gutachter und Antragsteller jedoch in einigen Fällen erweitert werden. Pro Antrag werden schriftliche Stellungnahmen von meist drei Fachgutachtern eingeholt.

Die vom Wissenschaftlichen Beirat geforderten Verfahrensänderungen befolgend, faßt die PBWU die Einzelgutachten zusammen und legt sie anonymisiert mitsamt den Forschungsanträgen dem Projektrat vor. Einzelne Anträge, die nach Einschätzung der PBWU ein uneinheitliches Begutachtungsergebnis aufweisen, werden auch dem Sprecher des Wissenschaftlichen Beirats zur Stellungnahme übermittelt (s. z.B. i.Q.: PBWU, 28.1. und 4.3.1987). Die Schreiben, mit denen die Unterlagen zur Stellungnahme übersandt werden, enthalten meist auch eigene Einschätzungen der PBWU zu den Anträgen. Weiterhin wird das geplante weitere Vorgehen für den Fall, daß keine Einwände erhoben würden, bereits in Aussicht gestellt (z.B. (i.Q.: $P B W U$, 28.1. und 7.11.1987).

Insgesamt werden aufgrund des bestehenden Antragsüberhangs im Jahr 1987 allerdings nur sehr wenige Anträge bewilligt. Neben einem Fortsetzungsantrag werden lediglich reine Serviceleistungen finanziert, die für die Etablierung des Forschungsschwerpunktes Wank von der PBWU für unverzichtbar gehalten werden (Waldzustandserhebung und Infrastrukturmaßnahmen). Die positiv begutachteten Neuanträge werden vom Umweltministerium zur Entschei- 
dung auf die nächste Gremiensitzung zu Beginn 1988 zurückgestellt (i.Q.: PBWU, 17.12.1987 und 21.1.1988).

\subsection{Weitere Kritik der PBWU-Gremien am Förderverfahren}

Daß ein Fortsetzungsantrag trotz positiven Begutachtungsergebnisses wegen Finanzierungsproblemen zurückgestellt worden sei, wird im Oktober 1987 vom Sprecher des Wissenschaftlichen Beirats zum Anlaß nach wie vor bestehenden Verfahrenskritik genommen (i.Q.: Wissenschaftlicher Beirat, 19.10.1987). In der Zurückstellung werde wiederum eine unberechtigte Bevorzugung von Wank-Anträgen gegenüber vereinbarungsgemäß prioritär zu behandelnden Fortsetzungsanträgen gesehen; dies mache eine Beiratssitzung erforderlich.

Auch vom Kultusressort wird Verfahrenskritik geäußert. Es nimmt Bezug auf die PBWU, die den Projektrat zu vier ,ausnahmslos positiv“ beurteilten Anträgen, denen die PBWU hohe Priorität zumißt, schriftlich um Zustimmung bittet (i.Q.: PBWU, 7.11.1987). Das Kultusministerium interveniert mit der Begründung, daß ,eine Äußerung ... ohne Kenntnis der Gutachten sowie der Namen der Gutachter nicht zumutbar und auch nicht möglich“ sei. Weitere Unregelmäßigkeiten werden der PBWU angelastet. Sie betreffen die unvollständige Antragsübermittlung an den Beiratssprecher, gerade im Fall finanzaufwendiger Anträge, sowie eigenmächtige Gutachteninterpretation (i.Q.: Bayer. Kultusministerium, 11.12.1987). Die PBWU versucht, die Kritik mit Hinweis auf die getroffenen Verfahrensvereinbarungen und mit Verständnisproblemen zu entkräften (i.Q.: Bayer. Kultusministerium, 16.12.1987) .

Neben der Verfahrenskritik besteht im PBWU-Projekt nach wie vor das Problem der Finanzmittelknappheit beim projektfinanzierenden Umweltministerium. Dies veranlaßt die GSFGeschäftsführung zu einer schriftlichen Reaktion. In enger Abstimmung mit der Projektgruppe wird der Amtschef des Bayerischen Umweltministeriums gebeten, über die künftige ,finanzielle Ausgestaltung der Waldschadensforschung ... Klarheit zu schaffen“, um damit eine „sinnvolle Forschungsplanung“ zu sichern. Ausführlich wird dabei die bisherige, durch Zurückstellungen geprägte Fördersituation rekapituliert. Auf die derzeit vorliegenden, förderungswürdigen Anträge sowie weitere erforderliche Forschungsmaßnahmen wird verwiesen, für die nach inoffizieller Mitteilung aus dem Ministerium für 1988 keine zusätzlichen Fördermittel bereit stünden. Es wird zu bedenken gegeben, daß die sich aufgrund der Mittelknappheit abzeichnenden Lükken im Forschungsprogramm eine sinnvolle Ergebnisinterpretation verhinderten und damit den Erfolg des Gesamtprojekts in höchstem Maße gefährdeten. Eine Forschungsplanung und Kooperation der Wissenschaftler sei auf dieser Basis kaum möglich. Als ,verlängerter Arm des Ministeriums" fiele in diesem Zusammenhang ein Teil des Unmuts und der Verstimmung auf die GSF zurück, von welcher Schaden abgewendet werden müsse (i.Q.: GSF, 22.10.1987).

In seiner Antwort bekräftigt das Ministerium die Zusicherung der Staatsregierung, auch weiterhin für die Waldschadensforschung und deren Koordinierung ausreichend Finanzmittel zur Verfügung zu stellen. Es verweist darauf, daß 1988 für laufende Vorhaben bereits knapp 6 Mio. DM eingeplant seien und bekundet sein Bemühen, zusätzliche Mittel für die Bewilligung weiterer Anträge freizumachen (i.Q.: Bayer. Umweltministerium, 21.1.1988). 


\subsubsection{Förderverfahren zu Forschungsanträgen des Jahres 1988}

Der lange Zeitverlauf seit der letzten Sitzung der PBWU-Gremien am 28.11.1986, die unbefriedigende Finanzmittelsituation im Jahr 1987, die Kritik am Förderverfahren und die anstehenden Förderentscheidungen zu zahlreichen, inzwischen aufgelaufenen Forschungsanträgen machen eine klärende Sitzung der PBWU-Gremien überfällig (s.a. i.Q.: Wissenschaftlicher Beirat, 19.10.1987). Zur Sitzung am 29.1.1988 werden der Projektrat und der Sprecher des Beirats kurzfristig eingeladen (i.Q.: $P B W U, 26.1 .1988)$. Bereits zwei Monate später, am 29.3.1988, findet eine weitere gemeinsame Sitzung der PBWU-Gremien statt.

\subsection{Fallweise schriftliche Begutachtung und Förderentscheidungen zu vorliegenden Anträgen}

Auf der Sitzung am 29.1.1988 nimmt der neue, gesprächsleitende Vertreter des Umweltministeriums im Projektrat ausführlich zur Fördersituation Stellung (i.Q.: PBWU, 29.1.1988). Aktuell würden 28 Forschungsvorhaben mit einem Mittelumfang von ca. 10 Mio. DM gefördert. Die bestehenden Finanzierungsschwierigkeiten werden vom mit unvorhergesehenen Maßnahmen des technischen Umweltschutzes sowie dem Wechsel der administrativen Zuständigkeit für die PBWU im Ministerium begründet. Für die Zukunft werde jedoch die Verbesserung der Mittelsituation und Kontinuität in der Bereitstellung erwartet.

Allerdings könnten für 1988 nur in begrenztem Umfang weitere Fördermittel zur Verfügung gestellt werden. Dabei ist über 21 Forschungsanträge zu beraten bzw. zu entscheiden, die sich zudem in unterschiedlichen Stadien des Verfahrensablaufes (bewilligungsreif, begutachtet, beantragt) befinden. Die Antragsstruktur entspricht weitgehend der vorangegangener Begutachtungsrunden. Es handelt sich überwiegend um die bisher bereits erwähnten AntragstellerInstitute, Anträge zur pflanzlichen Biochemie und Physiologie dominieren, knapp die Hälfte der Anträge betreffen den Forschungsschwerpunkt Wank. Insgesamt weisen die Anträge ein Gesamtmittelvolumen von rund 11 Mio. DM aus, allein für 1988 beläuft sich die Antragssumme auf knapp 5 Mio. DM. Die Anträge selbst sowie die PBWU-Zusammenfassungen zu bereits eingeholten Fachgutachten sind den Sitzungsteilnehmern bekannt.

Die Anträge werden im einzelnen behandelt. Für acht zum Teil finanzintensive Anträge, für die ein insgesamt positives Gutachtervotum vorliegt, wird eine positive Förderungsentscheidung getroffen. In den meisten Fällen können Bewilligungen bereits im April/Mai 1988 ausgesprochen werden. Die Entscheidungen zu den weiteren Anträgen werden ,zurückgestellt“ (i.Q.: PBWU, 29.1.1988, Anlage 2).

Auf der Sitzung vom 29.3.1988 stehen somit noch 14 Anträge mit einem Gesamtvolumen von rund 8 Mio. DM - für 1988 allein rund 3 Mio. DM - zur Behandlung an (i.Q.: PBWU, 29.3.1988). Die geringfügigen, verfügbaren Restmittel in Höhe von 0,7 Mio. DM erlauben lediglich, in 1988 einige wenig finanzaufwendige Fortsetzungsvorhaben zu bewilligen. Weiterhin kündigt der Sitzungsleiter an, durch interne Mittelumschichtungen ein finanziell aufwendiges, aber für wichtig erachtetes Forschungsangebot eines Beiratsmitgliedes am Wank zu ermöglichen. Die Finanzmittelsituation im Projekt stellt einen wesentlichen Kritikpunkt des Wissenschaftlichen Beirats dar. Während bei einem unterstellten Verwaltungskostenanteil der PBWU 
von ca. $3 \%$ für die ersten drei Projektjahre Fördermittel in Höhe von 50 - 60 Mio. kalkuliert worden waren, seien bislang lediglich ca. 12 Mio. DM abgeflossen. Der Sitzungsleiter rückt die Erwartungen der Wissenschaftler dahingehend zurecht, daß die gesamte Forschungsförderung des Freistaats Bayern zum Thema etwa eine derartige Größenordnung erreiche.

Bezogen auf die abfließenden Fördermittel des PBWU-Projektes stellt das Jahr 1988 mit etwas über 5 Mio. DM dennoch das Maximum und damit den Kulminationspunkt der PBWUForschungsförderung dar [s. REUTHER et al. 1993, Anhang 5; STEUER 1997, S. 15].

\subsection{Kritische Diskussion des Förderverfahrens in den PBWU-Gremien}

Neben der Entscheidung über vorliegende Forschungsanträge stellt die mit dem praktizierten Förderverfahren im PBWU-Projekt verbundene Kritik aus den Gremien einen wichtigen Tagesordnungspunkt der Sitzung vom 29.1.1988 dar (i.Q.: PBWU, 29.1.1988). Das Umweltressort sowie die Geschäftsführung der GSF bemühen sich um eine Aussprache zu Problemen bzw. Verstimmungen im PBWU-Projekt. Die GSF fordert den Sprecher des Wissenschaftlichen Beirats ausdrücklich zur Kritik an der PBWU auf. Dieser verweist in erster Linie auf die nach wie vor ,unzureichende Beteiligung an Grundsatzentscheidungen zur Arbeit der PBWU“. Weitere Kritik müsse allerdings erst intern besprochen werden. Der Sprecher des Beirats informiert seine Kollegen über die relevanten Ergebnisse der Sitzung. Seinem Eindruck nach sei der neue Projektratsvorsitzende ,gewillt, dem Beirat ein stärkeres Gewicht zu geben“ (i.Q.: Wissenschaftlicher Beirat, 1.2.1988).

Die „künftige Beteiligung des Wissenschaftlichen Beirats in der Waldschadensforschung“ entwickelt sich auf der folgenden Sitzung am 29.3.1988 denn auch zum zentralen Tagesordnungspunkt (i.Q.: PBWU, 29.3.1988). Als Ergebnis einer internen Vorbesprechung kritisiert der Wissenschaftliche Beirat, in der Vergangenheit zu wenig bei der Festlegung von Zielsetzung und Schwerpunkten, Ergebnisbewertung, Maßnahmenformulierung und Beratung von Projektrat und Projektgruppe beteiligt worden zu sein. Auch erschiene der Informationsfluß zwischen den Gremien und der PBWU verbesserungswürdig. Man habe sich bislang bewußt zurückgehalten, um nicht in den Verdacht der Selbstbedienung bzgl. Forschungsmitteln zu geraten und um möglicherweise entstehende Interessenkonflikte als Antragsteller und gleichzeitig Beiratsmitglied zu vermeiden.

Um Mithilfe gebeten fordere der Beirat nun ein ,neues Procedere im Bereich der Forschungsförderung“. Danach sollten unter Mitwirkung des Beirats „,Koordinierungszentren zu regionalen oder fachlichen Forschungsschwerpunkten" gebildet werden, dort jeweils erforderliche Forschungsarbeiten abgesteckt und hinsichtlich Zeit- und Finanzmittelbedarf konkretisiert werden. Dabei könne für den Standort Wank auf ein eben erst konstituiertes wissenschaftliches „Leitungskomitee“, dem Vertreter der am Standort tätigen Gruppen angehörten, zurückgegriffen werden.

Anschließend solle eine Ausschreibung zur Einreichung neuer Anträge bis zu einem bestimmten Stichtag vorgenommen werden, der eine gemeinsame mündliche Begutachtungsrunde folgen solle. Der Wissenschaftliche Beirat wäre bei der Auswahl der Gutachter, die vermehrt und 
damit situationsvertraut bayerischen Forschungsinstitutionen angehören sollten, beteiligt und nähme die anschließende Prioritätensetzung innerhalb der förderungswürdigen Anträge vor. Die letztendliche Förderentscheidung obläge nach wie vor dem Projektrat entsprechend den verfügbaren Finanzmitteln. Die Beteiligung des Beirats bei uneinheitlichen Gutachtervoten und finanzintensiven Anträgen (über 1 Mio. DM), sowie die Weitergabe der schriftlichen Gutachten in nicht anonymisierter Form an den Projektrat war, bereits auf der Sitzung vom 29.1.1988 beschlossen worden (i.Q.: PBWU, 29.1.1988).

Sowohl der Finanzgeber als auch die GSF-Geschäftsführung können sich dem Vorschlag des Beirats anschließen. Zur Umsetzung wird vereinbart, bis Herbst des laufenden Jahres die Förderschwerpunkte einerseits, das verfügbare Mittelvolumen des Bayerischen Umweltministeriums andererseits festzulegen. In einer weiteren Sitzung könnten dann Forschungskonzeption, Gutachterauswahl und Ausschreibung vorbereitet werden.

Für erwartete Fortsetzungsanträge, deren Begutachtung aus zeitlichen Gründen noch nicht nach dem vorgeschlagenen Neuverfahren in einer gemeinsamen Begutachtungsrunde abgewikkelt werden sollen, wird von der PBWU dem Umweltministerium und Beiratssprecher eine Interimslösung zur fallweisen Einzelbehandlung von Anträgen vorgeschlagen (s.a. i.Q.: $P B W U$, 13.5., 6.6., und 29.7.1988). Diese solle es der PBWU erlauben, unter Einbeziehung ihrer Gremien bei der Gutachterauswahl und Antragsberatung rechtzeitig zu entsprechenden Förderentscheidungen zu gelangen und eine nahtlose Fortsetzung der Forschungsarbeiten sicherzustellen. Der Vorschlag wird gebilligt.

\subsection{Behandlung von Forschungsberichten zu PBWU-Vorhaben}

Während das auf der Sitzung vom 29.3.1988 vorgeschlagene Verfahren die Behandlung von Anträgen betrifft, bleibt die weitere Vorhabenbetreuung und die Behandlung von Forschungsberichten außer Betracht. Bislang waren vorgelegte Zwischenberichte von der PBWU lediglich zur Kenntnis genommen worden. Erst im Oktober 1987 war vereinbart worden, die Fälligkeiten, Berichtsvorlagen und Maßnahmen bei Terminüberschreitung im Rahmen der halbjährlichen Arbeitsberichte der PBWU an das Ministerium mitzuteilen und auf die jeweils vorgelegten Berichte ,im Sinne fachlicher Stellungnahmen“ einzeln einzugehen (i.Q.: Bayer. Umweltministerium, 8.10.1987). Ergänzend wird festgelegt, daß „die Einschaltung des Wissenschaftlichen Beirats im Rahmen der Prüfung und Bewertung von Berichten ... nicht erforderlich erscheint“. Allerdings sollten die jährlichen PBWU-Zwischenberichte an das Ministerium, die auf den Stand der Untersuchungen eingehen, mit dem Beratungsgremium abgestimmt sein (i.Q.: PBWU, 7.6. und 24.6.1988).

In der Folge bearbeitet die PBWU bewilligungsgemäß vorgelegte Zwischen- und Schlußberichte fallweise in Eigenregie und legt sie dem Ministerium mitsamt einer Stellungnahme vor. Diese bezieht sich i.d.R. auf die Bewertung der durchgeführten Arbeiten hinsichtlich Arbeitsund Zeitplan, Zielsetzung des Vorhabens und Beitrag zur Ursachenforschung. In einigen Fällen werden von den Vorhabenleitern Nachbesserungen inhaltlicher oder formaler Art gefordert. Nur in wenigen Fällen kritisch beurteilter Schlußberichte wird ein fachlich kompetentes Mitglied des Wissenschaftlichen Beirats kontaktiert und um Stellungnahme gebeten. Nach Billi- 
gung der Schlußberichte durch das Umweltministerium versendet die PBWU Pflichtexemplare unter anderem an die Mitglieder des Wissenschaftlichen Beirats und Projektrats. Die Gutachter zu den entsprechenden Forschungsanträgen werden bei der Bewertung der Berichte sowie bei deren Verteilung - ausnahmlich im Falle von Fortsetzungsanträgen - nicht berücksichtigt.

\subsubsection{Neuordnung des PBWU-Projektes}

\subsection{Vorschlag der GSF-Geschäftsführung zur Reform der PBWU}

Die GSF-Geschäftsführung nimmt die von ihr selbst provozierte Kritik des Wissenschaftlichen Beirats an der PBWU (i.Q.: PBWU, 29.1. und 29.3.1988) zum Anlaß, eine Personal- und Organisationsreform der Projektgruppe zu empfehlen bzw. vorzunehmen. Begünstigend kommt hinzu, daß das zweite PBWU-Teilprojekt „Luftverunreinigungen und menschliche Gesundheit“ an der GSF nun begonnen werden soll, was ohnehin personelle Umstrukturierungen erforderlich macht.

Bereits kurz nach der Gremiensitzung vom 29.3.1988 sind die Vorstellungen der GSFGeschäftsführung über die Zukunft der PBWU intern konkretisiert (i.Q.: GSF, 12.4.1988). Darin wird die Stärkung des Wissenschaftlichen Beirats dahingehend interpretiert, daß dieser nun die Rolle eines „Lenkungsgremiums“ übernähme und praktisch „Fachaufsicht“ über das PBWU-Projekt hätte. Damit käme der PBWU lediglich die Funktion eines Projektstabes mit deutlich reduzierten Aufgaben zu. Die Geschäftsführung beabsichtige daher konsequenterweise eine personelle „Kapazitätsreduktion“. So solle die Projektgruppe für die künftige Bearbeitung des Teilprojekts „Wald“ auf 2 wissenschaftliche Mitarbeiter verringert werden.

Die Vorstellungen der GSF werden dem Vertragspartner Bayerisches Umweltministerium offiziell mitgeteilt (i.Q.: GSF, 26.4.1988). Die geforderten Funktionserweiterungen des Wissenschaftlichen Beirats werden als ,fundamentale Änderung“ bezeichnet, welche die Aufgaben der PBWU auf Sekretariatsfunktionen für den Beiratsvorsitzenden reduzierten. In Hinblick auf eine Verbesserung der Akzeptanz der PBWU bei ihrer wissenschaftlichen Klientel würden diese Änderungen von der GSF allerdings begrüßt. Es sei an der Zeit, der häufig geäußerten Kritik am Umfang der Projektgruppe Rechnung zu tragen. Die GSF habe daher beschlossen, zum 1.6.1988 den bisherigen Leiter der PBWU zurückzuziehen und einen bisherigen Projektgruppenmitarbeiter mit der Leitung der Gruppe, der noch ein weiterer, bisheriger wissenschaftlicher Mitarbeiter und eine Schreibkraft angehören solle, zu betrauen. Die beabsichtigten personellen Veränderungen seien mit dem Sprecher des Wissenschaftlichen Beirats abgestimmt. Das PBWU-Teilprojekt „Wald“ würde der Geschäftsführung unterstellt werden.

\subsection{Vorschlag des Umweltministeriums zu Förderverfahren und Organisations- struktur der PBWU}

Das Ministerium antwortet auf die Vorschläge der GSF (i.Q.: Bayer. Umweltministerium, 19.5.1988). Dabei wird vorausgeschickt, daß Übereinstimmung hinsichtlich der Akzeptanzprobleme der PBWU sowie einer erforderlichen Neustrukturierung bestünde. Nicht geteilt wird jedoch die Auffassung, daß die Aufgaben der PBWU auf die eines Sekretariats des Beiratsvor- 
sitzenden reduziert worden wären. Nach wie vor hätte die PBWU die Aufgaben der Konzeption von Forschungsprogrammen, der Abwicklung von Begutachtungsverfahren, der Erstellung von Berichten, Vorbereitung von Tagungen oder der Bearbeitung von Einzelfragestellungen des Auftraggebers. Hierzu sei wissenschaftliche Kompetenz auch bei der Projektgruppe erforderlich.

Die Befugnisse des Wissenschaftlichen Beirats zu erweitern, hält das Ministerium für sinnvoll. Allerdings sei es zweckmäßig, ,im Interesse der Objektivität und Transparenz des Auswahlverfahrens“ bei der Behandlung von Forschungsanträgen „Sicherungsmechanismen“ einzubauen. So sollten nur Gutachter benannt werden, die nicht gleichzeitig Mitglied des Wissenschaftlichen Beirats oder Angehörige entsprechender Arbeitsgruppen sind: Die Antragsteller sollten in einer Vortragsrunde zu Wort kommen, an der die Gutachter sowie die PBWU-Gremien teilnehmen.

Anschließend wird ein „Verfahren bei der Vergabe, Betreuung und Auswertung von Forschungsvorhaben“ im Detail vorgeschlagen, wobei jeweils auf die Beteiligung der PBWUGremien eingegangen wird (i.Q.: Bayer. Umweltministerium, 19.5.1988). Danach würde der Beirat von der Erstellung des Rahmenprogramms, der Gutachterbenennung, Prioritätensetzung und Formulierung von Fördervorschlägen bis hin zur Billigung der PBWU-Ergebnisberichte beteiligt werden. Dem Projektrat solle insbesondere die Billigung von Vorschlägen zu Rahmenprogramm, Gutachtern und Fördervorschlägen obliegen.

\subsection{Diskussion der Vorschläge zur Umstrukturierung des PBWU-Projekts}

In der Folge finden verschiedene Diskussionen zwischen GSF, dem Umweltministerium und Mitgliedern des Wissenschaftlichen Beirats zu den Verfahrensvorschlägen statt (z.B. am 23.6., 7.7., 19.7. und 2.8.1988). Die personell bereits neu organisierte PBWU wird in die Verhandlungen eng mit eingebunden.

Einen zentralen und kontrovers behandelten Diskussionspunkt stellen die Modalitäten des Begutachtungsverfahren zu Forschungsanträgen dar. So empfiehlt die GSF-Geschäftsführung entgegen den Vorstellungen des Umweltministeriums aus Praktikabilitätsgründen die schriftliche Begutachtung. Dabei müßten die Gutachter die Anträge ,in fachlicher Hinsicht und hinsichtlich einer Förderungswürdigkeit prüfen“. Die Prioritätensetzung in Hinblick auf das Rahmenprogramm und verfügbare Finanzmittel sollte nach Auffassung der GSF allerdings nicht von den Gutachtern, sondern vom Wissenschaftlichen Beirat vorgenommen werden. Auch ein Mitglied des Beirats äußert Bedenken „ob die den Gutachtern zugedachten Aufgaben von diesen auch tatsächlich übernommen werden können“ (i.Q.: Bayer. Umweltministerium, 2.8.1988). Demgegenüber plädiert dessen Sprecher für ein Verfahren, welches den Gutachtern durchaus - in Anlehnung an DFG-Schwerpunktprogramme - derartige Beurteilungen übertragen würde. Dabei müßten den Gutachtern allerdings entsprechende Informationen zum Programmrahmen zur Verfügung stehen. Die Nichteinmischung des Wissenschaftlichen Beirats in die fachliche Begutachtung von Anträgen werde unterstützt, die Empfehlung der Gutachter müßten dann allerdings eindeutig, präzise und gut dokumentiert dem Beirat vorgelegt werden (i.Q.: PBWU, 19.7.1988). 
Einigen Mitgliedern des Beirats geht der Reformentwurf des Umweltministerium zur Stärkung des Wissenschaftlergremiums nicht weit genug. Er entspräche nicht den Vorstellungen über eine effiziente Zusammenarbeit zwischen Wissenschaft, Projektträger und Ministerium, die für eine optimale Nutzung der vorhandenen finanziellen und personellen Ressourcen notwendig wäre (i.Q.: Wissenschaftlicher Beirat, 8.8. und 24.8.1988). Es wird vorgeschlagen, die Programmkonzipierung und -aktualisierung sowie die Ergebnisbewertung „,der Wissenschaft zu überlassen“. Über den erforderlichen Wissensstand verfügten in der Regel nur solche Wissenschaftler, die selbst aktiv auf dem betreffenden Gebiet forschten und international anerkannt seien, was in besonderem Maße für die interdisziplinäre Waldschadensforschung gelte.

Für das Umweltministerium zeichnet sich ab, daß im Beiratsgremium selbst unterschiedliche Meinungen zu dem vorgelegten Entwurf der Verfahrens-Neuregelung bestünden und es daher erforderlich sei, das Konzept auf der nächsten Sitzung der PBWU-Gremien zu diskutieren. Das Anliegen des Ministeriums, bis dahin eine abgestimmte Haltung des Wissenschaftlichen Beirats herbeizuführen (i.Q.: Bayer. Umweltministerium, 8.8.1988), kann aus verschiedenen personellen Verhinderungsgründen nicht realisiert werden. Allerdings räumt der Sprecher des Beirats ein, daß die Handlungsfähigkeit der PBWU zur Bearbeitung von Forschungsanträgen aber in jedem Fall erhalten bleiben müsse, eventuell noch nach bisherigem Verfahren (i.Q.: PBWU, 18.10.1988).

\subsection{Behandlung der PBWU-Neuregelung auf den Gremiensitzungen}

Die nächste, für Oktober 1988 vorgesehene PBWU-Sitzung, auf der auch die „Neuregelung der PBWU“ behandelt werden soll, wird aus terminlichen, personellen Gründen sowie noch bestehender Ungewißheit über die künftige Mittelsituation von Seiten des Ministeriums mehrfach verschoben (i.Q.: Bayer. Umweltministerium, 30.9. und 20.10.1988). Unter Leitung des neuen Abteilungsleiters am Umweltministerium kann die Sitzung dann am 15.12.1988 durchgeführt werden. Das Angebot an den Wissenschaftlichen Beirat, eine interne Vorbesprechung zur PBWU-Neuregelung vorzuschalten, wird nicht in Anspruch genommen. Der vorgesehene Tagesordnungspunkt bleibt unbehandelt und wird ohne nähere Begründung auf die nächste Sitzung verschoben.

Einige Fortsetzungsanträge werden auf der Sitzung diskutiert und entschieden. Diese hatte die PBWU inzwischen zur Vermeidung von Unterbrechungen der Forschungsarbeiten in Abstimmung mit ihren Gremien einer in Hinblick auf das künftige Verfahren ,,vorgezogenen schriftlichen Begutachtung zugeführt“.

Für das weitere Vorgehen fordern die Mitglieder des Beirats allerdings in Vorgriff auf das beabsichtigte neue Verfahren, künftig alle Neu- und Fortsetzungsanträge eines Jahres zeitgleich $\mathrm{zu}$ behandeln und damit in gegenseitige Förderkonkurrenz zu stellen. Für das kommende Jahr 1989 sollten für zu erwartende Anträge vorab jeweils Projektskizzen eingefordert werden, die einer grundsätzlichen Vorbewertung durch den Wissenschaftlichen Beirat unterzogen würden. Positiv befundene Projektskizzen würden dann in ausformulierter Antragsform externen Gutachtern zur Beurteilung vorgelegt werden (i.Q.: PBWU, 15.12.1988). 
Die Projektgruppe unter neuer Leitung ist bemüht, die Gremien eng in ihr Handeln einzubeziehen und intensiv Kontakte zu pflegen. Die Berichterstattung gegenüber dem Auftraggeber erfolgt ausführlicher als früher, die Mitglieder der Gremien werden über alle relevanten Vorgänge, insbesondere im Bereich der Antragsbehandlung, umfassend und mittels schriftlicher Unterlagen informiert und um Abstimmung bzw. Stellungnahme gebeten. Dabei verfährt die PBWU weiterhin in Ermangelung einer Verfahrensneuregelung nach einer Interimslösung, welche die einzelfallweise, schriftliche Begutachtung von Anträgen vorsieht. Das Verfahren ist vom Sprecher des Wissenschaftlichen Beirats und dem Bayerischen Umweltministerium akzeptiert (i.Q.: PBWU, 18.10. und. 10.11.1988).

Inzwischen ist allerdings das Umweltministerium mit dem Verhalten des Wissenschaftlichen Beirats unzufrieden. Dessen Vertreter kritisieren, daß der Beirat bisher seinen Aufgaben nicht nachgekommen sei und seine Beratung der PBWU in allen wissenschaftlichen Fragen künftig ernst nehmen müsse (i.Q.: $P B W U$, vom 15.12.1988). Nach wie vor steht eine abgestimmte Haltung des Beirats zur Neuregelung der PBWU aus.

Auf der nächsten Sitzung der PBWU-Gremien am 14.3.1989 wird das Thema Neuregelung der PBWU schließlich behandelt. Vertreter des Beirats beurteilen die Kooperation zwischen der Projektgruppe und ihren Gremien nun positiv und sind der Auffassung, daß die inzwischen geübte Beteiligung des Wissenschaftlichen Beirats an Beratung und Entscheidungen der PBWU ohnehin ihren Vorstellungen entspräche. Eine weitere Diskussion erübrige sich daher. Es wird beschlossen, das „,nunmehr erprobte Verfahren“ bei der Behandlung von Anträgen fortzusetzen und die „Neuregelung der PBWU vorerst nicht weiter zu verfolgen“(i.Q.: PBWU, 14.3.1989).

\subsubsection{Förderverfahren im PBWU-Projekt nach dessen Neuordnung}

Auf der Sitzung vom 14.3.1989 werden 11 Fortsetzungsanträge behandelt, die entsprechend den Festlegungen in den PBWU-Gremien einer Antragsrunde 1989 zeitlich vorgezogen werden sollten. Nach Rücksprache mit dem Beirat hatte die PBWU eine schriftliche Begutachtung durchgeführt. Der Beirat hatte auf deren Grundlage Förderempfehlungen erarbeitet, die nun dem Projektrat vorgestellt, gemeinsam diskutiert und mit einer Förderentscheidung zu jedem einzelnen Antrag abgeschlossen werden (i.Q.: PBWU, 14.3.1989).

\subsection{Vor-Sichtung von Anträgen durch den Wissenschaftlichen Beirat}

Angesichts der hohen Zahl von Fortsetzungsvorhaben bei nur geringer Mittelverfügbarkeit fordert der Sprecher des Wissenschaftlichen Beirats grundsätzlich, einen Mittel-Verteilungsschlüssel anzuwenden, der auch in gewissem Umfang (zu ca. 1/3) Fördermittel zur Bedienung neuer Forschungsansätze zulasse. Zudem sollten die Fördermittel nicht nur einem Standort, dem Wank, zufließen und die Grundlagenforschung nicht gänzlich zurückgefahren werden.

Die auf der Sitzung getroffenen Förderentscheidungen zu Fortsetzungsanträgen sowie die begrenzten verfügbaren Fördermittel beschränken derartige Steuerungsvorschläge in praxi jedoch erheblich. Nach Abzug aller vertraglichen Verpflichtungen stehen für 1989 und 1990 insgesamt lediglich ein Restbetrag von knapp 1,5 Mio. DM für Neuvorhaben zur Verfügung. Mit dieser 
Vorgabe sollen durch den Wissenschaftlichen Beirat alle beabsichtigten Neu- und Fortsetzungsvorhaben vorerst gesichtet werden (i.Q.: PBWU, 14.3.1989).

Hierzu wird am 3.5.1989 eine interne Sitzung durchgeführt. Auf dieser nehmen allerdings lediglich der Sprecher des Wissenschaftlichen Beirats, sein Vertreter sowie der Leiter der PBWU teil. Die geringe Teilnehmerzahl läßt nach Ansicht der Anwesenden auf Ermüdungserscheinungen bei verschiedenen Beiratsmitgliedern schließen; gegebenenfalls sei eine ,personelle Umbesetzung" durch das Bayerisches Umweltministerium erforderlich (i.Q.: PBWU, 3.5.1989).

Insgesamt liegen 12 Neuanträge mit einem Antragsvolumen in Höhe von insgesamt knapp 6 Mio. DM, allein für 1989 und 1990 knapp 3 Mio. DM, vor. Die enorme Diskrepanz zu den verfügbaren Fördermitteln für beide Jahre erfordert eine deutliche Selektion bereits bei der ersten Antragssichtung durch den Wissenschaftlichen Beirat. Als maßgebliche Beurteilungskriterien werden die „Beteiligung am Forschungsschwerpunkt Wank und der Bezug zur Waldschadensforschung“ herangezogen. Für 8 der 12 Neuanträge werden ,erhebliche Bedenken“, „geringe Priorität“ oder die Empfehlung, ,die Anträge nicht, zumindest nicht in der vorliegenden Form weiterzuverfolgen“, ausgesprochen. Das Sitzungsprotokoll wird beiden PBWU-Gremien als Unterlage zur nächsten gemeinsamen Sitzung übermittelt (i.Q.: PBWU, 29.5.1989).

Schließlich wird im Rahmen dieser Begutachtung für lediglich 2 durch externe Gutachter positiv beurteilte Neuanträge eine Finanzierung ausgesprochen (i.Q.: PBWU, 27.7.1989). Weiterhin wird der Antrag eines neu in das Gremium des Wissenschaftlichen Beirats aufgenommenen Institutsleiters der GSF zu Ende des Jahres 1989 bewilligt (i.Q.: PBWU, 7.11.1989). Einige der nicht geförderten Anträge werden in der Folge allerdings erneut vorgelegt und behandelt.

\subsection{Jahresweise Bündelung der Behandlung von Forschungsanträgen}

In Anbetracht der knappen Fördermittel setzt sich immer mehr durch, die vorliegenden oder angekündigten Forschungsanträge für das kommende Jahr gebündelt zu behandeln. Gegenüber fallweiser Behandlung und Förderentscheidung im Rahmen der aktuell verfügbaren Fördermittel werden alle Anträge nun gegeneinander in finanzielle wie fachliche Konkurrenz gestellt. Zudem kann durch die zeitlich parallele Bearbeitung mehrerer Vorhaben die Zusammenarbeit bei Planung, Durchführung und Auswertung verbessert werden. Neu- wie Fortsetzungsanträge werden gleichermaßen jahresweise zusammengefaßt und gemeinsam behandelt. Dies umfaßt die Vor-Sichtung durch den Wissenschaftlichen Beirat, die Durchführung der externer Begutachtung und die Förderentscheidung in gemeinsamen PBWU-Gremiensitzungen unter Berücksichtigung vorhandener Fördermittel.

Bei Fortsetzungsanträgen tritt die PBWU frühzeitig mit potentiellen Antragstellern in Kontakt und mahnt gegebenenfalls die rechtzeitige Antragsvorlage an (i.Q.: PBWU 3.5.1989). Hier muß berücksichtigt werden, daß in vielen Fällen die zeitliche Gleichschaltung von Fortsetzungsanträgen bzw. -vorhaben aufgrund verfahrensbedingt unterschiedlicher Laufzeiten der entsprechenden Erstvorhaben erschwert wird.

Im Zuge der jahresweisen Bündelung der Antragsbehandlung werden zu Beginn des Jahres 1990 insgesamt 13 Neu- und Fortsetzungsanträge - zu etwa gleichen Anteilen - behandelt. Die 
PBWU hatte zu den einzelnen Anträgen bereits externe, schriftliche Gutachten eingeholt. Bei einer Antragssumme von insgesamt rund 3,5 Mio. DM fallen auf das Jahr 1990 rund 1 Mio. beantragte Mittel. Dem stehen, wie die Vertreter des Bayerischen Umweltministeriums auf der Sitzung am 20.2.1990 mitteilen, 0,7 Mio. Fördermittel gegenüber. Diese vergleichsweise geringe Diskrepanz zwischen beantragten und verfügbaren Fördermitteln kann mit einer relativ geringen Ablehnungsrate von nur 2 Anträgen und gewissen Mittelkürzungen bei förderungswürdigen Anträgen leicht bewältigt, ja sogar überkompensiert werden. Gegenüber den in Aussicht gestellten Fördermittel werden für 1990 Festlegungen in Höhe von 0,6 Mio. DM getroffen (i.Q.: PBWU, 20.2.1990).

Die weitere Verlängerung des Mitte des Jahres 1990 auslaufenden PBWU-Vertrages, explizit auf die Tagesordnung der Sitzung am 20.2.1990 gesetzt, passiert die beiden PBWU-Gremien ohne Einwände, Anregungen und Vorschläge. Die Mittel hierfür hatte das Bayerisches Umweltministerium bereits außerhalb der für eigentliche Forschungsmaßnahmen vorgesehenen Fördermittel reserviert.

Der Vorschlag eines Beiratsmitglieds, eine mündliche Begutachtungsrunde unter Anwesenheit der Antragsteller durchzuführen, wie zu Beginn des Projektes erfolgt, findet keine Zustimmung. Als Gründe werden hohe Kosten und Terminfindungsprobleme genannt.

Aufgrund der zu Beginn des Jahres gegebenen Fördermittelsituation und der getroffenen Förderentscheidungen für 1990 kommt die nächste gemeinsame Sitzung der PBWU-Gremien am 17.7.1990 ohne den Tagesordnungspunkt der (Nach-)Behandlung vorliegender Anträge aus. Es wird wiederum festgelegt, für das kommende Jahr 1991 die erwarteten Neu- und Fortsetzungsanträge in ihrer Behandlung zeitlich zu bündeln und zeitgerecht einer Förderentscheidung zu Beginn des nächsten Jahres zuzuführen (i.Q.: PBWU, 17.7.1990).

In dieser, von „Antragsaltlasten“ freien Situation kann die Diskussion zur Umsetzung bestehender „Forschungslücken“ aufgegriffen werden. Hierzu besteht eine von der PBWU erstellte „Vorschlagsliste für weiteren Forschungsbedarf“" als Grundlage, die eine Verstärkung der bestehenden Förderschwerpunkte sowie die Neu-Etablierung der Laubwaldforschung vorsieht. Im Wissenschaftlichen Beirats besteht allerdings inhaltlich noch weiterer Diskussionsbedarf, auch das Bayerische Umweltministerium muß erst die Ergebnisse der Haushaltsverhandlungen im Herbst des laufenden Jahres abwarten. Auf die weitere Behandlung des Themas Neukonzeptionierung der Forschung wird an anderer Stelle (Kap. 5.3.4) eingegangen.

\subsection{Zurückhaltende Bewilligung von Neuvorhaben}

Zur Sitzung der PBWU-Gremien am 11.12.1990 liegen 5 Fortsetzungs- und 6 Neuanträge vor. Ausgenommen den Antrag zur Fortführung der Labor-Einrichtung „Zentrale Analytik“ an der GSF werden für das Jahr 1991 insgesamt Mittel in Höhe von knapp 2 Mio. DM für Neu- und Fortsetzungsanträge beantragt. Nach Durchführung der externen Begutachtung und interner Vorbesprechung durch Mitglieder des Wissenschaftlichen Beirats können auf der nächsten Gremiensitzung am 26.2.1991 Förderentscheidungen getroffen werden. Diese führen im Falle der Fortsetzungsanträge überwiegend zu einem positiven Ergebnis, z.T. mit finanziellen oder 
zeitlichen Beschränkungen. Im Falle der Neuanträge werden überwiegend fachlich begründete Ablehnungen ausgesprochen bzw. eine modifizierte Wiedervorlage der Anträge anheimgestellt. Die Finanzierung der positiv entschiedenen Anträge ist im Rahmen der verfügbaren Fördermittel. Auch die „Zentrale Analytik“ kann weitergeführt werden. Auf der Gremiensitzung am 10.7.1991, die vom neuen Leiter der Abteilung Umweltsicherung am Bayerischen Umweltministerium geleitet wird, werden 2 der zur Modifizierung empfohlenen Neuanträge nachverhandelt (i.Q.: PBWU, 11.12.1990, 26.2.1991 und 10.7.1991).

Das Verfahren, die für das kommende Jahr erwarteten Anträge gegen Ende des laufenden gemeinsam zu behandeln, soll auch für das Jahr 1992 angewendet werden. Auf der Sitzung der PBWU-Gremien am 31.10.1991 werden bereits grundsätzliche Stellungnahmen des Wissenschaftlichen Beirats zu den 6 vorliegenden Neu- bzw. Fortsetzungsvorhaben vorgetragen, die in einer internen Vorbesprechung formuliert worden waren. Diese führen z.T. zur Befürwortung, z.T. aber auch zu Ablehnung bzw. Modifizierungsauflagen für Anträge, noch bevor die externe Begutachtung durchgeführt wurde. Die Umsetzung der geforderten Antragsmodifizierungen durch die Antragsteller hat zeitliche Verzögerungen zur Folge, so daß erst auf der übernächsten Sitzung am 16.7.1992, der vorletzten Sitzung der PBWU-Gremien, Förderentscheidungen getroffen werden. Hierbei stehen als Ergebnis des internen wie externen Selektions- bzw. Begutachtungsprozesses nur noch 2 Anträge zur Entscheidung an, von denen einer als letzte Fördermaßnahme im PBWU-Projekt bewilligt wird (i.Q.: PBWU, 13.10.1991 und 16.7.1992).

\subsection{Neubesetzungen im Wissenschaftlichen Beirat}

Die geringe personelle Beteiligung des Wissenschaftlichen Beirats an der internen Antragsvorbegutachtung vom 3.5.1989 war Anlaß zur Umbesetzung des seit 1985 existierenden Beratungsgremiums gewesen. Im Protokoll zur Sitzung war dem Bayerischen Umweltministerium empfohlen worden, bei den einzelnen Mitgliedern nachzufragen, „ob eine weitere Beteiligung in dem Beratungsgremium der PBWU überhaupt noch erwünscht sei." Andernfalls erschiene eine personelle Umbesetzung notwendig. Auf der nächsten Sitzung der PBWU-Gremien vom 27.7.1989 wird das Thema aufgegriffen und Vorschläge zur Umbesetzung des Wissenschaftlichen Beirats diskutiert. Akzentverschiebungen in den wissenschaftlichen Fragestellungen, insbesondere die beabsichtigte Verstärkung der Laubwaldforschung, machten nach Ansicht des Bayerischen Umweltministeriums auch eine veränderte Zusammensetzung der im Beirat vertretenen Disziplinen erforderlich (i.Q.: PBWU, 3.5.1989 und 27.7.1989).

Im Verlauf des Jahres 1990 findet eine gewisse Umbesetzung statt, die allerdings in der Summe mehr einer Erweiterung des bisher achtköpfigen Gremiums als dessen fachlicher Umstrukturierung entspricht. Während zwei Mitglieder nordbayerischer Universitäten auf eigene Veranlassung ausscheiden, kommen vier Wissenschaftler neu hinzu. Diese vertreten die Fachgebiete allgemeine Botanik, Biochemie, Bioklimatologie und Bodenkunde. Die Auswahl orientiert sich nur bedingt an den geplanten, veränderten Forschungsschwerpunkten. Eine Rolle scheint sowohl der Ersatz der durch das Ausscheiden von Mitgliedern betroffenen Fachdisziplinen als auch die Vertretung bestimmter Forschungsinstitutionen zu spielen. Die neuen Mitglieder gehören bayerischen Universitäten, unter anderem der Forstwissenschaftlichen Fakultät der Uni- 
versität München, sowie der GSF an. Mit den neuen und zusätzlichen Vertretern von Bodenkunde und Botanik werden diese klassischen Naturwissenschaften um renommierte Repräsentanten der Grundlagenforschung verstärkt. Dies läßt sich mit dem Anliegen des Bayerischen Umweltministeriums, angewandte Waldschadensforschung zu fördern, kaum begründen und läßt auf die Dominanz des Beratungsgremiums bei Vorschlag und Auswahl neuer Beiratsmitgliedern schließen. Durch die Berufung eines neuen GSF-Institutsleiters in das Gremium kann der Einfluß des Forschungszentrums im Projekt nun auch in fachlicher Hinsicht - neben der Vertretung im Projektrat - verstärkt werden. Die fachlich-inhaltliche Beteiligung im Rahmen eines Forschungsprojektes des Instituts folgt alsbald.

\subsubsection{Theorieorientiertes Zwischenergebnis}

\subsubsection{1 „Entmachtung“ der PBWU durch die Wissenschaft?}

Die Kritik an der Projektgruppe wird von der GSF zur strukturellen und personellen Änderung genutzt. Im Ergebnis ist dieser Schritt, der zu einer verkleinerten PBWU unter der Leitung eines ihrer bisherigen Mitarbeiter führt, entscheidender als die geplanten Verfahrensänderungen, die letztendlich ohne verbindliche Festlegungen bleiben.

Die Absetzung des ehemaligen Leiters der PBWU bedeutet einerseits die Beseitigung von Kritik, die unmittelbar auf dessen Person zu beziehen ist. „Eigenmächtige“ Entscheidungen der PBWU, die fachliche Präjudizierung von Anträgen, wenig transparente Verfahrensweisen in der Antragsbehandlung waren weitere Punkte, welche der Gruppe und damit dem verantwortlichen Gruppenleiter den Vorwurf der Kompetenzüberschreitung bei gleichzeitig unzureichender Beteiligung ihrer Gremien einbrachte.

Allerdings läßt sich nur ein Teil der Kritik tatsächlich der Projektgruppe zuordnen. So war der Wissenschaftliche Beirat vertraglich nicht in dem Maße, wie es seinen Vorstellungen entsprach, am Verfahren zu beteiligen. Zudem entstand manche Kritik (z.B. Nichtentscheidung oder Verzögerung in der Antragsbehandlung) lediglich in Verbindung mit Finanzierungsproblemen des Umweltministeriums und wurde zu Unrecht der PBWU angelastet. Der Wissenschaftliche Beirat versucht interessengeleitet und nicht ausschließlich formal legitimiert, seinen Machteinfluß auf das Förderverfahren und die entsprechenden Entscheidungen zu vergrößern.

Bei der GSF stehen der Ruf der Forschungseinrichtung sowie personelle Konsequenzen im Mittelpunkt des Interesses. Der Leiter der PBWU, der für die Kritik am Handeln der PBWU und ihre mangelnde Akzeptanz verantwortlich gemacht wird, wird abgezogen, womit gleichzeitig der Effekt der personellen Kapazitätsverringerung eintritt. Die Besetzung der PBWULeitung mit einem Diplom-Forstwirt bedeutet zwar gegenüber dem früheren promovierten Agrarwissenschaftler einen fachspezifisch günstigeren Zuschnitt in Hinblick auf das bearbeitete Thema Waldschäden und kann zudem auf ein persönlich unbelastetes Verhältnis gründen. Allein die Verkleinerung der Gruppe und Übertragung der Leitung an einen bisherigen, zudem nicht promovierten Mitarbeiter kann aber als Machtverschiebung zugunsten des Wissenschaftlichen Beirats gewertet werden. Durch die Abstimmung der Personalmaßnahmen mit dessen Sprecher kann sich die GSF der Akzeptanz des Gremiums versichern und die Maßnahme als 
ein Entgegenkommen gegenüber der Wissenschaft gewertet wissen. Die formalen Voraussetzungen für die Fortführung des Projektes sind weiterhin gegeben. Insgesamt ist für die GSF kein wesentlicher Kompetenzverlust zu erwarten.

Die Personaländerungen kann die GSF in Eigenregie und ohne weitere Diskussion durchführen und entsprechende Fakten schaffen. Hinsichtlich der Neuregelung des Förderverfahrens und der Beteiligung der PBWU-Gremien muß jedoch ein Kompromiß ausgehandelt werden, der einerseits die Transparenz, Neutralität und Objektivität des Förderverfahrens sichert, andererseits den Forderungen des Wissenschaftlichen Beirats nach stärkerer Beteiligung Rechnung trägt.

Das Umweltministerium rückt in der anschließenden Diskussion jedoch den Aufgabenumfang der Projektgruppe und deren für erforderlich gehaltene, wissenschaftliche Kompetenz zurecht. Es möchte damit seinen politischen Einfluß über die intermediäre Koordinierungsstelle auf den Forschungsprozeß auch weiterhin sichern.

Für den Wissenschaftlichen Beirat steht neben der Kritik an der Finanzierung des PBWUForschungsprogramms eine Verfahrensreform unter stärkerer Eigenbeteiligung im Zentrum der Forderungen. Dabei ist zu berücksichtigen, daß sich etwa zeitgleich am alpinen Forschungsschwerpunkt unter maßgeblicher Beteiligung eines der die PBWU kritisierenden Beiratsmitglieder ein „Leitungskomitee“ etabliert. Dieses Komitee beabsichtigt unter anderem, forschungskonzeptionelle, -steuernde und -bewertende Funktionen zu übernehmen. Deren Forderung, in die Beratungs- und Entscheidungsvorgänge der PBWU-Gremien einbezogen zu werden, unterstreicht den steigenden Steuerungsanspruch der Wissenschaft im PBWU-Projekt.

Die Handlungsfähigkeit der PBWU unter neuer Leitung bleibt erhalten, da, legitimiert von Beirat und Umweltministerium, nach altem Verfahren der Antragsbehandlung vorgegangen wird. Die Projektgruppe nutzt inzwischen ihre Möglichkeiten, der bisherigen Kritik aus den Gremien gegenzusteuern und eine Basis für vertrauensvolle, kooperative Zusammenarbeit zu schaffen. Sie bemüht sich, die Behandlung von Forschungsanträgen möglichst transparent zu gestalten und die Gremien intensiv zu beteiligen. Sie kann damit ein Vertrauensverhältnis zu ihren Gremien aufbauen und ihre Akzeptanz erheblich verbessern. Auch das Vertrauen der GSFGeschäftsführung in die personell umbesetzte Projektgruppe scheint nunmehr zu bestehen. Der Vorschlag des Ministeriums, einen Repräsentanten der PBWU aus der GSF-Geschäftsführung $\mathrm{zu}$ bestellen, wird nicht mehr für nötig gehalten.

Mit diesem neuen, auf mehr Konsens und enge Zusammenarbeit ausgerichteten PBWUVerhalten wird erreicht, daß etwa ein Jahr nach dem Vortragen massiver Kritik an der Gruppe, nämlich im März 1989, sich der Beirat allgemein positiv über das nun eingespielte Verfahren äußert (i.Q.: PBWU, 14.3.1989). Die Phase der Kritik an der Projektgruppe und der fehlenden Akzeptanz ist damit endgültig überwunden. Weder hinsichtlich des Verfahrens der Antragsbehandlung, noch des PBWU-Aufgabenprofils besteht weiterer Regelungsbedarf, auch wenn konstatiert wird, daß der Gruppe in Detailfragen noch ein breiter Handlungsspielraum verbliebe. 
Dabei darf nicht übersehen werden, daß inzwischen eine fortgeschrittene, weniger programmatische Regelungen bedürfende Implementationsphase des Forschungsprojektes erreicht ist. Das überwiegend politisch orientierte Ziel des federführenden Ressorts, einen alpinen Forschungsschwerpunkt zu etablieren, ist weitgehend erfüllt. Grundlegende Einflußnahme des Beirats auf diesen Forschungsschwerpunkt, der von der Mehrheit seiner Mitglieder ohnehin mehr geduldet als inhaltlich argumentativ unterstützt wird, ist daher kaum mehr zu erwarten und zeigt sich auch in der zögerlichen Behandlung eines entsprechenden Forschungskonzeptes des am Wank gebildeten Leitungskomitees. Die nunmehr vorhandene Akzeptanz der PBWU ist also nicht nur ein Resultat der relativen Machterweiterung des Wissenschaftlichen Beirats aufgrund der personellen Abstufung der PBWU. Sie kann auch unter dem Aspekt geringer Steuerungsabsichten des Beirats auf das laufende Forschungsprogramm und unter dem Eindruck insgesamt zurückgehenden Mittelflusses für die Waldschadensforschung gesehen werden.

Weder die teilweise Umbesetzung des Beiratsgremiums im Jahr 1990, die im wesentlich eine personelle Erweiterung darstellt, noch das gemeinsame Ringen um die Erstellung und Umsetzung eines neues Forschungskonzeptes können in den Folgejahren den Fortbestand der PBWU-Waldschadensforschung sichern. Gegenüber den inzwischen veränderten Randbedingungen, nämlich geringerem politischen Handlungsbedarf zu einem abklingenden Thema mit der Konsequenz abnehmender staatlicher Fördermittelbereitstellung, kann sich auch ein funktional und personell gestärktes wissenschaftliches Beratungsgremium nicht durchsetzen.

\subsection{Wandel der Steuerungsdominanz im PBWU-Projekt}

Der Grad der Beteiligung der politischen und wissenschaftlichen Akteursgruppen an dem Implementationsprozeß der Waldschadensforschung im PBWU-Projekt spiegelt die gegebenen Machtverhältnisse und deren Veränderungen im Zeitverlauf bzw. während des Forschungsprozesses wider. Dabei läßt sich ein Wandel in der Steuerungsdominanz feststellen. Als problemlösungsorientiertes Programm angelegt, versucht das Umweltministerium bei hohem politischen Handlungsdruck zu Beginn, eine rasche Programmimplementation durchzuführen. Die hierfür erforderliche Dominanz der entsprechenden politischen Akteure Umweltressort und Projektgruppe wird hergestellt. Dies kann allerdings nur dadurch erfolgen, daß ein breit gefaßtes Rahmenprogramm einen forschungspolitisch akzeptablen Kompromiß zwischen Politik und Wissenschaft zuläßt, das wissenschaftliche Beratungsgremium verfahrensmäßig weitgehend übergangen und von der Finanzierungshoheit des Umweltressorts steuernd Gebrauch gemacht wird. Eine Interessenaushandlung findet nicht statt, Umweltressort und PBWU setzen sich - sogar gegen die Kritik weiterer politischer Akteure im Projektrat - durch. Das forschungspolitische Ziel, ein Waldschadensforschungsprogramm rasch zu implementieren, kann damit erreicht werden.

Die unterbleibende Vorauswahl der Forschungsanträge durch den Beirat in der richtungsgebenden Startphase der Programmimplementation enthebt das Projekt jedoch der wissenschaftlichen Steuerung und Schwerpunktsetzung innerhalb des breit angelegten Rahmenprogramms. Die maßgebliche Beteiligung der PBWU im Rahmen des Förderverfahrens der ersten beiden Antragsrunden bis hin zu deren Neuorganisation bedeutet einen erheblicher Steuerungseinfluß auf die zentralen Förderentscheidungen und damit den gesamten Forschungsprozeß im Projekt. 
Das fachliche Instrument der Antragsevaluierung durch externe Gutachter wird während der gesamten Implementationsphase eingesetzt. Damit wird zum einen generell die wissenschaftliche Qualität der Forschungsbeiträge sichergestellt, was die Akzeptanz und das Ansehen des Projektes intern und extern verbessert. Zum anderen ist hiermit jedoch auch ein wissenschaftlicher Steuerungsinput verbunden, der sich nicht zwangsläufig mit der forschungspolitischen Zielsetzung decken muß. Denn die fachliche Ausrichtung der Gutachter ist überwiegend auf Grundlagenforschung orientiert, das Bayerische Umweltministerium beabsichtigt problemlösungsorientierte, angewandte Forschung. Der Zielkonflikt kann anfangs dadurch überwunden werden, daß das Ministerium durch relativ großzügige Finanzierung auch allein aus wissenschaftlicher Sicht zu bevorzugende Vorhaben ,,mitträgt“, die nicht unbedingt prioritär für einen politisch beabsichtigten, problemorientierten Forschungsansatz sind.

Auf Dauer stellt sich das Bayerische Umweltministerium und die Projektgruppe nicht völlig gegen die Kritik des Wissenschaftlichen Beirats. Ohnehin sind die wichtigsten forschungspolitischen Eckpfeiler des PBWU-Programmes - insbesondere in Form des alpinen Forschungsschwerpunktes zum Thema Photooxidantien - bereits gesetzt. Entgegenkommendes Verhalten des Auftraggebers sowie der GSF-Geschäftsführung gegenüber dem Beirat hinsichtlich dessen künftiger Beteiligung und damit dessen Steuerungseinflusses im Projekt prägt daher zunehmend die weitere Programmimplementation.

Der Kompetenzzugewinn des Wissenschaftlergremiums geht zulasten der Projektgruppe. Die Vorauswahl der Anträge sowie die Erstellung von Förderempfehlungen auf Grundlage der eingeholten Gutachten werden nun vom Beirat durchgeführt. Zwar kann die Projektgruppe eine Interimsphase noch für ihre Rehabilitierung in den Gremien und Beteiligung am Förderverfahren nutzen. Spätestens nach der Neuordnung des PBWU-Projektes Ende 1988, in der sich die geschwächte Position der Projektgruppe auch über personelle Veränderungen manifestiert, dominieren jedoch die wissenschaftlichen Steuerungseinflüsse des Beirats das Projekt. Mit diesem Wendepunkt der PBWU-Neuordnung ist zwar die Konfliktphase zwischen Projektgruppe und Wissenschaftlichem Beirat und damit zwischen Politik und Wissenschaft beendet. Nahezu die gesamte Steuerung des Förderverfahrens erfolgt nun durch das oder zumindest in enger Abstimmung mit dem Wissenschaftlergremium.

\subsection{Forschungskoordination}

Speziell die Waldschadensforschung als interdisziplinär angelegte Forschung erfordert einen hohen Koordinationsaufwand. Eine Forschungsabstimmung durch die Wissenschaftler selbst kann jedoch allein aufgrund mangelnder außerfachlicher Interessen und Kompetenz meist nur innerhalb des eigenen Fachbereichs erwartet werden. Zu derartigen Ergebnissen kommen Studien über die österreichische und schweizerische Waldschadensforschung [KROTT 1994a, S. 119, 259; STARK 1991, S. 294; s.a. WiSSENSCHAFTSRAT 1994, S. 146/147].

Auftrag der PBWU war vertragsgemäß die Koordinierung der bayerischen Waldschadensforschung. Dies wurde vom Auftraggeber sowie der Projektgruppe selbst als über eine reine Koordination des Förderverfahrens hinausgehende Aufgabe der Forschungskoordination verstanden und bedeutet die Aufeinanderabstimmung der Forschung auch in fachlicher Hinsicht. For- 
schungskoordination setzt allerdings ein entsprechendes Koordinationsinstrumentarium, ausreichende fachliche Kompetenz, sowie die erforderliche Durchsetzungsmacht voraus.

Die Koordination als eine steuernde Einflußnahme von außen auf den Forschungsprozeß gerät jedoch schnell an verschiedene Grenzen. Diese setzen generell das Autonomiebedürfnis und entsprechende Handlungsspielräume der Wissenschaftler [MAYNTZ 1985, S. 20/21]. Entsprechend dem Selbstverständnis der Forscher werden die Forschungserfolge und somit die Erkenntnisproduktion innerhalb des eigenen Fachgebietes erhofft. Bei Bearbeitung und Synthese eines interdisziplinären Forschungsverbundes muß sich jedoch das informale Eigenständigkeitsstreben der Forscher dem integralen, formalen Forschungsziel des Gesamtprojektes unterordnen [KROTT 1994a, S. 156-172]. Dies erfordert, daß der Forscher akzeptiert, die Bedeutung seines eigenen Forschungsbeitrages in Hinblick auf die übergeordnete Fragestellung zu relativieren [STARK 1991, S. 296].

Zudem ist die Neigung der Forscher, im Rahmen eines abgestimmten interdisziplinären Projektes innerhalb wie außerhalb ihres Forschungsgebietes zu kooperieren, vergleichsweise gering. Interdisziplinarität würde - z.B. hinsichtlich gemeinsam oder zeitgleich durchzuführender Arbeiten, der Bereitstellung von Daten oder gemeinsamer Publikationen - eine enge und intensive Zusammenarbeit erfordern. Dies schafft Abhängigkeiten und Zwänge zulasten der Eigenständigkeit, verschärft Konkurrenzsituationen, ist mit erhöhten Risiken des Scheiterns verbunden, ist zeitaufwendig und wenig ergiebig für wissenschaftliche Reputation und Karriere [KAUFMANN 1987]. Gerade bei bestehenden Finanzmittel-Engpässen kommen zur fachlichen Konkurrenz finanzielle Konkurrenzsituationen um Fördermittel hinzu. Auch in diesem Fall wird die eigenständige Bearbeitung des eigenen Projektes und dessen Auswertung in den Vordergrund gestellt gegenüber einer Integration der Ergebnisse mit denen anderer Arbeitsgruppen im interdisziplinären Forschungsverbund. Zudem sind die Ergebnisse anderer Gruppen häufig selbst nicht überprüfbar und eventuell mit zeitlichen und inhaltlichen Unsicherheiten ihres $\mathrm{Zu}$ standekommens belastet [KROTT 1994a, S. 130/131]

Auch die Durchführung der meisten Forschungsarbeiten im Rahmen von Dissertationen trägt dazu bei, daß das Forschungsinteresse an Nachbarprojekten oder am Gesamtprojekt nicht im Vordergrund steht. Die Zielsetzungen sind primär an denen der durchführenden Forschungseinrichtung orientiert oder nur formal - um die Erfolgswahrscheinlichkeiten für eine Förderung zu erhöhen - auf das Gesamtprojekt abgestimmt. Auch steht für den Dissertanden naturgemäß die eigene Arbeit gegenüber dem Beitrag zum Gesamtprojekt im Mittelpunkt [STARK 1991, S. 294].

Letztlich setzt die Zusammenarbeit und Abstimmung der Forschung in einem Verbundprojekt die Integrations- und Kooperationsfähigkeit bei den Forschern voraus. Aufgrund unterschiedlicher Persönlichkeitsstrukturen der Forscher kommt daher der Personenkonstellation innerhalb und zwischen den beteiligten Arbeitsgruppen große Wichtigkeit hinsichtlich einer erfolgreichen Forschung zu. Dieser Aspekt wird jedoch bei der Forschungsplanung i.d.R wenig berücksichtigt [STARK 1991, S. 297/298]. 
Die genannten, koordinationserschwerenden Aspekte treten auch im PBWU-Projekt auf. Zwar gelingt es durch den Aufbau des alpinen Forschungsstandortes, die beteiligten Forschungsvorhaben auf gemeinsame Probeflächen, Meß- und Beprobungsaktionen (z.B. Blattprobennahmen) zu konzentrieren und als Serviceleistung Meßdaten zur Immissions- oder Witterungssituation oder zum Waldzustand allgemein zur Verfügung zu stellen. Darüber hinaus sind die durchgeführten Arbeiten jedoch weder in der Antragsphase noch in der Durchführung im wissenschaftlichen Detail aufeinander abgestimmt. Wenige Ausnahmen bestehen z.B. im Falle gemeinsam vorgelegter Sammelanträge aus ein und demselben Institut. Nahezu jedes Vorhaben ausgenommen reine Serviceleistungen - sieht in der eigenen Forschungsfragestellung den Lösungsbeitrag zum Thema, ohne sich in eine strukturelle Hierarchie eines Programmnetzwerkes einzuordnen. Die Forscher verstehen sich daher nicht als Lieferanten von Beiträgen, Daten und Teilergebnissen, sondern nutzen bestenfalls - und nur in wenigen Fällen - anderweitig erarbeitete Daten oder Ergebnisse für ihre in den Mittelpunkt gerückte Forschungsfragestellung. Einige wenige Forschungsabstimmungen mit entsprechendem Datenaustausch zwischen den Wissenschaftlern können im Bereich pflanzenphysiologischer/biochemischer und bodenkundlicher Arbeiten am alpinen Forschungsschwerpunkt belegt werden. Entsprechend sind auch gemeinsame Publikationen verschiedener Arbeitsgruppen die Ausnahme.

Zudem wurden einige, weitgehend standortsunspezifische Forschungsvorhaben an dem alpinen Forschungsstandort integriert, die ohnehin besser und kosteneffizienter an anderen, z.B. einfacher und unproblematischer zu bearbeitenden Standorten positioniert worden wären als am Wank. Mit der Strategie, sich an diesem Forschungsstandort zu beteiligen, auch wenn dies wissenschaftlich nicht unbedingt begründet war, konnten jedoch bei den gegebenen forschungspolitischen Prioritätensetzung die Chancen der Bewilligung erhöht werden.

Auch ein andersartiges Vorgehen, nämlich das wissenschaftliche Potential mittels geeigneter Koordinationsmaßnahmen zu einem abgestimmten und hierarchisch geordneten Forschungsnetzwerk zu verbinden, wäre an Barrieren geraten. Das Koordinations- und Integrationspotential der Projektgruppe selbst war für eine derartige Forschungsstrukturierung allein aus fachlicher Sicht zu gering. Hier wäre bereits zu Zeiten der Rahmenprogrammplanung eine engere Zusammenarbeit mit dem hierzu fähigen Wissenschaftlichen Beirat oder einzelnen herausragender Wissenschaftler-Persönlichkeiten mit entsprechender Integrationsfähigkeit erforderlich gewesen, die Vorhaben in ihrer jeweiligen Zielsetzung und Durchführung mitsamt entsprechender Datenerhebung und Datenweitergabe an Nachbarprojekte aufeinander abzustimmen.

Die von den Forschern negativ beurteilte, gegenseitige Abhängigkeit wäre im PBWU-Projekt jedoch eingetreten. Allein die zeitliche Gleichschaltung der einzelnen Vorhaben in ihrer Bewilligung, um die zeitgerechte Durchführung von anderen benötigter Forschungsarbeiten sicherzustellen, konnte bereits nach der zweiten Begutachtungsrunde aufgrund bestehender Finanzierungsengpässe und zeitlicher Förderdiskontinuitäten nicht mehr gewährleistet werden.

Die anfänglichen Begutachtungsrunden, welche die Durchführung eines zeitgleichen und inhaltlich optimal aufeinander abgestimmten Forschungsprogramms erlaubt hätten, blieben hinter diesem Anspruch erheblich zurück und konnten zudem das Gesamtprogramm nicht annähernd ausfüllen. Zeitlich nachgeschaltete Vorhaben konnten zwar das Programm fachlich sinnvoll er- 
gänzen und z.B. bei jahresweiser Antragsbündelung der späteren Förderphase aufeinander abgestimmt werden. Die Anbindung an bereits laufende Arbeiten war dann jedoch erheblich erschwert. So bestand für die PBWU hier bestenfalls noch die Möglichkeit, die fachlich erforderliche oder wünschenswerte Zusammenarbeit mit anderen, bereits etablierten Gruppen als mehr oder weniger allgemeine Auflage in der Bewilligung der Forschungsarbeiten zu formulieren. Den Ansprüchen an eine eng abgestimmte Forschungsnetzplanung wird dies periphere Koordinierungsmaßnahme allerdings nicht gerecht.

Insgesamt können im PBWU-Projekt die bestehenden Defizite in der fachlich adäquaten Forschungskoordination und -synthese durch verschiedene Koordinationsmaßnahmen der PBWU nicht beseitigt werden. Im Mittelpunkt der Maßnahmen stehen hier u.a. die Durchführung gemeinsamer Arbeitsgruppengespräche, Rundbriefe, Statusseminare und Exkursionen, die zentrale chemische Probenanalyse und das Vorhalten einer Datenbank für Immissions- und Klimadaten am Wank. Derartige Koordinationsmaßnahmen sind überwiegend auf organisatorischer Ebene sowie der Informationsebene wirksam und verbreitern die gemeinsame Basis bei der Durchführung der Forschungsarbeiten. Sie stellen jedoch keine Koordinationsmaßnahmen zur Steuerung des fachlich-interdisziplinären Forschungssystems dar. Auch der Wissenschaftliche Beirat sieht sich bestimmungsgemäß eher für grundsätzliche Programmentscheidungen zuständig als eine fachliche Detailkoordination in der Durchführung des Forschungsprojektes.

Neben den genannten suboptimalen Förderrahmenbedingungen ist eine weiterreichende fachliche Koordination durch die Projektgruppe aufgrund beschränkter Fachkompetenz ohnehin weder wissenschaftlich erwünscht noch möglich. Die Unmöglichkeit, als überwiegend im Organisatorischen wirkende und derart auch verstandene Institution die fachlichen Details eines interdisziplinären Forschungsverbundes adäquat beurteilen und damit koordinieren zu können, wird daher auch als Begründung einzelner Wissenschaftler herangezogen, eine überwiegend fachlich orientierte Koordinationsstelle für den alpinen Forschungsschwerpunkt zu schaffen.

Von dem selbst gestellten Aufgabenprofil der Forschergruppe, auch in das Förderverfahren einzugreifen, werden jedoch Ziel- und Interessenkonflikte mit den bereits bestehenden PBWUGremien befürchtet. Dieser Ansatz regionaler Koordinierungseinheiten, bestehend aus vor Ort tätigen Wissenschaftlern, kann sich daher gegenüber dem Auftraggeber Umweltministerium nicht durchsetzen. Abgesehen davon, daß dem Sprecher der Gruppe ein wenig wirksamer Gaststatus im Wissenschaftlichen Beirat eingeräumt wird, erwächst aus diesem Vorstoß keine fachlich steuernde Funktion im PBWU-Projekt.

Neben überwiegend organisatorisch wirksamen Maßnahmen findet eine vorhabenübergreifende, fachliche Gesamtkoordination des Forschungsprozesses im PBWU-Projekt während der Vorhabenbearbeitung weder durch das Gremium des Wissenschaftlichen Beirats, noch durch die Projektgruppe selbst statt. Hinsichtlich der Forschungssteuerung durch die verschiedenen politischen und wissenschaftlichen Akteure ist daher um so mehr Bedeutung der Antragsselektion und Erarbeitung von Förderentscheidungen zuzumessen. 


\subsubsection{Programmimplementation am alpinen Forschungsschwerpunkt Wank}

\subsubsection{Etablierung des Forschungsschwerpunktes „, Wank“}

Die Waldschadensforschung am alpinen Forschungsschwerpunkt Wank bei GarmischPartenkirchen stellt den zentralen und inhaltlich wie finanziell umfangreichsten Implementationsbestandteil des PBWU-Projektes dar. Das Kräftespiel zwischen politischen Steuerungsinteressen der Forschung, insbesondere in regionaler und thematischer Hinsicht, den wissenschaftlichen Interessen zur konzeptionellen und fachlichen Beteiligung und Einflußnahme, sowie der Programmdurchführungs- und Koordinierungsanspruch der intermediären Projektgruppe kann hier in einem eigenen Kapitel exemplarisch aufgezeigt werden.

\subsection{Forschungsrahmenplan und erste Anträge}

Die Absicht, die Waldschadensforschung im PBWU-Projekt standortbezogen zu betreiben und hierbei auch den Alpenraum zu berücksichtigen, wird frühzeitig artikuliert. Schon zur ersten Sitzung des Projektrats am 8.10.1984 schlägt die Projektgruppe in Abstimmung mit dem projektfinanzierenden Umweltressort einen alpinen, bayerischen Forschungsschwerpunkt als Bestandteil der künftigen Programmstruktur vor (i.Q.: PBWU, 8.10.1984).

Im ersten Entwurf des Rahmenprogramms der PBWU (i.Q.: PBWU, 1.3.1985) werden die Vorstellungen konkretisiert. Neben den bereits bestehenden, außerhalb des Projektes bearbeiteten und finanzierten Forschungsprogrammen im Fichtelgebirge, Bayerischen Wald und Höglwald ist der „Standortraum Kalkalpen“ als einziger neu zu schaffender Standort im Programm vorgesehen. Die Region um Garmisch-Partenkirchen wird dabei aufgrund langjähriger Messungen der Immissionssituation durch ein ortsansässiges, renommiertes Forschungsinstitut und nach Ortsbegehungen durch die Projektgruppe bereits favorisiert.

Die Bedeutung der Alpenwälder, deren Gefährdung durch verstärkt beobachtete Waldschäden sowie die unbefriedigende Forschungssituation werden als Begründung für einen alpinen Forschungsschwerpunkt angeführt. Dabei wird aufgrund der gegebenen Immissionssituation die „Überprüfung der Photooxidantien-Hypothese“ in den Mittelpunkt der dort geplanten Forschungsarbeiten gestellt. Das Rahmenprogramm sieht hypothesenorientiert eine breite Palette von Forschungsthemen zur Erfassung der wesentlichen Standortsparameter, zur differentialdiagnostischen Analyse von Schäden an Waldbäumen sowie zur Wirkung verschiedener Schadfaktoren auf die Pflanze vor. Wie im Ausschreibungstext vermittelt wird, ist beabsichtigt, die durchzuführenden Forschungvorhaben auf gemeinsame Untersuchungsflächen zu konzentrieren. Deren Auswahl sollte „durch die beteiligten Forschern im Einvernehmen“ erfolgen (Ausschreibungstext zum Rahmenprogramm, Stand 1.4.1985).

Auf der gemeinsamen Sitzung der PBWU-Gremien am 21.3.1985 wird der Entwurf des Rahmenprogramms diskutiert und ,grundsätzlich akzeptiert“ (i.Q.: PBWU, 21.3.1985). Dabei erhält laut Protokoll die Einrichtung eines Alpen-Forschungsschwerpunktes höchste Priorität gegenüber der Verstärkung der Forschung an bereits bestehenden Forschungsschwerpunkten und standortsunabhängigen Untersuchungen. 
Wie bereits an anderer Stelle dargelegt wurde (s. Kap. 5.2.2), besteht insbesondere seitens des Wissenschaftlichen Beirats jedoch erhebliche Kritik gegenüber dem Forschungsprogramm (i.Q.: Wissenschaftlicher Beirat, 2.5., 3.5., 8.5.1985 und ohne erkennbare Datumsangabe). Insbesondere der von der Projektgruppe verfaßte Ausschreibungstext zum Rahmenprogramm gäbe die auf der Gremiensitzung überarbeitete Gesamtkonzeption nicht adäquat wieder. Zudem würde die vorgegebene Vielzahl konkreter Forschungsthemen mehr zu einem „bunten Gemisch von Anträgen“ führen und die Kreativität der Wissenschaftler unterdrücken, als zu integrierter Forschung anregen. Das Rahmenprogramm wird von der PBWU daraufhin jedoch nur geringfügig modifiziert (Fassung vom 24.5.1985) und im Juni 1985 ausgeschrieben.

Auf die Ausschreibung hin werden der PBWU vorerst 11 Forschungsanträge vorgelegt, welche Arbeiten am geplanten Alpenstandort vorsehen (i.Q.: PBWU, Oktober 1985, ohne genaue Datumsangabe). 9 hiervon werden im Rahmen der ersten, mündlichen Begutachtungsrunde zur Förderung mit Mitteln in Höhe von insgesamt 6 Mio. DM empfohlen und im Verlauf des Jahres 1986 bewilligt (i.Q.: PBWU, 11.6.1986). Die Forschungsthemen betreffen

- das Ablagerungsverhalten von Niederschlägen,

- den pflanzlichen Gaswechsel und biochemische Parameter in Nadeln in Hinblick auf schadensbedingte Veränderungen,

- Wechselwirkungen zwischen Pflanze und Parasiten

- Elementanalysen in Jahrringen von Bäumen

- die Analyse von Witterungsdaten und

- pflanzensoziologische Aufnahmen der Bodenflora in Hinblick auf die Indikation immissionsbedingter Veränderungen.

Die zu bearbeitenden Themen führen bei den PBWU-Gremien zu der Feststellung, daß mit den vorliegenden Forschungsanträgen das Forschungskonzept bei weitem noch nicht ausgefüllt würde (i.Q.: $P B W U, 18.11 .1985$ ). Weitere potentielle Forschergruppen sollten hinsichtlich einer Beteiligung am alpinen Forschungsprogramm gezielt angesprochen werden. Der Wissenschaftliche Beirat, der am Förderverfahren selbst nicht beteiligt ist, verweist - neben der notwendigen Koordination der Forschungsarbeiten und der Zusammenführung der Arbeitsgruppen - auf die besondere Wichtigkeit der Standortsauswahl.

\subsection{Politischer Handlungsdruck zur Festlegung des Forschungsstandorts}

Noch bevor Förderentscheidungen getroffen werden, führt die PBWU mit den betreffenden Antragstellern sowie Vertretern des einschlägigen Forschungsinstitutes in Garmisch-Partenkirchen ein erstes „Informationsgespräch Alpenstandort“ durch (i.Q.: PBWU, 8.10.1985). Man einigt sich darauf, zur Überprüfung der Photooxidantien-Hypothese ein „Höhenprofil“, bestehend aus mehreren Untersuchungsflächen in den staatlichen Wäldern am Bergmassiv „Wank“ zu bearbeiten; weitere Geländebegehungen seien allerdings erforderlich.

Zu Beginn des Jahres 1986 lädt die PBWU zur Vorbereitung einer Ortsbegehung die Vorhabenleiter der zu bewilligenden Anträge zu einer weiteren Sitzung zum „Schwerpunktstandort Kalkalpen“ ein (i.Q.: PBWU 13.1.1986). Auch Mitglieder des Wissenschaftlichen Beirats, unter anderem der neue Leiter des ortsnahen Forschungsinstitutes, sind anwesend. Einige Sit- 
zungsteilnehmer sehen noch Bedarf, vorab die Konzeption des Forschungsansatzes bzw. die zu bearbeitende Arbeitshypothese zu diskutieren. „Wegen der zeitlichen Dringlichkeit der Ursachenklärung und der politischen Bedeutung" welche die PBWU unterstreicht, wird jedoch letztlich befürwortet, mit der Standortfestlegung und den eigentlichen Forschungsarbeiten unverzüglich zu beginnen, und nicht erst weitere, von Mitgliedern des Wissenschaftlichen Beirats geforderte Vorerkundungen abzuwarten. Abschließend wird der Wank als künftiger Forschungsstandort bestätigt. Dafür sprächen, wie aus dem Protokoll hervorgeht, insbesondere bereits vorhandene Daten, die Präsenz des Garmischer Forschungsinstituts sowie weitere standörtliche wie infrastrukturelle Gegebenheiten. Der Leiter des Forschungsinstituts stellt im Nachgang zu der Sitzung sein hohes Engagement in der alpinen Forschung am Wank in Aussicht (i.Q.: Wissenschaftlicher Beirat, 26.3.1986).

Ein Mitglied des Wissenschaftlichen Beirats und Vertreter der nordbayerischen Forschungsgruppe Forsttoxikologie äußert sich allerdings kritisch zu dem Sitzungsprotokoll der PBWU. Die Diskussion zu Untersuchungskonzept und Standortsfindung würde darin nicht zutreffend wiedergeben. Die im Protokoll aufgeführten Beschlüsse zum Forschungsstandort am Wank gingen über das hinaus, was im Verlauf der Sitzung tatsächlich vereinbart worden sei. Auf die Notwendigkeit entscheidungsvorbereitender Voruntersuchungen und Ortsbegehungen zur endgültigen Standortsfestlegung wird verwiesen (i.Q.: Wissenschaftlicher Beirat, 26.3.1986).

Die Ortsbegehung am 23.4.1986 verfolgt das Ziel, den Wank auf seine Eignung als Forschungsstandort hin zu überprüfen. Die Kritik des oben genannten Beiratsmitglieds, welches selbst an der Begehung teilnimmt, wird wiederholt und im Protokoll berücksichtigt. Es wird vermerkt, daß nach Meinung der Wissenschaftler die vorliegenden Informationen noch nicht ausreichten, die geplanten, umfangreichen Infrastruktur-Maßnahmen am Standort zu rechtfertigen. Detaillierte Vorerkundungen und Feinkartierungen zur fachlichen Absicherung des Forschungsstandortes würden daher gefordert. Bereits bewilligte Forschungsarbeiten müßten zwischenzeitlich auf vorläufig festzulegenden Probeflächen und noch ohne größere Infrastrukturmaßnahmen (Meßtürme), wie geplant sei, durchgeführt werden (i.Q.: PBWU, 23.4.1986). Die Vorerkundungen müßten abgewartet, erst entsprechend deren Ergebnissen dürfte mit den eigentlichen Untersuchungen begonnen werden (i.Q.: Wissenschaftlicher Beirat, 4.6.1986).

Während auf der Sitzung am 15.5.1986 Mitglieder des Wissenschaftlichen Beirats noch die Forderung wiederholen, die genaue Lage des Forschungsstandortes erst nach der Ergebnissen umfangreicher Voruntersuchungen zu definieren, kündigen die Vertreter des Umweltministeriums an, bereits in wenigen Wochen den Aufbau einer zentralen Fördermaßnahme „Basisstation“ am Wank finanzieren zu wollen. Sie verweisen dabei auf das Angebot des betreffenden Beiratsmitglieds, mit seinem Forschungsinstitut den Aufbau und Betrieb einer instrumentell und finanziell aufwendigen Meßstation auf einer Freifläche in mittlerer Hanglage am Wank (Basisstation) zu übernehmen und dies umgehend in die Wege zu leiten (i.Q.: PBWU, 15.5.1986). Die Station soll während der Laufzeit des Projektes relevante atmosphärische Meßdaten liefern, den beteiligten Forschergruppen zur Verfügung stellen und gleichzeitig als Labor- und Unterkunftsstation für den allgemeinen Forschungsbetrieb genutzt werden können (i.Q.: PBWU-Antrag 43-7/86, 16.7.1986) 
Wie bereits bei der Darstellung der Gesamt-Implementation des PBWU-Forschungsprogramms ausgeführt (Kap. 5.3.1), manifestiert sich die vom Umweltministerium betonte „höchste Priorität" des alpinen Forschungsstandorts im PBWU-Projekt (i.Q.: PBWU, 15.5.1986) in der Bevorzugung von Wank-Anträgen gegenüber anderweitigen Forschungsvorhaben. Ein erstes Bündel von Forschungsanträgen wird - ohne Einschaltung des Wissenschaftlichen Beirats in das Förderverfahren - bewilligt, noch bevor ein als geeignet ausgewiesener Standort gefunden ist. Der mehrfachen Forderung nach detaillierten Vorerkundungen wird lediglich über die Durchführung einer luftbildgestützten Kronenzustandserhebung durch einen Lehrstuhl der Forstwissenschaftlichen Fakultät nachgekommen. Ohne deren Ergebnisse jedoch abzuwarten, wird der Aufbau einer mit über 1 Mio. DM kostenintensiven und hinsichtlich der Lage des Forschungsstandorts Fakten schaffenden Meßstation am Wank von Projektgruppe und Ministerium vorgezogen und außerhalb der regulären Begutachtungsrunden behandelt.

Diese Vorgehensweise bewirkt bei zahlreichen Beiratsmitglieder erhebliche Kritik und Verärgerung über das Verfahren der Antragsbehandlung und gipfelt in einem entsprechenden Schreiben an den Bayerischen Umweltminister (i.Q.: Wissenschaftlicher Beirat, 11.7.1986). Der Minister unterstreicht zwar die politische Prioritätensetzung und bittet um Verständnis, daß nicht alle aus wissenschaftlicher Sicht wünschenswerten Vorhaben bewilligt werden könnten (i.Q.: Bayer. Umweltministerium, 13.8.1986). Er kann den Eindruck bei Teilen des Beirats, die den Forschungsstandort Wank als politische Vorzeigestation von Umweltministerium und PBWU sehen (i.Q.: Wissenschaftlicher Beirat, 22.10.1986), allerdings nicht entkräften.

Das Bewilligungsverfahren zu dem Antrag der Meßstation, zudem von einem Beiratsmitglied selbst vorgelegt, gestaltet sich denn auch problematisch. Zwar werden überwiegend positive, schriftliche Fachgutachten eingeholt. Angesichts des hohen Antragsvolumens und der zentralen Bedeutung für den Forschungsstandort möchten Mitglieder des Wissenschaftlichen Beirats das Vorhaben jedoch erst ausführlich diskutiert wissen; ohne dies könne keine Zustimmung gegeben werden (i.Q.: Wissenschaftlicher Beirat, 15.9., 22.9. 1986 und PBWU, 24.9.1986). Auch der Vertreter des Kultusministeriums im Projektrat rät an, den Wissenschaftlichen Beirat hierzu intensiver als über ein schriftliches Rundum-Verfahren zu beteiligen (i.Q.: Bayer. Kultusministerium, 1.10.1986).

Die PBWU empfiehlt dem Umweltministerium hingegen, „das Vorhaben so rasch wie möglich zu bewilligen“ (i.Q.: PBWU, 5.11.1986). Sie verweist auf die fachliche Notwendigkeit der Station für das Wankprojekt, die bereits erzielte allgemeine Akzeptanz des ForschungsGrundkonzeptes, die generelle Eignung des Standorts und letztlich verschiedene Sachzwänge. Auf der nächsten gemeinsamen Sitzung der PBWU-Gremien am 28.11.1986 wird die inzwischen vollzogene Bewilligung des Vorhabens aus den Reihen des Wissenschaftlichen Beirats zwar wiederum deutlich kritisiert, vom Vorhaben-leitenden Beiratsmitglied und dem Umweltministerium hingegen gerechtfertigt (i.Q.: PBWU, 28.11.1986).

Mit der Bewilligung des Vorhabens zum Aufbau und Betrieb einer Meßstation am Wank ist der Forschungsschwerpunkt faktisch etabliert. Bis zum Frühjahr 1987 kann der Meßbetrieb 
aufgenommen und die Station der allgemeinen Nutzung durch beteiligte Arbeitsgruppen zur Verfügung gestellt werden.

Ende 1986 kann die Projektgruppe in einem internen Papier zum Schwerpunktstandort Kalkalpen - Konzeption und geplante Arbeiten - vom November zwar auf die „stattliche Anzahl“ von insgesamt 10 Forschungsvorhaben am alpinen Forschungsstandort verweisen. Dennoch bestünden noch erhebliche Lücken im Untersuchungsprogramm. Das Papier wird daher zu einem Forschungskonzept ausgebaut, in dem die Arbeitshypothese dargestellt und laufende sowie geplante Forschungsarbeiten beschrieben werden (i.Q.: PBWU, Dez. 1986, ohne genaue Datumsangabe).

Die aufgrund des Vorerkundungscharakters mit Spannung erwarteten Ergebnisse der Luftbildauswertung zu den Waldschäden am Wank [AMMER et al. 1987] werden im Frühjahr 1987 vorgestellt (i.Q.: PBWU, 10.4.1987). An der Sitzung sind zahlreiche Wissenschaftler laufender oder beantragter Forschungsvorhaben, Vertreter des Projektrates und weitere Gäste beteiligt. Seitens des Gremiums des Wissenschaftlichen Beirats der PBWU nimmt lediglich der Vorhabenleiter der „Basisstation“ teil.

Als Ergebnis kann eine Höhenzonierung der Waldschäden am Wank mit Schadensmaxima in zwei Höhenstufen festgestellt werden. Auf die nur teilweise gegebene statistische Absicherung der Schadensmaxima wird im Rahmen der Diskussion kaum eingegangen. Vielmehr kann das Ergebnis vom Betreiber der Basisstation zur Stützung der am Forschungsstandort unterlegten Photooxidantien-Hypothese genutzt werden, wonach sich höhere Schadensniveaus mit Zonen höherer Photooxidantienbelastung an der Obergrenze winterlicher und sommerlicher Inversionslagen gut zur Deckung bringen ließen. Auch habe sich damit die bereits vorab getroffene Standortsfestlegung und insbesondere der Aufstellungsort der Basisstation im nachhinein weitgehend als richtig erwiesen. Der Leiter der PBWU schließt sich den Aussagen voll an.

\subsection{Etablierung des Wank als PBWU-Forschungsschwerpunkt}

Im Rahmen weiterer Ortsbegehungen am Wank im Frühling/Sommer 1987 wird die Detaileinrichtung des Forschungsstandortes durchgeführt. Es werden konkrete Untersuchungs- bzw. Probenahmeflächen definiert und Einzelbäume ausgewählt (i.Q.: $P B W U, 28.4$., 8.5. und 4.6.1987). Die an der Begehung vom 28.4. teilnehmenden Wissenschaftler hatten sich „nach ausführlicher und z.T. kontroverser Diskussion“ darauf geeinigt, den Ansatz zweier Intensivmeßflächen mit umfangreicher infrastruktureller Ausstattung durch ein Set von weiteren Probenahmeflächen im Rahmen eines Höhenprofils zu ergänzen. Dabei gestaltet sich die Auswahl geeigneter Versuchsflächen bzw. Einzelbäume aufgrund der verschiedenen zu erfüllenden Forschungskriterien einerseits, der heterogenen Bergwaldsituation andererseits, schwierig. So müssen erhebliche Kompromisse hinsichtlich der Eignung der Bestände eingegangen werden, z.T. wird das „Fehlen über Gebirgswald hinausgehender Krankheitssymptome“ bemängelt. Letztlich wird jedoch ein Kollektiv von Untersuchungsflächen fixiert, welches laut Protokoll zur Begehung vom 8.5.1987 die geforderten Kriterien „hinreichend erfüllt“ bzw. einen „durchaus akzeptablen Kompromiß“" darstellt. 
Die Bayerische Staatsforstverwaltung bietet Unterstützung forstlich-infrastruktureller Art (Zäunung, Wegebau etc.). Weitere Maßnahmen wie den Aufbau von Forschungsgerüsten zum Erreichen der Baumkronen und die Stromversorgung auf den Intensivmeßflächen übernimmt das die Basisstation betreibende Forschungsinstitut in einem gesonderten Auftrag. Die Arbeiten erfolgen in enger Abstimmung mit und unter Beteiligung der PBWU. Diese organisiert und führt Probenahmen durch und veranstaltet Arbeitsgruppenbesprechungen. Noch im Sommer 1987 zollt der Sprecher des Wissenschaftlichen Beirats den Aktivitäten der PBWU zur Organisation des Wank-Projekts „Anerkennung und Bewunderung “ (i.Q.: Wissenschaftlicher Beirat, 28.8.1987).

Wenngleich nach Meinung von Beiratsmitgliedern der Wank gegenüber anderen Forschungsschwerpunkten nach wie vor bevorzugt würde (i.Q.: Wissenschaftlicher Beirat, 19.10.1987), ist auch dieser von der das PBWU-Gesamtprojekt belastenden Finanzmittelknappheit der Jahre 1986/87 betroffen. Die Geschäftsführung der GSF moniert gegenüber dem Bayerischen Umweltministerium fehlende Fördermittel für fachlich notwendige Forschungsvorhaben, die den Erfolg des Programms gefährdeten (i.Q.: GSF, 22.10.1987). Auf der Projektratssitzung am 29.1.1988 unter Beisein des Beiratssprechers können jedoch weitere, den Wank betreffende Anträge aus der zweiten Begutachtungsrunde bewilligt werden. Insgesamt werden zu diesem Zeitpunkt nahezu 20 Forschungsvorhaben am Wank durchgeführt (i.Q.: PBWU, 29.1.1988).

\subsubsection{Wissenschaftliches Steuerungskomitee für den Wank}

Anläßlich einer der Wank-Begehungen im Frühjahr 1987 (i.Q.: PBWU, 28.4.1987) wird von den Teilnehmern ein leitender Mitarbeiter des ortsansässigen Forschungsinstituts ,pper Akklamation“ zum „Sprecher der Wissenschaftler“ gewählt. Außer der Anregung regelmäßiger Gespräche gehen nähere Ausführungen zu dessen Aufgaben aus dem Protokoll nicht hervor. Allerdings ergänzt der Leiter des Instituts in seiner Funktion als Mitglied des Beiratsgremiums gegenüber der PBWU, ob es nicht sinnvoll wäre, ein wissenschaftliches ,Steering Committee zu bilden“, welches bei der Erstellung des Forschungsprogramms sowie bei der Begutachtung der Forschungsanträge und der laufenden Arbeiten mitwirken könnte (i.Q.: Leitungskomitee, 29.4.1987).

Der neu gewählte Sprecher der Wissenschaftler, der gleichzeitig die Erstellung der Infrastruktur am Wank betreut, greift diese Idee auf. Konkret hätte dieses Gremium derzeit mindestens die als dringend erachteten Aufgaben, die bisher laufenden Vorhaben in Hinblick auf eventuell weiteren Forschungsbedarf zu sichten und die Verfahrensweise zur Probennahme am Wank neu zu regeln (i.Q.: Leitungskomitee, 13.8.1987).

\subsection{Kritik eines Wank-Forschers an der PBWU-Koordination}

Die relativ kurzfristige Einladung der PBWU zur ersten Probennahme der beteiligten Arbeitsgruppen am Wank ist für den Sprecher der Wissenschaftler Anlaß, Kritik an der Vorgehensweise der Projektgruppe zu formulieren (i.Q.: Leitungskomitee, 14.8.1987). Seiner Ansicht nach sei eine ,generalstabsmäßige Vorbereitung unbedingt erforderlich“, die noch verbleibende Frist zum Beprobungstermin zu kurz, so daß ,nicht mehr gutzumachende Folgen für das Ge- 
samtprojekt" befürchtet werden müßten. Die PBWU kommt der Anregung einer Vorbesprechung zur Beprobung nach (i.Q.: PBWU, 16.9.1987); die Probennahme selbst kann plangemäß stattfinden. Des weiteren bringt der Sprecher der Wissenschaftler im Rahmen eines Jahresberichtes, den er an die am Wank beteiligten Forscher übersendet, deutlich seine Besorgnis über die trotz Zusage der PBWU noch immer nicht vorliegenden Finanzmittel zur Durchführung von Infrastrukturmaßnahmen am Wank zum Ausdruck (i.Q. Leitungskomitee, 22.12.1987). Auch die erforderliche ,nachdrückliche Intervention beim Projektträger“" zur adäquaten Vorbereitung der ersten Probennahme wird erwähnt. Der Bericht schließt mit der Ankündigung, ein wissenschaftliches Leitungskomitee für den Wank einzurichten. Der Leiter der PBWU sieht sich veranlaßt, den Vorwürfen zu entgegnen und die Bemühungen der Projektgruppe zur Projektkoordination am Wank entgegenzuhalten (i.Q.: PBWU, 28.12.1987). Ein weiterer Schriftwechsel folgt (i.Q.: PBWU, 2812.1987; Leitungskomitee, 7.1.1988).

\subsection{Gründung eines wissenschaftlichen Leitungskomitees für den Wank}

Noch im Dezember 1987 bittet der Sprecher der Wissenschaftler fünf renommierte Forscher, die selbst am Wank beteiligt sind oder dies beabsichtigen und z.T. dem Wissenschaftlichen Beirat angehören, zur Mitarbeit in einem Leitungskomitee für den alpinen Forschungsschwerpunkt (i.Q.: Leitungskomitee, 21.12.1987). Auch der Leiter der PBWU wird hierzu aufgefordert. Vordringlichste Aufgabe sei die Überarbeitung der bestehenden Konzeption der PBWU und die Übernahme der wissenschaftlichen Koordination der Arbeiten am Standort.

Die angeschriebenen Wissenschaftler schließen sich dem Vorschlag gerne an. Die Bereitschaft zur Beteiligung wird z.T. mit den Argumenten eigener Forschungsarbeiten am Standort und möglicher Forschungskooperationen ergänzend begründet. Dabei werde hohes Niveau des wissenschaftlichen Konzepts sowie der finanziellen Ausstattung am Forschungsstandort gefordert (i.Q.: Wank-Forscher 7.1. und 6.3.1988).

Auf der konstituierenden Sitzung des wissenschaftlichen Leitungskomitees für den Schwerpunkt „Kalkalpen“ am 8.2.1988, an der auch der Leiter der PBWU teilnimmt, herrscht Übereinstimmung, daß die Forschungskonzeption am Wank klarer ausgerichtet und die Koordinierung der Forschungsarbeiten gestrafft werden müsse. Der Wissenschaftliche Beirat der PBWU solle damit in seiner Funktion nicht beeinträchtigt, sondern ergänzt werden (i.Q.: Leitungskomitee, 8.2.1988). Aufgaben des Komitees seien,

- ein mittelfristiges Forschungskonzept zu erstellen,

- die Forschung zu koordinieren,

- eingehende Anträge hinsichtlich ihrer Konzeptkonformität zu bewerten,

- Forschungslücken aufzudecken und Vorschläge zu deren Schließung zu unterbreiten,

- Arbeitstreffen und Workshops einzuberufen sowie

- gemeinsame Forschungsberichte etc. abzufassen und herauszugeben.

Zur Aufgabenerfüllung müsse das Komitee allerdings vom Wissenschaftlichen Beirat mit entsprechenden Entscheidungskompetenzen ausgestattet werden.

\subsection{Diskussion des Forschungskonzeptes des Leitungskomitees}


Den ersten Vorschlag eines Forschungskonzepts legt der Sprecher des Komitees im März 1988 vor (i.Q.: Leitungskomitee, 15.3.1988). Unter dem Dach der Photooxidantien-Hypothese enthält es verschiedene Detail-Hypothesen zu den Schadursachen im alpinen Bergwald. Eine allgemeine Zielsetzung, die geplanten Untersuchungsstrategien und ein detaillierter Forschungsplan folgen. Anläßlich der erster Diskussion des Konzeptvorschlags herrscht Übereinstimmung, daß die Arbeit in einem derartigen Leitungsgremium nur bei mittelfristiger Finanzierung, welche eine Forschungsplanung überhaupt ermöglichte, sinnvoll sei (i.Q.: Leitunmgskomitee, 25.3.1988).

In überarbeiteter Form wird das Forschungskonzept, Stand 28.3.1988, von dessen Sprecher den PBWU-Gremien vorgelegt. Im Anschreiben werden die PBWU-Gremien über die Konstituierung des Leitungskomitees in Kenntnis gesetzt. Es werde gebeten, das Forschungskonzept kritisch zu prüfen und bei Zustimmung die erforderlichen Mittel zu seiner Realisierung bereitzustellen (i.Q.: Leitungskomitee, 28.3.1988). Auf der am darauffolgenden Tag stattfindenden Sitzung der PBWU-Gremien wird hierzu lediglich konstatiert, daß das Vorgehen des Leitungsgremiums am Wank in etwa dem Vorschlag des Wissenschaftlichen Beirats entspräche, regionale oder fachliche Koordinationszentren zu bilden. Aufgrund weiteren Abstimmungsbedarfs wird das Papier bis zur nächsten Sitzung zurückgestellt (i.Q.: PBWU, 29.3.1988).

Unter den am Wank beteiligten Forschern wird das Konzeptpapier auf deren Arbeitsgruppenbesprechung am 13.4.1988 jedoch ausführlich diskutiert. Die PBWU hält es für erforderlich, auf ihr bereits bestehendes Rahmenprogramm aus dem Jahr 1995 sowie ihr Konzeptpapier zur Wank-Forschung hinzuweisen, in dem wesentliche Teile des neuen Forschungskonzeptes bereits enthalten seien. Von einigen Wank-Forschern werden der Entwurf des Konzeptpapiers fachlich-inhaltlich kritisiert und Modifizierungen gefordert. Diese betreffen insbesondere die zusätzliche Einbeziehung von Forschungsthemen, die deren Vertreter in dem Papier zu wenig repräsentiert sehen. Besonders kontrovers werden die beabsichtigte Antragsvorbewertung und Ergebnispräsentation durch das Komitee diskutiert. Dies wird von der Mehrzahl der Anwesenden als „Bevormundung“ und gegen die Unabhängigkeit der Forschung gerichtet empfunden. Die Entfernung entsprechender Passagen aus dem Aufgabenkatalog wird daher gefordert (i.Q.: $P B W U$, 13.4.1988).

$\mathrm{Da}$ das Konzeptpapier in seinen Grundlagen, nämlich der Hypothesenbildung und deren Begründung, sowie dem gewählten Untersuchungsansatz nicht unumstritten ist, zeigen auch kritische Anmerkungen von Wank-Forschern, Komitee-Mitgliedern und der PBWU, die im Sommer 1988 um schriftliche Änderungsvorschläge gebeten werden (i.Q.: Wank-Forscher, 16.8., 30.8. bzw. 31.8.1988).

Das vorgesehene Aufgabenspektrum des Komitees ist auch für das Bayerische Umweltministerium Anlaß zur Stellungnahme. In seinem Schreiben an den gesamten Wank-Adressatenkreis wird ausgeführt, daß die Organisation und Koordination der staatlich finanzierten, bayerischen Waldschadensforschung und damit insbesondere die Durchführung von Begutachtungsverfahren, Workshops, Ergebnisbewertung etc. der PBWU oblägen. Weitere Gremien neben dem Wissenschaftlichen Beirat und Projektrat sowie den Gutachtern seien weder vorgesehen, noch sei eine Aufgabenverlagerung von der PBWU auf andere Institutionen geplant. Im Rahmen der 
bestehenden Strukturen werde die Zusammenarbeit der beteiligten Arbeitsgruppen jedoch unterstützt (i.Q.: Bayer. Umweltministerium, 16.5.1988).

Auch gegenüber einem führenden Vorhabenleiter am Wank, Mitglied des Wissenschaftlichen Beirats und Leitungskomitees zugleich, äußert das Ministerium seine Sorge um eine Verlagerung von PBWU-Aufgaben auf das Leitungskomitee. Der Wissenschaftler betont jedoch die Notwendigkeit eines neuen Konzeptes und hält es für sinnvoll, daß die mit dem Forschungsstandort vertrauten Wissenschaftler auch Einfluß auf die Prioritätensetzung bei der Forschungsförderung und der Bewertung der Ergebnisse nehmen könnten (i.Q.: Bayer. Umweltministerium, 2.8.1988). Allein die vom Umweltministerium tolerierte Beteiligung des Gremiensprechers bei Rahmenprogrammerstellung und Behandlung von Forschungsanträgen erscheint dem Leitungskomitee jedoch nicht ausreichend. Es besteht weiterhin auf den im Konzept beschriebenen Aufgaben; die der PBWU seien demgegenüber vielmehr im administrativen Bereich anzusiedeln (i.Q.: Leitungskomitee, 22.9.1988).

Erst auf der Sitzung vom 15.12.1988 kann der Sprecher des Leitungskomitees den PBWUGremien die Forschungskonzeption für den Wank selbst vorstellen (i.Q.: PBWU, 15.12.1988). Vom Wissenschaftlichen Beirat wird eine grundsätzlich positive Stellungnahme zu dem Konzeptpapier abgegeben. Das Umweltministerium wiederholt allerdings die Aussage, daß es über die PBWU hinaus keiner weiteren koordinierenden Institution bedürfe, wenn der Wissenschaftliche Beirat seine beratende Funktion „ernst nähme“. Angesichts der angespannten Finanzlage wird die Diskussion jedoch auf die nächste Sitzung zurückgestellt.

Der Sprecher des Leitungskomitees teilt die Ergebnisse der Sitzung seinen Kollegen mit (i.Q.: Leitungskomitee, 21.12.1988). Er schildert die Bedenken des Umweltministeriums sowie die Vertagung des Themas, da vom Wissenschaftlichen Beirat noch keine Stellungnahme zu dem Konzeptpapier abgegeben worden sei, welche das Ministerium aber wiederum erwarte. Die als „katastrophal“ empfundene Situation zahlreicher Forschungsanträge einerseits, knapper Finanzmittel andererseits, lassen den Sprecher „ernsthaft die Frage“ stellen, „ob es überhaupt sinnvoll ist, das Forschungskonzept weiter zur Förderung über die PBWU vorzusehen“".

Auf der nächsten Sitzung der PBWU-Gremien steht das Forschungskonzept des Leitungskomitees wiederum auf der Tagesordnung. Dessen Sprecher unterstreicht, daß weder ein zu den PBWU-Gremien konkurrierendes Gremium, noch eine Vorbegutachtung von Anträgen beabsichtigt sei. Ansonsten werden weitgehend die bereits bekannten Standpunkte ausgetauscht (i.Q.: PBWU, 14.3.1989). Die abschließende Festlegung, wonach sich der Beirat erst dann mit dem Konzeptpapier befassen solle, wenn es aktualisiert sei, hält der Sprecher des Leitungskomitees allerdings für ,unzumutbar“. Im Schreiben vom Mai 1989 an die PBWU führt er aus, daß das Papier seit über einem Jahr dem Gremium vorläge, „ohne daß es zu einer detaillierten Stellungnahme (...) gekommen wäre“. Zwar sei eine Aktualisierung erforderlich, diese sollte jedoch auf der Basis von Empfehlungen des Beirats erfolgen (i.Q.: Leitungskomitee, 8.5.1989).

In seiner Übermittlung der Sitzungsergebnisse an die Kollegen kann der Sprecher des Leitungskomitees jedoch auch auf einige positive Aspekte verweisen. So schiene das Bayerische Umweltministerium ,jetzt eher die Namensgebung zu stören als das Gremium als solches“. 
Auch die finanzielle Situation der Forschungsförderung stelle sich ,nicht mehr ganz so hoffnungslos" dar wie früher. Nochmals hätte allerdings deutlich gemacht werden müssen, daß dringend die Empfehlungen des Wissenschaftlichen Beirats zu dem Forschungskonzept erwartet würden (i.Q.: Leitungskomitee, 9.5.1989).

Das erste Statusseminar der PBWU im Februar/März 1989 zum Forschungsschwerpunkt Waldschäden [PBWU 1989] nutzt der Sprecher des Leitungskomitees dazu, das Forschungskonzept öffentlich vorzustellen [S. 351-356]. Er verbindet die inhaltliche Präsentation mit deutlicher Kritik an der bisherigen Wank-Forschung und der zögerlichen Behandlung des Konzeptes in den PBWU-Gremien.

\subsubsection{Kritik externer Wissenschaftler an der Wank-Forschung}

Die weitere inhaltliche Auseinandersetzung mit dem Forschungskonzept wird maßgeblich durch einen Anstoß von außen in Gang gebracht. Er geht auf vier renommierte und z.T. in der Waldschadensforschung engagierte Münchner Botanik-Professoren zurück.

In einem gemeinsam unterzeichneten Schreiben der Wissenschaftler an die PBWU-Gremien werden ,ernste Befürchtungen für die weitere Entwicklung des Wank-Projekts“ geäußert (i.Q.: $P B W U$, 8.3.1989). Anlaß seien die Ausführungen des Sprechers des Wissenschaftlichen Beirats zur Hypothesenbildung und -überprüfung sowie zum Einsatz der Statistik in der Waldschadensforschung anläßlich seiner Schlußworte zum ersten Statusseminar der PBWU [PBWU 1989, S. 497-506]. Projiziert auf den Forschungsstandort Wank würden hieraus erhebliche Kritikpunkte an der dortigen Forschung erwachsen. Letztlich, so wird resümiert, würde das „Wankprojekt nur eine Meß- und Sammelaktion“ darstellen, „die zu einer nicht auswertbaren Datenflut führt“ Die PBWU-Gremien werden gebeten, die „Bedenken bei der weiteren Behandlung des Wankprojektes zu berücksichtigen“.

Der Sprecher des Beirats antwortet den Professorenkollegen (i.Q.: Wissenschaftlicher Beirat, 17.3.1989). Er verwahrt sich gegenüber den Vorwürfen, diskutiert verschiedene Kritikpunkte und bietet ein vertiefendes Gespräch an. Da der Beirat, ,ja nicht verantwortlich für die Versuchsplanung am Wank" sei, schlägt er vor, die Forschungskonzeption am Wank in einer gemeinsamen Sitzung der kritisierenden Münchner Professoren mit dem Wissenschaftlichen Beirat und dem Wank-Leitungskomitee zu diskutieren. Die längst fällige Aktualisierung des vorliegenden Konzeptpapiers durch letztgenanntes Gremium könnte gleichzeitig miteinbezogen werden (i.Q.: Wissenschaftlicher Beirat, 16.5.1989; PBWU, 22.5. und 8.6.1989).

Der Sprecher des Leitungskomitees zeigt Bereitschaft zu einer gemeinsamen Diskussion. Er empfiehlt allerdings, den Wissenschaftlichen Beirat von einer „Oberbegutachtung“ durch die Kritiker freizuhalten Das Umweltministerium sieht in der geplanten Vorgehensweise die Möglichkeit, unter Einbeziehung von Empfehlungen des Wissenschaftlichen Beirats zur Aktualisierung des Konzeptpapiers ,,in einem iterativen Verfahren zu einem aus Sicht des Forschungsschwerpunkts optimalen Projekt zu kommen“(i.Q.: PBWU, 24.5. 1989; Bayer. Umweltministerium, 27.6.1989). 
Die Münchner Professoren hingegen lehnen es im Grundsatz ab, während des internen Abstimmungsprozesses zwischen Leitungskomitee und Wissenschaftlichem Beirat in die Diskussion über die Wank-Konzeption einzutreten. Die Diskussionsbereitschaft bestünde nur gegenüber den PBWU-Gremien, die für die Forschung am Wank verantwortlich seien. Dennoch lieBen sie sich eine Beteiligung an der Diskussion offen (i.Q.: PBWU, 10.7.1989; i.Q.: externe Kritiker, 18.7.1989). Von den PBWU-Gremien hingegen wird diese Vorstellung nicht akzeptiert. Die Kritiker-Gruppe sollte durchaus zu einem konstruktiven Beitrag gewonnen werden; dies müsse aber in gemeinsamer Diskussion mit dem Leitungskomitee erfolgen (i.Q.: PBWU, 27.7.1989 und 24.7.1989).

Die gegebene Situation veranlaßt den Sprecher des Leitungskomitees, , noch vor einer weiteren Diskussion im Wissenschaftlichen Beirat" sein Forschungskonzept zu überarbeiten. Er legt einen aktualisierten Entwurf des Wank-Konzepts vor (Forschungskonzept des Schwerpunktstandorts Kalkalpen, Stand 20.12.1989; i.Q.: Leitungskomitee, 21.12.1989). Neue Forschungsergebnisse seien eingearbeitet und Änderungsvorschläge weitgehend berücksichtigt. Diese beträfen unter anderem die Umbenennung des Leitungskomitees in „Forschergruppe Wank“ und den Verzicht auf die ursprünglich formulierte Aufgabe, eine „,wissenschaftliche Vorbewertung der eingehenden Anträge“ vorzunehmen.

Nach Ansicht der PBWU würden in dem Konzeptpapier jedoch nach wie vor Zuständigkeiten und Kompetenzen der Forschergruppe gefordert, die zu Überschneidungen mit denen der PBWU führen und die „,Organisation der bayerischen Forschungskoordination wesentlich verändern würden“. So sei die Überprüfung der Anträge auf ihre Konzeptkonformität in dem Papier ebenso vorgesehen wie die Koordination der Arbeiten am Forschungsschwerpunkt Wank. Das Ministerium werde daher um eine Klarstellung gebeten (i.Q.: PBWU, 8.1.1990). Diese erfolgt noch vor der gemeinsamen Diskussion zum Forschungskonzept. Darin wird die Bildung der Forschergruppe Wank, an der alle betroffenen Wissenschaftler gleichberechtigt seien, zwar begrüßt. Die bisherige Struktur der PBWU-Gremien müsse jedoch erhalten bleiben. Es wird daher ein Vorschlag unterbreitet, welcher die entsprechenden Formulierungen im Konzeptpapier entsprechend modifiziert (i.Q.: Bayer. Umweltministerium, 7.2.1990).

Mit Übersendung eines aktualisierten Papiers lädt die PBWU zur gemeinsamen Diskussion der Konzeption für den alpinen Forschungsschwerpunkt ein (i.Q.: PBWU, 3.1. und 4.1.1990). Die Kritiker teilen zwar mit, terminlich verhindert zu sein, aber rechtzeitig vor der Sitzung eine „ohnehin klarere, schriftliche Stellungnahme“ vorzulegen (i.Q.: externe Kritiker, 10.1.1990). Diese, alsbald an die Sitzungsteilnehmer übermittelt, konzentriert sich auf die dem Forschungskonzept zugrundeliegenden Prämissen zur Bearbeitung der Photooxidantien-Hypothese. Nach deren kritischer Diskussion wird der Schluß gezogen, daß keine der unterstellten Voraussetzungen gegeben sei. Das Konzept sei als Grundlage für ein Schwerpunktprojekt daher ungeeignet, die Durchführung des Forschungsplans könne nicht empfohlen werden (i.Q.: externe Kritiker, 8.2.1990).

Die Stellungnahme steht neben dem Konzeptpapier der neu benannten Forschergruppe Wank im Mittelpunkt der Diskussionsrunde mit dem Wissenschaftlichen Beirat vom 13.2.1990. Einige der detaillierten wie grundlegenden Kritikpunkte könnten nach Auffassung der Teilnehmer 
entkräftet oder für die Forschungskonzeption als nicht relevant angesehen werden, andere müßten hingegen als berechtigt akzeptiert werden. Als Ergebnis der Diskussion werden einige Anregungen entwickelt oder aus der Stellungnahme der Kritiker übernommen, die die erhobene Kritik entkräften bzw. das Konzeptpapier ergänzen sollen (i.Q.: Wissenschaftlicher Beirat, 13.2.1990). Auf der kurz darauf stattfindenden Sitzung der PBWU-Gremien werden die Ergebnisse der Diskussion vorgestellt. Es wird festgelegt, daß das Forschungskonzept im Hinblick auf die nicht auszuräumenden Kritikpunkte revidiert und dem Wissenschaftlichen Beirat zur Abstimmung gegeben werden müsse (i.Q.: PBWU, 20.2.1990).

\subsubsection{Weitere Behandlung des alpinen Forschungskonzepts}

Entsprechend den Ergebnissen der PBWU-Gremiensitzung vom 20.2.1990 überarbeitet der Sprecher der Forschergruppe Wank das Forschungskonzept (i.Q.: Forschergruppe Wank, 26.4.1990). Bis zur nächsten Sitzung der PBWU-Gremien am 17.7.1990 liegt jedoch noch keine neue Fassung vor. Es hätten sich, wie der Sprecher auf der Sitzung ausführt, neue Aspekte ergeben, die ,zuerst noch der Prüfung und Diskussion“ bedürften. Entgegen den bisherigen Ergebnissen zur Höhenverteilung der Schäden, die mit dem Höhengradienten der Ozonverteilung synchronisiert und damit zur Begründung der Photooxidantien-Hypothese herangezogen werden konnten, zeigen die aktuellen Ergebnisse nunmehr einen nahezu gegengerichteten Verlauf. Die aktuellen Maxima der Schäden deckten sich nicht länger mit den früher ausgewiesenen Höhenstufen [MÖSSMER et al. 1990, S. 14ff.; s.a. REUTHER et al. 1993, S. 8]. Abgesehen von Zweifeln an der Verläßlichkeit der Ergebnisse der Waldzustandserhebungen am Wank würde mit einer derartigen Schadensverteilung der Photooxidantien-Hypothese eine wesentliche Argumantationsbasis entzogen (i.Q.: PBWU, 20.2.1990).

Auf den nächsten Sitzungen der PBWU-Gremien wird die Wank-Forschungskonzeption ausführlich und z.T. kontrovers diskutiert. Es wird eingeräumt, daß die aktuellen Erhebungsergebnisse „die Photooxidantien-Hypothese am Wank nicht erhärten“ könnten. Das Konzept müsse neu überdacht und gegebenenfalls allgemeiner formuliert werden (i.Q.: $P B W U$, 11.12.1990). Auch ein überarbeitetes Forschungskonzept (Stand 18.2.1991) focussierte nach Ansicht des Beirats noch immer zu stark auf die Koinzidenz zwischen Schadens- und Ozonverteilung. Als Kompromiß wird festgelegt, das Forschungskonzept sowie ein aktuelles Ergebnispapier der PBWU durch den Sprecher der Forschergruppe Wank, den Leiter der PBWU und ein Mitglied des Wissenschaftlichen Beirats ,zu einer gemeinsamen Dokumentation zusammenzufassen“, die das Bayerisches Umweltministerium als „Leitlinie für die künftigen Forschungsaktivitäten im Alpenraum“" verwenden könnte (i.Q.: PBWU, 26.2.1991).

Eine nochmals überarbeitete Version des Forschungskonzeptes (Stand: 17.5.1991) stellt die Diskussionsgrundlage für die Beiratssitzung am 20.6.1991 dar. Dort wird der Verlauf der Waldschadensforschung am Wank hinsichtlich der Abstimmung zwischen PBWU und Wissenschaftlichem Beirat in früheren Jahren, der Funktion des Leitungsgremiums und der Behandlung des Forschungskonzeptes durch den Beirat rückblickend als unbefriedigend bezeichnet. Das vorliegende Konzept repräsentiere nach wie vor überwiegend die ehemalige WankKonzeption. Da sich die Waldschadensforschung derzeit im Umbruch befände, müßte als Ausgangsbasis für eine zukunftsorientierte Konzeption jedoch ein neues Papier erarbeitet werden, 
mit dem auch „Politiker überzeugt und Fördermittel eingeworben werden könnten“. Trotz einiger wissenschaftlicher Bedenken wird jedoch zugestanden, mit dem vorliegenden Papier zumindest ein ,retrospektives“ Rahmenprogramm für die bisherige Wank-Forschung mit Ausblick auf künftige Forschungsschwerpunkte geschaffen zu haben (i.Q.: PBWU, 20.6.1991).

Der Beschluß des Beirats, das Forschungskonzept in Zusammenarbeit mit dem Leiter der PBWU zu einem konsensfähigen Papier zu modifizieren, gelingt letztlich doch, wenn auch unter Protest des Sprechers der Forschergruppe Wank (i.Q.: Forschergruppe Wank, 5.7.1991; PBWU, 18.7.1991). Nach Einarbeitung letzter Änderungen (i.Q.: PBWU, 7.8. und 14.8.1991; Forschergruppe Wank, 9.8.1991) kann das Forschungskonzept unter gemeinsamer Autorenschaft den PBWU-Gremien zur Kenntnis gegeben (i.Q.: PBWU, 4.9.1991) und in der Vortragssammlung zum zweiten PBWU-Statusseminar zum Thema „Waldschäden“ publiziert werden [PBWU 1991, S. 15-40]. An einer Übereinstimmung zwischen Höhenverteilung der Waldschäden und Inversionslagen ist nun nicht mehr explizit festhalten. Auch sind keine Ausführungen mehr zum Gremium der Forschergruppe Wank und den in der Kritik stehenden Aufgabenstellungen enthalten.

\subsubsection{Theorieorientiertes Zwischenergebnis}

\subsection{Forschungsprozeß}

Die fachliche Legitimation für einen Forschungsstandort im bayerischen Alpenraum ist gegeben. Die politische Vorgabe, daß der Etablierung eines alpinen Standorts höchste Priorität gegenüber anderen Fördermaßnahmen zukommt, wird bereits bei der Rahmenplanung zum PBWU-Projekt unterstrichen.

Die Beteiligung der Forscher am alpinen Standort kommt allerdings nur langsam in Gang. In der ersten Antragsrunde zum PBWU-Projekt stellen rund 1/3 aller Anträge Beiträge zur alpinen Waldschadensforschung dar. Noch vor der Entscheidung über Fördermaßnahmen wird der „Wank“ bei Garmisch-Partenkirchen als Forschungsstandort favorisiert. Anträge werden bewilligt, noch bevor eine über das Rahmenprogramm hinausgehende Detailplanung ausformuliert und der Standort selbst mit konkreten Untersuchungsflächen ausgewählt ist.

Einige Mitglieder des Wissenschaftlichen Beirats, insbesondere Vertreter nordbayerischer Waldschadensforschung, stehen dem geplanten Alpenstandort daher kritisch gegenüber. Die Prioritätensetzung läßt zudem annehmen, daß die Fördermittel zulasten der übrigen Forschungsstandorte verteilt werden dürften. Zwar fordern Beiratsmitglieder wiederholt forschungskonzeptionelle Diskussionen und die Durchführung von Vorerkundungen zur Auswahl des Forschungsstandorts. Mit fachlichen Indizien, günstigen infrastrukturellen Voraussetzungen sowie unter Hinweis auf zeitliche Dringlichkeit und die politische Bedeutung setzen sich jedoch die politischen Akteure Umweltressort und PBWU durch. Das Engagement des stark an der alpinen Forschung interessierten Leiters eines ortsansässigen Forschungsinstitutes, gleichzeitig Mitglied im Wissenschaftlichen Beirat, erleichtert fachlich-argumentativ und in der praktischen Umsetzung die Etablierung des Forschungsstandortes. Diesen gegen die Kritik aus dem 
wissenschaftlichen Beratungsgremium politisch durchgesetzt $\mathrm{zu}$ haben, stellt jedoch ein schwerwiegendes und anhaltendes Akzeptanzproblem der PBWU dar.

Spätestens durch die Bewilligung des Vorhabens zum Aufbau einer zentralen ImmissionsMeßstation ist der Forschungsstandort am Wank räumlich definiert. Eine wissenschaftliche Legitimation erfährt er im nachhinein dadurch, daß die Ergebnisse der Waldzustandserhebung am Wank die unterlegte Photooxidantien-Hypothese - zumindest vorerst - stützen. Die bestehende oder mutmaßliche Koinzidenz zwischen Schadensverteilung und Schadstoffbelastung paßt ins Konzept der Waldschädigung durch Photooxidantien. Sie bildet die Basis dafür, mit der Forschung am Standort fortzufahren und den Bedarf an weiteren Fördermitteln zu begründen. Auch die Einrichtung und Bearbeitung eines Wank-Höhenprofils, bestehend aus mehreren, z.T. infrastrukturell intensiv ausgestatteten Bestandes-Untersuchungsflächen erscheint plausibel. Die erheblichen Kompromisse, die aufgrund der standörtlichen Gegebenheiten eingegangen werden müssen, werden von den Wank-Befürwortern akzeptiert.

In der Folge können zahlreiche Forschungsvorhaben am Wank etabliert werden. Insgesamt wird nahezu die Hälfte aller Forschungsvorhaben des PBWU-Projektes am Wank durchgeführt. Die Fördersumme beträgt über die gesamte Projektlaufzeit nahezu 15 Mio. DM [REUTHER et al. 1993, Anhang 4].

Bald nach Etablierung der Wank-Forschung wird von seiten des stark involvierten Forschungsinstitutes in Garmisch-Partenkirchen gefordert, ein wissenschaftliches Leitungskomitee für den Forschungsschwerpunkt einzurichten. Kritik an der Koordinationsfunktion der PBWU am Wank ist geeignet, die Argumentationsgrundlage hierfür zu verbessern. Dem alsbald gegründeten Komitee gehören Vertreter der Wank-Forschung, Mitglieder des Wissenschaftlichen Beirats sowie der Leiter der PBWU an. Das Aufgabenspektrum des Komitees, zu dem unter anderem Forschungskoordination und Antragsvorbegutachtung gehören sollen, kollidieren jedoch nach Auffassung des Umweltministeriums mit denen der PBWU; die Verfahrensneutralität im Projekt wird als gefährdet angesehen. Die weitgehende Rücknahme derartiger Aufgaben und Reduzierung auf wissenschaftliche Kooperation führt erst zur Akzeptanz des Gremiums durch das Ministerium.

Das vom Sprecher des Gremiums ausgearbeitete Forschungskonzept für den alpinen Forschungsschwerpunkt bleibt in den PBWU-Gremien lange Zeit unbehandelt. Erst der kritische Input externer Wissenschaftler an der bisherigen Wank-Forschung rückt es in den Mittelpunkt der Diskussion. Die Auseinandersetzung mit der Kritik wird verlagert auf die inhaltliche Beschäftigung mit dem Konzeptpapier des Leitungsgremiums. Berechtigte Kritikpunkte sollen zur Optimierung des Papiers verwendet werden. Diese betreffen insbesondere die grundlegenden Annahmen zu Waldschadensverteilung und Immissionssituation für die Formulierung der Photooxidantien-Hypothese am Standort. Sie werden nicht nur von den externen Kritikern, sondern aufgrund aktueller Ergebnisse der Waldzustandserhebung am Wank auch innerhalb der PBWU-Gremien in Zweifel gezogen. Das Forschungskonzept kann daher nur unter Abschwächung bzw. Verallgemeinerung bestimmter Hypothesen-Grundlagen aufrecht erhalten werden. Es kann dann aber nach Ansicht des Wissenschaftlichen Beirats nicht mehr als Grundlage für künftige alpine Waldforschung dienen. Dem Papier wird nurmehr der Charakter eines retro- 
spektiven Rahmenprogramms unter Würdigung der Diskrepanz einstiger Hypothesengenerierung und aktueller Ergebnislage zugestanden.

Die Projektgruppe kann trotz der schwindenden Relevanz des Konzeptpapieres ihre Beteiligung daran und die damit signalisierte Akzeptanz in ihren Gremien als Erfolg verbuchen (s.a. i.Q.: Wissenschaftlicher Beirat, 22.7. und 1.8.1991). Für die Fortführung der Waldschadensforschung erscheint allerdings eine weitere konzeptionelle Phase unumgänglich. Für den bestehenden Standort Wank selbst sind aufgrund der Hypothesen- und Ergebnislage sowie aufgrund mangelnder Akzeptanz in den PBWU-Gremien künftig lediglich abschließende Arbeiten vorstellbar und insgesamt ein Auslaufen des Forschungsprogramms absehbar. Das langzeitige Festhalten an der ursprünglichen Hypothese bzw. der hierfür erforderlichen Prämissen hatte dem Schwerpunkt eher geschadet als genutzt.

\subsection{Top down- und bottom up-Merkmale der Programmimplementation am alpinen Forschungsschwerpunkt}

Der Wank als einziger neu gegründeter, regionaler Forschungsschwerpunkt im PBWU-Projekt weist am ehesten Züge eines problemlösungsorientierten Programmimplementation nach dem top down-Ansatz auf. Das Ziel, Waldschäden eines vorgegebenen Forschungsstandorts im Bergwald hinsichtlich der Wirkung von Photooxidantien zu erforschen, wird politisch vorgegeben. Es wird gegen z.T. erheblichen Widerstand des wissenschaftlichen Beiratsgremiums mit dem Argument politischen Handlungsdrucks und durch den Einsatz finanzieller Steuerungsmacht des auftraggebenden Ressorts durchgesetzt.

Mit der Erkenntnis, daß die breite Programmausschreibung den gewünschten Erfolg, einen neuen Forschungsschwerpunkt ausreichend mit Forschungsinhalten zu etablieren, nicht erzielen kann, werden im PBWU-Projekt in der Folge (zur zweiten Antragsbegutachtung) weitere Elemente des ,top down“-Ansatzes der Programmimplementation eingesetzt.

So sollen einzelne Antragsteller gezielt auf eine Beteiligung am Forschungsschwerpunkt Wank angesprochen werden. Einzelne, aber unsystematische Vorgespräche der Projektgruppe mit Antragstellern finden statt, die als Anzeichen für eine Abstimmung des Forschungskonzeptes und das aktive Bemühen gewertet werden können, geeignete Beiträge für ein problemlösungsorientiertes Forschungsprogramm zu akquirieren. Sie beschränken sich allerdings eher darauf, bereits bestehende, standörtlich flexible Anträge auf den Wank zu lenken, als neue programmorientiert zu entwickeln. Zudem bezieht sich nur ein geringer Anteil der Anträge, die in der zweiten Begutachtungsrunde 1986 vorgelegt werden, auf den Alpenstandort.

Erst das Vorgehen, ein renommiertes, in der Luftschadstofforschung engagiertes und am Projekt interessiertes Forschungsinstitut zur zentralen und weitreichenden Beteiligung am alpinen Forschungsschwerpunkt zu gewinnen, sichert die Durchsetzung des politischen Interesses bei gleichzeitig wissenschaftlicher Unterstützung. Der neue Forschungsschwerpunkt kann nun rasch und trotz Widerstandes in den PBWU-Gremien etabliert werden.

Das dortige Forschungsprogramm wird jedoch als ein Strauß separat geplanter und daher nicht vorab aufeinander abgestimmter Einzelvorhaben durchgeführt. Darin werden - neben durchaus 
relevanten Forschungsthemen - einerseits nicht prioritäre Forschungsthemen bearbeitet, andererseits fehlen für eine Komplettierung des anspruchsvollen Forschungsziels geeignete, angepaßte Vorhaben sowie Finanzmittel. Zudem erlaubt das offene Programm den Wissenschaftlern wiederum, zumindest anteilig Aspekte von grundlagenorientierter Forschung zu bearbeiten. Dies schmälert wiederum die Effektivität des Programmes hinsichtlich des problemlösungsorientierten politischen Ansatzes und hätte unter Umständen an anderen, leichter zu bearbeitenden Standorten kostengünstiger durchgeführt werden können.

Die PBWU greift mit nur geringer Steuerungswirkung über verschiedene koordinierende Maßnahmen wie die gemeinsame Bearbeitung von Probeflächen, Probennahmetermine, Datenbank etc. in die Programmimplementation ein. Der Mangel eines konkreten und detaillierten, mit Fördermitteln versehenen Forschungsplanes, der mit entsprechenden Forschungsvorhaben zielorientiert umgesetzt wird, kann dadurch jedoch nicht kompensiert werden. Die beteiligten Forschungsvorhaben müssen demgegenüber im nachhinein zu einem losen und lückigen Gesamtkonzept verbunden und das Gesamtprogramm mit noch in erheblichem Maße verbleibenden Forschungsdefiziten etikettiert werden.

Daß vor dem Hintergrund des bestehenden Wank-Forschungsprogramms nun um so größerer Steuerungsbedarf während der Implementationsphase selbst erforderlich ist, zeigt die Herausbildung eines Leitungskomitees für den Forschungsstandort. Das Ziel des Gremiums, mittels eines ex post aufgestellten Forschungskonzeptes wissenschaftlich steuernden Einfluß auf die Wank-Forschung zu nehmen, wird allerdings nicht erreicht. Die für die Konzeptumsetzung erforderlichen Steuerungsbefugnisse des Leitungsgremiums finden nicht die Akzeptanz des Auftraggebers. Das Umweltressort möchte seine politische Steuerungsmacht und die via PBWU für den Standort nicht zugunsten der örtlichen, wissenschaftlichen Interessenverfolgung aufgeben und an den bisherigen Selektionsmechanismen zur Vorhabenförderung festhalten. Zudem würde das Leitungsgremium eine - wissenschaftlich dominierte - Koordinierungskonkurrenz zur PBWU darstellen, die zwangsläufig den Koordinationsaufwand insgesamt erhöhen und die Leistungsfähigkeit der bestehenden Forschungskoordination schmälern würde.

Weiterhin kommt das Leitungsgremium, um die Akzeptanz der am Standort bereits beteiligten Forscher zu gewinnen, nicht ohne deren Einbeziehung in das Forschungskonzept aus. Dies führt dazu, daß darin retrospektiv bereits bestehende Forschungsinhalte festgeschrieben werden und prospektiv eine große Bandbreite von möglichen Forschungsthemen vorgelegt wird, die wiederum eine stark steuerungsbedürftige bottom up-Struktur der Implementation erfordern würde. Ein derartiger, die wissenschaftliche Steuerung begünstigender Ansatz liegt wiederum nicht im Interesse des Bayerischen Umweltministeriums, und auch das Beiratsgremium der PBWU kritisiert den eher inkrementalistischen Charakter des Konzeptes. Das Konzeptpapier wird zwar letztlich in den PBWU-Gremien im Jahr 1991 akzeptiert, seine Umsetzung unterbleibt jedoch, zumal sich die politischen Rahmenbedingungen für die Waldschadensforschung insgesamt verändert haben. 


\subsubsection{Neuformulierung des PBWU-Forschungsprogramms}

Die Forschung im PBWU-Projekt beruht konzeptionell auf dem Rahmenprogramm aus dem Jahr 1985. Dieses ist jedoch seit seiner Entstehung und insbesondere hinsichtlich seiner Implementierung in Form des alpinen Forschungsschwerpunktes mit erheblicher Kritik von Seiten der Wissenschaft belastet. Der Ansatz der am alpinen Forschungsschwerpunkt eigeninitiativ gegründeten Forschergruppe, zumindest für den alpinen Teilbereich eine detaillierte Forschungskonzeption auszuarbeiten, ist hinsichtlich einer Umsetzung nicht erfolgreich. Zeitlich parallel dazu werden in den PBWU-Gremien selbst Überlegungen zu einer konzeptionellen Neuorientierung der gesamten PBWU-Waldschadensforschung angestellt.

\subsubsection{Diskussion über weiteren Forschungsbedarf}

Bereits auf der PBWU-Gremiensitzung vom 29.1.1988 war die Intensivierung der Laubwaldforschung von den Vertretern des Bayerischen Umweltministeriums als „Ziel der Bayerischen Staatsregierung“ genannt und der Wissenschaftliche Beirat um Forschungsvorschläge gebeten worden. Allerdings zeigt sich im Beirat Zurückhaltung. Die Schadensentwicklung müsse erst weiter beobachtet werden; zum jetzigen Zeitpunkt seien allenfalls „Voruntersuchungen“ angebracht (i.Q.: PBWU, 29.1.1988). Der auf der nächsten Sitzung vorgetragene Vorschlag des Beirats zur Laubwaldforschung fällt entsprechend knapp aus, wird in der Folge jedoch hinsichtlich des Vorschlags einer landesweiten, rasterartigen Pilot-Inventur konkretisiert (i.Q.: PBWU, 29.3. und 27.7.1989).

Das Umweltministerium fordert wiederum die stärkere Berücksichtigung der Laubwaldforschung bei künftigen Schwerpunktsetzungen und bittet um die Zusammenstellung bestehenden Forschungsbedarfs. Hierzu wird die Projektgruppe initiativ und legt zur nächsten Sitzung ein „Forschungslücken-Papier“ (Stand November 1989) vor (i.Q.: PBWU, 7.11.1989). Das Papier wird allerdings erst auf der übernächsten Gremiensitzung am 17.7.1990 - in aktualisierter Form - behandelt.

Die darin enthaltene Vorschlagsliste zum Forschungsbedarf wird im Beirat grundsätzlich zwar akzeptiert, im Detail herrscht aber Diskussionsbedarf (i.Q.: PBWU, 17.7.1990). So wird generell empfohlen, „neue Forschungsansätze viel mehr von bisherigen Ergebnissen abhängig“ zu machen und gewisse Forschungsprioritäten neu zu setzen. Für künftige Laubwaldforschung, deren Wichtigkeit bestätigt wird, seien „erhebliche Vorausplanungen“ erforderlich. Vom Bayerischen Umweltministerium wird die Vorschlagsliste der PBWU jedoch als eine „,wichtige Grundlage und Hilfestellung für die Haushaltsverhandlungen“ bezeichnet.

Besonderen politischen Anstoß für die Auseinandersetzung mit der Zukunft der Waldschadensforschung liefert der Wechsel des Bayerischen Umweltministers im Jahr 1990. Die Einstellung des neuen Ministers gegenüber der Waldschadensforschung sei, wie den PBWUGremien anläßlich einer Sitzung mitgeteilt wird, ,positiv-kritisch“; künftige Forschung müßte „problemorientiert, pragmatisch und erfolgversprechend“ sein (i.Q.: PBWU, 11.12.1990). Auch unter Berücksichtigung anderweitiger finanzieller Verpflichtungen rechnen die Vertreter des Umweltministeriums im Projektrat daher mit einer künftigen Verringerung der Fördermittel für die Waldschadensforschung. Nach Ansicht des Sprechers des Wissenschaftlichen Beirats 
erforderte dies nun um so mehr, dem Bayerisches Umweltministerium „gute Argumente“ für weitere Forschungsaktivitäten an die Hand zu geben.

Gegenüber der dringlichen Behandlung des vorliegenden Forschungskonzeptes für den alpinen Forschungsschwerpunkt Wank tritt die generelle Diskussion über der Zukunft der bayerischen Waldschadensforschung auf den Gremiensitzungen zeitlich zurück. Hinsichtlich künftiger Laubwaldforschung solle zudem erst eine für Oktober 1991 geplante wissenschaftliche Veranstaltung zum Thema abgewartet werden (i.Q.: PBWU, 11.12.1990 und 26.2.1991). Der Wissenschaftliche Beirat fordert im Zusammenhang mit der Diskussion der WankForschungskonzeption, daß für künftige Schwerpunkte der bayerischen Waldschadensforschung eine völlig neues Papier entwickelt werden müßte, welches zur Überzeugung von Politikern und zur Einwerbung von Fördermitteln geeignet sei (i.Q.: PBWU, 20.6.1991).

\subsubsection{Entwicklung eines Forschungskonzeptes zur künftigen Waldschadensforschung}

Auf der Sitzung der PBWU-Gremien vom 10.7.1991, auf der die Behandlung des Forschungskonzeptes für den Wank abgeschlossen wird, steht die Erstellung zukünftiger Forschungskonzepte für die bayerische Waldschadensforschung auf der Tagesordnung. Das Bayerische Umweltministerium betont die „Wichtigkeit eines klaren Konzeptes für die Zukunft“. Zudem sei noch für das laufende Jahr eine Landtagsanhörung zur Zwischenbilanz der Waldschadensforschung anberaumt (i.Q.: PBWU, 10.7.1991).

Nach Auffassung des Beirats müsse ein Forschungskonzept erarbeitet werden, dessen Umsetzung zeitlich, inhaltlich und finanziell realisierbar sei. Es wird beschlossen, daß einzelne Mitglieder des Wissenschaftlichen Beirats zu verschiedenen, als wichtig erkannten Teilbereichen kurzgefaßte Konzeptentwürfe erarbeiten sollen. Diese betreffen differentialdiagnostische Transekt- bzw. Gradientenstudien im Alpenraum und Nordbayern, Forschung zu Schäden an Laubbäumen und Untersuchungen zu den Auswirkungen von Klimaveränderungen.

Noch im Herbst 1991 werden von Beiratsmitgliedern nach einer internen Arbeitsverteilung entsprechende Teilkonzept-Entwürfe vorgelegt (i.Q.: Wissenschaftlicher Beirat, 30.7., 25.9. und 30.9.1991). Die PBWU ist bei der Erstellung der Entwürfe selbst mitbeteiligt bzw. liefert hierzu Stellungnahmen (i.Q.: PBWU, 22.8. und 26.9.1991) an die betreffenden Autoren. Die inhaltliche Diskussion der Papiere erfolgt im Gremium des Wissenschaftlichen Beirats, die PBWU nimmt an den Sitzungen teil (i.Q.: PBWU, 9.10.1991). Sie muß dabei aus dem Umweltministerium mitteilen, daß dort eine gewisse Unsicherheit über die Zukunft bzw. inhaltliche Ausrichtung der Waldschadensforschung bestünde. Mittel in Höhe von ca. 5 Mio. DM pro Jahr könnten zwar voraussichtlich auch weiterhin zur Verfügung gestellt werden. Angewandte Forschung und ein deutlicher Bezug zur Luftschadstoff-Thematik würden jedoch vorausgesetzt.

Die Ergebnisse der Diskussion im Wissenschaftlichen Beirat zu den einzelnen Teilkonzepten werden auf der nächsten gemeinsamen Sitzung der PBWU-Gremien im Herbst 1991 vorgestellt (i.Q.: PBWU, 31.10.1991). Danach stellten Luftschadstoffe zwar auch weiterhin Gegenstand der Untersuchungen dar, wenn aber bevorzugt auch „subtilere Wirkungsmechanismen“ untersucht werden sollten. Statt punktueller Schwerpunktstandorte würden verstärkt regionale For- 
schungsansätze vorgesehen. Die Laubwaldforschung sollte intensiviert werden. Zur Umsetzung dieser Vorgaben werden verschiedene Teilkonzepte vorgeschlagen. Dabei handelt es sich um

- ein Nord-Süd-Transekt im Alpenraum zur Durchführung einer epidemiologischen Inventur,

- ein Ost-West-Transekt in Nordbayern zur Laubwaldforschung,

- die Fortführung des Forschungsschwerpunktes Bayerischer Wald mit Schwerpunkt Stoffeintrag,

- Expositionskammerexperimente als Ergänzung zu standortbezogenen Untersuchungen.

Für die Transektstudien in Nordbayern und im Alpenraum würden Voruntersuchungen zur Standortsfestlegung für erforderlich gehalten. Hinsichtlich der Aussichten für eine künftige Finanzierung der Teilkonzepte müßten allerdings noch weitere ressortabstimmende Gespräche abgewartet werden, wie die Vertreter des Umweltministeriums einschränkend anmerken. Es wird beschlosssen, daß der Sprecher des Beirats gemeinsam mit dem Leiter der PBWU eine Homogenisierung der bestehenden Teilkonzepte vornimmt. Das daraus resultierende Positionspapier sollte zur Präsentation gegenüber den Ministerien geeignet sein.

Die Überarbeitung und Zusammenführung der vorliegenden Teilkonzepte mündet in das Forschungskonzept „Waldschadensforschung und Wirkung von Umweltchemikalien“, Stand 8.11.1991. Es wird als „Fortschreibung des Rahmenprogramms der PBWU vom 24.5.1985“ bezeichnet. Darin werden einleitend die künftigen Forschungsziele dargestellt, welche durch die Bearbeitung der in den Teilkonzepten enthaltenen Forschungsschwerpunkte erreicht werden sollen. Anschließend an eine generalisierende Übersicht über bisherige Ergebnisse der Forschung wird das Forschungskonzept detailliert ausgeführt. Es schließt mit der an das Umweltministerium gerichteten Empfehlung des Wissenschaftlichen Beirats, die Umsetzung des Konzeptes zu unterstützen und dafür notwendige Mittel in etwa in bisheriger Höhe zur Verfügung zu stellen. Es werde erwartet, daß damit konkretere Empfehlungen zur Schadstoffreduktion möglich würden.

Der Konzeptentwurf wird nicht nur mit den Mitgliedern des Beirats (i.Q.: PBWU, 8.11. und 29.11.1991), sondern auch mit Fachvertretern des Umweltministeriums abgestimmt. Ressortspezifische Aspekte sollten mit einfließen, um die Erfolgsaussichten und politische Durchsetzbarkeit des Papiers zu erhöhen (i.Q.: PBWU, 11.11.1991).

Das Umweltministerium unterbreitet umfangreiche Änderungsvorschläge, die die Begründung für die Durchführung der Forschungsarbeiten verbessern helfen sollen (z.B. infrastrukturelle, wissenschaftliche und ökonomische Voraussetzungen, Bezug zu bisherigen Ergebnissen, Kooperation mit Landesbehörden, grenzüberschreitende Zusammenarbeit) (i.Q.: Bayer. Umweltministerium 21.11.1991). Generell seien, wie aus dem Ministerium verlautet, Zielsetzung und Abgrenzung des Forschungsplans gegenüber bisherigen Arbeiten und Ergebnissen noch zu wenig konkret formuliert (i.Q.: PBWU, 29.11.1991).

Die Erfolgsaussichten für das Konzept werden vom Sprecher des Wissenschaftlichen Beirats bereits zu diesem Zeitpunkt skeptisch beurteilt. Es bestünde der Eindruck, das Umweltressort wolle sich „aus der Waldschadensforschung zurückziehen“. Unabhängig davon wolle sich die 
im Projektrat vertretene Fachabteilung des Ministeriums jedoch für die Umsetzung des neuen Konzepts einsetzen (i.Q.: Wissenschaftlicher Beirat, 23.11. und 8.12.1991).

\subsubsection{Vorstellung des Forschungskonzepts gegenüber dem Bayerischen Umweltminister}

Anfang des Jahres 1992 übersendet der Beiratssprecher das neue PBWU-Forschungskonzept an den Bayerischen Umweltminister (i.Q.: Wissenschaftlicher Beirat, 14.1.1992). Darin wird die Hoffnung auf weitere finanzielle Unterstützung der von der PBWU koordinierten Forschung zum Ausdruck gebracht und bestehende Forschungsdefizite hervorgehoben, deren Beseitigung das Konzeptpapier dienen solle. Auf die kontroverse Ursachendiskussion anläßlich einer Landtagsanhörung vom 5.12.1991 [BAYERISCHER LANDTAG 1991] wird verwiesen, der Klärung der Luftschadstoffeinwirkung werde allerdings nach wie vor hohe Bedeutung beigemessen. Ein erläuterndes Gespräch wird angeboten.

Im Antwortschreiben des Ministers wird die aus umweltpolitischer Sicht große Bedeutung der anwendungsorientierten Forschung betont. Sie sei als Grundlage für sachgerechte politische und administrative Entscheidungen unverzichtbar. In Anbetracht der angespannten Haushaltslage bedürfe die Realisierung des vorgelegten Konzeptes allerdings noch der Abstimmung mit dem Landwirtschaftsressort (i.Q.: Bayer. Umweltministerium 3.3.1992).

Auf der nächsten Sitzung der PBWU-Gremien kann seitens des Bayerischen Umweltministeriums über ein entsprechendes Gespräch zwischen Umwelt- und Landwirtschaftsminister berichtet werden (i.Q.: PBWU, 27.2.1992). Es bestünde die Absicht, die Ressortabstimmung zu optimieren, wobei das Umweltministerium auch weiterhin einschlägige, ressortspezifische Forschungsarbeiten finanzieren wolle. Von reiner Grundlagenforschung würde aber künftig Abstand genommen werden zugunsten anwendungsorientierter Untersuchungen, die sich ,in politische Handlungsempfehlungen umsetzen ließen“. Die interministerielle Abstimmung sei noch nicht abgeschlossen, so daß hinsichtlich der Umsetzung des Forschungskonzeptes noch keine Festlegungen getroffen werden könnten.

Ein Gespräch zwischen dem Bayerischen Umweltminister und dem Sprecher des Wissenschaftlichen Beirats findet am 23.3.1992 statt. Nach Einschätzung des Beiratssprechers sei das Gespräch „grundsätzlich positiv“ gewesen, der Minister hätte sich „,durchaus offen für Forschung zu Luftschadstoffen / Waldschäden“" gezeigt. Als besonders wichtig erschiene allerdings die bessere Koordinierung und Abstimmung die Forschung, und hier insbesondere die Ressortabstimmung zum Landwirtschaftsministerium (i.Q.: PBWU, 23.3., 21.5. und 16.7. 1992).

\subsubsection{Vorstudien zur Umsetzung des Forschungskonzeptes}

Wenn auch gewisse Bedenken geäußert werden, noch vor einer Entscheidung des Ministers zum Forschungskonzept bereits tätig zu werden, erklären sich die Vertreter des Umweltministeriums im Projektrat bereit, Vorstudien zur Umsetzung von Teilbereichen des neuen Konzeptes zu finanzieren. Dies geschieht auf Empfehlung von Mitgliedern des Wissenschaftlichen Beirats anläßlich einer Gremiensitzung (i.Q.: PBWU, 27.2.1992). Die PBWU wird in Kooperation mit zwei Mitgliedern des Beirats mit der Organisation und Betreuung der Vorstudien 
beauftragt. Diese betreffen Vorerkundungen zur weiteren Forschung im Alpenraum und in Nordbayern. Die Finanzierung mit vergleichsweise geringem Umfang erfolgt über eine Aufstockung bereits laufender PBWU-Forschungsvorhaben (i.Q.: PBWU, 25.5.1992).

Auf der letzten Sitzung der PBWU-Gremien am 9.3.1993 werden die Ergebnisse der Vorstudien den PBWU-Gremien vorgestellt und ausführlich diskutiert (i.Q.: $P B W U$, 9.3.1993). Eine weitere Beschlußfassung hierzu unterbleibt allerdings. Von den Vertretern des Umweltressorts angekündigte Sparmaßnahmen, welche die Waldschadensforschung existentiell beträfen, verdeutlichen, daß an eine Umsetzung der Forschungskonzeption zum jetzigen Zeitpunkt nicht zu denken ist. Nach Ansicht des Wissenschaftlichen Beirats machte die weitere Ausarbeitung der Vorstudien sowie das Initiieren von entsprechenden Forschungsanträgen nur dann Sinn, wenn „positive Signale des Bayerischen Umweltministeriums zur Fortführung der Forschungsförderung erkennbar seien“.

\subsubsection{Theorieorientiertes Zwischenergebnis}

\subsection{Prozeß der Neukonzeptionierung der PBWU-Waldschadensforschung}

Die Konzeption der PBWU-Waldschadensforschung geht auf das Rahmenprogramm aus dem Jahr 1985 zurück. Anstöße, das Programm und insbesondere den Bereich der Laubwaldforschung zu intensivieren, kommen überwiegend von politischer Seite. Das wissenschaftliche Beratungsgremium der PBWU ist anfangs zurückhaltend. Dennoch wird, von politischen Rahmenbedingungen gedrängt, ein neues wissenschaftliches Forschungskonzept erarbeitet, welches auch und gerade für die Vorlage gegenüber der Politik Bestand haben soll.

Der Vorsitzende des Beirats selbst übersendet das Forschungskonzept direkt an den Bayerischen Umweltminister, um den politischen Stellenwert des Papiers zu erhöhen. Die Umsetzungschancen sollen dadurch verbessert werden, daß Bezug auf das ministeriell akzeptierte Rahmenprogramm der PBWU zu Gründungszeiten genommen wird, die geplanten Themen als effiziente Fortführung bisheriger Forschung verstanden werden und dringender Forschungsbedarf deutlich gemacht wird. In Hinblick auf ressortspezifische Belange des Umweltministeriums wird zudem der Bezug zu Luftschadstoffen deutlich herausgestellt.

Seitens des Umweltministeriums wird jedoch eine Entscheidung für das Konzept und damit für die Fortführung der Waldschadensforschung verzögert. Zwar wird ein grundsätzlich positiver Eindruck signalisiert, erwartete Mittelkürzungen sowie erklärter Abstimmungsbedarf zwischen Umwelt- und Landwirtschaftsressort führen jedoch zur „Nichtentscheidung“. Hinzu kommt das eher verwirrende Bild des Waldschadens-Problems und der Beteiligung von Luftschadstoffen, welche eine Landtagsanhörung Ende 1991 geboten hatte und nicht geeignet ist, das Umweltministerium in seinem Engagement in der Waldschadensforschung zu bestärken. Daß trotz nicht erfolgter Entscheidung zu dem Forschungskonzept auf höchster politischer Ebene jedoch auf Fachreferatsebene Vorstudien zu dessen Umsetzung vereinbart werden, bringt die Hoffnung zum Ausdruck, daß die derzeitige Situation als nur vorübergehend eingeschätzt und zumindest dort die Fortführung der Waldschadensforschung für sinnvoll erachtet wird. 


\subsection{Verschmelzung wissenschaftlicher und politischer Interessen}

Das Rahmenprogramm der PBWU aus dem Jahr 1985 ist nach wie vor gültig. Es gilt formal als fachliche Grundlage für die Einpassung von Forschungsbeiträgen in das PBWU-Projekt und dient dem Umweltministerium als politische Legitimation bei der Bewilligung von Fördermitteln. Die Schwächen des Rahmenprogramms wurden bereits an anderer Stelle (Kap. 5.2) herausgearbeitet. Zudem weist das realisierte Forschungsprogramm auch zu vorgerückter Implementationsphase noch erhebliche Umsetzungslücken auf. Diese betreffen nicht nur Detailfragestellung innerhalb bearbeiteter Forschungsbereiche, sondern auch gesamte Forschungskomplexe, wie z.B. die bislang vollkommen unbearbeitete Laubwaldforschung. Zudem sind im Verlauf der Waldschadensdiskussion und der diesbezüglichen Forschung zusätzliche Forschungsaspekte, wie z.B. die Belastung der Wälder durch Stickstoffeinträge aus Verkehr und Landwirtschaft, hinzugekommen, oder die bisherigen Prioritätensetzungen haben sich aufgrund von Veränderungen (Verbesserungen wie Verschlechterungen) im Waldzustand oder des inzwischen erreichten Kenntnisstandes zu Schäden und Ursachen verschoben.

Politisch wird zu Beginn der 90er Jahre insbesondere durch den neuen Bayerischen Umweltminister deutlich problemorientierte, angewandte und ressortspezifische Forschung gefordert. Der politische Legitimationsdruck auf die Waldschadensforschung steigt angesichts der angespannten staatlichen Mittelsituation einerseits, des inzwischen allgemein geringeren Problemdrucks des Themas andererseits. Unter den inzwischen politisch wie wissenschaftlich gegebenen Bedingungen ist es unhaltbar geworden, sich in der Forschungsförderung nach wie vor auf das Rahmenprogramm aus dem Jahr 1985 zu beziehen. Dies reicht bei weitem nicht mehr aus, um Forschung zu legitimieren. Ein neues Konzept ist daher überfällig.

Erste Anstöße zur Neukonzeptionierung der bayerischen Waldschadensforschung im PBWUProjekt Ende der 80er Jahre entspringen überwiegend politischem Interesse der Vertreter des Umweltressorts im Projektrat. Die Verstärkung der Forschung zu Schäden an Laubbäumen wird als umzusetzendes Ziel der Bayerischen Staatsregierung bezeichnet. Als wesentlicher Grund hierfür kann angenommen werden, daß nach den offiziellen Ergebnissen der jährlichen Waldzustandserhebungen bei der bisher im Mittelpunkt stehenden Baumart Fichte Verbesserungstendenzen dokumentiert werden, während Laubbäume nach wie vor gleichbleibend hohes Schadensniveau verzeichnen. Zudem war nach der hinsichtlich künftiger Umsetzung erfolglosen Neukonzeptionierung der Wank-Forschung ein konzeptionelles Vakuum im PBWUProjekt entstanden. Dieses verlangt nach wissenschaftliche Aktualisierung und Neuorientierung Forschung im Hinblick auf eine politische Legitimation und damit Überlebensfähigkeit des Projekts.

Das wissenschaftliche Beratungsgremium der PBWU verhält sich vorerst zurückhaltend bzgl. einer Verstärkung der Laubwaldforschung. Hierfür können fachlich-wissenschaftliche Gründe sowie eine erfahrungsbedingte Skepsis gegenüber der Umsetzung von Forschungsschwerpunkten im PBWU-Projekt maßgeblich gewesen sein. Der Beirat war gerade bei der Etablierung des alpinen Forschungsschwerpunktes mit den organisatorischen und finanziellen Rahmenbedingungen unzufrieden gewesen und hatte entsprechende Vorstudien bemängelt; Fehler, die nun vermieden werden müßten und daher eher zu bedächtigem Handeln anraten lassen. In 
weiten Teilen der Wissenschaft kann zu Ende der 80er Jahre zudem eine gewisse Ernüchterung hinsichtlich der Problemschwere und damit hinsichtlich des zu erwartenden Forschungserfolges zum Thema Waldschäden unterstellt werden, die gegen ein intensives Engagement wie zu Beginn der Waldschadensforschung spricht. Hierunter fallen insbesondere auch Schäden an Laubbäumen, die in ihrem Ausmaß sowie ursächlichen Zusammenhang mit Luftschadstoffen von einigen Wissenschaftlern in Frage gestellt werden. Letztlich sind die für Laubwaldforschung als wichtig erkannten Aspekte forstpathologischer Forschung im Beirat personell nicht vertreten, was andernfalls zu einem früheren Aufgreifen dieses Themas geführt haben könnte.

Dennoch will sich der Beirat der ministeriellen Aufforderung, ein neues Forschungskonzept zu erstellen, nicht entziehen. Zu befürchten ist, daß engere und strengere politische Rahmenbedingungen zu Einschränkungen in der Forschungsfinanzierung führen könnten. Dies erfordert es um so mehr, der Politik überzeugende Argumente und ein klares Konzept zur künftigen Waldschadensforschung vorzulegen. Es wird deutlich, daß es sich hierbei mehr um ein politisches als ein wissenschaftliches Papier handeln müsse. Im Beirat beschleunigt dies die Erarbeitung und Abstimmung eines neuen PBWU-Forschungskonzepts und führt $\mathrm{zu}$ hoher Bündnisneigung. Zudem können eigene Forschungsinteressen der Wissenschaftler miteinbezogen werden, was die Bereitschaft, sich an der Konzepterstellung zu beteiligen, erhöht.

Im Gegensatz zur Erarbeitung des PBWU-Rahmenprogramms 1985 sind nun wissenschaftliche wie politische Akteure gleichermaßen an der Ausarbeitung des neuen Forschungskonzeptes beteiligt, wobei die Federführung bei Mitgliedern des Wissenschaftlichen Beirats liegt. PBWU und die Fachvertreter des Bayerischen Umweltministeriums beteiligen sich an der Ausgestaltung. Mit dieser Vorgehensweise können zentrale Kritikpunkte des ersten Rahmenprogramms, welches mit dem Ziel rascher Umsetzung von der Projektgruppe federführend erstellt worden war, vermieden werden. Im Ergebnis wird ein von der Wissenschaft getragenes, fachlich differenziertes wie anspruchsvolles und politisch akzeptables Konzept vorgelegt.

Die gemeinsame Erarbeitung sichert hohe Kompromißfähigkeit und Akzeptanz zwischen den politischen und wissenschaftlichen Konzeptbeiträgen. Politische Zielvorstellungen und wissenschaftliche Programminhalte können aufgrund des inzwischen erreichten Kenntnisstandes wesentlich stärker konkretisiert und akzentuiert in das Konzept eingebracht werden. Umfangreiche Vorstudien sollen die Forschungsansätze auf eine wissenschaftlich abgesicherte Basis stellen und somit Erfolg und Effektivität künftiger Forschung erhöhen. Damit würde zudem der programmkoordinierende Steuerungsaufwand zur inhaltlichen Ausrichtung und Durchführung der Forschung erleichtert, die Antragsselektion im Rahmen des Begutachungsverfahrens könnte straffer gehandhabt werden.

Erwartungsgemäß trägt das Konzept zwar in wissenschaftlicher Hinsicht die Handschrift ihrer Verfasser. Es ordnet sich jedoch den thematischen wie regionalen Zielvorgaben der Forschungspolitik unter, die in den PBWU-Gremien vereinbart worden waren. Der Beirat selbst stellt fest, daß das neue Forschungskonzept überwiegend als politisches Papier verstanden werden und hohen politischen Überzeugungswert aufweisen müßte, sollte die Waldschadensforschung auch in Zukunft weitergeführt werden. Damit erhöht sich der Druck auf die Wissen- 
schaft, sich einer problemlösungsorientierten Forschung zulasten der Forscherfreiheit zu beugen.

Insgesamt wird Ende 1991 ein Forschungskonzept vorgelegt, das im Vergleich zum Rahmenprogramm der PBWU des Jahres 1985 zweifelsfrei als wissenschaftlich fundierter, politisch und wissenschaftlich-konzeptionell stärker abgestimmt und daher als erfolgversprechender bezeichnet werden kann. Dennoch bleibt diesem Programm die Implementation aus politischen Gründen versagt.

Obwohl sich die Fachabteilung am Ministerium noch für die Umsetzung bis hin zur Genehmigung einzelner Vorstudien einsetzt, wird eine Entscheidung zu dem Konzeptpapier durch die politische Spitze des Ministeriums zurückgestellt und geht zeitgleich in der Terminierung des PBWU-Projektes im Jahr 1993 auf. Begründet mit dem Hinweis auf die Bedeutung stark anwendungsorientierter Forschung und erforderliche Ressortabstimmungen kommt zum Ausdruck, daß es der Wissenschaft nicht gelingt, die Umweltpolitik auch weiterhin für die Bearbeitung des Themas Waldschäden zu gewinnen.

Während zu Beginn der Waldschadensforschung im PBWU-Projekt die Politik z.T. gegen erheblichen Widerstand der Wissenschaft die Umsetzung eines stark kritisierten Forschungsprogramms durchsetzt, lehnt sie nun - paradoxerweise - ein von hochrangigen Wissenschaftlern erarbeitetes und abgestimmtes Forschungskonzept und damit letztlich die Fortsetzung der bayerischen Waldschadensforschung ab. Da die Gründe für die Ablehnung des Forschungskonzeptes weitgehend mit der Terminierung des PBWU-Projektes insgesamt verkoppelt sind, werden sie in dessen Rahmen analysiert.

\subsubsection{Förder- und Begutachtungsverfahren im PBWU-Projekt}

Die Durchführung von Forschungsvorhaben ist das Kernstück der Implementation des Forschungsprogrammes. Sie bestimmen mit den durchgeführten Arbeiten und erzielten Ergebnissen letztlich die Qualität und den Erfolg des PBWU-Gesamtprojektes. Dem Auswahl- und Entscheidungsprozeß zur Förderung von Forschungsanträgen kommt somit in forschungspolitischer, inhaltlicher wie finanzieller Hinsicht eine zentrale Stellung im Implementationsgeschehen zu. Es ist naheliegend, daß dieser Prozeß von den im Projekt beteiligten wissenschaftlichen wie politischen Akteure zur Gestaltung und Steuerung der Forschung entsprechend der jeweiligen Interessenlage zu beeinflussen versucht wird.

Das Verfahren der Selektion von Forschungsbeiträgen zur finanziellen Förderung im Rahmen des Projektes ist daher bei der empirischen Darstellung und Analyse von besonderem Interesse und soll im folgenden gesondert behandelt werden. Vorangestellt wird eine quantitativ orientierte Gesamtübersicht über relevante Programmstruktur-Daten im PBWU-Projekt. Diese sowie die wiederum überwiegend quantitative Analyse des Begutachtungsprozesses stützt sich maßgeblich auf die Aufbereitung und statistische Bearbeitung des betreffenden Dokumentenmaterials im Rahmen der Diplomarbeit von STEUER [1997], die im Rahmen der vorliegenden Arbeit durchgeführt wurde. Weiterhin bietet sich aufgrund der institutionellen, organisatorischen und thematischen Ähnlichkeit an, die Studie von HILL \& RIESER [1983] der For- 
schungsförderungspolitik des schweizerischen Nationalfonds vergleichend zu Analysezwecken heranzuziehen. Die Autoren charakterisieren ausführlich die dortige Verfahrensweise der Projektselektion, bewerten diese und geben Vorschläge zur Verfahrensoptimierung [S. 176ff, 247ff].

\subsubsection{Programmstruktur im PBWU-Projekt}

In Abbildung 5 ist das Förderverfahren im PBWU-Projekt dargestellt. Es reicht von der Antragstellung bis zur Vorhabenentlastung und berücksichtigt schematisch und idealtypisch die an den jeweiligen Verfahrensschritten beteiligten PBWU-Akteure. Auf das Verfahren wird hier im Rahmen der Implementationsanalyse allerdings nur hinsichtlich der Antragsbewilligung Bezug genommen. Das Verfahren der Vorhabenbetreuung und der ex post-Evaluation (Berichtsbeurteilung) wird im Rahmen des Kapitels Terminierung und Evaluierung (Kap. 5.4) behandelt.

Die Beteiligung von PBWU-Akteuren zu bestimmten Verfahrensschritten, die daraus resultierenden Kräfteverhältnisse zwischen Politik und Wissenschaft sowie der Wandel in der Steuerungsdominanz wurden bereits ausführlich behandelt.

Im Grundsatz wurden unter dem skizzierten Verfahrensablauf im PBWU-Projekt insgesamt knapp 120 Forschungsanträge mit einem Gesamt-Antragsvolumen von etwa 43 Mio. DM in den Jahren 1984 bis 1993 behandelt. Davon werden über 70 Forschungsvorhaben mit rund 25 Mio. DM Fördervolumen finanziert. Darin sind der eigentliche PBWU-Vertrag zur Finanzierung der Projektgruppe sowie die Serviceleistung der Zentralen Analytik einschließlich der jeweiligen Fortsetzungsaufträge zwischen Bayerischem Umweltministerium und GSF als weitere, finanzaufwendige Maßnahmen noch nicht mit enthalten. Die Serviceleistungen und zentrale Dienste einbezogen erhöht sich der Finanzierungsumfang im PBWU-Projekt auf 35 Mio. DM bei ca. 80 Vorhaben [STEUER 1997, S. 14; REUTHER et al. 1993, S. 2].

Die Förderquote, das Verhältnis zwischen geförderten und eingereichten Forschungsanträgen, beträgt insgesamt $65 \%$. Sie liegt zu Implementationsbeginn zwar niedriger als im weiteren Verlauf. Dennoch führt die anfänglich große Zahl eingereichter Forschungsanträge zu vergleichsweise zahlreichen Antragsbewilligungen und Festlegung überproportional hoher Fördermittel. Somit sind bereits im zweiten Förderjahr 1986 rund 30\% aller Fördermittel bzw. Einzelvorhaben des PBWU-Projektes festgelegt. 


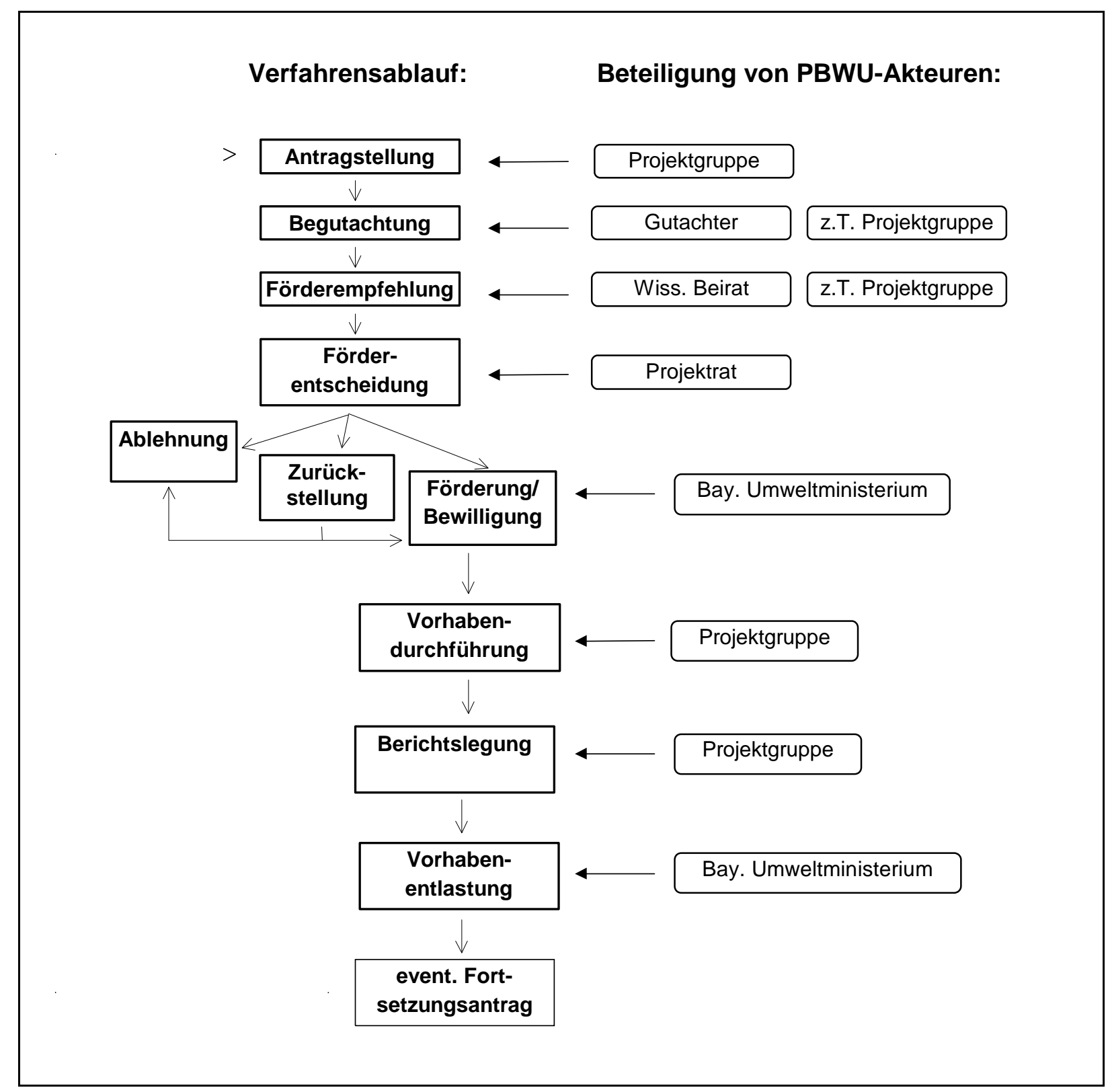

Abb. 5: Ablauf des Förderverfahrens (verändert nach STEUER [1997, S. 6])

Der reale, jährliche Mittelabfluß gestaltet sich allerdings ausgeglichener. Bis zum Jahr 1988 steigen die jährlich verausgabten Fördermittel auf 5,5 Mio. DM an und gehen anschließend bis zum Projektende 1993 (und geringfügig darüber hinaus) stetig zurück (s. Abbildung 6). Bemerkenswert ist der mit 40\% hohe Anteil von Fortsetzungsvorhaben [STEUER 1997, S. 14-19; REUTHER et al. 1993, Anhang 5]. 


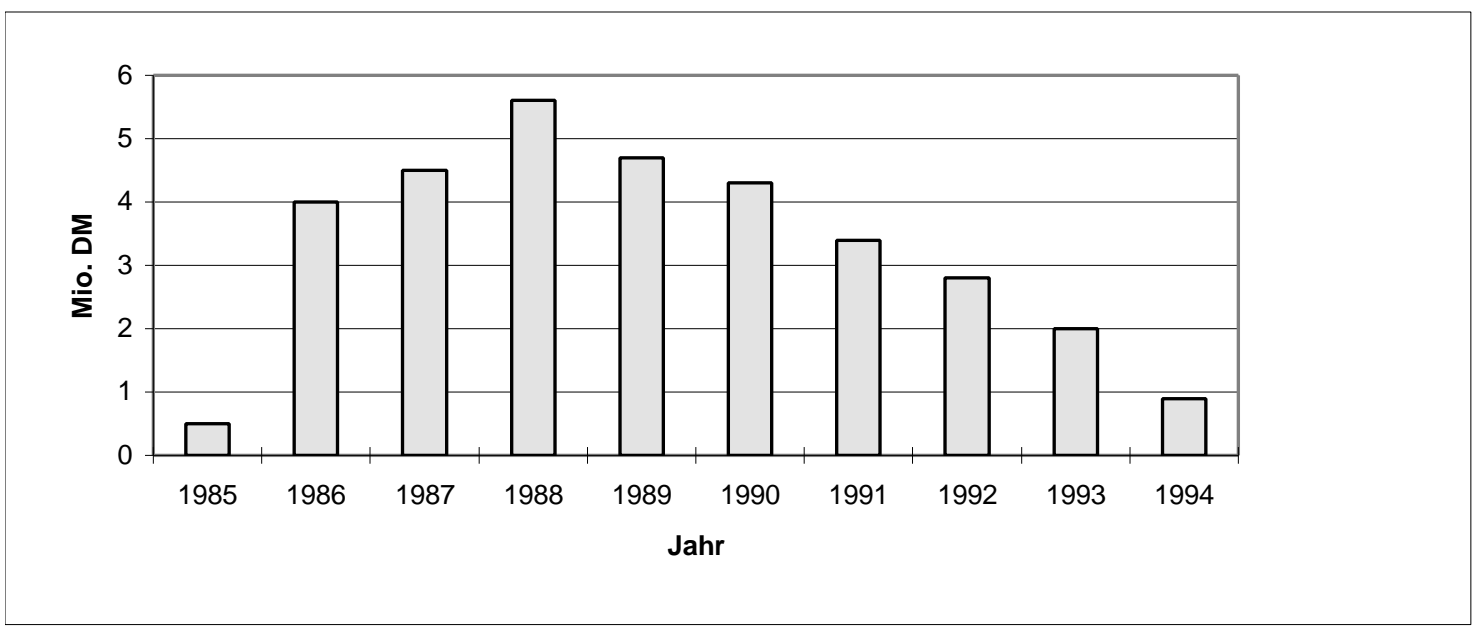

Abb. 6: Jährlicher Fördermittelabfluß [PBWU 1992, S. 6]

Das PBWU-Programm kann weiterhin in seiner Forschungs-institutionellen, fachlichen und regionalen (Freiland- und Labor-) Struktur charakterisiert werden [STEUER 1997, S. 20-24]. In institutioneller Hinsicht wird der Forschungsprozeß im wesentlichen von sechs in Bayern angesiedelten Forschungsinstitutionen getragen. Dabei dominieren die Münchner Universitäten sowie die außeruniversitären Forschungseinrichtungen GSF Neuherberg und das FraunhoferInstitut in Garmisch-Partenkirchen (FhG-IFU) (Tabelle 11). Die besondere Finanzierungsstruktur letztgenannter Institutionen (z.B. Einbeziehung verschiedener Verwaltungskosten, Mehrwertsteuer etc. in die Fördersumme) bedingt den im Vergleich zu universitärer Forschung hohen Förderaufwand mit.

Die beteiligten Forschungsdisziplinen entsprechen im wesentlichen dem Auftrag des PBWUProjektes. Es dominiert der biologische Forschungsbereich der Schadstoff-Wirkungsforschung mit Vorhaben aus pflanzlicher Biochemie und Physiologie einschließlich Cuticula-Forschung. Hierauf entfallen im PBWU-Projekt insgesamt 44 Forschungsvorhaben (s. Tabelle 12). Entsprechend der im Zentrum stehenden Luftschadstoff-Hypothese bei der Verursachung von Waldschäden stellen Forschungsarbeiten im Bereich Immission, Schadstoffeintrag etc. einen weiteren Schwerpunkt dar.

Entsprechend der Zielsetzung des PBWU-Projektes wird der neue, alpine Forschungsschwerpunkt am stärksten mit Forschungsbeiträgen unterstützt. Nahezu die Hälfte aller Forschungsvorhaben und Fördermittel sind dem Wank zuzuordnen. Während die bereits bestehenden, überwiegend anderweitig finanzierten bayerischen Schwerpunktstandorte der Waldschadensforschung aus dem PBWU-Projekt nur geringfügig verstärkt werden, stellen Laborarbeiten zur Wirkungsforschung einen Großteil der Fördermaßnahmen dar, ohne jedoch einen gleichermaßen hohen Förderaufwand wie die Freilandarbeiten zu bedingen (s. Tabelle 13). 


\begin{tabular}{|l|c|c|}
\hline Antragsteller & $\begin{array}{c}\text { Anzahl Vorha- } \\
\text { ben }\end{array}$ & $\begin{array}{c}\text { Kosten } \\
\text { (Mio. DM) }\end{array}$ \\
\hline Uni. München & 19 & 5,6 \\
\hline TU München & 19 & 5,4 \\
\hline Uni. Würzburg & 12 & 1,7 \\
\hline FhG-IFU Garmisch-P. & 10 & 5,4 \\
\hline GSF Neuherberg & 7 & 4,2 \\
\hline Uni. Bayreuth & 4 & 2,5 \\
\hline Sonstige & 3 & 0,3 \\
\hline Summe: & $\mathbf{7 4}$ & $\mathbf{2 5 , 1}$ \\
\hline
\end{tabular}

Tab. 11: Geförderte Forschungsvorhaben und Finanzmittel (Daten aus STEUER [1997, S. 21/22])

\begin{tabular}{|l|c|c|}
\hline Fachdisziplin & $\begin{array}{c}\text { Anzahl Vorha- } \\
\text { ben }\end{array}$ & $\begin{array}{c}\text { Kosten } \\
\text { (Mio. DM) }\end{array}$ \\
\hline Physiologie/Biochemie & 35 & 8,3 \\
\hline Cuticula & 9 & 1,9 \\
\hline Immission/Luftchemie & 17 & 7,3 \\
\hline Bodenkunde & 8 & 5,2 \\
\hline sonstige & 5 & 2,4 \\
\hline Summe: & $\mathbf{7 4}$ & $\mathbf{2 5 , 1}$ \\
\hline
\end{tabular}

Tab. 12: Beteiligte Fachdisziplinen (Daten aus STEUER [1997, S. 20])

\begin{tabular}{|l|c|c|}
\hline Standort & $\begin{array}{c}\text { Anzahl Vorha- } \\
\text { ben }\end{array}$ & $\begin{array}{c}\text { Kosten } \\
\text { (Mio. DM) }\end{array}$ \\
\hline Wank (Kalkalpen) & 35 & 14,6 \\
\hline Fichtelgebirge & 8 & 3,0 \\
\hline Bayer. Wald & 8 & 3,6 \\
\hline Höglwald & - & - \\
\hline $\begin{array}{l}\text { Labor od. nicht zuorden- } \\
\text { bar }\end{array}$ & 23 & 3,9 \\
\hline Summe: & $\mathbf{7 4}$ & $\mathbf{2 5 , 1}$ \\
\hline
\end{tabular}

Tab. 13: Forschungsstandorte und standortsunabhängige Laborarbeiten (Daten nach REUTHER et al. [1993, Anhang 4], ergänzt durch PBWU [1992])

\subsubsection{Grundlagen der Antragsbegutachtung}

Wie für vergleichbare Forschungsprogramme üblich, beruht auch im PBWU-Projekt die wissenschaftliche Legitimierung und Auswahl zu fördernder Forschungsanträge über die gesamte Projektlaufzeit hinweg auf einer Beurteilung durch externe Fachgutachter, einem sog. Peer Review-System (siehe unten) [s.a. JASANOFF 1990, S. 61ff; FELT et al. 1995, S. 235-243; HILL \& RIESER 1983, S. 22ff, 251ff]. Aufgrund von Gutachtervoten wird eine Förderempfehlung in 
den PBWU-Gremien getroffen, die wiederum Grundlage für eine Förderentscheidung durch das finanzierende Umweltministerium ist (s.a. Abbildung 5).

Auf das Verfahren, die wissenschaftliche Qualität und andere Kriterien der einzelnen Forschungsbeiträge über externe Wissenschaftler überprüfen zu lassen, hatten sich die PBWUGremien im Grundsatz bereits zu Zeiten der Rahmenprogrammerstellung geeinigt. Von einer Begutachtung ausgenommen sind lediglich ausgesprochene Serviceleistungen wie die Durchführung verschiedener Grundlagenerhebungen (wie z.B. terrestrische oder luftbildgestützte Waldzustandserhebungen, Standortskartierung oder Infrastruktur-Maßnahmen am Forschungsschwerpunkt Wank) oder die Einrichtung des zentralen Analyselabors, welche nicht Forschungsarbeiten im eigentlichen Sinne darstellen.

An und für sich kann die Antragsbegutachtung als Bestandteil der Programmevaluierung verstanden werden. Da wie bereits erwähnt, die Implementation des PBWU-Projektes und damit auch die Steuerung des Forschungsprozesses wesentlich durch die einzelnen Forschungsvorhaben determiniert wird, wird der Selektionsprozeß der Forschung jedoch bereits bei der Beschreibung und Analyse der Programmimplementation - und nicht erst bei der Evaluationsphase zum Projekt - behandelt.

Die Besonderheit ist, daß die Beurteilung als ex ante-Analyse durchgeführt wird, mit dem Ziel, eine Prognose über die künftige, tatsächliche Entwicklung und das erwartete bzw. vom Antragsteller in Aussicht gestellte Forschungsergebnis abzugeben [s.a. HILL \& RIESER 1983, S. 248, KURKI 1991, S. 71]. Generell wird die Evaluation als Verfahren zur Leistungsbewertung wissenschaftlicher Forschung stark diskutiert [s. z.B. EVERED \& HARNATT 1989] und inzwischen kritisch beurteilt. So weisen FELT et al. [1995, S. 240/241] darauf hin, daß weder Objektivitätsanspruch noch Wertfreiheit im Bewertungsurteil gegeben sind und Evaluation immer als sozialer Aushandlungsprozeß verstanden werden muß. Auch JASANOFF [1990, S. 61-79] kann für verschiedene US-amerikanische Forschungsprogramme zeigen, daß Begutachtungsverfahren (am Beispiel des Peer Reviews) keinesfalls fehlerfreie, objektive Verfahren zur Sicherung von Qualität und Integrität wissenschaftlicher Ziele sind, sondern vielmehr die Problematik verläßlicher Wissenschaft aufzeigen. Zudem hängt das Ergebnis der Begutachtung von der Auswahl der Gutachter und deren persönlicher wissenschaftlicher Einschätzung ab [FELT et al. 1995, S. 239]. Als Teil der wissenschaftlichen Gemeinschaft seien bei Gutachtern Interessenkonflikte und -koalitionen niemals völlig auszuschalten.

\subsubsection{Begutachtung im PBWU-Projekt}

Abgesehen von der ersten, als mündliche Gutachterdiskussion (Panel) durchgeführten Antragsrunde im Jahr 1985 wird das weitere Verfahren ausschließlich in Form schriftlicher Begutachtung (Mail Review) durchgeführt. Im Rahmen der zweiten Begutachtungsrunde im Jahr 1986 werden alle zu einem Stichtag eingereichten Anträge den Gutachtern vorgelegt. In der anschließenden Übergangsphase bis hin zur Neustrukturierung des PBWU-Projektes im Jahr 1988 findet die schriftliche Antragsbegutachtung einzelfallweise statt. In der Folge - bis zu letzten, im Jahr 1992 eingereichten Anträgen - werden diese wiederum einzelfallweise begutachtet, jedoch für den Zeitraum eines Jahres gebündelt in einer Art Antragsrunde in den 
PBWU-Gremien gemeinsam und damit zeitgleich behandelt. In der Regel werden zu jedem Antrag 2-3 schriftliche Stellungnahmen externer Gutachter eingeholt.

\subsection{Begutachtungspanel}

Angesichts der Zahl von rund 30 Forschungsanträgen der ersten Begutachtungsrunde 1985 erscheint die zeit- und kostenaufwendige, mündliche Gutachterdiskussion (Begutachtungspanel) der ersten Runde im Jahr 1985 zielführend. Sie kann zudem als akzeptanz- und vertrauensbildende Maßnahme im Verständigungsprozeß zwischen PBWU, Projektrat und externen Gutachtern gewertet werden. Kombiniert mit der mündlichen Vorstellung der Einzelprojekte durch die Antragsteller können mit diesem Verfahren nicht nur Verständnisprobleme oder sonstige Unklarheiten ausgeräumt werden. Die anschließende, interne Gutachtersitzung - unter Beisein von PBWU und Projektratsmitgliedern - bietet die Möglichkeit, bei fachlich kompetenter Auseinandersetzung über ggf. bestehende Beurteilungsdiskrepanzen eine optimalere Selektion und ein eindeutiges Gutachtervotum zu erarbeiten. Letzeres minimiert wiederum den Auswertungsspielraum - und damit den möglichen Steuerungseinfluß - eines Dritten hinsichtlich des Beurteilungsergebnisses [HILL \& RIESER 1983, S. 274/275]. So ist denn auch der Anteil kontrovers beurteilter Anträge im ersten - mündlichen - Begutachtungsverfahren mit unter 10\% verglichen mit dem anschließendem, schriftlichen Verfahrensmodus (über 20\%) gering [STEUER 1997, S. 32]

Das Gremium des Wissenschaftlichen Beirats bleibt bei Durchführung der ersten Begutachtungsrunde vollkommen unbeteiligt. Über die Ergebnisse der Begutachtungssitzung in Kenntnis gesetzt, einigt es sich ,nach intensiver Diskussion“ darauf, die Voten zu akzeptieren und möchte explizit von einer „Nach-Begutachtung“ Abstand nehmen (i.Q.: PBWU, 18.11.1985).

Während die fachliche Diskussion der zahlreichen Fachgutachter zwar maßgebliche Grundlage für das Gesamtergebnis der Beurteilung darstellt, ist hier der forschungspolitische Einfluß von Projektgruppe und Projektrat bereits impliziert. Beide Akteure sind bei der gutachterlichen Diskussion der Anträge beteiligt, die PBWU hat die Gesprächsführung inne und beeinflußt die Diskussion insbesondere durch die von ihr zu jedem Antrag verfaßten und auf der Gutachtersitzung präsentierten Stellungnahmen.

Dadurch, daß wissenschaftliche wie politische Akteure an der Sitzung beteiligt sind, kommt es bereits während der Diskussion zu einer Durchmischung beider Interessenlagen in der Bewertung der Anträge. Zwar kann aufgrund des Protokolls zur Gutachtersitzung von fachlicher Dominanz der Gutachter in der Diskussion ausgegangen werden, die politischen Akteure konnten ihre Interessenlagen jedoch einbringen. Die Trennung zwischen fachlicher Empfehlung der Gutachter und Entscheidung durch das politische Gremium des Projektrats verschwimmt, die Projektgruppe, welcher laut Vertrag (z.B. i.Q.: PBWU, 12.10.1987) keinerlei Entscheidungskompetenz eingeräumt wird, kann sich um so mehr mit ihren Vorstellungen einbringen. Es wird zwar die Einigung auf ein Gesamturteil erzielt. Da dieses - auch aufgrund der fachlichen Breite der beteiligten Gutachter - im Detail über eine generelle Förderempfehlung häufig nicht hinausgeht, verbleiben der PBWU im Rahmen der Umsetzung des Begutachtungsergebnisses zu Vorhabenbewilligungen noch Interpretationsspielräume bei deren Ausgestaltung. 
Die quantitative Analyse des PBWU-Förderverfahrens zeigt für die erste, mündliche Begutachtungsrunde mit etwa 30 Forschungsanträgen, daß aufgrund der bereits während der Sitzung getroffener Förderempfehlungen bei meist eindeutigen Voten nachträglich nur ein insgesamt geringer Interpretationsspielraum verbleibt [STEUER 1997, S. 46ff]. Die Anzahl kontroverser bzw. uneinheitlicher Förderempfehlungen ist gering. Im Rahmen der nachgeschalteten Projektratssitzung sind die zu treffenden Förderentscheidungen reine Formsache. Auf weitere Spezifizierungen der Förderempfehlung wie eventuelle Antragsmodifizierungen oder Mittelkürzungen wird in der Regel verzichtet. Bei uneinheitlichen, kontroversen oder zu Antragsmodifizierung ratenden Gutachterempfehlungen (etwa 10\% aller Anträge [STEUER 1997, S. 32]) nutzen PBWU und Projektrat allerdings den verbleibenden Interpretationsspielraum. Dabei fällt auf, daß die Voten tendenziell positiv interpretiert werden. Dies könnte mit dem förderpolitischen Drängen nach einer zügigen Programmimplementation erklärt werden.

\subsection{Mail Review}

Die Begutachtungsform des Mail Reviews zur zweiten Begutachtungsrunde 1986 sowie in der Folge der einzelfallweisen Antragsbewertung wurde insbesondere wegen des vergleichsweise geringeren Durchführungsaufwandes gewählt. Während die Empfehlung mehrerer externer Gutachter zwar wiederum die Grundlage für eine Förderentscheidung darstellt, kommt hier dem Interpretationsspielraum insbesondere bei der zusammenfassenden Bewertung der Gutachten eine wesentliche Bedeutung hinsichtlich eines möglichen Steuerungspotentials zu. Die Einzelgutachten liegen im Gegensatz zur gemeinsamen Ergebniserarbeitung im Rahmen eines Begutachtungspanels voneinander unabhängig und in schriftlicher Form vor. Sie lassen sich nicht nur für den Extremfall kontroverser Begutachtung, sondern auch bei lediglich tendenzieller Gutachterempfehlung oder hinsichtlich einzelner Bewertungsdetails kaum ohne eigene Gewichtung zu einem Gesamturteil zusammenfassen. So beträgt der Anteil „kontrovers“ begutachteter Anträge im PBWU-Projekt bei schriftlicher Begutachtung bei knapp 20\%, der Anteil mit „eindeutiger Bewertungstendenz“ bei über 20\% [STEUER 1997, S. 32]. Dieser Interpretationsspielraum fällt um so größer aus, als im PBWU-Projekt auf eine formalisierte Bewertung mit der expliziten Vorgabe der Beurteilungskriterien und deren Interpretation, z.B. in Form eines sog. „Scoring-Verfahrens“ [HILL \& RIESER 1983, S. 254ff], verzichtet wird. Damit eröffnet die Ableitung eines Gesamturteils der externen Begutachtung dem durchführenden Akteur eine erhebliche Einflußnahme auf das Ergebnis.

Diese Einflußnahme nimmt während der zweiten Begutachtungsrunde 1986 sowie der 1987/88 folgenden, einzelfallweisen Antragsbehandlung die Projektgruppe für sich in Anspruch. Sie gibt den Gutachtern nicht nur eine eigene Einschätzung als Empfehlung vor, sondern führt die Einzelgutachten zu einer Gesamtbeurteilung zusammen. Gerade bei uneindeutigen Einzelgutachen oder bei uneinheitlicher externer Gesamtbeurteilung kann somit die PBWU als „Zünglein an der Waage" fungieren. Dadurch, daß anfangs die Einzelgutachten aus Anonymitätsgründen dieser Gesamtbewertung nicht beigelegt werden, entbehrt diese Vorgehensweise jedweder Kontrollmöglichkeit. Entsprechend heftig wird sie auch von den PBWU-Gremien kritisiert.

Eine zusätzliche Einflußnahme verbleibt dem politischen Gremium Projektrat auf der nächsten Ebene; nämlich dann, wenn die von der PBWU erarbeiteten und damit bereits verdichteten 
Gutachtenzusammenfassungen und Förderempfehlungen zu einer Förderentscheidung umgesetzt werden. Auch hier können als kontrovers oder uneindeutig resümierte Empfehlungen modifiziert oder eindeutige Empfehlungen umgekehrt werden. Explizit weist der Vertreter des Kultusressorts im Projektrat darauf hin, daß die Fachgutachter ausschließlich die wissenschaftliche Begutachtung der Anträge zu leisten hätten, nicht jedoch die Einpassung in das Programm (i.Q.: PBWU, 15.5.1986). Der Wissenschaftliche Beirat bleibt während dieser Phase wiederum vollkommen unbeteiligt und kann die vom Projektrat letztlich getroffenen Förderentscheidungen lediglich zur Kenntnis nehmen.

Wie für die erste Runde bestätigt die Analyse des Förderverfahrens auch für die zweite, schriftliche Begutachtungsrunde des Jahres 1986 mit über 20 Anträgen die Verfahrensdominanz und damit den Steuerungseinfluß der politischen Akteure gegenüber dem Wissenschaftlichen Beirat [STEUER 1997, S. 46-50]. Zwar waren subjektive Auslegungen und Interpretationen der Gutachten bei der Zusammenfassung durch die Projektgruppe im Detail nicht Gegenstand der Analyse. Deutliche Abweichungen der politischen Akteure von dem Gutachtervotum wurden jedoch dokumentiert. Sie betreffen insgesamt 25\% aller Anträge. Während lediglich in 2 Fällen gegen das Gutachtervotum entschieden wurde, wurden 5 uneinheitlich bis kontrovers begutachtete Anträge finanziert, weitere Anträge wurden unabhängig vom Begutachtungsergebnis aufgrund politischer oder finanzieller Prioritätensetzung zurückgestellt. Der Bezug mehrerer Gutachter in ihrer Stellungnahme auf die von der PBWU vorgegebenen Empfehlungen ist Beleg dafür, auch hieraus einen frühzeitigen, präjudizierenden Steuerungseinfluß auf die Förderempfehlung abzuleiten.

In der Folge wird die Vorauswahl der Anträge, die Gesamtbewertung der Einzelgutachten und die Erarbeitung einer Förderempfehlung aufgrund der Kritik an dem Antragsbehandlungsverfahren durch die Projektgruppe zunehmend auf den Wissenschaftlichen Beirat verlagert. Die eingeholten Einzelgutachten stellen immer mehr die Grundlage für die weitere Beratung in den PBWU-Gremien dar. Die Gutachten können bewertet und nach Kriterien des Beirats gewichtet werden [s.a. STEUER 1997, S. 47]. Damit geht der potentielle Gestaltungs- bzw. Steuerungseinfluß auf den Forschungsprozeß auf dieses Wissenschaftlergremium über, was eine neue Phase des Begutachtungsverfahren sowie des Forschungsprozesses im PBWU-Projekt kennzeichnet. Der Beirat formuliert auf Grundlage der externen Gutachten Förderempfehlungen, aufgrund derer der Projektrat, und dort insbesondere das finanzierende Umweltressort, Förderentscheidungen fällt und mittels der Projektgruppe umsetzt.

Das markanteste und hinsichtlich der Forschungssteuerung relevanteste Unterscheidungsmerkmal zwischen anfänglichem und nun angewandtem Verfahren innerhalb der schriftlichen Begutachtung ist, daß das Ergebnis wissenschaftlich-fachlicher Antragsbegutachtung nun nicht mehr von einem oder mehreren politischen Akteuren (Projektgruppe und Projektrat), sondern wiederum von einem wissenschaftlichen Akteur, dem Wissenschaftlichen Beirat, bewertet wird. Zudem nimmt der Beirat bereits eine Vorauswahl der für eine Begutachtung vorzusehenden Anträge vor. Daß die Beurteilungsdiskrepanzen zwischen wissenschaftlichen Akteursgruppen Gutachter / Beirat geringer ausfallen als zwischen wissenschaftlicher und politischer Akteursgruppe Gutachter / Projektgruppe bzw. Projektrat, und daraus ein entsprechender Steuerungsinput resultiert, ist anzunehmen. 
Noch nahezu 60 Anträge können der Beteiligung des Wissenschaftlichen Beirats am Förderverfahren ab 1987 bis zu Ende des PBWU-Projektes zugeordnet werden [STEUER 1997, S. 47/48]. Deren Analyse zeigt, daß die Abweichungsquote der Empfehlungen des Beirats von den Gutachtervoten allein bei eindeutig positiven Voten wiederum insgesamt rund $25 \%$ beträgt. Dabei sind unter einer Abweichung vom positiven Begutachtungsergebnis nicht nur eine Förderabsage, sondern auch kontroverse Beiratsempfehlungen sowie Förderung mit Modifikationen zu verstehen. Generell ist die Einschätzung des Wissenschaftlichen Beirats - im Gegensatz zu der der politischen Akteure im Rahmen der ersten beiden Begutachtungsrunden - allerdings eher restriktiv. Bei kontroverser, Modifizierung fordernder oder nur tendenziell förderempfehlender Gutachtenlage wird der verbleibende Entscheidungsspielraum teils zugunsten, teils zulasten der Antragsteller genutzt. Immerhin sind davon etwa die Hälfte aller Anträge betroffen. Der Projektrat folgt in der Regel den Empfehlungen des wissenschaftlichen Beratungsgremiums.

Insgesamt ist das vom Wissenschaftlichen Beirat maßgeblich dominierte Förderverfahren gekennzeichnet durch eine auffallend hohe Förderquote (Bewilligungsrate). Sie beträgt $77 \%$ [STEUER 1997, S. 48], demgegenüber die der ersten Bewilligungsphase ohne Beteiligung des Beirates 48\%. Da der Beirat in ähnlichem Maße wie früher die politischen Akteure die externen Fachgutachten befolgte, und tendenziell sich sogar noch förderungskritischer verhielt, kann dieser Befund nicht allein mit dem Steuerungseinfluß des Beirats bei der Förderempfehlung erklärt werden. Vielmehr können folgende Gründe angeführt werden [s.a. STEUER 1997, S. 48]:

- Es fand inzwischen eine wirksame Vorauswahl der eingereichten Anträge hinsichtlich der Einpassung in das Rahmenprogramm durch den Wissenschaftlichen Beirat statt. Dies verschärft die Selektion noch vor der eigentlichen Begutachtung und verbessert die FörderChancen der im Grundsatz akzeptierten und begutachteten Anträge.

- Im Projektverlauf nimmt einerseits der Anteil von Fortsetzungsvorhaben gegenüber Erstvorhaben, andererseits der von Mitgliedern des Wissenschaftlichen Beirats selbst zu. Derartige Anträge haben, wie noch ausgeführt werden wird (s. Kap. 5.3.5.3.7), vergleichsweise gute Bewilligungschancen.

- Von dem Instrument der Antragsmodifizierung und insbesondere -kürzung statt einer Antragsablehnung wurde zunehmend Gebrauch gemacht.

- Es wurden vermehrt hochqualifizierte, auf das bereits laufende Forschungsprogramm abgestimmte und konkrete Forschungslücken abdeckende Forschungsanträge eingereicht. Die „Goldgräberstimmung“ in der anfänglichen Waldschadensforschung war vorüber.

\subsection{Auswahl der externen Fachgutachter}

Bei der Bedeutung für das Begutachtungsergebnis [HILL \& RIESER 1983, S. 251/252] kann die Auswahl der externen Gutachter für die Ausrichtung des Programmes insgesamt als Machtund Einflußpotential genutzt werden. Zu Beginn des PBWU-Projektes wird die Gutachterwahl aufgrund einer Empfehlung des Wissenschaftlichen Beirats vorgegeben (i.Q.: Bayer. Umweltministerium 11.2.1985). Der Fachgutachterkreis setzt sich anfangs aus DFG-Gutachtern zu- 
sammen, welche zugleich die Begutachtung des vom Kultusressort geförderten, nordbayerischen Forschungsschwerpunktes der Forschungsgruppe Forsttoxikologie vornehmen.

Damit ist zwar der fachliche Zuschnitt und entsprechende fachliche Kompetenz gewährleistet. Eine Interessenkollision auf der Ebene der Fachgutachter hinsichtlich der Vorhaben- bzw. Mittelzuteilung zwischen beiden Forschungsverbünden ist jedoch zu erwarten. Die nordbayerischen Forscher können aufgrund der informalen positiven Stellung der Gutachter zu ihrem Forschungsverbund bzw. den dort beteiligten Forschergruppen auf deren Unterstützung hoffen. Eine umfangreiche - zumindest antragsmäßige - Beteiligung dieser Forschergruppen am PBWU-Projekt sowie die forschungsseitige Unterstützung des nordbayerischen Forschungsschwerpunktes im PBWU-Projekt ist die Folge.

Zudem werden überwiegend der Grundlagenforschung zuzuordnende Gutachter eingesetzt. Dies muß die vom Auftraggeber beabsichtigte, problemlösungsorientierte Forschung in Richtung grundlagenorientierter, von weitgehender wissenschaftlicher Freiheit geprägten Forschung ablenken. Es liegt nahe, daß zumindest im Rahmen der externen Begutachtung die wissenschaftliche Qualität und Relevanz des Antrags mehr im Vordergrund stehen als die Einpassung in das politisch dominierte Gesamt(Rahmen)programm [HILL \& RIESER 1983, S. 171]. Damit sichert sich die Wissenschaft die Einbeziehung grundlagenorientierter Forschung in ein grundsätzlich anwendungsorientiertes Programm.

Von der Vorgabe, die genannten DFG-Gutachter zu beteiligen, muß die Projektgruppe in der Folge antragsthematisch oder terminlich bedingt allerdings immer öfter abweichen. Sie kommt damit in den Genuß eines potentiellen Steuerungseinflusses aufgrund der Auswahl von Gutachtern. Dies wird von den Gremien entsprechend kritisiert, zumal die Namen der Gutachter sowie die Gutachten selbst mit dem Hinweis auf erforderliche Anonymität von der Projektgruppe anfangs nicht preisgegeben werden. Nach Neuordnung der PBWU im Jahr 1988 muß diese ihre Gutachterauswahl mit dem Wissenschaftlichen Beirat - in der Regel mit dessen Sprecher - abstimmen, woraus aufgrund der inzwischen bestehenden Akzeptanz der Projektgruppe allerdings keine Interessenkonflikte entstehen. Der Beirat akzeptiert die Gutachter-Vorschläge der PBWU. Letztlich baut die Projektgruppe über die gesamte Projektlaufzeit einen Gutachterstamm von rund 80 Fachwissenschaftlern auf (s. interne Gutachterlisten der PBWU; i.Q.: PBWU, ohne Datumsangabe).

Eine absichtliche Begünstigung oder Benachteiligung konkreter Anträge durch die Auswahl bestimmter Gutachter kann der PBWU weder unterstellt noch nachgewiesen werden. Die PBWU ließ sich in ihrer Auswahl von der fachlichen Expertise der Gutachter leiten und versuchte, durch die Beteiligung von 2-3 Gutachtern die Bewertung auf eine fachlich abgesicherte und mehrheitliche Basis zu stellen. Sie war auf Gutachternennungen von außen angewiesen und kaum in der Lage, die Qualifikation der jeweiligen Gutachterperson zu beurteilen und eventuell bestehende persönliche Beziehungen zum Antragsteller zu (er)kennen. Es wurden Wissenschaftler ausgewählt, die von den bereits involvierten Gutachtern oder anderen Förderinstitutionen der PBWU nach dem Kriterium fachlich-thematischer Kompetenz genannt worden waren. Gegenseitige Begutachtungen oder innerhalb desselben Instituts wurden grundsätzlich ausgeschlossen [s.a. HILL \& RIESER 1983, S. 209]. Im Falle von Fortsetzungsanträgen 
wurde versucht, auf dasselbe Gutachterkollektiv wie zur Erstbegutachtung zurückzugreifen. Bekannte oder vermutete Gutachter/Antragsteller-Beziehungen wurden soweit möglich durch die Wahl von Alternativ-Gutachtern berücksichtigt. Von einzelnen Antragstellern selbst empfohlene Gutachter blieben unberücksichtigt.

Der aktive Steuerungseinfluß der PBWU aufgrund der Funktion der Gutachterauswahl sollte daher nicht überschätzt werden. Zwar kann unterstellt werden, daß in wenigen Einzelfällen die Projektgruppe nach eigener Neigung bzgl. eines Forschungsantrags bekanntermaßen kritische oder aber positiv gestimmte Gutachter absichtlich am Verfahren zu beteiligen oder auszuschließen versuchte. Was nachweislich bleibt, ist die Nähe zu DFG-Gutachtern und damit die Bevorzugung grundlagenforschungsorientierter Wissenschaftler. Damit und mit der Bewertung der Gutachtervoten durch das wissenschaftliche Beiratsgremium der PBWU steht generell die Beurteilung fachlich-inhaltlicher Aspekte von Forschungsanträgen mehr im Mittelpunkt der Beurteilung als die forschungspolitisch problemlösungsrelevanter Aspekte zum Thema Waldschäden.

\subsection{Gering formalisierte Begutachtungsmodalitäten}

Hervorgehoben werden muß, daß die externe Begutachtung der Anträge im PBWU-Projekt ohne wesentliche strukturelle, inhaltliche und sonstige Vorgaben an die Gutachter erfolgte. Nach Umfang, Inhalt, Beurteilungskritierien und deren Gewichtung sowie der Bildung des Gesamturteils blieb die Ausgestaltung des Gutachtens dem Autor selbst überlassen. Die PBWU bat lediglich um ,fachlichen Stellungnahme“ zu dem jeweiligen Antrag, wobei dem Gutachter das Rahmenprogramm der PBWU bereits bekannt war oder bekannt gemacht wurde. Meist wurde die Einpassung des Antrages in das Rahmenprogramm explizit mit abgefragt, auch wenn innerhalb der PBWU-Gremien Uneinigkeit darüber bestand, ob diese Aufgabe den Gutachtern überhaupt zugemutet oder nicht besser innerhalb der PBWU-Gremien beurteilt werden sollte. Formelmäßig wurde mitgeteilt, daß i.d.R. mehrere eingeholte Fachgutachten Grundlage für die weitere Beratung und Förderentscheidung in den PBWU-Gremien darstellten. Im Falle von Begutachtungsrunden wurden den Gutachtern alle Anträge bzw. Kurzfassungen mitsamt einer finanziellen Zusammenstellung übermittelt. Von Finanzmittelknappheiten wurden die Gutachter im Anschreiben gegebenenfalls informiert und gelegentlich um das Anlegen eines kritischen Maßstabes hinsichtlich der Bewertung der beantragten Fördermittel gebeten (i.Q.: PBWU, verschiedenen Datums).

Der freizügige Begutachtungsmodus ohne weitere inhaltliche Vorgaben kann mit dem generellen und vertraglich fixierten Anliegen des PBWU-Projektes, im Auftrag des Bayerischen Umweltministeriums möglichst unbürokratisch Waldschadensforschung zu fördern, begründet werden (s. Verträge zwischen Umweltministerium und GSF zur Durchführung der PBWU; i.Q.: Bayer. Umweltministerium, verschiedenen Datums). Auch wurde unterstellt, dem weitgehend dem Gutachterkreis der Deutschen Forschungsgemeinschaft angehörenden Fachgutachter seien die dortigen Richtlinien geläufig und würden ohnehin zur Orientierung dienen. Letztlich könnte auch die Auffassung, die eingeholten Gutachterempfehlungen nur als Hilfsmittel zur Förderentscheidung der PBWU-Gremien zu verwenden [FELT et al. 1995, S. 242], zu dem wenig formalisierten Procedere geführt haben. 
Dies bedeutet, daß die Gutachter aufgrund unterschiedlicher Wahrnehmung und Interpretation individuell wichtig erscheinenden Kriterien verwenden, bewerten und gewichten konnten. Eine annähernd objektive Gewichtung der beurteilten Kriterien sowie die Vergleichbarkeit der jeweiligen Einzelgutachten und damit die Bildung einer Gesamtempfehlung ist somit erschwert. Zwar zeigen die Analyse-Ergebnisse im Rahmen der Arbeit von STEUER [1997, S. 29ff], daß auch mit diesem Vorgehen auf nahezu alle wesentlichen Bewertungskriterien eingegangen wird und insbesondere auch „qualitative“ und „nicht parametrisierbare Beurteilungsgrößen“ [FELT et al. 1995, S. 238] einfließen können. Die Gesamtempfehlung des Gutachters muß jedoch nicht strikt dem mathematisch herleitbaren Resultat aus den Einzelempfehlungen zu verschiedenen Kriterien folgen. Die unvermeidliche qualitative - wie quantitative - Heterogenität der Gutachten hat zur Folge, daß in deren Zusammenfassungen durch die Projektgruppe oder den Wissenschaftlichen Beirat überwiegend zwar die Gesamteinschätzung der Gutachter, aber eventuell auch einzelne markante Beurteilungsdetails unsystematisch herausgegriffen - durchaus zur Grundlage für weitere Entscheidungen erhoben werden konnten. Diese Vorgehensweise sichert im wesentlichen die Respektierung des Gesamturteils der Gutachter. Unterhalb der Ebene der Gesamtbeurteilung läßt sie allerdings erhebliche Interpretationsspielräume, z.B. in inhaltlicher oder finanzieller Hinsicht, zu.

\subsection{Kriterien der Antragsbewertung}

Idealvorstellung ist, die Antragsqualität hinsichtlich des erwarteten Forschungserfolgs mit Hilfe geeigneter, meßbarer und vergleichbarer Faktoren zu überprüfen, um begründete Förderentscheidungen treffen zu können. Geeignete Erfolgskriterien sind jedoch schwierig zu ermitteln und kritisch zu beurteilen; objektive ex ante-Beurteilungen von Anträgen durch meßbare Faktoren sind nicht möglich. Daher werden i.d.R. Sachverständige zugezogen, die mittels Hilfskriterien den Antrag verbal beurteilen [HILL \& RIESER 1983, S. 247ff].

Um Subjektivitätseinflüsse zu kontrollieren und zu minimieren, erscheint es den Autoren der Schweizerischen Untersuchung sinnvoll, mehrere Gutachter für die Beurteilung eines Antrags heranzuziehen, die Bewertung zu formalisieren und die Begutachtungskriterien einschließlich deren Interpretation explizit zu benennen. Bevorzugt wird ein Punktvergabe-Verfahren (Scoring-Verfahren), mit dem alle relevanten Kriterien in ihrer Ausprägung meßbar erfaßt, in einer Bewertungsmatrix gewichtet und zu einem Gesamturteil zusammengeführt werden können. Dieses Verfahren hat insbesondere den Vorteil der Vergleichbarkeit, hoher Systematik und Konsistenz, Klarheit in Fragen und Antworten und minimiert verschiedene Subjektivitätseinflüsse des Gutachters. Eine Rangfolge der Kriterien sowie der Gesamtbeurteilung kann ohne weiteres erstellt werden, welche unter anderem erlaubt, positiv beurteilte Anträge ihrer Rangfolge nach zu bedienen [HILL \& RIESER 1983, S. 254 ff].

Allerdings engt das Verfahren die Begutachtungsspielräume des Gutachters stark ein, erlaubt keine Nuancierung und ist wenig flexibel. Eine Kombination aus zahlenmäßiger Bewertung vorgegebener Begutachtungskriterien und verbaler Gesamtbeurteilung wird daher als Kompromiß empfohlen. Gegenüber einer formalen Rangfolgebewilligung wird allerdings die fachlich kompetente Beratung der Gutachten durch ein aus allen tangierten Fachbereichen zusammengesetztes Fachgremium vorgezogen, wobei die Diskussion auf uneindeutige Empfehlungen 
begrenzt werden kann. Deren Ergebnis kann dem Finanzgeber letztlich als Grundlage für Finanzmittelzuteilungen dienen [HILL \& RIESER 1983, S. 254ff, 266, 279ff]. Übertragen auf das PBWU-Projekt entspricht der Wissenschaftliche Beirat dem empfohlenen Fachgremium weitgehend.

Unabhängig von den Modalitäten des Begutachtungsverfahren im einzelnen kommt generell den verwendeten Beurteilungskriterien eine entscheidende Bedeutung für die fachliche Bewertung des Forschungserfolges zu. Daß bei der Beurteilung die fachliche Qualität des Antrags im Mittelpunkt der Bewertung steht, können auch die Schweizer Autoren mit Hinweis auf eine einschlägige amerikanische Studie belegen. Dort zeigte sich, daß bei der Begutachtung wissenschaftliche Gesichtspunkte gegenüber persönlichen Merkmalen des Antragstellers dominieren. Allerdings traten starke Differenzen bzw. fehlende Übereinstimmung in den verschiedenen Gutachterexpertisen zu einem Antrag auf, die unterschiedliche menschliche Wahrnehmungsbzw. Beurteilungsunterschiede dokumentieren [HILL \& RIESER 1983, S. 251/252].

Um die Antragsbeurteilung durch externe Fachgutachter im PBWU-Projekt zu analysieren, wurde im Rahmen der Arbeit von STEUER [1997, S. 9-12] alle Einzelgutachten erfaßt. Da die Begutachtung durch weitestgehende Freiheit in der Verwendung bestimmter Beurteilungskriterien gekennzeichnet war, mußte hierzu erst ein Kategoriensystem, bestehend aus inhaltlichen, Verfahrens- und Umfeldkriterien, entwickelt werden. Anschließend wurden die tatsächlich verwendeten Beurteilungskriterien dem Kategoriensystem zugeordnet und quantitativ erfaßt.

Folgende inhaltlichen Kriterien wurden, in Anlehnung an die der DEUTSCHEN FORSCHUNGSGEMEINSCHAFT (DFG) [1992, S. 5ff] und an ein Screening der im Rahmen des PBWUProjektes erstellten Gutachten definiert und zur Kartierungsgrundlage erhoben [STEUER 1997, S. 10-12]:

- Anpassung des Antrags an das Rahmenprogramm (Programmvorgaben)

- Theorie-Qualität (Forschungshypothese)

- Methodik-Qualität (Untersuchungsansatz)

- Qualifikation und Kompetenz des Antragstellers

- Effektivität (Durchführbarkeit und erwarteter wissenschaftlicher Erfolg)

- Finanzrahmen (quantitativ, Mittelumfang)

- Kosten (qualitativ; Begründung und Rechtfertigung der beantragten Mittel)

- Kooperation in einem Forschungsverbund

- Antragsformalia

- Folgeprojekt (Antragsbewertung mit Bezug auf Erst-Vorhaben)

- Persönliche Beziehung zwischen Antragsteller und Gutachter

Wie aus den Häufigkeiten der definierten Kriterien in den insgesamt über 250 ausgewerteten Einzelgutachten hervorgeht [STEUER 1997, S. 29ff], kann mit dem gewählten Kategoriensystem das Bewertungsspektrum der Gutachter ausreichend präzise und repräsentativ abgegriffen werden. 


\subsection{Inhalte der Gutachten}

Die Analyse bestätigt, daß im PBWU-Projekt die Beurteilung der fachlichen Qualität von Forschungsanträgen im Vordergrund steht. Sie dominiert gegenüber personenbezogenen, aber auch gegenüber kostenbezogenen Kriterien [STEUER 1997, S. 29ff]. So weist das Kriterium „Effektivität“, also die gutachterseits eingeschätzten Durchführungs- und Erfolgserwartungen mit über $80 \%$ aller maximal möglichen Nennungen die weitaus höchste Erwähnungsrate in den Gutachten auf. Es folgen mit jeweils über 60\% Erwähnungsrate die Kriterien „Theorie“ und „Methodik“ sowie die „Einpassung in das Rahmenprogramm“. Daß letztgenanntes Kriterium von den Gutachtern vergleichsweise häufig genannt wird, kann darauf zurückgeführt werden, daß im Anschreiben der PBWU an die Gutachter auf diese formale Voraussetzung regelmäßig hingewiesen wurde. Mit mittlerer Häufigkeit (55\%) wird der „Finanzrahmen“ erwähnt, während „Kosten“, „Kooperation in einem Forschungsverbund“ sowie „Qualifikation des Antragstellers“ mit je 36-37\% unterdurchschnittlich häufig erwähnt werden. Äußerungen zu Antragsformalia werden nur selten (7\%) und nur als Negativkriterium diesbezüglicher Mängel getroffen (s. Abbildung 7).

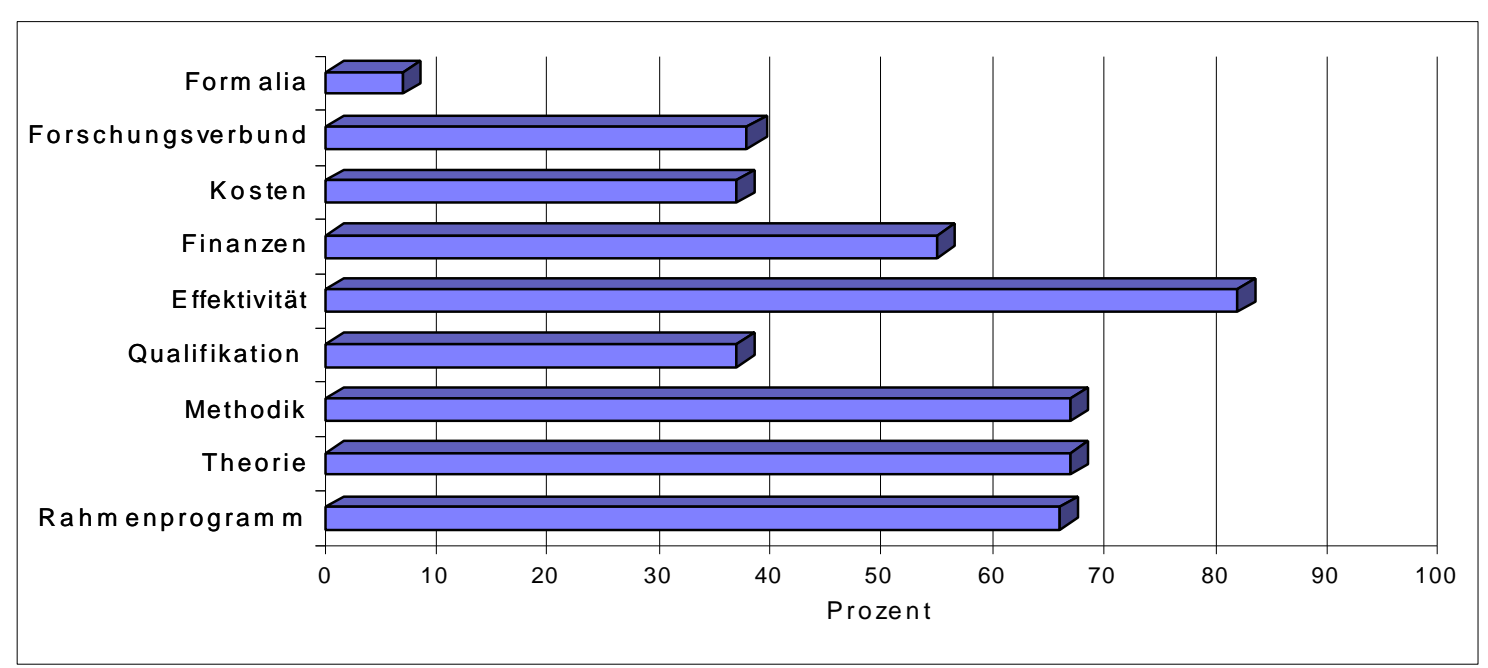

Abb. 7: Bewertungskriterien der Gutachten im PBWU-Projekt (in \% aller Vorhaben) [STEUER 1997, S. 30]

Der Befund, daß fachliche gegenüber personenbezogenen Kriterien in der Erwähnungshäufigkeit überwiegen, weist zwar auf die wissenschaftliche Betonung der Begutachtung hin. Er kann jedoch nicht mit einem Hinweis auf die Objektivität der Begutachtung gleichgesetzt werden. Abgesehen von der generell nicht gegebenen Wertfreiheit des Evaluationsurteils [s.a. FELT et al. 1995, S. 241] kann konkret durch die Wahl eines dem Antragsteller bekanntermaßen persönlich zu- oder abgeneigten Gutachters das Begutachtungsergebnis verzerrt werden. Für eine sachgerechte Beurteilung ist zwar Voraussetzung, Experten des tangierten Fachgebiets des Antragstellers einzusetzen. Mit der Nähe zum Fachgebiet und damit der Kompetenz zur fachlichen Beurteilung steigt jedoch die Wahrscheinlichkeit, daß Antragsteller und Gutachter in ein 
Beziehungsgeflecht wissenschaftlicher Konkurrenz oder Koalition eingebunden sind, sich jedenfalls nicht neutral gegenüber stehen.

Derartige Beziehungen werden bei der Beurteilung der Anträge von Wissenschaftlerkollegen in den seltensten Fällen explizit in den Gutachter ausgedrückt und müssen nicht zwangsläufig in dem Kriterium „Qualifikation des Antragstellers“ gesucht werden. Aufgrund nur sehr weniger derartiger Schriftwechsel und mündlicher Mitteilungen im PBWU-Projekt läßt sich zwar vermuten, daß einzelne Begutachtungen im PBWU-Projekt von Beziehungen zwischen beiden überprägt waren. Und auch die - allerdings nur unterdurchschnittlich häufige - Verwendung des genannten, personenbezogenen Kriteriums läßt eher auf subjektive Bewertungseinflüsse schließen als fachliche Kriterien.

Über die Häufigkeit hinausgehend kann bei der Kartierung der einzelnen Beurteilungskriterien auch hinsichtlich ihrer positiven, negativen oder nicht eindeutigen oder wertungsfreien Verwendung differenziert werden [STEUER 1997, S. 36-44]. Danach sind als Positivkriterien vor allem die bewertete „Effektivität“ der beabsichtigten Forschung, ,Einpassung in das Rahmenprogramm“ sowie „Kooperation im Forschungsverbund“ zu sehen. Abgesehen von dem erstgenannten Kriterium sind dies Aspekte, die sich von den Gutachtern auch vergleichsweise leicht und eindeutig bestimmen lassen. Auch die „Qualifikation des Antragstellers“ wurde fast ausschließlich in positiver Hinsicht verwendet. Trotz weitgehender Anonymität könnte hierbei ein grundsätzlich höflicher Umgang miteinander zum Ausdruck kommen. Das „Kosten“Kriterium wurden demgegenüber als hauptsächlicher Kritikpunkt (Negativkriterium) verwendet und spiegelt sich in entsprechenden, häufig vorgenommenen Kürzungsvorschlägen der Gutachter wider.

Diese Detailergebnisse lassen sich zu der Gesamtaussage verallgemeinern, „daß inhaltliche Konzeption, Anpassung an das Rahmenprogramm, wissenschaftliche Effektivität sowie positive Qualifikation des Antragstellers ausschlaggebende Gründe für die positive gutachterliche Einschätzung gewesen waren“. Ablehnungsempfehlungen der Gutachter zu Forschungsanträgen wurden hingegen überwiegend mit formalen und Kostenargumenten begründet [STEUER 1997, S. 37, 44]. Die Einpassung des Antrages in das Rahmenprogramm mußte, zumal bei diesbezüglicher Vorsortierung der Anträge anfangs durch die Projektgruppe, später den Wissenschaftlichen Beirat, konsequenterweise als Positivkriterium verwendet werden. Da gutachterseitige Negativ-Bewertungen naturgemäß eher einer kritischen Hinterfragung standhalten müssen als positive Bewertungen, ist hierbei die Verwendung von Argumenten naheliegend, die möglichst zuverlässig und leicht $\mathrm{zu}$ begründen sind. Daß im PBWU-Begutachtungsverfahren fachliche Aspekte zur „Theorie“ und „Methodik“ häufig wertneutral eingesetzt wurden und negative Beurteilungen überwiegend durch „harte“ formale wie finanzielle Kriterien begründet wurden, stützt diese Annahme.

Mit der Verwendung forschungsstruktureller Kriterien wie die Einpassung in den Programmrahmen und Einbeziehung in den interdisziplinären Forschungsverbund kann jedoch noch kein Anspruch auf eine interdisziplinäre Begutachtung im PBWU-Projekt erhoben werden. Das PBWU-Begutachtungsverfahren fußt im wesentlichen auf einer antragsweisen fachlichen Beurteilung durch in der Regel als intradisziplinär kompetent ausgewiesene Experten. Weder die 
anfangs durchgeführte Behandlung der Anträge in einer gemeinsamen, mündlichen Begutachtungsrunde, noch die Übermittlung auch aller weiteren Anträge des gesamten Antragsspektrums an den jeweiligen Fachgutachter, können dieses Manko beseitigen. Unter Umständen könnte hier die Bewertung personenbezogener Kriterien (z.B. bzgl. des Disziplinenübergreifenden Integrations-, und Kooperationsvermögens oder der Risikobereitschaft des Forschers) sogar weiter führen als die überwiegend fachspezifisch-inhaltliche Beurteilung des Antrags. Auf den ebenso wichtigen, interdisziplinären Abstimmungsbedarf auf Seiten der Antragsteller noch vor der Begutachtungsphase soll jedoch auch an dieser Stelle hingewiesen werden.

\subsection{Mitglieder des Wissenschaftlichen Beirats als Antragsteller}

Auch wenn der Wissenschaftliche Beirat ausnahmlich der ersten beiden Antragsrunden 1985/86 selbst bei der Vorbereitung von Förderentscheidungen mitwirkte, blieb es seinen Mitgliedern unbenommen, selbst Anträge oder solche aus dem Umfeld der eigenen Arbeitsgruppe einzubringen. Daß sie bei der konkreten Beratung eigener Anträge in den PBWU-Gremien ausgeschlossen werden, versteht sich von selbst. Auch gehört es nach eigener Auffassung zum Selbstverständnis der im Beirat vertretenen Wissenschaftler, sich im Begutachtungsverfahren zurückzuhalten, um nicht den Eindruck eines „Selbstbedienungsladens“ zu erwecken. Generell nehmen Anträge aus dem Kreis des Wissenschaftlichen Beirats aufgrund der Konstellation, Antragsteller und gleichzeitig Entscheidungsträger zu sein, eine Sonderstellung ein. Es kann angenommen werden, daß von seiten der Gutachter, vom Finanzgeber sowie von Kollegen des Gremiums trotz beabsichtigter Verfahrensneutralität zumindest informal eine gewisse Begünstigung der Anträge von Beiratsmitgliedern stattfand.

Nach der Analyse des Förderverfahrens [STEUER 1997, S. 61-66] ist ein hoher Anteil, nämlich rund $40 \%$ aller bewilligten Forschungsvorhaben, Mitgliedern des Wissenschaftlichen Beirats bzw. deren Arbeitsgruppen zuzuordnen. Auch finanziell beanspruchen die Forschungsvorhaben aus dem Kreis des Beirats mehr als die Hälfte (55\%) aller Fördermittel für Forschungsvorhaben im PBWU-Projekt. Die Anzahl (etwa 30 Vorhaben insgesamt) sowie die Fördermittel bleiben, absolut betrachtet, über die Jahre hinweg zwar in etwa gleich hoch. In Relation zur Gesamtzahl nimmt der Anteil bewilligter Vorhaben des Beirats jedoch dann von 26\% auf 51\% zu, als dieser - gegenüber den ersten beiden Begutachtungsrunden - am Förderverfahren beteiligt wird. Hinsichtlich der beanspruchten Fördermittel steigt der Anteil von $42 \%$ auf etwa 70\%. Zudem weisen die Anträge aus dem Kreis des Beirats eine vergleichsweise (um jeweils über 10 Prozentpunkte) höhere Förder- bzw. geringe Ablehnungsrate auf.

Daß ein erheblicher Anteil der Waldschadensforschung im PBWU-Projekt von Mitgliedern dessen wissenschaftlichen Beratungsgremiums oder aus dessen direktem Umfeld geleistet wurde, ist aufgrund der Zusammensetzung des Gremiums vorerst nicht überraschend. Der Schritt, mehrere renommierte, selbst in der Waldschadensforschung tätige Forscher in das Gremium zu berufen, implizierte dies zwangsläufig, wollte man nicht auf den Beitrag dieser wichtigen und fachlich hoch kompetenten Forschungsträger verzichten. 
Die Befunde zur quantitativen und zeitlichen Analyse des Begutachtungsverfahrens stützen jedoch die Vermutung, daß Anträge aus dem Kreis des Wissenschaftlichen Beirats einen bevorzugten Status besaßen, insbesondere zu Zeiten maßgeblicher Beteiligung des Gremiums am Förderverfahren.

Hierfür können folgende Gründe angeführt werden:

Bereits in der Phase der Rahmenprogrammgestaltung, an der der Wissenschaftliche Beirat zumindest in gewissem Umfang beteiligt war, konnte das Gremium seine Zielvorstellungen hinsichtlich relevanter Forschungsrichtungen und thematischer Schwerpunkte einbringen. Daß diese an den bearbeiteten Forschungsgebieten der einzelnen Beiratsmitglieder Anleihe nehmen, ist naheliegend und kann auch in verschiedenen Schriftstücken zur Modifizierung des PBWURahmenprogramms dokumentiert werden.

Weiterhin genossen die Mitglieder des Gremiums zwangsläufig erhebliche Informationsvorteile und zeitliche Vorteile gegenüber externen Antragstellern, wie z.B. Kenntnisse über Finanzen oder thematische Rahmenbedingungen, zu schließende Forschungslücken und fachlichkonzeptionelle Diskussionen während der Gremiensitzungen. Diese konnten bei der Antragstellung zur Verbesserung der Förderchancen genutzt werden.

Die gutachterseits vorgetragene Aufforderung, die im benachbarten Forschungsverbund der Bayerischen Forschergruppe Forsttoxikologie bearbeiteten Themen auch im PBWU-Projekt nicht zu vernachlässigen, kann als Bevorzugung von Beiratsmitgliedern verstanden werden. Mehrere führende Wissenschaftler dieses Forschungsschwerpunktes waren im Wissenschaftlichen Beirat vertreten; sie konnten somit von einer gutachterlicher Unterstützung dortiger Forschungsthemen auch im PBWU-Projekt nicht nur ausgehen, sondern forderten diese sogar.

Bei dem Versuch des Projektrats, die Kritik an der Projektgruppe und dem geübten Förderverfahren der ersten Projektphase zu beseitigen, wurde das Wissenschaftlergremium zu einer aktiveren Forschungsbeteiligung im PBWU-Projektes ausdrücklich aufgefordert.

Gerade für das Bayerische Umweltministerium kann das Bestreben unterstellt werden, die Mitglieder des Beirats dem PBWU-Projekt allgemein gewogen zu machen, wozu die verfahrensmäßige Beteiligung ebenso wie die Förderbeteiligung beitragen konnten. Angesichts der anfänglich bestehenden Kritik waren entsprechende Maßnahmen um so notwendiger, um die Akzeptanz des alpinen Forschungsschwerpunktes zu erreichen und den Forschungsprozeß dort in Gang zu bringen. Den Leiter eines einschlägigen Forschungsinstituts in den Beirat zu berufen und gleichzeitig - mit Erfolg - zur Einreichung von Forschungsanträgen zu animieren, unterstreicht diese Absicht.

In der fortgeschrittenen Begutachtungsphase unter Beteiligung des Beratungsgremiums muß berücksichtigt werden, daß Gutachter und Gutachten zwar gegenüber den externen Antragstellern im Regelfall anonym blieben, nicht jedoch gegenüber dem darüber beratenden Beirat, was den Gutachtern auch bekannt war. Auch wenn Antragsteller aus dem Beiratsgremium bei Behandlung eigener Anträge verfahrensmäßig ausgeschlossen waren, mußten Gutachter be- 
rechtigterweise annehmen, daß Details über Autor und/oder Inhalt von Gutachten dem Antragsteller bekannt wurden. Dies könnte von vorn herein ein Gutachterurteil beeinflußt haben.

Ein weitere Eigen-Begünstigung des Gremiums könnte sich daraus ergeben, daß der Beirat abgesehen von der ersten und zweiten Begutachtungsrunde - die Vorauswahl eingereichter Anträge hinsichtlich ihrer Forschungsinhalte und generellen Einpassung in das Rahmenprogramm vornahm. Dabei ist gerade vor dem Hintergrund knapper Fördermittel eine relative Bevorzugung dahingehend denkbar, daß Anträge externer Forscher nicht weiterverfolgt wurden, verbleibende Anträge aus dem Kreis des Wissenschaftlichen Beirats jedoch einer Begutachtung zugeführt wurden. Die hohe Förderquote von Anträgen aus dem Kreis des Beirats spricht hierfür [STEUER 1997, S. 62-64].

Schließlich konnten Beiratsmitglieder als Antragsteller bei den im Beirat bestehenden Forscherkoalitionen im Regelfall auf eine wohlwollende Empfehlung der Kollegen zum eigenen Antrag bauen. Dabei ist allerdings einzuschränken, daß innerhalb des Gremiums aufgrund unterschiedlicher fachlicher, forschungsstandörtlicher oder persönlich bedingter Koalitionsbildungen auch Konkurrenzerscheinungen auftreten konnten mit der Folge ablehnender Einstellung gegenüber Anträgen von Beiratskollegen. So wurden bspw. der unter anderem strukturell bedingt hohe Kostenansatz von Anträgen außeruniversitärer Beiratsmitglieder häufig Kritikpunkt der Koalition universitärer Beiratsmitglieder. Ähnliches gilt für das Verhalten von Befürwortern und Kritikern des alpinen Forschungsstandorts Wank, was sich bei der Behandlung entsprechender Anträge aus dem Kreis des Wissenschaftlichen Beirats niederschlagen konnte.

Können derartige Begünstigungsmomente angenommen haben, so wurden sie jedoch unterschiedlich genutzt. Auffällig ist, daß einige Mitglieder des Beirats bzw. die vertretenen Institute überproportional viele, andere überhaupt keine Vorhaben im PBWU-Projekt einbringen konnten oder wollten. Ausschlaggebend dafür scheint die generelle Interessenlage sowie die Verknüpfbarkeit der eigenen Forschungsrichtung mit den Themen oder regionalen Forschungsschwerpunkten des PBWU-Projektes zu sein. Zwangsläufig trägt die starke Beteiligung von Beiratsmitgliedern an der PBWU-Waldschadensforschung zu einer Konzentration der beteiligten Forschungsinstitutionen sowie damit der bearbeiteten Forschungsrichtungen bei.

Die dem Wissenschaftlichen Beirat vergleichbare Sonderstellung kann auch auf die von der GSF vorgelegten Anträge übertragen werden. Die GSF war nicht nur über ihren Wissenschaftlichen Geschäftsführer im Projektrat, sondern zu fortgeschrittener Projektphase auch im Wissenschaftlichen Beirat vertreten. Dort konnte sie ihre wissenschaftlichen sowie politischen Interessen einbringen bzw. auf die Klientelverbundenheit in den Gremien zählen. Zudem bestanden zwischen PBWU und GSF-Geschäftsführung sowie GSF-Instituten verschiedene formale wie informale Beziehungen. Diese konnten, da sie eine intensive und kurzfristige Rückkopplung ermöglichten, bspw. als Informationsvorteil gegenüber externen Akteuren genutzt werden. 


\subsection{Fortsetzungsvorhaben begünstigen Inkrementalismus}

Die Auswirkungen des hohen Anteils an Fortsetzungsvorhaben auf die Programmstruktur sollen hier herausgearbeitet werden. Nahezu 30 Vorhaben, das sind knapp 40\%, wurden als Fortsetzungsvorhaben im Anschluß an ein entsprechendes Erstvorhaben zum gleichen Thema durchgeführt [STEUER 1997, S. 19]. Da vom Finanzgeber die Förderperiode von Forschungsvorhaben grundsätzlich auf 2 bis maximal 3 Jahre begrenzt worden war, und eine kurzfristige Verlängerung nur um einige Monate möglich war, wurde von der Beantragung eines Fortsetzungsvorhabens häufig Gebrauch gemacht. Dieses wurde dann wie ein Neuantrag dem üblichen Förderverfahren unterworfen, wobei jedoch versucht wurde, auf die Gutachter des Erstvorhabens zurückzugreifen.

Die voraussichtliche Notwendigkeit wurde z.T. bereits zu Beantragung des Erstvorhabens angedeutet. Überwiegend stellte sich jedoch im Verlauf der Forschungsarbeiten heraus, daß entweder das vorgesehene Programm aufgrund von Verzögerungen erst in einer weiteren Vorhabenphase umfassend und abschließend bearbeitet werden könnte; oder es wären weitere, neue Forschungsaspekte aufgetreten, die in die Forschungsarbeiten notwendigerweise miteinbezogen werden müßten. Beiderlei Aspekte wurden von den Vorhabenleitern zur Begründung für eine Fortsetzung der Arbeiten in Form eines Folgevorhabens herangezogen.

Hinsichtlich der fachlichen Begutachtung bieten Fortsetzungsanträge den Vorteil, die erwartete Forschungsleistung bereits unter Berücksichtigung der Ergebnisse des Erstvorhabens bewerten zu können (s. auch Ausführungen zur Evaluierung von Forschungsberichten Kap. 5.3.5.4). Zudem ist im Falle weiterführender, auf den Arbeiten des Erstvorhabens aufbauender Forschungsaspekte mit einer vergleichsweiser hohen Erfolgswahrscheinlichkeit zu rechnen.

Die Fortsetzung eines verzögerten Vorhabens zu finanzieren, um doch noch zu dem regulär in Aussicht gestellten Forschungsergebnis zu gelangen, stellt nahezu einen Sachzwang dar. Andernfalls würde die laufzeitgemäße Beendigung der Arbeiten einen z.T. erheblichen Verzicht auf die Ergebnisse trotz bisheriger Finanzierung bedeuten. Vergleichbares kann für die Bearbeitung zusätzlicher, für unverzichtbar gehaltener Forschungsaspekte in einem Fortsetzungsvorhaben gelten. Daher wurde von den PBWU-Gremien im Förderverfahren grundsätzlich auch die Bevorzugung von Fortsetzungsanträgen gegenüber der Bearbeitung neuer Fragestellungen festgelegt.

Daß somit Förderentscheidungen der Vergangenheit aktuelle Entscheidungen weitgehend präjudizieren, hemmt die wissenschaftliche Innovationskraft, Neuentwicklungen und notwendige Umorientierungen in der Forschung. Damit dominieren Besitzstands- und Fortschreibungsmaximen das Programm [HILL \& RIESER 1983, S. 281, 222]. Die Wirkung aus der Bevorzugung von Fortsetzungsanträgen tritt naturgemäß mit fortschreitender Projektlaufzeit in zunehmendem Maße auf. Im PBWU-Projekt wurden erste Fortsetzungsvorhaben im Jahr 1988 bewilligt, schon ab 1989 übertreffen Fortsetzungsvorhaben sowohl nach Anzahl als auch nach Fördermitteln die Erstvorhaben [STEUER 1997, S. 18/19]. Die bereits etablierten Forscher und deren Arbeitsgebiete - unter besonderer Beteiligung von Vorhaben aus dem Bereich des Wissenschaftlichen Beirats - prägen zunehmend die Ausrichtung des Gesamtprojektes. 
Dies ist um so negativer zu bewerten, als damit auch derartige Forschungsthemen noch zu fortgeschrittener Implementationsphase und knapper werdenden Fördermitteln Vorzug gegenüber Neuanträgen genießen, welche zu Implementationsbeginn auf Grundlage eines suboptimalen Rahmenprogrammes und gleichzeitig großzügigem Förderverhalten beantragt worden waren. Nur ein neues Forschungsprogramm, oder zumindest die Fortschreibung und Aktualisierung des bisherigen, hätte das Manko, wissenschaftlich weitgehend festgefahren zu sein, beseitigen können. Dies wurde in der letzten Phase des PBWU-Projektes zwar auch bzgl. der alpinen Forschung bzw. der gesamten PBWU-Forschungskonzeption versucht. Allerdings war der politische Handlungsdruck sowie weitere Rahmenbedingungen nicht mehr gegeben, die neuen Forschungskonzepte umzusetzen.

Die Gefahr des „Forschungsinkrementalismus“, d.h. der weitgehenden Fortschreibung der bestehenden Forschungsthemen, ergibt sich nicht nur aus der Bevorzugung von Fortsetzungsvorhaben (zu Inkrementalismus s. KROTT, 1990, S. 67/68; LindBLOM et al. 1979, zit. in: KROTT 1993, S. 105; SCHUBERT 1991, S. 21ff]. Die generelle Schwierigkeit, wissenschaftlich risikoreiche Forschungsvorhaben nach den üblichen Bewertungskriterien hinreichend zuverlässig beurteilen zu können, führt dazu, daß vor allem Projekte mit sicherer Beurteilungsbasis, konventioneller Theorie und Methodik sowie voraussehbarem Erfolg in das Programm aufgenommen werden. Durch die Verwendung spezifischer Bewertungskriterien, wie z.B. die „Originalität“ der Forschung [HILL \& RIESER 1983, S. 287] - bzw. der Forscher -, oder die anteilige Reservierung von Fördermitteln zur Umsetzung neuer, risikoreicher Forscherideen kann die Einbeziehung derartiger Vorhaben erleichtert werden.

Auch im PBWU-Projekt wurde der Aspekt risikoreicher Forschung im Rahmen des Begutachtungsverfahrens mangels formalisierter Bewertungsvorgaben kaum thematisiert. Die Qualifikation des Antragstellers wurde zwar, wie die Analyse des Förderprozesses zeigt, im Rahmen des Begutachtungsverfahrens beurteilt [STEUER 1997, S. 30]. Eine Tendenz hinsichtlich der Berücksichtigung risikoreicher Forschung läßt sich hieraus jedoch mangels qualitativer Analyse dieses Kriteriums nicht ableiten, und würde zudem von dem hohen Anteil an Fortsetzungsvorhaben bei weitem überlagert.

Die Frage nach der Förderung risikoreicher Forschung sollte vor dem Hintergrund des forschungspolitischen Zieles problemlösungsorientierter Forschung gestellt werden. Hierbei ist einerseits zu berücksichtigen, daß risikoreiche Forschung überwiegend bei grundlagenorientierter Forschungsförderung ihre Legitimation erfahren wird, zu der sich im PBWU-Projekt das Umweltressort nicht verpflichtet sehen mußte. Andererseits konnte angenommen werden, daß auch für das komplexe Umweltproblem Waldschäden neuartige, unkonventionelle Forschungsansätze erforderlich wären, um die Ursachen zu klären. Dies betraf nicht nur die neuartige und risikobehaftete Interdisziplinarität der Forschung, sondern auch Aspekte der Forschungsmethodik und Theorie [KROTT 1998, S. 17/18].

Daß auch im Rahmen des PBWU-Projektes zumindest in beschränktem Umfang Risikobereitschaft in der Forschungsförderung besteht, belegen zum einen die Finanzierung einzelner, vergleichsweise unkonventioneller Vorhaben (wie z.B. zum Parasitenverhalten an schadstoffbelasteten Pflanzen oder zur Bearbeitung weitgehend unbekannter Stoffkomponenten). Zum ande- 
ren wurde in großem Umfang methodische Arbeiten mitfinanziert, die z.T. erhebliches Erfolgsrisiko aufwiesen oder eines erheblich längeren Zeitaufwandes bedurften als erwartet (wie z.B. Isolierung und Nachweis verschiedener Inhaltsstoffe oder Pflanzenkompartimente, Methodenübertragung von Modellpflanzen auf Waldbäume). Letztlich bleibt jedoch offen, ob es sich hierbei tatsächlich um beabsichtigte Förderung risikobehafteter Forschung handelte oder lediglich Ausfluß der Implementation eines offenen und wenig stringenten Forschungskonzeptes.

\subsubsection{Evaluierung von Forschungsberichten}

Die Bewertung der in der Regel jährlich vorzulegenden Zwischenberichte bzw. der Schlußberichte zu den einzelnen Forschungsvorhaben erfolgt vertragsgemäß durch die PBWU. Sie fertigt Stellungnahmen hierzu und legt diese mitsamt einer Billigungsempfehlung dem Bayerischen Umweltministerium vor. Inhalt der meist mehrseitigen Stellungnahmen ist eine Darstellung der Zielsetzung des Vorhabens und der bisherigen Arbeiten und Ergebnisse vor dem Hintergrund des bestehenden Arbeitsprogramms und Zeitplans. Abschließend werden - insbesondere im Falle von Schlußberichten - die Ergebnisse hinsichtlich des Beitrags zum Gesamtprogramm und des Klärungsbeitrages zu den Waldschadensursachen gewürdigt.

Im Mittelpunkt der Berichtsevaluierung stehen formale Aspekte eines Ist/Soll-Vergleiches zwischen Bewilligung/Vertrag und tatsächlich durchgeführten Arbeiten. Dies kann von der Projektgruppe selbst unschwer geleistet werden. Eine weitergehende, wissenschaftliche Überprüfung der Arbeiten und Ergebnisse findet nicht statt.

Wie aus der Analyse des Förderverfahrens in der Arbeit von STEUER [1997, S. 58-61] hervorgeht, werden die vorgelegten Berichte fast ausnahmslos von PBWU bzw. Umweltministerium gebilligt. In seltenen Fällen (weniger als $10 \%$ aller Zwischen- und Schlußberichte) werden Nachbesserungen von den entsprechenden Forschergruppen eingefordert, die jedoch überwiegend formalen Charakter aufweisen. Dabei steht die PBWU für erforderlich erklärten Programm-Modifikationen, Zielverschiebungen oder methodischen Neuorientierungen in der Durchführung des Forschungsprogrammes meist positiv gegenüber. Wissenschaftlich begründeten Modifizierungswünschen könnte die PBWU ohnehin kaum fundierte Ablehnungsgründe entgegenstellen. Nur in einigen Ausnahmefällen deutlicher Abweichungen vom ursprünglichen Programm, zu deren fachlicher Beurteilung sich die PBWU außerstande sieht, werden Mitglieder des Wissenschaftlichen Beirats hinzugezogen. In den Fällen, in denen zum Zeitpunkt der Berichtsvorlage die beschriebenen Modifizierungen längst vollzogen waren, wäre eine Ablehnung zudem wirkungslos.

Häufig sind im Bericht vorgetragene Modifizierungswünsche gleichzeitig mit einem Antrag auf entsprechende Mittelaufstockung oder notwendig gewordene Laufzeitverlängerung verbunden. Auch hier unterstützt die PBWU - bei entsprechender fachlichen Begründung und in einigen Fällen nach Rücksprache mit dem Wissenschaftlichen Beirat - das Anliegen der Projektleiter gegenüber dem Finanzgeber. In der Regel akzeptiert auch der Finanzgeber die Modifizierungswünsche. Insbesondere kostenneutrale Modifizierungen werden ohne weiteres akzeptiert. 
Insgesamt unterstützt das gegenüber den Projektleitern wohlwollende Verhalten der PBWU und des Auftraggebers gegenüber fachlichen Modifizierungswünschen die wissenschaftliche Selbststeuerung der Forschung. Sie trägt damit indirekt zu einer Stärkung des bottom upAnsatzes in der Waldschadensforschung bei. Die strenge Reglementierung der Forschung in Form eines strikten Festhaltens an den vertraglichen Vorgaben aus Arbeits- und Zeitplan würde den Charakteristika der Forschung nicht gerecht werden und deren durchaus effektivitätssteigernden Selbststeuerung zuwiderlaufen [HILL \& RIESER 1983, S. $281 \mathrm{ff}$ ].

Wesentlich kritischer fällt jedoch die Gesamteinschätzung der PBWU zum forschungspolitisch erwarteten bzw. erzielten Ergebnis des jeweiligen Forschungsvorhabens aus. Hier stellt sich die Projektgruppe auf die politische Ebene der Forschung und äußert sich zu dem Klärungsbeitrag im Sinne des auftraggebenden Umweltressorts. Beanstandet werden häufig der überwiegende Anteil an methodischen oder grundlagenforschungsorientierten Arbeiten und Ergebnissen zulasten eines nur geringen oder stark fachspezifischen Beitrags zur Ursachenklärung der Waldschäden. Die häufige Kartierung des Kriteriums „,Methodik“, „Effektivität der Forschung“ und „Einpassung in das Rahmenprogramm“ bei der Analyse der PBWU-Berichtsstellungnahmen in der Arbeit von STEUER [1997, S. 56/57 und 60] unterstreicht die forschungspolitische Bedeutung, die die PBWU diesen Aspekten beimißt.

Damit unterscheidet sich die Einschätzung der PBWU zum Forschungsergebnis in ihrer Stellungnahme von der des Projektleiters im Zwischen- oder Schlußbericht. Während die PBWU den politisch verwertbaren Beitrag des Forschungsergebnisses herausfiltriert, ist es in der Regel das Anliegen des Forschers, auch und gerade den wissenschaftlichen Erfolg der Forschung in der Berichtsdarstellung hervorzuheben. Gerade weil die Forschungsvorhaben häufig in Form von Dissertationen bearbeitet werden, die dann zentralen Bestandteil des Schlußberichtes bilden, ist der Erfolgsmaßstab der Forschung stärker wissenschaftlich gegenüber der scientific community, als forschungspolitisch gegenüber dem Ministerium formuliert. War noch im Forschungsantrag der Inhalt des Vorhabens auf das Ziel des Forschungsförderers focussiert, einen politisch verwertbaren Beitrag zur Waldschadensforschung zu liefern, verliert sich dieser Aspekt in den Berichten häufig immer mehr zugunsten des wissenschaftlichen Forschungserfolges. Die Diskrepanz zwischen forschungspolitischem Anspruch und Wirklichkeit kann von der PBWU bereits im Rahmen eines Ist/Soll-Vergleiches des Forschungsergebnisses dokumentiert werden.

Nahezu zwangsläufig muß das Forschungsergebnis von PBWU wie Bayerischem Umweltministerium jedoch akzeptiert werden. Auch wenn die Zahlung der Schlußrate von der Billigung des Berichtes abhängig gemacht wird, bestehen erforderlichenfalls kaum noch Nachbesserungsmöglichkeiten. Das Untersuchungsprogramm ist bei Schlußberichtsvorlage meist längst abgeschlossen, die Mittel verausgabt, das durchführende und vom Bayerischen Umweltministerium finanzierte Personal (Doktoranden, technische Angestellte etc.) bereits nicht mehr auf dem Projekt beschäftigt. Lediglich formalen, redaktionellen Änderungswünschen des Schlußberichtes könnte noch nachgekommen werden.

Letztlich kann das Evaluierungsobjekt der Forschungsberichte für eine Steuerung und Kontrolle des Forschungsprozesses in der gegebenen Form nicht sinnvoll eingesetzt werden. Den 
Befunden aus der Untersuchung der schweizerischen Forschung vergleichbar [HILL \& RIESER 1983, S. 200 ff und 280 ff], können damit überwiegend nur formale Aspekte der Forschung wie zeitliche und thematische Einhaltung bzw. Abweichungen im Forschungsplan berücksichtigt werden. Abweichungen von der Programmplanung können i.d.R. kaum rechtzeitig entdeckt werden. Der erste Zwischenbericht dokumentiert ohnehin meist nur die Erarbeitung methodischer Grundlagen. Steuernde Einflußmöglichkeiten auf den weiteren Forschungsverlauf sind im Falle erfolgter oder vermuteter Abweichungen nur gering, auch wenn die Zuteilung der jeweils nächsten Mittelrate die Billigung des fälligen Berichtes zur Voraussetzung hat.

Während bei Einphasenprojekten (d.h. ohne Fortsetzungsphase) dem Schlußbericht überwiegend nur dokumentarischer Charakter zukommt, ist dieser im Falle von Fortsetzungsanträgen beurteilungsrelevant [HILL \& RIESER 1983, S. 243/244]. Zwar waren im PBWU-Projekt die Vorlagetermine für Schlußberichte erst auf einige Monate nach Laufzeitende festgesetzt, was die Möglichkeiten einer ex post-Einflußnahme ohnehin stark reduziert. In den Folgeanträgen wurden allerdings die im Schlußbericht zu dokumentierenden Ergebnisse vorweggenommen und damit zum wesentlichen Bestandteil fachlicher Beurteilung gemacht.

Von der naheliegenden Möglichkeit, die externen Gutachter der Forschungsanträge - wie bei der Bewertung von Fortsetzungsanträgen - auch im Verlauf des Vorhabens zur Beurteilung der betreffenden Forschungsberichte einzusetzen, oder fachlich kompetente Mitglieder des Wissenschaftlichen Beirats regelmäßig zu beteiligen, wurde im PBWU-Projekt nicht Gebrauch gemacht. Gründe hierfür können sein, daß die wissenschaftliche Feinabstimmung, Steuerung und Beurteilung des Forschungsverlaufes über eine grundsätzliche Förderentscheidung aus politischen Gründen nicht für erforderlich gehalten wurde, oder daß man sich der generell geringen Steuerungswirkung des Kontrollinstrumentes Forschungsberichte bewußt war und daher auf seinen Einsatz verzichtete.

Die Weitergabe des gebilligten Schlußberichtes an die PBWU-Gremien, die an der Begutachtung beteiligten Gutachter, die Geschäftsstelle der Interministeriellen Arbeitsgruppe des Bundes und der Länder zum Thema Waldschäden/Luftverunreinigungen sowie verschiedene Zentralbibliotheken war nicht als Forschungskontrollinstrument angelegt, sondern diente nur zur Dokumentation und Information.

Den politischen Anforderungen des Auftraggebers an die Forschung war mit Billigung des Berichtes Genüge geleistet. Abgesehen von marginalen Berichtsmodifizierungen wurde der Schlußbericht als das Ergebnis und der Nachweis der Forschung akzeptiert. Die für den Auftraggeber relevanten, in der Berichtsstellungnahme sowie in den jährlichen PBWU-Ergebnisberichten zusammenfassend bewerteten Forschungsergebnisse stellen somit das Gesamtergebnis und den Forschungserfolg des PBWU-Projektes dar und bilden die Grundlage für eine Umsetzung in politisches Handeln. 


\subsection{Terminierung und Evaluierung des PBWU-Projekts}

Die Terminierung beschließt als letzte Phase des Policy-Zyklus den Forschungsprozeß [WINDHOFF-HÈRITIER 1987, S. 65]. Im folgenden wird der Prozeß der Terminierung des PBWU-Projektes dargestellt. Danach werden theorieorientiert die internen wie externen Faktoren analysiert, die zur Terminierung führten. Eine besondere Rolle wird dabei die Frage spielen, inwieweit die Terminierung sich argumentativ auf eine fachliche Evaluierung des Forschungserfolges stützte.

$\mathrm{Zu}$ Ende der 80er Jahre scheint der Zenit der Waldschadensthematisierung überschritten zu sein. Zumindest die Wissenschaft ist sich weitgehend einig, daß es entgegen den früheren Vermutungen ein „Waldsterben“ im eigentlichen Wortsinne nicht gibt (s. z.B. Diskussion zur Jahrestagung der Deutschen Botanischen Gesellschaft vom 1.-5.10.1990 in Regensburg und Presseecho in der Mittelbayerischen Zeitung Regensburg vom 10.10.1990). Die der Waldschadensforschung beständig unterlegte und für die Begründung von Forschungsbedarf herangezogene Luftschadstoff-Hypothese wird von immer mehr Wissenschaftlern kritisiert und in Zweifel gezogen [s. z.B. LANDMANn, UnSWORTH, SCHLAEPFER in: FORSCHUNGSBEIRAT WALDSCHÄDEN 1989b, S. 945-970].

Neue oder noch weitgehend unbearbeitete Forschungsbereiche bzgl. angewandter Waldschadensforschung betreffen neben Themen der breit gefächerten Waldökosystemforschung die bislang forschungsseitig weitgehend vernachlässigten Laubbäume, die Problematik von Stickstoffeinträgen in Waldökosysteme und deren langfristige Veränderungen. Entsprechender Forschungsbedarf war denn auch in dem neuen Forschungskonzept der PBWU zu Ende des Jahres 1991 aufgezeigt worden.

\subsubsection{Einschätzung der Waldschadensforschung durch das Bayerische Umweltministerium}

Noch im Februar 1989 zitiert der Bayerische Umweltminister anläßlich der Begrüßung zum ersten Statusseminar der PBWU den Ministerratsbeschluß vom 10.11.1987, wonach „die Staatsregierung der intensiven Forschungs- und Koordinierungstätigkeit in den kommenden Haushalten durch angemessene Mittelausstattung Rechnung trägt“. Zeitweise auftretende Finanzierungsengpässe für Neuvorhaben lägen an der großen Zahl laufender Vorhaben, stellten aber keinesfalls eine Strategie zur Reduzierung der Förderung der bayerischen Waldschadensforschung dar [PBWU 1989, S. 1-3].

Der Wechsel des Ministers im Jahr 1990 führt zu einer Neubewertung der Waldschadensforschung. Wie bereits bei der Neukonzeptionierung des PBWU-Forschungsprogramms erwähnt, wird die Einstellung des neuen Ministers gegenüber der Waldschadensforschung als ,positivkritisch" bezeichnet. Mit einer Verringerung der für die Waldschadensforschung zur Verfügung gestellten Finanzmittel wird gerechnet (i.Q.: PBWU, 11.12.1990).

Auch das Schreiben eines renommierten und dem Thema Waldschäden äußerst kritisch gegenüberstehenden Münchner Botanikers kann zu der Einschätzung des Ministers beigetragen ha- 
ben. Der Wissenschaftler sieht sich veranlaßt, dem neuen Minister bzw. der Bayerischen Staatsregierung eine Neubewertung des Themas „Waldsterben“ zu empfehlen (i.Q.: externe Kritiker, 8.11.1990). Seiner Auffassung nach sei ,nunmehr die Haltlosigkeit der Schreckensszenarien der 80er Jahre offensichtlich“, es ergäben sich „keine fundierten Hinweise auf ein Waldsterben und die Beteiligung der üblichen Luftschadstoffe“. Im Antwortschreiben des Ministers vom 14.12.1990 kommt eine Neubewertung allerdings nicht zum Ausdruck. Vielmehr sei man um eine sehr differenzierte Aufklärung bemüht, die für die kommenden Jahre noch erheblicher Forschung bedürfte.

Wie der Staatssekretär im Umweltministerium in seinem Grußwort zum zweiten Statusseminar der PBWU am 4.2.1991 versichert, sei man zwar bereit, auch in den kommenden Jahren ,die Mittel für die Waldschadensforschung in gleichbleibender Höhe einzusetzen“. Es werde aber um Verständnis gebeten, daß „zunehmend nur nach Schwerpunktbereichen“ der PBWUGremien finanziert werden würde, mit dem Ziel, die Erkenntnisse auch in konkrete Maßnahmen des Umweltschutzes umsetzen zu können [PBWU 1991, S. 1-4].

Im Zuge weiterer umweltministerieller Positionierungen zum Thema wird die PBWU im Juni 1991 kurzfristig beauftragt, zur Vorlage gegenüber dem Minister eine Zwischenbilanz der Waldschadensforschung in Bayern zu ziehen. Vom zuständigen Fachreferat wird empfohlen, ergebnisorientiert zu berichten, den weiteren Forschungsbedarf zu artikulieren und auf die Ressortabstimmung zwischen bayerischem Umwelt- und Landwirtschaftsministerium einzugehen (i.Q.: PBWU, Juni 1991, ohne genaue Datumsangabe). Die PBWU erstellt einen entsprechenden Bericht (,Waldschadensforschung in Bayern - eine Zwischenbilanz, Stand Juni 1991“), stimmt ihn mit der Fachabteilung ab und übersendet ihn an das Bayerische Umweltministerium (i.Q.: PBWU, 21.6.1991).

Daß das Umweltministerium eine Neuorientierung der Forschungsförderung beabsichtigt, wird der PBWU auch im Zusammenhang mit der Erstellung des neuen Forschungskonzeptes im Herbst 1991 übermittelt (i.Q.: PBWU, 2.10.1991). Fördermittel würden zwar voraussichtlich wie bisher zur Verfügung stehen, angewandte Forschung und deutlicher Bezug zur Luftschadstoff-Thematik seien aber wichtige Fördervoraussetzungen.

Beim Sprecher des Wissenschaftlichen Beirats besteht aufgrund verschiedener bilateraler Unterredungen mit Vertretern des Bayerischen Umweltministeriums bereits der Eindruck, daß sich das Ministerium ,aus der Waldschadensforschung zurückziehen“ wolle. Das Prinzip der Schadwirkung sei klar, es ginge nun nur noch um die politische Umsetzung. Wenn die Schäden ohnehin nur biotisch seien, gäbe es keinen Auftrag mehr für das luftschadstofforientierte Umweltministerium. Daher hinge sehr viel vom Ergebnis der für Ende des Jahres 1991 geplanten Anhörung im Landtag zum Thema ab (i.Q.: Wissenschaftlicher Beirat, 23.11.1991).

\subsubsection{Landtagsanhörung zur Waldschadensforschung}

An der Expertenanhörung im Bayerischen Landtag am 5.12.1991 zum Thema „Bilanz der Waldschäden und der Waldschadensforschung von 1983 bis 1991“ nehmen verschiedene füh- 
rende Wissenschaftler aus Bayern und dem benachbarten Ausland teil. Auch einige Mitglieder des Wissenschaftlichen Beirats sowie der Leiter der PBWU sind als Referenten beteiligt.

Die Erwartung der Politik, eindeutige und von der Mehrheit der Experten getragene Aussagen zum Thema Waldschäden an die Hand zu bekommen, kann die Anhörung nicht erfüllen. Aussagen der Wissenschaftler sind widersprüchlich, was das Problem an sich, dessen zeitliche Entwicklung und Ursachen betrifft [BAYERISCHER LANDTAG 1991]. Die Presse spricht bspw. von ,andauerndem Expertenstreit“ (Hofer Anzeiger vom 7.12.1991), Wissenschaftler von einer „Überforderung der Fachwissenschaft?“ [KRAPFENBAUER 1992].

Auch der Leiter der PBWU berichtet der GSF-Geschäftsführung über die Anhörung dahingehend, daß die heterogene Beurteilung des Problems Waldschäden durch die Experten bei den Abgeordneten wohl „,kein gutes Bild hinterlassen“ hätte und hinsichtlich einer weiteren Forschungsförderung eher kontraproduktiv gewesen sein dürfte. Die derzeit bestehenden „Probleme“" in der Waldschadensforschung und die internen „Sorgen“ der Projektgruppe hinsichtlich ihrer Weiterführung bleiben nicht unerwähnt (i.Q.: PBWU, 11.12.1991).

Die Landtagsanhörung trägt dazu bei, die ministeriellen Bedenken bzgl. eines weiteren Engagements in der Waldschadensforschung zu vergrößern. Man sei am Umweltministerium ohnehin der Meinung, es handle sich überwiegend um forstliche Probleme, für die das Umweltressort nicht zuständig sei. Zwar bestünde noch Forschungsbedarf im Bereich Laubwaldschäden, hier sei jedoch der ursächliche Zusammenhang mit Immissionen nicht deutlich genug (i.Q.: Wissenschaftlicher Beirat, 8.12.1991).

\subsubsection{Ressortabstimmungsversuche zwischen Umwelt- und Landwirtschaftsministerium}

Das Argument der Ressortabstimmung erhält im Zusammenhang mit der künftigen Finanzierung der Waldschadensforschung immer größere Bedeutung. In der erwähnten Zwischenbilanz der PBWU zur Waldschadensforschung vom Juni 1991 mußte auf die Förderbeteiligung der verschiedenen Ressorts an spezifischen Forschungsthemen bereits gesondert eingegangen werden. Auch bei der Behandlung des neuen Forschungskonzeptes hatte das Umweltministerium auf weitere ressortabstimmende Gespräche verwiesen, die erst abgewartet werden müßten (s. i.Q.: PBWU, 31.10.1991).

Auf der Sitzung der PBWU-Gremien am 27.2.1992 kann über ein entsprechendes Gespräch zwischen Umwelt- und Landwirtschaftsminister berichtet werden (i.Q.: PBWU, 27.2.1992). Es bestünde die Absicht, die Ressortabstimmung zu optimieren, wobei das Umweltministerium auch weiterhin ressortspezifische Forschungsarbeiten finanzieren wolle. Auf Verwaltungsebene sei die Abstimmung bislang jedoch noch nicht abgeschlossen. Insgesamt könne die Waldschadensforschung aufgrund der schwierigen Haushaltslage wohl nicht im bisherigen Umfang weitergeführt werden.

Wie ein weiteres Gespräch auf Abteilungsebene am Umweltministerium mit dem Beiratssprecher und dem PBWU-Leiter ergibt (i.Q.: PBWU, 21.5.1992), stockten die interministeriellen Verhandlungen hinsichtlich der Zukunft der Waldschadensforschung. Unumgänglich erscheint eine Reduzierung der Fördermittel und die gemeinsame Nutzung vorhandener Meßeinrichtun- 
gen und Versuchsflächen zu Forschungszwecken, um die Forschung kostengünstiger zu gestalten. Auch sollten künftig die Ergebnisse der Bayerischen Forstlichen Versuchs- und Forschungsanstalt der PBWU zur Verfügung gestellt werden. Einzelheiten hierzu werden auf Referatsebene zwischen Umwelt- und Landwirtschaftsressort am 29.6. und 6.8.1992 besprochen (i.Q.: PBWU, 16.7.1992; Bayer. Landwirtschaftsministerium, 6.8.1992). An der Finanzierung der PBWU-Forschung könne sich das Landwirtschaftsministerium ohne zusätzliche Mittel der Staatsregierung jedoch nicht beteiligen.

\subsubsection{Einspar-Maßnahmen des Umweltministeriums in der Forschung}

\subsubsection{Ausgabenstop und Einspar-Appell}

Ein Schreiben des Bayerischen Umweltministeriums vom Oktober 1992 zu konkreten Sparmaßnahmen in der Forschung deutet bereits auf die Beendigung des PBWU-Projektes hin (i.Q.: Bayer. Umweltministerium, 22.10.1992). Unter hohem Termindruck per Fax als eine Anordnung der politischen Spitze des Hauses übermittelt, ist es an alle Vertragspartner (Auftragnehmer) von PBWU-Vorhaben gerichtet. Vorangestellt wird, daß die angespannte Haushaltssituation der öffentlichen Hand auch am Umweltministerium Kürzungen erforderte. Daher könnten ,neue Forschungsvorhaben ... nur mehr in besonders gelagerten Fällen finanziert werden“, laufende Vorhaben seien ,auf Einsparungsmaßnahmen oder vorzeitige Beendigung zu überprüfen“, ab sofort sollten möglichst keine neuen finanziellen Verpflichtungen mehr eingegangen werden.

Das Schreiben wird von den betroffenen Waldschadensforschungern in erster Reaktion gegenüber Umweltministerium und PBWU mit Bedauern und Unverständnis zur Kenntnis genommen. Einsparungen scheinen indes kaum möglich, ohne einen ordnungsgemäßen und erfolgreichen Abschluß der Arbeiten zu gefährden (i.Q.: Waldschadensforscher, 26.10., 28.10. und 1.11.1992).

Auch die GSF-Geschäftsführung nimmt das Schreiben des Ministeriums mit ,großer Bestürzung" auf (i.Q.: GSF, 9.11.1992). Die angekündigten Sparmaßnahmen würden insbesondere bedeuten, daß der Mitte 1993 auslaufende 3. Vertrag zur Projektgruppe sowie der bereits Ende 1992 auslaufende Vertrag zur Zentralen Analytik als die zentralen PBWU-Projektbestandteile nicht weiter verlängert würden. Insgesamt seien an der GSF 13 Vorhaben von der Sparmaßnahme betroffen, das PBWU-Teilprojekt „Luftschadstoffe und menschliche Gesundheit" mit eingeschlossen.

Die GSF-Geschäftsführung geht ausführlich auf die Konsequenzen für den Fall der Nichtverlängerung des PBWU-Vertrags ein. So könnten innerhalb der verbleibenden Projektlaufzeit nur noch laufende Arbeiten durchgeführt werden, um eine ordentliche Projektabwicklung zu gewährleisten. Dabei wird insbesondere auf zwei inzwischen aufgenommene, EU-anteilfinanzierte Kooperationsprojekte der PBWU mit Nachbarländern verwiesen. Auch der Nutzenentgang für die in Zukunft erwarteten Forschungsergebnisse wird deutlich gemacht. Auf den entstehenden „enormen ideellen Schaden“, der für die GSF entstünde, wird ebenso hingewiesen wie auf die personellen Probleme mit den projektbezogen eingestellten Mitarbeiter. 


\subsubsection{Stellungnahme des Wissenschaftlichen Beirats zu den Sparmaßnahmen}

Die Projektgruppe lädt den Wissenschaftlichen Beirat umgehend zu einer Sondersitzung ein (i.Q.: PBWU, 6.11 und 13.11.1992). Wie im gleichermaßen betroffenen Teilprojekt ,,menschliche Gesundheit" bereits erfolgt, schlägt sie als Reaktion des Gremiums auf die geplanten Sparmaßnahmen eine Resolution an den Minister vor. An der kurzfristig einberufenen Sitzung am 20.11.1992 wird eine Stellungnahme entworfen und innerhalb weniger Tage mit den weiteren Beiratsmitgliedern sowie dem GSF-Geschäftsführer abgestimmt (i.Q.: PBWU, 20.11. und 23.11.1992).

Die Stellungnahme des Wissenschaftlichen Beirats bringt vor dem Hintergrund des bestehenden Problems der Waldschäden die Sorge über die beabsichtigten Kürzungsmaßnahmen bzw. Einstellung des PBWU-Projekts zum Ausdruck. Durch den damit verbundenen Verzicht auf die Nutzung der GSF-Expositionskammern und die Bearbeitung konkreter Forschungsfragestellungen sei der „hohe Standard der Umweltforschung in Bayern“ ernsthaft gefährdet. Dadurch entstehende „Forschungsnachteile“ werden ins Feld geführt. Die im Bereich der Umweltforschung unerläßliche Verbundforschung, zu der die PBWU neben ihren weiteren Querschnittsaufgaben einen wesentlichen Koordinierungsbeitrag leistete, würde wegfallen. Der Beirat empfiehlt eindringlich, ,laufende und künftig relevante Forschungsaktivitäten auch weiterhin zu unterstützen und die Forschungskoordinationsstelle PBWU aufrecht zu erhalten“. Abschließend wird angeführt, daß auch die Öffentlichkeit wenig Verständnis für die geplanten Kürzungsmaßnahmen finden würde. Vom Sprecher des Gremiums am 22.11.1992 unterzeichnet, wird die Stellungnahme an den Bayerischen Umweltminister übersandt (i.Q.: $P B W U$, 25.11.1992).

\subsubsection{Externe politische Unterstïtzung gegen die Sparmaßnahmen}

Die geplanten Sparmaßnahmen des Umweltministeriums werden von der GSF und der Projektgruppe selbst auf die politische Bühne gebracht. Seitens der GSF-Geschäftsführung wird der Besuch des CSU-Fraktionsvorsitzenden im Hause Ende des Jahres 1992 dazu genutzt, im Hinblick auf die Haushaltsberatungen des Bayerischen Landtags auch auf die geplanten Kürzungsmaßnahmen in der Umweltforschung hinzuweisen (i.Q.: GSF, ohne genaue Datumsangabe). Die PBWU wendet sich zudem schriftlich an den Fraktionsvorsitzenden. Weiterhin werden von ihr der Vorsitzende des Umweltausschusses im Bayerischen Landtag, der ehemalige Bayerische Umweltminister sowie die oppositionelle Landtagsfraktion angeschrieben und von den geplanten Sparmaßnahmen in Kenntnis gesetzt. Dabei wird jeweils um Unterstützung zur „Beibehaltung umweltrelevanter Forschung in Bayern“ anläßlich der Beratungen im Finanzausschuß des Landtags gebeten (i.Q.: PBWU, 30.11. und 4.12.1992).

Der umweltpolitische Sprecher der Opposition reagiert. Ihm erscheint angesichts der „erneuten Zunahme der Waldschäden ... die Absicht des Bayerischen Umweltministeriums, die Förderung der bayerischen Waldschadensforschung künftig einzustellen, mehr als befremdlich“. Zwar sei es an der Zeit, statt Grundlagenforschung ausschließlich angewandte Forschung zu fördern, das Einstellen der Waldschadensforschung wäre jedoch ,das falsche Signal“ (Sozialdemokratische Presse-Korrespondenz Nr. 68, 18.12.1992, S. 4-6). 
Auch aus dem Ausland erhält die PBWU in ihrem Anliegen Beistand. Externe Kooperationspartner der PBWU waren informell über die beabsichtigten Sparmaßnahmen unterrichtet und um Unterstützung aus dem politischen Raum gebeten worden. Daraufhin schreiben der Landeshauptmann der Provinz Bozen-Südtirol, die Salzburger Landesregierung sowie der Österreichische Vizekanzler und Bundesminister für Wissenschaft und Forschung an den Bayerischen Umweltminister (i.Q.: externe PBWU-Befürworter, 16.11., 23.11. bzw. 5.12.1992). Um die Fortführung und erfolgreiche Beendigung der laufenden Projektkooperation mit der PBWU werde trotz bestehender Spar-Notwendigkeiten gebeten; Österreich befürworte die weitere Kooperation mit Bayern auf verschiedenen Gebieten der alpinen Umweltforschung. Im Antwortscheiben des Bayerischen Umweltministers an seinen österreichischen Ministerkollegen wird auf die Bedeutung der bisherigen Arbeiten am Forschungsschwerpunkt Wank ebenso hingewiesen wie auf die Planungen im Rahmen des neuen PBWU-Forschungskonzeptes. Die Umsetzung des Konzeptes sei aufgrund unumgänglicher Mittelkürzungen jedoch fraglich (i.Q.: Bayer. Umweltministerium 18.1.1993).

\subsubsection{Terminierung der PBWU}

\subsubsection{Unsicherheit über die Zukunft der PBWU}

Zu Beginn des Jahres 1993 teilt das Bayerische Umweltministerium den Vorhabenleitern das Ergebnis des Spar-Apells mit (i.Q.: Bayer. Umweltministerium, 5.1.1993). Es sei zwar möglich gewesen, den Haushalt soweit zu konsolidieren, daß die laufenden Vorhaben nunmehr planmäBig weitergeführt und der finanzielle Verpflichtungsstop aufgehoben werden könnten. Wie die Projektgruppe gegenüber dem Wissenschaftlichen Beirat anmerkt, ließen sich daraus allerdings über die Zukunft des PBWU-Projekts mitsamt der Umsetzung seiner neuen Forschungskonzeption keine Aussagen ableiten (i.Q.: PBWU, 8.1.1993).

Die GSF-Geschäftsführung fragt daher am Ministerium dringend nach einer Entscheidung über die Zukunft der PBWU nach Vertragsende Juni 1993 an (i.Q.: GSF, 19.1.1993). In dem von der PBWU entworfenen Schreiben wird die inzwischen vollzogene Einstellung des zentralen Analysenlabors mit Bedauern zur Kenntnis genommen. Ein erheblicher Teil der PBWUAufgaben, wie z.B. die Koordination zweier länderübergreifender EU-Projekte, die Betreuung noch laufender Forschungsvorhaben sowie Aufgaben im Zusammenhang mit geplanten Symposien und eines Abschlußberichtes für den Forschungsstandort Wank reichten über die geltende Vertragsdauer hinaus. Daher werde für den Fall einer PBWU-Beendigung zumindest eine „Übergangslösung“ gefordert.

Am 9.3.1993 findet die letzte Sitzung der PBWU-Gremien statt. Die Zukunft der PBWUWaldschadensforschung stellt dabei den zentralen Tagesordnungspunkt dar (i.Q.: PBWU, 9.3.1993). Der Projektratsvertreter des Umweltministeriums rechtfertigt die Sparmaßnahmen in der Waldschadensforschung. Sie seien aufgrund eines erheblichen, von der Staatsregierung beschlossenen Einsparungssolls unumgänglich. Zwar böten die laufenden Vorhaben nur geringfügige Einsparpotentiale; interne Umschichtungen sowie der Verzicht auf weitere Forschungsvorhaben und die Zentrale Analytik hätten es aber ermöglicht, den Kürzungsvorgaben 
zu entsprechen. Für die Zukunft seien die Finanzmittel allerdings ,,nicht ausreichend, um im bisherigen Förderrahmen fortzufahren“.

Der Sprecher des Wissenschaftlichen Beirats berichtet über ein Gespräch mit dem Referenten des Umweltministers anläßlich der vorgelegten Resolution. Er hätte dort auf die Beibehaltung der vorhandenen Forschungs- und Koordinationsstrukturen zumindest ,auf kleiner Flamme“ gedrängt. Auch weitere Vertreter des Beirats unterstreichen die Notwendigkeit, das PBWUProjekt zumindest in seiner Grundstruktur zu erhalten, da nach wie vor Forschungsbedarf bestünde. Das Ministerium sichert eine Prüfung zu. Weder das Landwirtschaftsministerium noch die GSF sehen Möglichkeiten, zur Sicherung der Projektgruppe finanziell für das Bayerische Umweltministerium einzuspringen. Auf das für Oktober 1993 geplante dritte Statusseminar zum Thema Waldschäden soll angesichts der gegebenen Situation verzichtet werden. Ein weiterer Sitzungstermin der PBWU-Gremien wird nicht vereinbart.

Der Leiter der PBWU nimmt den Verzicht auf die Durchführung des Statusseminars zum Anlaß, dem Umweltministerium ,den Ausdruck des Bedauerns“ bei einer Vielzahl davon unterrichteter Wissenschaftler mitzuteilen. So erschiene es nach deren und eigener Ansicht gerade bei Beendigung der Waldschadensforschung wissenschaftlich sinnvoll und notwendig, die Forschung ergebnisorientiert zu präsentieren; zudem stellte dies eine Rechtfertigung gegenüber dem Geldgeber und der Öffentlichkeit dar (i.Q.: PBWU, 24.3.1993).

\subsubsection{Landtagsanfrage zur Zukunft der PBWU}

Auch anläßlich des ersten Statusseminars zum PBWU-Teilprojekt „Luftschadstoffe und menschliche Gesundheit" im Februar 1993 wird die unklare Zukunft der PBWU deutlich und publik. Der Geschäftsführer der GSF zeigt sich in seiner Begrüßung zur Veranstaltung besorgt über die künftige Finanzierung des PBWU-Projektes. Der Sprecher des Wissenschaftlichen Beirats dieses Teilprojektes, ein renommierter Münchener Arbeitsmediziner, appelliert in seiner Einführung an die Weiterförderung der umweltmedizinischen Wirkungsforschung. Bei gegebenem Verständnis für Sparmaßnahmen dürfte diese Forschung nicht „,durch eine überproportionale Mittelkürzung benachteiligt werden“ [PBWU 1993a, S. 1-3 bzw. 11-14].

Für den teilnehmenden Vertreter der politischen Opposition sind diese Appelle Anlaß für eine schriftliche Landtagsanfrage (Anfrage vom 10.3.1993). Die Staatsregierung wird darin insbesondere nach der derzeitigen Situation und dem Weiterbestehen der PBWU befragt. In deren Beantwortung durch das Bayerische Umweltministerium wird auf zwingende Mittelkürzungen verwiesen, die auch den Forschungsbereich der PBWU sowie die Koordinierungsstelle selbst beträfen (i.Q.: Bayer. Umweltministerium 19.4.1993). Angesichts des bisherigen hohen Forschungsengagements des Ministeriums und des damit erreichten ,erheblichen Beitrags zur Ursachenaufklärung" einerseits, der bestehenden forstlichen Forschungsprogramme des Landwirtschaftsressorts andererseits, sei im Bereich Waldschadensforschung „eine Dringlichkeit für neue Vorhaben seitens des Umweltministeriums derzeit nicht erkennbar“. Spätestens die Beantwortung der Landtagsanfrage macht deutlich, daß eine Verlängerung der PBWU über den bisherigen Vertragszeitraum hinaus unrealistisch ist. 


\subsubsection{Beendigung der $P B W U$}

Anläßlich der letzten von der PBWU veranstalteten, internationalen Fachtagung im April 1993 (Symposium „Stoffeinträge aus der Atmosphäre und Waldbodenbelastung in den Ländern von ARGE ALP und ALPEN-ADRIA“ am 27.-29.4.1993 in Berchtesgaden) appellieren sowohl der Geschäftsführer der GSF als auch der Leiter der PBWU in ihren Eröffnungsansprachen an die weitere Finanzierung der koordinierten Umweltforschung durch den Staat. Daß die über die PBWU finanzierten Forschungsmaßnahmen zum Thema Waldschäden künftig ,haushaltsbedingten Einsparnotwendigkeiten zum Opfer fallen müssen“, wird herausgestellt [PBWU 1993b, S. 10-12 bzw. 16-18].

Auch der Referent des Schlußwortes zur Veranstaltung, ein Mitglied des Wissenschaftlichen Beirats, bringt sein Bedauern zum Ausdruck, daß das PBWU-Projekt und damit die Projektgruppe beendet würden. Für den abrupten Abbruch der Förderung fehle ihm das Verständnis. Zumindest für interessante und erfolgversprechende Forschungsansätze sollte auch weiterhin Fördermöglichkeit bestehen, nicht nur, um die getätigten Investitionen und den inzwischen erlangten Sachverstand noch sinnvoll ausnützen zu können [PBWU 1993b, S. 426-434).

Gespräche mit den Fachbeamten des Umweltministeriums signalisieren anfangs noch die Möglichkeit, zumindest eine PBWU in reduziertem Umfang für den Teilbereich ,,menschliche Gesundheit“ zu finanzieren. Einer von der Projektgruppe vorgeschlagenen Neuorientierung hinsichtlich der Bearbeitung anderer Forschungsbereiche steht das Ministerium hingegen negativ gegenüber (i.Q.: PBWU, 4.3.1993).

Nach mehreren Vorgesprächen legt die GSF noch im März 1993 einen Antrag auf Fortführung der PBWU vor (i.Q.: GSF, 17.3. und 6.4.1993). Dieser bezieht sich allerdings ausschließlich auf das Teilprojekt „Luftschadstoffe und menschliche Gesundheit“. Eine Fortführung des Teilprojektes „Waldschadensforschung“ wird als aussichtslos eingeschätzt. Um eine Betreuung der beiden dort noch bis 1994 laufenden EU-Projekte zu gewährleisten, wird allerdings beantragt, die Verträge diesbezüglich geringfügig aufzustocken und zeitlich zu verlängern. Anläßlich einer Besprechung vom 27.5.1993 am Umweltministerium wird der PBWU mitgeteilt, daß auch dem reduzierten Antrag der GSF zur Fortführung der PBWU im Bereich Gesundheitsforschung aufgrund von Finanzierungsproblemen nicht entsprochen werden könne (s. i.Q.: $P B W U$, 28.5.1993). Lediglich die Aufstockung der beiden EU-Projekte könne genehmigt werden.

Für den vertragsgemäß zu erstellenden PBWU-Schlußbericht wird aufgrund der von der GSF als überraschend empfundenen Projektbeendigung lediglich eine ,reduzierte“ Fassung, bestehend aus einem „,kurzen Resümee über Entwicklung und Stand der Waldschadensforschung“ vereinbart. Die Aktenübergabe sowie die Zukunft der am Wank bestehenden ForschungsInfrastruktur wird im Grundsatz geregelt.

Die GSF-Geschäftsführung bringt gegenüber dem Umweltministerium ihr Bedauern zum Ausdruck über die ,inzwischen neunjährige, vertrauensvolle“ und ihrer Ansicht nach „,erfolgreiche Zusammenarbeit in der Forschungskoordination durch die PBWU“, die ,nun so abrupt beendet werden muß“ (i.Q.: GSF, 28.5.1993). Der ehemalige Leiter der Gruppe legt den Projekt- 
schlußbericht für die gesamte Projektlaufzeit 1984 - 1993 im November 1993 dem Ministerium vor [REUTHER et al. 1993].

Von den während der gesamten PBWU-Projektdauer von 1984 bis 1993 finanzierten, rund 80 Forschungsvorhaben sind zum Zeitpunkt der PBWU-Beendigung Juni 1993 etwa 15 Vorhaben laufzeitbedingt noch nicht abgeschlossen [REUTHER et al. 1993, Anhang 2]. Die Fälligkeiten der entsprechenden Schlußberichte reichen bis in das Jahr 1994. Die Abwicklung dieser Vorhaben wird ab Juli 1993 von Fachbeamten des Bayerischen Umweltministeriums in Eigenregie durchgeführt.

Auch die bereits seit dem Jahr 1992 in Bearbeitung befindliche Veröffentlichung der Forschungsarbeiten und -ergebnisse am Wank im Rahmen einer renommierten, englischsprachigen Buchreihe wird fallen gelassen. Nicht nur die Beendigung des PBWU-Projektes, sondern auch grundsätzliche Bedenken von Seiten der Herausgeber gegen die Publikation und die damit verbundene Arbeitsbelastung spielen hierbei eine Rolle. Ein Mitherausgeber führt hierbei die fehlende ,richtige Entfaltung“ des Forschungsschwerpunkts ebenso wie den ,geordneten Abschluß“ an, womit letztlich nur ein „Torso“ mit z.T. nicht publikationswürdigen und interpretierbaren Ergebnissen bestünde (i.Q.: $P B W U$, 3.6.1993). Allerdings gehen die vorbereitenden Arbeiten zumindest ausschnittsweise in einer später erfolgenden, internationalen Publikation zur Thematik Waldschäden und Ozon auf [SANDERMANN et al. 1997]. Der Sprecher der Forschergruppe Wank und eine Mitarbeiterin seines Instituts beteiligen sich zusammen mit dem Leiter der PBWU mit einem zusammenfassenden und auf die Ozonwirkung focussierenden Kapitel über die Waldschadensforschung am Wank an dem Buch.

\subsubsection{Theorieorientiertes Zwischenergebnis}

\subsubsection{Terminierungsprozeß}

Die Terminierung des PBWU-Projektes wird durch die allgemein abnehmende Thematisierung und Problematisierung der Waldschäden seit etwa Ende der 80er Jahre erleichtert. Letztlich geben finanzielle Engpässe im Staatshaushalt als Begründung den Ausschlag.

Auch die Person des amtierenden Bayerischen Umweltministers prägt die Situation. Während sein Vorgänger an der PBWU-Gründung aktiv beteiligt war und sich für diese eingesetzt hatte, muß sich sein Nachfolger keiner derartigen Beziehung zu dem Projekt verpflichtet fühlen. Kann die Forderung angewandter, in politische Maßnahmen umsetzbarer Forschung im Grundsatz auch seinem Vorgänger unterstellt werden, so wird sie nun zumindest deutlicher hervorgehoben und läßt auf eine Einschränkung oder Straffung der Waldschadensforschung schlieBen. Dennoch werden auch unter dem neuen Minister anfangs noch Mittel in gleichbleibender Höhe für die Waldschadensforschung in Aussicht gestellt.

Die Mitte 1991 eingeforderte Zwischenbilanz zur Waldschadensforschung signalisiert, daß der neue Minister sich der Waldschadensforschung konkret annehmen werde. Auch die Vorlage der neuen Forschungskonzeption an den Minister zu Beginn 1992 zwingt zu einer politischen Auseinandersetzung mit und Entscheidung über die Zukunft der umweltministeriell finanzierten Waldschadensforschung. 
Gegen Ende des Jahres 1991 verstärkt sich die Unsicherheit über die Zukunft der PBWUWaldschadensforschung. Dabei wird angesichts wachsender Finanzierungsprobleme das Argument der Ressortabgrenzung immer mehr in den Mittelpunkt gestellt. Während das Bayerische Umweltministerium luftschadstoffbezogene Forschungsförderung nach wie vor für sich in Anspruch nimmt, wird für die forstliche Forschung das Landwirtschaftsministerium für zuständig erklärt. Mit der Interpretation der Ergebnisse einer Landtagsanhörung, wonach ohnehin überwiegend nur mehr Laubwaldschäden forstlichen Forschungsbedarf aufwiesen, kann das Umweltressort weitere Aktivitäten auf das Nachbarressort abwälzen.

Insbesondere die schwierige Haushaltslage, die sich zu Beginn des Jahres 1992 abzeichnet, wird formal als Begründung herangezogen, die vom Umweltministerium finanzierte Waldschadensforschung zu reduzieren. Eine wissenschaftliche Diskussion, Begründung und Evaluierung weiterer Forschungsnotwendigkeiten wird damit überflüssig.

Die im Oktober 1992 ministeriell verhängte Sparmaßnahme zu allen laufenden Forschungsvorhaben hat überwiegend symbolischen Charakter. Sie führt erwartungsgemäß zu keiner nennenswerten finanziellen Einsparung, die Konsolidierung des Haushalts kann überwiegend über abteilungsinterne Umschichtungen erfolgen. Mit der Ankündigung, grundsätzlich keine weiteren Forschungsmaßnahmen zu initiieren und damit auch auf die Fortführung der Projektgruppe zu verzichten, signalisiert die Sparmaßnahme aber das Ende des PBWU-Projekts.

Die Waldschadensforscher sind bestürzt. Der Abbruch der vom Umweltministerium finanzierten Forschung steht im Gegensatz zu den wissenschaftlichen Anforderungen einer detaillierten Klärung spezifischer Forschungsfragestellungen. Mit Beendigung des PBWU-Projekts würde ein erheblicher Finanzierungsstrom versiegen, was gerade in Hinblick auf den inzwischen erarbeiteten Methodik- und Erkenntnisstand, aber auch in Hinblick auf aufgebaute infrastrukturelle und personelle Kapazitäten als gravierend angesehen werden kann.

Der Wissenschaftliche Beirat reagiert mit einer Resolution zu den Einsparbeschlüssen des Ministers. Dabei wird mit Besorgnis auf forschungspolitische Aspekte wie die Gefährdung des hohen Standards der Umweltforschung in Bayern und den Nutzenentgang aus den Ergebnissen geplanter Forschung, die für umweltpolitische Maßnahmen unverzichtbar seien. Der Beirat spricht sich nicht nur für die Fortführung der Forschung, sondern auch der Projektgruppe als Koordinierungsinstrument aus, die seit Jahren erfolgreich einen Beitrag zur interdisziplinären Forschung geleistet hätte. Daß der Beirat die Projektgruppe mit einbezieht, soll dazu dienen, das Projekt in seiner bisherigen Gesamtstruktur zu sichern. Es verdeutlicht aber auch das inzwischen gute Verhältnis zwischen Projektgruppe und Beirat. Auch von der PBWU veranstaltete Symposien im Jahr 1993 werden vom Wissenschaftlichen Beirat dazu genutzt, der PBWU für ihre Arbeit zu danken und ihren Erhalt zu fordern.

Die GSF hebt in ihrer Reaktion auf die Sparmaßnahmen nicht nur auf forschungsseitige Gesichtspunkte, sondern auch auf die aus der als abrupt verstandenen Projektbeendigung resultierenden Image-Nachteile sowie Personalprobleme ab. Zumindest eine Übergangslösung zur geordneten Projektabwicklung wird daher gefordert. Die Projektgruppe selbst fühlt sich in ihrer Existenz bedroht. Ihre Hilferufe richten sich an die GSF-Geschäftsführung, den Wissenschaftli- 
chen Beirat, ausländische Kooperationspartner sowie einzelne Politiker, ohne damit letztlich jedoch Erfolg am Bayerischen Umweltministerium zu verbuchen. Das dortige Fachreferat nimmt eine gewisse Zwitterstellung ein. Einerseits sind die Anordnungen des Ministers, die zur Terminierung der PBWU führen, umzusetzen. Andererseits setzt man sich informal für das PBWU-Projekt befürwortend ein.

Die Beendigung der PBWU kann trotz der verschiedenen Stützungsversuche nicht verhindert werden. Der Bedarf für neue Forschungsaktivitäten wird seitens des Umweltministers nicht gesehen, folglich erübrigt sich auch die Berechtigung für eine forschungskoordinierende Stelle. Mit einer Gesamtauswertung und Evaluierung wird die Projektterminierung jedoch nicht verbunden. Auf die Durchführung eines abschließenden Statusseminars wird verzichtet, der von der PBWU zu liefernde Schlußbericht muß nur den Minimalanforderungen entsprechen. Die Erstellung einer Publikation über den Forschungsschwerpunkt Wank wird den Wissenschaftlern selbst überlassen und in keiner Weise forciert. Sie kommt letztlich auch nicht in der geplanten Form zustande, da die hierfür erforderlichen Strukturen und Motivation nicht mehr gegeben sind und Programmdefizite wie Kritikpunkte am Forschungsschwerpunkt ungern präsentiert werden möchten.

Nach neunjähriger Projektlaufzeit wird die Koordinierungsstelle PBWU und damit die vom Bayerisches Umweltministerium finanzierte, interdisziplinäre Verbundforschung zum Thema Waldschäden beendet. Die noch laufenden Vorhaben werden vom Ministerium selbst abgewikkelt. Auch der Versuch, die PBWU lediglich für das erst vor wenigen Jahren hinzugekommene Teilprojekt „Luftschadstoffe und menschliche Gesundheit“ zu verlängern, scheitert mit dem Argument finanzieller Gründe. Für den Vorschlag, die PBWU als Koordinierungsstelle für anderweitige, außerhalb der Waldschadensforschung liegende relevante Bereiche der Umweltforschung zumindest in ihren Grundstrukturen zu sichern, fehlte der politische Wille.

\subsubsection{Mangel an politischer Problemrelevanz}

Das zu Beginn der Waldschadensforschung greifende Argument, die Ursachenforschung zu verstärken, um aus Sicht der Luftreinhaltung gezielt konkrete, politische Gegenmaßnahmen gegen die Waldschäden ergreifen zu können, erscheint spätestens zu Beginn der 90er Jahre nicht mehr gültig. Die Thematik Waldschäden hat einerseits an Bedeutung und Brisanz inzwischen deutlich verloren, die jährlichen Waldzustandsberichte mit insgesamt kaum dramatischen Verschlechterungen, ja im einzelnen sogar Verbesserungen des Waldzustands, erregen kaum mehr das öffentliche Interesse. Die vorhergesagten Szenarien eines flächenhaften Waldsterbens sind in keiner Weise Realität geworden, vielmehr wird allgemein eine erhöhte Wuchsleistung der Wälder konstatiert.

Zudem sind die wissenschaftlichen Forschungsergebnisse überwiegend wirkungsmechanistisch und grundlagenforschungsorientiert. Sie sind damit kaum geeignet, konkretere Handlungsempfehlungen für die Politik zu Luftreinhaltemaßnahmen abzugeben, als zu Beginn der Waldschadensforschung möglich waren und im Rahmen der Vorsorgepolitik auch umgesetzt wurden. So kann am Ende der Forschungsära kaum ein umweltpolitisch relevanter, wissenschaftlich eindeutig gesicherter Zusammenhang zwischen Waldschäden und Luftschadstoffwirkung 
vor Ort bzw. unter umweltrelevanten Bedingungen hergestellt werden, der anfänglich nahezu unumstößlich zu sein schien.

Das $\mathrm{SO}_{2}$-Problem ist allein aufgrund der erzielten Emissionsminderungserfolge hierzulande kaum mehr ein Thema. Die Forschungsergebnisse zu Photooxidantien mit der Leitsubstanz Ozon lassen zu Projektende noch keine eindeutigen Schlüsse zu. Zahlreiche, zum großen Teil alpine Untersuchungsergebnisse aus In- und Ausland sprechen eher dagegen, unter den gegebenen Belastungsbedingungen eine direkte Schadwirkung auf die wenig ozonempfindliche Hauptbaumart Fichte abzuleiten. Vielmehr scheint natürlichen Belastungsfaktoren, resultierend z.B. aus ungünstigen Witterungsverhältnissen, Nährstoffverhältnissen und weiteren anthropogenen Bewirtschaftungseinflüssen (Streunutzung, etc.), allgemein größere Bedeutung zuzukommen als bisher angenommen [REUTHER et al. 1993, S. 15/16; s.a. RENNENBERG et al. 1997]. Auch eine Landtagsanhörung von Wissenschaftlern Ende 1991 hinterläßt den Eindruck wissenschaftlicher Uneinigkeit in der Problemeinschätzung, der Notwendigkeit, Schadphänomene und Ursachenfaktoren stark regionalisieren zu müssen, vermittelt den hohen Komplexitätsgrades des Problems und fördert daher kaum politisch konkret umsetzbare Ergebnisse zutage [BAYERISCHER LANDTAG 1991].

Die persönlich kritische Einschätzung des Themas Waldschäden durch den neuen Bayerischen Umweltminister, die bereits erzielten Erfolge in der Luftreinhaltepolitik, die nach wie vor uneinheitliche Einschätzung der Problemschwere und der Schadensursachen in der Wissenschaft nach über zehnjähriger Forschung reichen aus, um sich politisch für eine Beendigung des PBWU-Forschungsprojektes auszusprechen. Eine explizite Evaluation des Programmerfolges erscheint nicht erforderlich. Der Hinweis auf Finanzmittelknappheit und die Abwälzung eventuell noch erforderlichen Forschungsbedarfs auf das für den Wald eigentlich zuständige Nachbarressort erleichtern die Argumentation. Konsequenterweise ist die Auflage eines neuen, vom Wissenschaftliche Beirat zur Förderung empfohlenen Forschungskonzepts für das Bayerische Umweltministerium ebenso wenig erforderlich wie das Fortbestehen der Koordinierungsstelle PBWU.

\subsubsection{3 „Effizienzparadoxon“ der Forschung}

Zu Beginn der Waldschadensforschung, bei hohem Problem- und Handlungsdruck des Ministeriums, bestand nur geringer wissenschaftlicher Legitimationsbedarf für die Forschung. Der hohe Implementationsdruck kann von den Waldschadensforschern genutzt werden, sich am Programm zu beteiligen. Bei dem entwickelten wissenschaftlichen Kenntnisstand, dem Abbau des politischen Problem- und Handlungsdrucks sowie bei knapper werdenden Finanzen steigt allerdings der Legitimationsbedarf für Forschung. Dafür reicht die fachlich/sachliche Legitimation der Forschung nicht aus. Ist die Forschungsleistung nicht nur in wissenschaftlicher, sondern auch politischer Hinsicht ohnehin schwer zu messen, so schlägt dieser anfängliche Vorteil für die Forscher nun zu deren Nachteil um [MAYNTZ 1985, S. 19].

Die Fragestellung scheint aus Sicht des Umweltressorts inzwischen ausreichend geklärt zu sein, entsprechend politische Gegenmaßnahmen seien ergriffen worden, das ehemalige Entlastungsargument - Forschung vor Gegenmaßnahmen - ist nicht mehr erforderlich. Das Angebot zu 
weiterer, leistungsfähiger und effizienter Forschung, zu der die Wissenschaft bereit und in der Lage ist, muß nun nicht mehr, zumindest nicht vom Umweltressort, aufgegriffen und umgesetzt werden.

Daß die Wissenschaft an der Fortführung der Waldschadensforschung interessiert ist, ist ebenfalls verständlich, und das nicht nur aus finanziellen Gründen. Aus wissenschaftlicher Sichtweise hat die Waldschadensforschung nicht nur eine Menge an Grundlagenverständnis über das komplexe Ökosystem Wald, an Ursachen- und Wirkungszusammenhängen zwischen Luftschadstoffkomponenten und den verschiedenen Kompartimenten des Ökosystems Wald (Boden, Pflanze, Atmosphäre) erarbeitet. Vielmehr sind einzelne Fragen nach wie vor ungeklärt, oder neue im Verlauf des Erkenntnisfortschritts hinzugekommen (z.B. Stickstoffproblematik, Klimaeffekte, Langzeit- und Kombinationswirkungen, pflanzliche Anpassungsstrategien). Diese bedürfen aus wissenschaftlicher Sicht einer Bearbeitung und wurden entsprechend auch in die neue Forschungskonzeption der PBWU eingebaut.

Hinzu kommt, daß die an der Waldschadensforschung beteiligten Forschungsinstitutionen aufgrund der bisherigen Förderprogramme enorme fachliche, personelle und institutionellinstrumentelle Kapazitäten aufbauen konnten, denen bei Beendigung der Förderung die finanzielle Basis entzogen und der Verlust drohen würde. Zudem kann erwartet werden, daß aufgrund der inzwischen bestehenden fachlichen Kenntnisfülle und hohen methodischer Entwicklungsstandes die Effektivität sowie die finanzielle Effizienz der Forschung bei deren Weiterführung in Zukunft gesteigert werden könnte.

Die Nichtausnutzung des vorhandenen Forscherpotentials trotz Kenntnis des Forschungsbedarfs und dessen forschungskonzeptioneller Aufbereitung wie im neuen Forschungskonzept der PBWU kann - analog zum „Katastrophenparadoxon“ [VON PRITTWITZ 1990] - als „Effizienzparadoxon" der Forschung bezeichnet werden. Erstgenanntes Phänomen der Umweltpolitik wird dadurch charakterisiert, daß die Entstehung von Umweltkatastrophen und politische Schutzmaßnahmen in ihrer Intensität einander nicht entsprechen, sondern sich vielmehr gegenläufig verhalten, also vergleichsweise geringe Probleme ihrer politischen Antwort darauf inadäquat, d.h. stark überzeichnet werden und umgekehrt [VON PRITTWITZ 1990, S. 26]. Übertragen auf den Forschungsprozeß im PBWU-Projekt tritt nun die Situation auf, daß voraussichtlich erfolgversprechende, effiziente wissenschaftliche Erkenntnisproduktion auf der Grundlage eines ausgereiften Forschungsprogramms - paradoxerweise - politisch nicht umgesetzt wird, während zu Projektbeginn auf Grundlage eines breiten, weniger qualifizierten Rahmenprogramms umfangreiche Forschungsaktivitäten großzügig finanziert wurden.

Der Schlüssel zum Verständnis dieses „Effizienzparadoxons der Forschung“ liegt in der unterschiedlichen Zweckdienlichkeit und Legitimation wissenschaftlicher Forschung durch Politik und Wissenschaft. Während bei der Wissenschaft die Erkenntnisproduktion im Vordergrund steht, ist es prioritäres Ziel der Forschungsprogramme, entsprechend dem bestehenden politischen Problemdruck die Durchführung eines problemlösungsorientierten Forschungsprogrammes überhaupt zu initiieren, dieses zu implementieren und daraus eventuell Handlungsempfehlungen ableiten zu können. Demnach bewertet der Finanzgeber die Forschungsleistung weniger an Wissenschaftskriterien, sondern mehr an politischen Kriterien wie dem raschen Nachweis 
von Aktivitäten in der Forschung und dem politischen Druck des zu lösenden Problems. Das Kritierium der wissenschaftlichen Qualität der Forschung ist formal durch die fachliche Begutachtung gesichert.

\subsubsection{Terminierungsstrategien}

Die Terminierung der PBWU wird dadurch erleichtert, daß der zugrundeliegende Vertrag zwischen GSF und Bayerischem Umweltministerium lediglich nicht weiter verlängert $\mathrm{zu}$ werden braucht. Somit ist nicht einmal ein aktiver Schritt zur Beendigung des Projektes erforderlich. Zudem hält man es sich offen, auch ohne die PBWU-Konstruktion künftig umweltministeriell für erforderlich und sinnvoll gehaltene Forschungsmaßnahmen zu finanzieren, wovon vereinzelt auch Gebrauch gemacht wird.

Auch der Verweis auf das Nachbarressort, zuständigkeitshalber noch erforderliche Fördermaßnahmen zu übernehmen, kann als Strategie zur Erleichterung des Terminierungsverfahrens gesehen werden. Mit der der Wissenschaft entliehenen Aussage zur Ursachendiskussion, weitere Waldschadensforschung hätte gegenüber Luftschadstoffaspekten insbesondere forstliche Aspekte zu berücksichtigen, kann der ,schwarze Peter“ der Forschungsförderung dem Landwirtschaftsressort zugeschoben werden, ohne daß dieses sich jedoch zu einer verstärkten Finanzierung bereit erklärt. Diese Ressortzuständigkeitsfrage hat ihre Parallelität zu Beginn der Waldschadensforschung. Aufgrund der damaligen Ursachendiskussion nahm das Umweltressort die Forschungszuständigkeit für sich in Anspruch; dies erfolgte allerdings zu Zeiten hoher politischer und öffentlicher Thematisierung und Problemrelevanz, ausreichend verfügbarer Fördermittel und der strengen Ursachen-Focussierung auf Luftschadsstoffe.

Daß sich neben den Wissenschaftlern, denen bei Ende des PBWU-Projektes eine wichtige Fördermittelressource entzogen wird, auch die GSF sowie die Projektgruppe selbst für die Fortführung der PBWU einsetzt, ist durch deren informale institutionelle Interessen zu erklären. Bei der GSF geht es um das Renommee der Großforschungseinrichtung, finanzielle Aspekte der Drittmittelforschung sowie zu erwartende Personalprobleme mit den PBWU-Mitarbeitern. Für diese ist die Terminierung des Projektes z.T. existentiell. Änderungen der Tätigkeitsfelder sind zu erwarten, bestehende, an das Projekt gekoppelte Beschäftigungsverhältnisse sind betroffen.

Alle Organisationen bergen in sich die Tendenz, sich zu vergrößern und an Einfluß und Prestige etc. zuzugewinnen oder bei extern bedingter Verringerung diese zu minimieren bzw. durch andere Funktionen zu ersetzen [Downs 1967, S. 5 ff, S. 191 ff u.a.a.O.]. Dies wird von der PBWU auch versucht, allerdings ohne Erfolg. So kann letztlich weder die Beibehaltung des zweiten PBWU-Teilprojektes (,Luftschadstoffe und menschliche Gesundheit“) gesichert, noch die Forschungskoordination für andere Umweltbereiche erschlossen werden.

Jedoch auch die Fachabteilung am Bayerischen Umweltministerium setzt sich - zumindest zu Beginn der Terminierungsphase - für den Erhalt des PBWU-Projektes ein. Dies kann aus bestehenden persönlichen fachlichen Interessen sowie persönlichen Gewogenheiten gegenüber der GSF und der Projektgruppe resultiert haben, wie sie sich allein aus der jahrelangen $\mathrm{Zu}$ - 
sammenarbeit ergaben. Für die Mitarbeiter im Fachreferat sind zudem - ähnlich der Projektgruppe - inner-organisatorische Konsequenzen zu erwarten, wie z.B. interne Umsetzungen oder die Zuteilung neuer Aufgaben.

Daß sich die Terminierung häufig als schleichender Prozeß vollzieht, der zudem durch andere Policies substituiert und damit letztlich eine Schein-Terminierung darstellen kann [WINDHOFFHÉRITIER 1987, S. 105 ff], trifft auf das PBWU-Projekt nicht zu. Die PBWU-Terminierung ist weitgehend final. Nicht nur das Waldschadensforschungsprogramm des Bayerischen Umweltministeriums wird damit beendet, sondern auch die Organisationsstruktur der Stabsstelle selbst. Die institutionelle wie strukturelle Verankerung der Projektgruppe innerhalb der GSF, Wissenschaft, Politik bzw. Öffentlichkeit ist zu gering, um einer Terminierung zu widerstehen. Auch die Solidarisierung der Wissenschaftler mit der Projektgruppe bzw. dem PBWU-Projekt, die sich in einer entsprechenden Resolution gegenüber den Sparmaßnahmen des Umweltministeriums verdichtet, reicht hierfür nicht aus.

Hierbei ist zu erwähnen, daß das Umweltministerium auch nach Beendigung der PBWU in Eigenregie einzelne Forschungsvorhaben zum Thema Waldschäden finanziert und ehemalige PBWU-Mitarbeiter - innerhalb und außerhalb der GSF - mit der Koordination und Betreuung dieser Vorhaben betraut. Auch der PBWU-Schlußbericht [REUTHER et al. 1993] wird nach Beendigung restlicher, über die PBWU-Laufzeit hinausgehender Forschungsvorhaben fortgeschrieben [REUTHER et al. 1996]. Von einer - verdeckten - Weiterführung eines bayerischen Waldschadensforschungsprojektes kann aufgrund der als Einzelmaßnahmen zu bezeichnenden Fördermaßnahmen sowie der fehlenden Einbindung in eine übergreifende Förderkonzeption bzw. -struktur jedoch nicht gesprochen werden.

Außerhalb des Ministeriums übernehmen allerdings thematisch ähnliche Programme wie ein Bayerisches Klimaforschungsprogramm unter der maßgeblichen Finanzierung des Kultusressorts oder das vom Bund finanzierte Bayreuther Ökosystemforschungszentrum substituierende Funktion. Zahlreiche Forschungsthemen der ehemaligen Waldschadensforschung können - bei inhaltlicher Umstrukturierung - in diese aktuelleren Programme eingebracht werden.

Ohnehin kann die Terminierung eines staatlichen Programmes aus Anlaß der Zweckerfüllung oder der Problemlösung eher als die Ausnahme gelten [WINDHOFF-HÉRITIER 1987, S. 107]. Finanzielle Engpässe, Effektivitätsüberlegungen und politisch-ideologische Motive werden häufig allgemein als ausschlaggebende Gründe genannt und können nach den obigen Ausführungen auch für die Forschungspolicy des PBWU-Projektes geltend gemacht werden.

\subsubsection{Nutzenentgang durch kurzfristige Programmterminierung}

Auch wenn verschiedene Indizien wie die kritische Einschätzung des Umweltministers, die Nichtentscheidung des neuen PBWU-Konzeptes, die Sparmaßnahmen bei Forschungsvorhaben und die Ergebnisse der Landtagsanhörung zum Thema insgesamt zu Skepsis gegenüber einer Weiterführung des PBWU-Projektes Anlaß bieten, wird die Terminierung von den Betroffenen, insbesondere Projektgruppe und GSF-Geschäftsführung, als vergleichsweise überraschend empfunden. Von einer Weiterführung der PBWU zumindest in reduziertem Umfang war aus- 
gegangen worden. Das Forschungsprogramm ist noch nicht ausreichend implementiert, weiterer Forschungsbedarf besteht. Die innerhalb von 9 Jahren an der Koordinierungsstelle aufgebauten Strukturen der Forschungsförderung und des -managements zwischen Politik und Wissenschaft erscheinen wertvoll und erhaltenswert. Eine Phase der geordneten und strukturierten Beendigung der Programmimplementation ist allerdings nicht zu erkennen.

Die Restabwicklung des Projektes muß sehr kurzfristig erfolgen. Ein adäquater Projekt- bzw. Programmabschluß über 9 Jahre Waldschadensforschung kann in der Kürze der Zeit nicht erstellt werden. Das Gesamtergebnis des Projektes wird daher lediglich in Form eines kurzen Berichtes der PBWU dem Ministerium mitgeteilt; die GSF bedingt sich dies gerade angesichts mit Projektende auslaufender Arbeitsverträge von PBWU-Mitarbeitern aus. Damit entgeht dem Projekt ein wesentlicher Bestandteil des Programms, nämlich der programmatisch-inhaltliche Abschluß des Projektes mitsamt einer ausführlichen Zusammenführung und Evaluierung des Programms und seiner Ergebnisse. Im PBWU-Projekt kann weder innerhalb der Projektdauer, noch über diese hinausgehend, eine "Synthesephase“ realisiert werden, wie sie bspw. vom Waldschadensforschungsprogramm des Schweizerischen Nationalfonds einschließlich eigens reservierter Zeit- und Finanzressourcen gefordert wird [STARK 1991, S. 298].

Auch ein bereits in Aussicht genommenes drittes Statusseminar zum Thema Waldschäden, welches den Ergebnisstand der Forschungsarbeiten der Politik und Wissenschaft auftragsorientiert hätte vorstellen können, wird kurzerhand abgesagt. Die mit der Erstellung einer Buchveröffentlichung zum alpinen Forschungsschwerpunkt betrauten Wissenschaftler verzichten angesichts der Beendigung der PBWU auf die Weiterverfolgung ihres Vorhabens. Somit wird weder Wissenschaft, Politik noch Öffentlichkeit adäquat über das Forschungsergebnis des PBWU-Projektes informiert. Es bleibt den einzelnen Wissenschaftlern überlassen, ihre Forschungsergebnisse der scientific community zu präsentieren, was nicht im Sinne eines interdisziplinären, koordinierten Verbundprojektes sein kann. Damit stellt die Programmterminierung in ihrer überraschenden, kurzfristigen und weitgehend ungeregelt ablaufenden Form eine wesentliche Effizienzeinbuße des Gesamtprojektes dar.

\subsubsection{Evaluierung der Forschung}

\subsection{Grundlagen}

Wie bereits ausgeführt, erfolgt die Terminierung des PBWU-Projekts ohne eine die Terminierung legitimierende, wissenschaftliche Evaluierung des Programmerfolgs. Dies überrascht nicht. Die Evaluation eines Programmes durch die Wissenschaft oder der fachliche Nachweis der tatsächlichen Problemlösung als Voraussetzung für die Programmterminierung ist in der politischen Praxis die seltene Ausnahme [WINDHOFF-HÈriTIER 1987, S. 65 und 107].

Die Abschätzung des Programmnutzens kann sich, rationalen Modellen folgend, auf die Effektivität oder die Effizienz des Programmes [KURKI 1991, S. 72ff; RosSI et al. 1988, S. 12/13] bzw. des einzelnen Forschungsvorhabens beziehen. Dabei erfordern Effizienzanalysen, die den Erfolg eines Programmes gegenüber alternativen Programmen festzustellen versuchen, meist die Kosten-Nutzen-Analyse, die die Programmwirkungen monetär bewertet und mit den finan- 
ziellen Aufwendungen in Beziehung setzt. Die Voraussetzungen hierfür, nämlich die Vergleichbarkeit mit alternativen Programmen sowie die monetäre Bewertbarkeit des Programmergebnisses, sind in der Forschung allerdings kaum gegeben. Effektivitätsanalysen beschäftigen sich ebenfalls mit den Programmwirkungen und vergleichen Zielsetzung und Zielerreichungsgrad [KURKI 1991 S. 72ff; s.a. HELLSTERN \& WOLLMANN 1984, S. 24].

Der im Projekt durchgeführte Soll/Ist-Vergleich bei der Berichtsevaluierung zu den einzelnen Forschungsvorhaben (Zwischen- und Schlußberichten) durch die PBWU (siehe Kap. 5.3.5.4) zielt in diese Richtung, wenn auch die eigentliche wissenschaftliche Bewertung des Forschungsergebnisses außerhalb der Förderinstitution, nämlich in der scientific community erfolgt [HILL \& RIESER 1983, S. 281]. Auf den Sonderfall der ex ante-Evaluierung von Forschungsanträgen mit Hilfe des Instrumentariums externer Begutachtung wurde ebenfalls bereits ausführlich eingegangen (s. Kap. 5.3.5.4). Auf der Ebene des Gesamtprojektes dient die regelmäBige Berichterstattung der PBWU gegenüber dem Auftraggeber Bayerisches Umweltministerium in Form von Tätigkeits- und Ergebnisberichten oder anläßlich von Gremiensitzungen der Kontrolle des Projektes.

Ein weiteres Instrument zur Überwachung und Kontrolle der Forschungsarbeiten, der Besuch in den geförderten Forschungseinrichtungen oder die persönliche Unterrichtung über die Forschungsarbeiten [HILL \& RIESER 1983, S. 202], wurde hinsichtlich der beteiligten Gutachter im PBWU-Projekt nicht vereinbart. Persönliche Besuche der Projektgruppe oder des Finanzgebers bei den Forschungsinstituten fanden zwar vereinzelt statt, besaßen allerdings eher den Charakter des gegenseitigen Kennenlernens, der Kontaktpflege oder der Erläuterung der Forschungsarbeiten als den einer Forschungsbewertung oder -kontrolle und wurden auch nicht dahingehend verwendet.

Letztlich können die beiden Statussminare der PBWU zum Thema Waldschadensforschung genannt werden, im Rahmen derer, für die Auftragnehmer verpflichtend, über den Stand und die Ergebnisse der Forschungsarbeiten berichtet wurde. Diese Veranstaltungen, an denen neben den PBWU-Gremien sich ein großes wissenschaftliches Fachpublikum und auch Fachgutachter zu den einzelnen Vorhaben beteiligten, waren geprägt von der fachlichen Diskussion innerhalb der scientific community. Sie leisteten mehr einen Beitrag zur wissenschaftsinternen Steuerung und Kontrolle der Erkenntnisproduktion als zur speziellen, forschungspolitischen Kontrolle des PBWU-Programms. Der positive Effekt, der sich aus der wissenschaftlichkritischen Diskussion auf die Optimierung und damit den Erfolg und die Effektivität der einzelnen Forschungsarbeiten ergibt, sollte jedoch nicht unterschätzt werden.

Diese Veranstaltungen fördern gerade im Falle von gemeinsam bearbeiteten Forschungsthemen oder -standorten die Abstimmung der Forschungsarbeiten untereinander und dienen somit der Koordination des Gesamtprojekts. Auf politischer Ebene können die Statusseminare als Rechtfertigung und Vollzugsnachweis für den Auftraggeber gegenüber der Staatsregierung, die das Bayerisches Umweltministerium mit der Durchführung des PBWU-Projektes beauftragte, genutzt werden.

\subsection{Politische und wissenschaftliche Evaluierung des Gesamtprojekts}


Auf Grundlage der vorgelegten Forschungsberichte zu den einzelnen Forschungsvorhaben erstellt die PBWU jährliche Ergebnisberichte zum aktuellen Stand der Forschung. Diese werden ab der Phase der Konsolidierung der PBWU im Jahr 1988 - mit dem Gremium des Wissenschaftlichen Beirats abgestimmt und dem Bayerischen Umweltministerium vorgelegt. Die Berichte fassen lediglich die Forschungsergebnisse aus den einzelnen Vorhaben zusammen und versuchen, diese in einen Kontext zu stellen. Dabei wird auf den problemlösungsorientierten Ansatz des Projektes Bezug genommen, indem auf die für den politischen Auftraggeber bedeutsamen Forschungsaspekte besonders eingegangen wird.

Die PBWU versucht damit, die politische Relevanz des Forschungsprojektes zu verdeutlichen und gleichzeitig ihre eigene Position zu stärken. Sie versucht, die Einzelergebnisse aus dem Projekt integrierend darzustellen und zu einer Gesamtschau zu verdichten, um dem Anspruch an ein koordiniertes und aufeinander abgestimmtes Gesamtprojekt gerecht zu werden. Das Rahmenprogramm der PBWU könnte dabei eine gewisse Orientierung darstellen, da dort bereits im Detail die Bearbeitung einzelner Forschungsfragestellungen ausgewiesen worden war. Angesichts der sonstigen Offenheit des Programms und der von einer stringenten Programmausfüllung stark abweichenden Programmimplementation ist jedoch abzusehen, daß sich die nachträgliche Verschmelzung der Forschungsarbeiten und -ergebnisse zu einem abgestimmten und abgerundeten Gesamtprojektergebnis problematisch gestaltet. Sie wird zudem durch die z.T. asynchrone Durchführung zeitlich, inhaltlich und räumlich benachbarter Forschungsvorhaben erschwert.

Dem politischen Auftraggeber reichen die von der PBWU zusammengestellten Ergebnisse jedoch aus. Dort werden Aussagen formuliert, die die Relevanz verschiedener LuftschadstoffKomponenten und anderer Belastungsfaktoren sowie maßgeblicher Wirkungspfade unter politisch zulässiger Vergröberung wissenschaftlicher Details darstellen. Sie können als Grundlagen für politische Maßnahmen herangezogen werden und machen den politischen Erfolg des PBWU-Projektes aus.

Instrumente zur Kontrolle über das PBWU-Projekt an sich stellen vertragsgemäß regelmäßig vorzulegende Tätigkeitsberichte der PBWU an das Bayerische Umweltministerium sowie die mündliche Berichterstattung zum Stand der Arbeiten anläßlich der PBWU-Gremiensitzungen dar. Die Kontrollwirkung dieser Aktivitäten ist jedoch faktisch als gering anzusehen. Bei den vorgelegten Berichten kommt es in keinem Falle zu Rücksprache, Nachbesserungsbedarf oder sonstige Korrekturen (z.B. i.Q.: Bayer. Umweltministerium 20.5.1992, 3.5.1993); die mündlichen Berichterstattungen der PBWU auf den Gremiensitzungen bleibt meist unkommentiert (i.Q.: PBWU, Protokolle zu den Gremiensitzungen verschiedenen Datums).

Die parlamentarische Kontrolle des PBWU-Projektes läßt sich an Vollzugsberichten der Staatsregierung zu Inhalt, Umfang und Ergebnis der bayerischen Waldschadensforschung festmachen, in denen explizit auf die PBWU Bezug genommen wird. Dabei handelt es sich allerdings lediglich um Berichterstattungen der Staatsregierung zum Thema und über die PBWU erfolgte Forschungsaktivitäten, und weniger um Ansätze einer Evaluierung des Forschungsprojektes. 
Als eine Ausnahme können gezielt auf eine wissenschaftlich sowie politische Erfolgsbewertung des Projekts ausgerichtete Landtagsanfragen gewertet werden. So fragt z.B. die politische Opposition in einer Landtagsanfrage vom 15.5.1986 die Staatsregierung nach dem konkreten Koordinierungsbeitrag der PBWU, Details zum Forschungsprogramm, Fortschritten des abgesicherten Wissensstandes zu den Waldschadensursachen sowie zu Gegenmaßnahmen an (Anfrage und Antwort der Staatsregierung, s. Landtagsdrucksache 10/1084 vom 14.7.1986). Eine weitere Landtagsanfrage eines Abgeordneten der Opposition vom 10.3.1993 befaßt sich unter anderem mit der Beurteilung der von der PBWU koordinierten Forschungsvorhaben und deren Ergebnisse (s. Anfrage vom 10.3.1993 und Antwort des Bayerischen Umweltministers vom 19.4.1993). Beabsichtigt ist hier jedoch die kritische Durchleuchtung des Projektes oder dessen Bestandteile durch einzelne Vertreter der politischen Opposition, eventuell verursacht durch bestehende oder vermutete Defizite in der Programmimplementation. Von einer systematischen, umfassenden Programmevaluierung kann keine Rede sein.

Somit muß konstatiert werden, daß eine politische Gesamtevaluierung des PBWU-Projektes weder innerhalb der Phase der Programmimplementation, noch zu deren Ende, wie zur Ableitung einer Terminierungsentscheidung angenommen werden könnte, und auch nicht in Form einer erfolgskontrollierenden ex post-Analyse stattfindet.

Dennoch kann unterstellt werden, daß für die Entscheidung des Bayerischen Umweltministeriums, die Projektgruppe und damit das PBWU-Forschungsprojekt zu beenden, zumindest eine interne Bewertung zugrunde gelegen haben mußte. Diese Bewertung konnte sich formal unter anderem auf die von der PBWU eingeforderte schriftliche Zwischenbilanz zur bayerischen Waldschadensforschung vom Juni 1991, das neue Forschungskonzept der PBWU gegenüber dem Umweltminister zu Beginn des Jahres 1992 sowie die Resolution des Wissenschaftlichen Beirats vom Oktober 1992 stützen. Auch die Ergebnisse der Landtagsanhörung Ende 1991 zur Bilanz der Waldschäden und der Waldschadensforschung mögen eine Rolle gespielt haben sowie informal die Einstellung des Umweltministers zum Thema.

Letztlich äußert sich die Bewertung des Ministeriums zum PBWU-Projekt in der Antwort auf die Landtagsanfrage vom 10.3.1993. Danach hätten die bisherigen Forschungsergebnisse einen erheblichen Beitrag zur Ursachenaufklärung der Waldschäden geleistet, welche Grundlage für die Durchführung wirksamer umweltpolitischer Maßnahmen darstellten. Eine Dringlichkeit für neue Vorhaben sei in Anbetracht des bereits erfolgten, hohen Mitteleinsatzes und forstlicher Programme des Nachbarressorts nicht erkennbar (i.Q.: Bayer. Umweltministerium 19.4.1993).

Damit wird dem Projekt grundsätzlich wissenschaftlicher wie politischer Erfolg attestiert. Das Ziel des PBWU-Projektes, über problemorientierte Forschung Handlungsempfehlungen zu weiteren Gegenmaßnahmen im Bereich Luftreinhaltung zu den Waldschäden ableiten zu können, scheint aus politischer Sicht erreicht. Eine weitergehende politische Analyse des Programmergebnisses in Form einer Effektivitäts- oder Effizienzanalyse unterbleibt, auch wenn in der Begründung für die Beendigung des PBWU-Projektes auf diese beiden Aspekte (Zielerreichung und Mitteleinsatz) abgehoben wird. 
Dies verwundert angesichts der gegebenen und bereits erwähnten Schwierigkeiten in der politischen Bewertung des Erfolges sowie der Ziel-Mittel-Relation wissenschaftlicher Forschung allerdings nicht. Zum einen waren die Programmziele lediglich grob beschrieben und erschweren somit eine entsprechend qualifizierte Beurteilung der Programmeffektivität. Zum anderen ist die monetäre Bewertung der politischen Programmwirkung Waldschadensforschung ebenfalls kaum zu leisten. Der Kostenaufwand für die Forschung könnte nur vage zu dem daraus entstehenden politischen Nutzen in Beziehung gesetzt werden.

Auch in wissenschaftlicher Hinsicht unterbleibt die Evaluierung des gesamten PBWUForschungsprogrammes. Während - wie bereits dargestellt - die einzelnen Forschungsvorhaben als Bestandteile des Programms zumindest einem formalen Soll/Ist-Vergleich durch die PBWU unterworfen werden und sich im Rahmen der Selbststeuerung der Forschung über wissenschaftliche Publikationen etc. einer Evaluierung in der scientific community stellen, trifft dies für das Gesamtprojekt nicht zu.

Die jährlichen, an das Bayerische Umweltministerium gerichteten Ergebnisberichte sowie der Schlußbericht der PBWU zum Gesamtprojekt stellen kein qualitativ geeignetes Instrument zur wissenschaftliche Evaluierung des Forschungserfolges dar. Das Gremium des Wissenschaftlichen Beirats sieht hierzu weder Auftrag noch Veranlassung und ist ohnehin in den Forschungsprozeß selbst stark involviert. $\mathrm{Zu}$ den Berichten, die die Projektgruppe dem Beirat zur Abstimmung vorlegt, werden allenfalls marginale Änderungswünsche angemerkt (z.B. i.Q.: Wissenschaftlicher Beirat, 27.1., 30.1.1989, 15.7.1991, 19.3.1992, 1.3., 2.3.1993).

Das geplante und als Evaluationsinstrument potentiell geeignete dritte Statusseminar wird aufgrund bzw. trotz der bevorstehenden Terminierung des PBWU-Projektes storniert. Die Möglichkeit, die Ergebnisse des gesamten Forschungsprojektes vor Wissenschaft, Politik und Öffentlichkeit abschließend zu präsentieren, zu evaluieren und den Forschungsaufwand zu legitimieren, wird vom Auftraggeber nicht genutzt.

Letztlich scheitert auch die Erarbeitung einer qualifizierten wissenschaftlichen Buchveröffentlichung der Wank-Forschung. Die Ablehnung erfolgt durch die Wissenschaftler des Herausgebergremiums selbst, welche z.T. Mitglieder des Wissenschaftlichen Beirats sind. Mit einer derartigen Publikation hätte das Projekt nicht nur der Wissenschaft ihre Forschungsinhalte und ergebnisse in einem integrierenden Ansatz präsentiert, es hätte sich damit auch der wissenschaftlichen Evaluierung stellen können bzw. müssen. Es sind - neben zeitlichen Problemen Befürchtungen gerade hinsichtlich einer negativen Bewertung in der scientific community selbst, die zum Verzicht auf die Buchveröffentlichung führen (i.Q.: PBWU, 3.6.1993). Der Verzicht auf die Publikation wird allerdings mit Defiziten in den politischen Förderrahmen- und Durchführungsbedingungen begründet, die die erfolgreiche Umsetzung des Forschungsprogrammes behindert hätten, nicht mit den einzelnen wissenschaftlichen Forschungsergebnissen an sich.

Daß das wissenschaftliche Gesamtergebnis des Wank-Projektes in der scientific community weitgehend unverwertet bleibt, beeinträchtigt den wissenschaftlichen Erfolg, die wissenschaftliche Evaluation sowie die Legitimation des Gesamtprojektes erheblich. Die Publikation nur ei- 
niger weniger Forschungsaspekte durch beteiligte Arbeitsgruppen kompensiert dies keinesfalls. Der Befund, daß die Wank-Forschung kaum einen zweifelsfreien Schädigungsnachweis der Luftschadstoffwirkung auf den Bergwald liefert, auf den die meisten darauf hin focussierten Forschungsvorhaben ausgerichtet waren, könnte - politisch wie wissenschaftlich - als Mißerfolg des Forschungsprojektes empfunden worden sein und damit auch die Neigung zur Publikation gedämpft haben. 


\section{$6 \quad$ Politische Faktoren der Entwicklung und Steuerung des Forschungsprojektes}

Bezugnehmend auf die empirie- und theoriegestützte Analyse des PBWU-Projektes können die Faktoren, welche die Entwicklung und Steuerung des Forschungsprojektes und den damit verbundenen Forschungsprozeß prägen, zusammenfassend dargestellt werden. Dabei sind im Folgenden die jeweiligen Kernaussagen den Ausführungen vorangestellt.

\subsection{Handlungsdruck der Politik}

- Politischer Handlungsdruck kann als wichtiger, sachlich-fachliche Gesichtspunkte überlagernder Durchsetzungsfaktor für eine Forschungspolicy angesehen werden. Er erleichtert den Aufbau einer administrativen Vormachtstellung des maßgeblich betroffenen Ressorts im Falle der luftschadstofforientieren Waldschadensforschung des Umweltressorts - und begünstigt die rasche Realisation einer Forschungspolicy auch gegen politisch-administrative wie fachlich-wissenschaftliche Widerstände.

Das zu Ende der 70er, Beginn der 80er Jahre in allen Gesellschaftsbereichen aktuelle Thema „Waldsterben“ übt einen hohen Handlungsdruck auf die Politik aus. Mit der Focussierung der Ursachen auf den Faktor Luftschadstoffe sieht sich die Politik zur Verstärkung von Emissionsminderungen einerseits, zur Verstärkung problemorientierter Waldschadensforschung andererseits, gezwungen. Aufgrund deren Ergebnisse könnten konkrete Gegenmaßnahmen um so gezielter und effektiver ergriffen werden. Zudem verschafft die Durchführung der Forschung einen Zeitgewinn für die Politik bei der Umsetzung der aus den Forschungsergebnissen erst abzuleitenden Maßnahmen.

Bei der anfangs luftschadstoffdominierten Ursachendiskussion ist es naheliegend, daß sich das bayerische Umweltministerium um die Federführung in der Organisation und Betreuung der zu verstärkenden Waldschadensforschung bemüht. Der gegebene politische Handlungsdruck erleichtert es dem Umweltressort, die Durchführungskompetenzen einschließlich Finanzierungshoheit an sich zu ziehen und somit einen Zugewinn an Renommee, Macht und Einflußnahme auf die Politik zu verbuchen. Daneben sichert es sich über das PBWU-Projekt einen direkten Zugang zur Wissenschaft und die zumindest potentielle Möglichkeit, über die Steuerung der Forschung die Einflußnahme in Richtung der eigenen, ressortspezifischen Interessenverfolgung zu verstärken [s.a. BRAUN 1997, S. 181, 315/316].

Das objektseitig maßgeblich betroffene Landwirtschafts-(Forst-)ressort kann sich bezüglich eines eigenen Schadensverursacher-Beitrags weitgehend exkulpieren. Es kann über seine eigene ressortspezifische Förderstrukturen Forschungsbereiche wie Schadenserhebung und -beobachtung sowie ausgewählte Bereiche forstlich orientierter Waldschadensforschung nahe$\mathrm{zu}$ unbeeinflußt fortführen. Somit reicht der politische Handlungsdruck zwar dafür aus, daß Fördermittel-Ressourcen für die Waldschadensforschung dem Umweltressort zusätzlich zur Verfügung gestellt werden. Für eine Umverteilung der Fördermittel vom Landwirtschafts- auf 
das Umweltressort und damit für die Möglichkeit, daß das Umweltressort in den Zuständigkeitsbereich des Forstressorts eindringen kann, ist der Druck jedoch nicht groß genug. Die Frage, ob eine aktivere Forstpolitik zu Beginn der Forschungsdiskussion den bestehenden Handlungsdruck für eigene Forschungsfinanzierung verstärkt und zulasten des Umweltressorts hätte nutzen können, soll hier nur spekulativ gestellt werden. Daß davon in der Phase der PBWU-Terminierung, als das Ressort zur Übernahme der PBWU-Finanzierung und Verstärkung forstlicher Forschung gebeten wurde, nicht mehr Gebrauch gemacht wurde, läßt sich allerdings mit dem insgesamt nurmehr geringen Problemdruck begründen.

Der Versuch Bayerns, angesichts des bestehenden Handlungsdrucks die Koordination für die gesamte, bundesweite Waldschadensforschung zu übernehmen und damit auch in den Genuß von Fördermitteln des Bundes zu gelangen, mißlingt zwar. Er stellt jedoch die Weichen dafür, daß mit der bayernweiten Koordination eine in Bayern angesiedelte Großforschungseinrichtung des Bundes, die GSF, betraut wird. Diese Konstruktion ist bei den weiteren beteiligten Ressorts nicht widerspruchsfrei, jedoch ganz im Sinne des betreibenden bayerischen Umweltressorts, welches bereits frühzeitig und eng mit der GSF kooperiert.

Damit sind die Voraussetzungen für eine rasche Implementierung des durch Ministerratsbeschluß fixierten PBWU-Projektes, einschließlich der Projektgruppe selbst, weitestgehend gegeben. Administrative, organisatorische sowie fachlich-wissenschaftliche Kritikpunkte und Widerstände können mit Hilfe des politischen Argumentes Handlungsdruck überspielt oder zumindest zurückgestellt werden. Im Rahmen der durch politische Fakten geschaffenen Strukturen belasten sie jedoch in der Folge die Implementation der Forschungspolicy.

\subsection{Vermengung politischer und fachlicher Beiträge der Akteure}

- Die politisch-administrativ und wissenschaftlich geprägten Akteurskonstellationen in dem PBWU-Projekt führen aufgrund der jeweiligen interessenorientierten Handlungsstrategien zu einer Vermengung politischer und fachlich-wissenschaftlicher Argumente bei Programmdefinition und Forschungsimplementation. Dies hat zur Folge, daß weder das fachliche Potential maximal ausgeschöpft, noch die Verantwortung für politische Entscheidungen eindeutig sichtbar wird.

Die These von der Vermengung politisch-interessengeleiteter und fachlich-objektiver Beiträge durch Vertreter der Politik und der Wissenschaft wird durch verschiedene Befunde gestützt. Bereits bei der Erstellung des Forschungsrahmenprogramms treffen nicht nur politischadministrative Interessen auf wissenschaftlich orientierte Interessen der Forscher. Die jeweiligen Akteure bedienen sich auch der Argumente der Gegenseite. Mit Argumenten, die der wissenschaftlichen Diskussion entnommen sind, legitimieren die politisch-administrativen Akteure die Festschreibung konkreter Forschungsthemen im Programm. Beratende Fachwissenschaftler nutzen die politischen Rahmenvorgaben für ihre Argumentation, um die Einbeziehung bestimmter thematischer wie regionaler Forschungsgebiete in das Programm zu fordern.

Allein die Breite der Forschungsthemen in dem von der Projektgruppe entworfenen Rahmenprogramm stellt das Ergebnis einer Vermengung politischer mit fachlicher Argumentation dar. 
Der überwiegend politisch agierende Akteur Projektgruppe versucht, den breiten thematischen Forschungsrahmen fachlich zu begründen. Damit kann unter anderem ein hoher Finanzmittelaufwand für das Projekt legitimiert und eine stabile und existenzfähige Projektgröße erzielt werden. Möglichst wenig potentielle Antragsteller bzw. Forschungsbereiche a priori ausschließen zu müssen, kann auch dem Bestreben der Gruppe nach Akzeptanz durch die Forscher einschließlich des wissenschaftlichen Beratungsgremiums - dienen.

So werden gerade zu Beginn des PBWU-Projektes auch nicht im Focus des politischen Interesses stehende Forschungsthemen bewilligt. Dies dient weniger dem Interesse an Kenntnisverbreiterung im fachlichen Detail, als vielmehr der politischen Absicht, das Forschungsprogramm rasch zu implementieren und durch großzügige, wissenschaftsfreundliche Förderung die Akzeptanz der Wissenschaftsvertreter im Beirat für das Projekt zu gewinnen. Für den alpinen Forschungsstandort Wank bedienen sich die politischen Akteure sogar gegen Kritik aus dem wissenschaftlichen Beratungsgremium fachlicher Argumente bzgl. Waldzustand und Immissionssituation, um den politisch erwünschten Schwerpunkt unverzüglich zu etablieren. Diese Argumente werden wiederum von Forschergruppen bevorzugt aufgegriffen, um sich - konform der Argumentation der Politik - am Standort mit entsprechenden Forschungsbeiträgen legitimieren und ansiedeln zu können.

Vergleichbares kann auch bei dem in Eigeninitiative einer Forschergruppe erstellten Forschungskonzept für den alpinen Forschungsschwerpunkt beobachtet werden. Etwa dann, wenn eine Vielzahl am Standort bereits etablierter Forschergruppen im Konzept mitbeteiligt werden soll, werden wissenschaftlich-fachliche Argumente überwiegend zur Verfolgung forschungspolitischer Interessen verwendet. Weniger die fachliche Notwendigkeit zur Bearbeitung all der genannten Forschungsthemen, als die Absicht, mit deren Einbeziehung die Akzeptanz der betreffenden Forscher für das Programm zu erwirken, scheint das interessenleitende, forschungspolitische Moment einer scheinbar fachlichen Argumentation zu sein.

Auch bei der Argumentation zur Einpassung von Forschungsanträgen in das Rahmenprogramm kann die Vermengung fachlicher mit politischer Argumentation beobachtet werden. In erster Linie kann den Forschern zwar das originäre Interesse an der wissenschaftlichen Fragestellung unterstellt werden. Die Verknüpfung mit politischen Argumenten kann aber dazu dienen, die Erfolgsaussichten für die Finanzierung des Antrags zu verbessern. So erfolgt bspw. die Beantragung einiger Forschungsvorhaben am alpinen Forschungsschwerpunkt verknüpft mit der Argumentation des politisch gegebenen Handlungsdruckes oder des Beitrags zum interdisziplinären Forschungsprogramm. Dabei ist die wissenschaftliche Notwendigkeit, die Forschungsarbeiten am Standort durchführen zu müssen, insbesondere dann, wenn es sich um Grundlagenforschung handelt, häufig gar nicht gegeben.

In der Regel stellen die Forscher dabei in Aussicht, die Ergebnisse ihrer Forschungsarbeiten könnten maßgebliche Beiträge zur - politischen - Problemlösung liefern. Sie setzen somit Fachargumente politisch für ihre Forschungsinteressen ein. Dabei ist jedoch ein erheblicher Teil der Arbeiten der Grundlagenforschung zuzuordnen oder betrifft, bezogen auf das problemorientierte Forschungsprogramm, eher periphere Themen. $\mathrm{Zu}$ nennen sind hier Beispiele biochemischer oder pflanzenphysiologischer Wirkungsforschung ebenso wie die Bearbeitung 
von eher randständigen Schadstoffspezies, aufgrund deren vermuteter, problembezogener Relevanz ein Forschungsantrag begründet wird. Dabei kommt die anfänglich geringe Kenntnislage zur Beurteilung der tatsächlichen Relevanz den Forschern natürlich entgegen.

Wie bei der Erstellung des Rahmenprogramms eingangs bereits angedeutet, trifft auch für die Projektgruppe selbst die Vermengung politischer und wissenschaftlicher Argumentation zu. Neben formalen Koordinationsaufgaben aus ihrem politischen Auftrag kann sie aufgrund ihrer intermediären Stellung und der gegebenen Eigeninteressen, die auf Etablierung, Programmumsetzung, Bestandssicherung, Akzeptanz und Anerkennung im politischen, aber auch im wissenschaftlichen Lager gerichtet sind, beide Argumentationstypen gleichermaßen einsetzen. Dabei kann sie wie kein anderer Akteur sowohl auf fachliche Argumente der von ihr betreuten Forscherklientel sowie des Beratungsgremiums, als auch auf politische Argumente ihres Auftraggebers sowie des Projektrats zurückgreifen und beide zur Verfolgung der eigenen Interessen gegenüber den jeweiligen Akteuren nutzen.

Die Nutzung wissenschaftsexterner Argumente durch eben die Wissenschaft sowie umgekehrt politisch-externer Argumente durch Politik bzw. Verwaltung ist angesichts der gegebenen Akteurskonstellation im PBWU-Projekt zu erwarten. Insgesamt beeinträchtigt die Vermengung politischer und fachlicher Argumente jedoch die Effizienz des Forschungsprogramms. Das PBWU-Programm ist ,belastet“" mit Forschungsvorhaben, die nicht im Focus der Forschungspolicy stehen. Die fachliche oder lokale Fehlplazierung einzelner Vorhaben führt dazu, daß das zur Verfügung stehende Forscherpotential bzw. der Finanzmitteleinsatz im Sinne der problemorientierten Forschungspolicy nicht optimal ausgeschöpft werden. Die idealtypischen Zuständigkeiten und Handlungsrationalitäten von Politik und Wissenschaft, ausschließlich politische bzw. wissenschaftliche Beiträge zu leisten und Entscheidungen zu treffen, sind somit nicht gegeben. Dadurch werden die jeweiligen Potentiale - Kenntnis der Forschungsmöglichkeiten und Formulierung der Verwertungsmöglichkeiten - nicht voll genutzt. Zudem wird die Verantwortlichkeit für getroffene Entscheidungen in Hinblick auf die politische wie wissenschaftliche Argumentation verschleiert.

\subsection{Territorial-Verhalten als Hindernis für Koordination}

- Die in den Gremien des PBWU-Projektes beteiligten politischen wie wissenschaftlichen Akteure zeigen deutlich ein Territorialverhalten in Hinblick auf Ressortzuständigkeiten, wissenschaftliche Fachbereiche und regionale Zuständigkeiten. Die Akteurskonstellationen im PBWU-Projekt erlauben die Durchsetzung entsprechender politischer wie wissenschaftlicher Interessen und gestatten den Akteuren förderpolititsche Einflußnahme, ohne selbst beeinflußt zu werden. Diese Strategie geht jedoch zulasten einer ressortübergreifenden und interdisziplinären Forschungskoordination.

Die Beteiligung benachbarter Ressortvertreter im politischen Entscheidungsgremium des Projektrats, die führender Vertreter bestimmter Forschungsrichtungen und -schwerpunkte im wissenschaftlichen Beratungsgremium der PBWU führen zur Ausprägung eines territorialen Verhaltens der Akteure. Dies wird durch spezifische Kooperationsstrukturen zwischen beiden Akteursgruppen aufgrund anderweitig bestehender Förderprogramme noch verstärkt. Das im 
vorhandenen Forschungsterritorium neu auftretende Umweltressort mitsamt der Projektgruppe sieht sich bereits bestehenden Verflechtungen aus Förderer- und Forscherklientela, der Besetzung bestimmter Förder- und Forschungsbereiche sowie Forschungsstandorte gegenüber.

Dem Downs'schen Modell des behördlichen Territorialverhaltens entsprechend [DownS 1967, S. 211ff] streben die am PBWU-Projekt beteiligten Förderressorts, insbesondere das Kultusund das Landwirtschaftsressort danach, ihren Einflußbereich gegen den des neu hinzugekommenen Umweltressorts nicht nur zu verteidigen, sondern zu erweitern. Sie werden darin von ihrer jeweiligen Forscherklientel unterstützt. Gegenüber der zentralen Beteiligung der Nachbarressorts im Projektrat der PBWU wird der Projektgruppe selbst keine vergleichbare Beteiligung in anderen Fördergremien zugebilligt.

Förderpolitisch Einfluß zu nehmen ohne selbst beeinflußt zu werden, sichert den Nachbarressorts somit die Möglichkeit, sich am PBWU-Forschungsprogramm steuernd zu beteiligen und damit indirekt eine zusätzliche Finanzierung für ihr eigenes Programm erlangen zu können, ohne daß die Ressorteigenständigkeit und die Finanzierungshoheit aufgegeben werden müssen. Bis zuletzt sind die Nachbarressorts darin erfolgreich, eine erwähnenswerte finanzielle Beteiligung am PBWU-Projekt abzuwehren, was letztlich die Terminierung des Projektes auch aus ressort-territorialen Gründen miterklärt.

Die Beteiligung bestehender Forschungsverbünde am PBWU-Programm wollen die Nachbarressorts - insbesondere für den nordbayerischen Forschungsverbund des Kultusressorts zutreffend - durch mehrere strategische Schritte sichern. Die ressortspezifischen forschungspolitischen Interessen sowie die der betreuten Forscherklientel sollen bereits in das Rahmenprogramm einbezogen werden. Die Berufung mehrerer Vertreter des Forschungsverbundes in den Wissenschaftlichen Beirat verspricht um so mehr, die forschungsthematische wie regionale Interessenwahrung der Wissenschaft im PBWU-Projekt zu gewährleisten. Letztlich soll auch dasselbe Gutachtergremium im PBWU-Projekt eingesetzt werden, welches dem bereits bestehenden Forschungsverbund zugeordnet ist. Aufgrund der anfänglichen Verfahrensdominanz des Umweltressorts sowie der Projektgruppe können jedoch nicht alle der genannten Maßnahmen im beabsichtigten Umfang realisiert werden.

Die PBWU sichert sich über die Zufinanzierung von Forschungsprogrammen anderer Ressorts zwar formal eine förderseitige Beteiligung an dem gesamtbayerischen Förder- und Forschungskomplex. Ohne faktisches Einflußnahmepotential auf die Gestaltung externer Programme oder deren strukturelle und inhaltliche Verschmelzung $\mathrm{zu}$ einem gesamten Forschungskontext wird sie ihrem Auftrag konzeptionell und programmatisch abgestimmter, bayernweiter Forschungskoordination und Programmsteuerung allerdings nicht gerecht. Zudem gehen die Fördermittel dem eigenen Programm verloren. Das Fortbestehen mehrerer separater Förderprogramme bzw. Förderverfahren verschiedener Finanzgeber und dementsprechender ressortspezifisch-forschungspolitischer Interessen auch nach Einrichtung der PBWU signalisiert, daß die bayernweite, ressortübergreifende Forschungskoordination durch die neue Projektgruppe unter den gegebenen Rahmenbedingungen nicht realisierbar ist. Als entscheidender Faktor kann hierfür die fehlende übergreifende Finanzhoheit der Koordinierungsstelle bzw. des Umweltressorts bei Nichtbeteiligung der Nachbarressorts an einer Forschungsfinanzierung im 
PBWU-Projekt angesehen werden. Ungeachtet weiterer Probleme interdisziplinärer Forschung (s.a. Kap. 5.3.2.5.3) erschwert bereits die zersplitterte Finanzierung die bayerische Forschungskoordination in hohem Maße. 


\subsection{Politische Dominanz in der Etablierung und Terminierung der Forschungspolicy}

- Die Etablierungs- sowie die Terminierungsphase der Policy PBWU-Waldschadensforschung ist politisch dominiert. Der relativ geringe Steuerungseinfluß der Wissenschaft auf die konzeptionelle Rahmengebung, anfängliche Implementation und Terminierung des Forschungsprogramms resultiert jedoch nicht aus einem freien Kräftespiel politischer und wissenschaftlicher Steuerungsinteressen. Er repräsentiert vielmehr den dominanten Einsatz politischer Machtressourcen und Durchsetzungsstrategien nach dem Modellansatz einer top downSteuerung.

Die strukturelle Konzeption des PBWU-Projektes stellt über die Projektgruppe selbst sowie über die beiden politischen und wissenschaftlichen Beratungsgremien die Vertretung beider politischer und wissenschaftlicher - Interessen sicher. Sie bildet die Basis für eine Interessenaushandlung im Rahmen des Projekts. Nach dem Ansatz neuerer Steuerungstheorien [BRAUN 1997, S. 315/316] ist diese geprägt von dem Bestreben der Wissenschaft, Definitionsmacht über die Mittelverteilung und damit auf den Forschungsprozess zu erlangen oder zu erweitern. Die politischen Akteure hingegen wollen unter Einsatz des Steuerungsinstrumentes finanziellen Fördermittelanreizes im Forschungsprogramm Einfluß auf die Wissenschaft gewinnen, um eigene forschungspolitische Ziele zu verfolgen und umzusetzen. Der intermediären Koordinierungseinheit, im Fallbeispiel der Projektgruppe, ist das Interesse an einer gewissen Verselbständigung gegenüber politischen und wissenschaftlichen Einflüssen und damit der Entwicklung einer Eigendynamik zu unterstellen. Dieses Modell zeichnet sich auch im PBWU-Projekt ab. Dabei können auch klassische Steuerungstheorien des top down- bzw. bottom up-Ansatzes [VON PRITTwitz 1990, S. 96; WindHOFF-HÉRITIER 1987; S. 96/97; HILl \& RIESER 1983, S. 335ff], welche die jeweils politische bzw. wissenschaftliche Perspektive der Handlungsrationalität repräsentieren, hinsichtlich der bestehenden oder beabsichtigten Steuerungsverhältnisse analytisch miteinbezogen werden [s.a. BRAUN 1997, S. 19; JACHTENFUCHS et al. 1993, S. 213].

Nur geringe Formalisierungs- und Normierungsvorgaben im Abstimmungsverfahren zur Forschungskonzeption sowie speziell im Förderverfahren können von den betreibenden politischen Akteuren, dem federführenden Umweltressort und der Projektgruppe selbst, als aktueller Machtvorteil genutzt, bei Umgehung oder unzureichender Beteiligung der Gremien sogar mißbraucht werden. Somit überwiegen gerade zu Beginn der Forschungspolicy, als die Wissenschaft nur indirekt über die sie protegierenden Ressorts im Projektrat Einfluß nehmen kann, politische Interessen und dominieren den forschungspolitischen Steuerungseinfluß im Projekt. Der entscheidende Machtfaktor ist hierbei - insbesondere zu Zeiten umfangreich verfügbarer Fördermittel - weniger die Finanzierungshoheit als vielmehr die politische Verfahrensdominanz. Die Akteure Umweltressort und nachgeordnete Projektgruppe sind bestrebt, ein in Richtung Auftrags- oder Programmforschung gehendes, problemorientiertes Forschungsprojekt zu etablieren.

Die Festschreibung des von der Projektgruppe entworfenen Forschungs-Rahmenprogramms wird damit bereits zur ersten ernsthaften Auseinandersetzung zwischen politischer und wissen- 
schaftlicher Einflußnahme. Die forschungspolitischen Hauptzielsetzungen des Umweltressorts sollen in das Programm implementiert, die Interessen der Nachbarressorts und der Wissenschaft aber hinreichend berücksichtigt werden, um die allgemeine Akzeptanz des Programmes und Mitarbeit der Akteure sicherzustellen. Mit dem formalen Ziel der Offenheit für Forscherideen werden die informalen Ziele rascher Programmimplementation und Beteiligung zahlreicher Forscher gekoppelt.

Mit den Nachbarressorts kann das Rahmenprogramm in fachlicher Hinsicht weitgehend unproblematisch abgestimmt werden. Die ohnehin fachliche Breite und Offenheit des Programmentwurfes nimmt durch die, wenn auch nur beschränkte, Einbeziehung des wissenschaftlichen Beiratsgremiums noch zu, indem dessen generellen Forderungen nach Selbstbestimmung und Ideenfreiheit der Wissenschaft zumindest teilweise entsprochen wird. Die weitergehende Berücksichtigung der Wissenschaftsinteressen an der Ausgestaltung des konzeptionellen Forschungsrahmens wird schlichtweg durch formale Argumentation wie Zeitverzug und durch unzulängliche Übernahme der Modifizierungsvorschläge in das Programm umgangen. Dieses rigide Vorgehen von Umweltressort und Projektgruppe sichert zwar die forschungspolitische Steuerungsdominanz, belastet aber die Akzeptanz und das Vertrauensverhältnis gerade des Wissenschaftlichen Beirats gegenüber der PBWU erheblich, was auf Dauer nicht tragbar sein wird.

Die Steuerungsdominanz wird um so konfliktträchtiger, je mehr die Verteilung von Fördermitteln den Charakter einer Umverteilung annimmt [BRAUN 1997, S. 22, 386]. Der Konkurrenz- und Verteilungskampf um knappe Fördermittel, der durch den politischen Auftraggeber nach dessen Zielsetzungen entschieden wird, wird bspw. bei der Bevorzugung von Vorhaben für den alpinen Forschungsstandort gegenüber anderweitigen offensichtlich und führt zu einer erheblichen Zunahme der ohnehin bereits vorhandenen Kritik von seiten des Wissenschaftlichen Beirats.

Die Vorgehensweise läßt sich insgesamt als politisch beabsichtigter top down-Ansatz der Programmsteuerung interpretieren. Der Beitrag des Programms zur politischen Problemlösung Waldschäden ist beabsichtigt, die politischen Akteure sind in Form des Projektrats an der konzeptionellen Programmformulierung beteiligt, eine steuernde Koordinierungsgruppe wird eigens eingerichtet. Dieser Ansatz wird jedoch durch die gegebene Programmbreite, die Mitwirkung der Wissenschaft an der Programmformulierung, die zudem Grundlagenforschung in erheblichem Maße zuläßt, und die Vertagung der weiteren Aushandlung zwischen politischen und wissenschaftlichen Interessen in die anschließende Phase der Programmimplementation, im Ansatz konterkarriert [s.a. HILl \& RIESER 1983, S. 349; WINDHOFF-HÉRITIER 1987, S. 83; KROTT 1990, S. 66]. Für die strikte Einhaltung eines top down-Ansatzes ist die Projektgruppe $\mathrm{zu}$ schwach, ihre vom Auftraggeber formal zugedachten Kompetenzen werden bereits vor Projektbeginn stark kritisiert. Anforderungen an die konsequente Durchführung der Forschung nach dem top down-Ansatz wie straffes Programmanagement, starke Programmorganisation, klare Rahmenbedingungen und Forschungsplanung sowie eine unabhängige, kompetente und akzeptierte Koordinierungsstelle, bestehen entweder nicht in ausreichendem Maße oder können auf Dauer nicht eingehalten werden [HILL \& RIESER 1983, S. 170ff; 351ff; BRAUN 1997, S. 352ff]. Die kompromißartige Aufweichung eines problemorientierten Forschungsansatzes 
durch die Berücksichtigung von Forscherfreiheit und wissenschaftlicher Selbstbestimmung läßt Steuerungsprobleme und - zumindest aus politischer Sicht - Einbußen in der Programmeffizienz erwarten.

Der dominierende politische Steuerungseinfluß und damit ein Vorgehen in Richtung des top down-Ansatzes der Steuerung kann jedoch noch in der ersten Implementationsphase des PBWU-Forschungsprogramms beibehalten werden. Die Förderverfahren der ersten beiden Antragsrunden im Projekt, welche mit der Bewilligung 1/3 aller Forschungsvorhaben und Fördermittel inhaltlich wie finanziell das Gesamtprojekt bereits wesentlich prägen [STEUER 1997, S.17], beinhalten zwar das Instrumentarium externer Gutachter, sie finden jedoch ohne Beteiligung des Beirats statt. Demgegenüber nutzt die Projektgruppe selbst gegen die Kritik aus ihren Gremien, die sich bereits zu Gründungszeiten gegen die vertraglich festgeschriebenen Lenkungs- und Koordinationsfunktionen der Projektgruppe richten, forschungspolitisch, ja sogar wissenschaftlich ihre Einflußmöglichkeiten. Sie bestimmt, gedeckt durch das Bayerische Umweltministerium, maßgeblich die Durchführung des Förderverfahrens und beteiligt sich an der fachlichen Bewertung der Forschungsanträge.

Damit sichern sich auftraggebendes Umweltressort wie Projektgruppe die Durchsetzung ihrer Interessen und Ziele. Der politische Auftrag, ein bayernweites Waldschadensforschungsprogramm zu etablieren, kann rasch erfüllt werden. Einschlägige bayerische Forschungsinstitutionen werden an der Programmimplementation beteiligt, ein bayernspezifischer, alpiner Forschungsschwerpunkt kann aufgebaut werden. Die fachliche Qualität der Forschung ist im einzelnen durch die Einbeziehung externer Fachgutachter gewährleistet. Neben der Verfahrenshoheit dominiert nun - um so mehr bei auftretenden Finanzierungsengpässen - die Finanzierungshoheit die Förderentscheidungen. Die potentiell mögliche Einflußnahme während mehrerer Verfahrensschritte der Begutachtung, die geringe Verfahrenstransparenz und die finanzielle Prioritätensetzung bestimmter Vorhaben (insbesondere am alpinen Forschungsschwerpunkt) versetzen Umweltressort bzw. Projektgruppe in die Lage, die Finanzmittelhoheit und Begutachtung als Machtfaktoren gegen den Wissenschaftlichen Beirat ,auszuspielen“.

Die forschungspolitische Dominanz soll - in Verbindung mit der Antragsselektion durch externe Gutachter - zudem sicherstellen, daß das PBWU-Projekt nicht als „Selbstbedienungsladen“ für den Wissenschaftlichen Beirat genutzt werden kann; ein Anspruch, dem sich der Beirat selbst unterordnet. Ein wissenschaftlicher Steuerungs- und Selektionseinfluß zur Verfolgung eines - idealtypisch - stringenten Forschungsplanes wird durch zumindest anfangs großzügige Finanzmittelbereitstellung für nahezu alle fachlich für förderungswürdig empfohlenen Anträge zudem hinfällig.

Vergleichbar der Initiierungs- ist auch die Terminierungsphase des PBWU-Projekts von der Dominanz politischer Steuerung geprägt. Eine wissenschaftliche Evaluierung des Gesamtprojektes hinsichtlich einer Entscheidung über Beendigung oder Weiterfuihrung findet nicht statt. Seitens der Wissenschaft wird mittels eines neuen Konzeptes die Fortführung der Forschung gefordert, und auch die Projektgruppe selbst ist existentiell an ihrem Weiterbestehen interessiert. Daher müssen politische Argumente und Machtressourcen dominant gewesen sein, um das Projekt zu beenden. 
Die entsprechende politische Willensbegründung kann dabei weitgehend aus dem nurmehr geringen allgemeinen Problem- und Handlungsdruck zum Thema abgeleitet werden. Nach politischer Interpretation sind die inzwischen erzielten Forschungsergebnisse ausreichend, um über die Notwendigkeit weiterer umweltpolitischer Maßnahmen zu entscheiden. Forschung als Entlastungsinstrument und zur Konkretisierung von Gegenmaßnahmen ist demnach nicht mehr erforderlich. Die geringe politische Legitimation für Waldschadensforschung kann nicht einmal durch ein hochrangiges, wissenschaftlich legitimiertes und durch wissenschaftliche Resolutionen gestütztes Forschungskonzept überwunden werden.

Der politische Auftrag zum PBWU-Projekt, die Waldschadensforschung zu verstärken, um zu umsetzbaren Ergebnissen zu gelangen, wird politisch als erfüllt erklärt. Als politische Entscheidung muß diese Aussage, in welchem Maße auch zutreffend, von der Wissenschaft hingenommen werden. Diese politische Strategie, der Forschungspolicy ihre Zielerreichung zu attestieren, erlaubt es zum einen, das Projekt als insgesamt erfolgreich zu bezeichnen und damit nachträglich die politische Legitimation für seine Durchführung zu dokumentieren. Zum anderen liefert sie die politisch konsequente Begründung, durch Finanzierungsprobleme und Notwendigkeiten der Ressortabstimmung formal noch unterstützt, das Forschungsprojekt und damit auch die Projektgruppe zu beenden.

Insgesamt entwickeln sich im Policy-Verlauf die politische und wissenschaftliche Legitimation für Waldschadensforschung gegenläufig. Zu Beginn stützt sich das Forschungsprogramm auf hohe politische Legitimation. Politisch ist vorentschieden, daß diese Forschung zur Problemlösung beitragen wird. Wissenschaftlich ist diese Erwartung dagegen zu Beginn noch sehr fraglich. Es besteht ein nur suboptimales Forschungsprogramm der Projektgruppe, das weitgehend hypothetisch ist und in der Wissenschaft auf Kritik stößt. In der Phase der Terminierung ist mit einem hochqualitativen, wissenschaftlich akzeptierten Forschungsprogramm, eingespieltem Förderverfahren und leistungsfähigen Forschergruppen zwar die wissenschaftliche Legitimation für das Projekt in hohem Maße gegeben. Paradoxerweise fehlt jedoch nun die politische Legitimation für dessen Fortführung.

Dem aus wissenschaftsrationaler Sicht negativen Resultat, ein wissenschaftlich kritisiertes Forschungsprogramm zu fördern, demgegenüber ein wissenschaftlich akzeptiertes zu stoppen, steht das politikrational positive Resultat gegenüber, eine Forschungspolicy rechtzeitig und zielorientiert begonnen und nach Erreichung der politischen Zielsetzung beendet zu haben. Diese Sichtweise verdeutlicht die Diskrepanz zwischen politischer und wissenschaftlicher Interessenlage und macht die unterschiedlichen Steuerungsintentionen zwischen Politik und Wissenschaft verständlich. Bei der hier gegebenen Konstellation ist die Förderung suboptimaler Forschung die Konsequenz.

\subsection{Wissenschaftliche Dominanz im Verlauf der Programmimplementation}

- Die vertragsgemäße Einbeziehung der Wissenschaft in das Förderverfahren durch die Beteiligung des Beirats führt in der Folge zu einer Dominanz des wissenschaftlichen Steuerungseinflusses. Dem Auftraggeber verbleibt im wesentlichen seine Finanzierungshoheit als nur 
begrenzt wirksames Steuerungsinstrument, der Projektgruppe die Forschungsverwaltung, Organisation und Durchführung des Förderverfahrens.

Die anfängliche Programmsteuerungsdominanz der forschungspolitischen Akteure Umweltressort und Projektgruppe, welche im wesentlichen durch Umgehen des wissenschaftlichen Beratungsgremiums erfolgt, wird vom Beirat selbst lange, wenn auch unter Kritik und Protest, geduldet. Sie wird spätestens dann nicht mehr akzeptiert, als sich am Umweltressort Fördermittelknappheiten bemerkbar machen und forschungspolitisch-monetär begründete Entscheidungen fachlich begründete Förderempfehlungen deutlich zu dominieren drohen, wovon zudem Mitglieder des Beirats selbst betroffen sind.

Die Umgehung des wissenschaftlichen Beratungsgremiums kann langfristig nicht durchgehalten werden, sollen die PBWU-Vertragsgrundlagen erfüllt und - neben der rein fachlichen Begutachtung - eine wissenschaftliche Zuarbeit (Kooptation) und Abstimmung auf programmatischer Ebene stattfinden [s.a. BRAUN 1997, S. 350].

Aufgrund der mit Rücktrittsandrohungen vorgebrachten Kritik des Beirats wird diesem die stärkere, ja dominierende Beteiligung am Förderverfahren zulasten der PBWU-Kompetenzen eingeräumt. Entsprechend dem Machtzuwachs des Beirats wird die Projektgruppe personell und funktional neu geordnet und damit reduziert.

Während der Aushandlungsphase der Verfahrensneuordnung entsprechend den Vorstellungen des Wissenschaftlichen Beirats kommt es übergangsweise allerdings zu einer Durchmischung der Dominanzverhältnisse. Die PBWU führt unter neuer personeller Leitung das Förderverfahren zwar weiterhin federführend durch, beteiligt den Beirat aber bei allen Verfahrensschritten. Dadurch kann sie sich zumindest eine bislang fehlende Akzeptanz- und Vertrauensbasis erarbeiten und gewisse Kompetenzen im Förderverfahren erhalten, die ihr eine forschungspolitische Mitsprache bei der Abgabe von Förderempfehlungen zu Forschungsanträgen beläßt.

Dennoch geht mit der Verschiebung der Kompetenzen von der Projektgruppe auf den Beirat die Steuerungsdominanz im PBWU-Projekt im weiteren Verlauf der Programmimplementation an die Wissenschaft über. Das Förderverfahren ist nun geprägt von einer Vorselektion der Forschungsanträge durch den Wissenschaftlichen Beirat, den Einsatz externer Fachgutachter wie bisher und auf den Gutachtervoten basierenden Förderempfehlungen des Beirats für Förderentscheidungen durch den politischen Auftraggeber. Zudem nehmen dieser und die weiteren beteiligten, politischen Ressorts im Projektrat generell nur wenig forschungspolitischen Einfluß auf das Förderverfahren. Der Projektgruppe obliegt nach wie vor die konkrete Auswahl der Fachgutachter, wenn auch formal in Abstimmung mit dem Beirat.

Während in der politisch dominierten Phase der Programmsteuerung lediglich die fachliche Begutachtung an die Wissenschaft in Form der Fachgutachtung übergeben wurde, aber alle vorund nachgeschalteten Selektions-, Empfehlungs- und Entscheidungsprozesse innerhalb politischer Gremien bzw. durch den Auftraggeber und die Projektgruppe stattfanden, liegt nun das gesamte Förderverfahren mit Ausnahme der eigentlichen Förderentscheidung in Händen der Wissenschaft. Die vorher von der PBWU nutzbaren Steuerungseinflüsse bei der Vorbewertung der Anträge, Interpretation oder Gewichtung der Gutachten, Förderempfehlung kontrovers 
beurteilter Anträge oder Prioritätensetzung gleichwertig positiv bewerteter Anträge, gehen nun an den Wissenschaftlichen Beirat über [s.a. STEUER 1997, S. 50].

Weiterhin beteiligen sich einige Beiratsmitglieder selbst oder über angehörige Arbeitsgruppen an der Forschung im PBWU-Projekt. Aufgrund der Beiratszugehörigkeit können sie sich trotz unterstellter neutraler Begutachtung durch externe Gutachter und persönlichem Ausschluß vom Entscheidungsverfahren dennoch bereits ab Beginn der Programmimplementation informaler Vorteile (z.B. hohen Informationsstandes über Förderrahmenbedingungen zum Projekt) bedienen. Dies erleichtert den Zugang zum Programm und verbessert die Erfolgsaussichten für eingereichte Anträge. Der hohe Anteil an Forschungsvorhaben aus dem Personenkreis des Beirats und dessen Umgebung insbesondere in der fortgeschrittenen Implementationsphase [STEUER 1997, S. 61-66] ergänzt damit den dominierenden Steuerungseinfluß des wissenschaftlichen Beratungsgremiums im Förderverfahren um eine weitere Komponente, nämlich die erhebliche Beteiligung am und damit Gestaltung des Forschungsprozesses selbst.

Insgesamt trägt die Programmimplementation ab der Verfahrensneuregelung zugunsten der Wissenschaft und zu Lasten der koordinierenden Projektgruppe verstärkt Züge eines wissenschaftsfreundlichen bottom up-Ansatzes der Forschungssteuerung. Die wissenschaftliche Relevanz des einzelnen Forschungsantrags gerät dabei stärker in den Mittelpunkt der Bewertung und Selektion als dessen Beitrag zum problemlösungsorientierten Gesamtprogramm [HILL \& RIESER 1983, S. 171]. Der nach wie vor gültige Forschungsrahmen kann als politisches Steuerungsinstrument nicht dienen [s. a. BRAUN 1997, S. 47ff]. Er läßt in einer Art „Alibifunktion“ [HILL \& RIESER 1983, S. 349] weitgehende fachlich-thematische Steuerungs- und Ausgestaltungsmöglichkeiten durch die Wissenschaft im Verlauf der Implementationsphase zu und fördert damit zwangsläufig die Ausweitung politisch unerwünschter Grundlagenforschung.

Die forschungspolitische Steuerung beschränkt sich demgegenüber auf eine Entscheidungshoheit über die Vergabe der Fördermittel. Dieser Steuerungsaspekt ist jedoch nur wenig wirksam, weil der Wissenschaft vorab die fachliche Vorselektion (Begutachtung) eingeräumt worden war, innerhalb deren Rahmen nun die Politik durch Vorhabenfinanzierung auswählen kann. Ein derart abgestuftes Verfahren stellt einen für die Wissenschaft starken Steuerungsvorteil dar. Konkret ist somit allenfalls die finanzpolitisch begründete Ablehnung wissenschaftlich befürworteter Forschungsarbeiten legitimiert, nicht jedoch die finanzpolitische Förderempfehlung wissenschaftlich nicht empfohlener Forschung.

Für die politisch zielorientierte Durchführung eines problemorientierten Forschungsprogrammes ist die Steuerungsdominanz wissenschaftlicher Gremien hinderlich. Die strenge Verfolgung politischer Programmvorgaben und damit auch die Programmkoordination werden beeinträchtigt. Die wissenschaftliche Legitimation sowie die fachliche Qualität der durchgeführten Forschung profitieren hiervon jedoch. Auch bewirkt das hohe Ansehen und die herausragende Stellung sowohl der Fachgutachter, als auch der Beiratsmitglieder, einen positiven Effekt der Kenntnisverbreitung über das Projekt und der Projektwerbung im Wissenschaftssystem [s.a. BRAUN 1997, S. 348ff]. 


\subsection{Externe Fachbegutachtung zur Sicherung der Forschungsqualität}

- Der Einsatz des Instrumentariums fachlichen Qualitätsbeurteilung der Forschung durch externe Gutachter trägt dazu bei, hochqualitative, effiziente Forschung zu sichern und das Forschungsprojekt insgesamt zu legitimieren. Gleichzeitig geringe forschungspolitische Steuerungseinflüsse führen insgesamt zur Dominanz wissenschaftlicher Akteure bei der Erarbeitung von Förderentscheidungen. Der hohe Anteil an Fortsetzungsvorhaben bindet das Programm an erste Entscheidungen und behindert in der Folge Neuorientierung und Ideenvielfalt.

Basis für die Förderentscheidungen im Projekt ist die Bewertung der Forschungsanträge durch externe Fachgutachter im Rahmen mündlicher oder schriftlicher Verfahren. Dabei stehen nach den Ergebnissen das Gutachtenanalyse zum PBWU-Projekt fachliche (inhaltlich-thematische) Kriterien bei der Beurteilung im Vordergrund [STEUER 1997 S. 30]. Die externe Begutachtung kann daher als wirksames fachliches Instrumentarium zur Qualitätssicherung der Forschung gesehen werden. Sie trägt dazu bei, die Erfolgswahrscheinlichkeit und Effektivität des Projektes zu erhöhen und es in Wissenschaft und Politik zu legitimieren [BRAun 1997, S. 339, 344]. Auch die Bewertung des US-amerikanischen Forschungsprogramms zu säurehaltigen Niederschlägen in den 80er und 90er Jahren weist dem Begutachtungsverfahren im Forschungsprozeß eine bedeutende Rolle zu [NATIONAL ACIDIFICATION PRECIPITATION ProgRAM (NAPAP) 1991, S. 26/27].

Mit der fachlichen Begutachtung sind jedoch verschiedene Einflußnahme-Potentiale außerhalb der rein fachlichen Forschungsbewertung verbunden. Diese werden von den politischen und wissenschaftlichen Akteuren im PBWU-Projekt in unterschiedlichen Umfang und zu unterschiedlichen Projektphasen zur interessengeleiteten Forschungssteuerung genutzt. Im theorieorientierten Zwischenergebnis zum Förderverfahren im PBWU-Projekt wurde auf das Begutachtungsverfahren bereits ausführlich eingegangen (s. Kapitel 5.3.5), so daß hier nur einige wesentliche, steuerungsrelevante Aspekte in Hinblick auf das Kräftespiel zwischen Politik und Wissenschaft herausgegriffen werden.

Der Einsatz der Grundlagenforschung generell zugeneigter DFG- oder DFG-naher Gutachter im PBWU-Projekt gewährleistet einen strengen Beurteilungsmaßstab und hohen Qualitätsstandard der Forschung. Diese im politischen Projektrat fixierte und von Mitgliedern des wissenschaftlichen Beratungsgremiums ebenfalls empfohlene Rahmenvorgabe begünstigt die Durchführung grundlagenorientierter Forschung. In der vom Auftraggeber beabsichtigten, problemorientiert-angewandter Forschung beeinträchtigt dieser Typ der DFG-Begutachtung jedoch die Effizienz des Forschungserfolges. Der Anteil an grundlagenorientierter Forschung im PBWUProjekt ist mit nahezu 2/3 aller Forschungsarbeiten erheblich und dient damit nicht unbedingt der Zielerfüllung des Programms. Unterstützt wird diese Tendenz insbesondere durch das wenig stringente und die Forscherfreiheit berücksichtigende Rahmenprogramm als generell gering wirksame Eintrittshürde zum Waldschadensforschungsprogramm.

Der politische Auftrag- und Finanzgeber akzeptiert bzw. toleriert den hohen Anteil an Grundlagenforschung. Anfangs kann hierfür der hohe politische Handlungsdruck, das Programm bei ausreichenden Fördermitteln rasch zu implementieren, geltend gemacht werden. In der Folge 
kann dies als Zugeständnis gegenüber dem verfahrensmäßig zeitweilig übergangenen Wissenschaftlichen Beirats verstanden werden, der zudem die Steuerungsdominanz im Förderverfahren übernimmt. Angemerkt werden muß, daß sich die Durchführung von zumindest anteilmäBiger Grundlagenforschung auch in einem anwendungsorientierten Forschungsprogramm allein mit fehlenden wissenschaftlichen Grundlagen legitimieren läßt, wie sie im Falle der Waldschäden in erheblichem Umfang bei vielen Forschungsdisziplinen gegeben waren.

Die bereits geschilderten, wechselhaften Dominanzverhältnisse im PBWU-Projekt zwischen politischen und wissenschaftlichen Akteuren finden in der Beteiligung am Förderverfahren maßgeblich ihren Niederschlag. So ist die erste Phase der Programmimplementation unter Dominanz der politischen Akteure konsequenterweise geprägt durch die aktive Beteiligung der Politik am Begutachtungsverfahren (Vorbegutachtungen durch die Projektgruppe, Teilnahme am Begutachtungspanel, Auswertung und Interpretation der Gutachtervoten, Verfassung von Förderempfehlungen). Mit der Stärkung des Beiratsgremiums der PBWU treten derartige politische Steuerungseinflußmöglichkeiten jedoch zurück und werden zumindest zum Teil auf den Beirat verlagert (Sichtung der Anträge bzgl. einer Begutachtung, Bewertung der Gutachtervoten und Abgabe von Förderempfehlungen). Politische Steuerung reduziert sich nunmehr weitgehend auf die Finanzierungshoheit des Umweltressorts, deren Grenzen bereits aufgezeigt wurden, sowie auf das ohnehin nur schwach wirksame Filter-Kriterium der Einpassung des Forschungsbeitrages in das Rahmenprogramm.

Die Auswahl der Gutachter obliegt über das Gesamtprojekt hinweg im wesentlichen und abgesehen von der generellen Vorgabe, grundsätzlich DFG-Gutachter zu beteiligen, der Projektgruppe. Ihr kann hierbei allein aufgrund ihres beschränkten Einblicks in die soziale Welt der scientific community weitestgehend fachlich geleitetes Interesse unterstellt werden, so daß sich hieraus kein wesentlicher Steuerungseinfluß ableiten läßt. Der Interpretationsspielraum für die der Begutachtung nachgeschalteten Akteure ist gering. Sowohl die Phase politischer wie auch später - wissenschaftlicher Dominanz sind nach der Analyse von STEUER [1997, S. 49] durch eine insgesamt hohe Befolgungsrate der Gutachtervoten hinsichtlich Förderempfehlungen und entscheidungen gekennzeichnet. Abweichungen ergeben sich überwiegend im uneinheitlich bzw. kontrovers bewerteten Sektor.

Letztlich überlassen die politischen Akteure die weitere fachlich-inhaltliche Ausgestaltung des PBWU-Projektes weitgehend der Wissenschaft. Dies erfolgt allerdings erst dann, als unter politischer Verfahrensdominanz das Forschungsprogramm bereits in Grundzügen implementiert ist. Mit dem Einsatz des Instrumentes der fachlichen Begutachtung und weiteren - allerdings nur peripheren - Steuerungseinflüssen durch das Beiratsgremium akzeptiert und unterwirft sich der politische Auftraggeber weitgehend der Selbstverwaltung und Autonomie der Wissenschaft. Das gutachterzentrierte Förderverfahren unterstreicht gerade durch seine Merkmale der Verteilung von Reputation, Selektion geeigneter Forscher und Optimierung der Forschung durch thematische, personelle, institutionelle und finanzielle Steuerung [BRAUN 1997, S. 344345] den fachlich-wissenschaftlichen gegenüber dem politisch-problemorientierten Einfluß im PBWU-Projekt. 
Die Bindungswirkung erster Förderentscheidungen zu Beginn der Programmimplementation prägt allerdings weitgehend den Programmverlauf. Der hohe Anteil an Fortsetzungsvorhaben und das institutionell begrenzte Forscherkollektiv mit maßgeblicher Beteiligung von Mitgliedern des Wissenschaftlichen Geratungsgremiums an der Forschung im PBWU-Projekt lassen insgesamt wenig Raum für fachlich-inhaltliche sowie institutionelle Neuorientierungen.

\subsection{Koordinierungsstelle zwischen Programmsteuerung und Kooperation}

- Der Anspruch der intermediären Projektgruppe auf Programmsteuerung ist insbesondere aufgrund der starken Stellung der Wissenschaft unrealistisch und trotz Einsatzes formaler wie informaler Machtmittel auf Dauer nicht haltbar. Erst die in der späteren Implementationsphase verfolgte Strategie, die jeweiligen Kompetenzen weitgehend zu belassen und vertrauensvolle und freiwillige Kooperation von Wissenschaft und Politik herzustellen, schafft ein funktionierendes Verhandlungssystem.

Bei der Frage nach der politischen Steuerung problemorientierter, multidisziplinärer Forschung spielt die an der Schnittstelle zwischen Politik und Wissenschaft eigens installierte, intermediäre Projektgruppe eine zentrale Rolle. Der Auftrag der Forschungskoordination wird von den politischen Akteuren Umweltressort und Projektgruppe zu Zeiten der Gründung des Projektes synonym zum Anspruch auf politische wie wissenschaftliche Steuerung verstanden und zumindest anfangs dementsprechend umgesetzt. Diese Handlungsweise folgt einem als ,,steuerungsoptimistisch“ zu bezeichnenden Ansatz politischer Steuerung der Wissenschaft. Er unterstellt, daß zentral koordinierte Planungsverfahren eigene forschungspolitische Ziele umsetzen können [BRAUN 1997, S. 47 ff, S. 316].

Mit diesem Steuerungsanspruch politischer Akteure unter Einsatz einer intermediären Organisationseinheit wird tief in originär wissenschaftlich Intereressenpositionen eingegriffen. Das steuerungspolitische Kräftespiel zwischen Wissenschaft und Politik wird damit erheblich einseitig zugunsten der Politik gestärkt. Von der Wissenschaft wird dieser Steuerungsanspruch nach deren Selbstverständnis bzgl. Wissensproduktion und Forschungsfreiheit und dem gewohnten wie erwarteten Typ staatlicher Forschungsförderung jedoch abgelehnt [s.a. MARKL 1989, S. 31-52; DEUTSCHE FORSCHUNGSGEMEINSCHAFT (DFG) 1996].

Daher richtet sich bereits frühzeitig erhebliche Kritik nicht nur seitens der Wissenschaft, sondern auch seitens benachbarter politischer Ressorts und der GSF-Geschäftsführung gegen die Aufgabenstellung der Projektgruppe in dieser Form. Die Rückendeckung durch das auftraggebende Umweltressort, der politische Handlungsdruck, die Strategie, das Beratungsgremium zu übergehen und auch selbst fachlich in das Begutachtungsverfahren einzugreifen, ermöglichen es der Projektgruppe jedoch, gegen bestehende Widerstände das Projekt in Hinblick auf die politische Zielsetzung rasch zu etablieren. Die in der Initialphase des Projektes unter politischer Steuerungsdominanz getroffenen Festlegungen in forschungsthematischer, -standörtlicher, institutionell-personeller sowie finanzieller Hinsicht reichen aufgrund ihrer Bindungswirkung noch weit über diese Phase hinaus und beschränken den anschließend dominierenden wissenschaftlichen Steuerungseinfluß erheblich. 
Der direkte Eingriff der Politik durch die Koordinationsstelle in die Domäne der Wissenschaft stößt auf harte Ablehnung. Nach für das Gesamtprojekt existentiellen Auseinandersetzungen um die Zusammenarbeit zwischen Politik und Wissenschaft muß die in Anspruch genommene Aufgabe der Forschungssteuerung durch die Projektgruppe fallen gelassen und die offizielle Funktion der Projektkoordinierung formal auf die überwiegend organisatorische Projektbetreuung und Durchführung des Förderverfahrens reduziert werden.

Mit der funktionalen und personellen Neuorganisation der Projektgruppe ist auch ein Wechsel im strategischen Verhalten der Projektgruppe verbunden. Die PBWU-Gremien werden nicht nur formal in alle Teilbereiche des Förderverfahrens umfassend mit eingebunden. Die Projektgruppe versucht erfolgreich, die bisher entbehrten, informalen Kooperationsvoraussetzungen der Akzeptanz- und Vertrauensbasis bei dem wissenschaftlichen Beratungsgremium zu schaffen. Auch die Kompetenz und Anerkennung auf dem den modifizierten Aufgaben entsprechend reduzierten fachlichen Niveau und im organisatorischen Bereich kann nun leichter realisiert werden.

Mit der Rücknahme politischer Steuerung, der damit korrespondierenden Stärkung wissenschaftlicher Einflußnahme auf den Forschungsprozess und nicht zuletzt der Durchführung vertrauensbildender Maßnahmen sind die wesentlichen Kooperationsgrundlagen zwischen Politik und Wissenschaft im PBWU-Projekt neu geschaffen. Über die Nichteinmischung in das Begutachtungsverfahren und die Autoritätszuweisung an den Beirat respektiert die Politik die Selbstorganisation und das Autonomiestreben der Wissenschaft. Die Wissenschaft hingegen akzeptiert die Verfahrens- und Finanzierungshoheit und die anwendungsorientierten Steuerungsambitionen der Politik über die Projektgruppe. Die gegenseitige Akzeptanz und die Kooperationsfähigkeit manifestieren sich im weiteren Verfahrensverlauf zur Forschungsförderung und im Prozeß der späteren Neuformulierung des Forschungskonzeptes. Mit der Akzeptanz der Projektgruppe als Schnittstelle kann der „Antagonismus“ von wissenschaftlichen und politischen Interessen nicht überwunden, aber in ein funktionierendes Verhandlungssystem eingebettet werden [s.a. BRAUN 1997, S. 181, 383].

\subsection{Vorhabenbetreuung statt Forschungskoordination}

- Intensive, fachlich kompetente und integrative Forschungskoordination kann von der Projektgruppe nicht ausreichend geleistet werden. Strukturell und personell bedingt zeigt sie ihre Stärke in überwiegend organisatorischen Koordinationsmaßnahmen und Serviceleistungen zur Durchführung und Betreuung von Programm und Einzelvorhaben und der Zusammenstellung der Forschungsergebnisse für die Politik.

Die problemorientierte Bearbeitung des vielschichtigen Problems Waldschäden kann nur mit Hilfe Forschungsdisziplinen-übergreifender Forschungsansätze erfolgversprechend geleistet werden. Dies erfordert erheblichen Koordinierungsaufwand in der Phase der Programmbildung, Programmdurchführung und letztlich bei der integrierenden, synthetischen Aus- und Bewertung der Forschungsergebnisse. Die Forschungspolicy der bayerischen Staatsregierung, die Waldschadensforschung durch finanzielle Förderung zu verstärken, bezog den Aspekt der Forschungskoordination mit Gründung der PBWU mit ein. Die gezielte Etablierung der Pro- 
jektgruppe an einer Großforschungseinrichtung, ihre Besetzung mit mehreren Naturwissenschaftlern, die Begleitung durch politische und wissenschaftliche Gremien soll die erforderliche Koordination des Projektes sowie der Forschung gewährleisten.

Je nach dem Anspruch an die Intensität und fachliche wie organisatorische Dimension der Forschungskoordination wird der Koordinierungsauftrag im Projekt in unterschiedlichem Maße erfüllt. So erstellt die PBWU im Zuge der Programmbildung zwar ein in sich schlüssiges, thematisch strukturiertes und aufeinander abgestimmtes Rahmenprogramm, welches die forschungspolitisch erwünschten Eckpfeiler berücksichtigt und von fachlicher Neutralität geprägt ist. Es focussiert jedoch mehr auf die im einzelnen aufgelisteten Forschungsthemen als auf deren interdisziplinäre Verknüpfung im Gesamtrahmen des Verbundprojektes. Es ist daher von einem detaillierten und als Koordinierungsinstrument einzusetzenden, verbindlichen Forschungsnetzplan weit entfernt.

Die Ausarbeitung einer derartigen Forschungsplanung als Koordinierungsgrundlage hätte der intensiven Zusammenarbeit der Forschungspolitik mit der Wissenschaft in Form des Beirats und der am Projekt zu beteiligenden Forschergruppen bedurft. Aufgrund des hohen politischen Handlungsdrucks und Durchsetzungswillens sowie des Selbstverständnisses der Projektgruppe wird die Wissenschaft nur wenig für konzeptionelle Arbeiten, fachliche Abstimmungen und Vorstudien in der Planungsphase genutzt. Damit entsteht letztlich ein Programm, das zwar auf die gesamte Projektlaufzeit den gültigen Forschungsrahmen darstellt und im Grundsatz über die politische Legitimation der zur Förderung anstehenden Forschungsbeiträge entscheidet. In seiner thematischen Breite und Offenheit gegenüber weiteren Forschungsaspekten ist es als Koordinationsinstrument für fachbereichsübergreifende Forschung und die Abstimmung der einzelnen Beiträge allerdings nur wenig geeignet.

Das Rahmenprogramm ermöglicht einen leichten Einstieg für nahezu alle am Thema orientierten Antragsteller. Die Einpassung der Anträge als Voraussetzung für eine Beteiligung am Projekt ist leicht herzustellen. Folge ist, daß sich das Gesamtprogramm aus einer Vielzahl von Forschungsvorhaben zusammensetzt, die aufgrund der vorausgegangenen fachlichen, jedoch in der Regel disziplinären Begutachtung hohe Forschungsqualität besitzen, aber inhaltlich sowie zeitlich weitgehend unabhängig voneinander konzipiert, umgesetzt und ausgewertet werden. Sie lassen sich zwar als Einzelbausteine verschiedenen Themenbereichen oder regionalen Forschungsstandorten zuordnen und formal konzeptionell verknüpfen. Dem Anspruch an koordinierte, interdisziplinäre Forschung wird das Vorhabenmosaik nicht gerecht. Die Forschungsarbeiten sowie die erzielten Einzelergebnisse sind inhaltlich nicht miteinander verflochten. Entsprechend unzureichend ist daher auch die integrale, kontextuelle Gesamtauswertung für regionale oder thematische Forschungsschwerpunkte.

Die Koordination der Forschung im Zuge der Programmimplementation durch die Projektgruppe betrifft die technische Durchführung der Forschungsvorhaben. Die Bereitstellung infrastruktureller, technischer Leistungen wie zentrales Analyselabor, zentrale Datenhaltung und Datentransfer an beteiligte Forschergruppen, die Organisation und Durchführung von gemeinsamen Veranstaltungen wie Probennahmen, Arbeitsbesprechungen und Statusseminaren, weitere Informationsvermittlungen oder die z.T. individuelle Betreuung bei der Etablierung, Ab- 
wicklung und Beendigung einzelner Vorhaben stellen zwar wertvolle Beiträge einer Durchführungskoordination dar. Eine fachübergreifende Verknüpfung der Forschung in Planungs-, Durchführungs- und Auswertungsstadium wird damit jedoch nicht erbracht. Zudem erschweren ungünstige forschungspolitische Rahmenbedingungen wie die Unsicherheit und Diskontinuität in der Bereitstellung der Fördermittel, organisatorisch, technisch wie personell bedingte Verzögerungen bei der Bewilligung sowie die mangelnde zeitliche Gleichschaltung fachlich benachbarter Vorhaben die Koordination.

Die Projektgruppe ist mit einer intensiven inhaltlich-fachlichen Koordination, der Synthese sowie dem Assessment der Forschungsarbeiten überfordert, der fachlich kompetente Wissenschaftliche Beirat hingegen vertraglich nur für Grundsatz- oder konzeptionelle Entscheidungen zuständig. Letztlich bleibt bei der gegebenen Struktur die fachliche Koordination den Forschern weitgehend selbst überlassen. Deren Koordinationsneigung bemißt sich jedoch vornehmlich an dem Nutzen für die eigene wissenschaftliche Arbeit und weniger an übergeordneten Koordinationszielen, die zudem forschungspolitisch-problemorientiert ausgerichtet ist. Sie wird aufgrund dieser Eigeninteressen nur dann hoch sein, wenn die Koordinierungseffekte zur eigenen Forschungsfragestellung beitragen oder aus gemeinsamer, koordinierter Vorgehensweise ein sonstiger wissenschaftlicher oder Renommee-betreffender Nutzen gezogen werden kann [s.a. STARK 1991, S. 294]

Die „Forschergruppe“ für den alpinen Standort Wank belegt derartige Interessenstrukturen. Der über die Leistung der Durchführungskoordination durch die Projektgruppe hinausgehende Bedarf an fachlicher Koordinierung der beteiligten Arbeitsgruppen wird von maßgeblichen Forschern vor Ort gefordert. Er wird in der Bildung eines hochrangig besetzten Steuerungskomittees und in einer entsprechenden Neukonzeption der Forschung niedergelegt und zur Umsetzung beantragt. Die Forscher versprechen sich neben dem Erkenntniszugewinn über die fachliche Koordination einen erheblichen fachlichen und forschungspolitischen Steuerungseinfluß auf den Forschungsverbund insgesamt. Die forschungspolitisch befürchtete Steuerungsdominanz der Forscher und Überschneidungen mit den Koordinationsfunktionen der bereits bestehenden Projektgruppe führen neben fachlich-inhaltlicher Kritik letztlich dazu, daß die beantragte Koordinationsstruktur sowie das fachliche Konzept nicht akzeptiert werden.

Zumindest bei der fachlichen Auswertung der geleisteten Forschung kann die Projektgruppe ihre intermediäre Kompetenz sowie ihre fachlich-wissenschaftliche Neutralität in Hinblick auf eine politisch-problemorientierte Evaluierung der Forschung sinnvoll einsetzen. In den jährlichen Ergebnisberichten zum Gesamtprojekt sowie den Stellungnahmen zu Forschungsberichten aus dem Projekt gegenüber dem politischen Auftraggeber vermag sie die wissenschaftlichen Ergebnisse zusammenzufassen und auf die potentiell politisch relevanten Aussagen hin zu übersetzen. Dies erinnert in der Aufgabenstellung an das US-amerikanische Forschungsprogramm NAPAP [1991]. Die Zusammenfassung der Ergebnisse aus den verschiedenen Forschungsdisziplinen und deren kritische Beurteilung in Hinblick auf die problemorientierte Zielsetzung des Forschungsprogramms kann als eine wesentliche Leistung der Projektgruppe gesehen werden, auch wenn sie belastet ist mit vorangegangenen Defiziten fachlicher Koordinierung und Abstimmung während der Planungs-, Implementations- und Evaluationsphase. 


\subsection{Mangelnde Erfolgsbeurteilung der Forschungspolicy}

- Eine adäquate Präsentation und Erfolgsbeurteilung der Forschungsergebnisse des PBWUProjektes in Politik und Wissenschaft unterbleibt. Aus Sicht der Politik ist dies zur Projektlegitimation, die sich an den für Umweltpolitik relevanten Ergebnissen orientieren will, nicht erforderlich und erleichtert dessen Terminierung. Über die politische Zielsetzung des Projektes hinausgehend muß der Erfolg jedoch im Gesamtrahmen der Wald- und Umweltforschung gesehen werden.

Eine weiterführende Evaluation des Projektes mit dem Ziel, dessen Erfolg zu messen und auf dieser Grundlage über Fortführung oder Terminierung zu entscheiden, findet nicht statt. Dies unterstreicht die politische - gegenüber einer fachlich-wissenschaftlichen - Begründung für die Beendigung des Projektes. Gleichermaßen unterbleibt eine adäquate fachliche Ergebnisauswertung und -dokumentation, die über den knapp gehaltenen, zusammenfassenden und für die Politik ausgelegten Schlußbericht der Projektgruppe hinausginge. Das Projekt hält aus politischen Gründen weder die Durchführung eines abschließenden Statuskolloquiums noch die Erstellung eines wissenschaftlichen Forschungsberichtes für erforderlich.

Betroffen ist davon nicht nur die Politik, die sich mit nach wie vor relativ allgemeinen, unspezifizierten Formulierungen und verbleibenden Vermutungen zur Waldschadensursache sowie zur Ableitung von Gegenmaßnahmen zufrieden gibt bzw. zwangsläufig geben muß. Auch der scientific community bleiben das Ergebnis der PBWU-Waldschadensforschung als Gesamtprojekt sowie die vielfältigen, hinsichtlich eines Gesamtbildes zum Teil noch als diffus zu bezeichnenden Detailergebnisse zum Erkenntnisfortschritt weitgehend vorenthalten. Einige Publikationen zu ausgewählten Themen, die im Eigeninteresse einzelner Wissenschaftler entstehen, schränken diesen Befund nur marginal ein.

Die fehlende Bekanntgabe und Umsetzung der - positiven wie negativen - Forschungsergebnisse in Wissenschaft und Politik sind generell nicht ungewöhnlich. Auch für das US-amerikanische Forschungsprogramm NAPAP wird festgestellt, daß die Programmergebnisse nicht für die Formulierung politischer Maßnahmen herangezogen wurden [VoGT et al. 1996, S. 259]. Dies bedeutet einen erheblichen Effektivitätsverlust für das Projekt und die darin geleistete Forschungsarbeit. Das Projekt entbehrt einer fachlichen ex post-Legitimation. Die knappe und nicht aus den Ergebnissen des Projektes abgeleitete Argumentation der Politik, wonach die erzielten Ergebnisse für die Ableitung politischer Maßnahmen ausreichten, dienen zur Erfolgsbewertung, Projektlegitimation und Terminierung gleichermaßen. Gegenüber den nach wie vor noch offenen Forschungsfragen erleichtert eine derartige politische Argumentation die Terminierung des Projektes. Sie läßt aber die fachliche Evaluierung offen.

Unabhängig von den Beiträgen zur politischen bzw. wissenschaftlichen Klärung des Problems hat insbesondere die Wissenschaft von dem PBWU-Projekt profitiert. Durch die umfangreiche Fördermittelbereitstellung und die geringen fachlichen Vorgaben in Form des Rahmenprogramms konnten sich zahlreiche Forschungsrichtungen, -institutionen und Forscherpersonen an der Bearbeitung des Themas Waldschäden beteiligen und bestehende Forschungskapazitäten und -strukturen in materieller, institutioneller wie personeller Hinsicht ausbauen. Das PBWUProjekt konnte dazu genutzt werden, nicht nur Beiträge zu einer politisch beabsichtigten Pro- 
grammforschung zu leisten, sondern auch den Forschungskenntnisstand einschließlich methodischer Entwicklungen in den entsprechenden Fachgebieten wesentlich voranzutreiben.

Neben der verstärkten wissenschaftlichen Erkenntnisproduktion konnten die bestehenden Kompetenzen und Kapazitäten anschließend andere Forschungsthemen, wie z.B. Ökosystemforschung oder Klimaforschung, aufgreifen. Damit geht der wissenschaftliche Erfolg des Projektes weit über die eigentlich beabsichtigte Themenbearbeitung hinaus. Auch der politische Erfolg ließe sich somit letztlich nur im Gesamtkontext der gesamten Umweltforschung bewerten. 


\title{
7 Exkurs: Empfehlungen zu Durchführung und Koordination von Forschungsprogrammen
}

\begin{abstract}
Abschließend sollen aufgrund der in der Studie erzielten Analyseergebnisse praxistaugliche Empfehlungen für das Forschungsmanagement gegeben werden. Die ausgewählten Handlungsstrategien beziehen sich auf ein ausgewähltes Idealmodell, das sich an Zielen orientiert, wie sie häufig von der Politik und von der Forschung vorgeschlagen werden. Soweit sich Forschungsmanager mit den Zielen dieses Idealmodells identifizieren, können sie sich unmittelbar an den Empfehlungen orientieren.
\end{abstract}

\subsection{Frei gewähltes Idealmodell}

Vor dem Hintergrund des in der Studie bearbeiteten Fallbeispiels wird als Idealmodell ein staatlich finanziertes, auf angewandt-problemorientierte Forschung ausgerichtetes Forschungsprogramm angenommen. Dieses soll der Bearbeitung eines politisch relevanten Problems dienen und hierfür Lösungsbeiträge liefern. Die Komplexität des Problems erfordert inhaltlich und finanziell umfangreiche, zumindest mittelfristig anzulegende Forschungsaktivitäten, die nur im Rahmen eines multi- bzw. interdisziplinären Forschungsprogramms erfolgversprechend bearbeitet werden können. Zur Abwicklung des Projektes besteht eine zwischen Politik bzw. Finanzgeber und Forschung intermediär angesiedelte Koordinierungseinheit.

Das Idealmodell für angewandte, problemorientierte Forschung läßt sich im einzelnen folgendermaßen kennzeichnen:

- Die Politik initiiert und finanziert über ein Förderprogramm Forschung, deren Ergebnisse die wissenschaftlichen Grundlagen zur Lösung des politischen Problems darstellen sollen.

- Grundlage des Förderprogramms ist ein Forschungsprogramm-Rahmen, der den politischen Zielsetzungen entspricht, fachlich detailliert und wissenschaftlich fundiert ist.

- Das Programm bevorzugt entsprechend der politischen Zielsetzung angewandt-problemorientierte Forschungsbeiträge, die einen Beitrag zur Zielerreichung des Programms erwarten lassen.

- Ein geeignetes Begutachtungsverfahren zur Antragsselektion sichert die wissenschaftliche Qualität der Forschung.

- Die Wissenschaft füllt mit der Bearbeitung thematisch geeigneter Einzel-Forschungsbeiträge den Programmrahmen umfassend aus. Es findet eine enge Vernetzung und Kooperation der Forscher innerhalb und zwischen den beteiligten Fachdisziplinen statt (Interdisziplinarität).

- Die Programmabwicklung, -koordination und Verfahrensdurchführung erfolgt durch eine intermediäre Koordinierungsstelle. 
- Die wissenschaftliche, programmatische und organisatorische Koordination des Projektes soll die zielorientierte Programmeffizienz und den Programmerfolg sichern.

- Die Beendigung des Forschungsprogramms erfolgt nach Evaluierung des Gesamt-Forschungsergebnisses bei Erreichung der Programmzielsetzung.

\subsection{Empfehlungen zur Programmumsetzung}

Vor dem Hintergrund der Ergebnisse der vorliegenden Studie können die im Folgenden dargestellten Empfehlungen angeboten werden, um ein solches Modell in die Praxis umzusetzen.

\subsubsection{Politische Gestaltung zeitgerechter Forschung}

Die Anfangsphase eines politischen Problems ist durch hohen politischen Handlungsdruck und dessen treibende Kräfte gekennzeichnet. In Hinblick auf die Durchführung von Forschungsmaßnahmen führt der Handlungsdruck häufig dazu, daß rasch, jedoch kurzfristig und wissenschaftlich nicht adäquat gehandelt wird. Die Gefahr besteht, daß fachlich unausgereifte Forschungsprogramme über erhebliche Fördermittel nur kurzfristig durchgeführt und instabile, vorübergehende Forschungsstrukturen geschaffen werden, die bei nachlassendem politischem Handlungsdruck wieder aufgelöst werden, noch bevor sie zu den möglichen Ergebnissen gelangt sind. Diese Fehlsteuerung führt zu ineffizienter Forschung (s.a. „Effizienzparadoxon" der Forschung in der vorliegenden Studie, Kapitel 5.4.6.3 und 6.4).

In Abbildung 8 ist die gegenläufige Entwicklung von Fördermitteln und Programm- bzw. Forschungsqualität über die Zeit aufgetragen. Sie bringt schematisch zum Ausdruck, daß in der Initialphase hoher Fördermitteleinsatz zur Finanzierung vergleichsweise qualitativ geringwertiger Forschungsprogramme dient. Der im Projektverlauf üblicherweise zunehmenden Qualität der Forschung stehen aufgrund sinkenden politischen Handlungsdruckes in den Folgephasen nur mehr Fördermittel in geringerem Umfang zur Verfügung. Gerade im mittleren Projektverlauf erscheint daher eine Finanzierungsbrücke wichtig. Detailaspekte zur zeitlichen Dynamik der Forschung, wie bspw. abnehmender Grenznutzen der Forschung zu fortgeschrittener Programmphase, sollen bei dieser schematischen Betrachtung ausgeklammert werden.

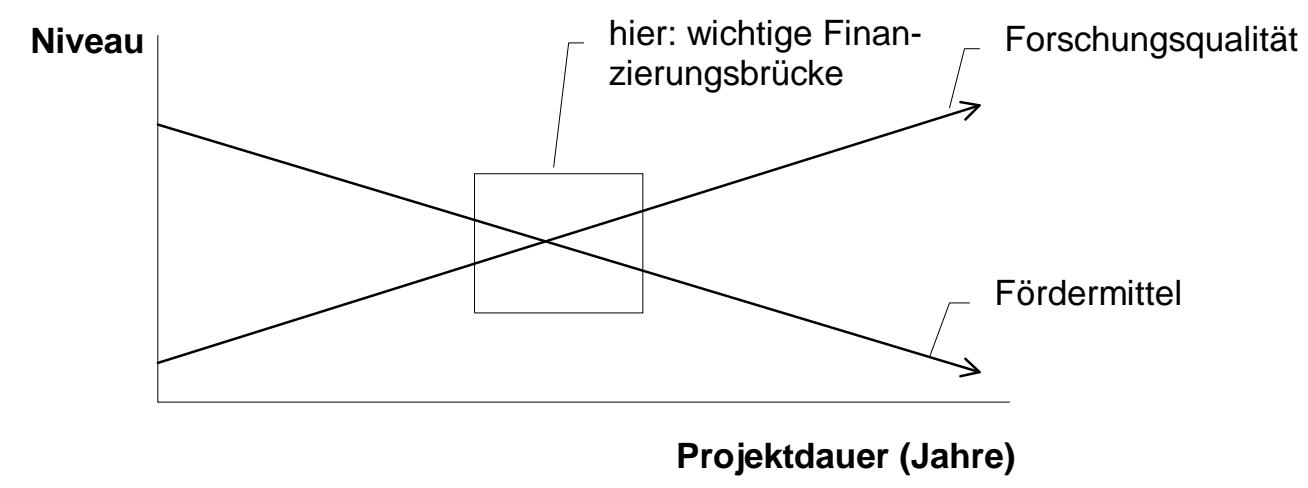

Abb. 8: Zusammenhang zwischen Forschungsqualität und Fördermitteln im Projektverlauf (schematisch) 
Als Strategie kann empfohlen werden, den - kurzfristigen - politischen Druck zu nutzen, um auch langfristig beständige und damit effiziente Umschichtungen in den Forschungsressourcen zu erreichen. So sollten Fördermittel nicht nur zur zeitlich begrenzten Finanzierung von Forschungsprogrammen zur Verfügung gestellt werden. Erforderlich erscheint auch die Veränderung bestehender sowie die Neuschaffung geeigneter Forschungsstrukturen. Dies kann über die institutionelle, finanzielle und personelle Umwidmung bereits bestehender Resourcen in Hinblick auf das $\mathrm{zu}$ bearbeitende Forschungsthema erfolgen, sowie durch die Schaffung neuer, fachlich einschlägiger Forschungsstrukturen auf Dauer (z.B. Neugründung einer fachlich einschlägigen Institution wie Lehrstuhl, Institut Professur o.ä.). Letztere Teilstrategie machte allerdings hinsichtlich der langfristig anfallenden Mehrkosten eine Regelung über die Übernahme der Folgekosten erforderlich. Eine Mischfinanzierung dieser dreistufigen Strategie aus (1) Forschungsfinanzierung, (2) Umwidmung bestehender Forschungsressourcen und (3) Neuschaffung von Forschungsstrukturen, z.B. zu je 1/3, wäre denkbar.

Dadurch kann letztlich problemorientiert wissenschaftliche Kompetenz aufgebaut werden, die auch bei Nachlassen des politischen Drucks und Versiegen der initialen Förderprogramme erhalten bleibt. Gleichzeitig entsteht Erneuerungsdruck auf die bestehenden Forschungsstrukturen [s.a. WiSSENSCHAFTSRAT 1994, S. 149ff.]

Der Ermittlung des Zeithorizontes für ein Forschungsprogramm und damit des Zeitbedarfs für die Forschung kommt große Bedeutung zu. In Abhängigkeit von der bestehenden Problemdimension sollte diese Frage bereits vor Beginn der Programmerstellung abgeschätzt und festgelegt werden. Dabei muß der fachlich erforderliche Zeitbedarf entscheidend sein und nicht bürokratische Aspekte staatlichen Haushaltswesens, die zu den weitverbreiteten 3 oder 6 jährigen Programmen führen. Je nach Forschungsproblem können 3 Jahre viel zu kurz oder viel zu lange sein.

Der zeitlichen Dynamik politischen Handlungsdrucks folgend werden insbesondere die Anfangs- und die Endphase des Zyklus von Forschungsprogrammen durch die Politik wesentlich geprägt. Daher ist gerade in diesen Phasen die Interaktion zwischen Politik und Wissenschaft von besonderer Bedeutung für das Zustandekommen sowie die Beendigung eines Forschungsprozesses. Politisches Lobbying kann geradezu als Voraussetzung für das Zustandekommen von politischen Programmen gesehen werden.

Aufgrund dieser Eigendynamik von Politik vermag Wissenschaft nur durch professionelles Lobbying ihre Chancen zu wahren. Sie kann durch prominente Interessenvertretung insbesondere zu Zeiten hohen politischen Handlungsdrucks mit großer Erfolgswahrscheinlichkeit für die Initiierung und Etablierung eines Forschungsprogramms werben. Dazu können bspw. Medienund Verbandsarbeit geleistet oder Kontakte zu politischen Institutionen wahrgenommen werden.

\subsubsection{Integration eigenständiger Forschungsfinanziers}

Nicht eindeutige Regelungen der politischen Verantwortlichkeiten können zu Konkurrenzerscheinungen der Ressorts um die Erstellung von Forschungszielen und -inhalten, um die Pro- 
grammdurchführung und die Finanzierung führen. Jeweils entsprechend der eigenen Interessenlage versuchen die beteiligten Finanziers, das Programm zu gestalten, ohne selbst Einschränkungen hinsichtlich der Ressortsouveränität hinnehmen zu müssen. Damit behindert die Konkurrenz die Optimierung des Programms bei den gegebenen Ressourcen (zum „Territorialverhalten“ der Akteure s.a. Kapitel 5.1.3.3.6 und 6.3).

Inhaltlich optimale Programme lassen sich nur bei eindeutig geregelten Kompetenzen erarbeiten. Das oder die zuständigen Ressorts müssen mit entsprechender Kompetenz, Entscheidungsund Finanzierungshoheit ausgestattet werden. Alternativ können folgende Strategien angeboten werden:

- Beschränkung des Programms auf das eigene Ressourcenpotential

Das Forschungsprogramm, dessen Durchführung und Finanzierung werden auf ein Ressort begrenzt (Ressortforschung). Damit wird auch die Verantwortlichkeit dem direkten politischen Einflußbereich unterstellt. Inhaltlicher Programmrahmen und Finanzierungsumfang sind dann zwar in der Regel vergleichsweise gering, dafür können die Ressortinteressen jedoch bestmöglich berücksichtigt werden. Eine einfache, nicht durch die ressortexterne Abstimmung erschwerte Gestaltung des Programms ist zu erwarten. Insgesamt verzichtet diese Strategie auf den in der Verwaltungspraxis nur schwer erfüllbaren Anspruch, die Forschungsprogramme mehrerer Ressorts untereinander abzustimmen.

\section{- Auswahl aus Alternativen für Gesamtprogramme durch Mehrheitsentscheidungen}

Alle einschlägigen, am Forschungsprogramm interessierten und zur Finanzierung bereiten Ressorts werden an der Programmkonzeptionierung aktiv beteiligt. Somit entsteht ein gemeinsam getragenes Programm (ressortübergreifende Schwerpunktforschung, s. z.B. Umweltprogramm der Bundesregierung [BUNDESMINISTERIUM FÜR BILDUNG, WISSENSCHAFT, FORSCHUNG UND TeChnologie - BMBF - 1997, S. 15]). Das gemeinsame Programm bedarf eines finanziellen Beteiligungsschlüssels, der ex ante-festgelegt wird. Dabei sind in etwa gleich hohe finanzielle Beiträge aller Beteiligten am einfachsten zu handhaben.

Bei dieser Vorgehensweise besteht jedoch die Gefahr, daß aufgrund der ressortspezifischen Eigeninteressen bei dem gemeinsamen Aushandlungsprozeß programmatische Schwerpunktsetzungen des Programms behindert werden, zugunsten eines Nebeneinanders verschiedener Unterziele oder eines „kleinsten gemeinsamen Nenners“. Um die Ressorts zu einer Entscheidung über Schwerpunkte anzuhalten, sollte daher eine Mehrheitsentscheidung über Alternativen für das Gesamtprogramm herbeigeführt werden. Die beteiligten Ressorts entwerfen in beliebiger Abstimmung mit den Nachbarressorts jeweils Idealvarianten für ein vollständiges Forschungsprogramm. Aus den Varianten wird dann durch mehrheitliche Entscheidungsfindung eine Variante ausgewählt. In der Mehrheitsentscheidung verlieren einzelne Ressorts ihre Vetoposition, die ansonsten Schwerpunktsetzungen unmöglich macht.

- Delegation von Entscheidungsmacht und Ressourcen an eine neutrale Stelle 
Angesichts unterschiedlicher Ressortinteressen, die sich in Konkurrenz- und Territorialverhalten äußern, wird die Programmplanung und -verantwortlichkeit mitsamt der Finanzierungshoheit in die Hände einer weitgehend ressortneutralen, zu allen Ministerien äquidistanten Koordinierungsstelle gelegt. Die Neigung, Kompetenzen und Ressort-Fördermittel einer derartigen Stelle zu übertragen, dürfte höher sein als die Bereitschaft, diese Mittel direkt einem anderen Ressort zur Verfügung zu stellen. Voraussetzung ist, daß die Abgabe von Kompetenzen und Ressourcen auch dauerhaft eingehalten und nicht zum Gegenstand der Interessenaushandlung während der Programmimplementation wird.

Die Ressortneutralität der Koordinierungsstelle kann durch Auslagerung und Ansiedlung bei einer weitgehend ressortunabhängigen Forschungseinrichtung begünstigt werden (z.B. Universität, außeruniversitäre Forschungseinrichtungen der Grundlagenforschung oder spezielle Projektträger der Großforschungseinrichtungen). Hier ist zudem in hohem Maße Fach- und Managementkompetenz zu erwarten.

\subsubsection{Trennung politischer und wissenschaftlicher Interessen bei der Programmplanung}

Aufgrund ihrer informalen Interessenlage drängt die Politik auf rasche Problemlösungen mit möglichst geringem Fördermittelaufwand, während die Wissenschaft umfangreiche Fördermittel für eigene, grundlagenorientierte Forschungsziele anstrebt. Politik und Wissenschaft vertreten allerdings nicht nur ihre jeweils akteursspezifischen Interessen. Wissenschaftliche Interessen und Argumente werden politisch genutzt und umgekehrt politische Interessen für Zwekke der Wissenschaft, um die eigene Interessenverfolgung argumentativ zu verbessern. Dies ist bspw. dann der Fall, wenn die Politik auch wissenschaftliche Interessenpositionen zur Beurteilung der Forschungsqualität von Forschungsanträgen bezieht, andererseits wissenschaftliche Fachgutachter nicht nur Fachargumente, sondern auch politische Argumente der Finanzierbarkeit der Forschung einsetzen.

Sowohl politische als auch wissenschaftlich-fachliche Interessen sind legitim und berechtigt. Die Vermengung beider Ebenen in der Argumentation führt jedoch zu Fehlentscheidungen. Durch geeignete Verfahren zur Planung (und Durchführung) eines Forschungsprogramms können die bestehenden politischen und wissenschaftlichen Interessenverflechtungen getrennt und damit für die Entscheidungen fruchtbar genutzt werden.

Es ist unbestritten, daß die Politik den wissenschaftlichen Sachverstand nicht nur in der Forschungsdurchführung, sondern bereits in der Programmplanung nutzen muß. Dabei ergibt sich hinsichtlich der Programmplanung jedoch das Problem, daß die Wissenschaftler aus forschungspolitischem Interesse bestrebt sein werden, die von ihnen vertretenen Fach- bzw. Forschungsrichtungen nach Möglichkeit auch über die wissenschaftliche Erfordernis hinausgehend in das Programm einzubeziehen und sich damit ihre Zutrittschancen erheblich zu verbessern.

Der Strategie, das Forschungsprogramm durch die politische Fachverwaltung oder eine intermediäre Koordinierungsstelle gänzlich ohne Beteiligung der Wissenschaft zu erstellen, stehen insbesondere fachliche Schwächen und Defizite sowie die mangelnde Akzeptanz solcher Programme durch die Wissenschaft entgegen. Der Forschungsbedarf kann nur von der Wissen- 
schaft optimal ermittelt werden. Andererseits wird aber die Strategie, allein die Wissenschaft mit der Erstellung des Programms zu beauftragen, trotz hoher Qualität zu sehr die Eigeninteressen der Forscher begünstigen, nicht aber den politischen Anforderungen zur Problemlösung ausreichend gerecht werden. Die Einschränkung, die an der Programmerstellung beteiligten Forscher grundsätzlich von der Beteiligung an dessen Umsetzung auszuschließen, würde zwar der Interessenneutralität entgegenkommen, dem Projekt jedoch erhebliches wissenschaftliches Potential und Sachverstand vorenthalten.

Empfohlen wird daher, daß grundsätzlich die Politik ein politisches Problem (Praxisproblem z.B. aus dem Bereich der Umwelt) zum Forschungsproblem erklärt. Dieser Festlegung sollte jedoch eine Anhörung von Experten (Hearing, Landtagsanhörung o.ä.) vorausgehen. Die Politik legt dann die - problemorientierte - Zielsetzung und fachliche Ausrichtung des Programms ebenso fest wie den beabsichtigten Typ der Forschung (z.B. Auftrags- oder Grundlagenforschung). Je ausgeprägter der politische Anspruch an Problemorientiertheit des Programmes ist, desto deutlicher muß dies formuliert und in die weitere Programmerstellung oder -umsetzung implementiert werden. Auch die Organisationsstrukturen sowie weitere Rahmenbedingungen, wie z.B. Modalitäten des Förderverfahrens, sollten eindeutig und verbindlich festgelegt werden. Damit können spätere Interessenkonflikte zwischen Politik und Wissenschaft vermieden und die Verfahrenstransparenz bei Programmerstellung und -durchführung gewährleistet werden.

Eine kompetente und weitgehend unabhängige (interessenneutrale) Koordinierungsstelle kann sinnvoll zur abgestuften Verfahrensdurchführung, Entflechtung der Interessenlagen und sachgerechten Entscheidungsfindung beitragen (zur Trennung von Zielsetzungs- und Durchführungsverfahren s.a. BUNDESMINISTERIUM FÜR WISSENSCHAFT UND VERKEHR - BMWV [1998, S. 23]). Geeignete Strukturen des Förderverfahrens können Synergieeffekte aus wissenschaftlichem Wettbewerb und Kooperation nutzen (z.B. über Ausschreibung des Programms, Kooperation als Auflage etc.) und die wissenschaftliche Qualität der Forschungsarbeiten sicherstellen (z.B. im Rahmen der Fachbegutachtung).

\subsubsection{Verfahren der Programmerstellung und Forschungsakquisition}

Zur weiteren Vorgehensweise der Programmerstellung und Antragsakquisition können verschiedene Strategien vorgeschlagen werden:

- Breite Forschungsziele und zielbezogenen Selektion von Antragsskizzen

Die Vorgabe breiter Rahmenvorgaben für ein Forschungsprogramm oder die bloße Nennung der politischen Hauptzielsetzung an die Forscher haben verschiedene Vorteile. Derartige Vorgaben sind durch die Politik oder eine intermediäre Koordinierungsstelle leicht zu bewerkstelligen. Sie sind insgesamt wissenschaftsfreundlich, indem sie im Programm von vorne herein kaum Forschungsbeiträge ausschließen und der Forschung reichlich Freiraum für Motivation und Ideenvielfalt belassen. Nachteile sind allerdings ein zu erwartendes Mosaik von zahlreichen Forschungs-Teilaspekten zum Thema, die sich weder bei Durchführung noch bei Endauswertung inhaltlich miteinander verknüpfen lassen. Das konkrete Forschungsprogramm entwickelt 
sich erst im Verlaufe des Forschungsprozesses und eher zufällig als Summe der Einzelbeiträge. Hinzu kommen aufwendige Selektionsverfahren der Forschungsanträge in politischer wie wissenschaftlicher Hinsicht.

Unter Nutzung der genannten Vorteile einer breiten Programm- bzw. Zielsetzungsausschreibung kann eine Strategie empfohlen werden, die als erste Selektionsphase die Einreichung von kurzen, aber inhaltsreichen Antragsskizzen (Proposals) vorschaltet. Dies ermöglicht allen interessierten Forschergruppen, mit noch vertretbarem Aufwand ihre beabsichtigte Beteiligung am Programm zu signalisieren und einen Lösungsbeitrag in Aussicht zu stellen. Die Politik bzw. die Koordinierungsstelle sind in der Lage, anschließend in einer Vorauswahl allein über den Zielbezug der erwartungsgemäß zahlreich eingereichten Skizzen noch ohne Prüfung der wissenschaftlichen Qualität zu befinden. Den Antragstellern der positiv selektierten Proposals wird anheimgestellt, entsprechende Forschungsanträge auszuformulieren. Diese werden nach Sicherstellung der wissenschaftlichen Qualität durch eine gutachterliche Evaluierung zur Finanzierung vorgesehen.

Die Phase der Antragsausformulierung durch ein nunmehr eingegrenztes Forscherkollektiv kann bspw. von der Koordinierungsstelle dazu genutzt werden, durch das Angebot zusätzlicher Fördermittel potentielle Forschungspartner frühzeitig zur Kooperation zu animieren bzw. über Auflagen zu verpflichten und so zu einem abgestimmten, interdisziplinären Forschungsprogramm zu gelangen. Damit könnten wesentliche der genannten Nachteile einer breiten Programmausschreibung im nachhinein beseitigt werden.

- Formulierung eines Forschungskonzeptes durch ein Expertengremium

Eine weitere Strategie für die Programmerstellung kann in der Erstellung eines Forschungsrahmenplans durch ein wissenschaftlich ausgewiesenes Expertengremium gesehen werden. Unter strenger Berücksichtigung der politischen Rahmenvorgaben und Zielsetzungen kann erwartet werden, daß diese in ein wissenschaftlich hochqualitatives Forschungsprogramm überführt werden, welches eine konkrete Rahmenvorgabe für einzureichende Forschungsanträge darstellt. Das Programm kann dann z.B. über eine Ausschreibung an alle interessierten Forschergruppen weitergegeben werden. Die Antragsselektion erfolgt anschließend über das Kriterium der Einpassung in den Programmrahmen und weiterhin über das Instrument der Antragsbegutachtung zur Überprüfung der wissenschaftlichen Qualität. Die Überprüfung, ob ein Beitrag zur politischen Problemlösung zu erwarten ist, wird bereits über die Einpassung in den Programmrahmen abgedeckt.

Ein wesentlicher Nachteil besteht allerdings darin, daß das Programm die Handschrift der Experten trägt und damit deren Forschungsinteressen repräsentiert. Unter Berücksichtigung des wissenschaftlichen Renommees der Experten kann dieser Nachteil jedoch in Kauf genommen werden. Die Vorteile eines detailliert ausgearbeiteten, schlüssigen Forschungsprogramms überwiegen. Es unterstützt die Umsetzung in wissenschaftlich hochwertige, den politischen Programmzielen entsprechende Forschungsbeiträge, auch in Hinblick auf eine stringente Programmkoordination. 


\subsubsection{Kompetenz der Koordinierungsstelle zur Programmkoordination}

Mangelnde Kompetenz der Koordinierungsstelle behindert nicht nur formal fachgerechtes Projektmanagement einschließlich eines adäquaten Forschungs-Controllings, sondern auch informal die erforderliche Akzeptanz der Koordinierungsstelle bei den Forschern. Sie stärkt aber auch die Interessendominanz der Wissenschaft und drängt auf die Einrichtung eines begleitenden wissenschaftlichen Beratungskomitees, was wiederum Interessenkonflikte zwischen Koordination und Wissenschaft begünstigt. Daher muß die Koordinierungsstelle mit hoher fachlicher und verwaltungstechnischer Kompetenz bei Programmplanung, -durchführung und evaluierung ausgestattet werden.

Hierzu wird empfohlen, die koordinierende Stelle mit wissenschaftlich wie verwaltungsmäßig versierten Persönlichkeiten innerhalb einer gleichermaßen renommierten Institution (z.B. Forschungsinstitution) zu besetzen. Dies sichert die fachliche Kompetenz und Akzeptanz in Politik und Forschung.

Dennoch ist eine Aufgabentrennung zwischen Programmkoordination durch die Koordinierungsstelle und Forschungskoordination durch die Wissenschaft sicherzustellen. Mit der fachlichen Koordinierung vor Ort ist eine zentrale Koordinierungsstelle in der Regel überfordert. In Forschungsmodulen [KROTT 1997], thematischen Forschungsteilbereichen oder an regionalen Schwerpunktstandorten sollte die fachliche Koordination der Forschung daher eigenverantwortlich einzelnen geeigneten Vertretern der Wissenschaft übertragen werden.

Die Aufgabe der Programmkoordination durch die Koordinierungsstelle besteht vielmehr darin, eine enge Zusammenarbeit mit den Forschungsvertretern vor Ort zu pflegen, autonome Strukturen der fachlichen Forschungskooperation zu unterstützen und für die erforderliche Flexibilität in Programmvorgaben und Forschungsverwaltung zu sorgen. Durch verschiedene Serviceleistungen kann sie die Kooperation und Herausbildung interdisziplinärer Forschungsstrukturen verbessern. Dazu gehören

- die Durchführung von fachübergreifenden Veranstaltungen (Querschnittsseminare),

- Seminare zum aktuellen Stand des Forschungsprojektes (Statusseminare),

- umfassender Informations- und der Datentransfer zwischen den Forschergruppen sowie

- die integrierende, syntheseartige Auswertung der Forschungsergebnisse.

\subsubsection{Ausgleich zwischen Programmvorgaben und Forschungsflexibilität}

Entscheidende Weichenstellungen und Festlegungen erfolgen bei Planung und in der Anfangsphase der Implementation des Forschungsprogramms. Anfängliche Fehlentscheidungen prägen das Projekt und können später nur schwer revidiert werden.

Dies betrifft nicht nur die inhaltliche Programmplanung. Auch die Forschungskooperation und der Aufbau interdisziplinärer Forschungsstrukturen können zwar durch die frühzeitige Beteiligung von Forschern an der Forschungsplanung begünstigt werden. Sie legen aber auch die $\mathrm{Zu}-$ sammensetzung des anfangs beteiligten Forscherkollektivs und somit auch die fachliche Aus- 
richtung des Programms weitgehend fest. Mitsamt erheblicher zeitlicher und finanzieller Bindungswirkung neigt das Forschungsprogramm dann zu Inkrementalismus, nur geringer Flexibilität und geringer Innovationsfähigkeit. Forscher, die in der Etablierungsphase nicht zum Zuge gekommen waren oder solche, die erst später sich beteiligen wollen, finden nurmehr erschwerte Zutrittsmöglichkeiten vor.

Hierzu können folgende Empfehlungen gegeben werden: Der Programmplanung muß höchste Bedeutung beigemessen und trotz bestehendem politischen Handlungsdruck entsprechende Zeit- und Finanzressourcen reserviert werden. Pilotstudien oder vergleichbare Vorerkundungen sollten verpflichtend vorgeschaltet und z.B. im Rahmen des Selektionsprozesses zu Forschungsanträgen nachgewiesen werden. Sie verbessern oder schaffen erst die methodischen und theoretischen Grundlagen für die Wahl des am besten geeigneten Forschungsansatzes, stellen die Umsetzung des Forschungsprogramms auf eine verläßlichere Basis und verbessern die Aussichten auf Forschungserfolg.

Der Nutzen, der in Hinblick auf Motivation, Kooperation und Interdisziplinarität aus der frühzeitigen Beteiligung von Forschern an der Forschungsplanung gezogen kann, soll durchaus realisiert werden. Es müssen jedoch Sicherungsmechanismen verankert werden, die die nötige Flexibilität der Forschung in inhaltlicher wie institutioneller Hinsicht gewährleisten.

So können ein kontinuierlich begleitendes Forschungs-Controlling oder regelmäßige Zwischenbilanzierungen verbindlich vorgesehen werden. Mit diesen Instrumenten kann das Programm insbesondere auf den Ergebnisfortschritt hin evaluiert und erforderlichenfalls die Programmvorgaben modifiziert werden. Die Einbeziehung von neuen, zusätzlichen Forschungsaspekten unter Beteiligung neuer Forschergruppen wird ermöglicht.

Von der Vergabe von Mitteln für Fortsetzungsvorhaben ist nur im unbedingt notwendigen Rahmen und nach kritischer Überprüfung Gebrauch zu machen ( z.B. zur Erreichung des ursprünglichen Forschungsziels). Die Einbeziehung neuer Forschungsaspekte - und damit gegebenenfalls neuer Forschergruppen - ist demgegenüber zu bevorzugen und kann durch entsprechende Mittelkontingentierung sichergestellt werden. Hierzu können jahresweise bestimmte Fördermittelanteile oder -quoten nur für Neuvorhaben reserviert werden, die Anzahl oder das Finanzvolumen für Fortsetzungsvorhaben begrenzt und/oder Mittel für eine zweite Programmphase zurückgestellt werden.

Auch die Kreativität und Ideenvielfalt der Forscher soll ausreichend berücksichtigt werden. Unverzichtbare Grundlagenforschung sowie risikoreiche, innovative Forschungsthemen im Forschungsneuland sollten daher ebenfalls anteilig, auch im Rahmen überwiegend angewandter Forschungsprogramme, zugelassen werden [s.a. BMWV 1998, S. 19; BMBF 1997, S. 159]. Statt einer finanziellen Kontingentierung der genannten Fälle im einzelnen bietet sich zur Sicherung der nötigen Programmflexibilität an, hierfür generell einen bestimmten Anteil der Gesamtfördermittel (z.B. 20\%) zu reservieren. 


\subsubsection{Verfahren zur Begutachtung von Forschungsanträgen}

Die fachliche Begutachtung sichert üblicherweise die wissenschaftliche Qualität der Forschung. Sie findet auch bei sämtlichen der oben empfohlenen Verfahren zur Antragsselektion Berücksichtigung (zum Verfahren der Antragsbegutachtung s.a. Kapitel 5.3.5).

Tendenziell führt eine dominierende Stellung der Fachbegutachtung im Förderverfahren zu einer Verstärkung der Selbststeuerung der Forschung zulasten des Entscheidungsspielraumes der Politik. Zudem können informale Interessenkoalitionen oder Konkurrenzerscheinungen zwischen Gutachter und Antragsteller bestehen, welche die Objektivität der Beurteilung und letztlich der Förderentscheidung belasten.

Je stärker der Anspruch des Forschungsprogrammes an angewandt-problemlösungsorientierte Forschung gerichtet ist, umso mehr muß daher der diesbezügliche Beitrag - politisches Hauptentscheidungskriterium sein (s.a. BMWV 1998, S. 6, 9, 23). In einem abgestuften Verfahren trifft zuerst die Politik oder Koordinierungsstelle eine Entscheidung über Zielkonformität und zu erwartenden Problemlösungsbeitrag der Forschung. Anschließend beurteilen und sichern mehrere Fachgutachter die wissenschaftliche Qualität und Realisierbarkeit des Vorhabens.

Hinsichtlich der Optimierung des Begutachtungsverfahrens zur Selektion von Forschungsanträgen können folgende Empfehlungen angeboten werden:

- Die Auswahl der externen Gutachter sollte von der intermediären, weitgehend neutralen Koordinierungsstelle über fachkompetente Selbstverwaltungsorganisationen der Wissenschaft (z.B. Deutsche Forschungsgemeinschaft), nicht aber von Politik, beteiligten Forschergruppen oder Beiratsgremien vorgenommen werden. Die Einbeziehung internationaler Gutachter kann die Unabhängigkeit der Begutachtung - und die Begutachtungsqualität - erhöhen.

- Hinsichtlich der Gutachterneutralität ist zu berücksichtigen, daß mit der fachlichen Kompetenz des Gutachters zur Antragsbeurteilung auch die Wahrscheinlichkeit einer wie auch immer gearteten - persönlichen - Beziehung (Konkurrenz, Kooperation) zum Antragsteller steigt. Die Anonymisierung von Gutachter und/oder Antragsteller kann das Problem in der Regel nicht lösen. Die Unbefangenheit des Gutachters sollte nicht erst durch dessen verfahrensmäßige Anonymisierung hergestellt, sondern allein durch weitgehende formale Beurteilungskompetenz gegeben sein. Die Anonymisierung des Antragstellers gegenüber dem Gutachter ist bei dessen Kenntnis der spezifischen scientific community ohnehin wenig erfolgversprechend und würde verhindern, über verschiedene, personenbezogene Begutachtungskriterien befinden zu können (z.B. Publikationen, Kooperationen, Arbeitsgruppe, persönliche Eignung für die Forschungsaufgabe aufgrund bisheriger Arbeiten).

- Schriftliche Begutachtungsverfahren sind weniger kosten- und zeitaufwendig als mündliche. Sie lassen sich durch Formalisierung der Begutachtung, wie die Vorgabe von Bewertungskriterien und Punktevergabeverfahren, in Durchführung und Auswertung vereinfachen und operationalisieren. Die Fachexpertise der einzelnen Gutachter kann gezielt genutzt werden. 
Sie sind daher generell für umfangreiche Programme ebenso wie für einzelfallweise Begutachtung zu bevorzugen.

- Mündliche Begutachtungsverfahren führen zu einer stärkeren Bindung der Gutachter an das Projekt und zur Herausbildung eines multidisziplinär zusammengesetzten Gutachtergremiums. Dies kann insbesondere für die begleitende Betreuung und Bewertung interdisziplinärer Forschungsprojekte sowie für den Aufbau interdisziplinärer Gutachterkompetenzen genutzt werden.

- Die Vorhabenbegleitung durch die beteiligten Fachgutachter - ob nach schriftlichem oder mündlichem Verfahren - ersetzt weitgehend ein weiteres fachliches Beratungsgremium.

- Turnusmäßiger Wechsel in der personellen Zusammensetzung der Gutachter verringert die einseitige Verfolgung bestimmter wissenschaftlicher Interessen im Projekt.

- Zeitlich getaktete Antragsstellungs-, Begutachtungs- bzw. Bewilligungsrunden ermöglichen eine Bündelung der Forschungsbeiträge in inhaltlicher und zeitlicher sowie finanzieller Hinsicht. Dies erleichtert die Programmkoordination und Verfahrensabwicklung ebenso wie die Forschungskoordination und -kooperation unter den beteiligten Wissenschaftlern (Aufbau von Forschungsverbünden, synchronisierte Vorhabenlaufzeiten etc.).

- Die Einbeziehung von Interdisziplinaritätskriterien (z.B. interdisziplinäre Vernetzung der Forschungsarbeiten, Kooperationsbereitschaft, fachübergreifende Forschungsansätze) bei der Begutachtung sowie der Einsatz geeigneter interdisziplinärer Gutachterkapazitäten begünstigt die Etablierung interdisziplinärer Forschungsverbünde.

- Die Aufgabe der fachlich weitgehend neutralen Koordinierungsstelle muß sich bei der Antragsselektion auf außerfachliche Kriterien, politische und wissenschaftliche Rahmenbedingungen konzentrieren. Gerade im Falle anwendungsorientierter Forschungsprogramme kann sie - unbesehen der fachlichen Begutachtung - die Bewertung des Zielbezugs und Lösungsbeitrags sowie einer geforderten Interdisziplinarität fundiert vornehmen.

\subsubsection{Kontrolle und Erfolgsbeurteilung der Forschung}

Bei der Kontrolle und Erfolgsbeurteilung der Forschung ist zwischen den Einzelbeiträgen der Forschung und dem Gesamtprogramm zu unterscheiden. 


\section{- Erfolgskontrolle einzelner Forschungsbeiträge}

Der Erfolgskontrolle einzelner Forschungsbeiträge sind während der Forschungsförderung enge Grenzen gesetzt. Nachträgliche Kontrollen, bspw. in Form der Bewertung der Forschungsberichte, sind weitgehend (mit Ausnahme von Fortsetzungsvorhaben) wirkungslos (s.a. Ausführungen zur Evaluierung von Forschungsberichten, Kapitel 5.3.5.4). Hier kommt der ex ante-Antragsevaluierung große Bedeutung zu. Sie bewertet den zu erwartenden Forschungserfolg mit Hilfe der fachlichen Begutachtung und Überprüfung des Problemlösungsbeitrags und setzt deren Ergebnis als wissenschaftliche bzw. politische Grundlage förderentscheidend ein.

Forscher können jedoch dazu neigen, im Verlauf des Forschungsprogrammes teils aus forschungsseitiger (z.B. methodischer) Notwendigkeit, teils aus wissenschaftlichem Eigeninteresse, von den ursprünglichen Ziel- und Programmvorgaben abzuweichen. Insbesondere bei problemorientiert-angewandter Forschung im Sinne einer Auftragsforschung sollte dies nur im unbedingt nötigen Umfang (z.B. methodisch bedingt) toleriert werden. Eine Kontrolle ist jedoch schwierig. Die dem Forschungsprozeß zeitlich nachgeschalteten Zwischenberichte dokumentieren überwiegend bereits geschaffene Fakten oder halten - besonders in der ersten Vorhabenphase - den Stand der Forschungsarbeiten noch bedeckt. Bei bekanntgewordenen Abweichungen mit Abbruch oder Verringerung der Finanzierung zu drohen, ist häufig nicht angemessen und zu stark dirigistisch gegenüber dem Forschungssystem und dessen berechtigten Anforderungen an Flexibilität.

Gerade im Bereich problemorientiert-angewandter Forschung kann empfohlen werden, die inhaltliche Kontrolle während des Forschungsverlaufes zu verstärken, aber auf realisierbare, einfach zu erhebende und vorher vereinbarte Ziele zu beschränken (z.B. die programmgemäße Abarbeitung einzelner Programmpunkte, Herstellung des Bezugs zur Zielsetzung). Eine derartige Erfolgskontrolle ist durch eine Koordinierungsstelle durchaus fachlich adäquat, rasch und ohne größeren Verwaltungsaufwand, der zu Verzögerungen im Vorhabenverlauf führen könnte, zu erbringen. Die gutachterlich bereits beurteilte, wissenschaftliche Qualität des Vorhabens steht, mit Ausnahme erheblicher methodischer Abweichungen oder ProgrammNeuorientierungen, nicht mehr zur Diskussion.

Dabei kann die inhaltliche Kontrolle an die Auszahlung der regelmäßigen (z.B. jährlichen) Fördertranche gekoppelt, d.h. die weitere Mittelfreigabe von der Billigung des Zwischenberichts abhängig gemacht werden. In diesem Zusammenhang wird empfohlen, die gutachterlich für erforderlich gehaltenen Fördermittel nicht nur zu bewilligen, sondern auch dem Forscher in Form eines Globalhaushaltes mit weitgehenden Freiheiten inhaltlicher Umwidmung oder zeitlicher Übertragbarkeit zur Verfügung zu stellen. Eine finanzielle Forschungskontrolle über die strikte Einhaltung von Finanzierungsplänen und Ausgabenachweise (mit Ausnahme eines abschließenden Gesamt-Verwendungsnachweises) erweist sich in der Regel als eine sachlich nicht gerechtfertigte Scheinkontrolle und sollte nicht zuletzt aus Gründen der Verwaltungsvereinfachung fallen gelassen werden.

Ergänzt werden kann die fachlich-inhaltliche Forschungskontrolle durch Statusseminare, im Rahmen derer die einzelnen Forschergruppen gegenüber den Programmpartnern, der scientific 
community, den Gutachtern sowie gegenüber dem Auftrag- bzw. Finanzgeber den Stand ihrer Arbeiten präsentieren. Die weitere, wissenschaftliche Evaluierung der Forschungsleistung, die allerdings bei Betrachtung anwendungsorientierter Forschung gegenüber grundlagenorientierter Forschung hier weniger im Mittelpunkt steht, erfolgt innerhalb des Systems der scientific community selbst.

\section{- Erfolgskontrolle des Gesamtprogramms}

Die wissenschaftlich fundierte Evaluierung eines Forschungsprogramms findet in der Regel nicht statt. Meist befinden externe politische Argumente über den Erfolg, die Zukunft und insbesondere die Beendigung von Programmen.

Der ex post-Evaluierung des Gesamtprojektes sollte in Hinblick auf das erreichte Forschungsergebnis und die Zielsetzung des Programms jedoch besondere Bedeutung beigemessen werden. Sie sollte parallel zur Programmdurchführung in Form eines Forschungs-Controlling oder Zwischenbilanzierungen erfolgen und - gegenüber externen politischen oder finanziellen Kriterien - zur maßgeblichen Entscheidungsgrundlage für Beendigung, Weiterführung oder Modifikation des Forschungsprogramms erhoben werden. Dies setzt wiederum das Bestehen klarer politischer wie forschungsseitiger Zielvorgaben als Beurteilungskriterien voraus, die bereits bei der Programmplanung fixiert werden müssen.

Die umfassende Erfolgsbewertung des durchgeführten Forschungsprogramms verspricht eine qualifizierte und dem betriebenen Förderaufwand adäquate Auseinandersetzung mit den Inhalten, Ergebnissen und dem Nutzen des Programms. Dabei erscheint es sinnvoll, gegebenenfalls auch auf wenig erfolgreiche oder mißlungene Forschungsansätze einzugehen, um aus Fehlern zu lernen und Wiederholungen zu vermeiden. Der finanzielle, personelle und zeitliche Aufwand für eine derartige Bewertung muß im Programm zu Beginn berücksichtigt werden.

Die hier gegebenen Empfehlungen betreffen entsprechend dem gewählten Idealmodell überwiegend die politische Evaluierung eines anwendungsorientierten Forschungsprogramms. Die Evaluierung dessen wissenschaftlichen Ertrags findet - ähnlich wie für Einzelbeiträge der Forschung - weitgehend innerhalb der scientific community statt und ist Bestandteil deren Selbststeuerung. Im Hinblick auf die überwiegend noch disziplinäre, grundlagenorientierte Struktur der Forschung mit geringer Ausrichtung auf Forschungskooperationen und interdisziplinäre Forschungsprogramme kommt der Bewertung problemorientierter, staatlich finanzierter Forschungsprogramme in der scientific community jedoch bislang nur eine untergeordnete Bedeutung zu. 


\section{Zusammenschau}

Das Umweltthema „Waldschäden/Waldsterben“ führte zu Beginn der 80er Jahre zu einer Vielzahl von Forschungsmaßnahmen, die sich um die Beschreibung der beobachteten Phänomene und Ursachenaufklärung bemühten. In Bayern wurde auf Ministerratsbeschluß in der Zeit von 1984 bis 1993 ein staatlich finanziertes Forschungsprogramm durchgeführt, welches zum Ziel hatte, die bayerische Waldschadensforschung finanziell zu verstärken und zu koordinieren. Mit dem Projekt sollten die Wirkungen von Umweltschadstoffen auf den Wald im Rahmen eines interdisziplinären Forschungsprogramms erforscht und Ergebnisse geliefert werden, die als Handlungsempfehlungen für die Durchführung oder Konkretisierung umweltpolitischer Gegenmaßnahmen dienen sollten.

Das Projekt wurde im Auftrag des Bayerischen Umweltministeriums über eine eigens an der damaligen Gesellschaft für Strahlen- und Umweltforschung mbH München (GSF), eingerichtete Koordinierungsstelle, die „Projektgruppe Bayern zur Erforschung der Wirkung von Umweltschadstoffen“ (PBWU), durchgeführt. Mit einem Finanzmittelaufwand von rund 35 Mio. DM und der Durchführung von etwa 80 Einzelvorhaben durch universitäre oder außeruniversitäre, bayerische Forschungsinstitutionen nimmt das Projekt einen prominenten Platz in der bayerischen wie auch bundesweiten Waldschadensforschung ein.

Die organisatorische Struktur des Projektes mit der Beteiligung politischer und wissenschaftlicher Akteure öffnet das Spannungsfeld zwischen Politik und Wissenschaft im Bereich der Forschungsförderung. Sie schafft eine besondere Ausgangslage für das Management staatlich finanzierter Programmforschung. Diese wird im Rahmen der vorliegenden Arbeit empirisch erhoben und theorieorientiert analysiert. Dabei steht die Frage im Mittelpunkt, wie sich interdisziplinäre Forschung organisieren und gestalten läßt, damit sie zum einen angewandtproblemorientiert durchgeführt wird und Ergebnisse liefert, welche die politische Zielsetzung erfüllen, und zum anderen den Anforderungen der Wissenschaft entspricht.

Da es sich bei dem PBWU-Projekt um ein forschungspolitisches Großvorhaben handelt, folgt die Analyse einem politikwissenschaftlich orientierten Forschungsansatz. Das Projekt stellt einen sektoralen Ausschnitt aus dem gesamten Politikfeld Waldschäden dar und kann als Forschungspolicy bezeichnet werden. Der theoretische Ansatz der Politikfeld-(Policy)-Analyse kann auf den Prozeß der Durchführung des PBWU-Projektes sowie den eigentlichen Forschungsprozeß übertragen werden. Dem Ansatz liegt ein Phasenmodell zugrunde, das die Policy in verschiedene, zeitlich aufeinanderfolgende, aber inhaltlich miteinander verknüpfte $\mathrm{Ab}$ schnitte gliedert. Das PBWU-Projekt läßt sich ohne wesentliche Einschränkungen den idealtypischen Phasen Problemdefinition, Agenda-Gestaltung, Programmformulierung, Implementation, Evaluation und Programmterminierung zuordnen, chronologisch-empirisch beschreiben und theorieorientiert analysieren.

Zur Unterstützung werden organisations- und wissenschaftssoziologische Aspekte, steuerungstheoretische Grundlagen sowie ein akteurstheoretischer Bezugsrahmen in den Policy-Ansatz einbezogen. Dies ermöglicht es, die formalen und informalen Interessen, Strategien und Hand- 
lungspläne, Einfluß- und Machtpotentiale der im PBWU-Projekt beteiligten Akteursgruppen in Hinblick auf den Forschungsprozeß zu beschreiben und zu erklären.

Die empirische Datenerhebung erfolgt unter Einsatz verschiedener sozialempirischer Methoden. Im Mittelpunkt steht eine Kombination aus retrospektiver, teilnehmender Beobachtung mit einer Dokumentenanalyse. Der Verfasser der Arbeit gehörte der Projektgruppe an und hatte Zugang zu sämtlichem Dokumentenmaterial. Eine quantitative Inhaltsanalyse zum Förder- und speziell zum Begutachtungsverfahren der Forschung ergänzt das methodische Instrumentarium.

Die Gründung des PBWU-Projektes hat die Thematisierung der Waldschäden als schwerwiegendes Problem zur Voraussetzung. Erst hieraus kann Bedarf an Forschung zur Problemklärung und -lösung abgeleitet werden. Angesichts der Ende der 70er, Anfang der 80er Jahre vehement geführten gesellschaftlichen Diskussion zum Thema aufgrund der allgemein akzeptierten, mutmaßlich dramatischen Schädigung der Wälder entsteht großer Erwartungsdruck auf die Forschung. Dabei liefert die Wissenschaft rasch eine Vielzahl unterschiedlicher Ursachenhypothesen. Diese stellen zwar nahezu ausschließlich die Luftschadstoffe in den Mittelpunkt; dennoch besteht Klärungsbedarf, der nach entsprechenden Forschungsmaßnahmen bzw. deren Finanzierung drängt. Seitens der Politik besteht hoher Handlungsdruck, Maßnahmen in verschiedenen Bereichen zu treffen. Forschungsmaßnahmen können nicht nur umweltpolitische Vorsorgemaßnahmen ergänzen und konkretisieren, sondern auch entlastend dazu dienen, weitere politische Maßnahmen bis zur Vorlage konkreter wissenschaftlicher Erkenntnisse hinauszuschieben. Dem hohen politischen Handlungsdruck steht eine hohe Legitimation für die Waldschadensforschung gegenüber.

Die vergeblichen politischen Bemühungen der bayerischen Forschungspolitiker, den Finanzierungsbedarf zusätzlicher Waldschadensforschung an den Bund zu überwälzen, gleichzeitig aber aufgrund günstiger forschungsstruktureller Voraussetzungen bundesweite Koordinierungsfunktionen in Bayern etablieren zu können, führen letztlich zu der Gründung des PBWUProjektes mit bayernweiter, aber ressortübergreifender Koordinationsfunktion. Dabei übernimmt das Umweltressort die Federführung. Es kann im Gegensatz zu dem benachbarten Landwirtschafts-(Forst-) und Kultusressort noch auf kein bestehendes Forschungsprogramm verweisen und reklamiert aufgrund der luftschadstoffzentrierten Ursachendiskussion ressortspezifische Kompetenz und Zuständigkeit für sich. Die Beziehungen zum GSF-Forschungszentrum sowie dessen hohe Bereitschaft, sich zu engagieren, erleichtern es, das Projekt trotz politischer Widerstände an der dem Bund unterstehenden Großforschungseinrichtung anzusiedeln.

Noch vor Vertragsabschluß zwischen Umweltministerium und GSF zur Durchführung des Projektes wird Kritik an den beabsichtigten Steuerungsfunktionen der PBWU-Projektgruppe signalisiert. Die benachbarten politischen Ressorts befürchten eine Einflußnahme auf die von ihnen maßgeblich betreuten Forschungsverbünde und Forscherklientela; die Wissenschaft sieht sich in ihrer Forschungsfreiheit und Eigenverantwortlichkeit beeinträchtigt. Sie will sich nicht einer zentral gesteuerten Auftragsforschung unterordnen. Der Vertragsabschluß wird jedoch rasch vollzogen, die Kritik kann allenfalls nachverhandelt werden. 
Die politischen Ressorts versuchen über ihre Interessenvertretung im Gremium des PBWUProjektrats gegenüber dem finanzierenden Umweltministerium bzw. der Projektgruppe, Einfluß auf die Ausgestaltung des Projektes zu nehmen, ohne ihre eigenen Ressortaktivitäten aufgeben und der PBWU-Koordination unterordnen zu müssen. Im Wissenschaftlichen Beirat der PBWU werden führende Wissenschaftler im Bereich Waldschadensforschung etabliert. In ihrer grundsätzlich und konzeptionell beratenden Funktion verspricht dies ein erhebliches Einflußpotential auf die Ausgestaltung des Projektes bzw. des Forschungsprozesses. Durch den Einsatz externer Fachgutachter zur Beurteilung von Forschungsanträgen soll die wissenschaftliche Qualität und möglichst objektive Beurteilung der Forschung gesichert werden.

Die Phase der Programmformulierung sowie der anfänglichen Implementation des PBWUForschungsprogramms wird vom Auftrag- und Finanzgeber Umweltressort und der in ihrem Auftrag handelnden Projektgruppe dominiert. Ein offener Forschungsrahmen, welcher vielen Forschergruppen Zutritt erlaubt, wird rasch erstellt. Bereits bestehende Forschungsschwerpunkte anderer Programme und Förderer werden aus überwiegend strategischen Gründen miteinbezogen. Der Abstimmungsbedarf mit dem wissenschaftlichen Beratungsgremium der PBWU wird nur unzureichend berücksichtigt. Die programmatische Schwerpunktsetzung, einen alpinen Forschungsschwerpunkt zur Bearbeitung der Photooxidantien-Hypothese zu gründen, kann fixiert und das Rahmenprogramm zur Einreichung von Forschungsanträgen ausgeschrieben werden.

Über zwei Antragsrunden der Jahre 1985 und 1986 wird das PBWU-Programm mit zahlreichen Forschungsvorhaben implementiert. Das Gremium des Wissenschaftlichen Beirats wird im Rahmen des Förderverfahrens übergangen, die politischen Vertreter im Projektrat akzeptieren und übernehmen die Förderempfehlungen der Projektgruppe. Diese nimmt an verschiedenen Stellen Einfluß auf das Begutachtungsverfahren. Die Verfahrensdominanz von Umweltressort und Projektgruppe wird mit dem bestehenden Handlungsbedarf legitimiert. Letztlich können Auftraggeber und Projektgruppe damit das Forschungsprogramm sowie den beabsichtigten alpinen Forschungsschwerpunkt rasch realisieren. Das politische Ressort kann damit Aktion zeigen und Handlungsdruck abbauen, die Projektgruppe Handlungsfähigkeit und Aufgabenerfüllung nachweisen.

Diese Vorgehensweise entspricht im wesentlichen dem Modellansatz einer top down-Steuerung der Forschung von oben. Der politische Auftraggeber bestimmt maßgeblich nicht nur den Programmrahmen, sondern auch die anschließende Implementation des Programms. Die Bindungswirkung der Festlegungen in finanzieller wie inhaltlicher Hinsicht bestimmt zudem das Projekt weit über diese Anfangsphase hinaus. Das thematisch breite Rahmenprogramm zeigt jedoch, daß der strenge, politisch dominierte Steuerungsanspruch bereits einen Kompromiß darstellt. Als Kriterium zur Einpassung der Forschungsbeiträge ist es kaum als Selektionskriterium zur inhaltlichen Steuerung der Forschung geeignet. Die Forscher können sich mit ihren Interessen leicht an den Programmrahmen anpassen und von der Projektförderung profitieren. Die Bewertung der Forschungsqualität durch überwiegend grundlagenforschungsorientierte Gutachter trägt dazu bei, diesen wissenschaftsfreundlichen Forschungstyp entgegen dem angewandt-problemlösungsorientierten Typ der Auftrags- oder Programmforschung, welcher im Grundsatz politisch beabsichtigt ist, zu stärken. 
Die anfängliche, politische Verfahrensdominanz trotz des formalen Beteiligungsanspruchs der Wissenschaft am Förderprozeß führt zu einer ernsthaften und anhaltenden Vertrauenskrise im PBWU-Projekt. Hinzu kommen die geringe Transparenz des Förderverfahrens durch die Projektgruppe und die förderseitige Bevorzugung von Forschungsanträgen für den alpinen Forschungsschwerpunkt, dessen Etablierung ohnehin von erheblicher wissenschaftlicher Kritik begleitet ist. Die Krise kann letztlich nur durch die Neuordnung des PBWU-Projektes auf Veranlassung der GSF und des Wissenschaftlichen Beirats beseitigt werden. Zentrale Maßnahmen sind die mit der Neubesetzung des PBWU-Leiters verbundene personelle Verkleinerung der Projektgruppe und die Reduzierung ihrer Aufgaben zugunsten des Beirats.

Damit geht die Steuerungsdominanz im Förderverfahren an die Wissenschaft über. Der Beirat nimmt eine Vorauswahl der Forschungsanträge vor und spricht auf der Grundlage externer Gutachtervoten Förderempfehlungen gegenüber dem Projektrat bzw. finanzierenden Umweltressort aus. Die Förderempfehlungen werden nahezu vollständig befolgt. Durch ein auf Kooperation ausgerichtetes Verhalten und gestützt durch ihren Auftraggeber Umweltministerium kann die Projektgruppe jedoch erreichen, die Akzeptanz wieder herzustellen. Sie konzentriert ihre Tätigkeit auf überwiegend verfahrensorganisatorische und projektbetreuende Funktionen. Das Verfahren zur Forschungsförderung kann konsolidiert und transparent gestaltet werden. Die Projektgruppe erreicht dadurch letztlich, wenn auch mit geringerem Steuerungsanspruch, wieder in die fachlichen Beratungen im Förderverfahren und die konzeptionelle Neuorientierung der Forschung im Projekt miteinbezogen zu werden.

Angesichts vorgerückter Projektlaufzeit und veränderter gesellschaftlicher Problembewertung gerät die Neukonzeptionierung der Waldschadensforschung jedoch in ein inzwischen ungünstigeres politisches Umfeld. Es wurden erhebliche Fördermittel verausgabt; der mit und in dem PBWU-Projekt erzielte Stand der Forschung reicht der politischen Spitze des Umweltressorts als Ergebnis aus. Weiterer ressortspezifischer Forschungsbedarf wird nicht gesehen, auch wenn - oder gerade weil - die Wissenschaft im Detail sowohl über die Problemschwere der Waldschäden und über deren Ursachen nach wie vor uneinig ist. Anfänglich gegebener politischer Handlungsdruck ist kaum mehr erkennbar.

Nicht einmal ein neues, gegenüber dem ersten Rahmenprogramm der PBWU wissenschaftlich vergleichsweise hochqualifiziertes Forschungskonzept des Beirats kann sich bei nunmehr hohem politischem Legitimationsbedarf durchsetzen und zur Fortsetzung des PBWU-Programms führen. Finanzmittelknappheiten im Staatshaushalt sowie die aufgrund des wissenschaftlichen Kenntnisstandes kaum mehr zu erkennende Zuständigkeit des Umweltressorts erleichtern die Terminierung des PBWU-Projektes und damit auch der Projektgruppe im Jahr 1993. Eine adäquate Evaluierung des Projektes bzw. des durchgeführten Forschungsprogramms als Terminierungsbegründung unterbleibt.

Aus der empirie- wie theoriegestützten Darstellung und Analyse des PBWU-Projektes können relevante Faktoren abgeleitet werden, welche die Steuerung des Projektes bzw. des Forschungsprozesses maßgeblich prägen. Dabei handelt es sich insbesondere um 
- den politischen Handlungsdruck als wichtigen, fachliche Aspekte überlagernden Legitimationsfaktor für Forschung,

- die Gegenläufigkeit von politischem Handlungsdruck bzw. Fördermittelbereitstellung und wissenschaftlicher Programm- bzw. Forschungsqualität im Zeitverlauf (,Effizienzparadoxon"),

- die Vermengung politischer und wissenschaftlich-fachlicher Beiträge der verschiedenen Akteure, welche verhindert, daß das fachliche Potential maximal ausgeschöpft und die Verantwortlichkeit für politische Entscheidungen eindeutig sichtbar wird,

- das Territorialverhalten der politischen Ressorts und wissenschaftlichen Klientela, welches die Sicherstellung ressortspezifischer Interessen und Einflußnahme im Projekt erlaubt, ohne sich selbst der ressortübergreifenden Forschungskoordination unterwerfen zu müssen,

- die Dominanz der Politik gegenüber wissenschaftlichen Steuerungsinteressen zur Etablierung, aber auch Terminierung einer Forschungspolicy nach dem Modell eines top downSteuerungsansatzes,

- die Steuerungsdominanz der Wissenschaft im Verlauf der Programmimplementation, welche die politisch beabsichtigte Programmforschung in Richtung des forscherseitig bevorzugten Steuerung nach dem bottom up-Modell verändert,

- die externe Fachbegutachtung zur Sicherung der Forschungsqualität, welche im Grundsatz grundlagenorientierte Forschungsansätze begünstigt und die wissenschaftliche Dominanz im Förderverfahren verstärkt,

- die Bindungswirkung erster Förderentscheidungen, die mitsamt dem hohen Anteil an Fortsetzungsvorhaben in der Folge zu begrenzten Zutrittsmöglichkeiten für weitere Forscher, inhaltlicher Programmstagnation führt und wenig Raum für Neuortientierung bietet,

- den Versuch der Koordinierungsstelle, die Wissenschaft einer strengen Programmsteuerung zu unterwerfen, welche unter den gegebenen Rahmenbedingungen und dem Selbstverständnis der Wissenschaft langfristig jedoch nicht durchgesetzt werden kann,

- den Anspruch an fachlich kompetente, integrative Koordination interdisziplinärer Verbundforschung, dem die Koordinationsstelle aus Verfahrens-, Kompetenz- und Akzeptanzgründen jedoch nicht gerecht werden kann,

- die unterbleibende, adäquate Erfolgsbeurteilung des PBWU-Projektes; dies erleichtert politisch zwar die Terminierung des Projektes, bringt es jedoch um eine wissenschaftliche wie politische Legitimation und stellt eine Effizienzeinbuße dar.

Das Wissen um die Steuerungsfaktoren im Forschungsprozeß kann letztlich für praxistaugliche Verbesserungsvorschläge zur Durchführung und Koordination staatlich finanzierter, problemorientierter Programmforschung genutzt werden. Die Empfehlungen beziehen sich im Detail auf

- die Überführung des politischen Handlungsdruckes in angemessene Forschungsstrukturen und deren Finanzierung,

- die Integration eigenständiger Forschungsfinanziers in gemeinsame Programmstrukturen,

- die Interessentrennung zwischen Politik und Wissenschaft bei der Programmplanung, 
- verschiedene Verfahren für Programmerstellung und Akquisition von Forschungsbeiträgen,

- die Kompetenz der Koordinierungsstelle zur Programmkoordination,

- den Ausgleich zwischen Programmvorgaben und der Flexibilität der Forschung,

- die Verbesserung des wissenschaftlichen Begutachtungsverfahrens sowie die

- die Kontrolle und Erfolgsbeurteilung einzelner Forschungsbeiträge bzw. des Gesamtprogramms.

Das PBWU-Projekt zur bayerischen Waldschadensforschung stellt ein Fallbeispiel staatlich finanzierter Umweltforschung dar. Die theoretischen Grundlagen der sozialwissenschaftlichen Analyse erlauben es jedoch, die Ergebnisse und die daraus resultierenden Empfehlungen auch auf andere interdisziplinäre Forschungsprogramme bzw. Förderstrukturen zu übertragen. 


\section{Zusammenfassung}

Die vorliegende Studie analysiert den Prozeß staatlicher Forschungsförderung am Beispiel der bayerischen Waldschadensforschung. Sie geht der Fragestellung nach, welchen Einfluß die Forscher einerseits und die politischen Auftraggeber andererseits auf die Formulierung und Durchführung eines Forschungsprogrammes nehmen. Die Erkenntnisse über die Praxis der Forschungspolitik werden abschließend zur Entwicklung von Gestaltungsvorschlägen für die Auftragsforschung genutzt.

Als theoretische Grundlage zur Erfassung des Forschungsvorhabens Waldschadensforschung dient ein politikwissenschaftlicher Ansatz. Mit Hilfe der Politikfeld-Analyse wird ein Forschungsprogramm von der Entstehung bis zur Beendigung logisch und chronologisch in die einzelnen Phasen des Policy-Zyklus zerlegt. Unter Einbeziehung steuerungstheoretischer Grundlagen können die einzelnen Akteure aus Politik und Wissenschaft mit ihren jeweiligen Interessenlagen, Machtpotentialen und Handlungsstrategien beschrieben und ihre Handlungen erklärt werden.

Das bayerische Programm zur Waldschadensforschung mit einer eigens eingerichteten Koordinierungsstelle „Projektgruppe Bayern zur Erforschung der Wirkung von Umweltschadstoffen“ (PBWU) wird als Fallbeispiel gewählt. Mit Hilfe der teilnehmenden Beobachtung, Dokumentenanalyse und quantitativen Inhaltsanalyse kann der Förderprozeß des 1984 bis 1993 vom Bayerischen Umweltministerium federführend durchgeführten und von einem wissenschaftlichen sowie politischen Beratungsgremium begleiteten Großforschungsvorhabens erhoben werden.

Als Ergebnis der empirischen Darstellung und politikwissenschaftlichen Analyse werden die Faktoren aufgezeigt, welche die Entwicklung und Steuerung des Forschungsprojektes maßgeblich beeinflussen. Politischer Handlungsdruck stellt die treibende Kraft zur Durchsetzung des Forschungsprogramms dar und bestimmt weitgehend unabhängig von der wissenschaftlichen Qualität der Forschung die Etablierung und Beendigung des Programms. Während der Programmimplementierung dominiert die Wissenschaft, unterstützt durch das Instrument externe Fachbegutachtung, die Programmsteuerung. Ein offener Programmrahmen, Akzeptanzdefizite der Koordinierungsstelle, territoriales Verhalten der politischen Ressorts und der Selbststeuerungsanspruch der Wissenschaft erschweren die Projektkoordination des interdisziplinären Forschungsprogramms und die Einhaltung der politischen Ziele.

Die Empfehlungen zur Verbesserung anwendungsorientierter, interdisziplinärer Forschungsförderung betreffen die Schaffung geeigneter Forschungsstrukturen und effizienter Forschung unter Ausnutzung politischen Handlungsdrucks. Unterschiedliche Strategievorschläge der Beteiligung von Politik und Wissenschaft an der Forschungsförderung sollen dazu dienen, die jeweiligen Akteursinteressen zu entflechten, die Verantwortlichkeiten eindeutig zu regeln und die spezifischen Beiträge von Politik und Wissenschaft möglichst optimal für Programmplanung, durchführung, -koordination und -evaluation einzusetzen. 


\section{Summary}

\section{Managing Science between political interests and scientific standards}

\section{A case study of the Bavarian Research in Forest Decline}

The subject of forest decline is used as an example for analysing the process of state sponsored scientific research. The intervention of both, the scientists and political clients, in the formulation and implementation process of research programs, is investigated. The results of the research practice and research politics will be used for recommendations on improving customer oriented, applied research structures.

Using the theoretical approach of the "policy analysis" model the political resp. the research process can be separated chronologically into different cyclic phases from its initiation up to its termination. By including aspects of control, power, and action strategies the reaction of involved political and research actors can be described and analysed in detail.

The empirical and analytical work is carried out focusing on the Bavarian scientific research project and its administration and co-ordination body „Projektgruppe Bayern zur Erforschung der Wirkung von Umweltschadstoffen" as a case study. The project, financed by the Bavarian State Ministry for Regional Development and Environmental Affairs (Bayerisches Umweltministerium), was established from 1984 until 1993 to strengthen and to co-ordinate the research in the field of forest decline.

The project group was accompanied by a scientific and a political advisory board. Utilising the methodological approach of attending observation, document and content analysis the political program and the process of scientific research can be evaluated.

The crucial political factors that play a decisive role in the development and management of the research program become evident: Political pressure is the driving force for the implementation of the program and determines its initiation as well as its termination, mostly irrespective of the quality of the program. In contrast the implementation of the program is dominantly controlled by the scientific community, supported by the involved scientific review committee. The achievement of project co-ordination and political targets is stressed by a wide frame of scientific objectives, co-ordination deficiencies in the intermediate administration body, territorial behaviour of the different administration actors, and the high demand of the research bodies on scientific self control.

Different strategies for political actors and scientists to participate in state sponsored research programs are recommended. In order to improve applied, interdisciplinary research they focus on suitable research structures and research efficiency using the political pressure. Within that frame, clearly corresponding responsibilities should be adjusted, the different - political and 
scientific - interests decartelized, and brought into an optimal context of program planing, implementation, co-ordination, and evaluation. 


\section{Literatur}

AlemanN, U. von (Hrsg.) (1995): Politikwissenschaftliche Methoden. Grundriß für Studium und Forschung. Westdeutscher Verlag, Opladen $408 \mathrm{~S}$.

Ammer, U., Koch, B., Burgis, M. \& MARTin, K. (1987): Ergebnisse der Waldschadensinventur 1986 anhand von Farbinfrarotluftbildern für das Untersuchungsgebiet „Wank“. Projektbericht des Lehrstuhls für Landschaftstechnik der Univ. München. April 1987, 15 $\mathrm{S}$.

ATteslander, P. (1993): Methoden der empirischen Sozialforschung. de Gruyter, Berlin, New York; Sammlung Göschen; 2100; 407 S.

BAumgartner, P. (1991): Die Entwicklung der Waldschadensproblematik in den Medien während der letzten fünf Jahre. Schweizerische Zeitschrift für Forstwesen, 142 (1991), S. $1-17$

BAYERISCHE STAATSFORSTVERWALTUNG (1981): Gefährdung der Nadelwälder in Bayern. Information 2/81, Juli 1981, S. 1-4

BAYERISCHE StAATSFORSTVERWALTUNG (1982a): Staatsminister Dr. Eisenmann zum Thema „Ursachen und Ausmaß des Baumsterbens und Gegenmaßnahmen“. Information 1/82, April 1982, S. 1-3

BAYERISCHE STAATSFORSTVERWALTUNG (1982b): Sonderheft: Baumsterben in Bayern. Information 3/82, April 1982, $34 \mathrm{~S}$.

BAYERISCHE STAATSFORSTVERWALTUNG (1983a): Sonderheft: Waldsterben. Information 1/83, Januar 1983, 48 S.

BAYERISCHE STAATSFORSTVERWALTUNG (1983b): Sonderheft: Waldsterben - Waldschadensinventur 1983 -. Information 4/83, Januar 1983, 48 S.

BAYERISCHER LANDTAG (1991): Protokoll der gemeinschaftlichen informatorischen Sitzung der Ausschüsse für Landesentwicklung und Umweltfragen und Ernährung und Landwirtschaft zum Thema „Bilanz der Waldschäden und Waldschadensforschung von 1983 bis 1991“, Anhörung vom 5. Dezember 1991, 56 S.

BAYERISCHES STAATSMINISTERIUM FÜR ERNÄHRUNG, LANDWIRTSCHAFT UND FORSTEN (Hrsg.) (1995): Dokumentation Waldschäden. Stand der Forschung und Ausblick. Bericht über das Symposium am 3. Mai 1995 in München, 90 S.

BAYERISCHES STAATSMINISTERIUM FÜR LANDESENTWICKLUNG UND UMWELTFRAGEN UND FÜR ERNÄHRUNG, LANDWIRTSCHAFT UND FORSTEN (1982): Dokumentation zum Baumsterben in Bayern. $23 \mathrm{~S}$.

BRAUN, D. (1997): Die politische Steuerung der Wissenschaft. Ein Beitrag zum „kooperativen Staat“". Schriften des Max-Planck-Instituts für Gesellschaftsforschung Köln, Band 28. Campus-Verlag Frankfurt/New York, $450 \mathrm{~S}$.

BRAUN, G. (1982): Die Immissionsgefährdung bayerischer Wälder. Allgemeine Forst Zeitschrift 37. Jg., Heft 15, S. 446-447 
BUNDESMINISTERIUM FÜR BILDUNG, WisSENSCHAFT, FORSCHUNG UND TECHNOLOGIE (BMBF) (Hrsg.) (1997): Forschung für die Umwelt. Programm der Bundesregierung, $172 \mathrm{~S}$.

BUNDESMINISTERIUM FÜR ERNÄHRUNG, LANDWIRTSCHAFT UND FORSTEN (1995 und 1996): Waldzustandsbericht der Bundesregierung 1995 (bzw. 1996) - Ergebnisse der Waldschadenserhebung. 104 bzw. $100 \mathrm{~S}$.

BUNDESMINISTERIUM FÜR WISSENSCHAFT UND VERKEHR (Hrsg.) (1998): Umweltwissenschaft im öffentlichen Auftrag. Vom Konzept zur Forschungspraxis. Wien, 58 S.

DER BundeSMINISTER FÜR FORSCHUNG UND TECHNOLOGIE (Hrsg.) (1984): Umweltforschung zu Waldschäden, Erste Bilanz der Forschungsförderung, $68 \mathrm{~S}$.

DER BUNDESMINISTER FÜR FORSCHUNG UND TECHNOLOGIE (Hrsg.) (1985): Umweltforschung zu Waldschäden, 3. Bericht, März 1995, 134 S.

DER RAT VON SACHVERSTÄNDIGEN FÜR UMWELTFRAGEN (1983): Waldschäden und Luftverunreinigungen. Sondergutachten März 1983. 380 S.

DER SPIEGEL (1981): Säureregen: „Da liegt was in der Luft“ I-III. Serie 47/48/49, Nov. 1981

DEUTSCHE ForsChUNGSGEMEINSCHAFT (1992): Richtlinien für die Fachgutachter der Deutschen Forschungsgemeinschaft. DFG-Vordruck 1.21 - 8/92 - II 29, Bonn, 11 S.

DEUTSCHE FORSCHUNGSGEMEINSCHAFT (1996): Forschungsfreiheit. Ein Plädoyer für bessere Rahmenbedingungen der Forschung in Deutschland. VHC-Verlagsgesellschaft mbH. Weinheim, $156 \mathrm{~S}$.

DIE ZEIT (1981): Oh Tannenbaum! Wo sind deine Blätter? Beitrag von G. Haff im ZEITMagazin Nr. 53, 25.12.1981, S. 4-10

Downs, A. (1967): Inside Bureaucracy. Little, Brown and Company Boston. A Rand Corporation Research Study, 292 p

DRESSEL, K. (1995): Auf der Suche nach reflexivem Wissen - Wissensformen in 15 Jahren Waldschadensdiskurs. Diplomarbeit an der Sozialwissenschaftlichen Fakultät der LMU München, 165 S.

EAston, D. (1965): A Systems Analysis of Political Live. University of Chicago. J. Wiley \& Sons, Inc, New York, London, Sydney, $491 \mathrm{~S}$.

EISENMANN, H. (1983): Waldschäden in Bayern (Stand Ende September 1983). Rede von Staatsminister Dr. Eisenmann vor dem Wirtschaftsausschuß des Bayerischen Senats am 27.10.1983, $12 \mathrm{~S}$.

Ell, R. \& Luhmann, H.-J. (1996): Von den Schwierigkeiten der Entdeckung des Waldsterbens in Deutschland. Man sieht nur, was man versteht oder: Schäden ohne Ursache und Ursachen ohne Schäden. Forstarchiv 67, S. 103-107

ELLENBERG, H. (1994): Allgemeines Waldsterben - Ein Konstrukt?. Bedenken eines Ökologen gegen Methoden der Schadenserfassung. Naturwissenschaftliche Rundschau 48. Jhrg., 3/1995 S. 93-96

ElLENBERG, H. (1996): Vegetation Mitteleuropas mit den Alpen in ökologischer, dynamischer und historischer Sicht. 5. Aufl., UTB Ulmer-Verlag Stuttgart, 1096 S. 
Evered, D. \& Harnatt, S. (Eds) (1989): The Evaluation of Scientific Research. Ciba Foundation Conference on the Evaluation of Scientific Research, London 6-8 June 1988. A Wiley-Interscience Publication. J. Wiley and Sons, Chichester, UK, $276 \mathrm{p}$

FARTHMANN, F. (1983): Luftverunreinigungen und Waldsterben. Ansprache des nordrheinwestfälischen Arbeits- und Sozialministers am 21.12.1982 auf einer Pressekonferenz zum Thema. Der Forst- und Holzwirt, 38. Jg., Heft 3, 1983, S. 49-52

Felt, U., Nowotny, H. \& TAschwer, K. (1995): Wissenschaftsforschung. Eine Einführung. Campus Verlag, Bd. 1086, Frankfurt/New York. 322 S.

Flick, U., Kardorf, E. VOn, KeupP, H., Rosenstiel, L. VON \& WolfF, S. (Hrsg.) (1995): Handbuch Quantitative Sozialforschung. Grundlagen, Konzepte, Methoden und Anwendungen. Psychologie Verlags Union, Weinheim, 517 S.

FORSCHUNGSBEIRAT WALDSCHÄDEN/LUFTVERUNREINIGUNGEN DER BUNDESREGIERUNG UND DER LÄNDER - FBW - (1984): Zwischenbericht Dezember (Vorabdruck) 1984, 123 S.

FORSCHUNGSBEIRAT WALDSCHÄDEN/LUFTVERUNREINIGUNGEN DER BUNDESREGIERUNG UND DER LÄNDER (1986): 2. Bericht Mai 1986. Herausgeber: Kernforschungszentrum Karlsruhe, $257 \mathrm{~S}$.

FORSCHUNGSBEIRAT WALDSCHÄDEN/LUFTVERUNREINIGUNGEN DER BUNDESREGIERUNG UND DER LÄNDER (1989a): Dritter Bericht. Herausgeber: Kernforschungszentrum Karlsruhe., $611 \mathrm{~S}$.

FORSCHUNGSBEIRAT WALDSCHÄDEN/LUFTVERUNREINIGUNGEN DER BUNDESREGIERUNG UND DER LÄNDER (1989b): „Internationaler Kongreß Waldschadensforschung: Wissensstand und Perspektiven“ Ulrich, B. (Hrsg.), 2.-6.10.1989, Friedrichshafen, 2 Bände, 988 p

FRIEDRICHS, J. (1980): Methoden der empirishen Sozialforschung. Westdeutscher Verlag, Opladen, $430 \mathrm{~S}$.

GöTtLING, H. (1981): Die Neuorganisation der Bayerischen Forstlichen Versuchs- und Forschungsanstalt. Allgemeine Forst Zeitschrift 33, 36. Jg., S. 831-832

Hellstern, G.M. \& WollmanN H. (Hrsg.) (1984): Handbuch zur Evaluierungsforschung. Westdeutscher Verlag, Opladen, 134 S.

HÉRETIER, A. (Hrsg.) (1993a): Policy-Analyse. Kritik und Neuorientierung. Politische Vierteljahresschrift, Sonderheft 24/1993, Westdeutscher Verlag, Opladen, 485 S.

HÉRETIER, A. (1993b): Policy-Analyse. Elemente der Kritik und Perspektiven der Neuorientierung (Einleitung). In: A. Héretier (Hrsg.): Policy-Analyse. Kritik und Neuorientierung. Politische Vierteljahresschrift, Sonderheft 24/1993, Westdeutscher Verlag, Opladen, S. 9-36

HILL, W. \& RIESER, I. (1983): Die Förderungspolitik des Nationalfonds im Kontext der schweizerischen Forschungspolitik. Schriftenreihe der Betriebswirtschaftlichen Institute an der Univ. Basel, Band 11, 415 S.

HoHn, H.-W. \& SchimanK, U. (1990): Konflikte und Gleichgewichte im Forschungssystem. Akteurskonstellationen und Entwicklungspfade in der staatlich finanzierten außeruniversitären Forschung. Schriften des Max-Planck-Instituts für Gesellschaftsforschung Köln, Band 7. Campus Verlag, 444 S. 
HoLl, E.-A. (1993): Entwicklung und Situation der Forstlichen Forschung München von 1971 bis 1991. Diplomarbeit der Forstwissenschaftlichen Fakultät der Univ. München, Freising, März 1993, 96 S.

Holzberger, R. (1995): Das sogenannte Waldsterben. Zur Karriere eines Klischees: Das Thema Wald im journalistischen Diskurs. Schriftenreihe der Medien-Akademie Weingarten, Band 1. $336 \mathrm{~S}$.

HuCKe, J. \& Wollmann, H. (1980): Methodenprobleme der Implementationsforschung. In: R. Mayntz (Hrsg.): Implementation politischer Programme: empirische Forschungsberichte. Neue wissenschaftliche Bibliothek 97, Soziologie, Verlagsgruppe Athenäum, Hain, Scriptor, Hanstein, Königstein/Ts., S. 216 - 235

INTERMINISTERIELLE ARBEITSGRUPPE WALDSCHÄDEN/LUFTVERUNREINIGUNGEN - IMA(1983): Übersicht Forschungsvorhaben, Stand 19.5.1983. Quelle: UFORDAT - Datenbank für Umweltforschung sowie Sondererfassung Umweltbundesamt, UMPLIS, 47 S.

INTERMINISTERIELLE ARBEITSGRUPPE WALDSCHÄDEN/LUFTVERUNREINIGUNGEN - IMA (Hrsg.) (1993): Tätigkeitsbericht 1991 - 1993; überarb. Neuauflage 30.8.1994, 37 S.

JaCHTENFuChS. M., Hey, C. \& STRÜBEl, M. (1993): Umweltpolitik in der Europäischen Gemeinschaft. In: v. Prittwitz, V. (1993b): v. Prittwitz, V. (Hrsg.): Umweltpolitik als Modernisierungsprozess. Politikwissenschaftliche Umweltforschung und -lehre in der Bundesrepublik Deutschland. Verlag Leske u. Budrich, Opladen, S. 137-162

JAKOBS, J. (1994): Praxisversuch Energieproduktion und -verwertung in Baden-Württemberg (Teil 1 und 2). Beiträge für Forstwirtschaft und Landschaftsökologie 28 (1994) 3 und 4, S. 176-183 bzw. S. 119-125

JASANOFF, S. (1990): The Fifth Branch. Science Advisers as Policymakers. Harvard University Press, Cambridge, Mass., London, England, 302 p

KANDLER, O. (1985): „Waldsterben“: Immissions- versus Epidemie-Hypothesen. Waldsterben 1985. Kolloquium an der Universität Hohenheim, 24./25. 1.1985 Herausgeber: Kortzfleisch, Oldenburg Verlag, $27 \mathrm{~S}$.

KANDLER, O. (1994): Vierzehn Jahre Waldschadensdiskussion. Scenarien und Fakten. Naturwissenschaftliche Rundschau 47. Jahrgang, Heft 11/1994, S. 419-430

KAUFMANN, F.-X. (1987): Interdisziplinäre Wissenschaftspraxis. Erfahrungen und Kriterien. In: J. Kocka (Hrsg.): Interdisziplinarität: Praxis - Herausforderung - Ideologie. Suhrkamp, Frankfurt, S. 63-81

KNABE, W. (1981): Zu den Schadbildern des Fichtensterbens. Bericht eines Kolloquiums. Allgemeine Forst Zeitschrift, 36, S. 660-661

KROTT, M. (1987): Waldsterben zwischen Mythos und Medien. Österreichische Erfahrungen mit der Zeitungsberichterstattung über die neuartigen Waldschäden. Forstarchiv 58 (1987), S. 6-10

KROTT, M. (1989): Forstliche Raumordnungspolitik. Praxis und Zukunft des österreichischen Waldentwicklungsplans. Forstliche Schriftenreihe Univ. für Bodenkultur, Wien, Band 2, $203 \mathrm{~S}$.

KROTT, M. (1990): Öffentliche Verwaltung im Umweltschutz. Ergebnisse einer behördenorientierten Policy-Analyse am Beispiel Waldschutz. Studienreihe Konfliktforschung 5. Hrsg.: Pelinka, A. und Haerpfer, Chr., W. Braumüller Verlag Wien, 475 S. 
KrotT, M. (1993): Einführung in die Politik der natürlichen Ressourcen. Studienunterlagen 1993/1994 des Inst. für forstl. Betriebswirtschaft und Forstwirtschaftspolitik der Univ. für Bodenkultur, Wien, $132 \mathrm{~S}$.

KROTT, M. (1994a): Management vernetzter Umweltforschung. Wissenschaftspolitisches Lehrstück Waldsterben. Studien zu Politik und Verwaltung Bd. 49. Hrsg.: Brünner, Chr., Mantl, W. und Welan, M. Böhlau Verlag Wien, Köln, Graz, 325 S.

KROTT, M. (1994b): Arbeitsschwerpunkt „Kulturlandforschung“. Wissenschaftliche Beratung und Controlling. Interner Endbericht, Wien, Sept. 1994, 102 S., unveröffentlicht

KROTT, M. (1996): Forstpolitische Selbststeuerung als Herausforderung für Wissenschaft und Praxis. Forstwissenschaftl. Centralblatt 115, S. 97-107

KROTT, M. (1997): Evaluierung als Instrument der Forschungspolitik. Alternative Verfahren am Beispiel interdisziplinärer Projekte. SWS-Rundschau, Heft 3/1997, Wien, S. 269-283

KROTT, M. (1998): Erstellung des Forschungsprogrammes Kulturlandforschung 2. Internes Protokoll zur internen Beratung vom 31.3.1998, 4 S., unveröffentlicht

KRAPFEnBAUER, A. (1992): Waldschäden - Überforderung der „Fachwissenschaft“? - Kommentar und Zukunftsperspektiven. Holz-Zentralblatt Nr. 52/53, Stuttgart, S. 828-829

KURKI, M. (1991): Untersuchungen zur Förderung der Forstwirtschaft in Baden-Württemberg - Unter Berücksichtigung der Subventionstheorie und Evaluierungsforschung. Mitteilungen der Forstlichen Versuchs- und Forschungsanstalt Baden-Württemberg, Heft 155, Januar 1991, Freiburg, 275 S.

LANDMANN, G. (1989): Comments on the current state of knowledge and further needs of research into forest decline. In: B. Ulrich (Hrsg.): Vortragssammlung „Internationaler Kongreß Waldschadensforschung: Wissensstand und Perspektiven" des Forschungsbeirats Waldschäden/Luftverunreinigungen der Bundesregierung und der Länder. 2.6.10.1989, Friedrichshafen, S. 945-955

Landmann, G. (1995): Forest decline and air Pollution effects in the French mountains: A synthesis. In: Landmann G. \& Bonneau, M. (eds.): Forest decline and atmospheric deposition effects in the French mountains, Springer, Berlin, p 407-452

Langebartels, C., Ernst, D., Heller, W., Lütz, C., Payer, H.-D., \& Sandermann H. (1997): Ozone Responses of Trees: Results from Controlled Chamber Exposures at the GSF Phytotron. In: Sandermann et al. (eds.) (1997): Forest Decline and Ozone: A Comparison of Controlled Chamber and Field Experiments. Ecological Studies Vol 127, Springer-Verlag Berlin, Heidelberg, p 163-200

LEHRINGER, S. (1982): Die Schädigung des Waldökosystems erfordert sofortiges Handeln. Von der Landesversammlung des Bayerischen Landesverbandes des Bundes Deutscher Forstmänner. Allgemeine Forst Zeitschrift 37. Jg., Heft 49, S. 1486-1487

MARKL, H. (1987): Wissenschaft: Zur Rede gestellt. Über die Verantwortung der Forschung. Piper Verlag München, 1989, 185 S.

MAYNTZ, R. (1980): Die Implementation politischer Programme - Theoretische Überlegungen zu einem neuen Forschungsgebiet. In: R. Mayntz (Hrsg.): Implementation politischer Programme: empirische Forschungsberichte. Neue wissenschaftliche Bibliothek 97, Soziologie, Verlagsgruppe Athenäum, Hain, Scriptor, Hanstein, Königstein/Ts., S. 236 249 
MAYNTZ, R. (1983) (Hrsg.): Implementation politischer Programme II. Ansätze zur Theoriebildung. Westdeutscher Verlag, Opladen, 255 S.

MAYNTZ, R. (1985): Forschungsmanagement: Steuerungsversuche zwischen Scylla und Charybdis; Probleme der Organisation und Leitung von hochschulfreien, öffentlich finanzierten Forschungsinstituten. Westdeutscher Verlag, Opladen, 152 S.

MAYNTZ, R. (1985): Soziologie der öffentlichen Verwaltung. UTB-Taschenbücher 765. Juristischer Verlag, 3. Aufl., 265 S.

MoHR, H. (1981): Biologische Erkenntnis. Ihre Entstehung und Bedeutung. Teubner Taschenbücher der Biologie, Stuttgart. $222 \mathrm{~S}$.

MoHr, H. (1995): Qualitatives Wachstum. Losung für die Zukunft. Verlag Weitbrecht, Stuttgart, Wien, $240 \mathrm{~S}$.

MÖHRING, C. (1992): 10 Jahre Waldschadensforschung. Bilanz und Ausblick. Der Bundesminister für Forschung und Technologie, (Hrsg.), Bonn, Nov. 1992, 72 S.

MÖSSMER, R., TRÄNKNER, H. \& MAIER, G. (1990): Waldschadenserhebung 1989 am Schwerpunktstandort Kalkalpen. Projektbericht der Bayerischen Forstlichen Versuchs- und Forschungsanstalt München. Februar 1990, 38 S.

National Acidic Precipitation Assessment Program (NAPAP) (1991): The Experience and Legacy of NAPAP. Report of the Oversight Review Board, Washington D.C., $41 \mathrm{p}$

NETSCH, W. (1989): Entstehung und Umsetzung weiderechtlicher Bestimmungen des bayerischen Forstrechtegesetzes (FoRG) von 1958. Forstliche Forschungsberichte München Nr. 98, $436 \mathrm{~S}$.

Nowotny, H. (1998): Quo Vadis European Science and Technology Assembly (ESTA)? Interview der Koordinierungsstelle EG der Wissenschaftsorganisationen (kowi) mit $\mathrm{H}$. Nowotny. kowi-aktuell, No. 37, 27. Juli 1988, S. 2-4

PRITTwITZ, V. VON (1990): Das Katastrophenparadox. Elemente einer Theorie der Umweltpolitik. Verlag Leske u. Budrich, Opladen. $280 \mathrm{~S}$.

PROJEKTGRUPPE BAYERN ZUR ERFORSCHUNG DER WIRKUNG VON UMWELTSCHADSTOFFEN (Hrsg.) (1989): Proceedings zum „1. Statusseminar der PBWU zum Forschungsschwerpunkt Waldschäden“. 27.2.-1.3.1989, Neuherberg. GSF-Bericht 6/89. 522 S.

PROJEKTGRUPPE BAYERN ZUR ERFORSCHUNG DER WIRKUNG VON UMWELTSCHADSTOFFEN (Hrsg.) (1991): Proceedings zum ,2. Statusseminar der PBWU zum Forschungsschwerpunkt Waldschäden“. 4.-6.2.1991, Neuherberg. GSF-Bericht 26/91. 551 S.

PROJEKTGRUPPE BAYERN ZUR ERFORSCHUNG DER WIRKUNG VON UMWELTSCHADSTOFFEN (Hrsg.) (1992): Interne Vorhabenzusammenstellung zum Forschungsschwerpunkt „Waldschäden“ der PBWU, Aug. 1992, 88 S., unveröffentlicht

PROJEKTGRUPPE BAYERN ZUR ERFORSCHUNG DER WIRKUNG VON UMWELTSCHADSTOFFEN (Hrsg.) (1993a): Proceedings zum „1. Statusseminar der PBWU zum Forschungsschwerpunkt Luftverunreinigungen und menschliche Gesundheit“, 25.-26.2.1993, Neuherberg. GSF-Bericht 31/93. $245 \mathrm{~S}$.

PROJEKTGRUPPE BAYERN ZUR ERFORSCHUNG DER WIRKUNG VON UMWELTSCHADSTOFFEN (Hrsg.) (1993b): Proceedings zum internationalen Symposium „Stoffeinträge aus der 
Atmosphäre und Waldbodenbelastung in den Ländern von ARGE ALP/ALPENADRIA“, 27.-29.4.1993, Berchtesgaden. GSF-Bericht 39/93. 565 S.

REHFUESS, K. E. (1978): 100 Jahre Forstwissenschaft in München. In: Forschungsberichte der Forstlichen Forschungsanstalt München 42/1978, Symposium „100 Jahre Forstwissenschaft in München“, S. 1-8

REHFUESS, K. E. (1981): Über die Wirkungen der sauren Niederschläge in Waldökosystemen. Forstwissenschaftliches Centralblatt 100 (1981), S. 363-381

Rennenberg, H., Polle, A. \& Reuther, M. (1997): Role of Ozone in Forest Decline on Wank Mountain (Alps). In: Sandermann et al. (eds.) (1997) Forest Decline and Ozone: A Comparison of Controlled Chamber and Field Experiments. Ecological Studies Vol 127, Springer-Verlag Berlin, Heidelberg p 135-162

REUTHER, M. \& KiRCHNER, M. (1990): Waldschadensforschung in Bayern - Koordinierung durch die Projektgruppe Bayern zur Erforschung der Wirkung von Umweltschadstoffen (PBWU). Broschüre des Bayerischen Staatsministerium für Landesentwicklung und Umweltfragen (Hrsg.), Jan. 1990, München, 46 S.

REUTHER, M., KIRCHNER, M. \& RÖSEL, K. (1993): PBWU-Schlußbericht 1984-1993. Teilprojekt „Waldschäden“. Schlußbericht zum PBWU-Projekt an das Bayer. Staatsministerium für Landesentwicklung und Umweltfragen. Nov. 1993. 37 S.

Reuther M., Kirchner, M., RöSEl, K. \& Weissgerber, K. (1996): Forschung zum Schwerpunkt Luftverunreinigungen und Waldschäden 1984 - 1995. Ergebnisbericht. Hrsg.: Bayerisches Staatsministerium für Landesentwicklung und Umweltfragen, Febr. 1996, $52 \mathrm{~S}$.

RoQuePlo, P. (1986): Der saure Regen: ein „Unfall in Zeitlupe“. Ein Beitrag zu einer Soziologie des Risikos. Soziale Welt 37, 1986, Heft 4, S. 402-426

Rossi, P.H., Freeman, H.E. \& Hofmann, G. (1988): Programm-Evaluation - Einführung in die Methoden angewandter Sozialforschung. Enke Verlag Stuttgart, 229 S.

RuSS-MoHL, S. (1993): Konjunktur und Zyklizität in der Politik: Themenkarriere, Medienaufmerksamkeitszyklen und „lange Wellen“. In: A. Héretier (Hrsg.): Policy-Analyse. Kritik und Neuorientierung. Politische Vierteljahresschrift, Sonderheft 24/1993, Westdeutscher Verlag, Opladen, S. 356-368

Sandermann, H., Wellburn, A.L. \& Heath, R.L. (Eds.) (1997): Forest Decline and Ozone: A Comparison of Controlled Chamber and Field Experiments. Ecological Studies Vol 127, Springer-Verlag Berlin, Heidelberg, 400 pp

SCHLAEPFER, R. (1995): Ziele und Möglichkeiten der Waldschadensforschung. In: Bayerisches Staatsministerium für Ernährung, Landwirtschaft und Forsten (Hrsg.): Dokumentation Waldschäden. Stand der Forschung und Ausblick. Bericht über das Symposium am 3. Mai 1995 in München, S. 54-61

SCHLAEPFER, R. (ed) (1993): Long-term Implications of Climate Change and Air Pollution on Forest Ecosystems. Progress report of the IUFRO Task Force „Forest, Climate Change and Air Pollution“. IUFRO World Series Vol. 4, Vienna 1993

SchmidT-VoGT, H. (1981): Bemerkenswertes zur Baumart Fichte. Allgemeine Forst Zeitschrift 36. Jg., Heft 51/52/53, S. 1364-1373 
SCHNALKE, S. (1986): Das Waldsterben im Spiegel des Bayerischen Landtags und des Bayerischen Senats. Diplomarbeit der Forstwissenschaftl. Fakultät der Univ. München, Januar 1986, $66 \mathrm{~S}$.

SCHÖNBORN, A. VON \& WEBER, E. (1981): Untersuchungen über die Immissionsbelastung von Tannen- und Fichtennadeln im Bereich des Bayerischen Waldes. Forstwiss. Centralblatt 100 , S. $265-270$

SCHUBERT, K. (1991): Politikfeldanalyse. Eine Einführung. Leske \& Budrich, Opladen, 208 S.

SCHULZE, E.-D. (1989): Überlegungen für eine zukünftige Waldschadensforschung. In: Projektgruppe Bayern zur Erforschung der Wirkung von Umweltschadstoffen (PBWU) (Hrsg.): Proceedings ,1. Statusseminar der PBWU zum Forschungsschwerpunkt Waldschäden“, 27.2.-1.3.1989, Neuherberg, GSF-Bericht 6/89, S.497-506

Schulze, E.-D., LANGe, O.L., \& OREn, R. (Eds) (1989): Forest Decline and Air Pollution. A Study of Spruce (Picea abies) on Acid Soils. Ecological Studies 77, Springer Verlag, 475 $\mathrm{p}$

SchÜTT, P. (1977): Das Tannensterben. Der Stand unseres Wissens über eine aktuelle und gefährliche Komplexkrankheit der Weißtanne (Abies alba Mill.). Forstwissenschaftliches Centralblatt 96 (1977), S. 177-186

SchÜTT, P. (1978): Ungelöste Probleme der Forstpathologie - 100 Jahre nach Robert Hartig. In: Forschungsberichte der Forstlichen Forschungsanstalt München 42/1978, Symposium „100 Jahre Forstwissenschaft in München“, S. 177 - 1875

SchÜTт, P. (1981a): Ursache und Ablauf des Tannensterbens - Versuch einer Zwischenbilanz. Forstwissenschaftliches Centralblatt 100 (1981), S. 286-287

SchÜтT, P. (1981b): Folgt dem Tannensterben ein Fichtensterben? Holz-Zentralblatt Nr. 11, 26.1.1981, S. 159-160

SchÜTT, P. (1982): Aktuelle Schäden am Wald - Versuch einer Bestandsaufnahme. Holz-Zentralblatt Nr. 25, 26.2.1982, S. 369-372

SchÜTT, P. (1983): Die Forschung wird nicht koordiniert: Warten auf den Zufall. In: Bild der Wissenschaft, Heft 12/1983, S. 80 - 86

SEITSCHEK, O. (1982): Die gegenwärtige Waldschutzsituation in Bayern. Allgemeine Forst Zeitschrift, 15/1982, S. 423-428

SKELLY, J.M. \& INNES, J.L. (1994): Waldsterben in the Forests of Central Europe and Eastern North America: Fantasy or Reality. Plant Disease Vol 78 No 11, Nov 1994, pp 10211032

SPIEKER, H., MielikÄInen, K., KÖHL, M. AND SkovsgaARD, J.P. (Eds.) (1996): Growth Trends in European Forests. Studies from 12 Countries. European Forest Institute Research Report No. 5, Springer-Verlag Berlin, Heidelberg, $372 \mathrm{p}$

StaHL, P. (1981): Wie ist das Sterben der Nadelwälder zu stoppen? Holz-Zentralblatt, Stuttgart, Nr. 145, S. 2247

STARK, M. (Hrsg.) (1991) Luftschadstoffe und Wald. Lufthaushalt, Luftverschmutzung und Waldschäden in der Schweiz. Ergebnisse aus dem Nationalen Forschungsprogramm 14. Programmleitung NFP 14, Band 5. Verlag der Fachvereine Zürich, 298 S. 
STEUER, D. (1997): Management von Forschungsprojekten am Beispiel der PBWU-Waldschadensforschung von 1984 bis 1993“. Diplomarbeit an der Forstwissenschaftlichen Fakultät der Univ. München, 74 S.

SudA, M. (1984): Das Thema Waldsterben in den Massenmedien. Inhaltsanalyse der Süddeutschen Zeitung. Diplomarbeit der Forstwiss. Fakultät der Univ. München, März 1984, 70 S.

Ulrich, B., MAYER, R. \& KHANNA, P.K. (1979): Deposition von Luftverunreinigungen und ihre Auswirkungen in Waldökosystemen im Solling. Schriften aus der Forstlichen Fakultät der Universität Göttingen. Band 58. Sauerländer Verlag, Frankfurt a.M., 291 S.

UMWELTBUNDESAMT (Hrsg.) (1996): Auswertung der Waldschadensforschungsergebnisse (1982 - 1992) zur Aufklärung komplexer Ursache-Wirkungsbeziehungen mit Hilfe systemanalytischer Methoden. Abschlußbericht Fortbildungszentrum Gesundheits- und Umweltschutz, Berlin e.V. UBA-Forschungsbericht Nr. 6/97, Erich Schmidt Verlag, 635 $\mathrm{S}$.

VEREIN DEUTSCHER INGENIEURE, KOMMISSION REINHALTUNG DER LuFT (Hrsg.) (1983): Säurehaltige Niederschläge - Entstehung und Wirkungen auf terrestrische Ökosysteme. Düsseldorf, 1983, $277 \mathrm{~S}$.

Vogt, K., Gordon, J., Wargo, J., Vogt, D., Asbornsen, H., Palmiotto, P., Clark, H., O’Hara, J., Keaton, W., Patel-Weynand, T. \& Witten, E. (1996): Ecosystems Balancing Science with Management, Springer Verlag New York, $470 \mathrm{p}$

WACHTER, A. (1977): Zum Weißtannensterben. Erfahrungsaustausch zwischen baden-württembergischen und bayerischen Forstleuten. Allgemeine Forst Zeitschrift Nr. 39, S. 966

WAGNER, F. (1978): Tannensterben in Ostbayern. Allgemeine Forst Zeitschrift Nr. 35, S. 992994

Weber, M. (1980): Wirtschaft und Gesellschaft. Tübingen, Mohr, 5. Auflage, 945 S.

WindHOFF-HÉRITIER, A. (1987): Policy-Analyse: Eine Einführung. Campus Studium Bd. 570; Campus Verlag Frankfurt a.M., New York; 184 S.

WiSSENSCHAFTSRAT (Hrsg.) (1994): Stellungnahme zur Umweltforschung in Deutschland. Band I, Köln, 252 S.

ZANG, P. (1981): Zur geschichtlichen Entwicklung der Bayerischen Forstlichen Versuchs- und Forschungsanstalt. Allgemeine Forst Zeitschrift 33, 36. Jg., S. 829-831

ZIERHOFER, W. (1998): Umweltforschung und Öffentlichkeit. Das Waldsterben und die kommunikativen Leistungen von Wissenschaft und Massenmedien. Westdeutscher Verlag, Opladen, Wiesbaden, 266 S.

ZimmeRmanN, W. (1991): Zur politischen Karriere des Themas Waldsterben. Schweizerische Zeitschrift für Forstwesen, 142 (1991), S. 19-31 


\section{Lebenslauf}

\section{Persönliche Daten:}

Name:

Joachim Markus Reuther

Adresse:

An der Fähre 6, 85737 Ismaning bei München

geboren am:

7. Mai 1956 in Hof a.d. Saale (Bayern)

Familienstand: verheiratet, 2 Kinder

\section{Ausbildung, Bundeswehr:}

\section{Schule:}

Sept. 1962 - Juni 1975 Volksschule und Humanistisches Gymnasium Hof. Abschluß: Allgemeine Hochschulreife

\section{Wehrdienst:}

Juli 1975 - Sept. 1976 Bundeswehr-Grundwehrdienst in Cham/Oberpfalz

\section{Studium:}

Okt. 1976 - Mai 1981 Studium der Forstwissenschaft an der Ludwig-MaximiliansUniversität (LMU) München.

Abschluß: Dipl.-Forstwirt univ. (Diplomprüfung April 1981)

Juni 1981 - Nov. 1983 Referendariat in der Bayerischen Staatsforstverwaltung. Abschluß: Große Forstliche Staatsprüfung (Juni 1983)

Dez. 1983 - Mai 1984 Gasthörer zum Referendariat an der Oberforstdirektion München

\section{Berufliche Tätigkeit:}

Juli 1984 - Aug. 1988 wissenschaftlicher Mitarbeiter bzw.

Sept. 1988 - Juni 1993 Leiter der Koordinierungsstelle „Projektgruppe Bayern zur Erforschung der Wirkung von Umweltschadstoffen“" am GSF-Forschungszentrum für Umwelt und Gesundheit $\mathrm{GmbH}$, Neuherberg

Juli 1993 - Aug. 1996 Wissenschaftlicher Mitarbeiter (Projektkoordination) an der Bayerischen Landesanstalt für Wald und Forstwirtschaft, Freising, zum Forschungsprojekt „Waldzustandserfassung im Fichtel- und Erzgebirge mit Hilfe der Fernerkundung"

Juli 1993 - Dez. 1998 Wissenschaftliche Nebentätigkeit (Projektkoordination) am GSF-Forschungszentrum zu den EU-Projekten „Eichenschäden in den Donauländern“ und „Fallstudie Buche im Dreiländereck Böhmen/Oberösterreich/Bayern“"

Apr. 1994 - Feb. 1995 Studien zu Politikwissenschaft, Verwaltungslehre und Wissenschaftsforschung an der Universität für Bodenkultur bzw. Universität Wien

seit Sept. 1996

Wissenschaftlicher Mitarbeiter (Projektkoordination) am Lehrstuhl für Bodenkunde und Standortslehre der LMU München zum Forschungs- 
projekt „Monitoring von Schäden in Waldökosystemen des Bayerischen Alpenraumes“" 